Boyer Pascal

\title{
FAISCEAUX PERVERS DES CYCLES ÉVANESCENTS DES VARIÉTÉS DE DRINFELD ET GROUPES DE COHOMOLOGIES DU MODĖLE DE DELIGNE-CARAYOL
}


Boyer Pascal

E-mail : boyer@math.jussieu.fr

Institut de mathématiques de Jussieu, UMR 7586, université Paris 6, 175 rue du Chevaleret Paris 13.

Classification mathématique par sujets (2000). — 14G22, 14G35, 11G09, 11G35, 11R39, 14L05, 11G45, 11Fxx.

Mots clefs. — Variétés de Drinfeld, modules formels, correspondances de Langlands, correspondances de Jacquet-Langlands, faisceaux pervers, cycles évanescents, filtration de monodromie, conjecture de monodromie-poids, variété de Shimura. 
A mes parents, à Olivia et à Nathan 



\title{
FAISCEAUX PERVERS DES CYCLES ÉVANESCENTS DES VARIÉTÉS DE DRINFELD ET GROUPES DE COHOMOLOGIES DU MODĖLE DE DELIGNE-CARAYOL
}

\author{
Boyer Pascal
}

\begin{abstract}
Résumé. - Dans la première moitié du livre, on traduit, dans la situation géométrique des variétés de Drinfeld, les principaux résultats du livre d'Harris et Taylor. On explicite notamment la restriction aux strates ouvertes des faisceaux des cycles évanescents en fonction de certains systèmes locaux dits d'Harris-Taylor dont on calcule la somme alternée des groupes de cohomologie à supports compacts. Dans la deuxième moitié du livre, on décrit les gradués de la filtration de monodromie du faisceau pervers des cycles évanescents ainsi que la suite spectrale correspondante. D'après le théorème de comparaison de Berkovich-Fargues, on obtient alors une description de la filtration de monodromie-locale du modèle de Deligne-Carayol.
\end{abstract}

\footnotetext{
Abstract (Perverse sheaf of vanishing cycles of Drinfeld varieties and cohomology group of Deligne-Carayol model)

In the first half of the book, we translate in the geometric situation of Drinfeld varieties, the principal results of the Harris and Taylor's book. We give in particular the restriction to the open strata of the vanishing cycles sheaves in terms of some local systems named Harris-Taylor's local systems which we calculate the alternated sum of the cohomology group with compact supports. In the last half of the book, we describe the monodromy filtration of the vanishing cycles perverse sheaf and the spectral sequence associated to it. Thanks to the Berkovich-Fargues' theorem, we obtain the description of the local monodromy filtration of the Deligne-Carayol model.
} 



\section{TABLE DES MATIÈRES}

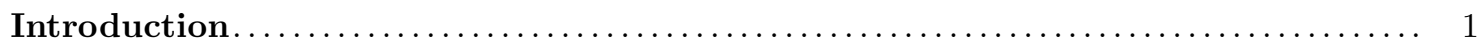

I. Variétés d'Igusa, systèmes locaux d'Harris-Taylor et cycles évanescents des

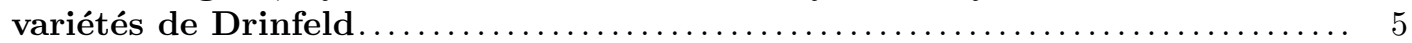

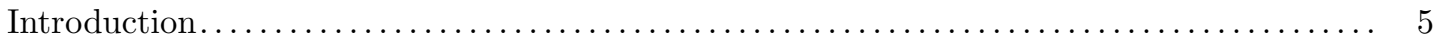

I.1. Modules formels et variétés de Drinfeld : rappels $\ldots \ldots \ldots \ldots \ldots \ldots \ldots \ldots \ldots \ldots \ldots \ldots \ldots$

I.1.1. Rappels sur les déformations des $\mathcal{O}_{o}$-modules formels $\ldots \ldots \ldots \ldots \ldots \ldots \ldots \ldots \ldots$

I.1.2. $\mathcal{D}$-faisceau elliptiques et structures de niveau $\ldots \ldots \ldots \ldots \ldots \ldots \ldots \ldots \ldots \ldots \ldots \ldots$

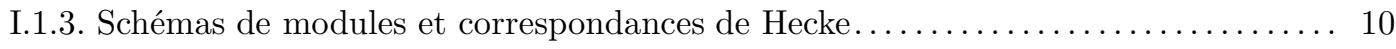

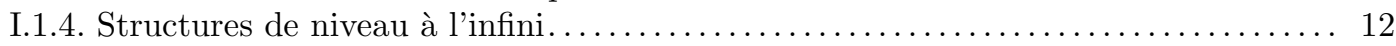

I.1.5. Stratification de la fibre spéciale $\ldots \ldots \ldots \ldots \ldots \ldots \ldots \ldots \ldots \ldots \ldots \ldots \ldots \ldots \ldots \ldots \ldots$

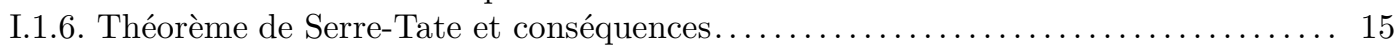

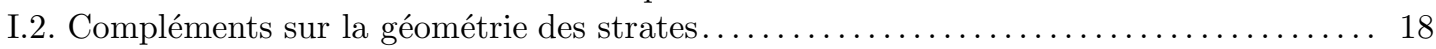

I.2.1. Scindage en partie étale et connexe des $\mathcal{O}_{o}$-modules de Dieudonné........... 18

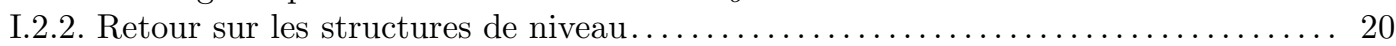

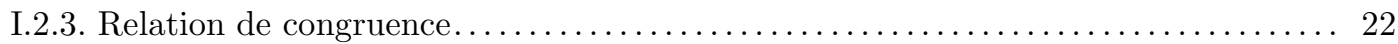

I.3. Variétés d'Igusa de première espèce $\ldots \ldots \ldots \ldots \ldots \ldots \ldots \ldots \ldots \ldots \ldots \ldots \ldots \ldots \ldots \ldots 24$

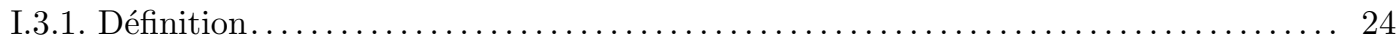

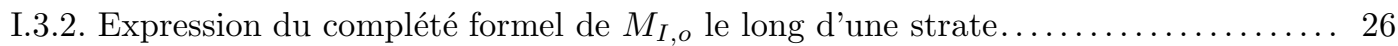

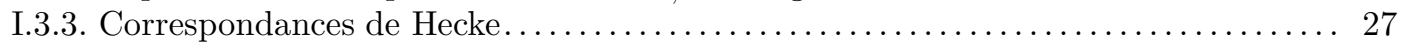

I.4. Variétés d'Igusa de seconde espèce $\ldots \ldots \ldots \ldots \ldots \ldots \ldots \ldots \ldots \ldots \ldots \ldots \ldots \ldots \ldots \ldots \ldots . \ldots \ldots$

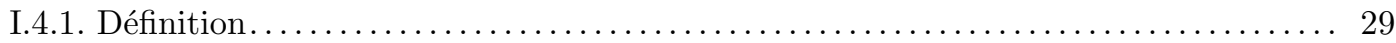

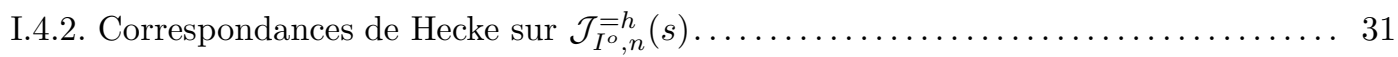

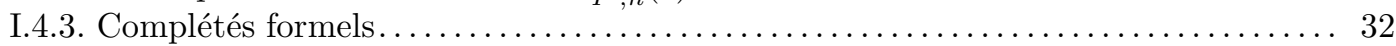

I.5. Cycles évanescents et systèmes locaux d'Harris-Taylor .................... 38

I.5.1. Rappels sur les cycles évanescents associés à $\operatorname{Def}_{n}^{h} \ldots \ldots \ldots \ldots \ldots \ldots \ldots \ldots \ldots \ldots . \ldots \ldots$

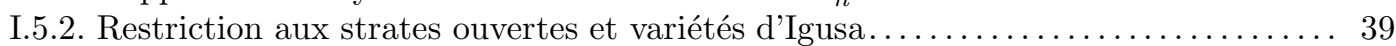

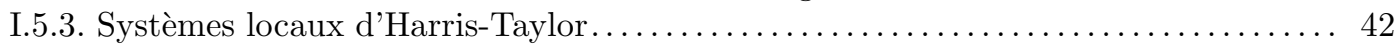

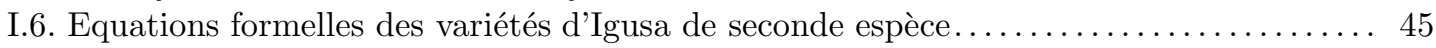

II. Groupes de cohomologie du modèle local : cas Iwahori................ 49

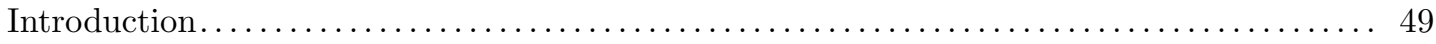

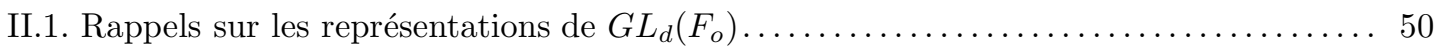

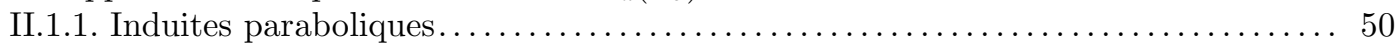

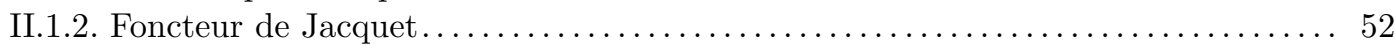

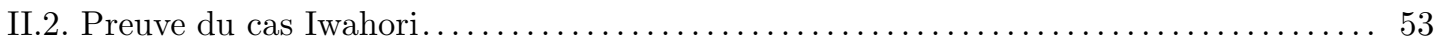

III. Cohomologie des faisceaux de Harris-Taylor $\ldots \ldots \ldots \ldots \ldots \ldots \ldots \ldots \ldots \ldots \ldots \ldots$

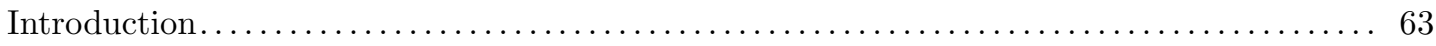




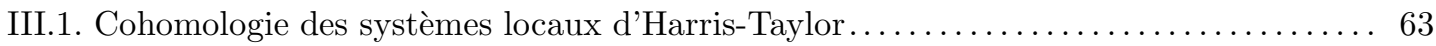

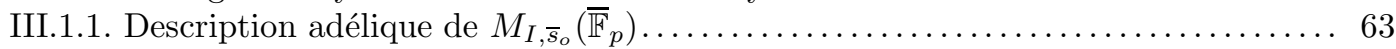

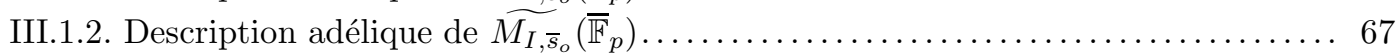

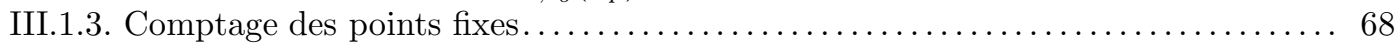

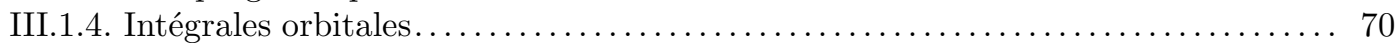

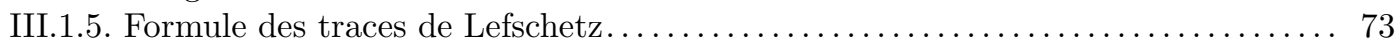

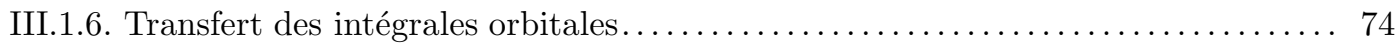

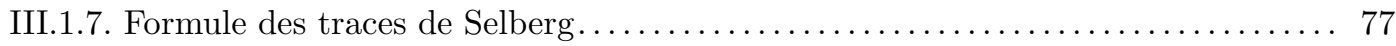

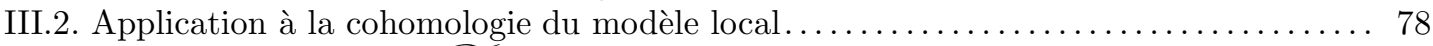

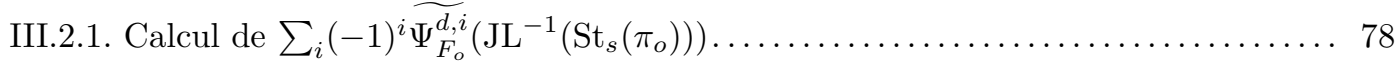

III.2.2. Retour sur le cas Iwahori................................. 80

IV. Filtration de monodromie des cycles évanescents $\ldots \ldots \ldots \ldots \ldots \ldots \ldots \ldots \ldots \ldots$

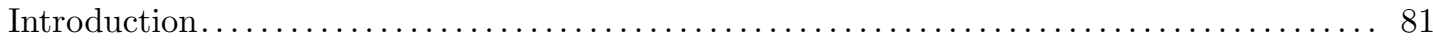

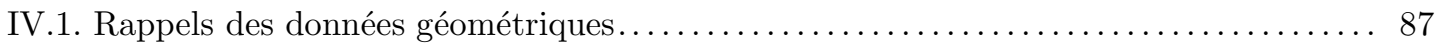

IV.1.1. Le modèle local de Deligne-Carayol. . $\ldots \ldots \ldots \ldots \ldots \ldots \ldots \ldots \ldots \ldots \ldots \ldots \ldots .88$

IV.1.2. Les variétés globales et les systèmes locaux d'Harris-Taylor . . . . . . . . . 88

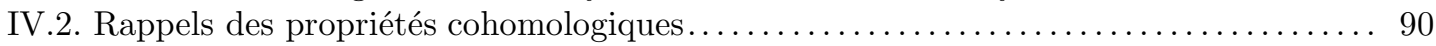

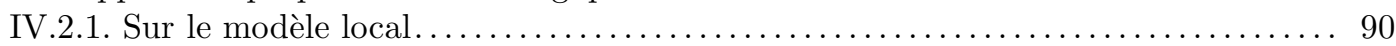

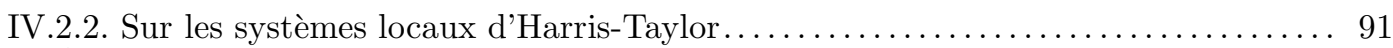

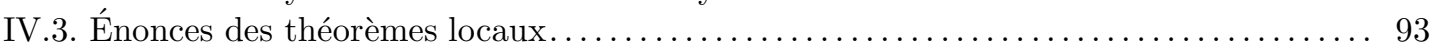

IV.3.1. Groupes de cohomologie des modèles de Deligne-Carayol. ............... 93

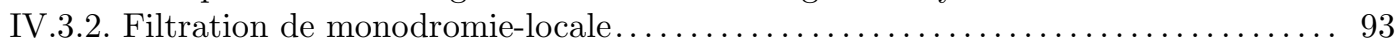

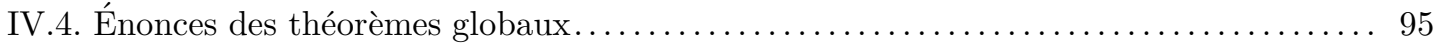

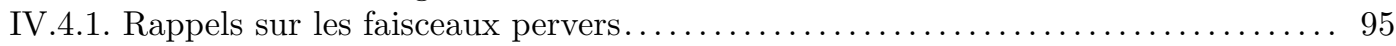

IV.4.2. Définition de la catégorie des faisceaux pervers de Hecke sur $X_{\mathcal{I}} \ldots \ldots \ldots \ldots 6$

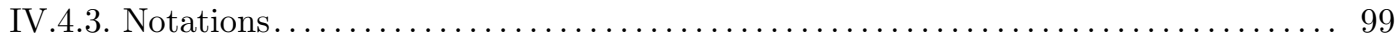

IV.4.4. Filtration de monodromie $\ldots \ldots \ldots \ldots \ldots \ldots \ldots \ldots \ldots \ldots \ldots \ldots \ldots \ldots \ldots \ldots \ldots \ldots \ldots$

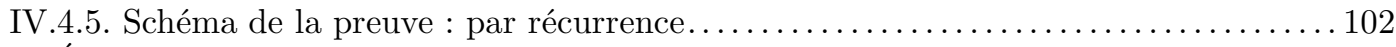

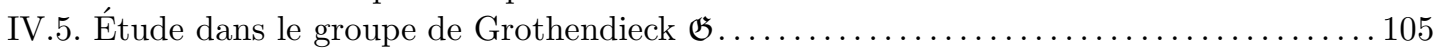

IV.5.1. Groupe de Grothendieck des faisceaux pervers : généralités.............. 105

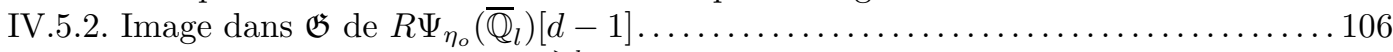

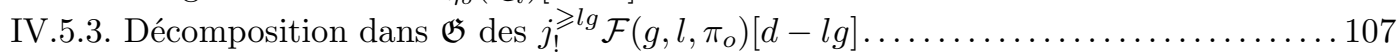

IV.5.4. Étude aux points supersinguliers des $j_{!}^{\geqslant l g} \mathcal{F}\left(g, l, \pi_{o}\right)[d-l g] \ldots \ldots \ldots \ldots \ldots \ldots 113$

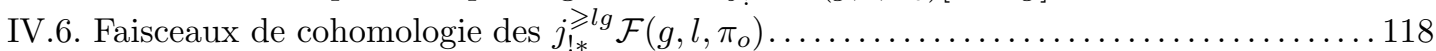

IV.6.1. Une écriture dans $\mathfrak{G}$ de $j_{l_{*}}^{\geqslant i g} \mathcal{F}\left(g, l, \pi_{o}\right)[d-l g] \ldots \ldots \ldots \ldots \ldots \ldots \ldots \ldots \ldots \ldots \ldots$

IV.6.2. Filtration de monodromie-locale en hauteur non maximale............... 119

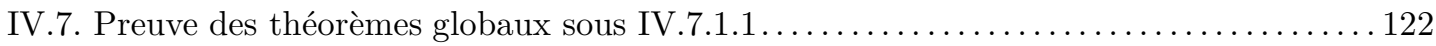

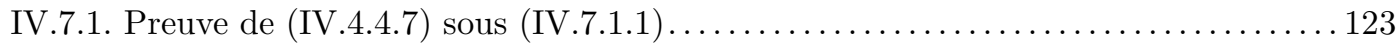

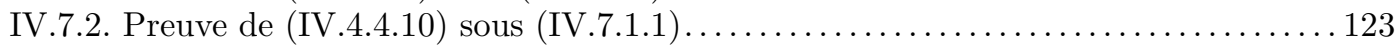

IV.7.3. Pureté de la filtration de monodromie de $R \Psi_{\eta_{o}}\left(\overline{\mathbb{Q}}_{l}\right) \ldots \ldots \ldots \ldots \ldots \ldots \ldots \ldots \ldots$

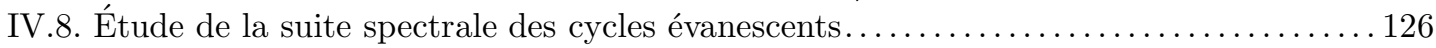

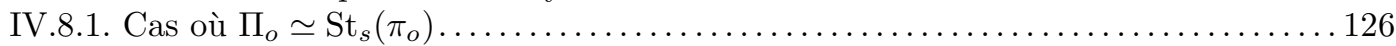

IV.8.2. Involution de Zelevinski et première preuve de $($ IV.7.1.1) $\ldots \ldots \ldots \ldots \ldots \ldots \ldots 128$

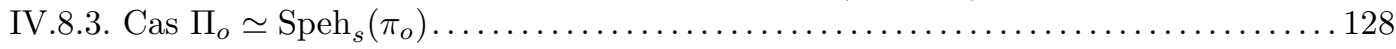

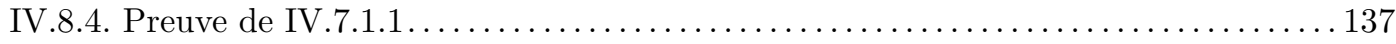

V. Compléments sur la cohomologie globale et applications.............. 139

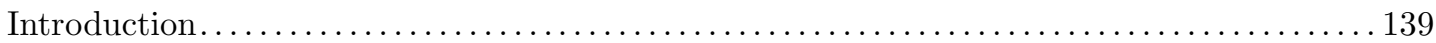

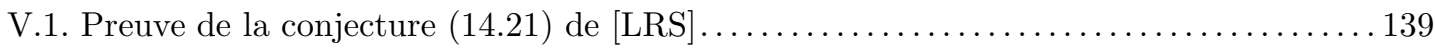

V.2. Correspondances de Jacquet-Langlands globales. . . . . . . . . . . . . . . . . . . 143

V.3. Composantes locales des représentations automorphes vérifiant $\operatorname{Hyp}(\infty) \ldots \ldots \ldots \ldots 145$

V.4. Pureté de la filtration de monodromie des $H_{\eta_{o}}^{i} \ldots \ldots \ldots \ldots \ldots \ldots \ldots \ldots \ldots \ldots \ldots \ldots \ldots$ 


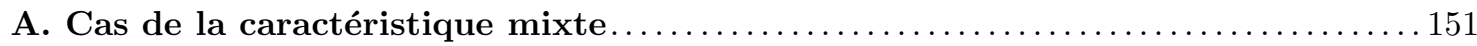

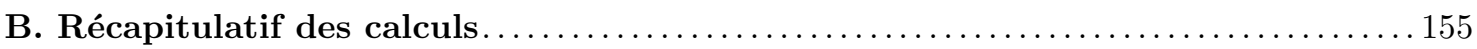

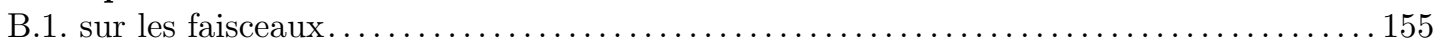

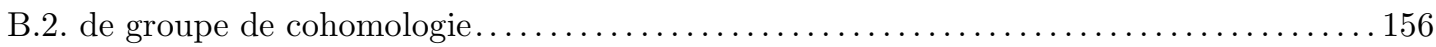

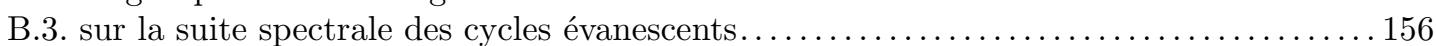

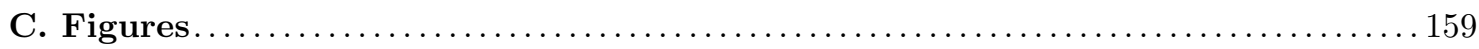

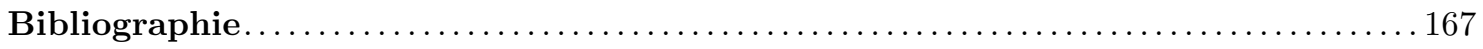




\section{INTRODUCTION}

0.1. - Soient $d$ un entier strictement positif et $K$ un corps local complet d'égale caractéristique $p$, d'anneau des entiers $\mathcal{O}_{K}$. On considère le groupe $D_{K, d}^{\times}$(resp. $W_{K}$ ) des éléments inversibles de "l"'algèbre à division centrale sur $K$ d'invariant $1 / d$ (resp. le groupe de Weil de $K$ ). Pour un nombre premier $l \neq p$, Langlands (resp. Jacquet-Langlands) a (resp. ont) conjecturé l'existence d'une bijection $L_{d}$ (resp. d'une injection JL) entre les $\overline{\mathbb{Q}}_{l}$-représentations irréductibles admissibles de $G L_{d}(K)$ et les représentations $l$-adiques indécomposables de $W_{K}$ (resp. entre les représentations admissibles irréductibles de $D_{K, d}^{\times}$et les représentations essentiellement de carré intégrable de $\left.G L_{d}(K)\right)$ qui sont compatibles à la formation des fonctions $L$ de paires; on renvoie à [16] pour des énoncés précis.

A l'aide de la cohomologie étale, Deligne a alors construit une série de représentations $\mathcal{U}_{K}^{d, i}$ du produit de ces trois groupes. Pour $d=2$ et pour $\rho$ une représentation irréductible de $D_{K, 2}^{\times}$ telle que $\pi:=\operatorname{JL}(\rho)$ soit une représentation cuspidale de $G L_{2}(K)$, Carayol, dans [8], montre que la composante $\rho$-isotypique $\mathcal{U}_{K}^{2,1}(\rho)$ de $\mathcal{U}_{K}^{2,1}$ réalise les correspondances de Langlands et de Jacquet-Langlands, i.e.

$$
\mathcal{U}_{K}^{2,1}\left(\mathrm{JL}^{-1}\left(\pi^{\vee}\right)\right) \simeq \pi \otimes L_{d}(\pi)\left(-\frac{1}{2}\right) .
$$

Le cas $d$ quelconque est traité dans [5]. En outre pour $d=2$, Carayol décrit également ce qui se passe pour les autres représentations.

Le but premier de ce travail est de faire de même pour $d$ quelconque, i.e. calculer complètement les $\mathcal{U}_{K}^{d, i}(\rho)$ pour $\rho$ une représentation irréductible quelconque de $D_{K, d}^{\times}$. Dans le cas où $\rho$ est la représentation triviale, le résultat se formule comme suit.

Théorème Pour $0 \leqslant i \leqslant d-1$, on a

$$
\mathcal{U}_{K}^{d, i}(1)=\pi_{i} \otimes 1(-i)
$$

où $\pi_{i}$ est l'unique quotient irréductible de l'induite parabolique

$$
\operatorname{Ind}_{P_{d-i, d}(K)}^{G L_{s g}(K)} 1\left(\frac{i(g-1)}{2}\right) \otimes \operatorname{St}_{i}\left(-\frac{(s-i)(g-1)}{2}\right)
$$

où $P_{d-i, d}$ est le parabolique standard associée aux d-i premiers vecteurs et $\mathrm{St}_{i}$ est la représentation de Steinberg de $G L_{i}(K)$.

L'énoncé du cas général, théorème (IV.3.1.1), s'énonce de manière similaire et fait intervenir les correspondances de Langlands et Jacquet-Langlands. En particulier dans le cas Iwahori, via l'isomorphisme de Faltings, cf. [10], on retrouve le résultat principal de [21]. 
0.2. - La preuve est de nature globale et repose sur le théorème de comparaison de Berkovich (1) des cycles évanescents locaux et globaux. Ainsi le deuxième résultat de ce texte est la description explicite du complexe des cycles évanescents des variétés de Drinfeld-Stuhler ainsi que de sa filtration de monodromie-poids et de la suite spectrale associée.

Par ailleurs les techniques s'appliquent dans le cadre de la caractéristique mixte pour les variétés de Shimura associés à certains groupes unitaires étudiées dans [14] ce qui fournit en particulier les conjectures de monodromie-poids versions faisceautique et cohomologique. Ce cadre est l'objet de [6], et nous donnons dans l'appendice A les grandes lignes des modifications formelles à apporter.

0.3. - Soit $X$ une courbe projective lisse, irréductible et géométriquement connexe définie sur "le" corps fini à $q=p^{r}$ éléments, $\mathbb{F}_{q}$ et soit $F$ son corps des fonctions. On fixe deux places distinctes $\infty$ et $o$ de $X$ que l'on peut supposer par simplification, rationnelles sur $\mathbb{F}_{q}$ de sorte que le complété $F_{o}$ du localisé de $F$ en $o$ est le corps local précédemment noté $K$. On note $A$ l'anneau des fonctions sur $X$, régulières en dehors de $\infty$. Étant donné un entier $d \geqslant 1$, on fixe une algèbre à division centrale $D$ sur $F$ de dimension $d^{2}$, non ramifiée en $\infty$ et $o$, ainsi qu'un ordre maximal $\mathcal{D}$.

Dans [19], les auteurs construisent pour un idéal non trivial $I$ de $A$, un schéma $M_{I}$ défini sur $F$, classifiant les $\mathcal{D}$-faisceaux elliptiques sur $X$, munis d'une structure de niveau $I$. Pour $o \notin V(I)$, $M_{I}$ a un modèle entier $M_{I, o}$ lisse sur le complété $\mathcal{O}_{o}$ de $A$ en la place $o$. Un tel modèle non lisse dans le cas où $o \in V(I)$ est construit dans [5]. Les schémas $M_{I, o}$ sont naturellement munis d'une action, par correspondances, de $\left(D_{\mathbb{A}}^{\infty}\right)^{\times}$.

0.4. - On s'intéresse alors à la fibre spéciale $M_{I, s_{o}}$ de $M_{I, o}$. Dans [5], je stratifie $M_{I, s_{o}}$ par des sous-schémas localement fermés $M_{I, s_{o}}^{=h}$ pour $1 \leqslant h \leqslant d$, de pure dimension $d-h$ tels que l'on ait un équivalent du théorème de Serre-Tate pour les $\mathcal{D}$-faisceaux elliptiques à savoir : le complété de l'hensélisé strict de l'anneau local de $M_{I, o}$ en un point géométrique de $M_{I, s_{o}}^{=h}$ est isomorphe à $\operatorname{Def}_{n}^{h}\left[\left[x_{1}, \cdots, x_{d-h}\right]\right]$ où $n$ est la multiplicité de $o$ dans $I$ et $\operatorname{Def}_{n}^{h}$ représente le foncteur des déformations de niveau $n$ d'un $\mathcal{O}_{o}$-module formel de hauteur $h$ sur $\overline{\mathbb{F}}_{p}$.

Par ailleurs pour $1 \leqslant h<d$, il existe un sous-schéma fermé $M_{I, s_{o}, 1}^{=h}$ de $M_{I, s_{o}}^{=h}$ stable sous les correspondances associées aux éléments du sous-groupe parabolique $P_{h, d}^{o p}\left(F_{o}\right)$ de $G L_{d}\left(F_{o}\right)$ (cf. la définition (II.1.1.1)) et tel que

$$
M_{I, s_{o}}^{=h}=M_{I, s_{o}, 1}^{=h} \times{ }_{P_{h, d}^{o p}\left(\mathcal{O}_{o} / \mathcal{M}_{o}^{n}\right)} G L_{d}\left(\mathcal{O}_{o} / \mathcal{M}_{o}^{n}\right)
$$

où $n$ est la multiplicité de $o$ dans $I$ : on dit que les strates non supersingulières sont géométriquement induites.

0.5. - Dans le premier chapitre, en suivant [14], on étudie la restriction aux strates $M_{I, s_{o}}^{=h}$ des faisceaux des cycles évanescents $R^{i} \Psi_{\eta_{o}}\left(\overline{\mathbb{Q}}_{l}\right)$. Pour cela on introduit selon loc. cit. les variétés d'Igusa de première et seconde espèce.

Les premières sont des revêtements galoisiens de $M_{I^{o}, s_{o}}^{=h}$ de groupe de Galois $G L_{d-h}\left(\mathcal{O}_{o} / \mathcal{M}_{o}^{n}\right)$, définies par la donnée d'une structure de niveau $\mathcal{M}_{o}^{n}$ sur la partie étale des $\mathcal{D}$-faisceaux elliptiques de $M_{\bar{I}^{o}, s_{o}}^{\bar{h}}$ : on dispose alors d'un morphisme radiciel de $\mathcal{I}_{\bar{I}^{o}, n}^{=h}$ vers $M_{\bar{I}, s_{o}, 1}^{=h}$.

Les variétés d'Igusa de seconde espèce sont des revêtements galoisiens $\mathcal{J}_{I^{o}, n}^{=h}(s) \longrightarrow \mathcal{I}_{I^{o}, n}^{=h}$ de groupe de Galois $\left(\mathcal{D}_{o, h} /\left(\Pi_{o, h}^{s}\right)\right)^{\times}$où $\mathcal{D}_{o, h}$ est l'ordre maximal de l'algèbre à division $D_{o, h}$ centrale sur $F_{o}$ d'invariant $1 / h$, avec $\Pi_{o, h}$ une uniformisante : elles sont définies via une rigidification modulo $\Pi_{o, h}^{s}$ de la partie connexe des $\mathcal{D}$-faisceaux elliptiques de $\mathcal{I}_{I^{o}, n}^{=h}$.

On dispose alors pour toute représentation $\tau$ irréductible et admissible de $D_{o, h}^{\times}$, d'un système local $\mathcal{F}_{\tau}$ dit de Harris-Taylor, sur $M_{I, s_{o}}^{=h}$. Suivant [14], la restriction à $M_{I, s_{o}, 1}^{=h}$ des faisceaux des cycles évanescents s'exprime alors en termes des systèmes locaux d'Harris-Taylor et des faisceaux des cycles évanescents du modèle local.

${ }^{(1)}$ en fait sur une version raffinée fournie par Fargues (cf. le théorème principal de l'appendice de [6]) 
0.6. - Au deuxième chapitre on prouve le cas Iwahori du théorème local. On commence par des rappels sur les représentations elliptiques de $G L_{d}\left(F_{o}\right)$. On raisonne ensuite par récurrence en supposant connu le cas Iwahori du théorème local en hauteur strictement inférieure à $d$. L'hypothèse de récurrence et le fait que les strates non supersingulières soient induites, nous permettent alors d'étudier combinatoirement la suite spectrale des cycles évanescents et de prouver le résultat.

0.7. - Au troisième chapitre, en suivant [19], il s'agit de calculer la somme alternée des groupes de cohomologie des systèmes locaux d'Harris-Taylor. La démarche est classique : il s'agit tout d'abord d'utiliser la formule des traces de Lefschetz et donc de compter les points fixes sous l'action d'une correspondance de Hecke tordue par une puissance assez grande du Frobenius et ensuite de transférer les intégrales orbitales obtenues afin de reconnaître le coté géométrique de la formule des traces de Selberg.

On en déduit alors un calcul de la somme alternée des groupes de cohomologie du modèle local de Deligne-Carayol. En particulier, dans le cas Iwahori, des arguments de pureté nous redonnent les résultats obtenus à la fin du chapitre précédent. Pour ce qui est du cas général, le théorème local correspond à dire qu'il n'y a pas d'annulation dans la représentation virtuelle $\sum_{i=0}^{d-1}(-1)^{i}\left[\mathcal{U}_{F_{o}}^{d, i}\left(\rho_{o}\right)\right]$ où $\mathcal{U}_{F_{o}}^{d, d-i}\left(\rho_{o}\right)$, pour $1 \leqslant i \leqslant d$, est donné par le $i$-ème terme de plus haut poids.

0.8. - Dans le quatrième chapitre on étudie la filtration de monodromie du faisceau pervers $R \Psi_{\eta_{o}}\left(\overline{\mathbb{Q}}_{l}\right)[d-1]$ dont on notera $g r_{k}$ les gradués ainsi que la fibre en un point supersingulier des termes $E_{1}^{i, j}$ de la suite spectrale

$$
E_{1}^{i, j}=h^{i+j} g r_{-i} \Rightarrow R^{i+j+d-1} \Psi_{\eta_{o}}\left(\overline{\mathbb{Q}_{l}}\right)
$$

et en particulier la fibre en un point supersingulier des termes $E_{1}^{i, j}$.

On décrit tout d'abord les $g r_{k}$ dans la catégorie des faisceaux pervers sur la tour des $\left(M_{I, s_{o}}\right)_{I}$ munis d'une action par correspondances de $\left(D_{\mathbb{A}}^{\infty}\right)^{\times} \times W_{o}$, en fonction des extension intermédiaires $j_{! *}^{\geqslant l g} \mathcal{F}_{\mathrm{JL}^{-1}\left(\mathrm{St}_{l}\left(\pi_{o}\right)\right)}[d-l g]$ des systèmes locaux d'Harris-Taylor, où $j \geqslant l g$ désigne l'injection de la strate $M_{I, s_{o}}^{=l g}$ et $\pi_{o}$ est une représentation irréductible cuspidale de $G L_{g}\left(F_{o}\right)$ avec $1 \leqslant l \leqslant d / g$.

On calcule ensuite les faisceaux de cohomologie de ces derniers ce qui donne le théorème local d'après le théorème de comparaison de Berkovich. En fait Fargues, cf. l'appendice de [6], améliore les résultats de Berkovich ce qui nous permet finalement de décrire la filtration de monodromielocale du modèle de Deligne-Carayol.

0.9. - Dans le dernier chapitre on donne divers compléments. On prouve en particulier la conjecture (14.21) de [19], des cas particuliers de correspondances de Jacquet-Langlands par ailleurs connus des experts. On montre aussi que les composantes locales de représentations automorphes de $D_{\mathbb{A}}^{\times}$sont ce que l'on attend à savoir ce que l'on devrait obtenir à partir des résultats de MoeglinWaldspurger sur les composantes locales des carrés intégrables de $G L_{d}(\mathbb{A})$ via une correspondance de Jacquet-Langlands globale. On montre enfin comment en caractéristique mixte, on peut prouver la conjecture de monodromie-poids version cohomologique.

0.10. - Dans l'appendice A, on donne le dictionnaire entre les notations de [14] et les nôtres et on renvoie à loc. cit. pour les résultats géométriques et cohomologiques que l'on utilise. Via ce dictionnaire, les résultats et les preuves du chapitre IV sont alors valables telles quelles; en particulier on obtient une preuve de la conjecture de monodromie-poids dans le cadre des variétés de Shimura de loc. cit.

0.11. - L'appendice B résume certains des résultats obtenus au cours de la preuve et l'appendice C présente diverses figures des nombreuses suites spectrales que l'on utilise, essentiellement pour $s=4$.

Les chapitres I et III sont essentiellement une traduction en égale caractéristique des résultats de [14]. Le chapitre II n'est pas utile mais il nous a semblé agréable de présenter une preuve du 
cas Iwahori qui n'utilise pas toute la machinerie. Pour les lecteurs intéressés par les résultats du chapitre IV, nous avons fait en sorte que celui-ci soit lisible indépendamment des deux premiers, sauf pour ce qui est des rappels de [24] donnés §II.1.

Remerciements Je tiens à exprimer ma profonde gratitude envers Gérard Laumon pour son soutien constant tout au long de ces années ainsi que pour sa perspicacité à dépister les fausses bonnes idées et à mettre en avant les autres. Merci aussi à Laurent Fargues de m'avoir fourni une bonne notion de monodromie locale qui m'a permis de simplifier grandement les preuves. J'adresse des remerciements à Jean-François Dat pour m'avoir fourni l'habillage catégoriel des faisceaux de Hecke ainsi que pour ses nombreux conseils ainsi qu'à Thomas Hausberger pour m'avoir indiqué une erreur dans mes précédents travaux au niveau des actions sur le modèle local. 


\section{CHAPITRE I}

\section{VARIÉTÉS D'IGUSA, SYSTÈMES LOCAUX D'HARRIS-TAYLOR ET CYCLES ÉVANESCENTS DES VARIÉTÉS DE DRINFELD}

\section{Introduction}

0.1. - Rappelons la situation globale. Soit $X$ une courbe projective lisse, irréductible et géométriquement connexe définie sur le corps fini à $q=p^{r}$ éléments, $\mathbb{F}_{q}$ et soit $F$ son corps des fonctions. On fixe deux places $\infty, o$ distinctes de $X$ que l'on peut supposer par simplification, rationnelles sur $\mathbb{F}_{q}$. On note $A$ l'anneau des fonctions sur $X$, régulières en dehors de $\infty$. Étant donné un entier $d \geqslant 1$, on fixe une algèbre à division centrale $D$ sur $F$ de dimension $d^{2}$, non ramifiée en $\infty$ et $o$, ainsi qu'un ordre maximal $\mathcal{D}$.

Dans [19], les auteurs construisent pour un idéal non trivial $I$ de $A$, un schéma $M_{I}$ défini sur $F$, classifiant les $\mathcal{D}$-faisceaux elliptiques sur $X$, munis d'une structure de niveau $I$. Pour $o \notin V(I)$, $M_{I}$ a un modèle entier $M_{I, o}$ lisse sur le complété $\mathcal{O}_{o}$ de $A$ en la place $o$. Dans [5], on construit un tel modèle non lisse dans le cas où $o \in V(I)$. Les schémas $M_{I, o}$ sont naturellement munis d'une action, par correspondances, de $\left(D_{\mathbb{A}}^{\infty}\right)^{\times}$.

0.2. - Dans [5], la fibre spéciale $M_{I, s_{o}}$ de $M_{I, o}$ est stratifiée par des sous-schémas localement fermés $M_{\bar{I}, s_{o}}^{=}$pour $1 \leqslant h \leqslant d$, de pure dimension $d-h$ tels que l'on ait un équivalent du théorème de Serre-Tate pour les $\mathcal{D}$-faisceaux elliptiques à savoir : le complété de l'hensélisé strict de l'anneau local de $M_{I, o}$ en un point géométrique de $M_{I, s_{o}}^{=h}$ est un anneau de séries formelles Def ${ }_{n}^{h}$ où ce dernier représente les déformations de niveau $n:=$ mult $_{o}(I)$ d'un $\mathcal{O}_{o}$-module formel de hauteur $h$.

Par ailleurs ces strates pour $h \neq d$, sont géométriquement induites au sens où il existe un sous-schéma fermé $M_{I, s_{o}, 1}^{=h}$ de $M_{I, s_{o}}^{=h}$ stable sous les correspondances associées aux éléments du sous-groupe parabolique $P_{h, d}^{o p}\left(F_{o}\right)$ de $G L_{d}\left(F_{o}\right)$, opposé au parabolique standard associé aux $h$ premiers vecteurs de la base canonique et tel que

$$
M_{I, s_{o}}^{=h}=M_{I, s_{o}, 1}^{=h} \times_{P_{h, d}^{o p}\left(\mathcal{O}_{o} / \mathcal{M}_{o}^{n}\right)} G L_{d}\left(\mathcal{O}_{o} / \mathcal{M}_{o}^{n}\right)
$$

0.3. - Il s'avère que l'adhérence $M_{I, s_{o}, 1}^{\geqslant h}$ des $M_{I, s_{o}, 1}^{=h}$ est lisse de sorte que la non lissité des strates $M_{I, s_{o}}^{\bar{h}}$ provient des intersections entre ces différentes composantes et donc de l'intrication des parties connexes et étales des $I$-structures de niveau. Afin de démêler la situation on introduit les variétés d'Igusa de première et seconde espèce. Pour tout place $o$ divisant $I$, on note $I=I^{o} \mathcal{M}_{o}^{n}$ où $n$ est la multiplicité de $o$ dans $I$ de sorte que $o$ ne divise pas $I^{o}$.

Les variétés d'Igusa de première espèce $\mathcal{I}_{I^{o}, n}^{=h}$ sont des revêtements galoisiens de $M_{\bar{I}^{o}, s_{o}}^{=h}$ de groupe de Galois $G L_{d-h}\left(\mathcal{O}_{o} / \mathcal{M}_{o}^{n}\right)$, définis par la donnée d'une structure de niveau $\mathcal{M}_{o}^{n}$ sur la partie étale des $\mathcal{D}$-faisceaux elliptiques de $M_{I^{o}, s_{o}}^{\bar{h}}$ : on dispose alors d'un morphisme radiciel de $\mathcal{I}_{I^{o}, n}^{=h}$ vers $M_{I, s_{o}, 1}^{=h}$.

Les variétés d'Igusa de seconde espèce sont des revêtements galoisiens $\mathcal{J}_{I^{o}, n}^{=h}(s) \longrightarrow \mathcal{I}_{I^{o}, n}^{=h}$ de groupe de Galois $\left(\mathcal{D}_{o, h} /\left(\Pi_{o, h}^{s}\right)\right)^{\times}$où $\mathcal{D}_{o, h}$ est l'ordre maximal de l'algèbre à division $D_{o, h}$ centrale 
sur $F_{o}$ d'invariant $1 / h$, avec $\Pi_{o, h}$ une uniformisante : elles sont définies via une rigidification modulo $\Pi_{o, h}^{s}$ de la partie connexe des $\mathcal{D}$-faisceaux elliptiques de $\mathcal{I}_{I^{o}, n}^{=h}$.

On dispose alors pour toute représentation irréductible et admissible de $\mathcal{D}_{o, h}^{\times}$, d'un système local dit de Harris-Taylor, sur $M_{I, s_{o}, 1}^{=h}$ qu'il est aisé de propager à toute la strate via l'action de $G L_{d}\left(\mathcal{O}_{o}\right)$. Afin de mieux suivre les actions, on partira plutôt d'une représentation irréductible $\tau_{o}$ de $D_{o, h}^{\times}$dont on prendra la restriction à $\mathcal{D}_{o, h}^{\times}$de sorte que le système local obtenu $\mathcal{F}_{\tau_{o}}$ ne sera pas irréductible.

0.4. - Suivant [14], on est en mesure de décrire assez simplement le complété formel de $M_{I, o}$ le long de la strate $M_{I, s_{o}, 1}^{=h}$. D'après les résultats de Berkovich, on en déduit alors une expression de la restriction à $M_{I, s_{o}, 1}^{=h}$ des faisceaux des cycles évanescents en termes des systèmes locaux d'Harris-Taylor et des faisceaux des cycles évanescents $\Psi_{F_{o}, n}^{h, i}$ associés par Berkovich aux anneaux $\operatorname{Def}_{n}^{h}$ tout en suivant l'action de $\left(D_{\mathbb{A}}^{\infty, o}\right)^{\times} \times W_{o}$.

0.5. - Décrivons succinctement le contenu des différents paragraphes. Le premier paragraphe est consacré aux rappels de $[\mathbf{1 9}]$ et $[\mathbf{5}]$ sur les $\mathcal{O}_{o}$-modules formels, $\S I .1 .1$, sur les $\mathcal{D}$-faisceaux elliptiques et leurs structures de niveau, §I.1.2, les variétés de modules $M_{I, o}$ munis d'une action par correspondances de $\left(D_{\AA}^{\infty}\right)^{\times}, \S I$ I.3. On rappelle ensuite, $\S$ I.1.4, selon [19], la construction des systèmes locaux $\mathcal{L}_{\rho_{\infty}}$ sur les variétés de $M_{I, o}$ associés à une représentation irréductible $\rho_{\infty}$ des inversibles de l'algèbre à division $\bar{D}_{\infty}$ centrale sur $F_{\infty}$ d'invariant $-1 / d$. On rappelle enfin, §I.1.5, la stratification de la fibre spéciale et comme application du théorème de Serre-Tate, §I.1.6, on montre la lissité des $M_{I, s_{o}, 1}^{\geqslant h}$.

0.6. - Le deuxième paragraphe s'intéresse plus en détail aux structures de niveau en y démêlant parties étales et connexes. A tout $S$-point de $M_{I, s_{o}}$ est associé un $\phi$-faisceau : si $S \longrightarrow M_{I, s_{o}}$ se factorise par $M_{I, s_{o}}^{=h}$, le $\phi$-faisceau associé se décompose alors en partie étale et connexe. L'un des résultats clef de ce premier paragraphe est la proposition (I.2.1.1) qui montre qu'après extension des scalaires par une puissance assez grande du Frobenius, la décomposition en composantes étale et connexe du $\phi$-faisceau d'un $S$-point de $M_{I, s_{o}}^{=h}$ est scindée modulo $\pi_{o}^{n}$. On a aussi des résultats au niveau des déformations de points de $M_{I, s_{o}}^{=h}$, corollaire (I.2.1.3) et proposition (I.2.2.6). On montre enfin la relation de congruence, proposition (I.2.3.1), qui permet de vérifier, §I.3.3, la compatibilité des diverses définitions des correspondances de Hecke.

0.7. - On introduit, $\S I .3$, suivant [14], les variétés d'Igusa de première espèce $\mathcal{I}_{I^{o}, n}^{=h}$. On peut comprendre la démarche des définitions de celles-ci, de leurs versions formelles $\hat{\mathcal{I}}_{I^{o},=h, m}(t)$ et de leurs liens aux variétés de Drinfeld, comme la différence entre la donnée d'une structure de niveau sur un $\phi$-faisceau et la donnée de structures de niveaux sur les parties étale et connexe du même $\phi$-faisceau. Les résultats dans ce sens sont les propositions (I.3.1.2) et (I.3.2.1). En particulier on a un morphisme radiciel de $\mathcal{I}_{\bar{I}^{o}, n}^{=h}$ vers $M_{\bar{I}, s_{o}, a}^{=h}$ où $n$ est la multiplicité de $o$ dans $I$. Au niveau des complétés formels, le résultat clef est la proposition (I.3.2.1) qui donne l'existence d'un isomorphisme canonique de $\hat{\mathcal{I}}_{I^{o},=h, n}(n)$ vers $\widehat{M_{I, o,=h, a}}$. On définit ensuite, $\S I .3 .3$, les correspondances de Hecke associées aux éléments de $G L_{h}\left(F_{o}\right) \times G L_{d-h}\left(F_{o}\right)$ sur la tour des $\hat{\mathcal{I}}_{I^{o},=h, m}(t)$, de manière compatible au théorème de Serre-Tate et à l'isomorphisme de la proposition (I.3.2.1).

0.8. - Les variétés d'Igusa de seconde espèce $\mathcal{J}_{I^{o}, m}^{=h}(s)$ de niveau $s$, sont introduites $\S$ I.4 : le revêtement $\mathcal{J}_{I^{o}, m}^{=h}(s) \longrightarrow \mathcal{I}_{\bar{I}^{o}, m}=h$ est étale de groupe de Galois $\mathcal{D}_{o, h, s}^{\times}:=\left(\mathcal{D}_{o, h} /\left(\Pi_{o, h}^{s+1}\right)\right)^{\times}$où $\mathcal{D}_{o, h}$ est l'ordre maximal de l'algèbre à division centrale sur $F_{o}$ d'invariant $1 / h$ et où $\Pi_{o, h}$ est un uniformisante de $\mathcal{D}_{o, h}$. On définit $\S$ I.4.2, les correspondances de Hecke sur la tour des $\mathcal{J}_{I^{o}, m}^{=h}(s)$ associées aux éléments de $\left(D_{\mathbb{A}}^{\infty, o}\right)^{\times} \times G L_{d-h}\left(F_{o}\right) \times \widetilde{\mathcal{N}}_{o}$ où $\widetilde{\mathcal{N}}_{o}$ est le noyau du morphisme $G L_{h}\left(F_{o}\right) \times$ $D_{o, h}^{\times} \times W_{F_{o}} \longrightarrow \mathbb{Z}$ qui au triplet $\left(g_{o}^{c}, \delta_{o}, c_{o}\right)$ associe la valuation $\operatorname{de} \operatorname{det}\left(g_{o}^{c}\right) \operatorname{rn}\left(\delta_{o}\right) \operatorname{cl}\left(c_{o}\right)$ où rn : $D_{o, h}^{\times} \longrightarrow F_{o}^{\times}$est la norme réduite et $\mathrm{cl}: W_{F_{o}} \longrightarrow F_{o}^{\times}$est l'application de la théorie du corps 
de classe. Les résultats principaux sur les variétés d'Igusa sont ceux de §I.4.3. Brièvement on se sert des $\mathcal{J}_{I^{o}, m}^{=h}(s)$ pour "détordre" le schéma formel $\hat{\mathcal{I}}_{I^{o},=h, m}(t)$ (cf. la proposition (I.4.3.4) et la remarque qui suit). Ce paragraphe est très proche du texte de [14]; en particulier en suivant loc. cit., on montre à la proposition (I.4.3.7), une version à niveau fini de ces résultats.

0.9. - Le cinquième paragraphe traite des cycles évanescents. A partir de ce point et jusqu'à la fin, sauf mention expresse du contraire, on considère l'action naturelle, i.e. celle de [5], de $G L_{d}\left(F_{o}\right)$ sur Def ${ }_{n}^{d}$ tordue par l'application $g_{o} \mapsto{ }^{t} g_{o}^{-1}$; on pointera cette modification par un tilde sur les espaces concernés. On s'intéresse en premier lieu à la restriction du faisceau des cycles évanescents $R^{i} \Psi_{\eta_{o}}\left(\overline{\mathbb{Q}}_{l}\right)$ de $M_{I, o} \longrightarrow \operatorname{Spec}\left(\mathcal{O}_{o}\right)$ à la strate $M_{I, s_{o}, a}^{=h}$, en fonction du faisceau des cycles évanescents $\widetilde{\Psi_{F_{o}, n}^{h, i}}$ du modèle local, i.e. de $\operatorname{Def}_{n}^{h}$ (cf. $\S$ I.5.1). L'énoncé principal, théorème (I.5.2.7), décrit cette restriction munie de son action de $\left(\mathcal{D}_{\mathbb{A}}^{\infty, o}\right)^{\times} \times P_{h, d}\left(F_{o}\right) \times W_{F_{o}}$. Formulons en rapidement l'énoncé : soit Twist ${ }_{\mathcal{J}_{I^{o}, n}^{h}(\infty)}\left(\widetilde{\Psi_{F_{o}, n}^{h, i}}\right)$ le faisceau sur $\mathcal{I}_{I^{o}, n}^{=h}$ tel que sa restriction à $\mathcal{J}_{I^{o}, n}^{=h}(s)$ muni de l'action naturelle de $\mathcal{D}_{o, h}^{\times}$est le faisceau constant $\widetilde{\Psi_{F_{o}, h}^{h, i}}$ muni de l'action "diagonale" de $\mathcal{D}_{o, h}^{\times}$. Ce faisceau est naturellement muni d'une action de $\left(\mathcal{D}_{\mathbb{A}}^{\infty, o}\right)^{\times} \times P_{h, d}^{o p}\left(F_{o}\right) \times W_{F_{o}}$ et on a un isomorphisme équivariant naturel

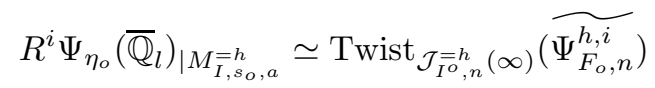

On relie ensuite $\S$ I.5.3 ces faisceaux aux systèmes locaux ${ }^{(1)} \mathcal{F}_{\tau_{o}}$ sur $M_{I, s_{o}, a}^{=h}$ associés aux revêtements d'Igusa de seconde espèce et à une représentation admissible, irréductible $\tau_{o}$ de $D_{o, h}^{\times}$. Cette description est donnée à la proposition (I.5.3.6).

0.10. - Enfin dans le dernier paragraphe, on donne, au niveau des complétés formels, des équations explicites définissant les variétés d'Igusa de seconde espèce.

\section{I.1. Modules formels et variétés de Drinfeld : rappels}

\section{I.1.1. Rappels sur les déformations des $\mathcal{O}_{o}$-modules formels. -}

I.1.1.1. Définition. - Étant donnés une $\mathcal{O}_{o}$-algèbre $R$ et $i: \mathcal{O}_{o} \rightarrow R$ son morphisme structural, un $\mathcal{O}_{o}$-module formel sur $R$ est un couple $G=\left(F,\left(f_{\lambda}\right)_{\lambda \in \mathcal{O}_{o}}\right)$ où $F \in R[[X, Y]]$ et $f_{\lambda} \in R[[X]]$ avec $f_{\lambda}(X)=i(\lambda) X$ modulo $\left(X^{2}\right)$, vérifiant les propriétés usuelles pour en faire un $\mathcal{O}_{o}$-module, $F$ représentant l'addition et $f_{\lambda}$ la multiplication par $\lambda$.

Remarques : - Si $R$ est un corps sur $\mathbb{F}_{q}$, il existe un entier $d$, appelé la hauteur de $G$, tel que $F(X, Y)=X+Y$ et $f_{\varpi_{o}}(X)$ est une série formelle en $X^{q^{d}}$.

- Sur $\overline{\mathbb{F}}_{q}$, il existe à isomorphisme près un unique $\mathcal{O}_{o}$-module formel de hauteur $d$ donnée que l'on notera $\Sigma_{d}$.

- L'anneau des endomorphismes de ce $\mathcal{O}_{o}$-module formel de hauteur $d$ sur $\bar{\kappa}$, est l'ordre maximal $\mathcal{D}_{o, d}$ de l'algèbre à division centrale sur $F_{o}, D_{o, d}$, d'invariant $1 / d$.

I.1.1.2. Définitions. - On note $\hat{\mathcal{O}}_{o}^{n r}=\overline{\mathbb{F}}_{q}\left[\left[\varpi_{o}\right]\right]$ et on considère la catégorie $C$ dont les objets sont les $\hat{\mathcal{O}}_{o}^{n r}$-algèbres artiniennes. Une déformation sur $R \in \operatorname{Ob}(C)$ du $\mathcal{O}_{o}$-module formel de hauteur $d$ sur $\overline{\mathbb{F}}_{q}, \Sigma_{d}$, est un $\mathcal{O}_{o}$-module formel $G=\left(F,\left(f_{\lambda}\right)_{\lambda \in \mathcal{O}}\right)$ sur $R$ dont la réduction modulo l'idéal maximal $\mathcal{M}$ de $R$ est $\Sigma_{d}$. Une structure de niveau $n$ sur $G$, est la donnée d'un homomorphisme de $\mathcal{O}_{o}$-module,

$$
\iota_{n}:\left(\varpi_{o}^{-n} / \mathcal{O}_{o}\right)^{d} \longrightarrow \mathcal{M}
$$

tel que $f_{\varpi_{o}}(X)$ est divisible par $\prod_{\alpha \in\left(\varpi_{o}^{-1} \mathcal{O}_{o} / \mathcal{O}_{o}\right)^{d}}\left(X-\iota_{n}(\alpha)\right)$. Une déformation de niveau $n$ définie sur $R$, est par définition une déformation sur $R$ munie d'une structure de niveau $n$.

${ }^{(1)}$ non irréductibles car la restriction de $\tau_{o}$ à $\mathcal{D}_{o, h}^{\times}$est une somme directe de $e_{\tau_{o}}$ représentations irréductibles. 
I.1.1.3. Proposition. - (cf. [9] proposition 4.3) Le foncteur des déformations de niveau $n$ du $\mathcal{O}_{o}$-module formel de hauteur d sur $\overline{\mathbb{F}}_{q}, \Sigma_{d}$, est représenté par l'anneau $\operatorname{Def}_{n}^{d}$ vérifiant les propriétés suivantes:

- pour $n=0$, le $\mathcal{O}_{o}$-module formel universel sur $\operatorname{Def}_{0}^{d}$ est de la forme $\left(X+Y,\left(f_{\lambda}\right)_{\lambda \in \mathcal{O}_{o}}\right)$ avec $f_{\varpi_{o}}(X)=\varpi_{o} X+a_{1} X^{q}+\cdots+a_{d-1} X^{q^{d-1}}+X^{q^{d}}$ et $\operatorname{Def}_{0}^{d} \simeq \overline{\mathbb{F}}_{q}\left[\left[a_{0}, a_{1}, \cdots, a_{d-1}\right]\right]$ avec $a_{0}=\varpi_{o}$;

- soit pour $1 \leqslant i \leqslant d, e_{i}$ est le $i$-ème vecteur de la base canonique. Pour $n>0$, le morphisme

$$
\phi: \overline{\mathbb{F}}_{q}\left[\left[v_{1}^{n}, \cdots, v_{d}^{n}\right]\right] \longrightarrow \operatorname{Def}_{n}^{d}
$$

défini par $\phi\left(v_{i}^{n}\right)=\iota_{n}\left(e_{i}\right)$ où $\iota_{n}$ est la structure de niveau universelle, est un isomorphisme.

Remarque : Dans la suite quand on écrit

$$
\operatorname{Def}_{n}^{d} \simeq \overline{\mathbb{F}}_{q}\left[\left[v_{1}^{n}, \cdots, v_{d}^{n}\right]\right] \quad\left(\text { resp. } \operatorname{Def}_{0}^{d} \simeq \overline{\mathbb{F}}_{q}\left[\left[a_{0}, a_{1}, \cdots, a_{d-1}\right]\right], a_{0}=\varpi_{o}\right)
$$

on sous-entend qu'il s'agit de l'isomorphisme ci-dessus.

Remarque : Le morphisme caractéristique $\overline{\mathbb{F}}_{q}\left[\left[\varpi_{o}\right]\right] \longrightarrow$ Def $_{n}^{d}$ peut se calculer de la façon suivante. Le morphisme $\operatorname{Def}_{0}^{d} \longrightarrow \operatorname{Def}_{1}^{d}$ est donné par l'égalité de polynômes

$$
\left|\begin{array}{ccccc}
v_{1}^{1} & v_{2}^{1} & \cdots & v_{d}^{1} & X \\
\left(v_{1}^{1}\right)^{q} & \left(v_{2}^{1}\right)^{q} & \cdots & \left(v_{d}^{1}\right)^{q} & X^{q} \\
\vdots & \vdots & \vdots & \vdots & \vdots \\
\left(v_{1}^{1}\right)^{q^{d}} & \left(v_{2}^{1}\right)^{q^{d}} & \cdots & \left(v_{d}^{1}\right)^{q^{d}} & X^{q^{d}}
\end{array}\right|=\lambda\left(\varpi_{0} X+a_{1} X^{q}+\cdots+a_{d-1} X^{q^{d-1}}+X^{q^{d}}\right)
$$

où $\lambda$ est le coefficient dominant du polynôme de gauche. Ainsi l'image de $\varpi_{o}$ dans $\operatorname{Def}_{1}^{d}$ est

$$
\left|\begin{array}{cccc}
v_{1}^{1} & v_{2}^{1} & \cdots & v_{d}^{1} \\
\left(v_{1}^{1}\right)^{q} & \left(v_{2}^{1}\right)^{q} & \cdots & \left(v_{d}^{1}\right)^{q} \\
\vdots & \vdots & \vdots & \vdots \\
\left(v_{1}^{1}\right)^{q^{d-1}} & \left(v_{2}^{1}\right)^{q^{d-1}} & \cdots & \left(v_{d}^{1}\right)^{q^{d-1}}
\end{array}\right|^{q}
$$

En notant $a_{i}^{n}$ pour $0 \leqslant i<d$, les images de $a_{i} \in \operatorname{Def}_{0}^{d}$ dans $\operatorname{Def}_{n}^{d}$, les morphismes $f_{n}: \operatorname{Def}_{n}^{d} \longrightarrow$ $\operatorname{Def}_{n+1}^{d}$ se calculent de proche en proche par l'égalité suivante :

$$
f_{n}\left(v_{i}^{n}\right)=f_{n}\left(a_{0}^{n}\right) v_{i}^{n+1}+f_{n}\left(a_{1}^{n}\right)\left(v_{i}^{n+1}\right)^{q}+\cdots+f_{n}\left(a_{d-1}^{n}\right)\left(v_{i}^{n+1}\right)^{q^{d-1}}+\left(v_{i}^{n+1}\right)^{q^{d}}
$$

I.1.1.4. Proposition. - (Deligne, Carayol : cf. [8] ou [5] 2.3) L'action naturelle de $G L_{d}\left(\mathcal{O}_{o}\right) \times$ $\mathcal{D}_{o, d}^{\times} \times I_{o}$ sur $\operatorname{Def}_{n}^{d}$, se prolonge au noyau de l'application

$$
\begin{array}{cll}
G L_{d}\left(F_{o}\right) \times D_{o, d}^{\times} \times W_{o} & \longrightarrow & \mathbb{Z} \\
(g, \delta, w) & \mapsto & \operatorname{val}\left(\operatorname{det}\left(g^{-1}\right) \operatorname{rn}(\delta) c l(w)\right)
\end{array}
$$

où $\mathrm{rn}: D_{o, d}^{\times} \rightarrow F_{o}^{\times}$est la norme réduite.

On rappelle que l'action de $g^{-1} \in G L_{d}\left(F_{o}\right) \cap \mathbb{M}_{d}\left(F_{o}\right)$ se déduit de l'action naturelle à gauche de $g$ sur la structure de niveau $\iota:\left(F_{o} / \mathcal{O}_{o}\right)^{d} \longrightarrow \mathcal{M}$; par ailleurs celle de $\mathcal{D}_{o, d}^{\times}$est définie via l'identification avec $\operatorname{Aut}\left(\Sigma_{d}\right)$.

\section{I.1.2. $\mathcal{D}$-faisceau elliptiques et structures de niveau. -}

I.1.2.1. Définition. - (cf. [19]) Un $\mathcal{D}$-faisceau elliptique $\left(\mathcal{E}_{i}, j_{i}, t_{i}\right)$ sur un schéma $S$ est d'après [19] un diagramme commutatif

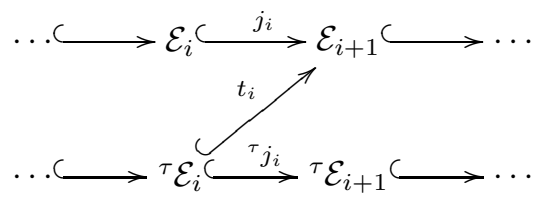


où :

- $\mathcal{E}_{i}$ est un $\mathcal{D}_{X \times S}$-module à droite localement libre de rang 1 , et donc un $\mathcal{O}_{X \times S}$-module localement libre de rang $d^{2}$;

$-{ }^{\tau} \mathcal{E}_{i}$ est égal à $\left(\operatorname{Id}_{X} \times \operatorname{Frob}_{S}\right)^{*} \mathcal{E}_{i}$;

- $j_{i}$ et $t_{i}$ sont des injections $\mathcal{D}_{X \times S}$-linéaires;

- $\mathcal{E}_{i+d} \simeq \mathcal{E}_{i}(\infty):=\mathcal{E}_{i} \otimes_{\mathcal{O}_{X}} \mathcal{O}_{X}(\infty)$ et le composé $\mathcal{E}_{i} \rightarrow \mathcal{E}_{i+1} \rightarrow \cdots \rightarrow \mathcal{E}_{i+d}$ est induit par l'injection canonique $\mathcal{O}_{X} \hookrightarrow \mathcal{O}_{X}(\infty)$;

- $\left(p r_{S}\right)_{*}\left(\mathcal{E}_{i} / \mathcal{E}_{i-1}\right)$ est un $\mathcal{O}_{S}$-module localement libre de rang $d$ où $p r_{S}: X \times S \rightarrow S$ est la projection canonique. De manière équivalente, $\mathcal{E}_{i} / \mathcal{E}_{i-1}$ est isomorphe à l'image directe $\left(\widetilde{i_{\infty}}\right)_{*}\left(\Gamma_{\infty, i}\right)$ d'un $\mathcal{O}_{S}$-module $\Gamma_{\infty, i}$ localement libre de rang $d$, par la section $\infty:\left(\widetilde{i_{\infty}}\right): S \longrightarrow$ $X \times S, s \longmapsto(\infty, s)$;

- l'image directe de Coker $t_{i}$ est un $\mathcal{O}_{S}$-module localement libre de rang $d$. Le support de Coker $t_{i}$ est disjoint de Bad $\cup\{\infty\} \times S$, où Bad désigne l'ensemble des places $x$ de $X$ telles que $\mathcal{D}_{x}$ n'est pas isomorphe à $\mathbb{M}_{d}\left(\mathcal{O}_{x}\right)$. De manière équivalente, Coker $t_{i}$ est isomorphe à l'image directe $\left(\widetilde{i_{0, i}}\right)_{*}\left(\Gamma_{0, i}\right)$ d'un $\mathcal{O}_{S}$-module $\Gamma_{0, i}$ localement libre de rang $d$, par la section

$$
\left(\widetilde{i_{0, i}}\right): S \stackrel{\left(i_{0, i}, i d_{S}\right)}{\longrightarrow} X \times S
$$

induite par un morphisme $i_{0, i}: S \rightarrow X$ tel que $i_{0, i}(S) \subset\left|X^{\prime}\right|$.

Remarque : Les inclusions $\mathcal{E}_{i} \hookrightarrow \mathcal{E}_{i+1}$ étant des isomorphismes sur $(X \backslash\{\infty\}) \times S$ et le support de Coker $t_{i}$ étant disjoint de $\infty \times S$, on en déduit que la donnée des morphismes $\left(t_{i}\right)_{i}$ est équivalente à la donnée d'un seul $t_{i}$. Les morphismes $i_{0, i}$ sont indépendants de $i$; on le note $i_{0}$, le morphisme caractéristique du $\mathcal{D}$-faisceau elliptique.

I.1.2.2. Définition. - (cf. [19]) Étant donnés $\left(\mathcal{E}_{i}, j_{i}, t_{i}\right)$ un $\mathcal{D}$-faisceau elliptique défini sur $S$ et $I$ un idéal de $A$ tel que $V(I) \cap i_{0}(S)=\emptyset$, une $I$-structure de niveau sur $\left(\mathcal{E}_{i}, j_{i}, t_{i}\right)$ est un isomorphisme de $\mathcal{D}_{I \times S}$-modules à droite, $\tilde{\iota}_{I}: \mathcal{D}_{I} \otimes \mathcal{O}_{S} \stackrel{\sim}{\longrightarrow} \mathcal{E}_{I \times S}$ tel que le diagramme suivant est commutatif

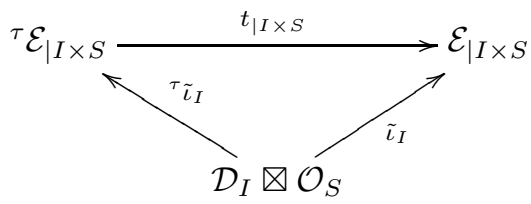

Pour définir la notion de structure de niveau en une place $v \in i_{0}(S)$ d'après [5], on est amené à considérer les objets suivants. Pour alléger les notations on prend $v=o$ ce qui sera d'ailleurs le cas dans la suite.

I.1.2.3. Définitions. - - Le $\left(\mathcal{O}_{o} \otimes \mathcal{O}_{S}\right)$-module $\mathcal{E}_{i} \otimes \mathcal{O}_{o}$ est indépendant de $i$; on le note $\mathcal{E}_{o}$. Un isomorphisme $\mathcal{D}_{o} \simeq \mathbb{M}_{d}\left(\mathcal{O}_{o}\right)$ étant fixé, soit $\mathcal{F}_{o}$ le $\left(\mathcal{O}_{o} \otimes \mathcal{O}_{S}\right)$-module libre de rang $d$ défini par $E_{1,1} \cdot \mathcal{E}_{o}$, où $E_{1,1}$ est l'idempotent de $\mathbb{M}_{d}\left(\mathcal{O}_{o}\right)$ associé au premier vecteur de la base canonique. Par équivalence de Morita, on a un isomorphisme $\mathcal{D}_{o}$-équivariant $\mathcal{E}_{o} \simeq \mathcal{F}_{o}^{d}$. Les morphismes $t_{i}$ induisent alors un morphisme $t_{o}^{\prime}:{ }^{\tau} \mathcal{F}_{o} \longrightarrow \mathcal{F}_{o}$. Le $\phi$-faisceau associé est par définition le couple $\left(\mathcal{F}_{o}, \phi_{o}\right)$ avec $\phi_{o}=t_{o}^{\prime}:{ }^{\tau} \mathcal{F}_{o} \longrightarrow \mathcal{F}_{o}$.

- Le $\mathcal{O}_{o}$-module de Dieudonné sur $B$ associé à $\left(\mathcal{E}_{i}, j_{i}, t_{i}\right)$ est le couple $\left(V_{o}, \varphi_{o}\right)$ où $V_{o}$ est le $\left(\mathcal{O}_{o} \hat{\otimes}_{\kappa(o)} \mathcal{O}_{S}\right)$-module localement libre de rang d,

$$
\mathcal{F}_{o} \otimes_{\left(\mathcal{O}_{o} \otimes_{\kappa(o)} \mathcal{O}_{S}\right)}\left(\mathcal{O}_{o} \hat{\otimes}_{\kappa(o)} \mathcal{O}_{S}\right)
$$

et $\varphi_{o}:\left(\operatorname{Id}_{\mathcal{O}_{o}} \hat{\otimes}_{\kappa(o)} \operatorname{Frob}_{\kappa(o)}\right)^{*} V_{o} \longrightarrow V_{o}$ est l'application induite par $\phi_{o}$ Pour tout $n$, on notera $V_{o, n}:=V_{o} \varpi_{o}^{n} \backslash V_{o}$. 
- Pour tout $n$, on note $\mathcal{F}_{o, n}=\mathcal{F}_{o} \otimes_{\mathcal{O}_{o}}\left(\mathcal{O}_{o} \backslash \mathcal{M}_{o}^{-n}\right)$ et on considère $\operatorname{Gr}\left(\mathcal{F}_{o, n}\right)$, le S-schéma en $\mathcal{O}_{o}$-modules finis, d'ordre $q^{n d}$ tués par $\varpi_{o}^{n}$, qui représente le foncteur de la catégorie des $S$-schémas dans celle des ensembles:

$$
S^{\prime} \mapsto\left\{u \in \operatorname{Hom}_{\mathcal{O}_{S}}\left(\mathcal{F}_{o, n}, S^{\prime}\right) / u\left(\phi_{o, n}(x)\right)=u(x)^{q} \forall x \in \mathcal{F}_{o, n}\right\}
$$

On a alors la suite exacte

$$
0 \longrightarrow \operatorname{Gr}\left(\mathcal{F}_{o, n}\right) \stackrel{i_{n}}{\longrightarrow} \operatorname{Gr}\left(\mathcal{F}_{o, n+1}\right) \stackrel{\varpi_{o}^{n}}{\longrightarrow} \operatorname{Gr}\left(\mathcal{F}_{o, n+1}\right) .
$$

- Si $P$ est un $R$-point d'un schéma $Y$, on note $[P]$ le sous-R-schéma de $Y$ qu'il définit. Pour $\left(P_{i}\right)$ une famille finie de tels points, on note $\sum\left[P_{i}\right]$ le sous-schéma de $Y$ défini par le faisceau d'idéaux produit des faisceaux d'idéaux définissant les $\left[P_{i}\right]$.

- Une $\mathcal{M}_{o}^{n}$-structure de niveau sur $\left(\mathcal{E}_{i}, j_{i}, t_{i}\right) / S$ est un homomorphisme de $\mathcal{O}_{o}$-modules $\iota_{o, n}^{\prime}$ : $\left(\mathcal{M}_{o}^{-n} / \mathcal{O}_{o}\right)^{d} \longrightarrow \operatorname{Gr}\left(\mathcal{F}_{o, n}\right)(S)$ tel que le sous-schéma

$$
\sum_{z \in\left(\mathcal{O}_{o} / \mathfrak{M}_{o}^{n}\right)^{d}}\left[\iota_{o, n}^{\prime}(z)\right]
$$

de $\operatorname{Gr}\left(\mathcal{F}_{o, n}\right)$ coïncide avec $\operatorname{Gr}\left(\mathcal{F}_{o, n}\right)$.

Remarque : Pour tout élément $z$ de $\left(\mathcal{O}_{o} / \mathcal{M}_{o}^{n}\right)^{d}, \iota_{o, n}^{\prime}(z)$ est un élément de $\mathcal{F}_{o, n}^{*}$ tel que $\phi_{o, n}^{*}\left(\iota_{o, n}^{\prime}(z)\right)=\iota_{o, n}^{\prime}(z)^{q}$. Le morphisme $\iota_{o, n}^{\prime}$ fournit alors un homomorphisme de $\mathcal{O}_{o}$-modules $\left(\mathcal{O}_{o} / \mathcal{M}_{o}^{n}\right)^{d} \longrightarrow \mathcal{F}_{o, n}^{*}$ qui après équivalence de Morita, donne un homomorphisme de $\mathcal{D}_{o}$-modules $\iota_{o, n}: \mathcal{D}_{o, n} \longrightarrow \mathcal{E}_{o, n}^{*}$ tel que le diagramme ci-dessous commute

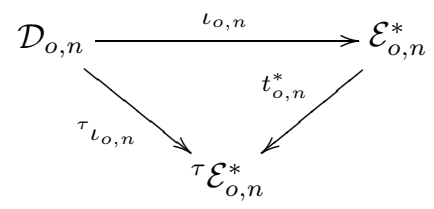

Dans la suite, $\iota_{o, n}$ désignera la structure de niveau après équivalence de Morita.

I.1.2.4. Proposition. - (cf. [5] propositions (7.1.3) et (7.1.4)) Soit

$$
\iota_{o, n}^{\prime}:\left(\mathcal{M}_{o}^{-n} / \mathcal{O}_{o}\right)^{d} \longrightarrow \operatorname{Gr}\left(\mathcal{F}_{o, n}\right)(S)
$$

une $\mathcal{M}_{o}^{n}$-structure de niveau définie sur un schéma $S$ tel que $S^{o}$ l'ouvert complémentaire de $i_{0}^{-1}(\operatorname{Spec} \kappa(o))$, est non vide. Alors $\iota_{o, n}^{\prime} \otimes_{\mathcal{O}_{S}} \mathcal{O}_{S}\left(S^{o}\right)$ induit un isomorphisme de $\mathcal{O}_{o}$-modules

$$
\left(\mathcal{O}_{o} / \mathcal{M}_{o}^{n}\right)^{d} \otimes \mathcal{O}_{S}\left(S^{o}\right) \longrightarrow\left(\mathcal{F}_{o, n} \otimes_{\mathcal{O}_{S}} \mathcal{O}_{S}\left(S^{o}\right)\right)^{*}
$$

Réciproquement soit $S$ est un schéma intègre tel que $S^{o}$ est non vide. Si l'homomorphisme de $\mathcal{O}_{o}$-modules $\iota_{o, n}^{\prime}:\left(\mathcal{M}_{o}^{-n} / \mathcal{O}_{o} /\right)^{d} \longrightarrow G r\left(\mathcal{F}_{o, n}\right)(S)$ induit un isomorphisme sur $S^{o}\left(\mathcal{M}_{o}^{-n} / \mathcal{O}_{o}\right)^{d} \nabla$ $\mathcal{O}_{S}\left(S^{o}\right) \stackrel{\sim}{\longrightarrow}\left(\mathcal{F}_{o, n} \otimes_{\mathcal{O}_{S}} \mathcal{O}_{S}\left(S^{o}\right)\right)^{*}$, alors $\iota_{o, n}^{\prime}$ est une $\mathcal{M}_{o}^{n}$-structure de niveau.

\section{I.1.3. Schémas de modules et correspondances de Hecke. -}

I.1.3.1. Proposition. - (cf. [19]) Le classifiant des classes d'équivalence des $\mathcal{D}$-faisceaux elliptiques munis d'une $I$-structure de niveau, définit un schéma régulier (un champ si $I=A$ ), $M_{I} \rightarrow X^{\prime}$ de dimension relative $d-1$.

I.1.3.2. Définition. - On notera $M_{I, o}:=M_{I} \times_{X^{\prime}} \operatorname{Spec}\left(\mathcal{O}_{o}\right)$ et $M_{I, s_{o}}\left(\operatorname{resp} . M_{I, \eta_{o}}\right)$ la fibre spéciale (resp. générique) de $M_{I, o}$.

I.1.3.3 - Soient $\mathbb{A}$ l'anneau des adèles de $F$ et $D_{\mathbb{A}}^{\times}:=D^{\times} \otimes_{F} \mathbb{A}$. La limite projective $M_{o}:=$ $\lim _{I} M_{I, o}$ est munie d'une action par correspondance de Hecke de $\left(D_{\mathbb{A}}^{\infty}\right)^{\times}$. Au dessus de la fibre 
générique $M_{\eta_{o}}$ de $M_{o}$ et à niveau fini, pour $K_{\mathbb{A}}^{\infty}$ un sous-groupe compact ouvert de $\left(D_{\mathbb{A}}^{\infty}\right)^{\times}$, l'action d'un élément $g^{\infty}$ de $\left(D_{\mathbb{A}}^{\infty}\right)^{\times}$se décrit par la correspondance géométrique

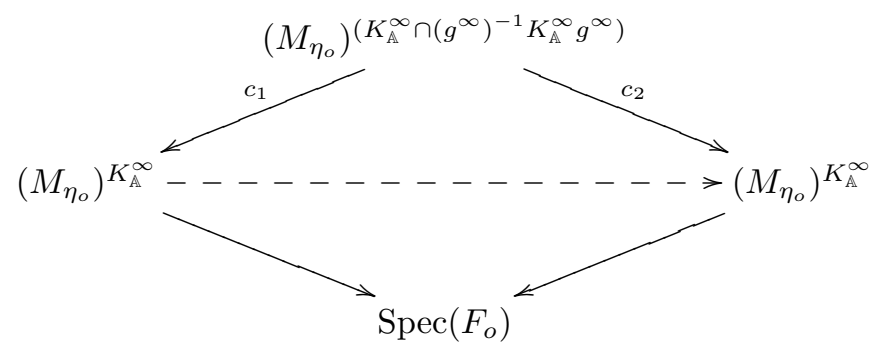

où le morphisme $c_{1}$ (resp. $c_{2}$ ) est induit par l'inclusion ${ }^{(2)}$

$$
\left(K_{\mathbb{A}}^{\infty} \cap\left(g^{\infty}\right)^{-1} K_{\mathbb{A}}^{\infty} g^{\infty}\right) \subset K_{\mathbb{A}}^{\infty}\left(\operatorname{resp} .\left(K_{\mathbb{A}}^{\infty} \cap\left(g^{\infty}\right)^{-1} K_{\mathbb{A}}^{\infty} g^{\infty}\right) \stackrel{A d\left(g^{\infty}\right)}{\longrightarrow} K_{\mathbb{A}}^{\infty}\right)
$$

En particulier on note $K_{\mathbb{A}, I}^{\infty}$ le noyau de $\left(\mathcal{D}_{\mathbb{A}}^{\infty}\right)^{\times} \longrightarrow \prod_{x \neq \infty} \mathcal{D}_{x}^{\times} \otimes_{\mathcal{O}_{x}} \mathcal{O}_{x} / \mathcal{M}_{x}^{n_{x}}$, où $n_{x}$ est la multiplicité de $x$ dans $I$. On a $\left(M_{\eta_{o}}\right)^{K_{\AA}, I}=M_{I, \eta_{o}}$.

I.1.3.4 - Dans [5] $§ 7.3$, on montre que ces correspondances s'étendent de manière unique sur $M_{o}$ tout entier. Si la composante $g_{o}^{\infty}$ de $g^{\infty}$ en $o$ est triviale, le résultat est évident. Précisons la situation pour $g_{o} \in G L_{d}\left(F_{o}\right) \cap \mathbb{M}_{d}\left(\mathcal{O}_{o}\right)$. On pose $S:=M_{o}$ et $S^{o}:=M_{\eta_{o}}$ et on considère le $\mathcal{D}$-faisceau elliptique universel sur $S$ muni de sa $I$-structure de niveau universelle pour tout idéal $I$ de $A$. La structure de niveau fournit un morphisme

$$
\iota_{o}^{\prime}:\left(F_{o} / \mathcal{O}_{o}\right)^{d} \times S \longrightarrow \mathcal{F}_{o}^{*} \otimes_{\mathcal{O}_{o}}\left(F_{o} / \mathcal{O}_{o}\right)
$$

selon les notations précédentes. Sur l'ouvert $S^{o}, \iota_{o}^{\prime o}:=\iota_{o}^{\prime} \otimes_{\mathcal{O}_{o}} F_{o}$ est un isomorphisme. A $g_{o}$ on associe, d'après loc. cit., la multiplication à gauche de ${ }^{t} g_{o} \times \operatorname{sur}\left(F_{o} / \mathcal{O}_{o}\right)^{d}$ et donc le morphisme

$$
\left[g_{o}^{*}\right]: \mathcal{F}_{o}^{*} \otimes_{\mathcal{O}_{o}}\left(F_{o} / \mathcal{O}_{o}\right) \longrightarrow \mathcal{F}_{o}^{*} \otimes_{\mathcal{O}_{o}}\left(F_{o} / \mathcal{O}_{o}\right)
$$

tel que sa restriction $\left[g_{o}^{*}\right]^{o}$ à $S_{o}$ est par définition induite, via l'isomorphisme $\iota_{o}^{\prime o}$, par la multiplication à gauche de ${ }^{t} g_{o}$ sur $\left(F_{o} / \mathcal{O}_{o}\right)^{d}$. On note $\left[g_{o}\right]$ l'endomorphisme de $\mathcal{F}_{o}$ déduit de $\left[g_{o}^{*}\right]$. L'image par $g_{o}$ du $\mathcal{D}$-faisceau elliptique universel $\left(\left(\mathcal{E}_{i}, j_{i}, t_{i}\right), \iota_{\infty}\right)$ est le $\mathcal{D}$-faisceau $\left(\left(\mathcal{E}_{i}^{\prime}, j_{i}^{\prime}, t_{i}^{\prime}\right), \iota_{\infty}^{\prime}\right)$ défini via les diagrammes cartésiens

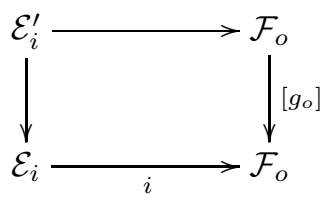

où $i$ est l'injection canonique, ou de manière duale par $\left(\mathcal{E}^{\prime}\right)^{*}:=\mathcal{E}^{*} \otimes_{\mathcal{E}_{o}^{*},\left[g_{o}^{*}\right.} \mathcal{E}_{o}^{*}$. La structure de niveau est alors définie de manière naturelle comme suit. Soient $n$ et $m$ tels que $\operatorname{Ker}^{t} g_{o} \subset\left(\mathcal{M}_{o}^{-n} / \mathcal{O}_{o}\right)^{d}$ et $\left(\mathcal{M}_{o}^{-m} / \mathcal{O}_{o}\right)^{d} \subset \operatorname{Im} g_{o}$. Si $\iota_{o, n}$ est la structure de niveau $n$ sur $\mathcal{F}_{o, n}$, la composée

$$
\left(\mathcal{M}_{o}^{-m} / \mathcal{O}_{o}\right)^{d} \longrightarrow\left(\mathcal{M}_{o}^{-n} / \mathcal{O}_{o}\right)^{d} / \operatorname{Ker} g_{o} \stackrel{\iota_{o, n}}{\longrightarrow} \mathcal{F}_{o, n}^{*} \stackrel{\left[g_{o}^{*}\right]}{\longrightarrow} \mathcal{F}_{o, m}^{*}
$$

définit une flèche $\iota_{o, m}^{\prime}:\left(\mathcal{M}_{o}^{-m} / \mathcal{O}_{o}\right)^{d} \longrightarrow\left(\mathcal{F}_{o, n}^{\prime}\right)^{*}$ dont on vérifie aisément en utilisant la proposition (I.1.2.4), qu'elle définit une structure de niveau $m$.

${ }^{(2)} \mathrm{cf.}[\mathbf{1 9}] \S 7$ 
I.1.4. Structures de niveau à l'infini. — On résume simplement ici le paragraphe 8 de [19]. I.1.4.1 - Étant donné un $\mathbb{F}_{q}$-schéma $S$ et $\left(\mathcal{E}_{i}, j_{i}, t_{i}\right)$ un $\mathcal{D}$-faisceau elliptique sur $S$, on considère pour tout $i \in \mathbb{Z}$, les $\mathcal{O}_{\infty} \hat{\otimes} \mathcal{O}_{S}$-modules localement libre de rang $d^{2}$ :

$$
\check{M}_{i}:=\mathcal{E}_{i}^{\vee} \mid(X \times S)_{\infty}^{\vee}
$$

où $\mathcal{E}_{i}^{\vee}$ est le faisceau dual de $\mathcal{E}_{i}$ et $(X \times S)_{\infty}^{\vee}$ est la complétion de $X \times S$ le long de $\{\infty\} \times S$. Les $j_{i}$ définissent alors un système inductif $\check{M}:=\cdots \hookrightarrow \check{M}_{i} \hookrightarrow \check{M}_{i+1} \hookrightarrow \cdots$ et l'on identifie les $\check{M}_{i}$ avec leur image dans $\check{M}$ qui est donc un $F_{\infty} \hat{\otimes} \mathcal{O}_{S}$-module de rang $d^{2}$. Les $t_{i}$ induisent alors un isomorphisme

$$
\check{M} \stackrel{\sim}{\longrightarrow}{ }^{\tau} \check{M}=\left(F_{\infty} \hat{\otimes} \operatorname{Frob}_{S}\right)^{*} \check{M}
$$

qui envoie $\check{M}_{i}$ surjectivement sur ${ }^{\tau} \check{M}_{i+1}$. On définit alors $\check{\psi}:{ }^{\tau} \check{M} \longrightarrow \check{M}$ comme l'inverse de l'isomorphisme ci-dessus; $\check{\psi}\left({ }^{\tau} \check{M}_{i}\right)=\check{M}_{i-1} \subset \check{M}_{i}$.

I.1.4.2 - L'action à droite de $\mathcal{D}$ sur les $\mathcal{E}_{i}$ induit une action à gauche sur $\check{M}$ qui commute à $\check{\psi}$ et qui stabilise $\check{M}_{i} \subset \check{M}$. Un isomorphisme $\mathcal{D}_{\infty} \simeq \mathbb{M}_{d}\left(\mathcal{O}_{\infty}\right)$ étant fixé, on a une équivalence de Morita

$$
(\check{M}, \check{\psi})=(\check{N}, \check{\psi})^{d} \text { et } \check{M}_{i}=\left(\check{N}_{i}\right)^{d}
$$

Ainsi $\check{N}$ est un $F_{\infty} \hat{\otimes} \mathcal{O}_{S}$-module de rang $d$ et $\check{N}_{i} \subset \check{N}$ en est un $\mathcal{O}_{\infty} \hat{\otimes} \mathcal{O}_{S}$-sous-module localement libre de rang $d$ vérifiant

$$
\varpi_{\infty} \check{N}_{i}=\check{N}_{i-(\operatorname{deg} \infty) d} \subset \cdots \subset \check{N}_{i-1} \subset \check{N}_{i}, \quad \check{\psi}\left({ }^{\tau} \check{N}_{i}\right)=\check{N}_{i-1}
$$

tel que le quotient $\check{N}_{i} / \varpi_{\infty} \check{N}_{i} \longrightarrow \check{N}_{i} / \check{\psi}\left({ }^{\tau} \check{N}_{i}\right)$ est un $\mathcal{O}_{\{\infty\} \times S}$-module supporté par le graphe d'un $\mathbb{F}_{q}$-morphisme de schéma $i_{\infty, i}: S \longrightarrow\{\infty\}$ et est localement libre de rang 1 sur son support avec $i_{\infty, i+1}=i_{\infty, i} \circ$ Frob $_{S}$.

I.1.4.3 - Soit $S$ un schéma muni d'un $\mathbb{F}_{q}$-morphisme de schéma $i_{\infty, 0}: S \longrightarrow\{\infty\}$. Tout autre $\mathbb{F}_{q}$-morphisme de schéma de $S$ vers $\{\infty\}$ est alors de la forme $i_{\infty, i}:=i_{\infty, 0} \circ \operatorname{Frob}_{S}^{i}$ pour un unique $i \in \mathbb{Z} / \operatorname{deg}(\infty) \mathbb{Z}$. On associe au couple $\left(S, i_{\infty, 0}\right)$ le triplet $\left(N_{d, 1}, \psi_{d, 1}, \mathcal{N}_{d, 1}\right)$ où

$$
N_{d, 1}=\left(F_{\infty} \hat{\otimes} \mathcal{O}_{S}\right)^{d}=\bigoplus_{i=0}^{\operatorname{deg}(\infty)-1}\left(F_{\infty} \hat{\otimes}_{\kappa(\infty), i_{\infty, i}^{*}} \mathcal{O}_{S}\right)^{d}
$$

de base canonique $\left(e_{i, j}\right)_{0 \leqslant i<\operatorname{deg}(\infty), 1 \leqslant j \leqslant d}, \psi_{d, 1}:{ }^{\tau} N_{d, 1} \stackrel{\sim}{\longrightarrow} N_{d, 1}$ est défini par $\psi_{d, 1}\left(e_{i, j}\right)=e_{i+1, j}$ pour $i \neq \operatorname{deg}(\infty)-1$ et $\psi_{d, 1}\left(e_{\operatorname{deg}(\infty)-1, j}\right)=e_{0, j-1}$ pour $j \neq 1$ et $\varpi_{\infty} e_{0, d}$ pour $j=1$, et

$$
\mathcal{N}_{d, 1}=\left(\mathcal{O}_{\infty} \hat{\otimes} \mathcal{O}_{S}\right)^{d}=\bigoplus_{i=0}^{\operatorname{deg}(\infty)-1}\left(\mathcal{O}_{\infty} \hat{\otimes}_{\kappa(\infty), i_{\infty, i}^{*}} \mathcal{O}_{S}\right)^{d}
$$

I.1.4.4 - Soit $\kappa(\infty)_{d}$ l'extension de degré $d$ de $\kappa(\infty)$ et soient $F_{\infty, d}=F_{\infty} \hat{\otimes}_{\kappa(\infty)} \kappa(\infty)_{d}, \mathcal{O}_{\infty, d}$ son anneau des entiers et $\sigma_{\infty, d}=F_{\infty} \hat{\otimes}_{\kappa(\infty)} \operatorname{Frob}_{q}^{\operatorname{deg}(\infty)}$. Soit $F_{\infty, d}\left[\tau_{\infty}\right]$ l'algèbre de polynômes sur $F_{\infty, d}$, non commutative avec la règle de commutation $\tau_{\infty} a=\sigma_{\infty, d}^{-1}(a) \tau_{\infty}$ pour tout $a \in F_{\infty, d}$. L'élément $\tau_{\infty}^{d}-\varpi_{\infty}$ est alors central dans $F_{\infty, d}\left[\tau_{\infty}\right]$ et $\bar{D}_{\infty}:=F_{\infty, d}\left[\tau_{\infty}\right] /\left(\tau_{\infty}^{d}-\varpi_{\infty}\right)$ est alors "l" 'algèbre à division centrale sur $F_{\infty}$ d'invariant $-1 / d$, d'ordre maximal $\overline{\mathcal{D}}_{\infty}:=\mathcal{O}_{\infty, d}\left[\tau_{\infty}\right] /\left(\tau_{\infty}^{d}-\varpi_{\infty}\right)$.

I.1.4.5 - Si $\lambda: S \longrightarrow \operatorname{Spec}\left(\kappa(\infty)_{d}\right)$ est un $\mathbb{F}_{q}$-morphisme de schémas, on peut construire une injection de $F_{\infty}$-algèbres

$$
\lambda^{*}: \bar{D}_{\infty} \hookrightarrow \operatorname{End}\left(N_{d, 1}, \psi_{d, 1}\right)
$$

de la façon suivante où $i_{\infty, 0}: S \stackrel{\lambda}{\longrightarrow} \operatorname{Spec}\left(\kappa(\infty)_{d}\right) \stackrel{\text { can }}{\longrightarrow}\{\infty\}$. Pour $\alpha \in \kappa(\infty)_{d}$, l'image de $1 \hat{\otimes} \alpha \in$ $F_{\infty, d} \subset \bar{D}_{\infty}$ est donnée par

$$
\lambda^{*}(1 \hat{\otimes} \alpha)\left(e_{i, j}\right)=\left(1 \hat{\otimes} \lambda^{*}\left(\operatorname{Frob}_{q}^{i-j \operatorname{deg}(\infty)}(\alpha)\right)\right) e_{i, j}
$$


et l'image de $\tau_{\infty} \in \bar{D}_{\infty}$ par

$$
\lambda^{*}\left(\tau_{\infty}\right)\left(e_{i, j}\right)=\left\{\begin{array}{l}
\varpi_{\infty} e_{i, d} \text { si } j=1 \\
e_{i, j-1} \text { sinon }
\end{array}\right.
$$

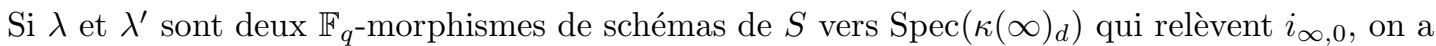
$\lambda^{\prime}=\lambda \circ \operatorname{Frob}_{S}^{n \operatorname{deg}(\infty)}$ pour $n \in \mathbb{Z} / d \mathbb{Z}$ et alors $\left(\lambda^{\prime}\right)^{*}=\lambda^{*} \circ \operatorname{Ad}\left(\tau_{\infty}^{-n}\right)$. On vérifie aussi que $\lambda^{*}\left(\bar{D}_{\infty}\right)$ laisse $\mathcal{N}_{d, 1}$ stable.

I.1.4.6. Définition. - Une structure de niveau à l'infini sur $\left(\mathcal{E}_{i}, j_{i}, t_{i}\right)$ est une paire $(\lambda, \alpha)$ où $\lambda: S \longrightarrow \operatorname{Spec}\left(\kappa(\infty)_{d}\right)$ est un $\mathbb{F}_{q}$-morphisme de schémas qui relève le pôle $i_{\infty, 0}$ et où $\alpha: \mathcal{N}_{d, 1} \stackrel{\sim}{\longrightarrow} \check{N}_{0}$ est un isomorphisme de $\mathcal{O}_{\infty} \hat{\otimes} \mathcal{O}_{S}$-modules tel que le diagramme suivant soit commutatif

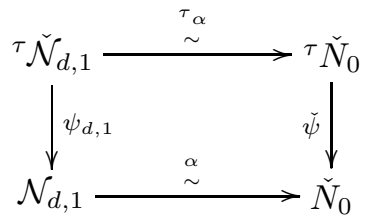

I.1.4.7 - On a une notion évidente d'isomorphisme entre $\mathcal{D}$-faisceaux elliptiques sur $S$ munis d'une structure de niveau à l'infini; on note $\mathcal{M}_{I}$ (resp. $\widetilde{\mathcal{M}_{I}}(S)$ ) la catégorie des classes d'isomorphismes des $\mathcal{D}$-faisceaux elliptiques sur $S$ munis d'une structure de niveau $I$ (resp. et d'une structure de niveau à l'infini). On obtient ainsi une catégorie fibrée $\widetilde{\mathcal{M}}_{I}$ qui est un pro-champ; en effet se donner un isomorphisme

$$
\alpha: \mathcal{N}_{d, 1} \stackrel{\sim}{\longrightarrow} \check{N}_{0}
$$

de $\mathcal{O}_{\infty} \hat{\otimes} \mathcal{O}_{S}$-modules, revient à se donner un système projectif

$$
\left(\alpha_{n}=\alpha \bmod \varpi_{\infty}^{n+1}\right)_{n \geqslant 0}
$$

d'isomorphismes de $\mathcal{O}_{\infty} /\left(\varpi_{\infty}^{n+1}\right) \hat{\otimes} \mathcal{O}_{S}$-modules et $\alpha$ commute avec les $\psi$ si et seulement si les $\psi_{n}$ commutent avec les $\psi$ modulo $\varpi_{\infty}^{n+1}$.

I.1.4.8 - On a en outre un morphisme d'oubli

$$
r_{\infty, I}: \widetilde{\mathcal{M}_{I}} \longrightarrow \mathcal{M}_{I}
$$

qui envoie $\left(\left(\mathcal{E}_{i}, j_{i}, t_{i}\right), \iota_{I},(\lambda, \alpha)\right)$ sur $\left(\left(\mathcal{E}_{i}, j_{i}, t_{i}\right), \iota_{I}\right)$, ainsi qu'un morphisme de structure

$$
\lambda_{I}: \widetilde{\mathcal{M}_{I}} \longrightarrow \operatorname{Spec}\left(\kappa(\infty)_{d}\right)
$$

qui envoie $\left(\left(\mathcal{E}_{i}, j_{i}, t_{i}\right), \iota_{I},(\lambda, \alpha)\right)$ sur $\alpha$ et qui relève $i_{\infty, 0} \circ r_{\infty, I}$. Pour $I \subset J \subset X \backslash\{\infty\}$, on a des 2-diagrammes commutatifs

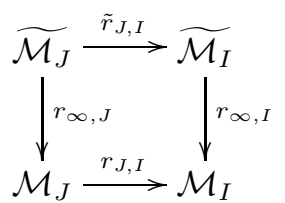

I.1.4.9 - Sur $\widetilde{\mathcal{M}_{I}}$, on a des actions continues à droites du groupe profini $\overline{\mathcal{D}}_{\infty}^{\times}$et de $\mathbb{Z} / d \mathbb{Z}: \delta \in \overline{\mathcal{D}}_{\infty}^{\times}$ (resp. $n \in \mathbb{Z} / d \mathbb{Z})$ envoie $\left(\left(\mathcal{E}_{i}, j_{i}, t_{i}\right), \iota_{I},(\lambda, \alpha)\right)$ sur

$$
\left(\left(\mathcal{E}_{i}, j_{i}, t_{i}\right), \iota_{I},\left(\lambda, \alpha \circ \lambda^{*}(\delta)\right)\right) \quad\left(\operatorname{resp} .\left(\left(\mathcal{E}_{i}, j_{i}, t_{i}\right), \iota_{I},\left(\lambda \circ \operatorname{Frob}_{S}^{n \operatorname{deg}(\infty)}, \alpha\right)\right)\right) .
$$

Comme on a

$$
\left(\lambda \circ \operatorname{Frob}_{S}^{n \operatorname{deg}(\infty)}\right)^{*}=\lambda^{*} \circ \operatorname{Ad}\left(\tau_{\infty}^{-n}\right)
$$

ces deux actions induisent une action à droite continue du groupe profini

$$
\overline{\mathcal{D}}_{\infty}^{\times} \rtimes \mathbb{Z} / d \mathbb{Z}
$$


où $n \in \mathbb{Z} / d \mathbb{Z}$ agit sur $\overline{\mathcal{D}}_{\infty}^{\times}$par $A d\left(\tau_{\infty}^{-n}\right)$. On identifie alors ce produit semi-direct avec

$$
\bar{D}_{\infty}^{\times} / \varpi_{\infty}^{\mathbb{Z}}
$$

en envoyant $(\delta, n)$ sur $\delta \tau_{\infty}^{-n}$. On obtient ainsi une action à droite de $\bar{D}_{\infty}^{\times} / \varpi_{\infty}^{\mathbb{Z}}$ qui commutent aux $r_{\infty, I}, \tilde{r}_{J, I}$ et $\lambda_{I}$ si on fait agir $\bar{D}_{\infty}^{\times} / \varpi_{\infty}^{\mathbb{Z}} \operatorname{sur} \operatorname{Spec}\left(\kappa(\infty)_{d}\right)$ à travers son quotient

$$
-\infty \circ \mathrm{rn}: \bar{D}_{\infty}^{\times} \longrightarrow \mathbb{Z} / d \mathbb{Z}
$$

$\left(\operatorname{Gal}\left(\kappa(\infty)_{d} / \kappa(\infty)\right)=\mathbb{Z} / d \mathbb{Z}\right)$.

I.1.4.10. Théorème. - (cf. [19] théorème (8.10) et proposition (8.8))

(i) Le morphisme de pro-champs

$$
r_{\infty, I}: \widetilde{\mathcal{M}_{I}} \longrightarrow \mathcal{M}_{I}
$$

est représentable et est un revêtement pro-galoisien de groupe de Galois $\bar{D}_{\infty}^{\times} / \varpi_{\infty}^{\mathbb{Z}} ; \widetilde{\mathcal{M}_{I}}$ est alors un schéma que l'on notera $\widetilde{M}_{I}$.

(ii) Les correspondances de Hecke sur les $M_{I}$ associées aux éléments de $\left(D_{\mathbb{A}}^{\infty}\right)^{\times} \times \mathbb{Z}$ se relèvent sur $\widetilde{M}_{I}$ et commutent à l'action de $\bar{D}_{\infty}^{\times} / \varpi_{\infty}^{\mathbb{Z}}$. De plus $F^{\times}$envoyé diagonalement dans $\left(\bar{D}_{\infty}^{\times} / \varpi_{\infty}^{\mathbb{Z}}\right) \times$ $\left(D_{\mathbb{A}}^{\infty}\right)^{\times}$agit trivialement sur les $\widetilde{M}_{I}$.

Remarque : La caractéristique étant disjointe de $\infty$, les arguments de loc. cit. s'appliquent sans modification sur les variétés $M_{I}$ même dans le cas de mauvaise réduction.

\section{I.1.5. Stratification de la fibre spéciale. -}

I.1.5.1. Définition. - Pour tout $1 \leqslant h \leqslant d$, on notera $P_{h, d}$ le parabolique standard associé aux $h$ premières coordonnées et soit $P_{h, d}^{o p}$ le parabolique opposé

I.1.5.2. Proposition. - (cf [20] proposition 2.4.6 et [5] lemme 6.2.3)

(a) Tout $\mathcal{O}_{o}$-module de Dieudonné $\left(V_{o}, \varphi_{o}\right)$ sur une extension $k$ de $\kappa(o)$ se décompose en une somme directe $\left(V_{o}^{c}, \varphi_{o}^{c}\right) \oplus\left(V_{o}^{e t}, \varphi_{o}^{e t}\right)$ où $\varphi_{o}^{c}$ est topologiquement nilpotent et $\varphi_{o}^{\text {et }}$ est bijective.

(b) Tout $\mathcal{O}_{o}$-module de Dieudonné $\left(V_{o}, \varphi_{o}\right)$ sur une $\mathcal{O}_{o}$ algèbre $R$ dans laquelle l'image de $\varpi_{o}$ est nilpotente, se dévisse en une suite exacte

$$
0 \longrightarrow\left(V_{o}^{e t}, \varphi_{o}^{e t}\right) \longrightarrow\left(V_{o}, \varphi_{o}\right) \longrightarrow\left(V_{o}^{c}, \varphi_{o}^{c}\right) \longrightarrow 0
$$

où $\varphi_{o}^{c}$ est topologiquement nilpotent et $\varphi_{o}^{e t}$ est bijective.

I.1.5.3. Définition. - Pour tout $1 \leqslant h \leqslant d$, on définit dans [5] un sous-schéma $M_{I, s_{o}}^{=h}$ de $M_{I, s_{o}}$ de pure dimension $d-h$, stabilisé par les correspondances de Hecke et caractérisé par l'une des propriétés équivalentes suivantes : en tout point géométrique de $M_{I, s_{o}}^{=h}$,

- le $\mathcal{O}_{o}$-module de Dieudonné qui lui est associé, a sa composante connexe de hauteur $h$.

- le $\phi$-faisceau $\left(\mathcal{F}_{o}, \phi_{o}\right)$ qui lui est associé est tel que si on note $M_{o, 1}$ la matrice de $\phi_{o, 1}:=$ $\phi_{o} \otimes_{\mathcal{O}_{o}}\left(\mathcal{O}_{o} \backslash \mathcal{M}_{o}^{-1}\right)$ dans une base quelconque de $\mathcal{F}_{o, 1}^{*}:=\mathcal{F}_{o}^{*} \otimes_{\mathcal{O}_{o}}\left(\mathcal{M}_{o} \backslash \mathcal{O}_{o}\right)$, alors d'après loc. cit.,

$$
M_{o, 1}^{! h}:=M_{o, 1}\left({ }^{\tau} M_{o, 1}\right) \cdots\left(\tau^{h-1} M_{o, 1}\right)
$$

a un mineure d'ordre $d-h$ inversible et tous ses mineures d'ordre $r>d-h$ sont nuls.

Remarque : Le schéma $M_{I, s_{o}}^{=h}$ n'est pas réduit sauf si la multiplicité de $o$ dans $I$ est nulle; on notera $M_{I, s_{o}, \text { red }}^{=h}$ le réduit associé.

I.1.5.4. Définitions. - Soit $I=I^{o} \mathcal{M}_{o}^{n}$ un idéal de A, $n$ étant la multiplicité de o dans $I$. L'ensemble

$$
G / P(d, h, n):=G L_{d}\left(\mathcal{O}_{o} / \mathcal{M}_{o}^{n}\right) / P_{h, d}\left(\mathcal{O}_{o} / \mathcal{M}_{o}^{n}\right)
$$


classifie les facteurs directs a de rang $h$ de $\left(\mathcal{M}_{o}^{-n} / \mathcal{O}_{o}\right)^{d}$. Pour tout $a \in G / P(d, h, n)$, on notera

$$
\begin{gathered}
P_{h, d ; a}\left(\mathcal{O}_{o} / \mathcal{M}_{o}^{n}\right):=a P_{h, d}\left(\mathcal{O}_{o}\right) a^{-1} \\
K_{o^{n}}:=\operatorname{Ker}\left(G L_{d}\left(\mathcal{O}_{o}\right) \longrightarrow G L_{d}\left(\mathcal{O}_{o} / \mathcal{M}_{o}^{n}\right)\right) \\
K_{o, h, d, n}(a)=K_{o^{n}} \cap P_{h, d ; a}^{o p}\left(\mathcal{O}_{o} / \mathcal{M}_{o}^{n}\right)
\end{gathered}
$$

I.1.5.5. Définition. - (cf. [5] définition (10.4.1)) Pour tout $a \in G / P(d, h, n)$, il existe un sousschéma fermé $M_{I, s_{o}, a}^{=h}$ de $M_{I, s_{o}}^{=h}$ stable sous l'action des correspondances de Hecke associées aux éléments de

$$
K_{\mathbb{A}}^{\infty} / K_{\mathbb{A}, I}^{\infty, o} \times K_{o, h, d, n}(a)
$$

Remarque : Concrètement si $\iota_{o, n}$ est la $\mathcal{M}_{o}^{n}$-structure de niveau universelle sur $M_{I, o}$ alors le noyau de $\iota_{o, n}^{e t}:=\left(\iota_{o, n} \times_{M_{I, o}} M_{I, s_{o}, a}^{=h}\right)_{\mid \mathcal{F}_{o}^{e t}}:\left(\mathcal{M}_{o}^{-n} / \mathcal{O}_{o}\right)^{d} \longrightarrow \mathcal{F}_{o}^{e t, *}$, où $\mathcal{F}_{o}^{e t}$ est la partie étale du $\phi$-faisceau universel sur $M_{I, s_{o}, a}^{=h}$ donnée par la proposition (I.1.5.2), est le facteur direct $a$ de $\left(\mathcal{M}_{o}^{-n} / \mathcal{O}_{o}\right)^{d}$.

I.1.5.6 - On en déduit alors le fait fondamental suivant.

Propriété géométrique fondamentale : les strates non supersingulières sont induites, i.e. ${ }^{(3)}$

$$
M_{I, s_{o}}^{=h}=M_{I, s_{o}, 1}^{=h} \times_{P_{h, d}^{o p}\left(\mathcal{O}_{o} / \mathcal{M}_{o}^{n}\right)} G L_{d}\left(\mathcal{O}_{o} / \mathcal{M}_{o}^{n}\right)
$$

où $M_{I, s_{o}, 1}^{=h}$ est la composante associée à la classe de $I_{d}$ dans $G / P(d, h, n)$.

I.1.5.7. Proposition. - L'ensemble des points géométriques de $M_{I, s_{o}, a}^{=h}$ au dessus d'un point géométrique donné de $M_{I^{\circ}, s_{o}}^{=h}$ est en bijection avec les isomorphismes

$$
\iota_{o, n}^{e t}:\left(\mathcal{M}_{o}^{-n} / \mathcal{O}_{o}\right)^{d} / a \longrightarrow \mathcal{F}_{o, n}^{e t, *}
$$

tels que $\phi_{o, n}^{e t, *} \circ \iota_{o, n}^{e t}={ }^{\tau} \iota_{o, n}^{e t}$ où $n$ est la multiplicité de o dans I. Cet ensemble est alors de cardinal $\# G L_{d-h}\left(\mathcal{O}_{o} / \mathcal{M}_{o}^{n}\right)$.

I.1.5.8. Définition. - On notera $M_{I, s_{o}}^{\geqslant h}\left(\operatorname{resp} . M_{I, s_{o}, a}^{\geqslant h}\right)$ l'adhérence schématique de $M_{I, s_{o}}^{=h}$ (resp. $\left.M_{I, s_{o}, a}^{=h}\right)$ dans $M_{I, s_{o}}$.

I.1.6. Théorème de Serre-Tate et conséquences. - D'après la théorie du module de coordonnées, cf. [12], Def ${ }_{n}^{d}$ représente aussi les déformations de niveau $n$, du $\mathcal{O}_{o}$-module de Dieudonné sur $\overline{\mathbb{F}}_{q}$ connexe de hauteur $d$. ${ }^{(4)}$

I.1.6.1. Définition. - Pour tout $1 \leqslant h \leqslant d$, soit $\operatorname{Def}_{n}^{d ;=h}$ la $\hat{\mathcal{O}}_{o}^{n r}$-algèbre qui classifie les déformations de niveau $n$ de $\Sigma_{d}$ telle que la composante connexe du $\mathcal{O}$-module de Dieudonné associé est de dimension $h$. On note $\operatorname{Def}_{n}^{d ; \geqslant h}$ son adhérence.

Remarque : Concrètement, cf. [5] $\S 9$, en notant $M_{1}$ la matrice modulo $\varpi_{o} \otimes 1$, de $\varphi$ dans une base du $\mathcal{O}_{o} \hat{\otimes}_{\kappa} \operatorname{Def}_{n}^{d}$-module $V$, $\operatorname{Def}_{n}^{d ; \geqslant h}$ est le quotient de $\operatorname{Def}_{n}^{d}$ par l'idéal engendré par tous les mineures d'ordre supérieur strictement à $d-h$ de

$$
M_{1}^{! h}:=M_{1}\left({ }^{\tau} M_{1}\right) \cdots\left({ }^{\tau^{h-1}} M_{1}\right) .
$$

${ }^{(3)}$ On rappelle que l'application canonique $G L_{d}\left(\mathcal{O}_{o}\right) \longrightarrow G L_{d}\left(\mathcal{O}_{o} / \mathcal{M}_{o}^{n}\right)$ est surjective.

(4) Plus généralement, on montre qu'il y a une équivalence de catégories, des déformations de niveau $n$ du $\mathcal{O}_{o}$-module divisible de type $(h, j)$ (la composante connexe est de hauteur $h$ et la partie étale de hauteur $j$ (cf. [9])) sur $\overline{\mathbb{F}}_{q}$ vers les déformations de niveau $n$ du $\mathcal{O}_{o}$-module de Dieudonné sur $\overline{\mathbb{F}}_{q}$ dont la partie connexe est de rang $h$ et la partie étale de rang $j$. Le foncteur de ces déformations est représentable (cf. [9] $)$ par l'anneau $\operatorname{Def}_{n}^{h, j} \simeq \operatorname{Def}_{n}^{h}\left[\left[w_{1}^{n}, \cdots, w_{j}^{n}\right]\right]$. 
I.1.6.2. Proposition-Définition. - (cf. [5] proposition (9.3.2)) On a une suite exacte

$$
0 \longrightarrow\left(V_{h}^{e t}, \varphi_{h}^{e t}\right) \longrightarrow(V, \varphi) \otimes_{\operatorname{Def}_{n}^{d}} \operatorname{Def}_{n}^{d ;=h} \longrightarrow\left(V_{h}^{c}, \varphi_{h}^{c}\right) \longrightarrow 0
$$

où $\left(V_{h}^{e t}, \varphi_{h}^{e t}\right)$ (resp. $\left.\left(V_{h}^{c}, \varphi_{h}^{c}\right)\right)$ est un $\mathcal{O}$-module de Dieudonné étale (resp. topologiquement nilpotent) de dimension $d-h$ (resp. $h$ ). La structure de niveau $n$ universelle sur $\operatorname{Def}_{n}^{d}$,

$$
\iota_{n}:\left(\mathcal{M}^{-n} / \mathcal{O}_{o}\right)^{d} \longrightarrow \mathcal{M}_{n}^{d}
$$

où $\mathcal{M}_{n}^{d}$ est l'idéal maximal de $\operatorname{Def}_{n}^{d}$, est alors telle que le noyau de

$$
\iota_{n}^{e t}:=\left(\iota_{n} \otimes_{\operatorname{Def}_{n}^{d}} \operatorname{Def}_{n}^{d ;=h}\right)_{\mid V_{h}^{e t}}:\left(\mathcal{M}^{-n} / \mathcal{O}\right)^{d} \longrightarrow \mathcal{M}_{n}^{d} \otimes_{\operatorname{Def}_{n}^{d}} \operatorname{Def}_{n}^{d ;=h}
$$

est un facteur direct a de $\left(\mathcal{M}_{o}^{-n} / \mathcal{O}_{o}\right)^{d}$, de sorte que

$$
\operatorname{Def}_{n}^{d ;=h}=\prod_{a \in G / P(d, h, n)} \operatorname{Def}_{n, a}^{d ;=h}
$$

où Def ${ }_{n, a}^{d ;=h}$ est muni d'une action de $K_{o^{n}} \cap P_{h, d ; a}\left(\mathcal{O}_{o} / \mathcal{M}_{o}^{n}\right)$.

I.1.6.3. Proposition. - (Théorème de Serre-Tate cf. [5] théorème 7.4.4) Le morphisme naturel du foncteur des déformations d'un point géométrique de $M_{I, o}$ vers le foncteur des déformations de niveau $n=\operatorname{mult}_{o}(I) d u \mathcal{O}_{o}$-module de Dieudonné qui lui est associé, est une équivalence de catégories. En outre l'action d'un élément $\left(g_{o}^{c}, \operatorname{Frob}_{o}^{r}\right) \in G L_{h}\left(F_{o}\right) \times W_{o}$ tel que $r=-\operatorname{val}\left(\operatorname{det} g_{o}^{c}\right)$ sur $M_{I, o}$, induit l'action de $\left({ }^{t}\left(g_{o}^{c}\right)^{-1}, \operatorname{Frob}_{o}^{r}\right) \in \mathcal{N}_{o}$ sur $\operatorname{Def}_{n}^{d}$.

Démonstration. - Par rapport à loc. cit., remarquons en effet que l'action de $g_{o}^{c}$ sur le $\mathcal{O}_{o}$-module de Dieudonné $\left(V_{o}, \varphi_{o}\right)$ est donnée par la multiplication à gauche par $g_{o}^{c}$, de sorte qu'étant donnée une structure de niveau $\iota_{o, n}:\left(\mathcal{M}_{o}^{-n} / \mathcal{O}_{o}\right)^{d} \longrightarrow G r\left(V_{o, n}, \varphi_{o, n}\right)$, l'action de $g_{o}^{c}$ est donnée par la multiplication à gauche de ${ }^{t} g_{o}^{c} \operatorname{sur}\left(\mathcal{M}_{o}^{-n} / \mathcal{O}_{o}\right)^{d}$ à comparer avec l'action définie au paragraphe précédent qui est donnée par la multiplication de $\left(g_{o}^{c}\right)^{-1}$ à gauche sur $\left(\mathcal{M}_{o}^{-n} / \mathcal{O}_{o}\right)^{d}$.

I.1.6.4. Corollaire. - (a) Le complété formel de l'anneau local de $M_{I, o}$ en un point géométrique quelconque de $M_{I, s_{o}}^{=h}$, est non canoniquement isomorphe ${ }^{(5)}$ à l'anneau qui représente les déformations d'un $\mathcal{O}_{o}$-module divisible de hauteur $d$, extension d'un $\mathcal{O}_{o^{-}}$ module formel de hauteur $h$ par sa partie étale, $\operatorname{Def}_{n}^{h, d-h}$ où $n$ est la multiplicité de o dans I.

(b) Le complété formel de l'anneau local de $M_{I, s_{o}}^{\geqslant h}$ en un point géométrique de $M_{I, s_{o}}^{=h^{\prime}}$ pour $h^{\prime} \geqslant h$ est isomorphe par le théorème de Serre-Tate à $\operatorname{Def}_{n}^{h^{\prime}, d-h^{\prime} ; \geqslant h}$ défini comme suit avec $j^{\prime}=d-h^{\prime}$ :

$$
\operatorname{Def}_{n}^{h^{\prime}, j^{\prime} ; \geqslant h} \simeq \operatorname{Def}_{n}^{h^{\prime} ; \geqslant h} \hat{\otimes}_{\overline{\mathbb{F}}_{q}} \overline{\mathbb{F}}_{q}\left[\left[w_{1}^{n}, \cdots, w_{j^{\prime}}^{n}\right]\right]
$$

où $\operatorname{Def}_{n}^{h^{\prime}}: \geqslant h$ est le réduit du quotient de $\operatorname{Def}_{n}^{h^{\prime}}$ par l'idéal engendré par $\varphi_{0 \rightarrow n}\left(u_{i}\right)$ pour $1 \leqslant$ $i<h$ où

$$
\varphi_{0 \rightarrow n}: \operatorname{Def}_{0}^{h^{\prime}}=\overline{\mathbb{F}}_{q}\left[\left[\varpi_{o}, a_{1}, \cdots, a_{h^{\prime}-1}\right]\right] \longrightarrow \operatorname{Def}_{n}^{h^{\prime}}
$$

est le morphisme d'oubli du niveau.

(c) Le complété formel de l'anneau local de $M_{I, s_{o}, a_{h}}^{\geqslant h}$ en un point géométrique de $M_{I, s_{o}, a_{h^{\prime}}}^{=h^{\prime}}$ pour $h^{\prime} \geqslant h$ et $a_{h} \subset a_{h^{\prime}}$ est isomorphe par le théorème de Serre-Tate à $\operatorname{Def}_{n, a_{h} \subset a_{h^{\prime}}}^{h^{\prime}, d-h^{\prime} \geqslant h}$ défini comme suit avec $j^{\prime}=d-h^{\prime}$ :

$$
\operatorname{Def}_{n, a_{h} \subset a_{h^{\prime}}}^{h^{\prime}, j^{\prime} ; \geqslant h} \simeq \operatorname{Def}_{n, a_{h}^{\prime}}^{h^{\prime} ; \geqslant h} \hat{\otimes}_{\overline{\mathbb{F}}_{q}} \overline{\mathbb{F}}_{q}\left[\left[w_{1}^{n}, \cdots, w_{j^{\prime}}^{n}\right]\right]
$$

${ }^{(5)} \mathrm{Au}$ paragraphe (I.4.3), on reviendra plus précisément sur ce point. 
où $a_{h}^{\prime}$ est le facteur direct de rang $h^{\prime}-h$ de $\left(\mathcal{M}_{o}^{-n} / \mathcal{O}_{o}\right)^{h^{\prime}}$ associé au quotient $a_{h^{\prime}} / a_{h}$ et où $\operatorname{Def}_{n, a_{h}^{\prime}}^{h^{\prime} ; h}$ est le réduit du quotient de $\operatorname{Def}_{n}^{h^{\prime}}$ par l'idéal engendré par les $\iota_{n}\left(e_{i}\right)$ pour $1 \leqslant i \leqslant h$ et $\left(e_{1}, \cdots, e_{h}\right)$ une base de $a_{h}^{\prime}$, où $\iota_{n}$ est la structure de niveau $n$ universelle sur $\operatorname{Def}_{n}^{h^{\prime}}$.

I.1.6.5. Proposition. - Pour tout $n \geqslant 0$ et tout $a \in G / P(d, h, n),\left(\operatorname{Def}_{n, a}^{d ; \geqslant h}\right)_{\text {red }}$ est régulier de dimension $d-h$.

Démonstration. - Soit $\left(w_{1}, \cdots, w_{h}\right)$ une base de $a$; pour $1 \leqslant i \leqslant h$, on écrit $w_{i}=\lambda_{1}^{i} e_{1}+\cdots+\lambda_{d}^{i} e_{d}$, où $\left(e_{1}, \cdots, e_{d}\right)$ est la base canonique de $\left(\mathcal{M}_{o}^{-n} / \mathcal{O}_{o}\right)^{d}$ et $\lambda_{k}^{i} \in \mathcal{O}_{o}$. Pour tout $1 \leqslant i \leqslant h$, l'idéal $\left(\lambda_{1}^{i}, \cdots, \lambda_{d}^{i}\right)$ est égal à $\mathcal{O}_{o}$. Notons $\left(F,\left(f_{\lambda}\right)_{\lambda \in \mathcal{O}_{o}}\right)$ le $\mathcal{O}_{o}$-module formel de hauteur $d$ universel avec

$$
F(X, Y)=X+Y+\cdots \text { et pour tout } \lambda \in \mathcal{O}_{o}, f_{\lambda}(X)=i(\lambda) X+\cdots
$$

où $i$ est l'injection naturelle $\mathcal{O}_{o} \rightarrow \operatorname{Def}_{n}^{d}$. Soit $\mathfrak{J}_{a}$ l'idéal de $\overline{\mathbb{F}}_{q}\left[\left[v_{1}^{n}, \cdots, v_{d}^{n}\right]\right]$ engendré par les éléments

$$
F\left(f_{\lambda_{1}^{i}}\left(v_{1}^{n}\right), \cdots, F\left(f_{\lambda_{d-1}^{j}}\left(v_{d-1}^{n}, f_{\lambda_{d}^{i}}\left(v_{d}^{n}\right)\right) \cdots\right), \quad 1 \leqslant i \leqslant h\right.
$$

Pour tout $1 \leqslant i \leqslant h$, les équations

$$
F\left(f_{\lambda_{1}^{i}}\left(v_{1}^{n}\right), F\left(f_{\lambda_{2}^{i}}\left(v_{2}^{n}\right), \cdots, F\left(f_{\lambda_{d-1}^{i}}\left(v_{d-1}^{n}, f_{\lambda_{h}^{i}}\left(v_{d}^{n}\right)\right)\right)\right) \cdots\right)=0 .
$$

s'écrivent sous la forme

$$
i\left(\lambda_{1}^{j}\right) v_{1}^{n}+\cdots+i\left(\lambda_{d}^{j}\right) v_{d}^{n}+\text { termes de degré }>1 .
$$

La matrice $(d \times h)$ des $\left(\bar{\lambda}_{k}^{j}\right)_{1 \leqslant{ }_{j} \leqslant{ }_{h}^{d}}$ étant de rang $h, \overline{\mathbb{F}}_{q}\left[\left[v_{1}^{n}, \cdots, v_{h}^{n}\right]\right] / \mathfrak{J}_{A}$ est alors isomorphe à $\overline{\mathbb{F}}_{q}\left[\left[u_{1}, \cdots, u_{d-h}\right]\right]$. En notant $\mathcal{I}_{a}$ l'idéal de définition de $\operatorname{Def}_{n, a}^{d ; h}$, on a clairement $\mathcal{I}_{a} \subset \mathcal{J}_{a}$. En outre $\mathcal{J}_{a} \subset \sqrt{\mathcal{I}_{a}}$ car d'après la proposition (9.3.3) de [5], pour tout $z \in a,\left(\iota_{n} \otimes_{\text {Def }_{n}^{d}}\left(\operatorname{Def}_{n, a}^{=h}\right)_{r e d}\right)(z)$ est nilpotent, d'où le résultat.

I.1.6.6. Corollaire. - Les schémas fermés réduits $\left(M_{I, s_{o}, a}^{\geqslant h}\right)_{\text {red }}$ sont réguliers pour tout a $\in$ $G / P(d, h, n)$ où $n$ est la multiplicité de o dans $I$.

I.1.6.7. Remarque. - Soit $Y^{h}$ une composante irréductible, donc connexe, de $M_{I^{o}, s_{o}}^{\geqslant h}$. On note ${ }^{(6)} Y^{h+i}=Y^{h} \times_{M_{I^{o}, s_{o}}^{\geqslant h}} M_{I^{o}, s_{o}}^{\geqslant h+i}$ et pour tout $n$ la multiplicité de $o$ dans $I, Y_{n}^{h}=$ $Y^{h} \times_{M_{I^{o}, s_{o}}^{\geqslant h}} M_{I, s_{o}}^{\geqslant h}=\bigcup_{a \in G / P(d, h, n)} Y_{n, a}^{h}$. Pour tout $a, a^{\prime} \in G / P(d, h, n)$, on a

$$
Y_{n, a}^{h} \cap Y_{n, a^{\prime}}^{h}=Y_{n, a+a^{\prime}}^{\operatorname{dim}\left(a+a^{\prime}\right)}
$$

où l'on considère $a, a^{\prime}$ comme des facteurs directs de rang $h$ de $\left(\mathcal{M}_{o}^{-n} / \mathcal{O}_{o}\right)^{d}$. En particulier si $Y^{d}$ est non vide, alors les $Y_{n, a}^{h}$ sont connexes, lisses et donc irréductibles.

Démonstration. - Au dessus de $M_{I^{o}, s_{o}}^{=h}$, on a $Y_{n}^{=h}=\bigsqcup_{a \in G / P(d, h, n)} Y_{n, a}^{=h}$. En outre au dessus de tout point géométrique de $Y^{=h}$, il y a exactement $|G / P(d, h, n)| \times\left|G L_{d-h}\left(\mathcal{O}_{o} / \mathcal{M}_{o}^{n}\right)\right|$ points géométriques de $Y_{n}^{=h}$, de sorte que pour tout $a \in G / P(d, h, n), Y_{n, a}^{=h}$ est la réunion disjointes de $\left|G L_{d-h}\left(\mathcal{O}_{o} / \mathcal{M}_{o}^{n}\right)\right|$ composantes irréductibles. L'inclusion $Y_{n, a}^{h} \cap Y_{n, a^{\prime}}^{h} \subset Y_{n, a+a^{\prime}}^{\operatorname{dim}\left(a+a^{\prime}\right)}$ est évidente. Montrons l'inclusion réciproque. Soit donc $z \in Y_{n, a+a^{\prime}}^{\operatorname{dim}\left(a+a^{\prime}\right)}$. Le complété de l'anneau local de $Y_{n}^{h}$ en $z$ est

$$
\operatorname{Def}_{n, a+a^{\prime}}^{\operatorname{dim}\left(a+a^{\prime}\right), d-\operatorname{dim}\left(a+a^{\prime}\right) ; h}=\bigcup_{b \in G / P\left(\operatorname{dim}\left(a+a^{\prime}\right), h, n\right)} \operatorname{Def}_{n, b \subset a+a^{\prime}}^{\operatorname{dim}\left(a+a^{\prime}\right), d-\operatorname{dim}\left(a+a^{\prime}\right) ; h}
$$

de sorte que $z \in Y_{n, \tilde{a}}^{h}$, pour tout $\tilde{a} \subset a+a^{\prime}$.

${ }^{(6)}$ On ne sait pas à priori si $Y^{h+i}$ est vide ou pas. 


\section{I.2. Compléments sur la géométrie des strates}

Nous verrons $\S I .3$ et $\S$ I.4, que les relations entre les variétés d'Igusa de première et seconde espèce avec les variétés $M_{I, o}$ de Drinfeld-Stuhler, sont directement en rapport avec les liens entre la structure de niveau $\iota_{o, n}$ et la donnée d'une structure de niveau sur la partie étale, $\iota_{o, n}^{e t}$, et connexe, $\iota_{o, n}^{c}$, séparément, ce qui sera abordée au paragraphe suivant. Pour définir précisément les parties connexes et étales de $\iota_{o, n}$, on est amené à étudier plus précisément le scindage en parties connexe et étale des $\mathcal{O}_{o}$-modules de Dieudonné sur un $\kappa(o)$-schéma ou sur un épaississement infinitésimal.

\section{I.2.1. Scindage en partie étale et connexe des $\mathcal{O}_{o}$-modules de Dieudonné. -}

I.2.1.1. Proposition. - Soit $S$ un $\kappa\left(\right.$ o)-schéma. Pour tout $S$-point de $M_{I, s_{o}}^{=h}$, on note $\left(V_{o}, \varphi_{o}\right)$, le $\left(\mathcal{O}_{o} \hat{\otimes}_{\kappa(o)} \mathcal{O}_{S}\right)$-module de Dieudonné qui lui est associé. Il existe alors un sous-module canonique $V_{o}^{e t}$ de $V_{o}$ de rang $(d-h)$ stable sous l'action de $\varphi_{o}$ tel que

- $\varphi_{o}^{e t}:=\left(\varphi_{o}\right)_{\mid V_{o}^{e t}}:{ }^{\tau} V_{o}^{e t} \longrightarrow V_{o}^{e t}$ est inversible;

- $\varphi_{o}^{c}:{ }^{\tau} V_{o}^{c} \longrightarrow V_{o}^{c}:=V_{o} / V_{o}^{e t}$, l'application induite par $\varphi_{o}$, est topologiquement nilpotente;

- pour tout $n \geqslant 0$, la suite exacte ${ }^{(7)}$

$$
0 \longrightarrow\left(V_{o, n}^{e t}, \varphi_{o, n}^{e t}\right) \longrightarrow\left(V_{o, n}, \varphi_{o, n}\right) \longrightarrow\left(V_{o, n}^{c}, \varphi_{o, n}^{c}\right) \longrightarrow 0
$$

se scinde après le changement de base $S \stackrel{\mathrm{Fr}_{o}^{n h}}{\longrightarrow} S$.

Démonstration. - Pour tout entier $i \geqslant 0$, on note $\tau^{i} \varphi_{o}: \tau^{i+1} V_{o} \longrightarrow \tau^{i} V_{o}$ l'application $\left(\mathcal{O}_{o} \hat{\otimes}_{\kappa(o)} \mathcal{O}_{S}\right)$-linéaire déduite de $\varphi_{o}:{ }^{\tau} V_{o} \longrightarrow V_{o}$. On pose

$$
\varphi_{o}^{! h}:=\varphi_{o} \circ{ }^{\tau} \varphi_{o} \circ \cdots \circ \tau^{\tau^{h-1}} \varphi_{o}: \tau^{h} V_{o} \longrightarrow V_{o} .
$$

Soit Spec $R \longrightarrow S$ un ouvert affine de $S$ tel que $V_{o} \times{ }_{S}$ Spec $R$ est libre. Si $M_{o, R}$ est la matrice de $\varphi_{o} \times{ }_{S} \operatorname{Spec} R$ par rapport à une base $\left(b_{i}\right)_{i}$ de $V_{o}$ alors

$$
M_{o, R}^{! h}=M_{o, R}\left({ }^{\tau} M_{o, R}\right) \cdots\left(\tau^{h-1} M_{o, R}\right)
$$

est celle de $\varphi_{o, R}^{! h}:=\varphi_{o}^{! h} \times_{S}$ Spec $R$ par rapport à cette même base $\left(b_{i}\right)_{i}$.

D'après (I.1.5.2) (b), pour tout $h^{\prime} \geqslant h, M_{o, R, 1}^{! h^{\prime}}$ a un mineure d'ordre $d-h$ inversible de sorte que $^{(8)} \varphi_{o, R, 1}^{! h^{\prime}}\left(\tau^{h^{\prime}} V_{o, R, 1}\right)$ est un facteur direct de $V_{o, R, 1}$ de rang $d-h$ car tous les mineures d'ordre supérieure à $d-h+1$ sont nuls. En remarquant que pour $h_{1} \leqslant h_{2}$, on a

$$
\varphi_{o, R}^{! h_{2}}\left(\tau^{h_{2}} V_{o, R}\right) \subset \varphi_{o, R}^{! h_{1}}\left(\tau^{h_{1}} V_{o, R}\right),
$$

on pose pour tout $h^{\prime} \geqslant h$

$$
V_{o, R, 1}^{e t}:=\varphi_{o, R, 1}^{! h^{\prime}}\left(\tau^{h^{\prime}} V_{o, R, 1}\right)=\varphi_{o, R, 1}^{! h}\left(\tau^{h} V_{o, R, 1}\right) .
$$

I.2.1.2. Lemme. - L'application $\varphi_{o, R, 1}$ induit une application $\varphi_{o, R, 1}^{e t}:{ }^{\tau} V_{o, R, 1}^{e t} \longrightarrow V_{o, R, 1}^{e t} q u i$ est bijective.

Démonstration. — Le diagramme commutatif suivant

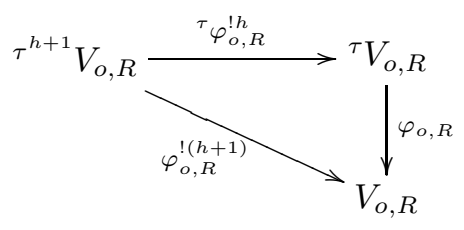

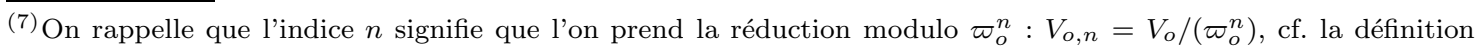
(I.1.2.3).

(8) l'indice 1 désigne la réduction modulo $\varpi_{o}$
} 
permet de définir $\varphi_{o, R, 1}^{e t}:{ }^{\tau} V_{o, R, 1}^{e t} \longrightarrow V_{o, R, 1}^{e t}$. En effet étant donné $v \in V_{o, R, 1}^{e t}$, il existe $w \in \tau^{h} V_{o, R, 1}$ tel que $v=\varphi_{o, R, 1}^{! h}(w)$, de sorte que

$$
\varphi_{o, R, 1}(v \otimes \lambda)=\varphi_{o, R, 1} \circ^{\tau} \varphi_{o, R, 1}^{! h}(w \otimes \lambda)=\varphi_{o, R, 1}^{! h+1}(w \otimes \lambda) \in V_{o, R, 1}^{e t},
$$

d'où l'existence de $\varphi_{o, R, 1}^{e t}$.

En ce qui concerne la surjectivité de $\varphi_{o, R, 1}^{e t}$, soit $v=\varphi_{o, R, 1}^{! h+1}(w) \in V_{o, R, 1}^{e t}$ avec $w \in \tau^{h+1} V_{o, R, 1}$. On a alors $v=\varphi_{o, R, 1} \circ^{\tau} \varphi_{o, R, 1}^{! h}(w)$ avec ${ }^{\tau} \varphi_{o, R, 1}^{! h}(w) \in{ }^{\tau} V_{o, R, 1}^{e t}$ d'où le résultat.

Pour l'injectivité soit $\left(e_{1, R}, \cdots, e_{j, R}\right)$ une base de $V_{o, R, 1}^{e t}$ en tant que $R$-module : $\left(e_{i, R} \otimes 1\right)_{i=1 \cdots j}$ est alors une base de ${ }^{\tau} V_{o, R, 1}^{e t}$ et $\left(\varphi\left(e_{i, R} \otimes 1\right)\right)_{i=1 \cdots j}$ est d'après la surjectivité de $\varphi_{o, R, 1}^{e t}$ une base de $V_{o, R, 1}^{e t}$. Ainsi si $v=\sum_{i=1}^{j} e_{i, R} \otimes \lambda_{j}$ appartient à $\operatorname{Ker} \varphi_{o, R, 1}^{e t}$, on en déduit que $\sum_{i=1}^{j} \lambda_{i} \varphi_{o, R, 1}\left(e_{i, R} \otimes\right.$ 1) $=0$ soit $\lambda_{i}=0$ pour $i=1, \cdots, j$.

On remarque par ailleurs que $\left(\varphi_{o, R, 1}^{c}\right)^{! h}: \tau^{h} V_{o, R, 1}^{c} \longrightarrow V_{o, R, 1}^{c}$ est nulle. On définit alors globalement $\operatorname{sur} S, V_{o, 1}^{e t}:=\varphi_{o, 1}^{! h}\left(V_{o, 1}\right)$ ainsi que $V_{o, 1}^{c}=V_{o, 1} / V_{o, 1}^{e t}$. Pour tout entier $n$, on définit de même

$$
V_{o, n}^{e t}:=\varphi_{o, n}^{! n h}\left(\tau^{n h} V_{o, n}\right)
$$

qui est un facteur direct de $V_{o, n}$ de $\operatorname{rang} n(d-h)$. De la même façon $\varphi_{o, n}^{e t}:{ }^{\tau} V_{o, n}^{e t} \longrightarrow V_{o, n}^{e t}$ est bijective et $\left(\varphi_{o, n}^{c}\right)^{! n h}$ est nulle. Pour $n \geqslant n^{\prime}$, on a une flèche naturelle

$$
V_{o, n}^{e t}=\varphi_{o, n}^{! n h}\left(\tau^{n h} V_{o, n}\right) \stackrel{r_{n^{\prime}, n}}{\longrightarrow} \varphi_{o, n^{\prime}}^{! n h}\left({ }^{n h h} V_{o, n^{\prime}}\right)=\varphi_{o, n^{\prime}}^{! n^{\prime} h}\left(\tau^{\tau^{\prime} h} V_{o, n^{\prime}}\right)=V_{o, n^{\prime}}^{e t}
$$

telle que pour $n_{1} \geqslant n_{2} \geqslant n_{3}$, on a $r_{n_{3}, n_{1}}=r_{n_{3}, n_{2}} \circ r_{n_{2}, n_{1}}$. On pose alors

$$
V_{o}^{e t}:=\underset{\leftarrow}{\lim _{\leftarrow}} V_{o, n}^{e t}
$$

qui est ainsi stable sous l'action de $\varphi_{o}$. Soit $V_{o}^{c}:=V_{o} / V_{o}^{e t}$ de sorte que $\varphi_{o}^{c}:{ }^{\tau} V_{o}^{c} \longrightarrow V_{o}^{c}$ est topologiquement nilpotente.

Montrons que le noyau $N_{o, n}$ de $\varphi_{o, n}^{! n h}:{ }^{n h} V_{o, n} \longrightarrow V_{o, n}$ est un relèvement de ${ }^{\tau^{n h}} V_{o, n}^{c}$. L'application $\varphi_{o, n}^{e t}$ étant injective, on a $N_{o, n} \cap \tau^{\tau^{n h}} V_{o, n}^{e t}=\{0\}$. Soit alors $x \in \tau^{n h} V_{o, n}$ et $v=\varphi_{o, n}^{! n h}(x)$. D'après la surjectivité de $\varphi_{o, n}^{e t}$, soit $w \in{ }^{\tau^{n h}} V_{o, n}^{e t}$ tel que $v=\varphi_{o, n}^{e t, ! n h}(w)$. On a alors $x-w \in N_{o, n}$ de sorte que

$$
{ }^{\tau^{n h}} V_{o, n}=N_{o, n} \oplus{ }^{\tau^{n h}} V_{o, n}^{e t}
$$

Par ailleurs l'égalité $\varphi_{o, n}^{! n h} \circ \varphi_{o, n}^{n h}=\varphi_{o, n}^{! n h+1}=\varphi_{o, n} \circ{ }^{\tau} \varphi_{o, n}^{! n h}$, montre que ${ }^{\tau^{n h}} \varphi_{o, n}:{ }^{n h+1} V_{o, n} \longrightarrow{ }^{\tau^{n h}} V_{o, n}$ induit une application $\varphi_{o, n}^{c}:{ }^{\tau} N_{o, n} \longrightarrow N_{o, n}$, d'où le résultat.

En ce qui concerne les déformations, on a la proposition suivante.

I.2.1.3. Corollaire. — Soit $S$ un $\operatorname{Spec} \mathcal{O}_{o}$ schéma artinien de réduit $\bar{S}$ et $S \rightarrow M_{I, o}$ tel que $\bar{S} \rightarrow$ $M_{I, o}$ se factorise par $M_{I, s_{o}}^{=h}$. En notant $\left(V_{o}, \varphi_{o}\right)$ le $\mathcal{O}_{o}$-module de Dieudonné associé à $S \rightarrow M_{I, o}$, $i l$ existe alors un sous- $\mathcal{O}_{o}-$ module de Dieudonné étale $\left(V_{o}^{\text {et }}, \varphi_{o}^{\text {et }}\right)$ tel que $V_{o, n}^{\text {et }}$ soit l'image de $\varphi_{o, n}^{! n h}$. De plus, si on définit $\left(V_{o}^{c}, \varphi_{o}^{c}\right)$ par la suite exacte

$$
0 \longrightarrow\left(V_{o}^{e t}, \varphi_{o}^{e t}\right) \longrightarrow\left(V_{o}, \varphi_{o}\right) \longrightarrow\left(V_{o}^{c}, \varphi_{o}^{c}\right) \longrightarrow 0
$$

$\varphi_{o}^{c}$ est topologiquement nilpotente.

Démonstration. - On raisonne localement sur un ouvert affine $\operatorname{Spec} R \rightarrow S$, pour $R$ une $\mathcal{O}_{o^{-}}$ algèbre artinienne et $\mathcal{M}$ un idéal nilpotent de $R$. Classiquement on peut se ramener à $\mathcal{M}^{2}=(0)$. 
Sur $\bar{R}$, on choisit une décomposition $\bar{V}_{o}=\bar{V}_{o}^{e t} \oplus \bar{V}_{o}^{c}$ ainsi que des bases $\left(\bar{e}_{1}, \cdots, \bar{e}_{d-h}\right)$ de $\bar{V}_{o}^{e t}$ et $\left(\bar{f}_{1}, \cdots, \bar{f}_{h}\right)$ de $\bar{V}_{o}^{c}$ de sorte que la matrice de $\bar{\varphi}_{o}$ par rapport à cette base est

$$
\left(\begin{array}{cc}
\bar{M}_{e t} & \bar{M}_{e x t} \\
0 & \bar{M}_{c}
\end{array}\right)
$$

On fixe des relèvements quelconques $\left(e_{1}, \cdots, e_{d-h}\right)$ et $\left(f_{1}, \cdots, f_{h}\right)$; la matrice de $\varphi_{o}$ dans ces bases est alors de la forme

$$
\left(\begin{array}{cc}
M_{e t} & M_{e x t} \\
\mathcal{M}_{1} & M_{c}
\end{array}\right)
$$

où $\mathcal{M}_{1}$ est à coefficient dans $\mathcal{M}$. En effectuant le changement de base via la matrice $\left(\begin{array}{cc}I d & 0 \\ P_{0} & I d\end{array}\right)$, où $P_{0}$ est à coefficients dans $\mathcal{M}$ de sorte que ${ }^{\tau} P_{0}$ est la matrice nulle, on obtient

$$
\left(\begin{array}{cc}
M_{e t} & M_{e x t} \\
P_{0} M_{e t}+\mathcal{M}_{1} & P_{0} M_{e x t}+M_{c}
\end{array}\right)
$$

de sorte qu'en choisissant $P_{0}=-\mathcal{M}_{1} M_{e t}^{-1}$, on obtient une décomposition $V_{o}=V_{o}^{e t} \oplus V_{o}^{c}$ dans laquelle la matrice de $\varphi_{o}$ est de la forme

$$
\left(\begin{array}{cc}
M_{e t} & M_{e x t} \\
0 & M_{c}^{\prime}
\end{array}\right)
$$

avec $M_{c}^{\prime}$ topologiquement nilpotente. On remarque par ailleurs que $V_{o, n}^{e t}$ est à nouveau défini globalement sur $S$ comme l'image de $\varphi_{o, n}^{! n h}$.

I.2.1.4. Définition. - Dans la suite, dans la situation du corollaire (I.2.1.3), on notera $\mathcal{F}_{o}^{*, c}$ le sous- $\mathcal{O}_{o}$-module de $\mathcal{F}_{o}^{*}$ des formes qui s'annulent sur $\mathcal{F}_{o}^{e t}$. Celui ci est stable sous $\phi_{o}^{*}$ et on notera $\phi_{o}^{*, c}$ la restriction de $\phi_{o}^{*}$ à $\mathcal{F}_{o}^{*, c}$. On remarque ainsi que $\mathcal{F}_{o}^{e t, *}$ est isomorphe au quotient $\mathcal{F}_{o}^{*} / \mathcal{F}_{o}^{*, c}$ et on note $\phi_{o}^{e t, *}$ l'application induite par $\phi_{o}^{*}$. On notera parfois $\mathcal{F}_{o}^{*, e t}$ un supplémentaire de $\mathcal{F}_{o}^{*, c}$, qui est isomorphe à $\mathcal{F}_{o}^{e t, *}$ mais qui n'est pas stable par $\phi_{o}^{*}$.

I.2.2. Retour sur les structures de niveau. - Soit $S$ un $\operatorname{Spec} \mathcal{O}_{o}$-schéma artinien, $\mathcal{I}$ un faisceau d'idéaux nilpotents et $\bar{S}$ le sous-schéma fermé associé. Soit $S \rightarrow M_{I, o}$ un $S$-point tel que $\bar{S} \rightarrow M_{I, o}$ se factorise par $M_{I, s_{o}, a}^{=h}$. On note $\left(\mathcal{F}_{o, n}, \phi_{o, n}\right)$ le $\phi$-faisceau associé sur $S$. D'après le paragraphe précédent, on écrit $\mathcal{F}_{o, n}^{*}$ comme une somme directe $\mathcal{F}_{o, n}^{*, e t} \oplus \mathcal{F}_{o, n}^{*, c}$ où la matrice de $\phi_{o, n}^{*}$ dans une base adaptée à cette décomposition; est de la forme

$$
M_{o, n}^{*}=\left(\begin{array}{cc}
M_{o, n}^{*, e t} & 0 \\
M_{o, n}^{*, e x t} & M_{o, n}^{*, c}
\end{array}\right)
$$

On note $\iota_{o, n}:\left(\mathcal{M}_{o}^{-n} / \mathcal{O}_{o}\right)^{d} \longrightarrow \mathcal{F}_{o, n}^{*}$ la structure de niveau $n$ associée. Pour tout élément $z \in$ $\left(\mathcal{M}_{o}^{-n} / \mathcal{O}_{o}\right)^{d}$, on a

$$
M_{o, n}^{*} \iota_{o, n}(z)=\iota_{o, n}(z)^{q},
$$

c'est à dire en écrivant $\iota_{o, n}(z)=\iota_{o, n}^{e t}(z)+\iota_{o, n}^{c}(z)$ :

$$
\begin{aligned}
M_{o, n}^{*, e t} \iota_{o, n}^{e t}(z) & =\iota_{o, n}^{e t}(z)^{q} \\
M_{o, n}^{*, e x t} \iota_{o, n}^{e t}(z)+M_{o, n}^{*, c} \iota_{o, n}^{c}(z) & =\iota_{o, n}^{c}(z)^{q}
\end{aligned}
$$

I.2.2.1 - Par définition, l'ensemble $\left\{z \in\left(\mathcal{M}_{o}^{-n} / \mathcal{O}_{o}\right)^{d} / \iota_{o, n}^{e t}(z)=0\right\}$ est égal au sous-module $a$. La condition de Drinfeld s'exprime alors comme suit :

- $\iota_{o, n}^{e t}:\left(\mathcal{M}_{o}^{-n} / \mathcal{O}_{o}\right)^{d} / a \times S \simeq\left(\mathcal{M}_{o}^{-n} / \mathcal{O}_{o}\right)^{d-h} \times S \longrightarrow \mathcal{F}_{o, n}^{e t, *} \simeq \mathcal{F}_{o, n}^{*, e t}$ est un isomorphisme vérifiant (I.2.2.1);

- $\iota_{o, n}^{c}: a \longrightarrow \mathcal{F}_{o, n}^{*, c}$ est une structure de niveau au sens de Drinfeld. 
Remarque : Réciproquement si on se donne $\iota_{o, n}^{e t}$ et $\iota_{o, n}^{c}$ comme ci-dessus, le choix d'un supplémentaire de $a$ permet de définir une application

$$
\iota_{o, n}=\iota_{o, n}^{e t} \oplus \iota_{o, n}^{c} .
$$

Le problème est qu'elle ne vérifiera pas obligatoirement la relation (I.2.2.2) sauf par exemple si $M_{o, n}^{e x t}$ est nulle. En outre il n'est pas certain que l'on puisse définir $\iota_{o, n}^{c}$ sur $b$ telle que (I.2.2.2) soit vérifiée.

I.2.2.2. Proposition. - Si R est un $\kappa\left(\right.$ o)-anneau réduit et $\left(\left(\mathcal{E}_{i}, j_{i}, t_{i}\right), \iota_{I}\right)$ un $R$-point de $M_{I, s_{o}, a}^{=h}$, alors $\iota_{o, n}^{c}: a \longrightarrow \mathcal{F}_{o, n}^{*, c}$ est nulle.

Démonstration. - Soit $\mathcal{M}$ un idéal maximal de $R ; \iota_{o, n}^{c} \otimes_{R} R / \mathcal{M}$ est alors nulle, de sorte que $\iota_{o, n}^{c}$ est à coefficients dans $\bigcap_{\mathcal{M} \text { maximal }} \mathcal{M}$ qui est l'idéal nul car $R$ est réduit.

I.2.2.3. Proposition. - Le morphisme d'oubli du niveau $M_{I, s_{o}, r e d}^{=h} \longrightarrow M_{I^{o}, s_{o}}^{=h}$ est fini et plat de degré

$$
\# G L_{d}\left(\mathcal{O}_{o} / \mathcal{M}_{o}^{n}\right) / \# G L_{h}\left(\mathcal{O}_{o} / \mathcal{M}_{o}^{n}\right)
$$

où $n$ est la multiplicité de o dans $I$.

Démonstration. - La platitude découle de l'équivalent du théorème de Serre-Tate, proposition (I.1.6.3), et des résultats de Drinfeld rappelés au paragraphe (I.1.1). Calculons alors les degrés. L'ensemble des points géométriques de $M_{\bar{I}, \overline{s_{o}}}^{\bar{h}}:=M_{\bar{I}, s_{o}}^{=h} \times_{\operatorname{Spec} \kappa(o)} \operatorname{Spec} \bar{\kappa}(o)$ au dessus d'un point géométrique donné de $M_{\bar{I}^{o}, \bar{s}_{o}}^{\bar{h}}$, est de cardinal

$$
\# G / P(d, h, n) \# G L_{d-h}\left(\mathcal{O}_{o} / \mathcal{M}_{o}^{n}\right) .
$$

Soit $s_{I}$ un tel point géométrique de $M_{I, s_{o}}^{=h}$ au dessus d'un point $s$ de $M_{I^{o}, s_{o}}^{=h}$. Soient alors $\left(M_{I, s_{o}, \text { red }}^{=h}\right)_{s_{I}}$ et $\left(M_{I^{o}, s_{o}}^{\bar{h}}\right)_{s}^{\hat{s}}$ les complétés formels de respectivement $M_{I, s_{o}, \text { red }}^{=h}$ et $M_{I^{o}, s_{o}}^{=h}$ aux points $s_{I}$ et $s$. D'après l'équivalent du théorème de Serre-Tate, et avec les notations du paragraphe (I.1.1) et plus particulièrement de la note (4), on a

$$
\left(M_{I^{o}, s_{o}}^{=h}\right)_{s} \simeq \overline{\mathbb{F}}_{q}\left[\left[w_{1}^{0}, \cdots, w_{d-h}^{0}\right]\right] \quad \text { et } \quad\left(M_{I, s_{o}, \text { red }}^{=h}\right)_{s_{I}} \simeq \overline{\mathbb{F}}_{q}\left[\left[w_{1}^{n}, \cdots, w_{d-h}^{n}\right]\right] .
$$

D'après $[\mathbf{9}]$, le degré de $\overline{\mathbb{F}}_{q}\left[\left[w_{1}^{0}, \cdots, w_{d-h}^{0}\right]\right] \longrightarrow \overline{\mathbb{F}}_{q}\left[\left[w_{1}^{n}, \cdots, w_{d-h}^{n}\right]\right]$ est égal à

$$
q^{n h(d-h)}=\#\left(\mathcal{O}_{o} / \mathcal{M}_{o}^{n}\right)^{h(d-h)},
$$

d'où le résultat.

I.2.2.4. Corollaire. - Le morphisme $M_{I, s_{o}, a, r e d}^{=h} \longrightarrow M_{I^{o}, s_{o}}^{=h}$ est fini et plat de degré

$$
\#\left(\mathcal{O}_{o} / \mathcal{M}_{o}^{n}\right)^{h(d-h)} \# G L_{d-h}\left(\mathcal{O}_{o} / \mathcal{M}_{o}^{n}\right)
$$

où $n$ est la multiplicité de o dans $I$.

I.2.2.5 - La proposition suivante justifie l'existence du morphisme de la proposition (I.3.2.1). En langage clair, étant donné un point $\bar{S} \longrightarrow M_{\bar{I}, s_{o}, a}^{=h}$ et une déformation de $\bar{S} \longrightarrow M_{\bar{I}^{o}, s_{o}}^{=h}$, obtenu via le morphisme d'oubli $M_{I, s_{o}, a}^{=h} \longrightarrow M_{I^{o}, s_{o}}^{=h}$, ainsi que des déformations des parties étale et connexe de la structure de niveau en la place $o$, on construit une déformation canonique de $\bar{S} \stackrel{\mathrm{Frob}^{n n}}{\longrightarrow} \bar{S} \longrightarrow M_{\bar{I}, s_{o}, a}^{=h}$, où $n$ est la multiplicité de $o$ dans $I$.

I.2.2.6. Proposition. - Soit $S$ un Spec $\mathcal{O}_{o}$-schéma artinien, un sous-faisceau d'idéaux nilpotents $\mathcal{I}_{S}$ de $\mathcal{O}_{S}$ et $\bar{S}$ le fermé de $S$ associé. Soit $\bar{S} \longrightarrow M_{I, s_{o}, a}^{=h}$ et $S \longrightarrow M_{I^{o}, o}$ une déformation du morphisme composé

$$
\bar{S} \longrightarrow M_{I, s_{o}, a}^{=h} \longrightarrow M_{I^{o}, s_{o}}^{=h} .
$$


Soit $\left(V_{o}^{\text {et }}, \varphi_{o}^{e t}\right)$ le sous- $\mathcal{O}_{o}$-module de Dieudonné étale de $\left(V_{o}, \varphi_{o}\right)$ associé à $S \longrightarrow M_{I^{o}, o}$, donné par le corollaire (I.2.1.3). On note $V_{o}^{*, c}$ le sous- $\mathcal{O}_{o}$-module des éléments de $V_{o}^{*}$ qui s'annulent sur $V_{o}^{\text {et }}$. Soient $b$ un supplémentaire de a dans $\left(\mathcal{M}_{o}^{-n} / \mathcal{O}_{o}\right)^{d}$, où $n$ est la multiplicité de o dans $I$, et

$$
\iota_{o, n}^{e t}: b \longrightarrow V_{o, n}^{e t, *}=V_{o, n}^{*} / V_{o, n}^{*, c} \quad \text { et } \quad \iota_{o, n}^{c}: a \longrightarrow V_{o, n}^{*, c}
$$

des déformations respectives de

$$
\bar{\iota}_{o, n}^{e t}: b \longrightarrow \bar{V}_{o, n}^{e t, *}=\bar{V}_{o, n}^{*} / \bar{V}_{o, n}^{*, c} \quad \text { et } \quad \bar{\iota}_{o, n}^{c}: a \longrightarrow \bar{V}_{o, n}^{*, c}
$$

Associée à toutes ces données, il existe alors une déformation canonique de $\bar{S}^{\operatorname{Frob}_{o}^{n h}} \bar{S} \longrightarrow M_{I, s_{o}, a}^{=h}$ telle que son $\mathcal{O}_{o}$-module de Dieudonné $\left(\tilde{V}_{o}, \tilde{\varphi}_{o}\right)$ se scinde modulo $\varpi_{o}^{n}$ en partie étale et connexe.

Démonstration. - D'après la proposition (I.1.6.3), il suffit de donner une déformation de $\left(\tau^{n h} \bar{V}_{o}, \tau^{n h} \bar{\varphi}_{o}\right)$ munie de sa structure de niveau $n,{ }^{\tau^{n h}} \bar{\iota}_{o, n}$. Soit $\left(V_{o}, \varphi_{o}\right)$ le $\mathcal{O}_{o}$-module de Dieudonné associé à $S \longrightarrow M_{I^{o}, o}$. D'après le corollaire (I.2.1.3), on a $V_{o}=V_{o}^{e t} \oplus V_{o}^{c}$, où $V_{o}^{e t}$ est défini canoniquement et est stable par $\varphi_{o}$ dont la matrice dans une base associée à cette décomposition est de la forme

$$
\left(\begin{array}{cc}
M_{o}^{e t} & M_{o}^{e x t} \\
0 & M_{o}^{c}
\end{array}\right)
$$

avec $M_{o}^{e t}$ inversible. On considère alors $\tilde{V}_{o}=V_{o}^{e t} \oplus V_{o}^{c} \varpi_{o}^{n}$ défini canoniquement et muni de l'application $\tilde{\varphi}_{o}$ déduite de $\varphi_{o}$, dont une matrice dans une base adaptée à l'écriture ci-dessus est

$$
\left(\begin{array}{cc}
M_{o}^{e t} & \varpi_{o}^{n} M_{o}^{e x t} \\
0 & M_{o}^{c}
\end{array}\right)
$$

de sorte que $\left(\tilde{V}_{o, n}, \tilde{\varphi}_{o, n}\right)$ se scinde en partie étale et connexe. En outre comme $\bar{\varphi}_{o}^{! n h}$ induit un isomorphisme $\tau^{\tau^{n h}} \bar{V}_{o} \simeq \bar{V}_{o}^{e t} \oplus \bar{V}_{o}^{c} \varpi_{o}^{n}$, on en déduit que $\left(\tilde{V}_{o}, \tilde{\varphi}_{o}\right)$ est une déformation de $\left(\tau^{n h} \bar{V}_{o}, \bar{\varphi}_{o}^{n h}\right)$. La structure de niveau $\tilde{\iota}_{o, n}$ est alors définie comme la composée de $\iota_{o, n}^{c} \oplus \iota_{o, n}^{e t}$ et de l'inclusion $V_{o}^{*} \hookrightarrow \tilde{V}_{o}^{*}$, définition licite car $\left(\tilde{V}_{o, n}, \tilde{\varphi}_{o, n}\right)$ se scinde en partie étale et connexe.

I.2.2.7. Remarque. - On reprend les notations de la proposition précédente. A $f: S \longrightarrow$ $M_{I, s_{o}, a}^{=h}$, on associe $S \longrightarrow M_{I^{o}, o}$ ainsi que des déformations $\iota_{o, n}^{e t}$ et $\iota_{o, n}^{c}$ de respectivement $\bar{\iota}_{o, n}^{e t}$ et $\bar{\iota}_{o, n}^{c}$. La proposition précédente fournit alors une déformation $S \rightarrow M_{I, o}$ de $\bar{S} \stackrel{\operatorname{Frob}_{a}^{n h}}{\longrightarrow} \longrightarrow M_{I, s_{o}, a}^{=h}$. Le morphisme ainsi défini n'est alors rien d'autre que $f \circ$ Frob $_{o}^{n h}$.

I.2.2.8. Définition. - On note $\widehat{M_{I, o,=h, a}}$ l'ouvert au dessus de $M_{I, s_{o}, a}^{=h}$ du complété formel $\widehat{M_{I, o, h, a}}$ de $M_{I, o}$ le long de $M_{I, s_{o}, a}^{\geqslant h}$.

\section{I.2.3. Relation de congruence. -}

I.2.3.1. Proposition. - L'action d'un élément $g_{o}=\left(g_{o}^{c}, 1\right) \in G L_{h}\left(F_{o}\right) \times G L_{d-h}\left(F_{o}\right)$ (resp. $\left.\left(\varpi_{o}, 1\right)\right)$ sur $\varliminf_{I} M_{I, s_{o}, 1}^{=h}$ (resp. $\widehat{M_{I, o,=h, 1}}$ ) est donnée par Frob $\operatorname{vol}_{o}^{\left.\operatorname{vat} g_{o}^{c}\right)}\left(\right.$ resp. Frob $\left.{ }_{o}^{h}\right)$.

Démonstration. - On reprend les notations du paragraphe (I.1.3). Remarquons en premier lieu qu'il suffit de montrer le résultat pour $g_{o}^{c} \in G L_{h}\left(F_{o}\right) \cap M_{h}\left(\mathcal{O}_{o}\right)$. On rappelle que l'action de $W_{o}$ sur $M_{I, o}$ est telle que l'image du $\mathcal{D}$-faisceau elliptique $\left(\mathcal{E}_{i}, j_{i}, t_{i}\right)$ par un frobenius géométrique est $\left({ }^{\tau} \mathcal{E}_{i},{ }^{\tau} j_{i},{ }^{\tau} t_{i}\right)$. On raisonne sur le $\phi$-faisceau universel $\left(\mathcal{F}_{o}, \phi_{o}\right)$ sur $\underset{\leftarrow}{\lim } S_{I}$ avec $S_{I}=M_{I, o}$. A la décomposition $\left(F_{o} / \mathcal{O}_{o}\right)^{d}=\left(F_{o} / \mathcal{O}_{o}\right)^{h} \oplus\left(F_{o} / \mathcal{O}_{o}\right)^{d-h}$ on associe la décomposition

$$
\left(\mathcal{F}_{o}^{*} \otimes_{\mathcal{O}_{o}}\left(F_{o} / \mathcal{O}_{o}\right)\right) \otimes_{\mathcal{O}_{S}} \mathcal{O}_{S^{o}}=\mathcal{F}_{o, h}^{o} \oplus \mathcal{F}_{o, d-h}^{o} .
$$

L'action de $g_{o}$ sur $S^{o}$ est alors induite par celle à droite de $\left({ }^{t} g_{o}^{c}\right.$, Id) sur $\mathcal{F}_{o, h}^{o} \oplus \mathcal{F}_{o, d-h}^{o}$. 
I.2.3.2. Lemme. - Il existe des faisceaux en $\mathcal{O}_{S}$-modules $\mathcal{F}_{o, h}$ et $\mathcal{F}_{o, d-h}$ contenus respectivement dans $\mathcal{F}_{o, h}^{o}$ et $\mathcal{F}_{o, d-h}^{o}$ tels que

$$
\mathcal{F}_{o}^{*} \otimes_{\mathcal{O}_{o}}\left(F_{o} / \mathcal{O}_{o}\right)=\mathcal{F}_{o, h} \oplus \mathcal{F}_{o, d-h}
$$

Démonstration. - On rappelle que $S$ étant régulier, $\mathcal{F}_{o}^{*} \otimes_{\mathcal{O}_{o}}\left(F_{o} / \mathcal{O}_{o}\right)$ s'injecte canoniquement dans $\left(\mathcal{F}_{o}^{*} \otimes_{\mathcal{O}_{o}}\left(F_{o} / \mathcal{O}_{o}\right)\right) \otimes_{\mathcal{O}_{S}} \mathcal{O}_{S^{o}}$. On note $\mathcal{F}_{o, h}$ (resp. $\left.\mathcal{F}_{o, d-h}\right)$ le faisceau en $\mathcal{O}_{S}$-modules défini localement pour tout ouvert affine Spec $R \rightarrow S$, comme le $R$-module engendré par l'ensemble des $f_{h, R}$ (resp. $\left.f_{d-h, R}\right)$ tels qu'il existe $f_{R} \in \mathcal{F}_{o}^{*} \otimes_{\mathcal{O}_{o}}\left(F_{o} / \mathcal{O}_{o}\right)$ tel que $f_{R} \otimes 1=f_{h, R}+f_{d-h, R}$ dans $\left(\mathcal{F}_{o}^{*} \otimes_{\mathcal{O}_{o}}\left(F_{o} / \mathcal{O}_{o}\right)\right) \otimes_{\mathcal{O}_{S}} \mathcal{O}_{S^{o}}$. Pour tout entier $n, g_{o}^{n}\left(f_{R}\right)$ est un élément de $\mathcal{F}_{o, R}^{*}$ qui se décompose sous la forme $f_{d-h, R}+\varpi_{o}^{n} f_{h, R}$. Or pour $n$ assez grand, $\varpi_{o}^{n} f_{h, R}$ appartient à $\mathcal{F}_{o, R}^{*}$ de sorte que $\mathcal{F}_{o, d-h}=\mathcal{F}_{o}^{*} \cap \mathcal{F}_{o, d-h}^{o}$ et donc $\mathcal{F}_{o, h}=\mathcal{F}_{o}^{*} \cap \mathcal{F}_{o, h}^{o}$ et finalement $\mathcal{F}_{o}^{*}=\mathcal{F}_{o, h} \oplus \mathcal{F}_{o, d-h}$.

I.2.3.3 - On note $\left(\left(\mathcal{E}_{i}^{\prime}, j_{i}^{\prime}, t_{i}^{\prime}\right), \iota^{\prime}\right)$ le $\mathcal{D}$-faisceau elliptique muni de sa structure de niveau infinie, obtenu comme l'image par $g_{o}$ du $\mathcal{D}$-faisceau elliptique universel sur $S,\left(\mathcal{E}_{i}, j_{i}, t_{i}, \iota\right)$. Il est alors défini par le diagramme commutatif

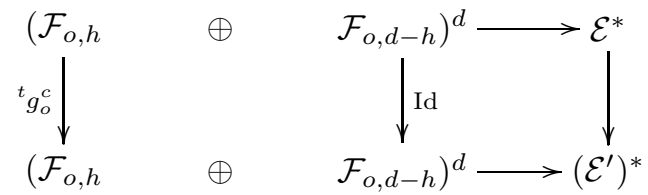

En termes de modules de Dieudonné, d'après la proposition (I.2.1.1), sur $M_{I, s_{o}, 1}^{=h}, \mathcal{F}_{o, d-h}$ correspond au dual de la composante étale $V_{o}^{\text {et }}$ de $V_{o}$. On note $m=\operatorname{val}\left(\operatorname{det} g_{o}^{c}\right)$. On rappelle que $\varphi_{o}^{! m}:{ }^{m} V_{o} \longrightarrow V_{o}$ est injective et a pour image $V_{o}^{e t} \oplus\left(g_{o}^{c} . V_{o}^{c}\right)$, de sorte que sur $M_{I, s_{o}, 1}^{=h}$, $t_{i}^{! m}:{ }^{\tau^{m}} \mathcal{E}_{i} \longrightarrow \mathcal{E}_{i}$ se factorise en un isomorphisme ${ }^{\tau^{m}} \mathcal{E}_{i} \longrightarrow \mathcal{E}_{i}^{\prime}$.

I.2.3.4 - En ce qui concerne les structures de niveaux, le seul problème se situe à la place $o$. Soient donc $\iota_{o, n+r}:\left(\mathcal{M}_{o}^{-n-r} / \mathcal{O}_{o}\right)^{d} \longrightarrow \mathcal{F}_{o, n+r}^{*}$ la structure de niveau $n+r$ en $o$ sur $\left(\mathcal{E}_{i}, j_{i}, t_{i}\right)$ où $r$ est assez grand. On rappelle qu'alors la structure de niveau $n$ en $o$ sur $\left(\mathcal{E}_{i}^{\prime}, j_{i}^{\prime}, t_{i}^{\prime}\right)$ est définie comme la composée de l'inclusion

$$
\left(\mathcal{M}_{o}^{-n} / \mathcal{O}_{o}\right)^{d} \hookrightarrow\left(\mathcal{M}_{o}^{-n-r} / \mathcal{O}_{o}\right)^{h} /\left(\mathcal{M}_{o}^{-r} / \mathcal{O}_{o}\right)^{h} \oplus\left(\mathcal{M}_{o}^{-n} / \mathcal{O}_{o}\right)^{d-h}
$$

avec $\iota_{o, n+r} \circ\left(\times{ }^{t} g_{o}\right)$ et de l'identification de $\left[g_{o}^{*}\right] \mathcal{F}_{o}^{*}$ avec $\left(\mathcal{F}_{o}^{\prime}\right)^{*}$. Sur la fibre spéciale, la partie connexe de la structure de niveau est nulle, il n’y a donc rien de plus à vérifier. Pour les déformations, on remarque que la matrice de $\phi_{o}^{! h}$ est de la forme $\left(\begin{array}{cc}\varpi_{o}^{\prime} & 0 \\ 0 & \mathrm{Id}\end{array}\right)$ pour une uniformisante $\varpi_{o}^{\prime}$ de $\mathcal{O}_{o}$, d'où le résultat.

I.2.3.5. Corollaire. - Soient $\left(g_{o}^{c}, g_{o}^{e t}\right) \in G L_{h}\left(F_{o}\right) \times G L_{d-h}\left(\mathcal{O}_{o}\right)$ et $n \geqslant m$ assez grand tel que $\left(g_{o}^{c}, g_{o}^{e t}\right)$ définit un morphisme

$$
M_{I^{o} \mathcal{M}_{o}^{n}, s_{o}, 1}^{=h} \stackrel{\left(g_{o}^{c}, g_{o}^{e t}\right)}{\longrightarrow} M_{I^{o} \mathcal{M}_{o}^{m}, s_{o}, 1}^{=h} .
$$

Le diagramme suivant est alors commutatif

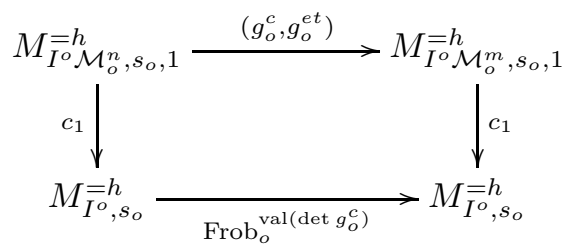

où $c_{1}$ est le morphisme de restriction du niveau. 
I.2.3.6. Corollaire. - L'action d'un élément de $G L_{d}\left(\mathcal{O}_{o}\right)$ de la forme

$$
\left(\begin{array}{cc}
I_{h} & 0 \\
* & I_{d-h}
\end{array}\right)
$$

est triviale $\operatorname{sur}\left(M_{\bar{I}, s_{o}, 1}^{=h}\right)_{\text {red }}$

\section{I.3. Variétés d'Igusa de première espèce}

I.3.1. Définition. - Soit $\mathcal{I}_{I^{o}, n}^{=h}(S)$ le $\kappa(o)$-schéma dont les $S$-points sont les $S$-points de $M_{I^{o}, s_{o}}^{=h}$ munis d'un isomorphisme

$$
\iota_{o, n}^{e t}:\left(\mathcal{M}_{o}^{-n} / \mathcal{O}_{o}\right)^{d-h} \times S \stackrel{\sim}{\longrightarrow} \mathcal{F}_{o, n}^{*, e t} \simeq \mathcal{F}_{o, n}^{e t, *}=\mathcal{F}_{o, n}^{*} / \mathcal{F}_{o, n}^{*, c}
$$

tel que $\phi_{o, n}^{*, e t} \circ \iota_{o, n}={ }^{\tau} \iota_{o, n}$, selon les notations habituelles.

I.3.1.1 - On a alors un morphisme d'oubli de la partie connexe de la structure de niveau

$$
i: M_{\bar{I}, s_{o}, a}^{=h} \longrightarrow \mathcal{I}_{I^{o}, n}^{=h}
$$

où $n$ est la multiplicité de $o$ dans $I$ et où $i$ est donné par

$$
\left(\left(\mathcal{E}_{i}, j_{i}, t_{i}\right), \iota_{I^{o}}, \iota_{o, n}\right) \longmapsto\left(\left(\mathcal{E}_{i}, j_{i}, t_{i}\right), \iota_{I^{o}}, \iota_{o, n}^{e t}\right) .
$$

I.3.1.2. Proposition. - Pour tout élément a de $G / P(d, h, n)$, il existe un morphisme $g_{n, a}$ : $\mathcal{I}_{I^{o}, n}^{=h} \longrightarrow M_{I, s_{o}, a}^{=h}$ qui rend le diagramme ci-dessous commutatif

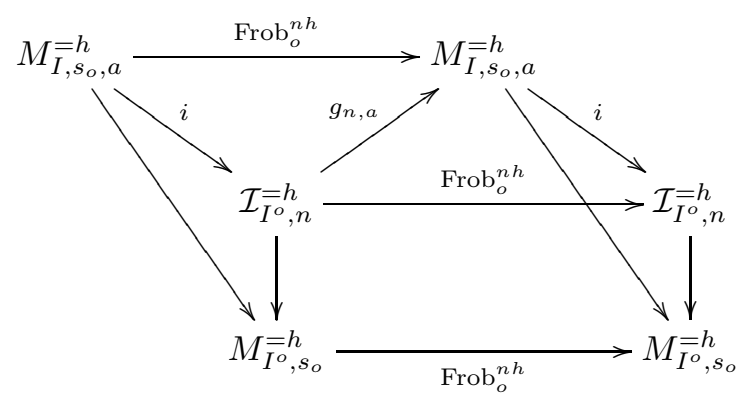

où $n$ est la multiplicité de o dans $I$. Ainsi le morphisme radiciel $g_{n, a}$ se factorise en un isomorphisme $g_{n, a, \text { red }}: \mathcal{I}_{I^{o}, n}^{=h} \longrightarrow M_{I, s_{o}, a, \text { red }}^{=h}$.

Démonstration. - Le morphisme de schéma $g_{m, a}$ est défini de la façon suivante. Étant donné un $S$-point de $\mathcal{I}_{\bar{I}^{o}, m}^{=h}$, il lui correspond un $\mathcal{D}$-faisceau elliptique $\left(\mathcal{E}_{i}, j_{i}, t_{i}\right) / S$ muni d'une $I^{o}$-structure de niveau et de l'isomorphisme $\iota_{o, m}^{e t} / S$. On lui associe alors le $S$-point de $M_{o^{m}, a}$ :

$$
\left\{\begin{array}{l}
\tau^{m h}\left(\mathcal{E}_{i}, j_{i}, t_{i}\right) \\
\tau^{m h} \iota_{I^{o}} \\
\iota_{o, m} \text { défini comme suit. }
\end{array}\right.
$$

D'après la proposition I.2.1.1, ${ }^{\tau^{m h}}\left(\mathcal{F}_{o, m}^{*}, \phi_{o, m}^{*}\right)$ se scinde en partie étale et connexe. Soit $b$ un supplémentaire de $a$ dans $\left(\mathcal{M}_{o}^{-m} / \mathcal{O}_{o}\right)^{d}$. On définit alors la structure de niveau $m$ :

$$
\iota_{o, m}:\left(\mathcal{M}_{o}^{-m} / \mathcal{O}_{o}\right)^{d}=a \oplus b \longrightarrow \tau^{m h}\left(\mathcal{F}_{o, m}\right)^{*}=\tau^{m h}\left(\mathcal{F}_{o, m}^{c}\right)^{*} \oplus \tau^{m h}\left(\mathcal{F}_{o, m}^{e t}\right)^{*}
$$

comme étant triviale sur $a$ et telle que sa restriction à $b$ soit donnée par $\tau^{m h}\left(\iota_{o, m}^{e t}\right)$. Vu la stabilité de ${ }^{\tau^{m h}}\left(\mathcal{F}_{o, m}^{c}\right)^{*}$ et de ${ }^{\tau^{m h}}\left(\mathcal{F}_{o, m}^{e t}\right)^{*}$ par ${ }^{\tau^{m h}} \phi_{o, m}^{*}$, on définit bien ainsi une $\mathcal{M}_{o}^{m}$-structure de niveau $\operatorname{sur} \tau^{m h}\left(\mathcal{E}_{i}, j_{i}, t_{i}\right)$ de telle sorte que la classe d'équivalence de $\left(\left(\mathcal{E}_{i}, j_{i}, t_{i}\right), \iota_{I}\right)$ est indépendante du choix de $b$ et de $\left(\mathcal{F}_{o, m}^{e t}\right)^{*}$. 
Vérifions alors la commutativité du triangle

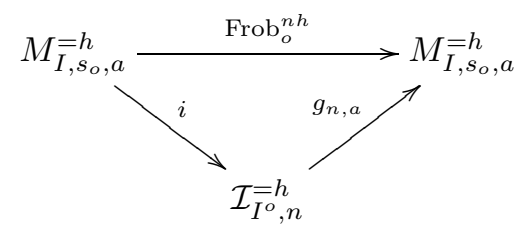

de l'énoncé, les autres commutativités en découlant de manière immédiate. Soit $S^{\prime}=\operatorname{Spec} R \rightarrow S$ un ouvert affine tel que $\mathcal{F}_{o, R}$ est libre et soit $\iota_{o, m}^{c} / S^{\prime}$ une structure de niveau $m$ sur $\left(\mathcal{F}_{o, R, m}^{c}\right)^{*}$. On peut choisir une base de $\left(\mathcal{F}_{o, R, m}^{c}\right)^{*}$ de sorte que la matrice de $\phi_{o, m}^{c, *}$ relativement à ce choix soit de la forme

$$
\left(\begin{array}{cccc}
0 & \cdots & \cdots & 0 \\
1 & \ddots & & \vdots \\
0 & \ddots & \ddots & \vdots \\
0 & \cdots & 1 & 0
\end{array}\right)
$$

Soit $z \in\left(\mathcal{M}_{o}^{-m} / \mathcal{O}_{o}\right)^{h}$, on pose $\iota_{o, m}^{c}(z)=\left(\begin{array}{c}x_{1} \\ \vdots \\ x_{h}\end{array}\right)$ avec $x_{i}=\sum_{k=0}^{m-1} x_{i}^{k} \varpi_{o}^{k}$. La relation $\left(\begin{array}{c}x_{1}^{q} \\ \vdots \\ x_{h}^{q}\end{array}\right)=$ $\phi_{o, m}^{c, *}\left(\begin{array}{c}x_{1} \\ \vdots \\ x_{h}\end{array}\right)$ permet d'exprimer tous les $x_{i}^{k}$ en fonction des $\left(x_{1}^{0}\right)^{q^{i}}$ pour $0 \leqslant i \leqslant m h$. De plus, comme $\left(M_{o, n}^{e x t, ! n h}\right)^{*}$ est nulle d'après (I.2.2.6), la condition de Drinfeld s'exprime par $\left(x_{1}^{0}\right)^{q^{m h}}=0$ de sorte que $\tau^{m h}\left(\iota_{o, m}^{c}\right)$ est triviale.

Le schéma $\mathcal{I}_{I^{o}, n}^{\bar{~} h}$ étant réduit, $g_{n, a}$ se factorise en un morphisme $g_{n, a, r e d}: \mathcal{I}_{I^{o}, n}^{=h} \longrightarrow M_{I, s_{o}, a, r e d}^{=h}$ qui est, d'après ce qui précède, une bijection au niveau des points géométriques et tel que le diagramme suivant est commutatif

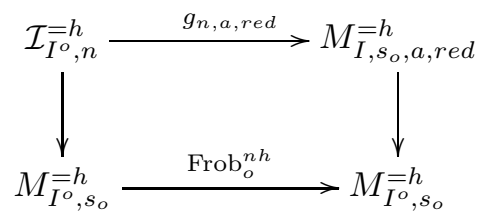

D'après le corollaire (I.2.2.4), $M_{I, s_{o}, a, r e d}^{=h} \longrightarrow M_{I^{o}, s_{o}}^{=h}$ est fini et plat de degré

$$
\#\left(\mathcal{O}_{o} / \mathcal{M}_{o}^{n}\right)^{h(d-h)} \# G L_{d-h}\left(\mathcal{O}_{o} / \mathcal{M}_{o}^{n}\right)
$$

où $n$ est la multiplicité de $o$ dans $I$. De même $\mathcal{I}_{I^{o}, n}^{=h} \longrightarrow M_{I^{o}, s_{o}}^{=h}$ est fini et plat de degré $\# G L_{d-h}\left(\mathcal{O}_{o} / \mathcal{M}_{o}^{n}\right)$ et $M_{I^{o}, s_{o}}^{\overline{=} h}$ étant régulier de dimension $d-h, M_{I^{o}, s_{o}}^{=} \stackrel{\text { Frob }_{o}^{n h}}{\longrightarrow} M_{I^{o}, s_{o}}^{\overline{=} h}$ est de degré $\#\left(\mathcal{O}_{o} / \mathcal{M}_{o}^{n}\right)^{h(d-h)}$. Comme $\mathcal{I}_{I^{o}, n}^{=h}$ et $M_{I, s_{o}, a, r e d}^{\bar{h}}$ sont lisses, on en déduit que $g_{n, a, r e d}$ est un isomorphisme.

I.3.1.3. Corollaire. - Pour tout $m \geqslant m^{\prime}$, on a des morphismes de transitions

$$
\mathcal{I}_{I^{o}, m}^{=h} \stackrel{\operatorname{Frob}_{o}^{\left(m-m^{\prime}\right) h}}{\longrightarrow} \mathcal{I}_{I^{o}, m^{\prime}}^{=h}
$$


et des diagrammes commutatifs

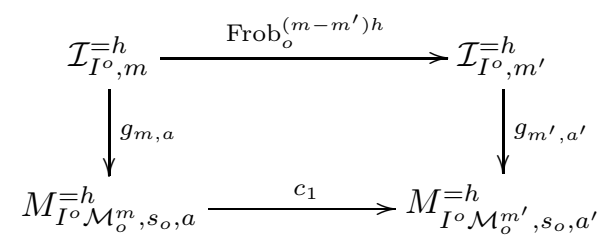

où $c_{1}$ est le morphisme de restriction du niveau.

I.3.2. Expression du complété formel de $M_{I, o}$ le long d'une strate. - Pour $I$ un idéal de $A$ et $m$ un entier, on considère l'extension étale

$$
\hat{\mathcal{I}}_{I^{o},=h, m} \longrightarrow \widehat{M_{I^{o},=h}}
$$

de fibre spéciale $\mathcal{I}_{I^{o}, m}^{=h} \longrightarrow M_{I^{o}, s_{o}}^{=h}$. On considère aussi

$$
\hat{\mathcal{I}}_{I^{o},=h, m}(t) \longrightarrow \hat{\mathcal{I}}_{I^{o},=h, m}
$$

l'espace classifiant des structures de niveau $t$ sur la partie connexe du $\varphi$-faisceau universel sur $\hat{\mathcal{I}}_{I^{o},=h, m}$.

I.3.2.1. Proposition. - Il existe un isomorphisme canonique

$$
\hat{g}_{n, a}: \hat{\mathcal{I}}_{I^{o},=h, n}(n) \longrightarrow \widehat{M_{I, o,=h, a}}
$$

qui prolonge le morphisme $g_{n, a}: \mathcal{I}_{I^{o}, n}^{=h} \longrightarrow M_{I, s_{o}, a}^{=h}$ défini au paragraphe précédent et tel que le diagramme ci-dessous soit commutatif

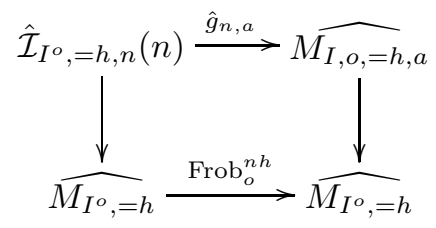

Démonstration. - La définition de $\hat{g}_{m, a}$ découle directement de la proposition (I.2.2.6), il ne reste plus qu'à voir qu'il s'agit d'un isomorphisme. Soit donc $S$ un $\operatorname{Spec} \mathcal{O}_{o^{-}}$-schéma muni d'un sous-faisceau d'idéaux nilpotents de $\mathcal{O}_{S}$ dont le fermé associé est $\bar{S}$ et $\left(S \stackrel{f_{i}}{\longrightarrow} \mathcal{I}_{I^{o}, m}^{=h},{ }_{i} \iota_{o, n}^{c}\right)$ pour $i=1,2$, dont les images par $\hat{g}_{m, a}$ sont des déformations isomorphes de $\bar{S} \longrightarrow \mathcal{I}_{I^{o}, m}^{=h} \longrightarrow M_{\bar{I}, s_{o}, a}^{=h}$. Notons $\left({ }_{i} V_{o},{ }_{i} \varphi_{o}\right)$ les $\mathcal{O}_{o}$-modules de Dieudonné sur $S$ associés à $f_{i}$ pour $i=1,2$. Par hypothèse, on a un isomorphisme

$$
h:{ }_{1} \tilde{V}_{o}={ }_{1} V_{o}^{e t}+\varpi_{o}^{n}{ }_{1} V_{o}^{c} \stackrel{\sim}{\longrightarrow}{ }_{2} \tilde{V}_{o}={ }_{2} V_{o}^{e t}+\varpi_{o}^{n}{ }_{2} V_{o}^{c}
$$

tel que $\bar{h}$ est l'identité et commute aux actions de ${ }_{i} \varphi_{o}$. Clairement $h$ induit un isomorphisme $h^{e t}:{ }_{1} V_{o}^{e t} \stackrel{\sim}{\longrightarrow}{ }_{2} V_{o}^{e t}$. Soit donc $v_{1} \in{ }_{1} V_{o}^{c}: h\left(\varpi_{o}^{n} v_{1}\right)=\varpi_{o}^{n} v_{2}+w$ avec $v_{2} \in{ }_{2} V_{o}^{c}$ et $w \in{ }_{2} V_{o}^{e t}$. On a

$$
\begin{array}{rlrl}
h\left({ }_{1} \varphi_{o}^{! n h}\left(\varpi_{o}^{n} v_{1}\right)\right) & =h\left(\varpi_{o}^{n}\left(\varpi_{o}^{n} u_{1}+u_{2}\right)\right) & u_{1} \in{ }_{1} V_{o}^{c}, & u_{2} \in{ }_{1} V_{o}^{e t} \\
& =\varpi_{o}^{n} w^{\prime} & w^{\prime} \in{ }_{2} V_{o} \\
& ={ }_{2} \varphi_{o}^{! n h}\left(\varpi_{o}^{n} v_{2}+w\right) &
\end{array}
$$

soit ${ }_{2}\left(\varphi_{o}^{e t}\right)^{! n h}(w) \in \varpi_{o}^{n} V_{o}^{e t}$, soit $w \in \varpi_{o}^{n} V_{o}^{e t}$. On en déduit donc un morphisme ${ }_{1} V_{o}^{c} \longrightarrow{ }_{2} V_{o}$ et finalement $h$ provient d'un isomorphisme ${ }_{1} V_{o} \simeq{ }_{2} V_{o}$. Si en outre $\iota_{o, n, 1}$ et $\iota_{o, n, 2}$ sont isomorphes, il est clair du fait que les ${ }_{i} \tilde{V}_{o, n}$, pour $i=1,2$, se scindent en partie étale et connexe, que les triplés $\left({ }_{i} V_{o},{ }_{i} \iota_{o, n}^{e t},{ }_{i} \iota_{o, n}^{c}\right)$ pour $i=1,2$, sont isomorphes. Pour des raisons de dimension, $\hat{g}_{m, a}$ induit alors un isomorphisme sur les espaces tangents, c'est donc un isomorphisme. 
Remarque : Soit $i: \widehat{M_{I, o,=h, a}} \longrightarrow \hat{\mathcal{I}}_{I^{o},=h, n}(n)$ la flèche donnée par

$$
i\left(\left(\mathcal{E}_{i}, j_{i}, t_{i}\right), \iota_{I^{o}}, \iota_{o, n}\right)=\left(\left(\mathcal{E}_{i}, j_{i}, t_{i}\right), \iota_{I^{o}}, \iota_{o, n}^{e t}, \iota_{o, n}^{c}\right)
$$

qui prolonge celle définie au paragraphe précédent. Le composé

$$
\widehat{M_{I, o,=h, a}} \longrightarrow \hat{\mathcal{I}}_{I^{o},=h, n}(n) \stackrel{\hat{g}_{n, a}}{\longrightarrow} \widehat{M_{I, o,=h, a}}
$$

est $\operatorname{Frob}_{o}^{n h}$.

\section{I.3.3. Correspondances de Hecke. -}

I.3.3.1. Proposition. - La tour $\left(\mathcal{I}_{I^{o}, n}^{=h}\right)_{n}$ est munie de correspondances de Hecke associées aux éléments de $G L_{d-h}\left(F_{o}\right) \times \mathbb{Z}$, compatibles aux morphisme $g_{m, a}$, c'est à dire que pour tout $n_{e t} \geqslant 0$, et tout élément $\left(g_{o}^{\text {et }}, r\right) \in G L_{d-h}\left(F_{o}\right) \times \mathbb{Z}$, il existe $m_{0} \geqslant n$ ainsi que des morphismes $\mathcal{I}_{I^{o}, m}^{=h} \stackrel{\left(g_{o}^{e t}, r\right)}{\longrightarrow} \mathcal{I}_{I^{o}, n}^{=h}$ pour tout $m \geqslant m_{0}$ compatibles aux morphismes de restriction du niveau, tels que le diagramme suivant soit commutatif

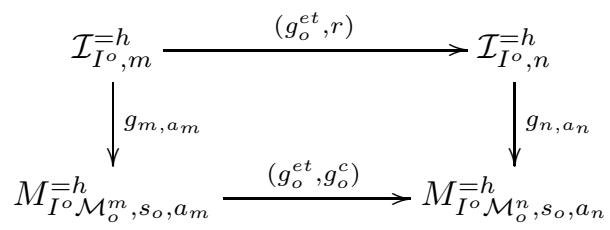

pour $a_{n}, a_{m}$ des éléments de $G / P(d, h, n)$ et $G / P(d, h, m)$ respectivement tels que $a_{n}$ soit l'image de $a_{m}$ par la surjection canonique, et où $g_{o}^{c}$ est un élément quelconque de $G L_{h}\left(F_{o}\right)$ tel que $r=$ $\operatorname{val}\left(\operatorname{det} g_{o}^{c}\right)$.

Démonstration. - On définit les correspondances de Hecke sur $\mathcal{I}_{I^{o}, m}^{h}$ associées aux éléments de $G L_{d-h}\left(F_{o}\right) \times \mathbb{Z}$, en procédant comme suit.

- Soit $g_{o}^{e t} \in G L_{d-h}\left(F_{o}\right)$ tel que $g_{o}^{e t} \in \mathbb{M}_{d-h}\left(\mathcal{O}_{o}\right)$. On choisit $m$ et $n$ tels que le noyau de ${ }^{t} g_{o}^{e t}:\left(F_{o} / \mathcal{O}_{o}\right)^{d-h} \longrightarrow\left(F_{o} / \mathcal{O}_{o}\right)^{d-h}$ est contenu dans $\left(\mathcal{M}_{o}^{-m} / \mathcal{O}_{o}\right)^{d-h}$ et tels que $\left(\mathcal{M}_{o}^{-n} / \mathcal{O}_{o}\right)^{d-h}$ est contenu dans

$$
\left.\operatorname{Im}\left({ }^{t} g_{o}^{e t}:\left(\mathcal{M}_{o}^{-m} / \mathcal{O}_{o}\right)^{d-h}\right) \rightarrow\left(\mathcal{M}_{o}^{-m} / \mathcal{O}_{o}\right)^{d-h}\right) .
$$

Soient $S=\mathcal{I}_{\bar{I}^{o}, m}^{=h}$ et $\left(\left(\mathcal{E}_{i}, j_{i}, t_{i}\right), \iota_{I^{o}}, \iota_{o, m}^{e t}\right)$ l'objet universel de $\mathcal{I}_{I^{o}, m}^{=h}$. A $g_{o}^{e t}$ on associe via la structure de niveau $\iota_{o, m}^{e t}$, le morphisme $\left[g_{o}^{e t}\right]: \mathcal{E}_{o, m}^{e t} \rightarrow \mathcal{E}_{o, m}^{e t}$ défini comme au paragraphe (I.1.3) du chapitre précédent.

- L'action de $\left(g_{o}^{e t},-k\right)$ pour $g_{o}^{e t} \in \mathbb{M}_{d-h}\left(\mathcal{O}_{o}\right)$ et $k$ un entier naturel assez grand tel que $\varpi_{o}^{[k / h]}\left(g_{o}^{e t}\right)^{-1} \in \mathbb{M}_{d-h}\left(\mathcal{O}_{o}\right)$, de sorte que $\varpi_{o}^{[k / h]} \mathcal{E}_{o, m}^{e t} \subset\left[g_{o}^{e t}\right] \mathcal{E}_{o, m}^{e t}$, est alors définie comme suit. Soit $\mathcal{E}_{i}^{\prime}$ défini par le carré cartésien

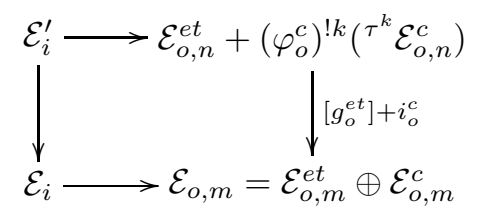

où $i_{o}^{c}$ est l'inclusion canonique.

On rappelle que modulo $\mathcal{E}_{o, m}^{e t}$, on a $\left(\varphi_{o}^{c}\right)^{! h}\left(\tau^{h} \mathcal{E}_{o}^{c}\right)=\varpi_{o} \mathcal{E}_{o}^{c}$, et donc l'image par $\varphi_{o}$ de $\tau\left(\left(\varphi_{o}^{c}\right)^{! k}\left(\tau^{k} \mathcal{E}_{o}^{c}\right)\right.$ est incluse dans $\left.\left[g_{o}^{e t}\right]\left(\mathcal{E}_{o, n}^{e t}\right)+\left(\varphi_{o}^{c}\right)^{! k}\left(\tau^{k} \mathcal{E}_{o, n}^{c}\right)\right)$, ce qui permet de définir des applications $t_{i}^{\prime}:{ }^{\tau} \mathcal{E}_{i}^{\prime} \longrightarrow \mathcal{E}_{i+1}^{\prime}$ déduites des $t_{i}$. Les $\mathcal{E}_{i}^{\prime}$ peuvent ainsi être organisés en un $\mathcal{D}$-faisceau elliptique et on peut de plus le munir de la $\mathcal{M}_{o}^{n}$-structure de niveau $\iota_{o, n}^{\prime}$ et sur la partie étale déduite de $\iota_{o, m}^{e t}$. Le $\mathcal{D}$-faisceau elliptique image par $\left(g_{o}^{e t}, k\right)$ de $\left(\left(\mathcal{E}_{i}, j_{i}, t_{i}\right), \iota_{I^{o}}, \iota_{o, m}^{e t}\right)$ est alors $\left(\left(\tau^{m-n} \mathcal{E}_{i}^{\prime}, \tau^{m-n} j_{i}^{\prime}, \tau^{m-n} t_{i}^{\prime}\right),{ }^{m-n} \iota_{I^{o}}^{\prime},{ }^{m-n} \iota_{o, n}^{\prime}\right.$ et $)$.

- On fait agir un élément $\varpi_{o}^{k}, k \geqslant 0$ du centre de $G L_{d-h}\left(F_{o}\right)$ en tordant $\mathcal{E}_{o}^{e t}$ par le faisceau inversible $\mathcal{O}_{X}(k . o)$, c'est à dire avec des notations similaires à celles introduites ci-dessus, $\mathcal{E}_{o}^{\prime}:=$ 
$\varpi_{o}^{k} \mathcal{E}_{o}^{e t} \oplus \mathcal{E}_{o}^{c}$. On peut de manière évidente définir des applications $t_{i}^{\prime}$ et donc organiser les $\mathcal{E}_{i}^{\prime}$ en un $\mathcal{D}$-faisceau elliptique muni d'une $\mathcal{M}_{o}^{n}$-structure de niveau sur la partie étale.

Soient $r \in \mathbb{Z}$ et $m \geqslant n$ tels que $m-n-r \geqslant 0$. On définit le morphisme $[r]: \mathcal{I}_{I^{o}, m}^{=h} \longrightarrow \mathcal{I}_{I^{o}, n}^{=h}$ comme étant égal à Frob ${ }_{o}^{m-n-r}$.

En remarquant que pour $k \geqslant 0, \varphi_{o}^{! k}$ induit un isomorphisme entre $\tau^{\tau^{k}}\left(\mathcal{E}_{o}^{e t} \oplus \mathcal{E}_{o}^{c}\right)$ et $\mathcal{E}_{o}^{e t}+\left(\varphi_{o}^{c}\right)^{k}\left(\mathcal{E}_{o}^{c}\right)$,

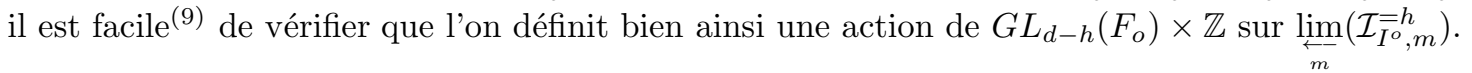

En vertu de la proposition (I.2.3.1) du chapitre précédent, il est immédiat de vérifier la commutativité du diagramme de l'énoncé.

I.3.3.2. Proposition. - La tour $\left(\hat{\mathcal{I}}_{I^{o},=h, n}(n)\right)_{n}$ est munie de correspondances de Hecke associées aux éléments de $G L_{d-h}\left(F_{o}\right) \times G L_{h}\left(F_{o}\right)$, compatibles aux morphisme $\hat{g}_{m, a}$, c'est à dire que pour tout $n \geqslant 0$, et tout élément $\left(g_{o}^{e t}, g_{o}^{c}\right) \in G L_{d-h}\left(F_{o}\right) \times G L_{h}\left(F_{o}\right)$, il existe $m_{0} \geqslant n$ ainsi que des morphismes

$$
\hat{\mathcal{I}}_{I^{o},=h, m}(m) \stackrel{\left(g_{o}^{e t}, g_{o}^{c}\right)}{\longrightarrow} \hat{\mathcal{I}}_{I^{o},=h, n}(n)
$$

pour tout $m \geqslant m_{0}$ compatibles aux morphismes de restriction du niveau, tels que les diagrammes suivants soient commutatifs

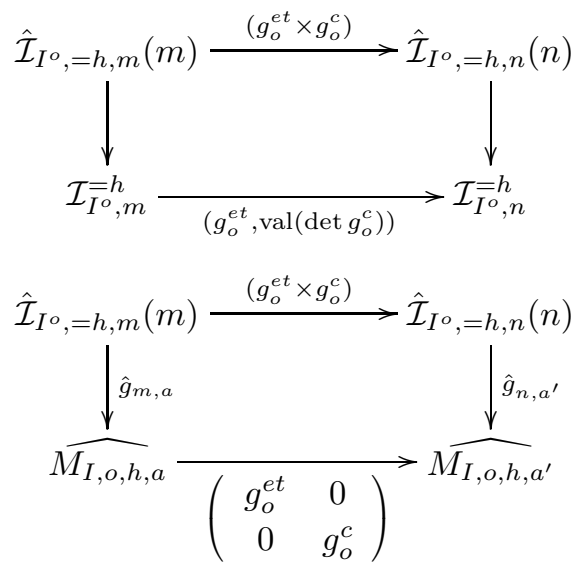

Démonstration. - On définit une correspondance de Hecke associée à un élément $\left(g_{o}^{e t}, g_{o}^{c}\right)$ de $G L_{d-h}\left(F_{o}\right) \times G L_{h}\left(F_{o}\right)$ sur $\hat{\mathcal{I}}_{I^{o},=h, m}(m)$, de telle sorte que l'action induite sur $\mathcal{I}_{I^{o}, m}^{=h}$ est donnée $\operatorname{par}\left(g_{o}^{e t}, \operatorname{val}\left(\operatorname{det} g_{o}^{c}\right)\right) \in G L_{d-h}\left(F_{o}\right) \times \mathbb{Z}$ telle qu'elle est définie ci-dessus.

- Soit $g_{o}^{c}$ (resp. $g_{o}^{e t}$ ) un élément de $G L_{h}\left(F_{o}\right)$ (resp. de $G L_{d-h}\left(F_{o}\right)$ ) tel que

$$
\begin{gathered}
g_{o}^{c} \in \mathbb{M}_{h}\left(\mathcal{O}_{o}\right) \\
\text { (resp. } \left.g_{o}^{e t} \in \mathbb{M}_{d-h}\left(\mathcal{O}_{o}\right)\right) \text { et } \varpi_{o}^{\operatorname{val}\left(\operatorname{det} g_{o}^{c}\right) / h}\left(g_{o}^{e t}\right)^{-1} \in \mathbb{M}_{d-h}\left(\mathcal{O}_{o}\right)
\end{gathered}
$$

de sorte que $\varpi_{o}^{\left[\operatorname{val}\left(\operatorname{det} g_{o}^{c}\right) / h\right]} \mathcal{E}_{o, m}^{e t} \subset\left[g_{o}^{e t}\right] \mathcal{E}_{o, m}^{e t}$. On choisit $m$ et $n$ tels que les noyaux de ${ }^{t} g_{o}^{c}:$ $\left.\left(F_{o} / \mathcal{O}_{o}\right)^{h} \longrightarrow F_{o} / \mathcal{O}_{o}\right)^{h}$ et ${ }^{t} g_{o}^{\text {et }}:\left(F_{o} / \mathcal{O}_{o}\right)^{d-h} \longrightarrow\left(F_{o} / \mathcal{O}_{o}\right)^{d-h}$ sont contenus dans $\left(\mathcal{M}_{o}^{-m} / \mathcal{O}_{o}\right)^{h}$ et tels que $\left.\left(\mathcal{M}_{o}^{-n} / \mathcal{O}_{o}\right)^{h}\left(\operatorname{resp} . \mathcal{M}_{o}^{-n} / \mathcal{O}_{o}\right)^{d-h}\right)$ est contenu dans

$$
\left.\operatorname{Im}\left({ }^{t} g_{o}^{c}:\left(\mathcal{M}_{o}^{-m} / \mathcal{O}_{o}\right)^{h} \longrightarrow \mathcal{M}_{o}^{-m} / \mathcal{O}_{o}\right)^{h}\right)
$$

(resp. dans $\left.\operatorname{Im}\left({ }^{t} g_{o}^{\text {et }}:\left(\mathcal{M}_{o}^{-m} / \mathcal{O}_{o}\right)^{d-h} \longrightarrow\left(\mathcal{M}_{o}^{-m} / \mathcal{O}_{o}\right)^{d-h}\right)\right)$. Soient $S$ un $\left(\hat{\mathcal{O}}_{o}^{n r}\right)$-schéma et ainsi qu'un $S$-point $\left(\left(\mathcal{E}_{i}, j_{i}, t_{i}\right), \iota_{I^{o}}, \iota_{o, m}^{c}, \iota_{o, m}^{e t}\right)$ de $\hat{\mathcal{I}}_{I^{o},=h, m}(m)$. Pour définir l'action du couple $\left(g_{o}^{e t}, g_{o}^{c}\right)$, il suffit de définir une structure de niveau $n \operatorname{sur}\left(\varphi_{o}^{c}\right)^{!}\left(\operatorname{val}\left(\operatorname{det} g_{o}^{c}\right)\right)\left(\mathcal{E}_{o}^{c}\right) \simeq \tau^{\operatorname{val}\left(\operatorname{det} g_{o}^{c}\right)} \mathcal{E}_{o}^{c}$. Soit $\left[g_{o}^{c}\right]: \mathcal{E}_{o, m}^{c} \rightarrow$ $\mathcal{E}_{o, m}^{c}$ le morphisme associé à $g_{o}^{c}$ défini via $\iota_{o, m}^{c}$, la structure de niveau $m$ (cf. le paragraphe (I.1.3)).

${ }^{(9)}$ essentiellement il s'agit de vérifier que l'action de $\left(g_{o}^{\text {et }}, k+s\right)$ est égale à celle de $\left(g_{o}^{\text {et }}, k\right)(1, s)$ pour $\left(g_{o}^{\text {et }}\right)^{-1} \in$ $\mathbb{M}_{d}\left(\mathcal{O}_{o}\right), s \geqslant 0$ et $k$ assez grand 
On a déjà vu que $\left[g_{o}^{c}\right]\left(\mathcal{E}_{o, m}^{c}\right) \simeq \tau^{\mathrm{val}\left(\operatorname{det} g_{o}^{c}\right)} \mathcal{E}_{o, m}^{c}$ de sorte que la structure de niveau $n$ déduite de $\iota_{o, m}^{c}$ convient.

- L'action d'un élément du centre de $G L_{d-h}\left(F_{o}\right)$ est définie de manière évidente. De même l'action de $\varpi_{o}^{k}$ vu comme élément du centre de $G L_{h}\left(F_{o}\right)$ se définit de manière naturelle $\hat{\mathcal{I}}_{I^{o},=h, m}(m) \longrightarrow \hat{\mathcal{I}}_{I^{o},=h, n}(n)$ pour $m$ et $n$ tels que $m-n+h k \geqslant 0$, et relève

$$
\operatorname{Frob}_{o}^{m-n+h k}: \mathcal{I}_{I^{o}, m}^{=h} \longrightarrow \mathcal{I}_{I^{o}, n}^{=h} .
$$

On vérifie aisément que l'on définit bien ainsi une action de $G L_{d-h}\left(F_{o}\right) \times G L_{h}\left(F_{o}\right)$ sur $\lim _{\leftarrow} \hat{\mathcal{I}}_{I^{o},=h, m}(m)$ de telle sorte que l'on a le diagramme commutatif suivant

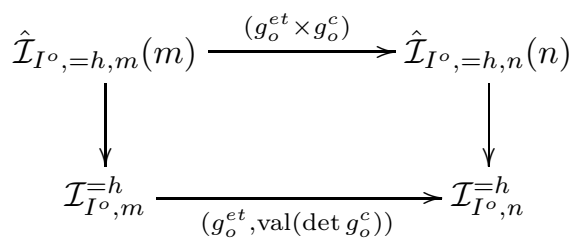

En vertu de la proposition (I.2.2.6) et de la définition de $\hat{g}_{m, a}$, le diagramme suivant est alors commutatif

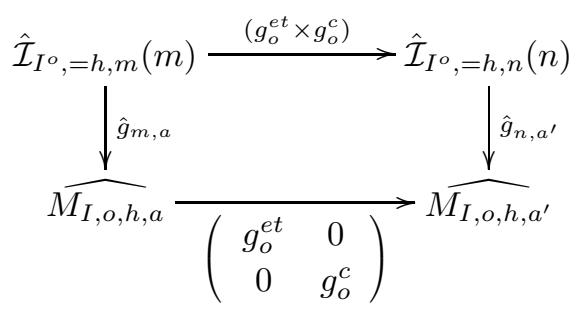

\section{I.4. Variétés d'Igusa de seconde espèce}

On rappelle que pour un idéal $I$ de $A$, on note $I=I^{o} \mathcal{M}_{o}^{n}$ avec $o \notin V\left(I^{o}\right)$. Soit $\Pi_{o, d}$ l'élément suivant de $G L_{d}\left(\mathcal{O}_{o}\right)$

$$
\left(\begin{array}{cccc}
0 & \cdots & 0 & \varpi_{o} \\
1 & 0 & \vdots & 0 \\
0 & \ddots & & \vdots \\
0 & 0 & 1 & 0
\end{array}\right)
$$

I.4.1. Définition. - Étant donnés un $\kappa(o)$-schéma $S$, ainsi qu'un $S$-point de $\mathcal{I}_{\bar{I}^{o}, m}^{=h}$, on a un $\mathcal{O}_{o} \otimes_{\kappa(o)} \mathcal{O}_{S}$-module localement libre de rang $d, \mathcal{F}_{o}$ ainsi qu'un morphisme linéaire injectif $\phi_{o}$ : ${ }^{\tau} \mathcal{F}_{o} \longrightarrow \mathcal{F}_{o}$.

Considérons la catégorie fibrée $\mathfrak{J}_{I^{o}, m}^{=h}(s)$ sur la catégorie des $\kappa(o)$-schémas dont les objets sont

$$
S \mapsto\left\{\begin{array}{l}
. S \longrightarrow \mathcal{I}_{\bar{I}^{o}, m}^{=h} \\
. \text { une section globale } \sigma \text { de } \mathcal{F}_{o} / \phi_{o}^{!(s)}\left(\tau^{s} \mathcal{F}_{o}\right) \text { telle que } \\
\phi_{o}^{! h}(\sigma \otimes 1)=\varpi_{o} \sigma \text { et telle que l'application } \mathcal{O}_{S} \longrightarrow \mathcal{F}_{o} / \phi_{o}\left({ }^{\tau} \mathcal{F}_{o}\right) \\
a \mapsto a \bar{\sigma}, \text { est inversible }
\end{array}\right.
$$

Dans la suite, on notera $\mathcal{F}_{o, / s}$ le quotient $\mathcal{F}_{o} / \phi_{o}^{! s}\left(\tau^{s} \mathcal{F}_{o}\right)$. 
I.4.1.1. Proposition. - Le morphisme naturel $\mathfrak{J}_{I^{o}, m}^{h}(s) \longrightarrow \mathcal{I}_{I^{o}, m}^{h}$ est relativement représentable par un schéma $\mathcal{J}_{I^{o}, m}^{=h}(s) \longrightarrow \mathcal{I}_{I^{o}, m}^{=h}$ étale galoisien de groupe de Galois $\mathcal{D}_{o, h, s}^{\times}:=\left(\mathcal{D}_{o, h} /\left(\Pi_{o, h}^{s}\right)\right)^{\times}$. En outre le diagramme suivant est cartésien

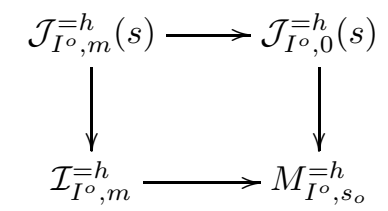

Démonstration. - La représentabilité est immédiate et le diagramme est clairement cartésien. Soit alors Spec $R \rightarrow S$ un ouvert affine tel que $\mathcal{F}_{o, R}:=\mathcal{F}_{o} \otimes_{\mathcal{O}_{S}} R$ est libre. D'après la proposition (I.2.1.1), il existe une décomposition

$$
\mathcal{F}_{o, R}=\mathcal{F}_{o, R}^{c} \oplus \mathcal{F}_{o, R}^{e t}
$$

dans laquelle la matrice de $\phi_{o}$ est de la forme $\left(\begin{array}{cc}M_{c} & 0 \\ M_{\text {ext }} & M_{e t}\end{array}\right)$ avec $M_{e t}$ inversible de taille $d-h$ de sorte que $\mathcal{F}_{o, R}^{e t} \subset \phi_{o}^{!(s+1)}\left(\tau^{s+1} \mathcal{F}_{o, R}\right)$ pour tout $s>0$. En outre $\mathcal{F}_{o, R} / \phi_{o}\left({ }^{\tau} \mathcal{F}_{o, R}\right)$ étant un $R$-module libre de rang 1 , il existe donc $e_{0} \in \mathcal{F}_{o, R}^{c}$ tel que

$$
\left(e_{0}, \phi_{o}\left(e_{0} \otimes 1\right), \cdots, \phi_{o}^{!(h-1)}\left(e_{0} \otimes 1\right)\right)
$$

soit une base $\operatorname{de} \mathcal{F}_{o, R}^{c}$ avec

$$
\phi_{o}^{! h}\left(e_{0} \otimes 1\right)=\varpi_{o} \otimes 1 \sum_{i=0}^{h-1} \gamma_{i} \phi_{o}^{! i}\left(e_{0} \otimes 1\right) \quad \text { modulo } \mathcal{F}_{o}^{e t}
$$

où $\gamma_{i} \in \mathcal{O}_{o} \hat{\otimes}_{\kappa(o)} R$. Il s'agit ainsi de trouver $\sigma=\sum_{i=0}^{h-1} \alpha_{i} \cdot \phi_{o}^{! i}\left(e_{0} \otimes 1\right)$ avec $\alpha_{i}=\sum_{\substack{0 \leq j \\ i+j . h \leqslant s}} \beta_{i}^{j} \varpi_{o}^{j}$ telle que $\phi_{o}^{! h}(\sigma \otimes 1)=\varpi_{o} \sigma$ et $\beta_{0}^{0}$ inversible. Notons

$$
\left(W_{h, S, o}, \phi_{h, S, o}\right)
$$

le $\phi$-faisceau $W_{h, S, o}:=\left(\mathcal{O}_{o} \otimes_{\kappa(o)} \mathcal{O}_{S}\right)^{h}$ tel que la matrice de

$$
\phi_{h, S, o}:{ }^{\tau} W_{h, S, o} \longrightarrow W_{h, S, o}
$$

dans la base canonique est donnée par $\phi_{h, S, o}\left(e_{i} \otimes 1\right)=e_{i+1}$ pour $1 \leqslant i<h$ et $\phi_{h, S, o}\left(e_{h} \otimes 1\right)=\varpi_{o} . e_{1}$. Le groupe $\operatorname{Aut}\left(W_{h, S, o}, \varphi_{h, S, o}\right)$ est

$$
\mathcal{D}_{o, h}^{\times} \simeq\left\{P \in G L_{h}\left(\mathcal{O}_{o}\right) / P \Pi_{o, h}=\Pi_{o, h} P\right\}
$$

Trouver $\sigma$ revient alors à rigidifier la partie connexe de $\mathcal{F}_{o}$, i.e. à donner un isomorphisme

$$
\left(\mathcal{F}_{o}^{c}, \phi_{o}^{c}\right) \simeq\left(W_{h, S, o}, \phi_{h, S, o}\right)
$$

modulo $\Pi_{o, h}^{s}$. On peut ainsi obtenir aisément des équations du revêtement à partir des éléments de la matrice $M_{c}$. On en déduit en outre que le revêtement est étale de groupe de Galois :

$$
\operatorname{Aut}\left(W_{h, S, o, / s}, \phi_{h, S, o, / s}\right) \simeq\left\{P \in G L_{h}\left(\mathcal{O}_{o}\right) /\left(1+\Pi_{o, h}^{s} \mathbb{M}_{h}\left(\mathcal{O}_{o}\right) / P \Pi_{o, h}=\Pi_{o, h} P\right\}\right.
$$

Remarque : Si $S$ est le spectre d'un corps ou d'un anneau artinien, il correspond, par la théorie du module de coordonnées (cf. [12]), à $\left(V_{o}^{c}, \varphi_{o}^{c}\right)$ un $\mathcal{O}_{o}$-module formel de hauteur $h$, de sorte que les $S$-points de $\mathcal{J}_{I^{o}, m}^{=h}(s)$ sont ceux de $\mathcal{I}_{I^{o}, m} \stackrel{h}{h}$ muni d'une rigidification à l'ordre $s$ du $\mathcal{O}_{o^{-}}$-module formel de hauteur $h$ qui leur est associé. Ainsi $\mathcal{J}_{I^{o}, m}^{=h}(s)$ est la variété d'Igusa de seconde espèce telle qu'elle est définie dans [14]. 


\section{I.4.2. Correspondances de Hecke sur $\mathcal{J}_{I^{o}, n}^{=h}(s)$. -}

I.4.2.1. Proposition. - La tour $\left(\mathcal{J}_{I^{\circ}, n}^{=h}(s)\right)_{s, n}$ est munie de correspondances de Hecke associées aux éléments du noyau $\widetilde{\mathcal{N}}_{o}$ de l'application

$$
\begin{array}{cl}
G L_{h}\left(F_{o}\right) \times D_{o, h}^{\times} \times W_{o} & \longrightarrow \mathbb{Z} \\
\left(g_{o}^{c}, \delta_{o}, \sigma_{o}\right) & \mapsto \operatorname{val}\left(\operatorname{det}\left(g_{o}^{c}\right) \operatorname{rn}\left(\delta_{o}\right) \operatorname{cl}\left(\sigma_{o}\right)\right)
\end{array}
$$

où $\mathrm{rn}: D_{o, h}^{\times} \longrightarrow F_{o}^{\times}$est la norme réduite, et $\mathrm{cl}: W_{F_{o}} \longrightarrow F_{o}^{\times}$est l'application de la théorie du corps de classe, de manière compatible aux morphismes $\mathcal{J}_{I^{o}, n}^{=h}(s) \longrightarrow \mathcal{I}_{I^{o}, n}^{=h}$, i.e. pour tout $n, s$, il existe $m_{0} \geqslant n$ et $t_{0} \geqslant s$ tels que pour tout $m \geqslant m_{0}$ et $t \geqslant t_{0}$, on ait des morphismes

$$
\mathcal{J}_{I^{o}, m}^{=h}(t) \stackrel{g_{o}^{e t} \times g_{o}^{c} \times \delta_{o} \times \sigma_{o}}{\longrightarrow} \mathcal{J}_{I^{o}, n}^{=h}(s)
$$

compatibles aux morphismes de restriction du niveau et tels que le diagramme suivant soit commutatif :

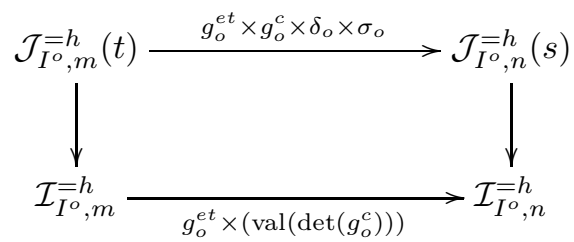

Démonstration. - - Les correspondances géométriques sur $\mathcal{I}_{\bar{I}^{o}, n}^{h}$ associées aux éléments de $\left(D_{\mathbb{A}}^{\infty, o}\right)^{\times} \times G L_{h}\left(\mathcal{O}_{o}\right) \times G L_{d-h}\left(F_{o}\right)$ se remontent aisément sur $\mathcal{J}_{I^{o}, n, o}^{=h}(m)$.

- Celles associées aux éléments de $\mathcal{D}_{o, h}^{\times}$sont données via l'isomorphisme $\operatorname{Aut}\left(V_{o}^{c}, \varphi_{o}^{c}\right) \simeq \mathcal{D}_{o, h}^{\times}$, de sorte que

$$
\mathcal{J}_{I^{o}, n}^{=h}(s)=\left(\lim _{\leftarrow} \mathcal{J}_{s^{\prime}}^{=h}\left(s^{\prime}\right)\right)^{\left(1+\Pi_{o, h}^{s} \mathcal{D}_{o, h}\right)} .
$$

- Soit $\left(g_{o}^{c}, \delta_{o}, 1\right)$ un élément de $\widetilde{\mathcal{N}}_{o}$ avec $g_{o}^{c} \in \mathbb{M}_{h}\left(\mathcal{O}_{o}\right)$. On choisit $m \geqslant n$ tels que

$$
\begin{gathered}
\operatorname{Ker}\left({ }^{t} g_{o}^{e t}\right) \subset\left(\mathcal{M}_{o}^{-m} / \mathcal{O}_{o}\right)^{d-h}, \\
\left(\mathcal{M}_{o}^{-n} / \mathcal{O}_{o}\right)^{d-h} \subset \operatorname{Im}\left({ }^{t} g_{o}^{e t}\right) \\
\varpi_{o}^{\operatorname{val}\left(\operatorname{det} g_{c}\right) / h}\left(g_{o}^{e t}\right)^{-1} \in \mathbb{M}_{d-h}\left(\mathcal{O}_{o}\right),
\end{gathered}
$$

de sorte que $\left(g_{o}^{e t}, g_{o}^{c}\right)$ définisse une correspondance de Hecke

$$
\mathcal{I}_{I^{o}, m}^{=h} \longrightarrow \mathcal{I}_{I^{o}, n}^{=h} \text {. }
$$

Il reste alors à définir, pour $t$ assez grand, à partir d'un isomorphisme

$$
\alpha_{t}:\left(\mathcal{F}_{o, / t}^{c}, \phi_{o, / t}^{c}\right) \simeq\left(W_{h, S, o, / t}, \phi_{h, S, o, / t}\right),
$$

un isomorphisme

$$
\alpha_{s}^{\prime}:\left(\mathcal{F}_{o, / s}^{c, '}, \phi_{o, / s}^{c, '}\right) \simeq\left(W_{h, S, o, / s}, \phi_{h, S, o, / s}\right),
$$

où $\left(\mathcal{F}_{o}^{c,}, \phi_{o}^{c, '}\right)$ est le $\phi$-faisceau connexe associé à $\left(\mathcal{F}_{o}^{c}, \phi_{o}^{c}\right)$ par ${ }^{t} g_{o}^{c}$. Rappelons que l'application

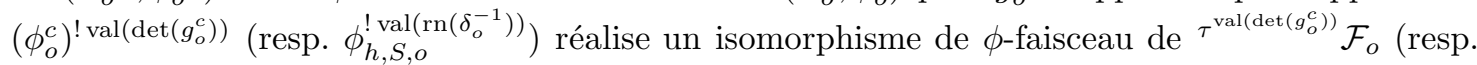
$\left.\tau^{\operatorname{val}\left(\operatorname{rn}\left(\delta_{o}^{-1}\right)\right)} W_{h, S, o}\right) \operatorname{vers} \mathcal{F}_{o}^{c,}\left(\operatorname{resp} . \delta_{o}^{-1}\left(W_{h, S, o}\right)\right)$, de sorte que

$$
\delta_{o} \circ \phi_{h, S, o}^{! \operatorname{val}\left(\operatorname{rn}\left(\delta_{o}\right)\right)} \circ \alpha_{t} \circ\left(\phi_{o}^{! \operatorname{val}\left(\operatorname{det}\left(g_{o}^{c}\right)\right)}\right)^{-1}
$$

restreint à $\mathcal{F}_{o, / t-\operatorname{val}\left(\operatorname{det}\left(g_{o}^{c}\right)\right)}^{c,}$ définit une rigidification à l'ordre $s:=t-\operatorname{val}\left(\operatorname{det}\left(g_{o}^{c}\right)\right)$ de la partie connexe du $\phi$-faisceau $\left(\mathcal{F}_{o}^{\prime}, \phi_{o}^{\prime}\right)$.

- pour $z \in F_{o}^{\times} \cap \mathcal{O}_{o}$, on fait agir $\left(z, z^{-1}, 1\right)$ de manière évidente, c'est à dire avec les notations ci-dessus, $\mathcal{F}_{o}^{c,}{ }^{\prime}=z \mathcal{F}_{o}^{c}$ avec $\alpha_{s}^{\prime}=z \circ \phi_{h, S, o}^{! h \operatorname{val}(z)} \circ \alpha_{t} \circ\left(\phi_{o}^{! h \operatorname{val}(z)}\right)^{-1}$. 
- Le procédé est exactement identique pour les éléments de $\widetilde{\mathcal{N}}_{o}$ de la forme $\left(1, \delta_{o}, c_{o}\right)$, en remarquant que $c_{o} \delta_{o}$ est un automorphisme de $\left(W_{h, S, o, / s}, \phi_{h, S, o, / s}\right)$.

I.4.3. Complétés formels. - On se sert des $\mathcal{J}_{I^{o}, m}^{=h}(s)$ pour "détordre" le schéma formel $\hat{\mathcal{I}}_{I^{o},=h, m}(t)$. Le lemme suivant est immédiat :

I.4.3.1. Lemme. - En notant $\mathcal{J}_{I^{o}, m}^{=h}(\infty):=\lim _{\leftarrow} \mathcal{J}_{I^{o}, m}^{=h}(s)$, un point fermé sur $\kappa$ de $\mathcal{J}_{I^{o}, m}^{=h}(\infty)$ correspond à la donnée d'un point fermé de $\mathcal{I}_{I^{o}, h}^{\stackrel{s}{m}}$ et d'un isomorphisme de la partie connexe $\left(V_{o}^{*, c}, \varphi_{o}^{*, c}\right)$ de son $\mathcal{O}_{o}$-module de Dieudonné associé, avec $\left(W_{h, \kappa, o}, \varphi_{h, \kappa, o}\right)$.

I.4.3.2. Définition. - Pour tout $s$, en accord avec les notations de $[14]$, on notera $\operatorname{Tw}_{I^{o}, h, m, t}(s)$ le quotient

$$
\mathcal{J}_{I^{o}, m}^{=h}(s) \times_{\operatorname{Spec} \kappa(o)} \operatorname{Spf} \operatorname{Def}_{t}^{h} / \mathcal{D}_{o, h}^{\times}
$$

où $\mathcal{D}_{o, h}^{\times}$agit diagonalement. L'espace topologique sous-jacent aux $\operatorname{Tw}_{I^{o}, h, m, t}(s)$ est $\mathcal{I}_{I^{o}, m}=h$. On notera aussi $\mathrm{Tw}_{I^{o}, h, m, t}(\infty)$ la limite $\lim _{\leftarrow} \operatorname{Tw}_{I^{o}, h, m, t}(s)$.

I.4.3.3. Proposition. - La tour $\mathrm{Tw}_{I^{o}, h, m, t}(\infty)$ est munie de correspondances de Hecke associées aux éléments de

$$
\left(D_{\mathbb{A}}^{\infty, o}\right)^{\times} \times G L_{d-h}\left(F_{o}\right) \times \widetilde{\mathcal{N}_{o}}
$$

vérifiant la propriété suivante : étant donné un point géométrique $x_{I^{\circ}, m}(\infty)$ de $\mathcal{J}_{I^{o}, m}^{=h}(\infty)$ au dessus d'un point $x_{I^{o}, m}$ de $\mathcal{I}_{I^{o}, m}^{=h}$, les morphismes canoniques

$$
f_{x_{I^{o}, m}(\infty)}:\left(\hat{\mathcal{I}}_{I^{o},=h, m}(t)\right)_{x_{I^{o}, m}} \longrightarrow \operatorname{Spf}_{\operatorname{Def}_{t}^{h}}^{h}
$$

ainsi que

$$
h_{x_{I^{o}, m}(\infty)}: \mathrm{Tw}_{I^{o}, h, m, t}(\infty)_{x_{I^{o}, m}} \longrightarrow \operatorname{Spf}_{\operatorname{Def}_{t}^{h}}^{h}
$$

sont tels que pour tout élément $\left(g^{\infty, o}, g_{o}^{\text {et }},\left(g_{o}^{c}, \delta_{o}, \sigma_{o}\right)\right) \in\left(D^{\infty, o}\right)^{\times} \times G L_{d-h}\left(F_{o}\right) \times \widetilde{\mathcal{N}_{o}}$, les diagrammes suivant sont commutatifs: $:(10)$

$$
\begin{aligned}
& \left(\hat{\mathcal{I}}_{J^{o},=h, m^{\prime}}\left(t^{\prime}\right)\right)_{x_{J^{o}, m^{\prime}}} \stackrel{f_{x_{J^{o}, m^{\prime}}(\infty)}}{\longrightarrow} \operatorname{Spf} \operatorname{Def}_{t^{\prime}}^{h} \\
& \left(g^{\infty, o}, g_{o}^{e t}, g_{o}^{c}\right) \downarrow \quad f_{\left(g^{\infty, o}, g_{o}^{e t},\left(g_{o}^{c}, \delta_{o}, \sigma_{o}\right)\right) x_{J^{o}, m^{\prime}}(\infty)} \quad \downarrow\left({ }^{t}\left(g_{o}^{c}\right)^{-1}, \delta_{o}, \sigma_{o}\right) \\
& \left(\hat{\mathcal{I}}_{I^{o},=h, m}(t)\right)_{x_{I^{o}, m}}^{f_{\left(g^{\infty}, o, g_{o}^{e t},\left(g_{o}^{c}, \delta_{o}, \sigma_{o}\right)\right) x} J^{o}, m^{\prime}(\infty)} \longrightarrow \operatorname{Spf}^{\operatorname{Def}_{t}^{h}}
\end{aligned}
$$

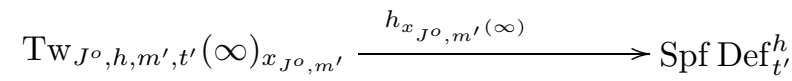

$$
\begin{aligned}
& \left(g^{\infty, o}, g_{o}^{e t},\left(g_{o}^{c}, \delta_{o}, \sigma_{o}\right)\right) \downarrow \quad h_{\left(g^{\infty, o}, g_{o}^{e t},\left(g_{o}^{c}, \delta_{o}, \sigma_{o}\right)\right) x_{J o} m^{\prime}(\infty)} \quad \downarrow\left({ }^{t}\left(g_{o}^{c}\right)^{-1}, \delta_{o}, \sigma_{o}\right) \\
& \operatorname{Tw}_{I^{o}, h, m, t}(\infty)_{x_{I^{o}, m} \stackrel{\left.h_{\left(g^{\infty}, o\right.}, g_{o}^{e t},\left(g_{o}^{c}, \delta_{o}, \sigma_{o}\right)\right) x}{J^{o}, m^{\prime}}(\infty)}^{\longrightarrow} \operatorname{Spf}_{\operatorname{Def}_{t}^{h}}^{h}
\end{aligned}
$$

où $\left(g^{\infty, o}, g_{o}^{e t}, g_{o}^{c}, \sigma_{o}\right) x_{J^{o}, m^{\prime}}=x_{I^{o}, m}$.

Démonstration. - Pour définir les correspondances sur la tour $\operatorname{Tw}_{I^{o}, h, m, t}(\infty)$, il suffit de faire agir $\widetilde{\mathcal{N}}_{o}$ diagonalement ${ }^{(11)}$. Concrètement, étant donné

$$
\left(g^{\infty, o}, g_{o}^{e t}, g_{o}^{c}, \delta_{o}, \sigma_{o}\right) \in\left(D_{\mathbb{A}}^{\infty, o}\right)^{\times} \times G L_{d-h}\left(F_{o}\right) \times \widetilde{\mathcal{N}}_{o},
$$

tel que $g_{o}^{e t} \in \mathbb{M}_{d-h}\left(\mathcal{O}_{o}\right), g_{o}^{c} \in \mathbb{M}_{h}\left(\mathcal{O}_{o}\right)$ et $\delta_{o} \in \mathcal{D}_{o, h}^{\times}$, soient $J^{o}, I^{o}$ des idéaux de $A$ de multiplicité nulle en $o$, et $m, m^{\prime}, t, t^{\prime}, s, s^{\prime}$ des entiers tels que :

(10) pour un choix convenables de $J^{o}, m^{\prime}, t^{\prime}$, comme ci-avant

${ }^{(11)}\left(g_{o}^{c}, \delta_{o}, \sigma_{o}\right) \in \widetilde{\mathcal{N}}_{o}$ agit sur $\operatorname{Def}_{t}^{h}$ via $\left({ }^{t}\left(g_{o}^{c}\right)^{-1}, \delta_{o}, \sigma_{o}\right) \in \mathcal{N}_{o}$ laquelle est définie par Deligne et Carayol, cf. le paragraphe (I.1.1) 
- $K_{\mathbb{A}, J^{o}}^{\infty, o} \subset K_{\mathbb{A}, I^{o}}^{\infty, o} \cap\left(g^{\infty, o}\right)^{-1} K_{\mathbb{A}, I^{o}}^{\infty, o} g^{\infty, o} ;$

- $\operatorname{Ker}\left({ }^{t} g_{o}^{e t}\right) \subset\left(\mathcal{M}_{o}^{-m^{\prime}} / \mathcal{O}_{o}\right)^{d-h}$ et $\left(\mathcal{M}_{o}^{-m} / \mathcal{O}_{o}\right)^{d-h} \subset \operatorname{Im}\left({ }^{t} g_{o}^{e t}\right)$;

- $\operatorname{Ker}\left({ }^{t} g_{o}^{c}\right) \subset\left(\mathcal{M}_{o}^{-t^{\prime}} / \mathcal{O}_{o}\right)^{h}$ et $\left(\mathcal{M}_{o}^{-t} / \mathcal{O}_{o}\right)^{h} \subset \operatorname{Im}\left({ }^{t} g_{o}^{c}\right)$;

- $\varpi_{o}^{\operatorname{val}\left(\operatorname{det}\left(g_{o}^{c}\right)\right) / h}\left(g_{o}^{e t}\right)^{-1} \in \mathbb{M}_{d-h}\left(\mathcal{O}_{o}\right)$;

- $s^{\prime}=s-\operatorname{val}\left(\operatorname{det}\left(\delta_{o}\right)\right)$.

On a alors une correspondance de Hecke $\left(g^{\infty, o}, g_{o}^{e t}, g_{o}^{c}, \delta_{o}, \sigma_{o}\right)$ :

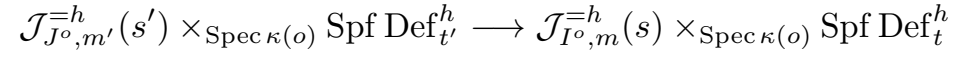

Les morphismes canoniques $f_{x_{I^{o}, m}(\infty)}$ et $h_{x_{I^{o}, m}(\infty)}$ sont définis à partir de l'isomorphisme fixé par le lemme (I.4.3.1) et la commutativité des diagrammes est alors évidente.

Remarque : En particulier, on notera que l'on peut munir les $\mathrm{Tw}_{I^{o}, h, m, t}(s)$ de correspondances de Hecke associées aux éléments

$$
\left(g^{\infty, o}, g_{o}^{e t}, g_{o}^{c}, \sigma_{o}\right) \in\left(D_{\mathbb{A}}^{\infty, o}\right)^{\times} \times G L_{d-h}\left(F_{o}\right) \times G L_{h}\left(F_{o}\right) \times W_{F_{o}},
$$

à partir de celles définies ci-dessus, en choisissant un élément quelconque $\delta_{o} \in D_{o, h}^{\times}$tel que $\left(g_{o}^{c}, \delta_{o}, \sigma_{o}\right) \in \widetilde{\mathcal{N}_{o}}$, le résultat ne dépendant pas de ce choix car on a quotienté par $\mathcal{D}_{o, h}^{\times}$.

I.4.3.4. Proposition. - Au dessus de tout ouvert affine $S$ de $\mathcal{I}_{I^{\circ}, m}^{h}$, il existe des isomorphismes

$$
\operatorname{Tw}_{I^{o}, h, m, t}(\infty) \times_{\mathcal{I}_{I^{o}, m}^{=h}} S \stackrel{\sim}{\longrightarrow} \hat{\mathcal{I}}_{I^{o},=h, m}(t) \times_{\mathcal{I}_{I^{o}, m}^{=h}} S
$$

tels qu'en tout point géométrique $x$ de $\mathcal{I}_{I^{\circ}, m}^{=h}$, on a le diagramme commutatif

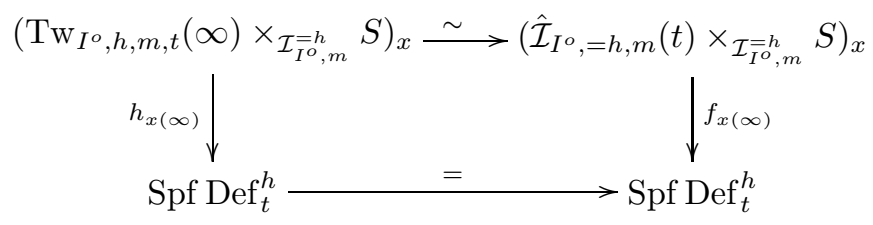

où $x(\infty)$ est un point géométrique de $\mathcal{J}_{I^{o}, m}^{=h}(\infty)$ au dessus de $x$.

Remarque : Cet énoncé peut paraître imprécis car les isomorphismes en question ne sont pas canoniques et dépendent comme on le verra du choix d'une extension de la partie connexe par la partie étale des $\mathcal{O}_{o}$-modules de Dieudonné. A priori il n'y a aucun moyen de les recoller, et donc bien sur il n'y a pas de sens à essayer de les rendre compatibles aux actions des correspondances de Hecke. La propriété essentielle est en fait la commutativité du diagramme de l'énoncé, qui nous permettra comme on le verra au paragraphe (I.5.2) de montrer que tous ces isomorphismes coïncident au niveau des cycles évanescents ${ }^{(12)}$ et se recollent de façon compatible aux actions de Hecke, en vertu de la proposition (I.4.3.3). On peut toutefois suspecter que le schéma tout entier est affine.

Démonstration. - Commençons par construire la flèche. Soit donc $R$ une $\mathcal{O}_{o}$-algèbre artinienne, $\mathcal{M}$ un idéal maximal de carré nul et $\bar{R}=R / \mathcal{M}$. Supposons donné un $\bar{R}$-point de $\mathcal{J}_{I^{o}, m}^{=h}(\infty)$. Étant donnée $\left(W_{h, R, o}, \varphi_{h, R, o}, \iota_{h, R, t}\right)$ une déformation de niveau $t$ de $\left(W_{h, \bar{R}, o}, \varphi_{h, \bar{R}, o}\right)$, il faut construire une déformation sur $R$, du $\bar{R}$-point correspondant de $\mathcal{I}_{I^{\circ}, m}^{h}$. D'après l'équivalent du théorème de Serre-Tate (cf. la proposition (I.1.6.3)), il suffit de construire une déformation de niveau $(m, t)^{(13)}$ du $\mathcal{O}_{o}$-module de Dieudonné $\left(\bar{V}_{o}, \bar{\varphi}_{o}\right)$ associé sur $\bar{R}$, cette construction devant être compatible à l'action diagonale de $\mathcal{D}_{o, h}^{\times}$sur le produit $\mathcal{J}_{I^{o}, m}^{=h}(\infty) \times_{\operatorname{Spec} \kappa(o)} \operatorname{Spf} \operatorname{Def}_{t}^{h}$.

(12) Moralement les cycles évanescents ne dépendent que de la partie connexe du $\mathcal{O}_{o}$-module de Dieudonné et pas de l'extension de cette partie par la partie étale.

${ }^{(13)}$ structure de niveau $m$ (resp. $t$ ) sur la partie étale (resp. connexe) 
I.4.3.5. Lemme. - Soit $R$ une $\mathcal{O}_{o}$-algèbre artinienne, $\mathcal{M}$ un idéal maximal de carré nul et $\bar{R}=R / \mathcal{M}$. Soit $\left(\bar{V}_{o}, \bar{\varphi}_{o}\right)$ un $\mathcal{O}_{o}$-module de Dieudonné sur $\bar{R}$, ainsi qu'une suite exacte

$$
0 \longrightarrow\left(\bar{V}_{o}^{e t}, \bar{\varphi}_{o}^{e t}\right) \longrightarrow\left(\bar{V}_{o}, \bar{\varphi}_{o}\right) \longrightarrow\left(\bar{V}_{o}^{c}, \bar{\varphi}_{o}^{c}\right) \longrightarrow 0
$$

avec $\left(\bar{V}_{o}^{e t}, \bar{\varphi}_{o}^{e t}\right)$ étale et $\left(\bar{V}_{o}^{c}, \bar{\varphi}_{o}^{c}\right)$ connexe. Supposons en outre donnée une déformation $\left(V_{o}^{c}, \varphi_{o}^{c}\right)$ sur $R$ de $\left(\bar{V}_{o}^{c}, \bar{\varphi}_{o}^{c}\right)$. Il existe alors une déformation $\left(V_{o}, \varphi_{o}\right)$ définie sur $R$ de $\left(\bar{V}_{o}, \bar{\varphi}_{o}\right)$ ainsi qu'une suite exacte

$$
0 \longrightarrow\left(V_{o}^{e t}, \varphi_{o}^{e t}\right) \longrightarrow\left(V_{o}, \varphi_{o}\right) \longrightarrow\left(V_{o}^{c}, \varphi_{o}^{c}\right) \longrightarrow 0
$$

dont la réduction modulo $\mathcal{M}$ est la suite exacte précédente sur $\bar{R}$.

Démonstration. - Soit $\left(V_{o}^{e t}, \varphi_{o}^{e t}\right)$ la déformation sur $R$ de $\left(\bar{V}_{o}^{e t}, \bar{\varphi}_{o}^{e t}\right)$. Le problème est alors de construire une extension de $\left(V_{o}^{e t}, \varphi_{o}^{e t}\right)$ par $\left(V_{o}^{c}, \varphi_{o}^{c}\right)$. La question est classique et découle des résultats de $[\mathbf{1 7}]$ chapitre $4 \S 3$. L'obstruction à l'existence d'une extension $V_{o}$ de $\bar{V}_{o}$ par $\mathcal{M} \otimes_{\bar{R}} \bar{V}_{o}$ réside ${ }^{(14)}$ dans

$$
\operatorname{Ext}_{\mathcal{O}_{o} \hat{\otimes}_{\kappa(o)}}^{2} \bar{R}\left(\bar{V}_{o}, \mathcal{M} \otimes_{\bar{R}} \bar{V}_{o}\right)
$$

Si cette obstruction est nulle, une extension étant choisie, l'obstruction à l'existence d'une flèche $V_{o}^{e t} \longrightarrow V_{o}$ (resp. $V_{o} \longrightarrow V_{o}^{c}$ ) réside dans le groupe

$$
\operatorname{Ext}_{\mathcal{O}_{o} \hat{\otimes}_{\kappa(o)}}^{1}\left(\bar{V}_{o}^{e t}, \mathcal{M} \otimes_{\bar{R}} \bar{V}_{o}\right) \quad\left(\text { resp. } \operatorname{Ext}_{\mathcal{O}_{o} \hat{\otimes}_{\kappa(o)}}^{1}\left(\bar{V}_{o}, \mathcal{M} \otimes_{\bar{R}} \bar{V}_{o}^{c}\right)\right)
$$

et l'ensemble de ces flèches est un torseur sous

$$
\operatorname{Hom}_{\mathcal{O}_{o} \hat{\otimes}_{\kappa(o)}} \bar{R}\left(\bar{V}_{o}^{e t}, \mathcal{M} \otimes_{\bar{R}} \bar{V}_{o}\right) \quad\left(\text { resp. } \operatorname{Hom}_{\mathcal{O}_{o} \hat{\otimes}_{\kappa(o)}}\left(\bar{V}_{o}, \mathcal{M} \otimes_{\bar{R}} \bar{V}_{o}^{c}\right)\right) .
$$

Si ces obstructions sont nulles, $V_{o}^{e t} \longrightarrow V_{o}$ est injective et $V_{o} \longrightarrow V_{o}^{c}$ est surjective. En outre l'ensemble des flèches $V_{o}^{e t} \longrightarrow V_{o}^{c}$ est un torseur sous

$$
\operatorname{Hom}_{\mathcal{O}_{o} \hat{\otimes}_{\kappa(o)} \bar{R}}\left(\bar{V}_{o}^{e t}, \mathcal{M} \otimes_{\bar{R}} \bar{V}_{o}^{c}\right),
$$

de sorte qu'il existe des flèches $V_{o}^{\text {et }} \longrightarrow V_{o}$ et $V_{o} \longrightarrow V_{o}^{c}$ tel que la suite $0 \longrightarrow V_{o}^{\text {et }} \longrightarrow V_{o} \longrightarrow$ $V_{o}^{c} \longrightarrow 0$ soit exacte. Montrons que toutes ces obstructions sont nulles. En fait on va montrer que les groupes dans lesquelles elles vivent sont nuls. L'argument est identique pour tous ces groupes, traitons par exemple le cas de $\operatorname{Ext}_{\mathcal{O}_{o} \hat{\otimes}_{\kappa(o)}}^{1}\left(\bar{V}_{o}^{e t}, \mathcal{M} \otimes_{\bar{R}} \bar{V}_{o}\right)$. Les $\mathcal{O}_{o} \hat{\otimes}_{\kappa(o)} \bar{R}$-modules $\bar{V}_{o}$ et $\bar{V}_{o}^{e t}$ étant localement libres, les faisceaux $\underline{\operatorname{Ext}}_{\mathcal{O}_{o} \hat{\otimes}_{\kappa(o)} \bar{R}}\left(\bar{V}_{o}^{e t}, \mathcal{M} \otimes_{\bar{R}} \bar{V}_{o}\right)$ sont nuls pour $i>0$. La suite spectrale locale-globale pour le $\operatorname{Ext}^{1}$ montre que $\operatorname{Ext}_{\mathcal{O}_{o} \hat{\otimes}_{\kappa(o)}}^{1}\left(\bar{V}_{o}^{e t}, \mathcal{M} \otimes_{\bar{R}} \bar{V}_{o}\right)$ est égal à

$$
H^{1}\left(\operatorname{Spec}(\bar{R}), \underline{\operatorname{Ext}}_{\mathcal{O}_{o} \hat{\otimes}_{\kappa(o)}}^{0}\left(\bar{V}_{o}^{e t}, \mathcal{M} \otimes_{\bar{R}} \bar{V}_{o}\right)\right.
$$

qui est nul car $\operatorname{Spec}(\bar{R})$ est affine.

Il faut alors construire $\varphi_{o}:{ }^{\tau} V_{o} \longrightarrow V_{o}$ qui soit compatible à $\varphi_{o}^{e t}$ et $\varphi_{o}^{c}$. On commence par remarquer que Frob $: R \longrightarrow R$ se factorise par $\overline{\operatorname{Frob}}_{o}: \bar{R} \longrightarrow R$, de sorte ${ }^{\tau} V_{o}=\bar{V}_{o} \otimes \bar{R}_{\bar{R}} \overline{\text { Frob }}_{o} R$. Comme précédemment il n'y a pas d'obstruction à l'existence d'une application $\varphi_{o}^{\prime}:{ }^{\tau} V_{o} \longrightarrow V_{o}$ et l'ensemble des telles applications est un torseur sous $\left.\operatorname{Hom}_{\mathcal{O}_{o} \hat{\otimes}_{\kappa(o)} \bar{R}}{ }^{\tau} \bar{V}_{o}, \mathcal{M} \otimes_{\bar{R}} \bar{V}_{o}\right)$. Fixons une telle application $\varphi_{o}^{\prime}$. Les applications $V_{o}^{e t} \longrightarrow V_{o}, \varphi_{o}^{\prime}$ et $\varphi_{o}^{e t}$ fournissent alors un élément de $\left.\operatorname{Hom}_{\mathcal{O}_{o} \hat{\otimes}_{\kappa(o)} \bar{R}}{ }^{\tau} \bar{V}_{o}^{e t}, \mathcal{M} \otimes_{\bar{R}} \bar{V}_{o}\right)$. En appliquant le foncteur $\operatorname{Hom}_{\mathcal{O}_{o} \hat{\otimes}_{\kappa(o)}}\left(\bullet, \mathcal{M} \otimes_{\bar{R}} \bar{V}_{o}\right)$ à la suite exacte courte

$$
0 \rightarrow{ }^{\tau} \bar{V}_{o}^{e t} \longrightarrow{ }^{\tau} \bar{V}_{o} \longrightarrow{ }^{\tau} \bar{V}_{o}^{c} \rightarrow 0
$$

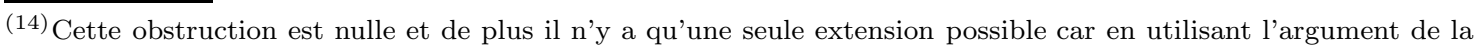

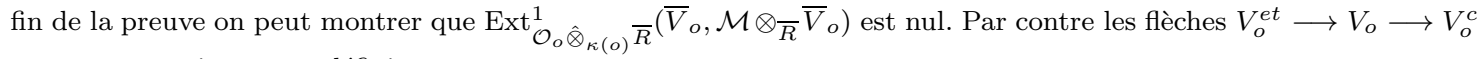
ne sont pas uniquement définies. 
on obtient la suite exacte

$$
\begin{aligned}
0 \rightarrow \operatorname{Hom}_{\mathcal{O}_{o} \hat{\otimes}_{\kappa(o)}}\left({ }^{\tau} \bar{V}_{o}^{c}, \mathcal{M} \otimes_{\bar{R}} \bar{V}_{o}\right) \longrightarrow \operatorname{Hom}_{\mathcal{O}_{o} \hat{\otimes}_{\kappa(o)} \bar{R}}\left({ }^{\tau} \bar{V}_{o}, \mathcal{M} \otimes_{\bar{R}} \bar{V}_{o}\right) \rightarrow \\
\longrightarrow \operatorname{Hom}_{\mathcal{O}_{o} \hat{\otimes}_{\kappa(o)}}\left(\tau \bar{V}_{o}^{e t}, \mathcal{M} \otimes_{\bar{R}} \bar{V}_{o}\right) \longrightarrow 0
\end{aligned}
$$

car, de manière identique à ce qui précède, $\left.\operatorname{Ext}_{\mathcal{O}_{o} \hat{\otimes}_{\kappa(o)}}^{1} \bar{R}^{\tau} \bar{V}_{o}^{c}, \mathcal{M} \otimes_{\bar{R}} \bar{V}_{o}\right)$ est nul. On modifie donc $\varphi_{o}^{\prime}$ en un $\varphi_{o}^{\prime \prime}$ qui soit compatible à $\varphi_{o}^{e t}$; l'ensemble des telles $\varphi_{o}^{\prime \prime}$ est alors un torseur sous $\operatorname{Hom}_{\mathcal{O}_{o} \hat{\otimes}_{\kappa(o)} \bar{R}}\left({ }^{\tau} \bar{V}_{o}^{c}, \mathcal{M} \otimes_{\bar{R}} \bar{V}_{o}\right)$. De même, $V_{o} \longrightarrow V_{o}^{c}, \varphi_{o}^{\prime \prime}$ et $\varphi_{o}^{c}$ définissent un élément de $\left.\operatorname{Hom}_{\mathcal{O}_{o} \hat{\otimes}_{\kappa(o)}} \bar{R}^{\tau}{ }^{\tau} \bar{V}_{o}^{c}, \mathcal{M} \otimes_{\bar{R}} \bar{V}_{o}^{c}\right)$. En appliquant le foncteur $\operatorname{Hom}_{\mathcal{O}_{o} \hat{\otimes}_{\kappa(o)}}\left({ }^{\tau} \bar{V}_{o}^{c}, \bullet\right)$, à la suite exacte

$$
0 \rightarrow \mathcal{M} \otimes_{\bar{R}} \bar{V}_{o}^{e t} \longrightarrow \mathcal{M} \otimes_{\bar{R}} \bar{V}_{o} \longrightarrow \mathcal{M} \otimes_{\bar{R}} \bar{V}_{o}^{c} \rightarrow 0,
$$

on obtient la suite exacte

$$
\begin{aligned}
0 \rightarrow \operatorname{Hom}_{\mathcal{O}_{o} \hat{\otimes}_{\kappa(o)}} \bar{R}^{\tau}\left(\bar{V}_{o}^{c}, \mathcal{M} \otimes_{\bar{R}} \bar{V}_{o}^{e t}\right) \longrightarrow \operatorname{Hom}_{\mathcal{O}_{o} \hat{\otimes}_{\kappa(o)} \bar{R}}\left({ }^{\tau} \bar{V}_{o}^{c}, \mathcal{M} \otimes_{\bar{R}} \bar{V}_{o}\right) \rightarrow \\
\left.\rightarrow \operatorname{Hom}_{\mathcal{O}_{o} \hat{\otimes}_{\kappa(o)}} \bar{R}^{\tau} \bar{V}_{o}^{c}, \mathcal{M} \otimes_{\bar{R}} \bar{V}_{o}^{c}\right) \longrightarrow 0
\end{aligned}
$$

car $\operatorname{Ext}_{\mathcal{O}_{o} \hat{\otimes}_{\kappa(o)}}^{1}\left({ }^{\tau} \bar{V}_{o}^{c}, \mathcal{M} \otimes_{\bar{R}} \bar{V}_{o}^{e t}\right)$ est nul. Il est alors possible de modifier $\varphi_{o}^{\prime \prime}$ en un $\varphi_{o}$ compatible à $\varphi_{o}^{e t}$ et $\varphi_{o}^{c}$.

Soit donc comme dans le lemme ci-dessus, $\left(V_{o}^{e t}, \varphi_{o}^{e t}\right)$ le relèvement sur $R$ de $\left(\bar{V}_{o}^{e t}, \bar{\varphi}_{o}^{e t}\right)$. Soit aussi $\iota_{o, m}^{e t}$ le relèvement sur $R$ de $\bar{\iota}_{o, m}^{e t}: c^{\prime}$ 'est une structure de niveau $m \operatorname{sur}\left(V_{o}^{e t}, \varphi_{o}^{e t}\right)$. De l'isomorphisme sur $\bar{R}$ :

$$
\left(\bar{V}_{o}^{c *}, \bar{\varphi}_{o}^{c *}\right) \simeq\left(W_{h, \bar{R}, o}, \varphi_{h, \bar{R}, o}\right)
$$

et de la déformation $\left(W_{h, R, o}, \varphi_{h, R, o}\right)$, on en déduit une déformation $\left(V_{o}^{c}, \varphi_{o}^{c}\right)$ définie sur $R$, de $\left(\bar{V}_{o}^{c}, \bar{\varphi}_{o}^{c}\right)$, munie de plus d'une structure de niveau $t$. Il suffit alors d'appliquer le lemme précédent. Il est en outre immédiat que cette construction est invariante par l'action diagonale de $\mathcal{D}_{o, h}^{\times}$sur le produit $\mathcal{J}_{I^{o}, m}^{=h}(\infty) \times_{\operatorname{Spec} \kappa(o)} \operatorname{Spf}_{\operatorname{Def}_{t}^{h}}^{h}$ et que le diagramme de l'énoncé est bien commutatif.

Montrons ensuite que la flèche en question est un isomorphisme. Le problème se ramène immédiatement au cas $t=0$. Il suffit alors de montrer que l'on obtient un isomorphisme au niveau des espaces tangents. Pour des raisons de dimension, il suffit de vérifier que l'on a une injection ce qui est clairement le cas.

I.4.3.6 - Dans la suite nous proposons suivant [14], une autre façon de voir cet isomorphisme en un cran fini. Notons

$$
\hat{\mathcal{J}}_{I^{o},=h, m}(s) \longrightarrow \hat{\mathcal{I}}_{I^{o},=h, m}
$$

l'extension étale de fibre spéciale $\mathcal{J}_{I^{o}, m}^{=h}(s) \longrightarrow \mathcal{I}_{I^{o}, m}^{=h}$. On note de même

$$
\hat{\mathcal{J}}_{I^{o},=h, m}(s, t) \longrightarrow \hat{\mathcal{J}}_{I^{o},=h, m}(s)
$$

le classifiant des structures de niveau $t$ sur la partie connexe du $\varphi$-faisceau universel sur $\hat{\mathcal{J}}_{I^{o},=h, m}(s)$. On définit de manière identique au paragraphe (I.4.2), des correspondances de Hecke $\operatorname{sur} \hat{\mathcal{J}}_{I^{o},=h, m}(s, t)$ associées aux éléments de $\left(D_{\mathbb{A}}^{\infty, o}\right)^{\times} \times G L_{d-h}\left(F_{o}\right) \times \widetilde{\mathcal{N}}_{o}$.

On note $\mathcal{M}_{t}^{h}$ l'idéal maximal de $\operatorname{Def}_{t}^{h}$ et soient pour $S$ un ouvert affine de $\mathcal{I}_{I^{o}, m}^{h}$ :

$$
S_{s}:=\mathcal{J}_{I^{o}, m}^{=h}(s) \times_{\mathcal{I}_{I^{o}, m}^{=h}} S, \quad \hat{S}_{s, t}:=\hat{\mathcal{J}}_{I^{o},=h, m}(s, t) \times_{\mathcal{I}_{I^{o}, m}^{=h}} S .
$$

Étant donné un point géométrique $x(\infty)$ de $\mathcal{J}_{I^{o}, m}^{=h}(\infty)$, on a des morphismes canoniques

$$
\left(S_{s} \times{ }_{\operatorname{Spec} \kappa(o)} \operatorname{Spf}\left(\operatorname{Def}_{t}^{h} /\left(\mathcal{M}_{t}^{h}\right)^{N}\right)\right)_{x(\infty)} \longrightarrow \operatorname{Spf}\left(\operatorname{Def}_{t}^{h} /\left(\mathcal{M}_{t}^{h}\right)^{N}\right)
$$


et

$$
\left(\hat{S}_{s, t}\right)_{x(\infty)} \longrightarrow \operatorname{Spf} \operatorname{Def}_{t}^{h}
$$

ainsi qu'une compatibilité aux correspondances de Hecke comme dans la proposition (I.4.3.3).

I.4.3.7. Proposition. - Pour tout $N \in \mathbb{N}$, il existe un $s_{0}$ assez grand tel que pour tout $s \geqslant s_{0}$, et $S$ un ouvert affine de $\mathcal{I}_{I^{\circ}, m}^{=}$, on ait un morphisme

$$
f_{N, s}: S_{s} \times_{\operatorname{Spec} \kappa(o)} \operatorname{Spf} \operatorname{Def}_{t}^{h} \longrightarrow \hat{S}_{s, t}
$$

tel qu'en tout point géométrique $x(\infty)$ de $\mathcal{J}_{I^{\circ}, m}^{=h}(\infty)$, on ait le diagramme suivant :

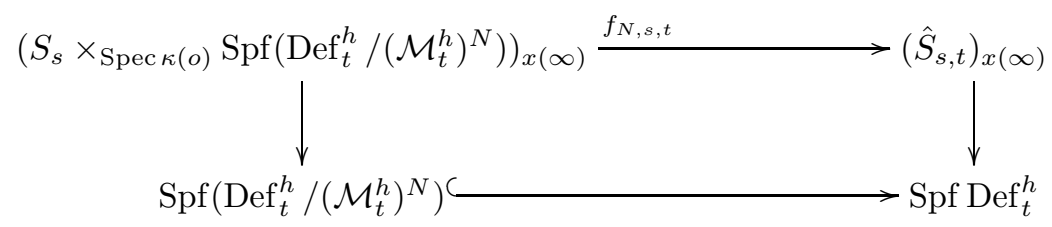

Démonstration. - On commence par prouver le lemme suivant qui est l'équivalent pour les $\mathcal{O}_{o^{-}}$ modules de Dieudonné du lemme (1.1.3) de [18].

I.4.3.8. Lemme. - Soit $R$ une $\mathcal{O}_{o}$-algèbre artinienne de morphisme structural $i: \mathcal{O}_{o} \rightarrow R$, et $r \in \mathbb{N}$ tel que $i\left(\varpi_{o}^{r}\right)=0$ dans $R$. Soient $\left(V_{o}, \varphi_{o}\right)$ et $\left(W_{o}, \psi_{o}\right)$ deux $\mathcal{O}_{o}$-modules de Dieudonné connexes sur $R$. Soit $\mathcal{M}$ l'idéal maximal de $R$ et $\bar{R}=R / \mathcal{M}$ : on suppose que $\mathcal{M}^{k}=(0)$. On notera $\bar{V}_{o}$ et $\bar{W}_{o}$ la réduction modulo $\mathcal{M}$ de $V_{o}$ et $W_{o}$. On a alors

- $\operatorname{Hom}\left(\left(V_{o}, \varphi_{o}\right),\left(W_{o}, \psi_{o}\right)\right) \longrightarrow \operatorname{Hom}\left(\left(\bar{V}_{o}, \bar{\varphi}_{o}\right),\left(\bar{W}_{o}, \bar{\psi}_{o}\right)\right)$ est injective ;

- pour toute application $\bar{f}:\left(\bar{V}_{o}, \bar{\varphi}_{o}\right) \longrightarrow\left(\bar{W}_{o}, \bar{\psi}_{o}\right)$, on peut trouver un relèvement de $\varpi_{o}^{N} \bar{f}$, où $N \geqslant r(\ln k / \ln q)$.

Démonstration. - Commençons par montrer que $\varpi_{o}^{N} \otimes 1$ annule $\mathcal{M} W_{o}$ pour $N \geqslant r(\ln k / \ln q)$. Dans $W_{o}$, en considérant $\psi_{o}: W_{o} \longrightarrow W_{o}$ comme une application $\tau$-linéaire, on a $\left(\varpi_{o}^{r} \otimes 1\right) \operatorname{Id}=$ $\left(1 \otimes i\left(\varpi_{o}^{r}\right)\right) \mathrm{Id}+\sum_{i=1}^{r h} a_{i}\left(\psi_{o}\right)^{i}$. Comme $i\left(\varpi_{o}^{r}\right)$ est nul dans $R$, on en déduit que pour $v \in \mathcal{M} W_{o}$, $\left(\varpi_{o}^{r} \otimes 1\right) v \in \mathcal{M}^{p} W_{o}$, d'où le résultat.

Soit donc $g:\left(V_{o}, \varphi_{o}\right) \longrightarrow \mathcal{M}\left(W_{o}, \psi_{o}\right):\left(\varpi_{o}^{N} \otimes 1\right) v \in \operatorname{Ker} g$. Or $\left(V_{o}, \varphi_{o}\right)$ étant connexe, on en déduit qu'il existe $w \in V_{o} / \varphi_{o}\left(V_{o}\right)$ tel que $\left(\varpi_{o}^{N} \otimes 1\right) v=\left(\varphi_{o}\right)^{i}(w)$. On a alors $\left(\psi_{o}\right)^{i}(g(w))=0$ soit $g(w)=0$ et donc $g=0$.

Pour le dernier point, on définit l'image d'un élément $v \in V_{o}$ comme $\varpi_{o}^{N} \otimes 1$ fois le relèvement quelconque de $\bar{f}(\bar{v})$. Cette définition ne dépend pas du choix de ce relèvement car $\mathcal{M} W_{o}$ est tué $\operatorname{par} \varpi_{o}^{N} \otimes 1$.

I.4.3.9. Lemme. - (cf. [14] lemme III.2.6) Il existe une fonction $\alpha: \mathbb{N} \longrightarrow \mathbb{N}$ croissante de limite l'infini, telle que :

- tout élément de $\varpi_{o}^{s-t} \mathcal{D}_{o, h}$ se relève en un endomorphisme de

$$
\left(W_{h, \operatorname{Def}_{t}^{h} /\left(\mathcal{M}_{t}^{h}\right)^{\alpha(s)}, o}, \varphi_{\left.h, \operatorname{Def}_{t}^{h} /\left(\mathcal{M}_{t}^{h}\right)^{\alpha(s)}\right)}\right.
$$

- tout élément de $\left(1+\varpi_{o}^{s} \mathcal{D}_{o, h}\right)$ agit trivialement sur $\operatorname{Def}_{t}^{h} /\left(\mathcal{M}_{t}^{h}\right)^{\alpha(s)}$.

Démonstration. - On remarque tout d'abord que pour $t>0, \varpi_{o} \in\left(\mathcal{M}_{t}^{h}\right)^{(q-1) q^{(t-1) h}}$. Le premier point découle alors du lemme précédent en prenant $\alpha(s)$ tel que

$$
\alpha(s) \ln (\alpha(s)) \leqslant(s-t)(\ln q)(q-1) q^{(t-1) h} .
$$

Le deuxième point est alors immédiat : en effet soit $\delta \in \mathcal{D}_{o, h}^{\times}$tel que $\delta \equiv 1 \bmod \varpi_{o}^{s}$. Alors $(\delta-1) / \varpi_{o}^{m}$ se relève en un endomorphisme de

$$
\left(W_{h, \operatorname{Def}_{t}^{h} /\left(\mathcal{M}_{t}^{h}\right)^{\alpha(s)}, o}, \varphi_{h, \operatorname{Def}_{t}^{h} /\left(\mathcal{M}_{t}^{h}\right)^{\alpha(s)}, o}\right) .
$$


Il en est de même de $\left(\delta^{-1}-1\right) / \varpi_{o}^{m}$, de sorte que $\delta$ se relève en un automorphisme de

$$
\left(W_{h, \operatorname{Def}_{t}^{h} /\left(\mathcal{M}_{t}^{h}\right)^{\alpha(s)}, o}, \varphi_{h, \operatorname{Def}_{t}^{h} /\left(\mathcal{M}_{t}^{h}\right)^{\alpha(s)}, o}\right)
$$

qui est l'identité sur les points de $\varpi_{o}^{m}$-torsion.

Soit alors $S_{s, t}(N):=S_{s} \times \times_{\operatorname{Spec} \kappa(o)} \operatorname{Spf}\left(\operatorname{Def}_{t}^{h} /\left(\mathcal{M}_{t}^{h}\right)^{N}\right)$. D'après le lemme ci-dessus, on a une action de $\left(1+\varpi_{o}^{s} \mathcal{D}_{o, h}\right)$ sur $\left(W_{h, \operatorname{Def}_{t}^{h} /\left(\mathcal{M}_{t}^{h}\right)^{\alpha(s)}, o}, \varphi_{h, \operatorname{Def}_{t}^{h} /\left(\mathcal{M}_{t}^{h}\right)^{\alpha(s)}, o}\right)$ telle que pour tout $a \geqslant t$, $\left(1+\varpi_{o}^{s-t+a} \mathcal{D}_{o, h}\right)$ agit trivialement sur

$$
\left(W_{h, \operatorname{Def}_{t}^{h} /\left(\mathcal{M}_{t}^{h}\right)^{\alpha(s)}, o, a}, \varphi_{h, \operatorname{Def}_{t}^{h} /\left(\mathcal{M}_{t}^{h}\right)^{\alpha(s)}, o, a}\right) .
$$

Ainsi $\left(1+\varpi_{o}^{s} \mathcal{D}_{o, h}\right) /\left(1+\varpi_{o}^{s+a-t} \mathcal{D}_{o, h}\right)$ agit diagonalement sur

$$
\left(W_{h, \operatorname{Def}_{t}^{h} /\left(\mathcal{M}_{t}^{h}\right)^{\alpha(s)}, o, a}, \varphi_{h, \operatorname{Def}_{t}^{h} /\left(\mathcal{M}_{t}^{h}\right)^{\alpha(s)}, o, a}\right) \times S_{s+a-t, t}(\alpha(s))
$$

et le quotient est un $\mathcal{O}_{o}$-module de Dieudonné tronqué à l'ordre $a$

$$
\left(V_{o, a}^{c, s}, \varphi_{o, a}^{c, s}\right)
$$

connexe sur $S_{s, t}(\alpha(s))$. La limite projective des $\left(V_{o, a}^{c, s}, \varphi_{o, a}^{c, s}\right)$ est alors un $\mathcal{O}_{o}$-module de Dieudonné $\left(V_{o}^{c, s}, \varphi_{o}^{c, s}\right) \operatorname{sur} S_{s, t}(\alpha(s))$ tel que

$$
\left(V_{o, t}^{c, s}, \varphi_{o, t}^{c, s}\right) \simeq\left(W_{h, \operatorname{Def}_{t}^{h}, o, t}, \varphi_{h, \operatorname{Def}_{t}^{h}, o, t}\right)
$$

de sorte que $\left(V_{o}^{c, s}, \varphi_{o}^{c, s}\right)$ est muni d'une structure de niveau $t$. Étant donné un $\bar{R}$-point de $\mathcal{J}_{I^{o}, m}^{=h}(s)$, une $\mathcal{O}_{o}$-algèbre artinienne $R_{s}, \mathcal{M}$ un idéal maximal de $R_{s}$ tel que $\bar{R}=R_{s} / \mathcal{M}$, ainsi qu'un $R_{s^{-}}$ point de $\operatorname{Spf}\left(\operatorname{Def}_{t}^{h} /\left(\mathcal{M}_{t}^{h}\right)^{\alpha(s)}\right)$, on construit une déformation sur $R_{s}$ de ce $\bar{R}$-point, en donnant une déformation de niveau $(m, t)$ de son $\mathcal{O}_{o}$-module de Dieudonné. On procède comme dans la preuve de la proposition précédente. Soit $\left(V_{o}^{e t, s}, \varphi_{o}^{e t, s}, \iota_{o, m}^{e t, s}\right)$ le relèvement sur $R$ de $\left(\bar{V}_{o}^{e t}, \bar{\varphi}_{o}^{e t}, \bar{l}_{o, m}^{e t}\right)$. D'après le lemme (I.4.3.5), il existe une extension $\left(V_{o}^{s}, \varphi_{o}^{s}\right)$ de $\left(V_{o}^{e t, s}, \varphi_{o}^{e t, s}\right) \operatorname{par}\left(V_{o}^{c, s}, \varphi_{o}^{c, s}\right) / R$ ainsi qu'une suite exacte

$$
0 \longrightarrow\left(V_{o}^{e t, s}, \varphi_{o}^{e t, s}\right) \longrightarrow\left(V_{o}^{s}, \varphi_{o}^{s}\right) \longrightarrow\left(V_{o}^{c, s}, \varphi_{o}^{c, s}\right) \longrightarrow 0
$$

sur $R_{s}$ qui se restreigne sur $R_{s} \otimes_{\operatorname{Def}_{t}^{h} /\left(\mathcal{M}_{t}^{h}\right)^{\alpha(s)}} \operatorname{Def}_{t}^{h} /\left(\mathcal{M}_{t}^{h}\right)^{\alpha(s-1)}$ au pull-back de

$$
0 \longrightarrow\left(V_{o}^{e t, s-1}, \varphi_{o}^{e t, s-1}\right) \longrightarrow\left(V_{o}^{s-1}, \varphi_{o}^{s-1}\right) \longrightarrow\left(V_{o}^{c, s-1}, \varphi_{o}^{c, s-1}\right) \longrightarrow 0
$$

On en déduit donc l'existence d'un ${ }^{(15)}$ morphisme pour tout ouvert affine $S$ de $\mathcal{I}_{\bar{I}^{o}, m}^{=h}$,

$$
f_{s, t}: S_{s, t}(\alpha(s)) \longrightarrow \hat{S}_{s, t}
$$

tel que le diagramme ci-dessous est commutatif

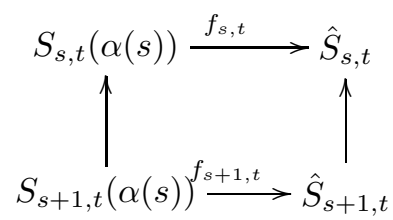

En particulier si $x(\infty)$ est un point fermé de $\mathcal{J}_{I^{o}, m}^{=h}(\infty)$, on a le diagramme commutatif

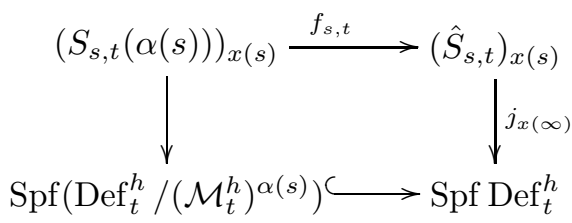

$\overline{(15)}$ non unique 
L'existence du morphisme $f_{N, s, t}$ de l'énoncé découle alors d'un lemme de Berkovich (cf. lemme 1 de l'annexe de [14], ou bien le lemme II.5.8 de loc. cit.)

\section{I.5. Cycles évanescents et systèmes locaux d'Harris-Taylor}

On rappelle que le but des variétés d'Igusa est en particulier de décrire la restriction à $M_{I, s_{o}}^{=h}$ du faisceau des cycles évanescents de $M_{I, o} \longrightarrow \operatorname{Spec}\left(\mathcal{O}_{o}\right)$. Cette question se pose dans le contexte suivant : la cohomologie de la fibre générique de la tour des $\left(M_{I, o}\right)_{I}$ est décrite dans [19] de manière "assez" précise. Cette cohomologie est aussi l'aboutissement de la suite spectrale des cycles évanescents

$$
E_{2}^{p, q}(I)=H^{p}\left(M_{I, \bar{s}_{o}}, R^{q} \Psi_{\eta_{o}}\left(\overline{\mathbb{Q}}_{l}\right) \Rightarrow H^{p+q}\left(M_{I, \eta_{o}}, \overline{\mathbb{Q}}_{l}\right)\right.
$$

Afin de séparer la contribution due aux différentes strates, entre en jeu la suite spectrale associée à la stratification

$$
E_{1}^{p, q}(I, i)=H_{c}^{p+q}\left(M_{I, \bar{s}_{o}}^{=d-p}, R^{i} \Psi_{\eta_{o}}\left(\overline{\mathbb{Q}}_{l}\right)\right) \Rightarrow H^{p+q}\left(M_{I, \bar{s}_{o}}, R^{i} \Psi_{\eta_{o}}\left(\overline{\mathbb{Q}}_{l}\right)\right.
$$

On cherche donc à avoir des renseignements sur ${\underset{I}{\leftarrow}}_{I} H_{c}^{i}\left(M_{\bar{I}, \bar{s}_{o}, 1}^{=h}, R^{j} \Psi_{\eta_{o}}\left(\overline{\mathbb{Q}}_{l}\right)\right)$ en tant que représentation de $\left(D_{\mathbb{A}}^{\infty, o}\right)^{\times} \times P_{h, d}\left(F_{o}\right) \times W_{F_{o}}$.

I.5.1. Rappels sur les cycles évanescents associés à $\operatorname{Def}_{n}^{h}$. - Pour tout $n \geqslant 0$, Def ${ }_{n}^{d}$ représente le foncteur des déformations de niveau $n$, par isogénies, du $\mathcal{O}_{o}$-module formel de hauteur $d$ sur $\overline{\mathbb{F}}_{q}$, cf. [9]. Soit alors $\Psi_{F_{o}, n}^{d, i}$ le $\overline{\mathbb{Q}}_{l}$-espace vectoriel de dimension finie obtenu via la théorie de Berkovich comme le $i$-ème foncteur des cycles évanescents associé au morphisme structural

$$
\operatorname{Spf} \operatorname{Def}_{n}^{d} \longrightarrow \operatorname{Spf} \hat{\mathcal{O}}_{o}^{n r} .
$$

Cet espace vectoriel est muni entre autre d'une action de $G L_{d}\left(\mathcal{O}_{o}\right)$ qui se factorise par le morphisme surjectif naturel $G L_{d}\left(\mathcal{O}_{o}\right) \longrightarrow G L_{d}\left(\mathcal{O}_{o} / \mathcal{M}_{o}^{n}\right)$ et on pose

$$
\Psi_{F_{o}}^{d, i}=\lim _{\substack{-\rightarrow n \\ m \geqslant n}} \Psi_{F_{o}, m}^{d, i}
$$

de sorte que pour $K_{o, n}:=\operatorname{Ker}\left(\mathcal{O}_{o}^{\times} \longrightarrow\left(\mathcal{O}_{o} / \mathcal{M}_{o}^{n}\right)^{\times}\right), \Psi_{F_{o}, n}^{d, i}=\left(\Psi_{F_{o}}^{d, i}\right)^{K_{o, n}}$. On introduit le groupe $\mathcal{N}_{o}$ (resp. $\left.\mathcal{N}_{o}^{\prime}\right)$ défini comme le noyau de

$$
\left(g_{o}, \delta_{o}, c_{o}\right) \in G L_{d}\left(F_{o}\right) \times D_{o, d}^{\times} \times W_{o} \mapsto \operatorname{val}\left(\operatorname{det}\left(g_{o}^{-1}\right) \operatorname{rn}\left(\delta_{o}\right) \operatorname{cl}\left(c_{o}\right)\right) \in \mathbb{Z}
$$

(resp. composé avec la projection canonique $\mathbb{Z} \longrightarrow \mathbb{Z} / d \mathbb{Z}$. Comme rappelé au paragraphe (I.1.1), pour $\xi_{o}$ un caractère d'ordre fini de $F_{o}^{\times}, \Psi_{F_{o}}^{d, i}\left(\xi_{o}^{\prime}\right)\left(\right.$ resp. $\left.\Psi_{F_{o}}^{d, i}\right)$, où $\xi_{o}^{\prime}$ est la restriction de $\xi_{o}$ à $\mathcal{O}_{o}^{\times}$, est muni d'une action de $\mathcal{N}_{o}^{\prime}$ (resp. de $\mathcal{N}_{o}$ ).

I.5.1.1 - Dans la définition de $\operatorname{Def}_{n}^{d}$, il est agréable de considérer plutôt les déformations par quasiisogénies de sorte que la construction précédente fourni des $\overline{\mathbb{Q}}_{l}$-espaces vectoriels $\mathcal{U}_{F_{o}, n}^{d, i} \simeq\left(\mathcal{U}_{F_{o}}^{d, i}\right)^{K_{o, n}}$ où

$$
\mathcal{U}_{F_{o}, \xi_{o}}^{d, i}:=\operatorname{Ind}_{\mathcal{N}_{o}^{\prime}}^{G L_{d}\left(F_{o}\right) \times D_{o, d}^{\times} \times W_{F_{o}}} \Psi_{F_{o}, \xi_{o}}^{d, i}
$$

est une représentation de $G L_{d}\left(F_{o}\right) \times D_{o, d}^{\times} \times W_{F_{o}}$.

Pour toute représentation admissible irréductible $\tau_{o}$ de $D_{o, d}^{\times}$, la réciprocité de Frobenius donne un isomorphisme

$$
\mathcal{U}_{F_{o}}^{d, i}\left(\tau_{o}\right) \simeq \operatorname{Hom}_{\mathcal{D}_{o, d}^{\times}}\left(\operatorname{res}_{\mathcal{D}_{o, d}^{\times}}^{D_{o, d}^{\times}} \tau_{o}, \Psi_{F_{o}, \xi_{o}}^{d, i}\right)
$$

où $\xi_{o}$ est le caractère central de $\tau_{o}$ et l'action de $\left(g_{o}^{c}, \sigma_{o}\right)$ est donnée par celle de $\left(g_{o}, \delta_{o}, \sigma_{o}\right) \in \mathcal{N}_{o}^{\prime}$ pour $\delta_{o} \in D_{o, h}^{\times}$quelconque. Afin d'avoir un théorème de Serre-Tate équivariant on introduit les modifications suivantes. 
I.5.1.2. Définition. - Pour tout $h, i, \widetilde{\mathcal{U}_{F_{o}}^{h, i}}$ est la représentation $\mathcal{U}_{F_{o}}^{h, i}$ où l'action de $G L_{h}\left(F_{o}\right)$ est tordue par $g_{o} \mapsto{ }^{t} g_{o}^{-1}$. On définit de même $\widetilde{\Psi_{F_{o}}^{h, i}}$ comme étant l'espace $\Psi_{F_{o}}^{h, i}$ muni d'une action de

$$
\widetilde{\mathcal{N}_{o}}:=\operatorname{Ker}\left(\left(g_{o}, \delta_{o}, c_{o}\right) \in G L_{d}\left(F_{o}\right) \times D_{o, d}^{\times} \times W_{o} \mapsto \operatorname{val}\left(\operatorname{det}\left(g_{o}\right) \operatorname{rn}\left(\delta_{o}\right) \operatorname{cl}\left(c_{o}\right)\right) \in \mathbb{Z}\right.
$$

via l'action de $\left({ }^{t} g_{o}^{-1}, \delta_{o}, c_{o}\right) \in \mathcal{N}_{o}$ sur $\Psi_{F_{o}}^{h, i}$.

I.5.2. Restriction aux strates ouvertes et variétés d'Igusa. - On rappelle le lemme suivant bien connu.

I.5.2.1. Lemme. - Soit $\mathcal{L}$ un $\overline{\mathbb{Q}}_{l}$-faisceau lisse sur $M_{I, o}$. On a alors

$$
R^{i} \Psi_{\eta_{o}}(\mathcal{L}) \simeq\left(R^{i} \Psi_{\eta_{o}}\left(\overline{\mathbb{Q}}_{l}\right)\right) \otimes \mathcal{L}_{s_{o}}
$$

où $\mathcal{L}_{s_{o}}$ est la restriction de $\mathcal{L}$ à la fibre spéciale $M_{I, s_{o}}$.

I.5.2.2 - Appliqué au faisceau $\mathcal{L}_{\rho_{\infty}}$ cela nous conduit à étudier $R^{i} \Psi_{\eta_{o}}\left(\overline{\mathbb{Q}}_{l}\right)_{M_{I, o}}$ le $i$-ème faisceau des cycles évanescents du morphisme structural

$$
M_{I, o} \longrightarrow \operatorname{Spec} \mathcal{O}_{o}
$$

On notera aussi $R^{i} \Psi_{\eta_{o}}\left(\overline{\mathbb{Q}}_{l}\right)_{M_{I, s_{o}, a}^{=h}}$ sa restriction à $M_{\bar{I}, s_{o}, a}^{=h}$. On considère le tiré en arrière $R^{i} \Psi_{\eta_{o}}\left(\overline{\mathbb{Q}}_{l}\right)_{\mathcal{I}_{I^{o}, n}^{h}}$ de $R^{i} \Psi_{\eta_{o}}\left(\overline{\mathbb{Q}}_{l}\right)_{M_{I, s_{o}, a}^{\bar{I}}}$ par le morphisme $\mathcal{I}_{\bar{I}^{o}, n}^{=h} \longrightarrow M_{I, s_{o}, a}^{=h}$, où $n$ est la multiplicité de $o$ dans $I$. On rappelle qu'étant donné un point géométrique $x(\infty)$ de $\mathcal{J}_{I^{o}, m}^{h}(\infty)$ au dessus d'un point $x$ de $M_{I, s_{o}}^{=h}\left(\right.$ resp. $y$ de $\left.\mathcal{I}_{I^{o}, n}^{=h}\right)$, on a des isomorphisme canoniques

$$
\begin{aligned}
& \widetilde{\Psi_{F_{o}, n}^{h, i}} \simeq\left(R^{i} \Psi_{\eta_{o}}\left(\overline{\mathbb{Q}}_{l}\right)\right)_{\left(M_{I, o,=h, a}\right)_{x}} \simeq\left(R^{i} \Psi_{\eta_{o}}\left(\overline{\mathbb{Q}}_{l}\right)_{M_{\bar{I}, s_{o}, a}^{=h}}\right)_{x} \\
& \widetilde{\Psi_{F_{o}, n}^{h, i}} \simeq\left(R^{i} \Psi_{\eta_{o}}\left(\overline{\mathbb{Q}}_{l}\right)\right)_{\left(\hat{\mathcal{I}}_{I^{o},=h, n}(n)\right)_{y}} \simeq\left(R^{i} \Psi_{\eta_{o}}\left(\overline{\mathbb{Q}}_{l}\right)_{\mathcal{I}_{I^{o}, n}^{h}, h}\right)_{y}
\end{aligned}
$$

que l'on note dans les deux cas $j_{x(\infty)}$. Le but de ce paragraphe et finalement des variétés d'Igusa est de décrire le faisceau $R^{i} \Psi_{\eta_{o}}\left(\overline{\mathbb{Q}}_{l}\right)_{M_{\bar{I}, s_{o}, a}^{=h}}$. Selon loc. cit. nous introduisons les notations suivantes :

- soient $\bar{Y}$ un schéma lisse sur $\overline{\mathbb{F}}_{q}$, et $\left(\bar{Y}_{n} \rightarrow \bar{Y}\right)$ un système projectif de revêtements étales de groupe de Galois $G=\underset{\leftarrow}{\lim _{n}} G_{n}$;

- soit $\mathfrak{L}$ un $\overline{\mathbb{Q}}_{l}$-faisceau lisse sur $\bar{Y}$, muni d'une action de $G$ se factorisant par un quotient fini $G_{N}$ de $G$.

Le faisceau $\mathfrak{L}_{\mid \bar{Y}_{n}}$ est ainsi muni d'une action de $G_{n} \times G_{n}$ et l'action diagonale de $G_{n}$ est compatible à son action sur $\bar{Y}_{n}$ de sorte qu'il existe un faisceau

$$
\operatorname{Twist}_{\left(\bar{Y}_{n}\right)}(\mathfrak{L})
$$

sur $\bar{Y}$ tel que sa restriction à $\bar{Y}_{n}$ munie de l'action naturelle de $G_{n}$ coïncide avec $\mathfrak{L}_{\mid \bar{Y}_{n}}$ muni de l'action diagonale de $G_{n}$.

I.5.2.3 - Rappelons l'énoncé suivant dû à Berkovich (cf. loc. cit. appendice). Supposons donné sur $\hat{\mathcal{O}}_{o}^{n r}$, un schéma formel $\mathcal{X}$ muni d'une action d'un groupe $G$ agissant trivialement sur la fibre spéciale $\mathcal{X}_{s}$. On note $\mathcal{X}(n)$ le schéma formel $\left(\mathcal{X}_{s}, \mathcal{O}_{\mathcal{X}} / \mathcal{I}_{\mathcal{X}}^{n}\right)$ où $\mathcal{I}_{\mathcal{X}}$ est un idéal de définition de $\mathcal{X}$. On suppose que $G=\lim _{n} G(n)$ tel que l'action de $G$ sur $\mathcal{X}(n)$ se factorise par $G(n)$. Soit alors $\mathcal{X}_{n} \longrightarrow \mathcal{X}$ des revêtements galoisiens de groupe de Galois $G(n)$ et on fait les hypothèses suivantes :

- le quotient de $\mathcal{X}_{n}(n)$ par l'action diagonale de $G(n)$ est un schéma formel $\mathcal{Y}^{n}$ (c'est le cas si par exemple $\mathcal{X}$ est quasi-projectif sur $\left.\operatorname{Spf} \hat{\mathcal{O}}_{o}^{n r}\right)$

- il existe un schéma formel spécial $\mathcal{Y}$ tel que $\mathcal{Y}(n)=\mathcal{Y}^{n}$;

- la famille des complétions formelles de $\mathcal{X}$ le long d'un point fermé de $\mathcal{X}_{s}$, a un nombre fini de classes d'isomorphismes. 
I.5.2.4 - On note $\Psi_{m}^{i}(\mathcal{X})$ (resp. $\left.\Psi_{m}^{i}(\mathcal{Y})\right)$ le $i$-ème faisceau des cycles évanescents du faisceau constant $\mathbb{Z} / p^{m} \mathbb{Z}$ associé à $\mathcal{X}$ (resp. $\mathcal{Y}$ ). D'après les travaux de Berkovich (cf. [4]), il existe un entier $N$, tel que tout automorphisme sur $\mathcal{X}$ trivial sur $\mathcal{X}(N)$, agit trivialement sur $\Psi_{m}^{i}(\mathcal{X})$. On fixe donc pour tout $m$, un tel entier $N(m)$, de sorte que l'action de $G$ sur $\Psi_{m}^{i}(\mathcal{X})$ se factorise par $G(N(m))$. En particulier les faisceaux Twist $\mathcal{X}_{n}\left(\Psi_{m}^{i}(\mathcal{X})\right)$ sont indépendants de $n \geqslant N(m)$; on le note Twist $\mathcal{X}_{\infty}\left(\Psi_{m}^{i}(\mathcal{X})\right)$. Berkovich démontre alors le résultat suivant.

I.5.2.5. Théorème. - (cf. l'appendice de Berkovich dans [14]) Il existe un système compatible d'isomorphismes canoniques de faisceaux :

$$
\Psi_{m}^{i}(\mathcal{Y}) \simeq \operatorname{Twist}_{\mathcal{X}_{\infty}}\left(\Psi_{m}^{i}(\mathcal{X})\right)
$$

I.5.2.6 - On cherche à appliquer le théorème ci-dessus à $\mathcal{Y}=\mathrm{Tw}_{I^{o}, h, m, t}(\infty)$. On pose donc $\mathcal{X}=\mathcal{I}_{I^{o}, m}^{=h} \times$ Spec $\kappa(o)$ Spf Def ${ }_{t}^{h}$ qui est muni d'une action de $G=\mathcal{D}_{o, h}^{\times}$agissant trivialement sur $\mathcal{X}_{s}=$

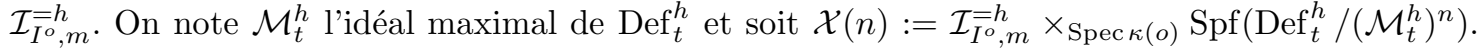
On considère une fonction $N: \mathbb{N} \longrightarrow \mathbb{N}$, telle que $\alpha \circ N=\mathrm{Id}$, où $\alpha$ est la fonction définie au lemme (I.4.3.9), de sorte que l'action de $G \operatorname{sur} \mathcal{X}(n)$ se factorise à travers $G(n):=\mathcal{D}_{o, h, N(n)}$. On considère ensuite $\mathcal{X}_{s}:=\mathcal{J}_{I^{o}, m}^{h}(N(s)) \times_{\operatorname{Spec} \kappa(o)} \operatorname{Spf} \operatorname{Def}_{t}^{h}$ de sorte que $\mathcal{X}_{s} \longrightarrow \mathcal{X}$ est galoisien de groupe de Galois $G(s)=\mathcal{D}_{o, h, N(s)}$. On est ainsi dans les conditions d'application du théorème ci-dessus d'où le résultat suivant.

I.5.2.7. Théorème. - Il existe, sur $\mathcal{I}_{I^{o}, m}^{=h} \otimes_{\kappa(o)} \bar{\kappa}(o)$, un isomorphisme canonique de $\overline{\mathbb{Q}}_{l^{-}}$faisceaux

$$
R^{i} \Psi_{\eta_{o}}\left(\overline{\mathbb{Q}}_{l}\right)_{\mathrm{Tw}_{I^{o}, h, m, t}(\infty)} \stackrel{\sim}{\longrightarrow} \operatorname{Twist}_{\mathcal{J}_{I^{\circ}, m} h(\infty)}\left(\widetilde{\Psi_{F_{o}, t}^{h, i}}\right)
$$

vérifiant les propriétés suivantes

(1) si $x$ est un point géométrique de $\mathcal{I}_{I^{o}, m}^{=h}$ et si $x(\infty)=(x(s))_{s}$ est un système projectif de points de $\mathcal{J}_{I^{o}, m}^{=h}(s)$ au dessus d'un point $x$ de $\mathcal{I}_{I^{o}, m}^{=h}$, on a un isomorphisme

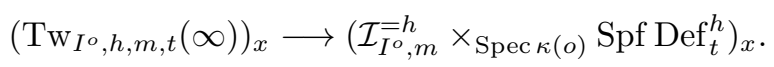

Par le théorème de changement de base lisse le faisceaux des cycles évanescents sur $\mathcal{I}_{I^{o}, m}^{=h} \times_{\operatorname{Spec} \kappa(o)} \operatorname{Spf} \operatorname{Def}_{t}^{h}$ est le faisceau constant $\widetilde{\Psi_{F_{o}, t}^{h, i}}$. On en déduit donc un isomorphisme

$$
f_{x(\infty)}: \widetilde{\Psi_{F_{o}, t}^{h, i}} \longrightarrow R^{i} \Psi_{\eta_{o}}\left(\overline{\mathbb{Q}}_{l}\right)_{\mathrm{Tw}_{h, m, t}(\infty)_{x}} .
$$

De même la donnée de $x(\infty)$, donne un isomorphisme

$$
h_{x(\infty)}: \widetilde{\Psi_{F_{o}, t}^{h, i}} \longrightarrow\left(\text { Twist }_{J_{I^{o}, m}^{h}(\infty)} \widetilde{\Psi_{F_{o}, t}^{h, i}}\right)_{x}
$$

et l'on a le diagramme commutatif

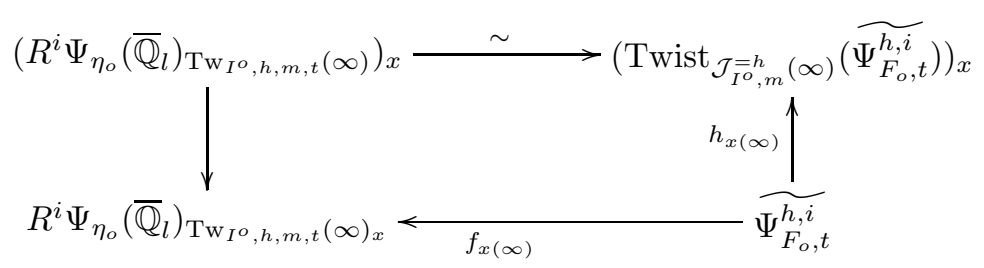

(2) l'action par correspondances d'un élément

$$
\left(g^{\infty, o}, g_{o}^{e t}, g_{o}^{c}\right) \in\left(D_{\mathbb{A}}^{\infty, o}\right)^{\times} \times G L_{d-h}\left(F_{o}\right) \times G L_{h}\left(F_{o}\right)
$$

sur $R^{i} \Psi_{\eta_{o}}\left(\overline{\mathbb{Q}}_{l}\right)_{\mathrm{Tw}_{I^{o}, h, m, t}(\infty)}$ donne par l'isomorphisme de l'énoncé, une action par correspondances sur Twist ${\mathcal{\mathcal { J } ^ { \circ } , h _ { m }}}_{(\infty)}\left(\widetilde{\Psi_{F_{o}, t}^{h, i}}\right)$ qui découle de l'action naturelle par correspondances d'un élément

$$
\left(g^{\infty, o}, g_{o}^{e t},\left(g_{o}^{c}, \sigma_{o}, \delta_{o}\right)\right) \in\left(D^{\infty, o}\right)^{\times} / K_{\mathbb{A}, I}^{\infty, o} \times G L_{d-h}\left(F_{o}\right) \times \widetilde{\mathcal{N}}_{o}
$$


(16) sur le produit $\mathcal{J}_{I^{o}, m}^{=h}(s) \times \operatorname{Spf}^{D_{e f}^{h}}{ }_{t}^{h}$ où l'on rappelle que $\left(g_{o}^{c}, \sigma_{o}, \delta_{o}\right) \in \widetilde{\mathcal{N}}_{o}$ agit diagonalement.

Démonstration. - Pour montrer que les correspondances de Hecke se décrivent comme indiqué au point (2), il suffit de travailler sur les germes aux points géométriques de $\mathcal{I}_{I^{o}, m}^{=h}$ en remarquant que les diagrammes suivant sont commutatifs, cf. la proposition (I.4.3.3) :

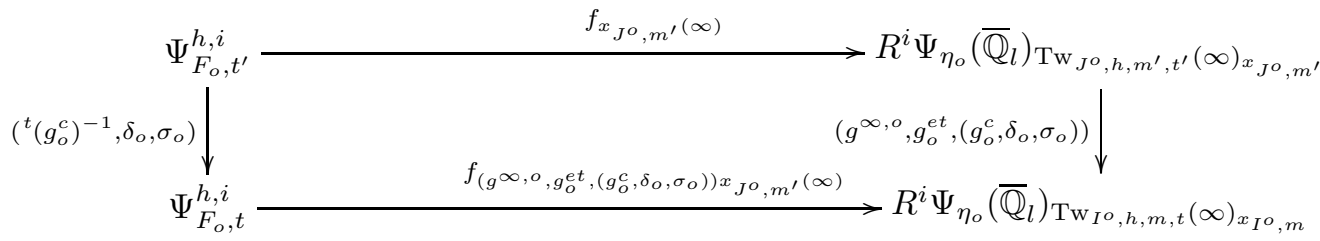

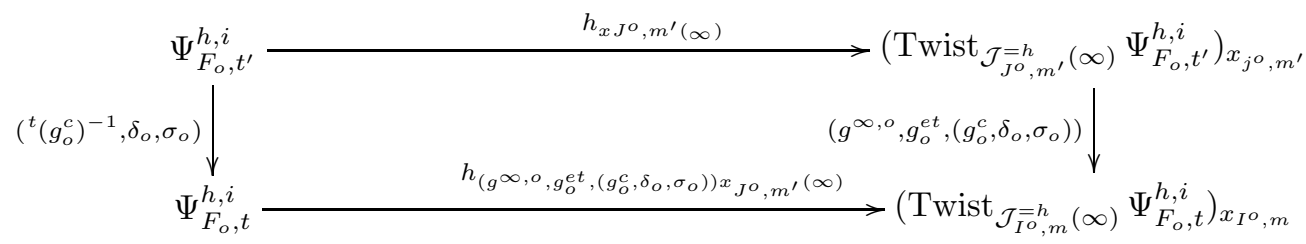

où $\delta_{o}$ est un élément quelconque de $D_{o, h}^{\times}$tel que $\left(g_{o}^{c}, \delta_{o}, \sigma_{o}\right) \in \widetilde{\mathcal{N}}_{o}$, et $J^{o}, I^{o}, m, m^{\prime}, t, t^{\prime}$ sont comme au paragraphe (I.4.3), avec

$$
\left(g^{\infty, o}, g_{o}^{e t},\left(g_{o}^{c}, \delta_{o}, \sigma_{o}\right)\right)\left(x_{J^{o}, m^{\prime}}(\infty)\right)=x_{I^{o}, m}(\infty) .
$$

I.5.2.8. Corollaire. - Il existe un isomorphisme canonique

$$
R^{i} \Psi_{\eta_{o}}\left(\overline{\mathbb{Q}}_{l}\right)_{\hat{\mathcal{I}}_{I^{o},=h, m}(t)} \longrightarrow \text { Twist }_{\mathcal{J}_{\bar{I}^{o}, m}^{h}(\infty)}\left(\widetilde{\Psi_{F_{o}, t}^{h, i}}\right)
$$

tel qu'en tout point fermé $x$ de $\mathcal{I}_{I^{o}, m}^{=h}$, le diagramme suivant soit commutatif

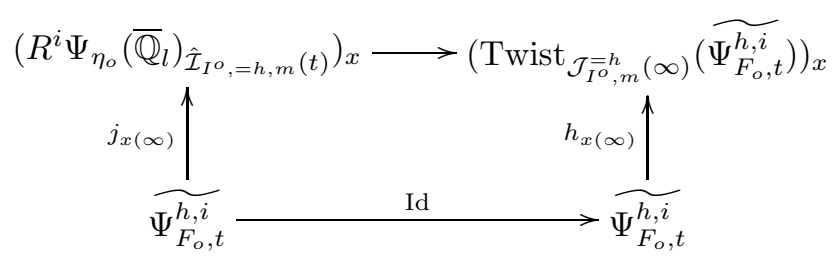

où les flèches verticales dépendent du choix d'un point fermé $x(\infty)$ de $\mathcal{J}_{I^{\circ}, h},(\infty)$ au dessus de $x$. En outre ces isomorphismes sont compatibles aux correspondances de Hecke associées aux éléments de $\left(D_{\mathbb{A}}^{\infty, o}\right)^{\times} \times G L_{d-h}\left(F_{o}\right) \times G L_{h}\left(F_{o}\right) \times W_{F_{o}}$ de la manière suivante : si $\left(g^{\infty, o}, g_{o}^{\text {et }}, g_{o}^{c}\right)$ est un élément de $\left(D_{\mathbb{A}}^{\infty, o}\right)^{\times} \times G L_{d-h}\left(F_{o}\right) \times G L_{h}\left(F_{o}\right)$, qui induit donc, en particulier, une correspondance de Hecke $[g] \operatorname{sur} \mathcal{I}_{I^{o}, m}^{=h}$, on a le diagramme commutatif suivant

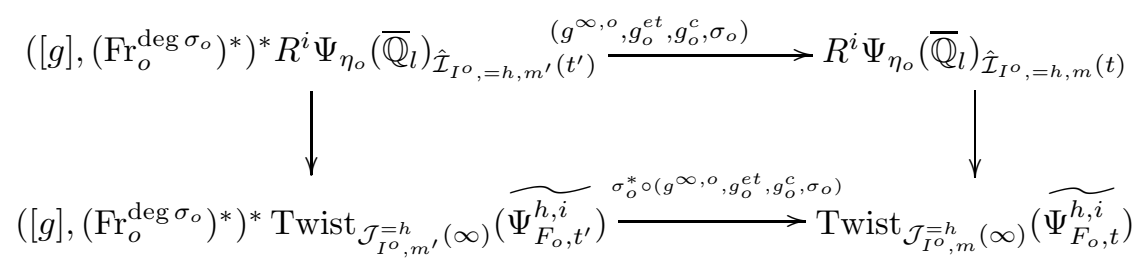

${ }^{(16)}$ le résultat ne dépend pas du choix de $\delta_{o}$ 
où l'action de $\left(g^{\infty, o}, g_{o}^{e t}, g_{o}^{c}, \sigma_{o}\right)$ sur Twist ${ }_{\mathcal{J}_{I^{o}, m}^{=h}(\infty)}\left(\widetilde{\Psi_{F_{o}, t}^{h, i}}\right)$ est précisée au point (2) du théorème précédent.

Démonstration. - La démonstration découle directement du résultat précédent et de la proposition (I.4.3.4) notamment la description des correspondances de Hecke se montre comme dans la preuve du théorème ci-dessus. On peut toutefois en donner une preuve directe à partir de la proposition (I.4.3.7). D'après le théorème 4.1 de [4], pour tout entier $r$, on peut choisir un entier $N$, tel que deux morphismes de schéma formels sur $\hat{\mathcal{O}}_{o}^{n r}$

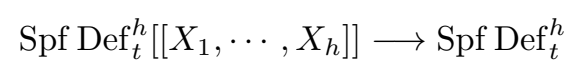

qui coïncide sur $\operatorname{Spf}\left(\operatorname{Def}_{t}^{h} /\left(\mathcal{M}_{t}^{h}\right)^{N}\right)\left[\left[X_{1}, \cdots, X_{h}\right]\right]$, induisent la même application sur les cycles évanescents du faisceau constant $\mathbb{Z} / l^{r} \mathbb{Z}$. De la proposition (I.4.3.7), on en déduit donc un morphisme

$$
f_{s}: R^{i} \Psi_{\eta_{o}}\left(\mathbb{Z} / l^{r} \mathbb{Z}\right)_{S_{s} \times \operatorname{Spec} \kappa(o)} \operatorname{Spf}_{\operatorname{Def}_{t}^{h}}=\widetilde{\Psi_{F_{o}, t}^{h, i}} \longrightarrow R^{i} \Psi_{\eta_{o}}\left(\mathbb{Z} / l^{r} \mathbb{Z}\right)_{\hat{S}_{s}}
$$

tel que pour tout point fermé $x(\infty)$ de $\mathcal{J}_{I^{o}, m}^{=h}(\infty)$, le morphisme

$$
\widetilde{\Psi_{F_{o}, t}^{h, i}} \longrightarrow\left(R^{i} \Psi_{\eta_{o}}\left(\mathbb{Z} / l^{r} \mathbb{Z}\right)_{\hat{S}_{s}}\right)_{x_{s}}
$$

est le morphisme $j_{x(\infty)}$, c'est en particulier un isomorphisme. Ce dernier se descend en un isomorphisme

$$
\operatorname{Twist}_{\mathcal{J}_{I^{o}, m}^{=h}(s)} \widetilde{\Psi_{F_{o}, t}^{h, i}} \longrightarrow R^{i} \Psi_{\eta_{o}}\left(\mathbb{Z} / l^{r} \mathbb{Z}\right)_{\hat{\mathcal{I}}_{I^{o},=h, m}(t)}
$$

tel qu'en tout point fermé $x(\infty)$ de $\mathcal{J}_{I^{o}, m}^{=h}(\infty)$ au dessus d'un point $x$ de $\mathcal{I}_{I^{o}, m}^{=h}$, on ait le diagramme commutatif suivant

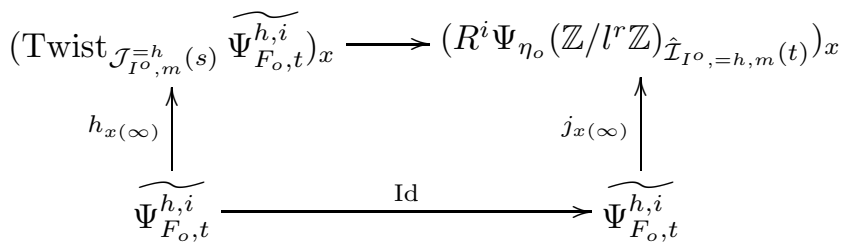

où les flèches verticales définies plus haut, dépendent du choix de $x(\infty)$. En travaillant au niveau des germes, on voit que ces isomorphismes sont compatibles lorsque $r$ varie, de sorte que l'on peut les recoller en un isomorphisme vérifiant les propriétés de l'énoncé.

I.5.3. Systèmes locaux d'Harris-Taylor. - Soit $\tau_{o}$ une représentation admissible irréductible de $D_{o, h}^{\times}$. L'espace que l'on souhaite étudier est le $\left(G L_{h}\left(F_{o}\right) \times W_{F_{o}}\right)$-module

$$
\operatorname{Hom}_{D_{o, h}^{\times}}\left(\tau_{o}, \mathcal{U}_{F_{o}}^{h, i}\right)=\mathcal{U}_{F_{o}}^{h, i}\left(\tau_{o}\right) \simeq \Psi_{F_{o}}^{h, i}\left(\tau_{o}\right):=\operatorname{Hom}_{\mathcal{D}_{o, h}^{\times}}\left(\tau_{o}, \Psi_{F_{o}}^{h, i}\right)
$$

ou de manière équivalente leur version avec un tilde, $\widetilde{\mathcal{U}_{F_{o}}^{h, i}\left(\tau_{o}\right)}$ qui désigne l'espace $\mathcal{U}_{F_{o}}^{h, i}\left(\tau_{o}\right)$ muni de l'action tordue de $G L_{h}\left(F_{o}\right) \times W_{o}$ par $g_{o}^{c} \mapsto{ }^{t}\left(g_{o}^{c}\right)^{-1}$.

I.5.3.1. Définition. - Soit $\tau_{o}$ une représentation irréductible de $D_{o, h}^{\times}$, sa restriction à $\mathcal{D}_{o, h}^{\times}$est une somme de représentations irréductibles

$$
\rho_{o, 1} \oplus \cdots \oplus \rho_{o, e_{\tau_{o}}}
$$

et on notera $e_{\tau_{o}}$ le nombre de celles ci. Étant donnée une représentation irréductible $\rho_{o}$ de $\mathcal{D}_{o, h}^{\times}$, soient alors $\tau_{o}$ et $\tau_{o}^{\prime}$ des sous-représentations irréductibles de l'induite de $\mathcal{D}_{o, h}^{\times}$à $D_{o, h}^{\times}$de $\rho_{o}$ : d'après la réciprocité de Frobenius, ce sont exactement celles telles que leur restriction à $\mathcal{D}_{o, h}^{\times}$ contienne $\rho_{o}$. On en déduit alors que $\tau_{o}$ et $\tau_{o}^{\prime}$ sont inertiellement équivalentes, i.e. $\tau_{o}^{\prime} \simeq \tau_{o} \otimes \xi_{o}$ 
avec $\xi_{o}: \delta \mapsto x^{v(\operatorname{det} \delta)}$ pour $x \in \overline{\mathbb{Q}}_{l}^{\times}$. On note $\mathfrak{C}_{h}$ l'ensemble des classes d'équivalences inertielles des représentations admissibles et irréductibles du groupe $D_{o, h}^{\times}$.

I.5.3.2 - Pour tout $\tau_{o}$, on a un morphisme naturel de $\mathcal{N}_{o}$-modules :

$$
\Psi_{F_{o}}^{h, i}\left(\tau_{o}\right) \otimes \tau_{o} \longrightarrow \Psi_{F_{o}}^{h, i}
$$

qui envoie $f \otimes v \operatorname{sur} f(v)$. On note $\Psi_{F_{o}}^{h, i}\left[\tau_{o}\right]$ l'image de ce morphisme et soit $\Psi_{F_{o}, m}^{h, i}\left[\tau_{o}\right]$ la préimage de $\Psi_{F_{o}}^{h, i}\left[\tau_{o}\right]$ dans $\Psi_{F_{o}, m}^{h, i}$. Le sous-module $\Psi_{F_{o}}^{h, i}\left[\tau_{o}\right]$ ne dépend que de la classe d'équivalence inertielle de $\tau_{o}$. Le groupe $\mathcal{D}_{o, h}^{\times}$étant compact, on a

$$
\Psi_{F_{o}}^{h, i}=\bigoplus_{\tau_{o} \in \mathfrak{C}_{h}} \Psi_{F_{o}}^{h, i}\left[\tau_{o}\right]
$$

I.5.3.3 - Soit $e_{\tau_{o}}$ le nombre de composantes irréductibles de $\left(\tau_{o}\right)_{\mid \mathcal{D}_{o, h}^{\times}}$; c'est aussi le nombre de caractère $\xi: \mathbb{Z} \longrightarrow \overline{\mathbb{Q}}_{l}^{\times}$tels que $\tau_{o} \simeq \tau_{o} \otimes(\xi \circ$ val $\circ$ det $)$. Comme $\Pi_{o, h}^{h}$ est dans le centre de $D_{o, h}^{\times}$, $e_{\tau_{o}}$ divise $h$. Soit $\Delta_{\tau_{o}}$ un ensemble d'éléments de $D_{o, h}^{\times}$tel que les congruences des $\operatorname{val}(\operatorname{det} \delta)$ pour $\delta \in \Delta_{\tau_{o}}$ forment un système de représentants de $\mathbb{Z} / e_{\tau_{o}} \mathbb{Z}$. On a alors le lemme suivant dont la preuve est claire.

\section{I.5.3.4. Lemme. - L'application}

$$
\begin{aligned}
\Psi_{F_{o}}^{h, i}\left(\tau_{o}\right) \otimes \tau_{o} & \longrightarrow \bigoplus_{\delta \in \Delta_{\tau_{o}}} \Psi_{F_{o}}^{h, i}\left[\tau_{o}\right]^{\delta} \\
f \otimes v & \mapsto\left(f\left(\delta^{-1} v\right)_{\delta}\right.
\end{aligned}
$$

où $\Psi_{F_{o}}^{h, i}\left[\tau_{o}\right]^{\delta}$ est l'espace $\Psi_{F_{o}}^{h, i}\left[\tau_{o}\right]$ muni de la structure de $\mathcal{N}_{o}$-module où $(g, d, w)$ agit via $\left(g, \delta^{-1} d \delta, w\right)$, est un isomorphisme de $\mathcal{N}_{o}$-modules.

I.5.3.5. Lemme. - Soit $\mathcal{F}_{\tau_{o}, 1}$ le système local sur $M_{I, s_{o}, 1}^{=h}$ associé à $\tau_{o}$ et au revêtement d'Igusa de seconde espèce. On a alors un isomorphisme naturel de $\left(D_{\mathbb{A}}^{\infty, o}\right)^{\times} \times G L_{d-h}\left(F_{o}\right) \times G L_{h}\left(F_{o}\right) \times W_{F_{o}}$ modules

$$
\left(\operatorname{Twist}_{\mathcal{J}_{I^{o}, m}=h}(\infty)\left(\widetilde{\Psi_{F_{o}, t}^{h, i}}\right)\right)^{h} \simeq \bigoplus_{\tau_{o} \in \mathfrak{C}_{h}}\left(\mathcal{F}_{\tau_{o}, 1} \otimes \widetilde{\Psi_{F_{o}, t}^{h, i}\left(\tau_{o}\right)}\right)^{h / e_{\tau_{o}}}
$$

Démonstration. - D'après le lemme précédent, on a

$$
\operatorname{Twist}_{\mathcal{J}_{\bar{I}^{o}, m}^{=h}(\infty)}\left(\widetilde{\Psi_{F_{o}, t}^{h, i}}\right)=\bigoplus_{\tau_{o} \in \mathfrak{C}_{h}} \operatorname{Twist}_{\mathcal{J}_{I^{o}, m}^{=h}(\infty)}\left(\widetilde{\Psi_{F_{o}, t}^{h, i}}\left[\tau_{o}\right]\right)
$$

et

$$
\left.\mathcal{F}_{\tau_{o}, 1} \otimes \widetilde{\Psi_{F_{o}, t}^{h, i}\left(\tau_{o}\right)} \simeq \bigoplus_{\delta \in \Delta_{\tau_{o}}} \operatorname{Twist}_{\mathcal{J}_{I^{o}, h}=h(\infty)}\left(\widetilde{\Psi_{F_{o}, t}^{h, i}} \tau_{\tau_{o}}\right]^{\delta}\right)
$$

D'après le point (2) du théorème (I.5.2.7), tous les Twist ${ }_{\mathcal{J}_{I^{o}, m}^{h}(\infty)}\left(\widetilde{\Psi_{F_{o}, t}^{h, i}}\left[\tau_{o}\right]^{\delta}\right)$ sont isomorphes en tant que $\left(D_{\mathbb{A}}^{\infty, o}\right)^{\times} \times G L_{d-h}\left(F_{o}\right) \times G L_{h}\left(F_{o}\right) \times W_{F_{o}}$-modules, d'où le résultat.

Remarque : Pour tout $t \leqslant t^{\prime} \leqslant \infty$, on a

$$
\begin{aligned}
& \operatorname{Twist}_{\mathcal{J}_{I^{o}, m}^{=h}(\infty)}\left(\widetilde{\Psi_{F_{o}, t}^{h, i}}\right)=\left(\text { Twist }_{\mathcal{J}_{I^{o}, m}^{=h}(\infty)}\left(\widetilde{\Psi_{F_{o}, t^{\prime}}^{h, i}}\right)\right)^{K_{o, t}} \\
& \operatorname{Twist}_{\mathcal{J}_{I^{\prime}, m}, h}(\infty)\left(\widetilde{\Psi_{F_{o}, t}^{h, i}}\left[\tau_{o}\right]\right)=\left(\text { Twist }_{\mathcal{J}_{I^{o}, m}=h}\left(\widetilde{\Psi_{F_{o}, t^{\prime}}^{h, i}}\left[\tau_{o}\right]\right)\right)^{K_{o, t}}
\end{aligned}
$$

où l'on rappelle que $K_{o, t}=\operatorname{Ker}\left(G L_{h}\left(\mathcal{O}_{o}\right) \longrightarrow G L_{h}\left(\mathcal{O}_{o} / \mathcal{M}_{o}^{t}\right)\right)$.

D’après le corollaire (I.5.2.8) et le théorème (I.5.2.7), on en déduit le résultat suivant. 
I.5.3.6. Proposition. -

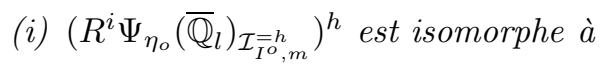

$$
\bigoplus_{\tau_{o} \in \mathfrak{C}_{h}}\left(\mathcal{F}_{\tau_{o}, 1} \otimes \mathcal{U}_{F_{o}, m}^{h, i}\left(\tau_{o}\right)\right)^{h / e_{\tau_{o}}} \text {. }
$$

(ii) L'action de $\left(D_{\mathbb{A}}^{\infty, o}\right)^{\times} \times G L_{h}\left(F_{o}\right) \times G L_{d-h}\left(F_{o}\right) \times W_{F_{o}}$ sur $\lim _{\leftarrow} \mathcal{I}_{I^{\prime}, m}^{=h}$ par les correspondances de Hecke, induit des morphismes $\left(g^{\infty, o} \times g_{o}^{c} \times g_{o}^{e t} \times \sigma_{o}\right)$ :

$$
\left(g^{\infty, o}, \operatorname{Frob}_{o}^{\operatorname{val}\left(\operatorname{det} g_{o}^{c}\right)+\operatorname{deg} \sigma_{o}}, g_{o}^{e t}\right)^{*} R^{i} \Psi_{\eta_{o}}\left(\overline{\mathbb{Q}}_{l}\right)_{\mathcal{I}_{I^{o}, m}^{=h}} \longrightarrow R^{i} \Psi_{\eta_{o}}\left(\overline{\mathbb{Q}}_{l}\right)_{\mathcal{I}_{J^{o}, m^{\prime}}}
$$

(pour $m, m^{\prime}$ et $I^{o}, J^{o}$ convenables) qui induisent par (i) le morphisme

$$
\begin{gathered}
\bigoplus_{\tau_{o} \in \mathfrak{C}_{h}}\left[\left(\operatorname{Frob}_{o}^{\operatorname{deg} \sigma_{o}}\right)^{*} \circ\left(g^{\infty, o}, g_{o}^{e t},\left(g_{o}^{c}, \delta_{o}, \sigma_{o}\right)\right)^{*}\right]\left(\mathcal{F}_{\tau_{o}, 1} \otimes \mathcal{U}_{F_{o}, m}^{h, i}\left(\tau_{o}\right)\right) \\
\downarrow^{\left(\sigma_{o}^{*} \circ\left(g^{\infty, o}, g_{o}^{e t}, g_{o}^{c}\right) \otimes\left(g_{o}^{c}, \delta_{o}, \sigma_{o}\right)\right)} \\
\bigoplus_{\tau_{o} \in \mathfrak{C}_{h}}\left(\mathcal{F}_{\tau_{o}, 1} \otimes \mathcal{U}_{F_{o}, m^{\prime}}\left(\tau_{o}\right)\right)
\end{gathered}
$$

où $\delta_{o}$ est un élément quelconque de $D_{o, h}^{\times}$tel que $\left(g_{o}^{c}, \delta_{o}, \sigma_{o}\right) \in \widetilde{\mathcal{N}}_{o}{ }^{(17)}$

(iii) on a

$$
R^{i} \Psi_{\eta_{o}}\left(\overline{\mathbb{Q}}_{l}\right)_{\mathcal{I}_{I^{o}, m}^{h}} \simeq\left(\operatorname{Twist}_{\mathcal{I}_{I^{o}, m}^{h}(\infty)}\left(\widetilde{\Psi_{F_{o}}^{h, i}}\right)\right)^{1+\varpi_{o}^{m} \mathbb{M}_{h}\left(\mathcal{O}_{o}\right)} .
$$

I.5.3.7. Remarques. - (i) Les systèmes locaux $\mathcal{F}_{\tau_{o}, 1}$ ne sont pas irréductibles mais plutôt une somme directe de $e_{\tau_{o}}$ systèmes locaux irréductibles. La complexité de l'écriture du point (i), est la contre-partie de la simplicité de la description de l'action de $G L_{d}\left(F_{o}\right) \times W_{o}$ qui tient au fait que l'on a fait apparaître $\widetilde{\mathcal{U}_{F_{o}}^{h, i}}\left(\tau_{o}\right)$ plutôt que $\operatorname{Hom}_{\mathcal{D}_{o, h}^{\times}}\left(\rho_{o}, \widetilde{\Psi_{F_{o}}^{h, i}}\right)$ qui est seulement une représentation de $\widetilde{\mathcal{N}}_{o} \cap\left(G L_{h}\left(F_{o}\right) \times D_{o, h}^{\times}\right)$.

(ii) Soit $\mathbb{Z} \stackrel{\sim}{\longrightarrow} \widetilde{\mathcal{N}}_{o} / \mathcal{D}_{o, h}^{\times}$l'isomorphisme défini par $n \mapsto \Pi_{o, h}^{n}$. On considère ainsi

$$
\lim _{\substack{I^{o}, n}} H_{c}^{j}\left(\mathcal{I}_{I^{o}, n}^{=h}, \mathcal{F}_{\tau_{o}, 1}\right)
$$

comme une représentation de

$$
\left(D_{\mathbb{A}}^{\infty, o}\right)^{\times} \times G L_{d-h}\left(F_{o}\right) \times \mathbb{Z} \text { ou de }\left(D_{\mathbb{A}}^{\infty, o}\right)^{\times} \times G L_{d-h}\left(F_{o}\right) \times\left(D_{o, h}^{\times} / \mathcal{D}_{o, h}^{\times}\right)
$$

En vertu du lemme (I.5.2.1), on en déduit alors le corollaire suivant

I.5.3.8. Corollaire. - On a un isomorphisme canonique

$$
H_{c}^{j}\left(M_{I, s_{o}, 1}^{=h}, R^{i} \Psi_{\eta_{o}}\left(\mathcal{L}_{\rho_{\infty}}\right)\right)^{h} \simeq \bigoplus_{\tau_{o} \in \mathfrak{C}_{h}}\left(H_{c}^{j}\left(M_{I, s_{o}, 1}^{=h}, \mathcal{F}_{\tau_{o}, 1} \otimes \mathcal{L}_{\rho_{\infty}}\right) \otimes \widetilde{\left.\mathcal{U}_{F_{o}, m}^{h, i}\left(\tau_{o}\right)\right)^{h / e_{\tau_{o}}}}\right.
$$

tel que l'action de $\left(g^{\infty, o}, g_{o}^{c}, g_{o}^{e t}, \sigma_{o}\right) \in\left(D_{\mathbb{A}}^{\infty, o}\right)^{\times} \times G L_{h}\left(F_{o}\right) \times G L_{d-h}\left(F_{o}\right) \times W_{F_{o}}$ sur la limite inductive indexée par les idéaux $I=I^{o} \mathcal{M}_{o}^{m}$ du membre de gauche, induit l'action de

$$
\left(g^{\infty, o}, g_{o}^{e t},-\operatorname{val}\left(\operatorname{det} g_{o}^{c}\right)-\operatorname{deg}\left(\sigma_{o}\right)\right) \otimes\left(g_{o}^{c}, \sigma_{o}\right)
$$

sur la limite inductive du membre de droite.

I.5.3.9. Définition. - On note $\mathcal{F}_{\tau_{o}}:=\mathcal{F}_{\tau_{o}, 1} \times_{P_{h, d}^{o p}\left(F_{o}\right)} G L_{d}\left(F_{o}\right)$ le faisceau sur $M_{I, s_{o}}^{=h}$ induit par $\mathcal{F}_{\tau_{o}, 1}$.

On déduit du corollaire précédent la proposition suivante dont la proposition (II.2.9) nous permettra au paragraphe suivant de donner le cas Iwahori du théorème local.

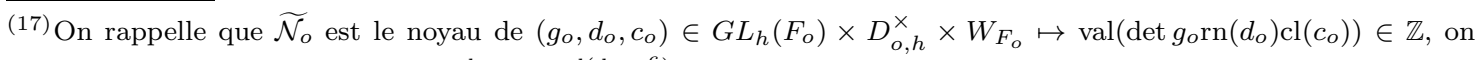
peut par exemple prendre $\delta_{o}=\Pi_{o, h}^{-\operatorname{deg} \sigma_{o}-\operatorname{val}\left(\operatorname{det} g_{o}^{c}\right)}$, où $\Pi_{o, h}$ est une uniformisante de $\mathcal{D}_{o, h}$ telle que $\Pi_{o, h}^{h}=\varpi_{o}$.
} 
I.5.3.10. Proposition. - Soient $1 \leqslant l g<d, \pi_{o}$ une représentation irréductible cuspidale de $G L_{g}\left(F_{o}\right)$ et $\Pi_{l}$ une représentation de $G L_{l g}\left(F_{o}\right)$. Pour $1 \leqslant i \leqslant l$ et pour tout $j$,

$$
\lim _{\vec{I}} H_{c}^{j}\left(M_{I, s_{o}}^{=l g}, \mathcal{F}_{\mathrm{JL}^{-1}\left(\mathrm{St}_{l}\left(\pi_{o}\right)\right)} \otimes \mathcal{L}_{\rho_{\infty}}\right) \otimes \Pi_{l} \otimes L_{g}\left(\pi_{o}\right)
$$

est muni d'une action naturelle de $\left(D_{\mathbb{A}}^{\infty, o}\right)^{\times} \times P_{l g, d}\left(F_{o}\right)^{o p} \times W_{o}$ telle que $\left(g^{\infty, o}, g_{o}^{c} \times g_{o}^{e t}, c_{o}\right)$ agisse via l'action naturelle de $\left(g^{\infty, o},-\operatorname{val}\left(\operatorname{det}\left(g_{o}^{c}\right)\right)-\operatorname{deg}\left(c_{o}\right)\right) \in\left(D_{\mathbb{A}}^{\infty, o}\right)^{\times} \times \mathbb{Z}$ et celle de $\left(g_{o}^{c}, c_{o}\right)$ sur $\Pi_{l} \otimes L_{g}\left(\pi_{o}\right)$. Cet espace, en tant que représentation de $G L_{d}\left(F_{o}\right) \times W_{o}$ est alors de la forme

$$
\bigoplus_{\xi}\left(\operatorname{Ind}_{P_{l g, d}^{\mathrm{op}}\left(F_{o}\right)}^{G F_{\mathrm{o}}\left(F_{o}\right)} \Pi_{l} \circ \xi(\operatorname{val}(\operatorname{det})) \otimes \pi_{\xi}\right) \otimes L_{g}\left(\pi_{o} \circ \xi(\operatorname{val}(\operatorname{det}))\right)
$$

où $\xi$ décrit les caractères $\mathbb{Z} \longrightarrow \overline{\mathbb{Q}}_{l}^{\times}$et $\pi_{\xi}$ est une représentation de $G L_{d-l g}\left(F_{o}\right)$.

Démonstration. - Le faisceau $\mathcal{F}_{\mathrm{JL}^{-1}\left(\mathrm{St}_{l}\left(\pi_{o}\right)\right)}$ étant induit à partir de $\mathcal{F}_{\mathrm{JL}^{-1}\left(\mathrm{St}_{l}\left(\pi_{o}\right)\right), 1}$ on a :

$$
\begin{aligned}
\lim _{\vec{I}} H_{c}^{j}\left(M_{I, s_{o}}^{=l g}, \mathcal{F}_{\mathrm{JL}^{-1}}\left(\operatorname{St}_{l}\left(\pi_{o}\right)\right)\right. & \left.\left.\otimes \mathcal{L}_{\rho_{\infty}} \otimes \Pi_{l}\right) \otimes L_{g}\left(\pi_{o}\right)\right) \simeq \\
\operatorname{Ind}_{P_{l g, d}^{\text {o }}\left(F_{o}\right)}^{G L_{d}\left(F_{o}\right)} & \lim _{\vec{I}} H_{c}^{j}\left(M_{I, s_{o}, 1}^{=l g}, \mathcal{F}_{\mathrm{JL}^{-1}\left(\mathrm{St}_{l}\left(\pi_{o}\right)\right), 1} \otimes \mathcal{L}_{\rho_{\infty}} \otimes \Pi_{l}\right) \otimes L_{g}\left(\pi_{o}\right)
\end{aligned}
$$

tel que l'action de $\left(\left(g_{o}^{c}, g_{o}^{e t}\right), c_{o}\right) \in P_{l g, d}\left(F_{o}\right)^{o p} \times W_{o}$ sur le membre de droite de (I.5.3.3), soit donnée par l'action de $\left(g_{o}^{e t},-\operatorname{val}\left(\operatorname{det} g_{o}^{c}\right)-\operatorname{deg}\left(c_{o}\right)\right) \times\left(g_{o}^{c}, c_{o}\right) \in G L_{d-l g}\left(F_{o}\right) \times \mathbb{Z} \times G L_{l g}\left(F_{o}\right) \times W_{o}$ où $\lim _{\vec{I}} H_{c}^{j}\left(M_{I, s_{o}, 1}^{=l g}, \mathcal{F}_{\mathrm{JL}^{-1}\left(\operatorname{St}_{l}\left(\pi_{o}\right)\right)} \otimes \mathcal{L}_{\rho_{\infty}}\right)\left(\right.$ resp. $\left.\Pi_{l} \otimes L_{g}\left(\pi_{o}\right)\right)$ est vue comme un $G L_{d-l g}\left(F_{o}\right) \times \mathbb{Z}$ module (resp. $G L_{l g}\left(F_{o}\right) \times W_{o}$-module). Le résultat découle alors de l'écriture

$$
\lim _{\vec{I}} H_{c}^{j}\left(M_{I, s_{o}, 1}^{=l g}, \mathcal{F}_{\mathrm{JL}^{-1}\left(\mathrm{St}_{l}\left(\pi_{o}\right)\right)} \otimes \mathcal{L}_{\rho_{\infty}}\right) \simeq \bigoplus_{\xi} \xi \otimes \pi_{\xi}
$$

où $\xi$ décrit les caractères $\mathbb{Z} \longrightarrow \overline{\mathbb{Q}}_{l}^{\times}$et où $\pi_{\xi}$ est une représentation de $G L_{d-l g}\left(F_{o}\right)$, à priori réductible.

\section{I.6. Equations formelles des variétés d'Igusa de seconde espèce}

On propose de donner au niveau des complétés formels des équations explicites du revêtement d'Igusa de seconde espèce, qui fournissent les systèmes locaux $\mathcal{F}_{\tau_{o}}$ associés à une représentation irréductible $\tau_{o}$ de $D_{o, d}^{\times}$.

I.6.1. Lemme. - Soit $z$ un point géométrique de $M_{I^{o}, s_{o}}^{h^{\prime}}$ avec $h^{\prime}>h$. Le revêtement $\mathcal{J}_{I^{o}, 0}^{h} \longrightarrow$ $M_{I^{o}, s_{o}}^{=h}$ donne au dessus du complété formel $\operatorname{Def}_{0}^{h^{\prime} d-h^{\prime} ; h}$ de $M_{I^{o}, s_{o}}^{h}$ en $z$, une extension

$$
\operatorname{Def}_{0}^{h^{\prime}, d-h^{\prime} ;=h} \hookrightarrow J_{0}^{h^{\prime}, d-h^{\prime} ;=h}(s)
$$

qui provient d'une extension $\operatorname{Def}_{0}^{h^{\prime} ;=h} \longrightarrow J_{0}^{h^{\prime} ;=h}(s)$; i.e. $J_{0}^{h^{\prime}, d-h^{\prime} ;=h}(s)$ est isomorphe à

$$
J_{0}^{h^{\prime} ;=h}(s) \hat{\otimes}_{\operatorname{Def}_{0}^{h^{\prime} ;=h}} \operatorname{Def}_{0}^{h^{\prime}, d-h^{\prime} ;=h}
$$

où l'on rappelle que $\operatorname{Def}_{0}^{h^{\prime}, d-h^{\prime} ;=h}=\operatorname{Def}_{0}^{h^{\prime} ;=h}\left[\left[w_{1}^{0}, \cdots, w_{d-h^{\prime}}^{0}\right]\right]$.

Démonstration. - Par définition des variétés d'Igusa de seconde espèce (cf. le paragraphe (I.4.1)), l'extension $\operatorname{Def}_{0}^{h^{\prime}, d-h^{\prime} ;=h} \hookrightarrow J_{0}^{h^{\prime}, d-h^{\prime} ;=h}(s)$ est donnée par la rigidification à l'ordre $s$ de la partie

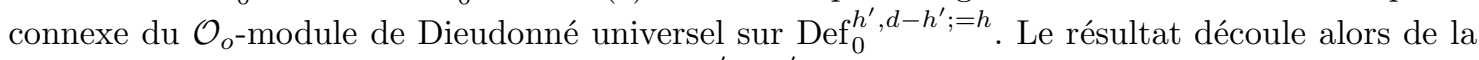
forme de ce $\mathcal{O}_{o}$-module de Dieudonné sur $\operatorname{Def}_{0}^{h^{\prime}, d-h^{\prime} ;=h}$ donnée à la proposition (9.9.1) de [5]. 
I.6.0.11 - Soit alors $z$ un point géométrique de $M_{I^{o}, s_{o}}^{=h^{\prime}}$ avec $h^{\prime}>h$. Le complété de l'anneau local de $M_{I^{o}, s_{o}}^{h}$ en $z$ est $\operatorname{Def}_{0}^{h^{\prime}, d-h^{\prime} ; h} \simeq \operatorname{Def}_{0}^{h^{\prime} ; h}\left[\left[w_{1}, \cdots, w_{d-h^{\prime}}\right]\right]$ avec $\operatorname{Def}_{0}^{h^{\prime} ; h} \simeq \hat{\mathcal{O}}_{o}^{n r}\left[\left[a_{h}, \cdots, a_{h^{\prime}-1}\right]\right]$. L'ouvert correspondant à $M_{\bar{I}^{o}, 0}^{=h}$ est

$$
\operatorname{Def}_{0}^{h^{\prime}, d-h^{\prime} ;=h} \simeq \operatorname{Def}_{0}^{h^{\prime} ;=h}\left[\left[w_{1}, \cdots, w_{d-h^{\prime}}\right]\right]
$$

$\operatorname{avecDef}_{0}^{h^{\prime} ;=h} \simeq \hat{\mathcal{O}}_{o}^{n r}\left(\left(a_{h}\right)\right)\left[\left[a_{h+1}, \cdots, a_{h^{\prime}-1}\right]\right]$. D'après le lemme précédent, l'extension $\operatorname{Def}_{0}^{h^{\prime} ;=h} \hookrightarrow$ $J_{0}^{h^{\prime} ;=h}(s)$ est donnée par l'équation

$$
\tau^{h} \circ \alpha=\alpha \circ\left(a_{h} \tau^{h}+\cdots+a_{h^{\prime}-1} \tau^{h^{\prime}-1}+\tau^{h^{\prime}}\right) \quad \text { modulo } \tau^{h+m}
$$

avec $\alpha=\sum_{i=0}^{m-1} \alpha_{i} \tau^{i}$ avec $\alpha_{0}$ non nul. On obtient alors le système suivant

$$
\left\{\begin{array}{l}
\alpha_{0}^{p^{h}-1}=a_{h} \\
\alpha_{1}^{p^{h}}=\alpha_{0} a_{h+1}+\alpha_{1} a_{h}^{p} \\
\cdots \\
\alpha_{h^{\prime}-h-1}^{p^{h}}=\alpha_{0} a_{h^{\prime}-1}+\alpha_{1} a_{h^{\prime}-2}^{p}+\cdots+\alpha_{h^{\prime}-h-1} a_{h}^{p^{h^{\prime}-h-1}} \\
\alpha_{h^{\prime}-h}^{p^{h}}=\alpha_{0}+\alpha_{1} a_{h^{\prime}-1}^{p}+\cdots+\alpha_{h^{\prime}-h} a_{h}^{p^{h^{\prime}-h}} \\
\cdots \\
\alpha_{m}^{p^{h}}=\alpha_{m-h^{\prime}+h}+\alpha_{m-h^{\prime}+h+1} a_{h^{\prime}-1}^{p^{m-h^{\prime}+h}}+\cdots+\alpha_{m} a_{h}^{p^{m-1}}
\end{array}\right.
$$

On peut effectuer le changement de variable $t_{i}=\alpha_{i} t_{0}^{-p^{i}}$ pour $i>0$ avec $t_{0}=\alpha_{0}$. Le système précédent s'écrit alors

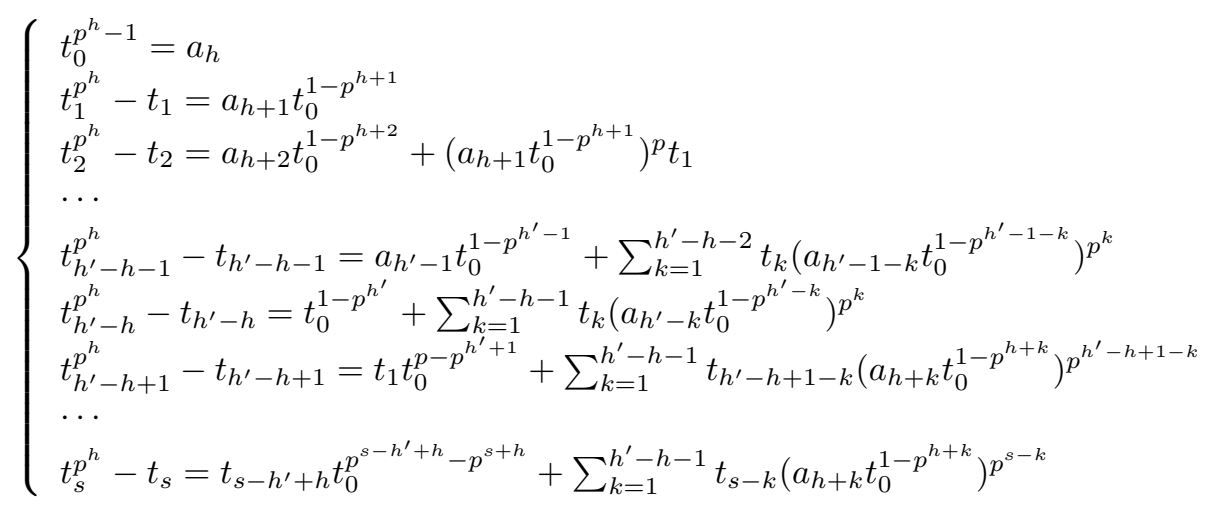

Pour $i \leqslant h^{\prime}-h$ (resp. $i \geqslant h^{\prime}-h$ ), on peut éliminer dans le membre de droite de l'équation dont le membre de gauche est $t_{i}^{p^{h}}-t_{i}$, les termes $t_{0}^{1-p^{h+k}} a_{h+k}$ pour $k<i$ (resp. $1 \leqslant k \leqslant h^{\prime}-h-1$ et $t_{0}^{1-p^{h^{\prime}}}$ ); on obtient alors le système suivant :

$$
\left\{\begin{array}{l}
t_{0}^{p^{h}-1}=a_{h} \\
t_{1}^{p^{h}}-t_{1}=a_{h+1} t_{0}^{1-p^{h+1}} \\
t_{2}^{p^{h}}-t_{2}=a_{h+2} t_{0}^{1-p^{h+2}}+r_{2} \\
\cdots \\
t_{h^{\prime}-h-1}^{p^{h}}-t_{h^{\prime}-h-1}=a_{h^{\prime}-1} t_{0}^{1-p^{h^{\prime}-1}}+r_{h^{\prime}-h-1} \\
t_{h^{\prime}-h}^{p^{h}}-t_{h^{\prime}-h}=t_{0}^{1-p^{h^{\prime}}}+r_{h^{\prime}-h} \\
t_{h^{\prime}-h+1}^{p^{h}}-t_{h^{\prime}-h+1}=r_{h^{\prime}-h+1} \\
\cdots \\
t_{s}^{p^{h}}-t_{s}=r_{s}
\end{array}\right.
$$

avec $r_{i} \in \mathbb{F}_{q}\left[\left[t_{1}, \cdots, t_{i-1}\right]\right]$ pour $1 \leqslant i \leqslant s$ définis par les relations suivantes :

$-r_{1}=0$ et pour $2 \leqslant i \leqslant h^{\prime}-h, r_{i}=\sum_{k=1}^{i-1} t_{i-k}\left(t_{k}^{p^{h}}-t_{k}-r_{k}\right)^{p^{i-k}}$ 
- pour $i>h^{\prime}-h, r_{i}=\sum_{k=1}^{h^{\prime}-h} t_{i-k}\left(t_{k}^{p^{h}}-t_{k}-r_{k}\right)^{p^{i-k}}$. 



\section{CHAPITRE II}

\section{GROUPES DE COHOMOLOGIE DU MODÈLE LOCAL : CAS IWAHORI}

\section{Introduction}

0.1. - On commence par des rappels sur les induites paraboliques d'après [24]. Les paraboliques que l'on considère sont les paraboliques opposés aux paraboliques standards. On s'intéresse tout particulièrement aux représentations elliptiques, d'après une terminologie de Dat, i.e. aux sousquotients irréductibles de l'induite normalisée du parabolique $P_{g, 2 g, \cdots, s g}^{\mathrm{op}}\left(F_{o}\right)$ à $G L_{s g}\left(F_{o}\right)$,

$$
\pi_{o}\left(\frac{s-1}{2}\right) \times \cdots \times \pi_{o}\left(\frac{1-s}{2}\right)
$$

où $\pi_{o}$ est une représentation irréductible cuspidale de $G L_{g}\left(F_{o}\right)$, celles-ci seront dites de type $\pi_{o}$. Ainsi on notera $[\overleftarrow{s-1}]_{\pi_{o}}$ (resp. $[\overrightarrow{s-1}]_{\pi_{o}}$ ) l'unique quotient (resp. sous-espace) irréductible de cette induite, noté plus habituellement $\operatorname{St}_{s}\left(\pi_{o}\right)$ (resp. $\left.\operatorname{Speh}_{s}\left(\pi_{o}\right)\right)$. On rappelle que d'après [24], ces sous-quotients irréductibles sont paramétrés par les orientations $\vec{\Gamma}^{s}$ du graphe linéaire complet à $s$ sommets

$$
\Gamma^{s}: \circ^{\frac{s-1}{2}}-\circ \cdots-0^{\frac{1-s}{2}}
$$

Étant données deux représentations $\pi_{1}$ et $\pi_{2}$, elliptiques de type $\pi_{o}$, de respectivement $G L_{s_{1} g}\left(F_{o}\right)$ et $G L_{s_{2} g}\left(F_{o}\right)$, les induites normalisées du parabolique $P_{s_{1} g,\left(s_{1}+s_{2}\right) g}^{\text {op }}\left(F_{o}\right)$ à $G L_{\left(s_{1}+s_{2}\right) g}\left(F_{o}\right)$,

$$
\pi_{1}\left(-\frac{s_{2}(g-1)}{2}\right) \times \pi_{2}\left(\frac{s_{1}(g-1)}{2}\right) \text { et } \pi_{1}\left(-\frac{s_{2}(g+1)}{2}\right) \times \pi_{2}\left(\frac{s_{1}(g+1)}{2}\right)
$$

sont de longueur 2, chacun des constituant étant une représentation elliptique de type $\pi_{o}$, dont l'orientation de $\Gamma^{s_{1}+s_{2}}$ est une des deux qui prolonge celle du graphe partiellement orienté obtenu par la concaténation du graphe de $\pi_{1}$ avec celui de $\pi_{2}$ (resp. du graphe de $\pi_{2}$ avec celui de $\pi_{1}$ )

$$
\begin{gathered}
\vec{\Gamma}^{s}: \overbrace{\longleftarrow \leftarrow \circ \rightarrow \circ}^{\vec{\Gamma}^{s_{1}}} \longleftrightarrow \overbrace{\circ \rightarrow 0 \cdots \circ}^{\vec{\Gamma}^{s_{2}}} \\
\text { (resp. } \vec{\Gamma}^{s}: \overbrace{\circ \longleftarrow 0 \cdots \rightarrow \circ}^{\vec{\Gamma}^{s_{2}}} \longleftrightarrow \overbrace{\circ \rightarrow 0 \cdots \circ}^{\vec{\Gamma}^{s_{1}}})
\end{gathered}
$$

On notera alors ces induites sous la forme $\pi_{1} \overrightarrow{\times} \pi_{2}$ (resp. $\pi_{1} \overleftarrow{\times} \pi_{2}$ ). Ces notations sont compatibles au calcul des foncteurs de Jacquet pour les paraboliques opposés au paraboliques standards tandis qu'elles sont inversement orientées pour les foncteurs de Jacquet pour les paraboliques standards, cf. $§ I I .1 .2$.

0.2. - On donne ensuite une preuve, théorème (II.2.5), de (IV.3.1.1) pour les représentations qui ont des vecteurs invariants sous le sous-groupe d'Iwahori standard $\mathrm{Iw}_{o}$, i.e. les représentations elliptiques de type $1_{o}$. Par un argument d'accouplement cohomologique, proposition (II.2.2), on obtient le résultat pour les représentations elliptiques de type $\xi_{o}$, où $\xi_{o}$ est un caractère de $\left(\overline{\mathbb{Q}_{l}}\right)^{\times}$. 
Pour prouver le cas Iwahori, on utilise que $\operatorname{Def}_{s t}^{d}:=\left(\operatorname{Def}_{n}^{d}\right)^{\mathrm{Iw}_{o} / K_{o, n}}$ muni de son morphisme structurel, est semi-stable (cf. la proposition (II.2.3)) et possède une interprétation modulaire très simple en termes de drapeaux (cf. (II.2.4)). On dispose alors d'une description explicite du complexe des cycles évanescents associé, rappelée au théorème (II.2.6), et on en déduit en particulier au corollaire (II.2.7) que pour toute représentation irréductible $\tau_{o}$ de $D_{o, d}^{\times},\left(\Psi_{F_{o}}^{d, i}\left(\tau_{o}\right)\right)^{\mathrm{Iw}}$ est un $\overline{\mathbb{Q}}_{l}$-espace vectoriel de dimension le coefficient binomial $\left(\begin{array}{c}i \\ d-1\end{array}\right)$, qui est pur de poids $2 i$. Le reste de la preuve est alors purement combinatoire à partir de la proposition (II.2.9).

\section{II.1. Rappels sur les représentations de $G L_{d}\left(F_{o}\right)$}

Tous les énoncés ainsi que leur preuve peuvent être trouvés dans [24].

\section{II.1.1. Induites paraboliques. -}

II.1.1.1. Définition. - Pour une suite $0<r_{1}<r_{2}<\cdots<r_{k}=d$, on note $P_{r_{1}, r_{2}, \cdots, r_{k}}$ le sousgroupe parabolique de $G L_{d}$ standard associé au sous-groupe de Levi $G L_{r_{1}}\left(F_{o}\right) \times G L_{r_{2}-r_{1}}\left(F_{o}\right) \times \cdots \times$ $G L_{r_{k}-r_{k-1}}\left(F_{o}\right)$ et soit $P_{r_{1}, \cdots, r_{k}}^{\mathrm{op}}$ le parabolique opposé dont on note $N_{r_{1}, \cdots, r_{k}}^{\mathrm{op}}$ le radical unipotent.

II.1.1.2 - Soient $\pi_{1}$ et $\pi_{2}$ des représentations de respectivement $G L_{n_{1}}\left(F_{o}\right)$ et $G L_{n_{2}}\left(F_{o}\right)$; on note selon la coutume, $\pi_{1} \times \pi_{2}$ l'induite parabolique

$$
\pi_{1} \times \pi_{2}:=\operatorname{Ind}_{P_{n_{1}, n_{1}+n_{2}}^{\text {op }}}^{G L_{n_{1}+n_{2}}\left(F_{o}\right)} \pi_{1}\left(-n_{2} / 2\right) \otimes \pi_{2}\left(n_{1} / 2\right)
$$

Remarque : Le symbole $\times$ est associatif, i.e. $\pi_{1} \times\left(\pi_{2} \times \pi_{3}\right)=\left(\pi_{1} \times \pi_{2}\right) \times \pi_{3}$ que l'on notera donc $\pi_{1} \times \pi_{2} \times \pi_{3}$.

II.1.1.3. Définitions. - Soit $g$ un diviseur de $d=s g$ et $\pi_{o}$ une représentation cuspidale irréductible de $G L_{g}\left(F_{o}\right)$ :

- les sous-quotients irréductibles de

$$
V\left(\pi_{o}, s\right):=\pi_{o}\left(\frac{s-1}{2}\right) \times \pi_{o}\left(\frac{s-3}{2}\right) \times \cdots \times \pi_{o}\left(\frac{1-s}{2}\right)
$$

seront dits elliptiques de type $\pi_{o}$;

- $V\left(\pi_{o}, s\right)$ possède un unique quotient (resp. sous-espace) irréductible que l'on notera

$$
[\overleftarrow{s-1}]_{\pi_{o}} \quad\left(\text { resp. }[\overrightarrow{s-1}]_{\pi_{o}}\right)
$$

c'est une représentation de Steinberg (resp. de Speh) généralisée notée habituellement $\operatorname{St}_{s}\left(\pi_{o}\right)$ (resp. $\operatorname{Speh}_{s}\left(\pi_{o}\right)$;

- pour $\pi_{1}$ et $\pi_{2}$ des représentations respectivement de $G L_{l_{1} g}\left(F_{o}\right)$ et $G L_{l_{2} g}\left(F_{o}\right)$, on notera

$$
\pi_{1} \overrightarrow{\times} \pi_{2} \quad\left(\operatorname{resp} . \pi_{1} \overleftarrow{\times} \pi_{2}\right)
$$

l'induite parabolique $\pi_{1}\left(l_{2} / 2\right) \times \pi_{2}\left(-l_{1} / 2\right)$ (resp. $\left.\pi_{1}\left(-l_{2} / 2\right) \times \pi_{2}\left(l_{1} / 2\right)\right)$.

La propriété habituelle de transitivité des induites paraboliques donne le lemme suivant.

II.1.1.4. Lemme. - Pour $\left(\pi_{i}\right)_{1 \leqslant i \leqslant 3}$ des représentations de $G L_{l_{i} g}\left(F_{o}\right)$, on a les égalités suivantes:

$$
\begin{aligned}
& \left(\pi_{1} \overrightarrow{\times} \pi_{2}\right)^{\vee} \simeq \pi_{1}^{\vee} \overleftarrow{\times} \pi_{2}^{\vee} \\
& \left(\pi_{1} \overrightarrow{\times} \pi_{2}\right) \overrightarrow{\times_{g}} \pi_{3}=\pi_{1} \overrightarrow{\times}\left(\pi_{2} \overrightarrow{\times} \pi_{3}\right) \\
& \left(\pi_{1} \overleftarrow{\times} \pi_{2}\right) \overleftarrow{\times} g \pi_{3}=\pi_{1} \overleftarrow{\times}\left(\pi_{2} \overleftarrow{\times} \pi_{3}\right)
\end{aligned}
$$

En outre si $\pi_{1}$ et $\pi_{2}$ sont elliptiques de type $\pi_{o}$, il en est de même de $\pi_{1} \overrightarrow{\times} \pi_{2}$ et donc de $\pi_{1} \overleftarrow{\times} \pi_{2}$. 
Remarque : Pour $\pi_{1}, \pi_{2}$ et $\pi_{3}$ des représentations elliptiques de type $\pi_{o}$, on a aussi

$$
\pi_{1} \overrightarrow{\times}\left(\pi_{2} \overleftarrow{\times} \pi_{3}\right)=\pi_{2} \overleftarrow{\times}\left(\pi_{1} \overrightarrow{\times} \pi_{3}\right)
$$

En effet d'après [24], on a $\pi_{1} \times \pi_{2} \simeq \pi_{2} \times \pi_{1}$ si les supports cuspidaux de $\pi_{1}$ et $\pi_{2}$ sont disjoints (1).

II.1.1.5. Proposition-Définition. - Pour $g$ un diviseur de $d=s g$ et $\pi_{o}$ une représentation irréductible cuspidale de $G L_{g}\left(F_{o}\right)$, on a pour $1 \leqslant l \leqslant s$ :

- $[\overleftarrow{l-1}]_{\pi_{o}} \overrightarrow{\times}[\overrightarrow{s-l-1}]_{\pi_{o}}$ (resp. $[\overrightarrow{l-1}]_{\pi_{o}} \overrightarrow{\times}[\overleftrightarrow{s-l-1}]_{\pi_{o}}$ ) est de longueur 2 ; on notera

$$
[\overleftarrow{l-1}, \overrightarrow{s-l}]_{\pi_{o}} \quad\left(\text { resp. }[\vec{l}, \overleftarrow{s-l-1}]_{\pi_{o}}\right)
$$

son unique sous-espace irréductible, et

$$
[\overleftarrow{l}, \overrightarrow{s-l-1}]_{\pi_{o}} \quad\left(\text { resp. }[\overrightarrow{l-1}, \overleftarrow{s-l}]_{\pi_{o}}\right)
$$

son unique quotient irréductible;

- par dualité $[\overleftarrow{l-1}]_{\pi_{o}} \overleftarrow{\times}[\overrightarrow{s-l-1}]_{\pi_{o}}\left(\right.$ resp. $[\overrightarrow{l-1}]_{\pi_{o}} \overleftarrow{\times}[\overleftarrow{s-l-1}]_{\pi_{o}}$ ) est de longueur 2 avec

$$
[\overrightarrow{s-l-1}, \overleftarrow{l}]_{\pi_{o}} \quad\left(\text { resp. }[\overleftarrow{s-l}, \overrightarrow{l-1}]_{\pi_{o}}\right)
$$

pour unique sous-espace irréductible et

$$
[\overrightarrow{s-l}, \overleftarrow{l-1}]_{\pi_{o}} \quad\left(\text { resp. }[\overleftarrow{s-l-1}, \vec{l}]_{\pi_{o}}\right)
$$

pour unique quotient irréductible;

- on notera $\lfloor\pi\rfloor$ (resp. $\lceil\pi\rceil$ ) l'unique, s'il existe, sous-espace (resp. quotient) irréductible de $\pi$. Pour $\pi_{1}$ et $\pi_{2}$ des représentations irréductibles elliptiques de type $\pi_{o}, \pi_{1} \overrightarrow{\times} \pi_{2}$ et $\pi_{1} \overleftarrow{\times} \pi_{2}$ ont un unique sous-espace et un unique quotient irréductible et on a :

$$
\begin{aligned}
\left\lfloor\pi_{1} \overrightarrow{\times} \pi_{2}\right\rfloor & =\left\lceil\pi_{2}^{\vee} \overleftarrow{\times} \pi_{1}^{\vee}\right\rceil^{\vee} \\
\left\lceil\pi_{1} \overrightarrow{\times} \pi_{2}\right\rceil & =\left\lfloor\pi_{2}^{\vee} \overleftarrow{\times} \pi_{1}^{\vee}\right\rfloor^{\vee}
\end{aligned}
$$

Pour $\pi_{3}$ une troisième représentation irréductible elliptique de type $\pi_{o}$, on a

$$
\begin{aligned}
& \left\lfloor\left\lfloor\pi_{1} \overrightarrow{\times} \pi_{2}\right\rfloor \overrightarrow{\times} \pi_{3}\right\rfloor=\left\lfloor\pi_{1} \overrightarrow{\times}\left\lfloor\pi_{2} \overrightarrow{\times} \pi_{3}\right\rfloor\right\rfloor=\left\lfloor\pi_{1} \overrightarrow{\times} \pi_{2} \overrightarrow{\times} \pi_{3}\right\rfloor \\
& \left\lceil\left\lceil\pi_{1} \overrightarrow{\times} \pi_{2}\right\rceil \overrightarrow{\times} \pi_{3}\right\rceil=\left\lceil\pi_{1} \overrightarrow{\times}\left\lceil\pi_{2} \overrightarrow{\times} \pi_{3}\right\rceil\right\rceil=\left\lceil\pi_{1} \overrightarrow{\times} \pi_{2} \overrightarrow{\times} \pi_{3}\right\rceil
\end{aligned}
$$

- Soient $r \geqslant 1$ et $\Gamma^{s}=\left(a_{i}, \epsilon_{i}\right)_{1 \leqslant i \leqslant r}$ tel que les $a_{i}$ sont des entiers strictement positifs avec $s-1=a_{1}+\cdots+a_{r}$ et $\epsilon_{i}= \pm 1$; on notera $\Gamma^{s}$ sous la forme $\left(\overleftarrow{a_{1}}, \cdots, \overrightarrow{a_{r}}\right)$ où pour tout $i$ la flèche au dessus de $a_{i}$ est $\overleftarrow{a_{i}}$ (resp. $\left.\overrightarrow{a_{i}}\right)$ si $\epsilon_{i}=-1$ (resp. $\left.\epsilon_{i}=1\right)$. On associe à $\Gamma^{s}$ un sous-quotient irréductible $\left[\Gamma^{s}\right]$ de $V\left(s, \pi_{o}\right)$ que l'on note aussi sous la forme $\left[\overleftarrow{a_{1}}, \cdots, \overrightarrow{a_{r}}\right]_{\pi_{o}}$. On convient par ailleurs des égalités suivantes :

$$
[\cdots, \overleftarrow{a}, \overleftarrow{b}, \cdots]_{\pi_{o}}=[\cdots, \overleftarrow{a+b}, \cdots]_{\pi_{o}} \quad[\cdots, \vec{a}, \vec{b}, \cdots]_{\pi_{o}}=[\cdots, \overrightarrow{a+b}, \cdots]_{\pi_{o}}
$$

D'après [24], on obtient alors une bijection, modulo les identifications ci-dessus, entre les sous-quotients irréductibles de $V\left(s, \pi_{o}\right)$ et l'ensemble des $\left[\Gamma^{s}\right]$ telle que l'on ait les relations suivantes :

$$
\begin{aligned}
& \left\lfloor[\cdots, \overleftrightarrow{a}]_{\pi_{o}} \overrightarrow{\times}[\overleftrightarrow{b}, \cdots]_{\pi_{o}}\right\rfloor=[\cdots, \overleftrightarrow{a}, \overrightarrow{1}, \overleftrightarrow{b}, \cdots]_{\pi_{o}} \\
& \left\lceil[\cdots, \overleftrightarrow{a}]_{\pi_{o}} \overrightarrow{\times}[\overleftrightarrow{b}, \cdots]_{\pi_{o}}\right\rceil=[\cdots, \overleftrightarrow{a}, \overleftarrow{1}, \overleftrightarrow{b}, \cdots]_{\pi_{o}} \\
& \left\lfloor[\cdots, \overleftrightarrow{a}]_{\pi_{o}} \overleftarrow{\times}[\overleftrightarrow{b}, \cdots]_{\pi_{o}}\right\rfloor=[\cdots, \overleftrightarrow{b}, \overleftarrow{1}, \overleftrightarrow{a}, \cdots]_{\pi_{o}} \\
& \left\lceil[\cdots, \overleftrightarrow{a}]_{\pi_{o}} \overleftarrow{\times}[\overleftrightarrow{b}, \cdots]_{\pi_{o}}\right\rceil=[\cdots, \overleftrightarrow{b}, \overrightarrow{1}, \overleftrightarrow{a}, \cdots]_{\pi_{o}}
\end{aligned}
$$

où $\overleftrightarrow{c}$ désigne arbitrairement $\overleftarrow{c}$ ou $\vec{c}$

\footnotetext{
(1) En fait loc. cit. donne un critère plus précis; dans le cas où $\pi_{1}$ et $\pi_{2}$ sont irréductibles, il faut et il suffit que leurs supports cuspidaux ne soient pas liés.
} 
II.1.1.6. Remarque. - Dans $[\mathbf{2 4}],\left(\overleftarrow{a_{1}}, \cdots, \overrightarrow{a_{r}}\right)$ est représenté sous la forme

$$
\circ^{\frac{s-1}{2}} \longleftarrow \circ \cdots \circ \longleftarrow \cdots \circ \rightarrow \circ^{\frac{1-s}{2}}
$$

où les $a_{1}$ premières flèches vont dans le sens de la flèche au dessus de $a_{1}$, les $a_{2}$ suivantes dans le sens de la flèche au dessus de $a_{2} \ldots$ Ainsi graphiquement on a

$$
\left[\Gamma_{1}^{s_{1}}\right] \overrightarrow{\times}\left[\Gamma_{2}^{s_{2}}\right]: \overbrace{\longleftarrow \leftarrow \cdots \rightarrow \circ}^{\Gamma_{1}^{s_{1}}} \longleftrightarrow \overbrace{0 \rightarrow 0 \cdots 0}^{\Gamma_{2}^{s_{2}}}
$$

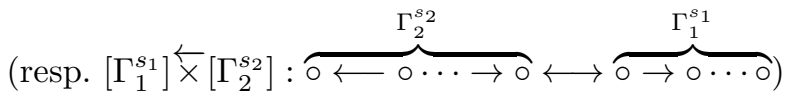

Exemples : la représentation cuspidale irréductible $\pi_{o}$ de $G L_{g}\left(F_{o}\right)$ étant fixé, avec nos notations, on a :

$$
\begin{aligned}
& {[\overleftarrow{s-1}]_{\pi_{o}}=[\overbrace{[\overrightarrow{0}]_{\pi_{o}} \overleftarrow{x} \ldots \overleftarrow{\times}[\overrightarrow{0}]_{\pi_{o}}}^{s}\rfloor=[\overbrace{\left.[\overleftarrow{0}]_{\pi_{o}} \vec{x} \ldots \overrightarrow{\times}[\overleftarrow{0}]_{\pi_{o}}\right\rceil}^{s}} \\
& {[\overrightarrow{s-1}]_{\pi_{o}}=[\overbrace{[\overleftarrow{0}]_{\pi_{o}} \vec{x} \ldots \vec{x}[\overleftarrow{0}]_{\pi_{o}}}^{s}=[\overbrace{[\overrightarrow{0}]_{\pi_{o}} \overleftarrow{x} \ldots \overleftarrow{\times}[\overrightarrow{0}]_{\pi_{o}}}^{s}} \\
& {[\overleftarrow{l-1}, \overrightarrow{s-l}]_{\pi_{o}}=\left[[\overleftarrow{l-1}]_{\pi_{o}} \overrightarrow{\times}[\overrightarrow{s-l-1}]_{\pi_{o}}\right\rfloor=\left\lceil[\overleftarrow{l-2}]_{\pi_{o}} \overrightarrow{\times}[\overrightarrow{s-l}]_{\pi_{o}}\right]} \\
& =\left\lceil[\overrightarrow{s-l-1}]_{\pi_{o}} \overleftarrow{\times}[\overleftarrow{l-1}]_{\pi_{o}}\right\rceil=\left\lfloor[\overrightarrow{s-l}]_{\pi_{o}} \overleftarrow{\times}[\overleftarrow{l-2}]_{\pi_{o}}\right\rfloor
\end{aligned}
$$

Notation : Dans la suite $[\overleftrightarrow{s-1}]_{\pi_{o}}$ désignera une représentation elliptique quelconque de type $\pi_{o}$ de $G L_{s g}\left(F_{o}\right)$.

II.1.2. Foncteur de Jacquet. - Soit $P=M N$ un parabolique de $G L_{d}$ de Lévi $M$ et de radical unipotent $N$.

II.1.2.1. Définition. - Pour $\pi$ une représentation admissible de $G L_{d}\left(F_{o}\right)$, l'espace des vecteurs $N\left(F_{o}\right)$-coinvariants est stable sous l'action de $M\left(F_{o}\right) \simeq P\left(F_{o}\right) / N\left(F_{o}\right)$. On notera $J_{N}(\pi)$ cette représentation tordue par $\delta_{P}^{-1 / 2}$.

II.1.2.2. Lemme. - Soit g un diviseur de $d=s g$ et $\pi_{o}$ une représentation irréductible cuspidale de $G L_{g}\left(F_{o}\right)$. Pour $1 \leqslant h \leqslant d$, le foncteur de Jacquet vérifie les propriétés suivantes :

- sig ne divise pas $h$, alors

$$
J_{N_{h, d}^{o p}}\left([\overleftarrow{s-1}]_{\pi_{o}}\right)=J_{N_{h, d}^{o p}}\left([\overrightarrow{s-1}]_{\pi_{o}}\right)=J_{N_{h, d}}\left([\overleftarrow{s-1}]_{\pi_{o}}\right)=J_{N_{h, d}}\left([\overrightarrow{s-1}]_{\pi_{o}}\right)=(0)
$$

- si $h=\lg$ alors

$$
\begin{gathered}
J_{N_{l g, s g}}\left([\overleftarrow{s-1}]_{\pi_{o}}\right)=[\overleftarrow{l-1}]_{\pi_{o}((s-l) / 2)} \otimes[\overleftarrow{s-l-1}]_{\pi_{o}(-l / 2)} \\
J_{N_{l g, s g}}\left([\overrightarrow{s-1}]_{\pi_{o}}\right)=[\overrightarrow{l-1}]_{\pi_{o}((l-s) / 2)} \otimes[\overrightarrow{s-l-1}]_{\pi_{o}(l / 2)} \\
J_{N_{l g, s g}^{o p}}\left([\overleftarrow{s-1}]_{\pi_{o}}\right)=[\overleftarrow{l-1}]_{\pi_{o}((l-s) / 2)} \otimes[\overleftarrow{s-l-1}]_{\pi_{o}(l / 2)} \\
J_{N_{l g, s g}^{o p}}\left([\overrightarrow{s-1}]_{\pi_{o}}\right)=[\overrightarrow{l-1}]_{\pi_{o}((s-l) / 2)} \otimes[\overrightarrow{s-l-1}]_{\pi_{o}(-l / 2)}
\end{gathered}
$$

Démonstration. - soit $\Gamma=\left(a_{i}, \epsilon_{i}\right)_{1 \leqslant i \leqslant r}$, les résultats découlent alors des propriétés suivantes que l'on trouve dans [24]:

- $J_{N_{g, 2 g, \ldots, s g}^{o p}}\left(\left[\Gamma^{s}\right]\right)$ est de la forme $\pi_{o}\left(\frac{1-s}{2}+\sigma(0)\right) \otimes \cdots \pi_{o}\left(\frac{1-s}{2}+\sigma(s-1)\right)$ où $\sigma$ est une permutation de l'ensemble $\{0, \cdots, s-1\}$ soumise à la règle suivante : soit $1 \leqslant i \leqslant r$ avec $\epsilon_{i}=1\left(\right.$ resp. $\left.\epsilon_{i}=-1\right)$ : pour tout $a_{1}+\cdots+a_{i-1} \leqslant r<r^{\prime} \leqslant a_{1}+\cdots+a_{i}$ alors $\sigma^{-1}(r)<\sigma^{-1}\left(r^{\prime}\right)\left(\right.$ resp. $\left.\sigma^{-1}(r)>\sigma^{-1}\left(r^{\prime}\right)\right)$. (2)

${ }^{(2)}$ Autrement dit $\sigma$ est compatible aux orientations des flèches. 
- en ce qui concerne $J_{N_{g, 2 g, \ldots, s g}}\left(\left[\Gamma^{s}\right]\right)$ la règle est inversée, i.e. $\sigma^{-1}(r)>\sigma^{-1}\left(r^{\prime}\right)\left(\right.$ resp. $\sigma^{-1}(r)<$ $\left.\sigma^{-1}\left(r^{\prime}\right)\right)$.

\section{II.2. Preuve du cas Iwahori}

On rappelle le lemme suivant :

II.2.1. Lemme. - Pour tout caractère $\psi: \mathbb{Z} \longrightarrow \overline{\mathbb{Q}}_{l}^{\times}$, on a un isomorphisme

$$
\widetilde{\mathcal{U}_{F_{o}}^{d, i}}\left(\tau_{o} \otimes(\psi \circ \text { val } \circ \text { det })\right) \simeq \widetilde{\mathcal{U}_{F_{o}}^{d, i}}\left(\tau_{o}\right) \otimes\left(\psi \circ d_{d}\right)
$$

où $d_{d}:(g, \sigma) \in G L_{d}\left(F_{o}\right) \times W_{F_{o}} \longrightarrow-\operatorname{val}(\operatorname{det} g)-\operatorname{deg}(\sigma) \in \mathbb{Z}$.

Le but est alors d'étendre le lemme précédent en la proposition suivante :

II.2.2. Proposition. - Pour tout caractère $\eta_{o} d e F_{o}^{\times}$, on a

$$
\widetilde{\mathcal{U}_{F_{o}}^{d, i}}\left(\tau_{o} \otimes \eta_{o}\right) \simeq \widetilde{\mathcal{U}_{F_{o}}^{d, i}}\left(\tau_{o}\right) \otimes\left(\eta_{o} \otimes \eta_{o} \circ \mathrm{cl}^{-1}\right)
$$

en tant que $\left(G L_{d}\left(F_{o}\right) \otimes W_{o}\right)$-module.

Démonstration. - L'isomorphisme de l'énoncé découle d'un accouplement cohomologique que l'on va expliciter dans les lignes qui suivent. On considère la catégorie des faisceaux en $\overline{\mathbb{Q}}_{l}$-modules sur les espaces considérés. Soit $\mathfrak{X}_{n}$ l'espace rigide analytique associé au schéma formel $\operatorname{Spf}_{\operatorname{Def}}^{d}$ sur $\hat{\mathcal{O}}_{o}^{n r}$ muni de la topologie étale de Berkovich (cf. [3]); on note en particulier $\mathfrak{X}_{s}$ (resp. $\mathfrak{X}_{\eta}$ ) sa fibre spéciale (resp. générique). Dans loc. cit., l'auteur construit le foncteur des cycles évanescents $\Psi_{\eta}$ tel que pour tout faisceau $\mathcal{F}$ sur $\mathfrak{X}_{n}, R^{q} \Psi_{\eta}(\mathcal{F})$ est le faisceau associé au préfaisceau qui à une extension étale $\mathfrak{N}_{s} \rightarrow \mathfrak{X}_{s}$ associe le $\overline{\mathbb{Q}}_{l}$-espace vectoriel de dimension finie $H^{q}\left(\mathfrak{N}_{\eta}, \mathcal{F}\right)$.

Ainsi pour tous faisceaux $\mathcal{F}_{1}$ et $\mathcal{F}_{2}$, on a une flèche :

$$
T^{0}: R^{0} \Psi_{\eta}\left(\mathcal{F}_{1}\right) \otimes_{\overline{\mathbb{Q}}_{l}} R^{0} \Psi_{\eta}\left(\mathcal{F}_{2}\right) \longrightarrow R^{0} \Psi_{\eta}\left(\mathcal{F}_{1} \otimes_{\overline{\mathbb{Q}}_{l}} \mathcal{F}_{2}\right)
$$

En outre étant donné un élément de $\mathcal{N}_{o}$, la correspondance $\left(c_{1}, c_{2}\right)$ associée fournit

$$
c_{1}^{*} R^{0} \Psi_{\eta}(\mathcal{F}) \longrightarrow R^{0} \Psi_{\eta}\left(c_{1}^{*} \mathcal{F}\right) \longrightarrow R^{0} \Psi_{\eta}\left(c_{2}^{!} \mathcal{F}\right) \longrightarrow c_{2}^{!} R^{0} \Psi_{\eta}(\mathcal{F})
$$

compatible avec $T^{0}$, c'est à dire que le diagramme suivant est commutatif :

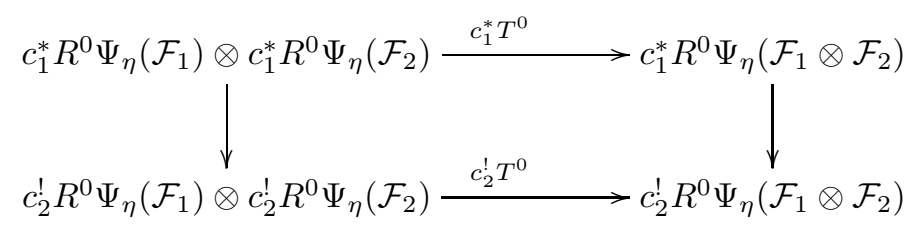

D'après, par exemple, [7] théorème II 6.2 , on en déduit pour tout $i \geqslant 0$, des flèches

$$
T^{i}: R^{0} \Psi_{\eta}\left(\overline{\mathbb{Q}}_{l}\right) \otimes_{\overline{\mathbb{Q}}_{l}} R^{i} \Psi_{\eta}\left(\overline{\mathbb{Q}}_{l}\right) \longrightarrow R^{i} \Psi_{\eta}\left(\overline{\mathbb{Q}}_{l}\right)
$$

qui, d'après la commutativité du diagramme ci-dessus et l'unicité des flèches $T^{i}$, sont compatibles à l'action de $\mathcal{N}_{o}$, i.e. $T^{i}\left(n s_{1}, n s_{2}\right)=n T^{i}\left(s_{1}, s_{2}\right)$ pour tout $n \in \mathcal{N}_{o}$.

On fixe $s \in R^{0} \Psi_{\eta}\left(\overline{\mathbb{Q}}_{l}\right)$ et on considère l'application

$$
T_{s}^{i}: R^{i} \Psi_{\eta}(\mathcal{F}) \longrightarrow R^{i} \Psi_{\eta}(\mathcal{F})
$$

définie par $T_{s}^{i}(t)=T^{i}(s, t)$. Pour tout $\mathcal{F}$, on vérifie aisément que $T_{s}^{0}$ est un isomorphisme, de sorte que d'après loc. cit. théorème 6.2 (c), qui n'est autre qu'une application du lemme des cinq, $T_{s}^{i}$ est un isomorphisme pour tout $\mathcal{F}$. On note encore $T^{i}$ l'application

$$
\widetilde{\mathcal{U}_{F_{o}}^{d, 0}} \otimes \widetilde{\mathcal{U}_{F_{o}}^{d, i}} \longrightarrow \widetilde{\mathcal{U}_{F_{o}}^{d, i}}
$$


En notant $R^{0} \Psi_{\eta}^{1}\left(\overline{\mathbb{Q}}_{l}\right)$ les cycles évanescents sur $\operatorname{Spf} \operatorname{Def}_{n}^{1}$, et $\widetilde{\mathcal{U}_{F_{o}}^{1,0}}$ la représentation de $F_{o}^{\times} \times F_{o}^{\times} \times W_{o}$ associée, on rappelle que l'on a

$$
\widetilde{\mathcal{U}_{F_{o}}^{1,0}}=\eta_{o} \otimes \eta_{o} \otimes \eta_{o} \circ \mathrm{cl}^{-1}
$$

La restriction de $T^{i}$ à

$$
\widetilde{\mathcal{U}_{F_{o}}^{1,0}} \otimes \widetilde{\mathcal{U}_{F_{o}}^{d, i}} \longrightarrow \widetilde{\mathcal{U}_{F_{o}}^{d, i}}
$$

est d'après ce qui précède un isomorphisme qui fournit l'isomorphisme de l'énoncé.

II.2.3. Proposition. - L'anneau $\operatorname{Def}_{n}^{d}$ est muni d'une action de $G L_{d}\left(\mathcal{O}_{o} / \mathcal{M}_{o}^{n}\right)$ et on définit

$$
\operatorname{Def}_{s t}^{d}:=\left(\operatorname{Def}_{n}^{d}\right)^{\mathrm{Iw}_{o} / K_{o, n}}
$$

où $\mathrm{Iw}_{o}$ est le sous-groupe d'Iwahori standard, i.e. l'ensemble des matrices de $G L_{d}\left(\mathcal{O}_{o}\right)$ triangulaires supérieures modulo $\mathcal{M}_{o}$. L'anneau ainsi défini ne dépend pas de l'entier $n>0$ choisi, il est régulier et semi-stable sur $\hat{\mathcal{O}}_{o}^{n r}$, i.e. il existe des coordonnées $\alpha_{1}, \cdots, \alpha_{d}$ tels que $\operatorname{Def}_{s t}^{d} \simeq \overline{\mathbb{F}}_{p}\left[\left[\alpha_{1}, \cdots, \alpha_{d}\right]\right]$ et l'image de $\pi_{o}$ par le morphisme structural $\hat{\mathcal{O}}_{o}^{n r} \simeq \overline{\mathbb{F}}_{p}\left[\left[\pi_{o}\right]\right] \longrightarrow \operatorname{Def}_{s t}^{d}$ est égale à $\prod_{i=1}^{d} \alpha_{i}$.

Démonstration. - L'indépendance de la définition de $\operatorname{Def}_{s t}^{d}$ relativement à d, découle simplement du fait que $K_{o, n}$ est un sous-groupe distingué de $\mathrm{Iw}_{o}$ pour tout $n>0$. Ainsi on a $\operatorname{Def}_{s t}^{d}=$ $\left(\operatorname{Def}_{1}^{d}\right)^{\mathrm{Iw}_{o} / K_{o, 1}}$ où Iw $\mathrm{Iw}_{o} / K_{o, 1}$ est isomorphe au sous-groupe des matrices triangulaires supérieures de $G L_{d}(\kappa(o))$. On rappelle en outre que $\operatorname{Def}_{0}^{d} \hookrightarrow \operatorname{Def}_{1}^{d}$ représente le foncteur des structures de niveau 1 sur le $\mathcal{O}_{o}$-module formel universel sur $\operatorname{Def}_{0}^{d}=\overline{\mathbb{F}}_{p}\left[\left[a_{0}, \cdots, a_{d-1}\right]\right]$ à savoir $\tau^{d}+a_{d-1} \tau^{d-1}+\cdots+a_{0}$ où $\tau=x^{q}$; la structure de niveau universelle sur $\operatorname{Def}_{1}^{d}$ est donnée par une application $\mathbb{F}_{q}$-linéaire $\iota_{o, 1}: \mathbb{F}_{q}^{d} \longrightarrow \operatorname{Def}_{1}^{d}$ avec $\iota_{o, 1}\left(e_{i}\right)=\alpha_{i}^{1} \in \operatorname{Def}_{1}^{d}$ où $\left(e_{i}\right)_{1 \leqslant i \leqslant d}$ est la base canonique de $\mathbb{F}_{q}^{d}$ qui vérifient la condition de Drinfeld

$$
\tau^{d}+a_{d-1} \tau^{d-1}+\cdots+a_{0}=\prod_{\left(\lambda_{i}\right)_{1 \leqslant i \leqslant d} \in \kappa(o)^{d}}\left(X-\sum_{i=1}^{d} \lambda_{i} \alpha_{i}^{1}\right)
$$

Par ailleurs, on a $\operatorname{Def}_{1}^{d} \simeq \overline{\mathbb{F}}_{p}\left[\left[\alpha_{1}^{1}, \cdots, \alpha_{d}^{1}\right]\right]$, l'action de $M \in G L_{d}(\kappa(o))$ étant donnée par la multiplication à droite de $M^{-1}$ sur $\mathbb{F}_{q}^{d}$. L'orbite sous l'action du Borel standard de $G L_{d}\left(\mathbb{F}_{q}\right)$ de la base canonique de $\mathbb{F}_{q}^{d}$ est le drapeau complet

$$
(0) \subset \operatorname{Vect}\left(e_{1}\right) \subset \operatorname{Vect}\left(e_{1}, e_{2}\right) \subset \cdots \subset \operatorname{Vect}\left(e_{1}, \cdots, e_{d}\right)=\mathbb{F}_{q}^{d}
$$

Ainsi l'inclusion $\operatorname{Def}_{0}^{d} \hookrightarrow \operatorname{Def}_{s t}^{d}$ représente le foncteur des "drapeaux complets" du noyau $\Sigma_{d}\left[\pi_{o}\right]$ de la multiplication par $\pi_{o}$ du $\mathcal{O}_{o}$-module formel universel sur $\operatorname{Def}_{0}^{d}$, i.e.

$$
\text { (0) } \subset G_{1} \subset G_{2} \subset \cdots \subset G_{d}=\Sigma\left[\pi_{o}\right]
$$

où les $G_{i}$ sont des sous- $\mathbb{F}_{q}$-modules de $\Sigma\left[\pi_{o}\right]$ de rang $i$. En posant

$$
P_{i}(X)=\prod_{\sum_{i} \lambda_{i} \alpha_{i}^{1} \in G_{i}}\left(X-\sum_{i} \lambda_{i} \alpha_{i}^{1}\right)
$$

dans $\operatorname{Def}_{1}^{d}[X]$, on a $P_{i}(X)=\left(X^{q}+\alpha_{i}\right) \circ P_{i-1}(X)$ et donc $\left(\tau+\alpha_{d}\right) \circ \cdots \circ\left(\tau+\alpha_{1}\right)=\tau^{d}+a_{d-1} \tau^{d-1}+$ $\cdots+a_{0}$. En outre la donnée des $\alpha_{i}$ définit complètement les $G_{i}$ de sorte que $\operatorname{Def}_{s t}^{d} \simeq \overline{\mathbb{F}}_{p}\left[\left[\alpha_{1}, \cdots, \alpha_{d}\right]\right]$ avec en particulier $a_{0}=\pi_{o}=\prod_{i=1}^{d} \alpha_{i}$.

II.2.4. Corollaire. - L'inclusion $\operatorname{Def}_{0}^{d} \hookrightarrow \operatorname{Def}_{\text {st }}^{d}$ représente le foncteur des "drapeaux complets" du noyau $\Sigma_{d}\left[\pi_{o}\right]$ de la multiplication par $\pi_{o} d u \mathcal{O}_{o}$-module formel sur $\operatorname{Def}_{0}^{d}$, i.e. la donnée de sous$\mathbb{F}_{q}$-modules de rang $i, G_{i}$ telles que $(0) \subset G_{1} \subset G_{2} \subset \cdots \subset G_{d}=\Sigma_{d}\left[\pi_{o}\right]$. Il existe alors des indéterminées $\alpha_{i}$ pour $1 \leqslant i \leqslant d$ tels que $\operatorname{Def}_{s t}^{d} \simeq \overline{\mathbb{F}}_{p}\left[\left[\alpha_{1}, \cdots, \alpha_{d}\right]\right]$ avec

$$
\left(\tau+\alpha_{d}\right) \circ \cdots \circ\left(\tau+\alpha_{1}\right)=\tau^{d}+a_{d-1} \tau^{d-1}+\cdots+a_{0} .
$$


II.2.5. Théorème. - Pour tout $0 \leqslant i<d$, on a

$$
\widetilde{\mathcal{U}_{F_{o}}^{d, i}}\left(1_{o}\right) \simeq[\overleftarrow{i}, \overrightarrow{d-i-1}]_{1_{o}} \otimes|\mathrm{cl}|^{-i}
$$

et pour $g \neq 1$ un diviseur de $d=s g$ et $\pi_{o}$ une représentation cuspidale de $G L_{g}\left(F_{o}\right)$, on a

$$
\widetilde{\left(\mathcal{U}_{F_{o}}^{d, i}\right.}\left(\mathrm{JL}^{-1}\left([\overleftarrow{s-1}]_{\pi_{o}}\right)\right)^{\mathrm{Iw}_{o}}=(0)
$$

où $\mathrm{Iw}_{o}$ désigne l'Iwahori standard de $G L_{d}\left(\mathcal{O}_{o}\right)$.

Démonstration. - On raisonne par récurrence sur $d$; on suppose donc le résultat acquis pour tout $h \leqslant d$ ce qui est vérifié pour $h=1$. Commençons par rappeler un résultat sur les cycles évanescents sur un schéma semi-stable.

II.2.6. Théorème. - (cf. [17]) Soit $X \longrightarrow S=\operatorname{Spec} \mathcal{O}_{o}$ un schéma semi-stable, i.e. tel que localement pour la topologie étale $\mathcal{O}_{X}$ est de la forme $\mathcal{O}_{o}\left[t_{1}, \cdots, t_{d}\right] /\left(\pi_{o}-\prod_{i=1}^{d} t_{i}\right)$; en notant comme d'habitude $\Psi_{\eta_{o}}$ le foncteur des cycles proches, on a

$$
\begin{gathered}
R^{i} \Psi_{\eta_{o}}\left(\overline{\mathbb{Q}}_{l}\right) \simeq \Lambda^{i} R^{1} \Psi_{\eta_{o}}\left(\overline{\mathbb{Q}}_{l}\right) \\
R^{1} \Psi_{\eta_{o}}\left(\overline{\mathbb{Q}}_{l}\right) \simeq \bigoplus_{i=1}^{d}\left(\overline{\mathbb{Q}}_{l}\right)_{\bar{X}_{s, i}} /\left(\overline{\mathbb{Q}}_{l} \text { diagonal }\right)(-1)
\end{gathered}
$$

avec $X_{i, s}$ localement défini par $t_{i}=0$ dans la fibre spéciale $X_{s}$ de $X$.

II.2.7. Corollaire. - Pour tout $0 \leqslant i<d$ et toute représentation irréductible admissible $\tau_{o}$ de $D_{o, d}^{\times},\left(\Psi_{F_{o}}^{d, i}\right)^{\mathrm{Iw} o}$ est un $\overline{\mathbb{Q}}_{l}$-espace vectoriel de dimension le coefficient binomial $\left(\begin{array}{c}i \\ d-1\end{array}\right)$ qui est pur de poids $2 i$.

Démonstration. - En effet avec les notations du théorème précédent, en tout point géométrique $z$ de $X_{s}$, les germes de $R^{i} \Psi_{\eta_{o}}\left(\overline{\mathbb{Q}}_{l}\right)_{z}$ sont purs de poids $2 i$, poids indépendant du relèvement choisi pour le Frobenius. On applique le théorème avec

$$
X=\operatorname{Spec}\left(\overline{\mathbb{F}}_{p}\left[\alpha_{1}, \cdots, \alpha_{d}\right]\right) \longrightarrow \operatorname{Spec} \overline{\mathbb{F}}_{p}\left[\pi_{o}\right],
$$

l'image de $\pi_{o}$ étant donnée par le produit des $\alpha_{i}$. Le théorème de comparaison de Berkovich donne alors que le $i$-ème faisceau des cycles évanescents $\Psi_{F_{o}, s t}^{d, i}$ de Spf $\operatorname{Def}_{s t}^{d}$ est pur de poids $2 i$. En outre en notant $\pi_{n \rightarrow s t}: \operatorname{Spf} \operatorname{Def}_{n}^{d} \longrightarrow \operatorname{Spf} \operatorname{Def}_{s t}^{d}$, le faisceau constant $\overline{\mathbb{Q}}_{l, s t, \eta_{o}}$ sur la fibre générique de l'espace analytique Spf $\operatorname{Def}_{s t}^{d}$ est isomorphe à $\pi_{n \rightarrow s t, \eta_{o}, *} \overline{\mathbb{Q}}_{l, n, \eta_{o}}$ avec des notations évidentes. On a

$$
R^{i} \Psi_{s t, \eta_{o}}\left(\pi_{n \longrightarrow s t, \eta_{o}, *} \overline{\mathbb{Q}}_{l}\right) \simeq \pi_{n \rightarrow s t, s_{o}, *} R^{i} \Psi_{n, \eta_{o}}\left(\overline{\mathbb{Q}}_{l, n, \eta_{o}}\right)
$$

soit donc

$$
R^{i} \Psi_{s t, \eta_{o}}\left(\overline{\mathbb{Q}}_{l, s t, \eta_{o}}\right) \simeq \pi_{n \rightarrow s t, s, *} R^{i} \Psi_{n, \eta_{o}}\left(\overline{\mathbb{Q}}_{l, n, \eta_{o}}\right)^{\mathrm{Iw} \mathrm{w}_{o}}
$$

Or comme les fibres spéciales sont réduites à un point, on obtient que $\Psi_{F_{o}, s t}^{d, i} \simeq\left(\Psi_{F_{o}}^{d, i}\right)^{\mathrm{Iw}_{o}}$, d'où le résultat.

II.2.8. Lemme. — (cf. [24]) L'espace des invariants sous l'Iwahori standard de

$$
[\overleftarrow{h-1}, \overrightarrow{d-h}]_{1}
$$

est de dimension le coefficient binomial $\left(\begin{array}{l}h-1 \\ d-1\end{array}\right)$.

Démonstration. - D'après un argument classique (cf. par exemple [5] lemme 4.7), cette dimension est égale au nombre de fois que $J_{N_{d}}\left([\overleftarrow{h}, \overrightarrow{d-h-1}]_{1_{o}}\right)$ contient la représentation triviale du tore maximal de $G L_{d}\left(F_{o}\right)$, où $N_{d}$ est le sous-groupe unipotent maximal du Borel standard et $J$ est le foncteur de Jacquet. Or d'après $[\mathbf{2 4}], J_{N_{d}}\left([\overleftarrow{h}, \overrightarrow{d-h-1}]_{1_{o}}\right)$ est la représentation triviale avec la multiplicité égale au cardinal de $\Lambda^{+}$, le sous-ensemble de l'ensemble des permutations, cf. la remarque (II.1.1.6), de $\{1, \cdots, d\}$ telles que $\lambda(i)<\lambda(i+1)$ pour tout $1 \leqslant i \leqslant h$ et $\lambda(i)>\lambda(i+1)$ 
pour tout $h \leqslant i \leqslant d$. Le cardinal de $\Lambda^{+}$est alors $\left(\begin{array}{l}h-1 \\ d-1\end{array}\right)$; en effet une fois choisi $h-1$ entiers distincts entre 2 et $d$, on les classe par ordre décroissant, $\alpha_{1} \geqslant \cdots \geqslant \alpha_{h-1}$; on classe de même par ordre croissant le complémentaire $\beta_{1} \leqslant \cdots \leqslant \beta_{d-h}$ et on pose $\lambda(i)=\alpha_{i}$ pour $1 \leqslant i<h, \lambda(h)=1$, $\lambda(h+i)=\beta_{i}$ pour $1 \leqslant i \leqslant d-h$. On vérifie aisément que l'on définit bien ainsi une bijection sur $\Lambda^{+}$.

II.2.0.3 - On commence dans un premier temps par traquer les sous-quotients irréductibles de

$$
n-\operatorname{Ind}_{B o p\left(F_{o}\right)}^{G L_{d}\left(F_{o}\right)}\left(|-|^{(d-1) / 2} \times \cdots \times|-|^{(1-d) / 2}\right)
$$

i.e. les représentations elliptiques de type $1_{o}$. On raisonne par récurrence, le cas $d=1$ correspondant à la théorie de Lubin-Tate. ${ }^{(3)}$

II.2.9. Proposition. - Les représentations elliptiques de type $1_{o}$ de

$$
\lim _{\vec{I}} H_{c}^{j}\left(M_{\bar{I}, \bar{s}_{o}}^{=h}, R^{i} \Psi_{\eta_{o}}\left(\overline{\mathbb{Q}}_{l}\right)\right)=\operatorname{Ind}_{P_{h ; d}^{o p}\left(F_{o}\right)}^{G L_{d}\left(F_{o}\right)} \underset{\vec{I}}{\lim _{\vec{I}}} H_{c}^{j}\left(M_{I, \bar{s}_{o}, 1}^{=h}, R^{i} \Psi_{\eta_{o}}\left(\overline{\mathbb{Q}}_{l}\right)\right)
$$

sont, avec les notations du §II.1.1, de la forme $[\overleftrightarrow{t}, \overleftarrow{i}, \overrightarrow{h-i-1}, \overleftrightarrow{d-h-t}]_{1_{o}} \otimes|\mathrm{cl}|^{-i-t}$ avec $0 \leqslant$ $t \leqslant d-h$; plus précisément pour $t=0$ (resp. $t=d-h$, resp. $0<t<d-h$ ) on obtient

$$
\begin{aligned}
& {[\overleftarrow{i}, \overrightarrow{h-i-1}]_{1_{o}} \overrightarrow{\times}[\overleftrightarrow{d-h-1}]_{1_{o}}} \\
& \text { (resp. }[\overleftarrow{i}, \overrightarrow{h-i-1}]_{1_{o}} \overleftarrow{\times}[\overleftrightarrow{d-h-1}]_{1_{o}} \\
& \left.\operatorname{resp.}\left([\overleftarrow{i}, \overrightarrow{h-i-1}]_{1_{o}} \overrightarrow{\times}[\overleftrightarrow{d-h-t-1}]_{1_{o}}\right) \overleftarrow{\times}[\overleftrightarrow{t-1}]_{1_{o}}\right)
\end{aligned}
$$

où par exemple l'unique quotient est de la forme

$$
[\overleftarrow{i}, \overrightarrow{h-i}, \overleftarrow{d-h-1}]_{1}
$$

$$
\begin{aligned}
\left(\operatorname{resp} .[\overleftrightarrow{d-h-1}, \overrightarrow{1}, \overleftarrow{i}, \overrightarrow{h-i-1}]_{1_{o}}\right. \\
\left.\quad \operatorname{resp} .[\overleftrightarrow{t-1}, \overrightarrow{1}, \overleftarrow{i}, \overrightarrow{h-i}, \overleftrightarrow{d-h-t-1}]_{1_{o}}\right)
\end{aligned}
$$

Par cela on entend tout d'abord que dans le groupe de Grothendieck correspondant, les 2 ou 4 représentations en question apparaissent simultanément; ensuite de manière plus précise, les induites de l'énoncé apparaissent comme sous-espace et comme quotient (quitte à changer les flèches non précisées).

Démonstration. - Elle est similaire à celle de la proposition (I.5.3.10). Soit $\xi_{o}$ un caractère (non ramifié) de $F_{o}^{\times}$. D'après l'hypothèse de récurrence on a

$$
\widetilde{\mathcal{U}_{F_{o}}^{h, i}}\left(\xi_{o}\right) \simeq[\overleftarrow{i}, \overrightarrow{h-i-1}]_{\xi_{o}} \otimes \xi_{o}(-i)
$$

en tant que représentation de $G L_{h}\left(F_{o}\right) \times W_{o}$. Les représentations elliptiques de type $1_{o}$ s'obtiennent exclusivement comme sous-quotient des induites normalisées

$$
n-\operatorname{Ind}_{B^{o p}\left(F_{o}\right)}^{G L_{d}\left(F_{o}\right)}|-|^{i_{1}} \times \cdots \times|-|^{i_{d}}
$$

avec $\left\{i_{1}, \cdots, i_{d}\right\}=\left\{\frac{1-d}{2}, \cdots, \frac{d-1}{2}\right\}$ ou encore avec des induites non normalisées comme les sousquotients de

$$
\operatorname{Ind}_{B^{o p}\left(F_{o}\right)}^{G L_{d}\left(F_{o}\right)}|-|^{i_{1}+(1-d) / 2} \times \cdots \times|-|^{i_{d}+(d-1) / 2} .
$$

Dans le groupe de Grothendieck des représentations admissibles de $G L_{d-h}\left(F_{o}\right) \times \mathbb{Z}$, on découpe $\lim _{\vec{I}} H_{c}^{j}\left(M_{I, s_{o}}^{=h}, \mathcal{F}_{\xi_{o}}\right)$ suivant les caractères $\chi_{o}$ de $\mathbb{Z}$ et ensuite suivant les supports cuspidaux pour $G L_{d-h}\left(F_{o}\right)$ ce qui s'écrit $\sum_{\eta_{o}, \chi_{o}} \eta_{o} \otimes \chi_{o}$ où $\eta_{o}\left(\right.$ resp. $\left.\chi_{o}\right)$ décrit les $\overline{\mathbb{Q}}_{l}$-représentations irréductibles

${ }^{(3)}$ Le cas $d=2$ est prouvé dans [8]. 
de $G L_{d-h}\left(F_{o}\right)$ (resp. $\left.\mathbb{Z}\right)$. On en déduit alors que pour $0 \leqslant i \leqslant h-1, \underset{\vec{I}}{\lim _{c}} H_{c}^{j}\left(M_{I, s_{o}}^{=h}, R^{i} \Psi_{\eta_{o}}\left(\overline{\mathbb{Q}}_{l}\right)\right)$ est de la forme

$$
\sum_{\xi_{o}, \eta_{o}, \chi_{o}} \operatorname{Ind}_{P_{h, d}^{o p}\left(F_{o}\right)}^{G L_{d}\left(F_{o}\right)}\left([\overleftarrow{i}, \overrightarrow{h-i-1}]_{\xi_{o} \otimes \chi_{o}} \otimes \eta_{o}\right) \otimes\left(\xi_{o} \chi_{o}\right)(-i)
$$

En ce qui concerne les représentations elliptiques de type $1_{o}$, on considère les $\chi_{o}$ de la forme $\xi_{o}^{-1}(-t)$, ce qui donne avec les notations ci-dessus $i_{1}=\frac{d-1}{2}-t, \cdots, i_{h}=\frac{d-1}{2}-h+1-t$, d'où le résultat.

Si on ne veut plus simplement raisonner dans le groupe de Grothendieck, on choisit $\eta_{o} \otimes \chi_{o}$ un sous-espace (resp. un quotient) irréductible du groupe de cohomologie précédent. Le reste du raisonnement est alors identique.

II.2.0.4 - On globalise $\mathrm{St}_{\infty} \otimes \mathrm{St}_{o}$ en une représentation automorphe $\Pi$ de $D_{\mathbb{A}}^{\times}$telle qu'il existe deux places distinctes de $\infty, o$ et des places de ramification Bad de $D$, pour lesquelles la composante locale de $\Pi$ est cuspidale; pour l'existence d'une telle globalisation cf. par exemple [1]. D'après le théorème (14.12) de [19], la composante isotypique $H_{\eta_{o}}^{i}\left[\Pi^{\infty}\right]$ est nulle pour $i \neq d-1$ et pour $i=d-1$ elle est isomorphe, en tant que représentation du groupe de Weil-Deligne local en $o$, à $\left.\operatorname{Sp}(d) \otimes|c l|\right|^{(1-d) / 2}$ où $\operatorname{Sp}_{d}$ est la représentation spéciale i.e. $\operatorname{Sp}(d)=\overline{\mathbb{Q}}_{l}((1-d) / 2) \oplus \cdots \oplus \overline{\mathbb{Q}}_{l}((d-1) / 2)$ en tant que représentation de $W_{o}$ avec l'action de la monodromie donnée par $N: \overline{\mathbb{Q}}_{l}(k / 2) \simeq$ $\overline{\mathbb{Q}}_{l}(k / 2+1){ }^{(4)}$

II.2.10. Proposition. - Pour tout $0 \leqslant i<d,[\overleftarrow{d-1}]_{1} \otimes|\mathrm{cl}|^{-i}$ est un sous-quotient de

$$
\left.\underset{I}{\left[\lim _{I}\right.} H^{i}\left(M_{I, \bar{s}_{o}}, R^{d-1-i} \Psi_{\eta_{o}}\left(\overline{\mathbb{Q}}_{l}\right)\right)\right]\left[\Pi^{\infty}\right] .
$$

Démonstration. - On utilise simplement que pour un schéma à réduction semi-stable, la filtration aboutissement de la suite spectrale des cycles évanescents coïncide avec la filtration des noyaux (cf. $[\mathbf{1 7}])$; le résultat découle alors directement de la description de la cohomologie générique donnée dans $[\mathbf{1 9}]$.

II.2.11. Lemme. - Soit $h_{0}$ le plus grand (s'il existe) $0<h \leqslant d$ tel qu'il existe $t, j$ pour lesquels

$$
[\overleftrightarrow{t}, \overleftarrow{i}, \overrightarrow{d-h-i-1}, \overleftrightarrow{h-t}]_{1_{o}} \otimes|\mathrm{cl}|^{-i-t}
$$

est un sous-quotient de $\lim _{\vec{I}} H_{c}^{j}\left(M_{I, \bar{s}_{o}}^{=-h}, R^{i} \Psi_{\eta_{o}}\left(\overline{\mathbb{Q}}_{l}\right)\right)\left[\Pi^{\infty}\right]$. Alors pour tout $h^{\prime}>h_{0}$,

$$
\left[\overleftrightarrow{t}, \overleftarrow{i}, \overrightarrow{d-h_{0}-i-1}, \overleftrightarrow{h_{0}-t}\right]_{1_{o}} \otimes|\mathrm{cl}|^{-i-t}
$$

n'est pas un sous-quotient de $\underset{\vec{I}}{\lim _{c}} H_{c}^{j+1}\left(M_{I, \bar{s}_{o}}^{=d-h^{\prime}}, R^{i} \Psi_{\eta_{o}}\left(\overline{\mathbb{Q}}_{l}\right)\right)\left[\Pi^{\infty}\right]$.

Démonstration. - Si $\pi_{1} \otimes|c 1|^{-i-t}$ est un sous-quotient de

$$
\lim _{\vec{I}} H_{c}^{j+1}\left(M_{I, \bar{s}_{o}}^{=d-h^{\prime}}, R^{i} \Psi_{\eta_{o}}\left(\overline{\mathbb{Q}}_{l}\right)\right)\left[\Pi^{\infty}\right]
$$

pour $\pi_{1}$ une représentation elliptique de type $1_{o}$, pour $h^{\prime}>h_{0}$ alors pour des raisons de poids $\pi_{1}$ est, d'après la proposition (II.2.9) de la forme $\left[\overleftrightarrow{t}, \overleftarrow{i}, \overrightarrow{d-h^{\prime}-i-1}, \overleftrightarrow{h^{\prime}-t}\right]_{1_{o}}$. On conclut alors par la maximalité de $h_{0}$.

(4) Dans le contexte de [14], ce résultat est prouvé dans [23]. 
II.2.12. Lemme. - Pour tout $h^{\prime}<h$ et tout $0 \leqslant t<h($ resp. $t=h)$,

$$
\begin{gathered}
{[\overleftrightarrow{t}, \overleftarrow{i}, \overrightarrow{d-h-i-1}, \overleftarrow{\alpha}, \overleftrightarrow{h-t-\alpha}]_{1_{o}} \otimes|\mathrm{cl}|^{-i-t}} \\
\left(\operatorname{resp} .[\overleftrightarrow{h}, \overleftarrow{i}, \overrightarrow{d-h-i-1}]_{1_{o}} \otimes|\mathrm{cl}|^{-h-i}\right)
\end{gathered}
$$

pour $0<\alpha \leqslant h-t$, n'est pas un sous-quotient de

$$
\lim _{\vec{I}} H_{c}^{j-1}\left(M_{I, \bar{s}_{o}}^{=d-h^{\prime}}, R^{i} \Psi_{\eta_{o}}\left(\overline{\mathbb{Q}}_{l}\right)\right)\left[\Pi^{\infty}\right] .
$$

Démonstration. — En effet d'après la proposition (II.2.9), les représentations elliptiques de type $1_{o}$ de poids $2(i+t)$ de $\lim _{\rightarrow} H_{c}^{j-1}\left(M_{I, \bar{s}_{o}}^{=}{ }^{-} h^{\prime}, R^{i} \Psi_{\eta_{o}}\left(\overline{\mathbb{Q}}_{l}\right)\right)\left[\Pi^{\infty}\right]$, pour $0 \leqslant t \leqslant h^{\prime}$, sont de la forme

$$
\left[\overleftrightarrow{t}, \overleftarrow{i}, \overrightarrow{d-h^{\prime}-i-1}, \overleftrightarrow{h^{\prime}-t}\right]_{1_{o}}
$$

de sorte qu'en numérotant comme d'habitude les sommets du graphe $\vec{\Gamma}^{d}$ de $\frac{d-1}{2}$ à $\frac{1-d}{2}$, on a toujours

$\ldots \bullet \frac{d-1-2(t+d-h-1)}{2} \longrightarrow \bullet \frac{d-1-2(t+d-h)}{2} \ldots$

alors que dans $[\overleftrightarrow{t}, \overleftarrow{i}, \overrightarrow{d-h-i-1}, \overleftarrow{\alpha}, \overleftrightarrow{h-t-\alpha}]_{1_{o}}$ cette flèche est orientée dans l'autre sens car $\alpha>0$ (pour $t=h$ un simple argument de poids suffit).

La proposition suivante montre que dans (II.2.9), on doit avoir $t=0$.

II.2.13. Proposition. — Pour $i>0$ fixé et pour tout $j, t>0$ et $h \neq 0$,

$$
[\overleftrightarrow{t}, \overleftarrow{i}, \overrightarrow{d-h-i-1}, \overleftrightarrow{h-t}]_{1}
$$

n'est pas un sous-quotient de $\underset{\vec{I}}{\lim _{c}} H_{c}^{j}\left(M_{I, \bar{s}_{o}}^{=d-h}, R^{i} \Psi_{\eta_{o}}\left(\overline{\mathbb{Q}}_{l}\right)\right)\left[\Pi^{\infty}\right]$.

Démonstration. - On raisonne par l'absurde; soit donc $h_{0}$ le plus grand, l'idée est de montrer que cette représentation se retrouverait, à travers la suite spectrale de stratification et celle des cycles évanescents dans la cohomologie de la fibre générique ce qui n'est pas d'après [19].

La suite spectrale associée à la stratification de la fibre spéciale $M_{I, s_{o}}$ :

$$
E_{1}(I, i)^{p, q}=H_{c}^{p+q}\left(M_{I, \bar{s}_{o}}^{=d-p}, R^{i} \Psi_{\eta_{o}}\left(\overline{\mathbb{Q}}_{l}\right)\right) \Rightarrow H^{p+q}\left(M_{I, \bar{s}_{o}}, R^{i} \Psi_{\eta_{o}}\left(\overline{\mathbb{Q}}_{l}\right)\right),
$$

le fait que $H^{0}\left(M_{I, s_{o}}^{d}, R^{i} \Psi_{\eta_{o}}\left(\overline{\mathbb{Q}}_{l}\right)\right)$ soit de poids $2 i$, la proposition (II.2.9), et les deux lemmes précédents, montrent que pour $0<t<h_{0}$ (resp. $t=h_{0}$ ) et tout $r \geqslant 1$,

$$
\begin{gathered}
\pi_{o}:=\left[\overleftrightarrow{t-1}, \overrightarrow{1}, \overleftarrow{i}, \overrightarrow{d-h_{0}-i-1}, \overleftarrow{1}, \overleftrightarrow{h_{0}-1}\right]_{1_{o}} \otimes|\mathrm{cl}|^{-i-t} \\
\left.\quad \text { resp. } \pi_{o}:=\left[\overleftrightarrow{h_{0}-1}, \overrightarrow{1}, \overleftarrow{i}, \overrightarrow{d-h_{0}-i-1}\right]_{1_{o}} \otimes|\mathrm{cl}|^{-i-t}\right)
\end{gathered}
$$

est un sous-quotient de $\left.\underset{\vec{I}}{\left(\lim _{r}\right.} E_{r}^{h_{0}, j-h_{0}}(I, i)\right)\left[\Pi^{\infty}\right]$. (5)

Montrons que pour tout $k \geqslant 2$, il n'existe pas de $j$ et $h$ tels que $\pi_{o} \otimes|c l|^{-i-t}$ soit un sous-quotient de $\lim _{\rightarrow} H_{c}^{j+k}\left(M_{I, \bar{s}_{o}}^{=d-h}, R^{i+1-k} \Psi_{\eta_{o}}\left(\overline{\mathbb{Q}}_{l}\right)\right)\left[\Pi^{\infty}\right]$. D'après la proposition (II.2.9), une représentation elliptique de type $1_{o}$ de cette dernière représentation de poids $2(i+t)$, est de la forme

$$
[\overleftrightarrow{t+k-1}, \overleftrightarrow{i-k+1}, \overrightarrow{d-h-i+k-2}, \overleftrightarrow{h-t-k+1}]_{1_{o}}
$$

en particulier on doit avoir $d-h-i+k-2 \leqslant d-h_{0}-i-1$ soit $h \geqslant h_{0}+k-1$ ce qui contredit la maximalité de $h_{0}$.

(5) En fait c'est un quotient. 
De même pour tout $k \geqslant 2$, il n'existe pas de $j, h$ tels que $\pi_{o} \otimes|c \mathrm{cl}|^{-i-t}$ soit un sous-quotient de $\lim _{\vec{I}} H_{c}^{j-k}\left(M_{I, \bar{s}_{o}}^{=-d-h}, R^{i+k-1} \Psi_{\eta_{o}}\left(\overline{\mathbb{Q}}_{l}\right)\right)\left[\Pi^{\infty}\right]$. En effet pour $h>0$, un tel sous-quotient elliptique de type $1_{o}$ de poids $2(i+t)$ de cet espace, est de la forme

$$
[\overleftrightarrow{t-k+1}, \overleftarrow{i+k-1}, \overleftrightarrow{d-h-i+k-2}, \overleftarrow{h-t+k-1}]_{1_{o}}
$$

de sorte que $i+k-1 \leqslant i$, contradiction. Pour $h=0$, pour des raisons de poids, il faut $t=j-1$; le résultat découle alors du lemme suivant.

II.2.14. Lemme. - La dimension des invariants sous $\mathrm{Iw}_{o}$ de

$$
[\overleftrightarrow{j-2}, \overrightarrow{1}, \overleftarrow{i}, \overrightarrow{d-h-i-1}, \overleftrightarrow{h-j+1}]_{1}
$$

est strictement supérieure à $\left(\begin{array}{c}i+j-2 \\ d-1\end{array}\right)\left(\begin{array}{c}i-1 \\ i+j-2\end{array}\right)$.

Démonstration. - On procède comme rappelé au lemme (II.2.8). Pour donner une numérotation qui induise l'orientation donnée, on peut commencer par donner le numéro 1 au sommet $(i+j-1)$ ème sommet, puis on choisit $i+j-2$ parmi $d-1$ qui serviront à numéroter les $i+j-2$ premiers sommets. Parmi ces $i+j-2$, on en choisit à nouveau $i-1$ pour numéroter les sommets de $j-1$ à $i+j-2$. On peut aussi numéroter avec 1 un des premiers sommets dans une configuration $\leftarrow \bullet \rightarrow$, il y en a forcément un parmi les $i+j-2$ premiers, d'où l'inégalité stricte.

Ainsi $\pi_{o} \otimes|c l|^{-i-t}$ apparaît dans l'aboutissement de la suite spectrale des cycles évanescents, ce qui n'est pas d'après [19].

II.2.15. Corollaire. - Tous les $\lim _{\vec{I}} H_{c}^{j}\left(M_{I, \bar{s}_{o}}^{=-h}, R^{i} \Psi_{\eta_{o}}\left(\overline{\mathbb{Q}}_{l}\right)\right)\left[\Pi^{\infty}\right]$ sont purs de poids $2 i$.

II.2.16. Lemme. $-\left.S i[\overleftarrow{i}, \overrightarrow{d-h-i}, \overleftarrow{h-1}]_{1_{o}} \otimes|c|\right|^{-i}$ est un sous-quotient de

$$
\lim _{\vec{I}} H_{c}^{j}\left(M_{I, \bar{s}_{o}}^{=d-h^{\prime}}, R^{i} \Psi_{\eta_{o}}\left(\overline{\mathbb{Q}}_{l}\right)\right)\left[\Pi^{\infty}\right]
$$

alors $h^{\prime}=h$ ou $h-1$.

Démonstration. - A nouveau si $[\overleftarrow{i}, \overrightarrow{d-h-i}, \overleftarrow{h-1}]_{1_{o}} \otimes|\mathrm{cl}|^{-i}$ est un sous-quotient de l'espace en question pour $h^{\prime}>h$, alors d'après (II.2.9)

$$
\left[\overleftarrow{i}, \overrightarrow{d-h^{\prime}-i-1}, \overleftarrow{1}, \overrightarrow{h^{\prime}-h}, \overleftarrow{h-1}\right]_{1_{o}} \otimes|\mathrm{cl}|^{-i}
$$

aussi. Soit alors $h_{0}$ le plus grand $h^{\prime}$ tel que $\lim _{I} \rightarrow H_{c}^{j}\left(M_{I, \bar{s}_{o}}^{=d-h^{\prime}}, R^{i} \Psi_{\eta_{o}}\left(\overline{\mathbb{Q}}_{l}\right)\right)\left[\Pi^{\infty}\right]$ contienne

$$
\left[\overleftarrow{i}, \overrightarrow{d-h^{\prime}-i-1}, \overleftarrow{\alpha_{1}}, \overrightarrow{\alpha_{2}}, \overleftrightarrow{h-\alpha_{1}-\alpha_{2}}\right]_{1_{o}} \otimes|\mathrm{cl}|^{-i}
$$

avec $\alpha_{1}$ et $\alpha_{2}$ strictement positifs. Celle-ci se retrouve alors par maximalité de $h_{0}$ et par un lemme analogue au précédent, dans l'aboutissement de la suite spectrale de stratification à savoir : $\lim _{I}^{\rightarrow} H^{j}\left(M_{I, \bar{s}_{o}}, R^{i} \Psi_{\eta_{o}}\left(\overline{\mathbb{Q}}_{l}\right)\right)\left[\Pi^{\infty}\right]$. Pour des raisons de poids, elle se retrouve aussi dans l'aboutissement de la suite spectrale des cycles évanescents; en effet elle ne peut pas être compensée par une contribution des points supersinguliers en vertu de (II.2.7), ni d'après (II.2.9) par des $R^{i^{\prime}} \Psi$ pour $i^{\prime}>i$ et ni d'après (II.2.13) pour $i^{\prime}<i$. On en déduit donc $h^{\prime} \leqslant h$.

Si $h^{\prime}<h-1$, d'après (II.2.9) [ $\left.\overleftarrow{i}, \overrightarrow{d-h-i-1}, \overleftarrow{h-1}\right]_{1}$, ne peut pas être un sous-quotient de $\lim _{\vec{I}} H_{c}^{j}\left(M_{I, \bar{s}_{o}}^{=d-h^{\prime}}, R^{i} \Psi_{\eta_{o}}\left(\overline{\mathbb{Q}}_{l}\right)\right)\left[\Pi^{\infty}\right]$ car $d-h^{\prime}-i-1>d-h-i ;$ d'où le résultat. 
II.2.17. Proposition. - Pour tout $0<h \leqslant d-i-1$,

$$
\underset{\vec{I}}{\lim _{\vec{I}}} H_{c}^{h}\left(M_{I, \bar{s}_{o}}^{=-h}, R^{i} \Psi_{\eta_{o}}\left(\overline{\mathbb{Q}}_{l}\right)\right)\left[\Pi^{\infty}\right]
$$

admet $[\overleftarrow{i}, \overrightarrow{d-h-i}, \overleftarrow{h-1}]_{1_{o}} \otimes|\mathrm{cl}|^{-i}$ comme sous-quotient

Démonstration. - On raisonne par récurrence descendante sur $h$; pour $h=d-i-1$, on utilise le fait que $\lim _{\rightarrow} H^{d-i-1}\left(M_{I, \bar{s}_{o}}, R^{i} \Psi_{\eta_{o}}\left(\overline{\mathbb{Q}}_{l}\right)\right)\left[\Pi^{\infty}\right]$ admet $\mathrm{St}_{o} \otimes|c \mathrm{l}|^{-i}$ comme sous-quotient. On rappelle que d'après (II.2.15)

$$
\lim _{\vec{I}} H_{c}^{d-i-1}\left(M_{I, \bar{s}_{o}}^{=i+1}, R^{i} \Psi_{\eta_{o}}\left(\overline{\mathbb{Q}}_{l}\right)\right)\left[\Pi^{\infty}\right]
$$

se calcule comme l'induite $\operatorname{Ind}_{P_{i+1}\left(F_{o}\right)}^{G L_{d}\left(F_{o}\right.}[\overleftarrow{i}]_{1} \otimes \pi^{\prime}$ pour une certaine représentation admissible $\pi^{\prime}$ de $G L_{d-i-1}\left(F_{o}\right)$ et que, d'après l'hypothèse de récurrence sur les $\Psi_{F_{o}}^{h, i}\left(1_{o}\right)$, pour tout $h \neq i+1$ la partie de poids $2 i$ de

$$
\lim _{\vec{I}} H_{c}^{d-i-1}\left(M_{I, \bar{s}_{o}}^{=i+1}, R^{i} \Psi_{\eta_{o}}\left(\overline{\mathbb{Q}}_{l}\right)\right)\left[\Pi^{\infty}\right]
$$

est soit nulle pour $h \leqslant i$, soit, pour $h>i+1$ de la forme $[\overleftarrow{i}, \overrightarrow{h-1-i}, \overleftrightarrow{d-h}]_{1_{o}} \otimes|\mathrm{cl}|^{-i}$. La suite spectrale associée à la stratification impose alors que $\pi^{\prime}$ admet la représentation de Steinberg $[\overleftarrow{d-i-2}]_{1_{o}}$ comme sous-quotient et donc $\lim _{\rightarrow} H_{c}^{d-i-1}\left(M_{I, \bar{s}_{o}}^{=i+1}, R^{i} \Psi_{\eta_{o}}\left(\overline{\mathbb{Q}}_{l}\right)\right)\left[\Pi^{\infty}\right]$ admet

$$
[\overleftarrow{i}, \overrightarrow{1}, \overleftarrow{d-i-2}]_{1_{o}} \otimes|\mathrm{cl}|^{-i}
$$

comme sous-quotient.

Supposons donc la propriété vérifiée pour $1<h<d-i$, i.e.

$$
\lim _{\vec{I}} H_{c}^{h}\left(M_{I, \bar{s}_{o}}^{=d-h}, R^{i} \Psi_{\eta_{o}}\left(\overline{\mathbb{Q}}_{l}\right)\right)\left[\Pi^{\infty}\right]
$$

admet $[\overleftarrow{i}, \overrightarrow{d-h-i}, \overleftarrow{h-1}]_{1} \otimes|\mathrm{cl}|^{-i}$ comme sous-quotient. En outre on sait cette dernière n'apparaît pas dans l'aboutissement de la suite spectrale des cycles évanescents de sorte qu'il existe $j, i^{\prime}, h^{\prime}$ tels qu'elle soit un sous-quotient de

$$
\lim _{\vec{I}} H_{c}^{j}\left(M_{I, \bar{s}_{o}}^{=d-h^{\prime}}, R^{i^{\prime}} \Psi_{\eta_{o}}\left(\overline{\mathbb{Q}}_{l}\right)\right)\left[\Pi^{\infty}\right]
$$

Pour des raisons de poids et d'après le corollaire (II.2.15), il faut $i^{\prime}=i$ de sorte que l'annulation doit se faire dans la suite spectrale associée à la stratification avec $j=h \pm 1$ et $h^{\prime} \neq h$, i.e. $[\overleftarrow{i}, \overrightarrow{d-h-i}, \overleftarrow{h-1}]_{1_{o}} \otimes|\mathrm{cl}|^{-i}$ est un sous-quotient de

$$
\lim _{\vec{I}} H_{c}^{h \pm 1}\left(M_{I, \bar{s}_{o}}^{=d-h^{\prime}}, R^{i} \Psi_{\eta_{o}}\left(\overline{\mathbb{Q}}_{l}\right)\right)\left[\Pi^{\infty}\right] .
$$

D'après le lemme précédent, on doit avoir $h^{\prime}=h-1$, soit en remarquant encore que

$$
\lim _{\vec{I}} H_{c}^{h-1}\left(M_{I, \bar{s}_{o}}^{=d-h+1}, R^{i} \Psi_{\eta_{o}}\left(\overline{\mathbb{Q}}_{l}\right)\right)\left[\Pi^{\infty}\right]
$$

est de la forme $\operatorname{Ind}_{P_{d-h+1}\left(F_{o}\right)}^{G L_{d}\left(F_{o}\right)}[\overleftarrow{i}, \overrightarrow{d-h-i}]_{1_{o}} \otimes \pi^{\prime}$, on obtient que $\pi^{\prime}$ admet $[\overleftarrow{h-1}]_{1_{o}}$ comme sousquotient et donc que

$$
\lim _{\vec{I}} H_{c}^{h-1}\left(M_{I, \bar{s}_{o}}^{=d-h+1}, R^{i} \Psi_{\eta_{o}}\left(\overline{\mathbb{Q}}_{l}\right)\right)\left[\Pi^{\infty}\right]
$$

admet $\left.[\overleftarrow{i}, \overline{d-h-i+1}, \overleftarrow{h-2}]_{1_{o}} \otimes|c|\right|^{-i}$ comme sous-quotient; d'où le résultat.

II.2.18. Corollaire. - Pour tout $0 \leqslant i<d, \Psi_{F_{o}}^{d, i}\left(1_{o}\right)$ admet $[\overleftarrow{i}, \overrightarrow{d-i-1}]_{1_{o}} \otimes|\mathrm{cl}|^{-i}$ comme sous-quotient. 
Démonstration. - D'après la proposition ci-dessus, $\underset{\vec{I}}{\lim } H_{c}^{1}\left(M_{I, \bar{s}_{o}}^{=-1}, R^{i} \Psi_{\eta_{o}}\left(\overline{\mathbb{Q}}_{l}\right)\right)\left[\Pi^{\infty}\right]$ admet comme sous-quotient $[\overleftarrow{i}, \overrightarrow{d-i-1}]_{1_{o}} \otimes|\mathrm{cl}|^{-i}$. Cette dernière représentation ayant des vecteurs fixes sous l'Iwahori $\mathrm{Iw}_{o}$, et comme

$$
\lim _{\vec{I}} H^{1}\left(M_{I, \bar{s}_{o}}, R^{i} \Psi_{\eta_{o}}\left(\overline{\mathbb{Q}}_{l}\right)\right)\left[\Pi^{\infty}\right]
$$

n'admet pas comme sous-quotient celle-ci, la suite spectrale associée à la stratification implique que $\lim _{\vec{I}} H^{0}\left(M_{I, \bar{s}_{o}}^{d}, R^{i} \Psi_{\eta_{o}}\left(\overline{\mathbb{Q}}_{l}\right)\right)\left[\Pi^{\infty}\right]$ admet $[\overleftarrow{i}, \overrightarrow{d-i-1}]_{1_{o}} \otimes|\mathrm{cl}|^{-i}$ comme sous-quotient. On utilise alors la proposition (15.2) de [5], cf. (IV.2.2.14), qui donne un isomorphisme $\left(D_{\mathbb{A}}^{\infty}\right)^{\times} \times W_{o^{-}}$-équivariant

$$
\lim _{\vec{I}} H^{0}\left(M_{I, s_{o}}^{d}, R^{i} \Psi_{\eta_{o}}\left(\overline{\mathbb{Q}}_{l}\right)\right) \simeq \operatorname{Hom}_{\bar{D}_{o}^{\times}}\left(\left(\mathcal{C}_{\bar{D}}^{\infty}\right)^{\vee}, \widetilde{\mathcal{U}_{F_{o}}^{d, i}}\right)
$$

où $\bar{D}$ est l'algèbre à division centrale sur $F$ dont les invariants sont ceux de $D$ excepté en les places $\infty, o$ où ils sont respectivement égaux à $-1 / d$ et $1 / d$, soit en particulier $\bar{D}_{o} \simeq D_{o, d}$, et où $\mathcal{C}_{\bar{D}}^{\infty}$ est l'algèbre de convolution des fonctions localement constantes sur $\bar{D}^{\times} \backslash\left(\bar{D}_{\mathbb{A}}^{\infty}\right)^{\times}$. Le résultat découle alors directement d'une correspondance de Jacquet-Langlands globale entre $\Pi$ et une unique sousreprésentation de $\mathcal{C} \frac{\infty}{D}$ de multiplicité 1 ; concrètement l'ensemble des sous-représentations $\tau^{\infty}$ de $\mathcal{C}_{\bar{D}}^{\infty}$ telles que $\tau^{\infty, o} \simeq \Pi^{\infty, o}$ est réduit à un élément de multiplicité 1 . Ainsi le lemme de Schur donne

$$
\widetilde{\mathcal{U}_{F_{o}}^{d, i}}\left(1_{o}\right)=\lim _{\vec{I}} H^{0}\left(M_{I, s_{o}}^{d}, R^{i} \Psi_{\eta_{o}}\left(\overline{\mathbb{Q}}_{l}\right)\right)\left[\Pi^{\infty, o}\right]
$$

en tant que représentation de $G L_{d}\left(F_{o}\right) \times W_{o}$, d'où le résultat.

Ainsi d'après (II.2.7) et (II.2.8), il ne reste plus de place dans $\widetilde{\mathcal{U}_{F_{o}}^{d, i}}\left(1_{o}\right)$ pour d'autres représentations ayant des vecteurs invariants sous l'Iwahori $\mathrm{Iw}_{o}$, autres que les

$$
[\overleftarrow{i}, \overrightarrow{d-i-1}]_{1_{o}} \otimes|\mathrm{cl}|^{-i}
$$

De la même façon pour toute représentation irréductible $\tau_{o}$ de $D_{o, d}^{\times}$qui n'est pas dans la classe d'équivalence inertielle de la représentation triviale, $\Psi_{F_{o}}^{d, i}\left(\tau_{o}\right)$ n'admet comme sous-quotient aucune représentation ayant des vecteurs invariants sous $\mathrm{Iw}_{o}$. 



\section{CHAPITRE III}

\section{COHOMOLOGIE DES FAISCEAUX DE HARRIS-TAYLOR}

\section{Introduction}

0.1. - Le but est de calculer la somme alternée des groupes de cohomologie des systèmes locaux d'Harris-Taylor. La démarche est classique : il s'agit tout d'abord d'utiliser la formule des traces de Lefschetz et donc de compter les points fixes sous l'action d'une correspondance de Hecke tordue par une puissance assez grande du Frobenius et ensuite de transférer les intégrales orbitales obtenues afin de reconnaître le coté géométrique de la formule des traces de Selberg. On en déduit alors un calcul de la somme alternée des groupes de cohomologie du modèle local de DeligneCarayol. Dans le cas Iwahori, des arguments de pureté nous redonne les résultats obtenus à la fin du chapitre précédent.

0.2. - Dans un premier temps, §III.1.1, on donne une description adélique des points géométriques des variétés d'Igusa de seconde espèce : on ne fait ici qu'adapter les résultats de [19]. On compte alors, §III.1.3, les points fixes sous l'action des correspondances de Hecke et d'une puissance arbitraire du frobenius en $o$. Ce comptage se fait en termes d'intégrales orbitales; à nouveau ces résultats sont une réadaptation de ceux de [19], les fonctions de transfert de la proposition (III.1.6.3) étant prises dans [14]. Par une application de la formule des traces de Lefschetz et de Selberg, on en déduit alors, au théorème (III.1.7.3), le calcul de $\left[H_{h, \rho_{\infty}, \tau_{o}}^{*}\right]$.

0.3. - Le dernier paragraphe est consacré au calcul, théorème (III.2.1), de la représentation virtuelle $\left[\widetilde{\Psi_{F_{o}}^{d, *}}\left(\tau_{o}\right)\right]$, où $\tau_{o}$ est une représentation irréductible quelconque de $D_{o, d}^{\times}$. Ce résultat est l'équivalent dans notre cadre du théorème VII.1.5 de [14]. En particulier comme d'après le corollaire (II.2.7), pour tout $0 \leqslant i<d$ et toute représentation irréductible admissible $\tau_{o}$ de $D_{o, d}^{\times}$, $\left(\Psi_{F_{o}}^{d, i}\right)^{\mathrm{Iw}_{o}}$ est pur de poids $2 i$, le calcul virtuel (III.2.1) redonne bien le théorème (II.2.5).

\section{III.1. Cohomologie des systèmes locaux d'Harris-Taylor}

L'essentiel des résultats est déjà présent dans [19], il suffit simplement de les adapter aux variétés d'Igusa.

III.1.1. Description adélique de $M_{I, \bar{s}_{o}}\left(\overline{\mathbb{F}}_{p}\right)$. -

III.1.1.1. Définition. - Un $\varphi$-espace $(V, \varphi)$ sur $\bar{\kappa}(o)$, est un $F \otimes_{\mathbb{F}_{q}} \bar{k}(o)$-espace vectoriel $V$ de dimension finie, muni d'une application $F \otimes_{\mathbb{F}_{q}}$ Frob $_{q}$-semi-linéaire bijective,

$$
\varphi: V \longrightarrow V \text {. }
$$

III.1.1.2 - Étant donné un $\mathcal{D}$-faisceau elliptique $\left(\mathcal{E}_{i}, j_{i}, t_{i}\right)$, soit $V_{i}=\mathcal{E}_{i, \eta}$ la fibre de $\mathcal{E}_{i}$ au point générique $\eta \otimes \overline{\mathbb{F}}_{q}$ de $X \otimes_{\mathbb{F}_{q}} \overline{\mathbb{F}}_{q}$; pour tout $i$, les $j_{i}$ induisent des isomorphismes $V_{i} \simeq V_{i+1}$. On 
note $V=V_{0}$ et $\varphi: V \longrightarrow V$ l'application bijective $F \otimes \operatorname{Frob}_{q}$-semi-linéaire induite par $t_{0}:(V, \varphi)$ est un $\varphi$-espace. L'action de $D$ sur $V$ commute à $\varphi$ et donne un homomorphisme de $F$-algèbre : $\iota: D^{o p} \longrightarrow \operatorname{End}(V, \varphi)$. Le triplet $(V, \varphi, \iota)$ est appelé la fibre générique du $\mathcal{D}$-faisceau elliptique $\left(\mathcal{E}_{i}, h_{i}, t_{i}\right)$.

III.1.1.3. Définition. — Deux $\mathcal{D}$-faisceaux elliptiques de caractéristique $o$ sur $\overline{\mathbb{F}}_{q}$ sont dits isogènes si leurs fibres génériques sont isomorphes.

III.1.1.4 — Si $x$ est une place de $F$, on considère le $F_{x}$-module de Dieudonné

$$
\left(V_{x}, \varphi_{x}\right):=\left(F_{x} \hat{\otimes}_{F} V, F_{x} \hat{\otimes}_{F} \varphi\right)
$$

muni du morphisme de $F_{x}$-algèbre $\lambda_{x}: D_{x}^{o p} \longrightarrow \operatorname{End}\left(V_{x}, \varphi_{x}\right)$. On pose

$$
M_{x}=H^{0}\left(\operatorname{Spec}\left(\mathcal{O}_{x} \hat{\otimes} \bar{\kappa}(o)\right), \mathcal{E}_{0}\right),
$$

qui est un $\mathcal{D}_{x}$-réseau de $V_{x}$ stable sous $\lambda_{x}\left(D_{x}^{o p}\right)$.

III.1.1.5. Proposition. - (cf. [19] proposition 9.4) La construction ci-dessus définit une bijection entre l'ensemble des classes d'isomorphismes des $\mathcal{D}$-faisceaux elliptiques sur $\bar{\kappa}(o)$ et l'ensemble des classes d'isomorphismes des paires

$$
\left((V, \varphi, \lambda),\left(M_{x}\right)_{x \in|X|}\right)
$$

où $(V, \varphi)$ est un $\varphi$-espace de rang $d^{2}$ sur $F \otimes \bar{\kappa}(o), \lambda: D^{o p} \rightarrow \operatorname{End}(V, \varphi)$ est un morphisme de $F$-algèbres et $\left(M_{x}\right)_{x \in|X|}$ est une collection de $\mathcal{D}_{x}$-réseaux des $F_{x}$-modules de Dieudonné $\left(V_{x}, \varphi_{x}\right)=$ $\left(F_{x} \hat{\otimes}_{F} V, F_{x} \hat{\otimes}_{F} \varphi\right)$ qui vérifient les propriétés suivantes :

- (i) si $x=\infty$, on $a^{(1)}$

$$
\begin{gathered}
\varphi_{\infty}\left(M_{\infty}\right) \supset M_{\infty}, \\
\varphi_{\infty}^{d}\left(M_{\infty}\right)=\varpi_{\infty}^{-1} M_{\infty}, \\
\operatorname{dim}_{\bar{\kappa}(o)}\left(\varphi_{\infty}\left(M_{\infty}\right) / M_{\infty}\right)=d,
\end{gathered}
$$

- (ii) si $x=o$, on $a$

$$
\varpi_{o} M_{o} \subset \varphi_{o}\left(M_{o}\right) \subset M_{o}
$$

le $\kappa(o) \otimes \bar{\kappa}(o)$-module $M_{o} / \varphi_{o}\left(M_{o}\right)$ est de longueur d et il est supporté par la composante connexe de $\operatorname{Spec}(\kappa(o) \otimes \bar{\kappa}(o))$ qui correspond à l'inclusion $\kappa(o) \hookrightarrow \bar{\kappa}(o)$;

- (iii) si $x \neq o, \infty$, on $a$

$$
\varphi_{x}\left(M_{x}\right)=M_{x}
$$

- (iv) toute base du $F \otimes \bar{\kappa}(o)$-espace vectoriel $V$ appartient et engendre le $\mathcal{O}_{x} \hat{\otimes} \bar{\kappa}(o)$-sous-module $M_{x}$ de $V_{x}$ pour presque toutes les places $x \neq o, \infty$ de $F$.

III.1.1.6. Définition. - Une $\varphi$-paire $(\tilde{F}, \tilde{\Pi})$ est un couple formé d'une $F$-algèbre $\tilde{F}$, commutative de dimension finie et d'un élément $\tilde{\Pi} \in \tilde{F}^{\times} \otimes \mathbb{Q}$ qui satisfait à la propriété suivante : pour toute $F$-sous-algèbre propre $F^{\prime}$ de $\tilde{F}, \tilde{\Pi}$ n'appartient pas à $F^{\prime} \times \otimes \mathbb{Q} \subset \tilde{F}^{\times} \otimes \mathbb{Q}$.

A tout $\varphi$-espace $(V, \varphi)$, Drinfeld associe une $\varphi$-paire (cf. [19] A.4).

III.1.1.7. Proposition. - (cf. [19] proposition 9.9) Soit $(\tilde{F}, \tilde{\Pi})$ la $\varphi$-paire associée au $\varphi$-espace $(V, \varphi)$. On a alors les propriétés suivantes :

- (i) $\tilde{F}$ est un corps et $[\tilde{F}: F]$ divise $d$;

- (ii) $F_{\infty} \otimes_{F} \tilde{F}$ est un corps et si $\tilde{\infty}$ est l'unique place de $\tilde{F}$ divisant $\infty$, on a l'égalité $\operatorname{deg}(\tilde{\infty}) \tilde{\infty}(\tilde{\Pi})=-[\tilde{F}: F] / d$;

- (iii) il existe une unique place $\tilde{o} \neq \tilde{\infty}$ de $\tilde{F}$ telle que $\tilde{o}(\tilde{\Pi}) \neq 0$; de plus ó divise o;

(1) où l'on a supposé pour simplifier $\operatorname{deg}(\infty)=1$ 
- (iv) on a l'égalité $h=d\left[\tilde{F}_{\tilde{o}}: F_{o}\right] /[\tilde{F}: F]$, où $h$ est l'indice de la strate à laquelle $\left(\mathcal{E}_{i}, j_{i}, t_{i}\right)$ appartient.

III.1.1.8. Corollaire. - L'algèbre $\operatorname{End}(V, \varphi, \lambda)$ est une algèbre à division centrale sur $\tilde{F}$ de dimension $(d /[\tilde{F}: F])^{2}$ dont les invariants sont donnés comme suit :

$$
\operatorname{inv}_{\tilde{x}}(\operatorname{End}(V, \varphi, \lambda))= \begin{cases}-[\tilde{F}: F] / d & \text { si } \tilde{x}=\tilde{\infty} \\ {[\tilde{F}: F] / d} & \text { si } \tilde{x}=\tilde{o} \\ {\left[\tilde{F}_{\tilde{x}}: F_{x}\right] \operatorname{inv}_{x}(D)} & \text { sinon }\end{cases}
$$

pour tout place $x$ de $F$ et toute place $\tilde{x}$ de $\tilde{F}$ divisant $x$.

III.1.1.9. Définition. - Un $(D, \infty, o)$-type est une $\varphi$-paire $(\tilde{F}, \tilde{\Pi})$ telle que :

- (i) $\tilde{F}$ est un corps et $[\tilde{F}: F]$ divise $d$ :

- (ii) $F_{\infty} \otimes_{F} \tilde{F}$ est un corps et si $\tilde{\infty}$ est l'unique place de $\tilde{F}$ divisant $\infty$, on a

$$
\operatorname{deg}(\tilde{\infty}) \tilde{\infty}(\tilde{\Pi})=-[\tilde{F}: F] / d ;
$$

- (iii) il existe une unique place $\tilde{o} \neq \tilde{\infty}$ de $\tilde{F}$ telle que $\tilde{o}(\tilde{\Pi}) \neq 0$; de plus $\tilde{o}$ divise $o$;

- (iv) pour toute place $x$ de $F$ et toute place $\tilde{x}$ de $\tilde{F}$ divisant $x$, on a

$$
\left(d\left[\tilde{F}_{\tilde{x}}: F_{x}\right] /[\tilde{F}: F]\right) \operatorname{inv}_{x}(D) \in \mathbb{Z} .
$$

III.1.1.10. Théorème. — (cf. [19] théorème 9.13) L'application composée

$$
\left(\mathcal{E}_{i}, j_{i}, t_{i}\right) \longmapsto(V, \varphi, \lambda) \longmapsto(\tilde{F}, \tilde{\Pi}),
$$

qui à un $\mathcal{D}$-faisceau elliptique défini sur $\bar{\kappa}(o)$ associe son $(D, \infty, o)$-type, induit une bijection de l'ensemble des classes d'isogénie des $\mathcal{D}$-faisceaux elliptiques définis sur $\bar{\kappa}(o)$ sur l'ensemble des classes d'isomorphismes des $(D, \infty, o)$-types.

III.1.1.11 - Toujours selon [19] (9.12), la bijection inverse est la suivante. Étant donné $(\tilde{F}, \tilde{\Pi})$, soit $(W, \psi)$ "le" $\varphi$-espace sur $\bar{\kappa}(o)$ qui lui correspond et soit $\Delta$ "l"'algèbre à division centrale sur $\tilde{F}$ dont les invariants sont

$$
\operatorname{inv}_{\tilde{x}} \Delta= \begin{cases}{[\tilde{F}: F] / d} & \text { si } \tilde{x}=\tilde{\infty} \\ -[\tilde{F}: F] / d & \text { si } \tilde{x}=\tilde{o} \\ {\left[\tilde{F}_{\tilde{x}}: F_{x}\right] \operatorname{inv}_{x}(D)} & \text { sinon }\end{cases}
$$

pour tout place $x$ de $F$ et toute place $\tilde{x}$ de $\tilde{F}$ divisant $x$. En particulier $\Delta$ est de dimension $(d /[\tilde{F}: F])^{2}$ sur $\tilde{F}$ et $D^{o p} \otimes_{F} \Delta$ et $\mathbb{M}_{d}(\operatorname{End}(W, \psi))$ sont des algèbres simples centrales sur $\tilde{F}$ de même dimension et possédant les mêmes invariants en toute place $\tilde{x}$ de $\tilde{F}$. En vertu du théorème de Skolem-Noether, on choisit un isomorphisme

$$
\alpha: D^{o p} \otimes_{F} \Delta \longrightarrow \mathbb{M}_{d}(\operatorname{End}(W, \psi))
$$

et on pose $(V, \varphi):=(W, \psi)^{d}$. On obtient alors un homomorphisme de $F$-algèbre

$$
\iota: D^{o p} \stackrel{\delta \mapsto \delta \otimes 1}{\longrightarrow} D^{o p} \otimes_{F} \Delta \stackrel{\alpha}{\longrightarrow} \mathbb{M}_{d}(\operatorname{End}(W, \psi))=\operatorname{End}(V, \varphi)
$$

tel que le commutant de $\iota\left(D^{o p}\right)$ dans $\operatorname{End}(V, \varphi)$ est l'image de $\Delta$ par l'homomorphisme de $\tilde{F}$ algèbre

$$
\Delta \stackrel{\delta \mapsto 1 \otimes \delta}{\longrightarrow} D^{o p} \otimes_{F} \Delta \stackrel{\alpha}{\longrightarrow} \operatorname{End}(V, \varphi)
$$

Remarque : $\mathrm{Si}(\tilde{F}, \tilde{\Pi})$ est un $(D, \infty, o)$-type associé à la strate $h$, on a les injections d'algèbres $\Delta^{\infty, o} \hookrightarrow D_{\mathbb{A}}^{\infty, o}, \Delta_{o}^{e t}:=\Delta_{o}^{\tilde{o}} \hookrightarrow \mathbb{M}_{d-h}\left(F_{o}\right)$ et $\Delta_{o}^{c}:=\Delta_{\tilde{o}} \hookrightarrow D_{o, h}$. 
III.1.1.12. Proposition. - Soit $(\tilde{F}, \tilde{\Pi})$ un $(D, \infty, o)$-type associé à la strate $h$. Il existe pour tout idéal I de A, une bijection de $\mathcal{J}_{I^{o}, m}^{=h}(s)(\bar{\kappa}(o))_{(\tilde{F}, \tilde{\Pi})}$ avec le quotient

$$
\Delta^{\times} \backslash\left[\left(D_{\mathbb{A}}^{\infty, o}\right)^{\times} / K_{\mathbb{A}, I^{o}}^{\infty, o} \times G L_{d-h}\left(F_{o}\right) / K_{o, m} \times D_{o, h}^{\times} /\left(1+\Pi_{o, h}^{s+1} \mathcal{D}_{o, h}\right)\right]
$$

De plus si $\left(\mathcal{J}_{J^{o}}^{=h}, m^{\prime}\left(s^{\prime}\right), c_{1}, c_{2}\right)$ est une correspondance de Hecke naturelle ${ }^{(2)}$ sur $\mathcal{J}_{I^{o}, m}^{=h}(s)$ associée $\grave{a}$ un élément $\left(g^{\infty, o}, g_{o}^{e t},\left(g_{o}^{c}, \delta_{o}, \sigma_{o}\right)\right)$ de $\left(D_{\mathbb{A}}^{\infty, o}\right)^{\times} \times G L_{d-h}\left(F_{o}\right) \times \widetilde{\mathcal{N}}_{o}{ }^{(3)}$ la correspondance induite par ces bijections est

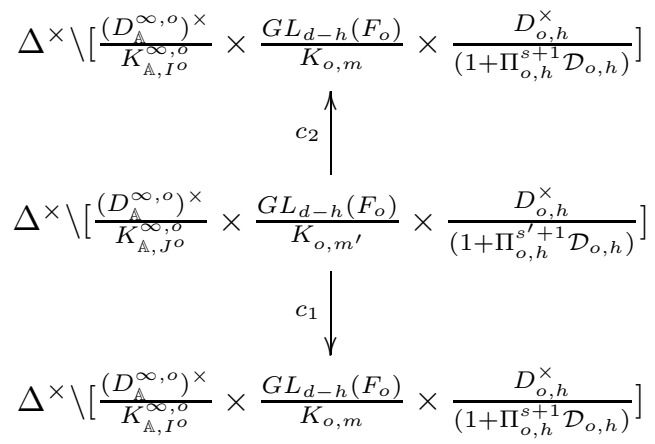

où $c_{1}$ est induit par les inclusions

$$
K_{\mathbb{A}, J}^{\infty, o} \subset K_{\mathbb{A}, I}^{\infty, o}, \quad K_{o, m^{\prime}} \subset K_{o, m}, \quad\left(1+\Pi_{o, h}^{s^{\prime}+1} \mathcal{D}_{o, h}\right) \subset\left(1+\Pi_{o, h}^{s+1} \mathcal{D}_{o, h}\right)
$$

et où $c_{2}$ est induit par la multiplication à droite de $\left(g^{\infty, o}\right)^{-1}$ sur $\left(D_{\mathbb{A}}^{\infty, o}\right)^{\times}$, de $\left(g_{o}^{\text {et }}\right)^{-1}$ sur $G L_{d-h}\left(F_{o}\right)$, et la multiplication à gauche de $\delta_{o}$ sur $D_{o, h}^{\times}$.

Démonstration. - En vertu de $[\mathbf{1 9}] \S 9$ et $\S 10, \mathcal{I}_{I^{o}, m}^{=h}(\bar{\kappa}(o))$ est en bijection avec

$$
\Delta^{\times} \backslash\left[\left(D_{\mathbb{A}}^{\infty, o}\right)^{\times} / K_{\mathbb{A}, I^{o}}^{\infty, o} \times G L_{d-h}\left(F_{o}\right) / K_{o, m} \times \mathbb{Z}\right]
$$

où l'action de $\left(D_{\mathbb{A}}^{\infty, o}\right)^{\times} \times G L_{d-h}\left(F_{o}\right)$ se décrit comme dans l'énoncé. L'action d'un élément $g_{o}^{c}$ de $G L_{h}\left(F_{o}\right)$ n'intervient que sur la composante $\mathbb{Z}$. Le groupe $P S L_{d}\left(F_{o}\right)$ étant simple, l'endomorphisme de $\mathbb{Z}$ associé à $g_{o}^{c}$ est la translation de valeur $k$. val $\left(\operatorname{det}\left(g_{o}\right)\right)$, pour un certain entier $k$ : en prenant pour $g_{o}^{c}$, l'élément $\varpi_{o}$ du centre, en utilisant la proposition B.10 de [19], on obtient $k=-1$. En outre d'après loc. cit., le frobenius géométrique en $o$ agit par translation de valeur -1 sur la composante $\mathbb{Z}$. Le résultat en découle alors de manière immédiate, en identifiant $\mathbb{Z} \times \mathcal{D}_{o, h}^{\times}$ avec $D_{o, h}^{\times}$où l'on envoie $\left(n, \delta_{o}\right)$ sur $\Pi_{o, h}^{n} \delta_{o}$.

III.1.1.13 - On rappelle, selon loc. cit., que le couple $(\tilde{F}, \tilde{\Pi})$ est construit de la manière suivante. Soit $D_{\natural}^{\times}$l'ensemble des classes de conjugaisons d'éléments de $D^{\times}$. Soit alors $\gamma \in D_{\natural}^{\times}$et $F^{\prime}=F[\gamma]$ tel qu'il existe une unique place $\infty^{\prime}$ au dessus de $\infty$ ainsi qu'une unique place $o^{\prime}$ au dessus de $o$ vérifiant $o^{\prime}(\gamma) \neq 0$ avec $h=\frac{d\left[F_{o^{\prime}}^{\prime}: F_{o}\right]}{\left[F^{\prime}: F\right]}$. Soit donc $\Pi^{\prime} \in F^{\prime}$ tel que $\infty^{\prime}\left(\Pi^{\prime}\right) \neq 0, o^{\prime}\left(\Pi^{\prime}\right) \neq 0$ et $x^{\prime}\left(\Pi^{\prime}\right)=0$ pour tout $x^{\prime} \neq \infty^{\prime}, o^{\prime}$. On définit alors $\tilde{F}=\cap_{\substack{n \in \mathbb{Z} \\ n \neq 0}} F\left[\left(\Pi^{\prime}\right)^{n}\right]$ et $\tilde{\Pi}$. Le couple $(\tilde{F}, \tilde{\Pi})$ est ainsi un $(D, \infty, o)$-type associé à la strate $h$ et tous ceux-ci sont obtenus par ce procédé. Un tel élément $\gamma \in D^{\times}$est dit elliptique en $\infty$ et de type $h$ en $o$ : son image dans $\Delta$ définit un élément $\delta \in \Delta_{\natural}^{\times}$. On a ainsi $\tilde{F} \subset F^{\prime}=F[\gamma]=\tilde{F}[\delta] \subset \Delta$ ainsi qu'une inclusion naturelle $: \Delta_{\mathbb{A}}^{\infty, o} \hookrightarrow D_{\mathbb{A}}^{\infty, o}$. Précisons la situation en la place $o$. A conjugaison près, on peut supposer que

$$
\gamma=\left(\gamma_{o}^{e t}, \gamma_{o}^{c}\right) \in G L_{d-h}\left(F_{o}\right) \times G L_{h}\left(F_{o}\right) \subset G L_{d}\left(F_{o}\right)
$$

(2) i.e. comme dans $[\mathbf{1 9}]$

(3) $J^{o}$ est donc tel que $K_{\mathbb{A}, J^{o}}^{\infty, o} \subset K_{\mathbb{A}, I^{o}}^{\infty, o} \cap\left(g^{\infty, o}\right)^{-1} K_{\mathbb{A}, I^{o}}^{\infty, o} g^{\infty, o}$ et $m^{\prime}$ tel que $K_{o, m^{\prime}} \subset K_{o, m} \cap\left(g_{o}^{e t}\right)^{-1} K_{o, m} g_{o}^{e t}$ 
avec $F_{o}\left[\gamma_{o}^{e t}\right]=\left(F^{\prime}\right)_{o}^{o^{\prime}} \subset \mathbb{M}_{d-h}\left(F_{o}\right)$ et $F_{o}\left[\gamma_{o}^{c}\right]=F_{o^{\prime}}^{\prime} \subset \mathbb{M}_{h}\left(F_{o}\right)$. On obtient ainsi des injections naturelles

$$
\left(\Delta_{o}^{e t}\right)^{\times}:=\left(\Delta_{o}^{\tilde{o}}\right)^{\times} \hookrightarrow G L_{d-h}\left(F_{o}\right) \text { et }\left(\Delta_{o}^{c}\right)^{\times}:=\Delta_{\tilde{o}}^{\times} \hookrightarrow D_{o, h}^{\times} .
$$

III.1.2. Description adélique de $\widetilde{M_{I, \bar{s}_{o}}}\left(\overline{\mathbb{F}}_{p}\right)$. - On considère désormais les structures de niveau à l'infini comme au paragraphe (I.1.4).

III.1.2.1 - En termes de la description (III.1.1.5), l'application sur les variétés sans niveau

$$
r_{\infty}: \widetilde{M_{\emptyset}} \longrightarrow M_{\emptyset}
$$

se décrit comme suit. Pour tout

$$
\left((V, \phi, \lambda),\left(M_{x}\right)_{x \in|X|}\right) \in M_{\emptyset}(\bar{\kappa}(o))
$$

soit $i_{\infty, 0}: \operatorname{Spec}(\bar{\kappa}(o)) \longrightarrow \operatorname{Spec}(\kappa(\infty))$ son pôle, i.e. le support du $\kappa(\infty) \otimes \bar{\kappa}(o)$-module $\varphi_{\infty}\left(M_{\infty}\right) / M_{\infty}$. Via l'identification $\mathcal{D}_{\infty} \simeq \mathbb{M}_{d}\left(\mathcal{O}_{\infty}\right)$, l'équivalence de Morita donne

$$
\left(V_{\infty}, \varphi_{\infty}\right)=\left(V_{\infty}^{\prime}, \varphi_{\infty}^{\prime}\right)^{d} \text { et } M_{\infty}=\left(M_{\infty}^{\prime}\right)^{d} .
$$

On note $\check{M}_{\infty}^{\prime}$ le dual du $\mathcal{O}_{\infty} \hat{\otimes} \bar{\kappa}(o)$-module libre $M_{\infty}^{\prime}$, de rang $d$, et soit $\check{\varphi}_{\infty}^{\prime}: \check{M}_{\infty}^{\prime} \longrightarrow \check{M}_{\infty}^{\prime}$ la restriction de l'application $\check{\psi}_{\infty}^{\prime}: \check{V}_{\infty}^{\prime} \longrightarrow \check{V}_{\infty}^{\prime}$ duale de $\varphi_{\infty}^{\prime}$.

III.1.2.2. Proposition. - (cf. [19] page 274) L'ensemble $\widetilde{M}_{\emptyset}(\bar{\kappa}(o))$ est en bijection avec l'ensemble des classes d'isomorphismes des triplets

$$
\left((V, \phi, \lambda),\left(M_{x}\right)_{x \in|X|},(\nu, \alpha)\right)
$$

où $\left((V, \phi, \lambda),\left(M_{x}\right)_{x \in|X|}\right)$ appartient à $M_{\emptyset}(\bar{\kappa}(o))$ et

$$
\nu: \operatorname{Spec}(\bar{\kappa}(o)) \longrightarrow \operatorname{Spec}\left(\kappa(\infty)_{d}\right)
$$

est un relèvement de $i_{\infty, 0}$ et

$$
\alpha: \mathcal{N}_{d, 1} \stackrel{\sim}{\longrightarrow} \check{M}_{\infty}^{\prime}
$$

est un isomorphisme de $\mathcal{O}_{\infty} \hat{\otimes} \bar{\kappa}(o)$-modules qui commute avec les $\psi$. L'application $r_{\infty}$ envoie $\left((V, \phi, \lambda),\left(M_{x}\right)_{x \in|X|},(\nu, \alpha)\right)$ sur $\left((V, \phi, \lambda),\left(M_{x}\right)_{x \in|X|}\right)$ et $\bar{D}_{\infty}^{\times} \simeq \overline{\mathcal{D}}_{\infty}^{\times} \rtimes \mathbb{Z} / d \mathbb{Z}$ agit comme décrit au paragraphe (I.1.4).

III.1.2.3 - On rappelle qu'étant donné un $(D, \infty, o)$-type, $(\tilde{F}, \tilde{\Pi})$, on a une inclusion d'algèbres

$$
\Delta \hookrightarrow \operatorname{End}\left(N_{d, 1}, \phi_{d, 1}\right)
$$

On identifie $\operatorname{End}\left(N_{d, 1}, \phi_{d, 1}\right)$ avec $\left(\bar{D}_{\infty}\right)^{o p}$ de sorte que l'on obtient une inclusion $\Delta^{\times} \hookrightarrow$ $\left(\left(\bar{D}_{\infty}\right)^{o p}\right)^{\times} \simeq \bar{D}_{\infty}^{\times}$. On note $\widetilde{\mathcal{J}_{I^{o}, m}^{=h}}(s)$ le produit fibré

$$
\widetilde{\mathcal{J}_{I^{o}, m}^{=h}}(s):=\mathcal{J}_{I^{o}, m}^{=h}(s) \times_{M_{I^{o}}} \widetilde{M_{I^{o}}} .
$$

III.1.2.4. Corollaire. - Soit $(\tilde{F}, \tilde{\Pi})$ un $(D, \infty, o)$-type associé à la strate $h$. Il existe pour tout idéal I de A, une bijection de $\widetilde{\mathcal{J}_{I^{o}}, m}(s)(\bar{\kappa}(o))_{(\tilde{F}, \tilde{\Pi})}$ avec le quotient

$$
\Delta^{\times} \backslash\left[\left(\bar{D}_{\infty}^{\times} / \varpi_{\infty}^{\mathbb{Z}}\right) \times\left(D_{\mathbb{A}}^{\infty, o}\right)^{\times} / K_{\mathbb{A}, I^{o}}^{\infty, o} \times G L_{d-h}\left(F_{o}\right) / K_{o, m} \times D_{o, h}^{\times} /\left(1+\Pi_{o, h}^{s+1} \mathcal{D}_{o, h}\right)\right]
$$

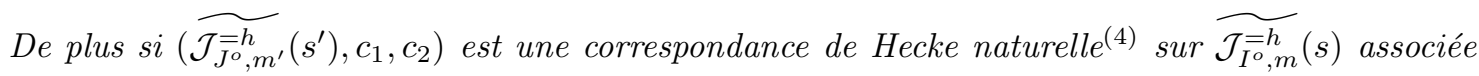
à un élément

$$
\left(\bar{g}_{\infty}, g^{\infty, o}, g_{o}^{e t},\left(g_{o}^{c}, \delta_{o}, \sigma_{o}\right)\right) \in\left(\bar{D}_{\infty}^{\times} / \varpi_{\infty}^{\mathbb{Z}}\right) \times\left(D_{\mathbb{A}}^{\infty, o}\right)^{\times} \times G L_{d-h}\left(F_{o}\right) \times \widetilde{\mathcal{N}_{o}}
$$

$\overline{(4)}$ i.e. comme dans $[\mathbf{1 9}]$ 
(5) la correspondance induite par ces bijections est

$$
\begin{aligned}
& \Delta^{\times} \backslash\left[\left(\bar{D}_{\infty}^{\times} / \varpi_{\infty}^{\mathbb{Z}}\right) \times \frac{\left(D_{\mathbb{A}}^{\infty, o}\right)^{\times}}{K_{\mathbb{A}, I^{o}}^{\infty, o}} \times \frac{G L_{d-h}\left(F_{o}\right)}{K_{o, m}} \times \frac{D_{o, h}^{\times}}{\left(1+\Pi_{o, h}^{s+1} \mathcal{D}_{o, h}\right)}\right] \\
& c_{2} \uparrow \\
& \Delta^{\times} \backslash\left[\left(\bar{D}_{\infty}^{\times} / \varpi_{\infty}^{\mathbb{Z}}\right) \times \frac{\left(D_{\AA}^{\infty, o}\right)^{\times}}{K_{\mathbb{A}, J^{o}}^{\infty, o}} \times \frac{G L_{d-h}\left(F_{o}\right)}{K_{o, m^{\prime}}} \times \frac{D_{o, h}^{\times}}{\left(1+\Pi_{o, h}^{s^{\prime}+1} \mathcal{D}_{o, h}\right)}\right] \\
& c_{1} \downarrow \\
& \Delta^{\times} \backslash\left[\left(\bar{D}_{\infty}^{\times} / \varpi_{\infty}^{\mathbb{Z}}\right) \times \frac{\left(D_{\mathbb{A}}^{\infty, o}\right)^{\times}}{K_{\mathbb{A}, I^{o}}^{\infty, o}} \times \frac{G L_{d-h}\left(F_{o}\right)}{K_{o, m}} \times \frac{D_{o, h}^{\times}}{\left(1+\Pi_{o, h}^{s+1} \mathcal{D}_{o, h}\right)}\right]
\end{aligned}
$$

où $c_{1}$ est induit par les inclusions

$$
K_{\mathbb{A}, J}^{\infty, o} \subset K_{\mathbb{A}, I}^{\infty, o}, \quad K_{o, m^{\prime}} \subset K_{o, m}, \quad\left(1+\Pi_{o, h}^{s^{\prime}+1} \mathcal{D}_{o, h}\right) \subset\left(1+\Pi_{o, h}^{s+1} \mathcal{D}_{o, h}\right)
$$

et où $c_{2}$ est induit par la multiplication à gauche par $g_{o}$ sur $\left(\bar{D}_{\infty}^{\times} / \varpi_{\infty}^{\mathbb{Z}}\right)$, à droite de $\left(g^{\infty, o}\right)^{-1}$ sur $\left(D_{\mathbb{A}}^{\infty, o}\right)^{\times}$, de $\left(g_{o}^{\text {et }}\right)^{-1}$ sur $G L_{d-h}\left(F_{o}\right)$, et la multiplication à gauche de $\delta_{o}$ sur $D_{o, h}^{\times}$.

Remarque : L'application

$$
\lambda(\kappa)_{(\tilde{F}, \tilde{\Pi})}: \widetilde{\mathcal{J}_{I^{o}, m}^{h}}(s)(\kappa)_{(\tilde{F}, \tilde{\Pi})} \longrightarrow \operatorname{Spec}\left(\kappa(\infty)_{d}\right)
$$

est induite par l'application

$$
\bar{D}_{\infty}^{\times} / \varpi_{\infty}^{\mathbb{Z}} \longrightarrow \mathbb{Z} / d \operatorname{deg}(\infty) \mathbb{Z}
$$

qui envoie $(n, \delta) \operatorname{sur} n+\operatorname{deg}(\infty) \infty(\operatorname{rn}(\delta)) \bmod d \operatorname{deg}(\infty) \mathbb{Z}$.

III.1.3. Comptage des points fixes. - On fixe un sous-groupe normal $\bar{K}_{\infty} \subset\left(\bar{D}_{\infty}^{\times} / \varpi_{\infty}^{\mathbb{Z}}\right)$ et on note

$$
M(s)=\widetilde{\mathcal{J}_{I^{o}, m}^{=h}}(s) / \bar{K}_{\infty}
$$

qui est donc muni d'une action du produit $\mathcal{D}_{o, h}^{\times} /\left(1+\Pi_{o, h}^{s+1} \mathcal{D}_{o, h}\right) \times\left(\bar{D}_{\infty}^{\times} / \varpi_{\infty}^{\mathbb{Z}}\right) / \bar{K}_{\infty}$ et d'une action par correspondances de Hecke associées aux éléments $g^{\infty, \tilde{o}}=\left(g^{\infty, o}, g_{o}^{e t}\right) \in\left(D_{\mathbb{A}}^{\infty, o}\right)^{\times} \times G L_{d-h}\left(F_{o}\right)$ :

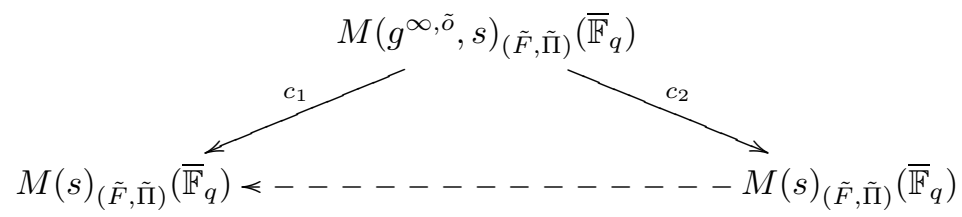

où $M\left(g^{\infty, \tilde{o}}, s\right)$ est le quotient

$$
\left({\underset{\leftarrow}{I}{ }_{i}, m}_{\lim _{I^{o}, m}} \widetilde{\mathcal{J}^{=h}}(s)\right) /\left(\left(K_{\mathbb{A}, I^{o}}^{\infty, o} \cap\left(g^{\infty, o}\right)^{-1} K_{\mathbb{A}, I^{o}}^{\infty, o} g^{\infty, o}\right) \times K_{o, m} \cap\left(g_{o}^{e t}\right)^{-1} K_{o, m} g_{o}^{e t}\right.
$$

et où la correspondance est induite par

$$
\begin{gathered}
\Delta^{\times} \backslash\left[\frac{\bar{D}_{\infty}^{\times} / \varpi_{\infty}^{\mathbb{Z}}}{\bar{K}_{\infty}} \times \frac{\left(D_{\mathbb{A}}^{\infty, o}\right)^{\times}}{K_{\mathbb{A}, I^{o}}^{\infty}}\right. \\
\left.\times \frac{G L_{d-h}\left(F_{o}\right)}{K_{o, m}} \times \frac{D_{o, h}^{\times}}{\left(1+\Pi_{o, h}^{s+1} \mathcal{D}_{o, h}^{\times}\right)}\right] \\
\uparrow c_{2}
\end{gathered}
$$

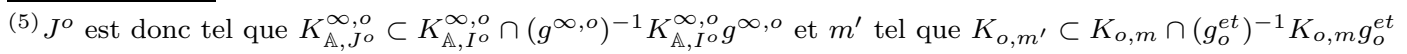




$$
\Delta^{\times} \backslash\left\{\frac{\bar{D}_{\infty}^{\times} / \varpi_{\infty}^{\mathbb{Z}}}{\bar{K}_{\infty}} \times \frac{\left(D_{\mathbb{A}}^{\infty, o}\right)^{\times}}{K_{\mathbb{A}, I^{o}}^{\infty, o} \cap\left(g^{\infty, o}\right)^{-1} K_{\mathbb{A}, I^{o}}^{\infty, o} g^{\infty, o}} \times\right.
$$

$$
\begin{array}{r}
\left.\frac{G L_{d-h}\left(F_{o}\right)}{K_{o, m} \cap\left(g_{o}^{e t}\right)^{-1} K_{o, m} g_{o}^{e t}} \times \frac{D_{o, h}^{\times}}{\left(1+\Pi_{o, h}^{s+1} \mathcal{D}_{o, h}\right)}\right\} \\
\downarrow c_{1} \\
\Delta^{\times} \backslash\left[\frac{\bar{D}_{\infty}^{\times} / \varpi_{\infty}^{\mathbb{Z}}}{\bar{K}_{\infty}} \times \frac{\left(D_{\mathbb{A}}^{\infty, o}\right)^{\times}}{K_{\mathbb{A}, I^{o}}^{\infty, o}} \times \frac{G L_{d-h}\left(F_{o}\right)}{K_{o, m}} \times \frac{D_{o, h}^{\times}}{\left(1+\Pi_{o, h}^{s+1} \mathcal{D}_{o, h}\right)}\right]
\end{array}
$$

où

$$
\begin{gathered}
c_{1}\left[h^{\infty, o}\left(K_{\mathbb{A}, I^{o}}^{\infty, o} \cap\left(g^{\infty, o}\right)^{-1} K_{\mathbb{A}, I^{o}}^{\infty} g^{\infty, o}\right), h_{o}^{e t}\left(K_{o, m} \cap\left(g_{o}^{e t}\right)^{-1} K_{o, m} g_{o}^{e t}\right), d_{o}\left(1+\Pi_{o, h}^{s+1} \mathcal{D}_{o, h}\right)\right] \\
=\left[h^{\infty, o} K_{\mathbb{A}, I^{o}}^{\infty, o}, h_{o}^{e t} K_{o, m}, d_{o}\left(1+\Pi_{o, h}^{s+1} \mathcal{D}_{o, h}\right)\right]
\end{gathered}
$$

et

$$
\begin{aligned}
c_{2}\left[h^{\infty, o}\left(K_{\mathbb{A}, I^{o}}^{\infty, o} \cap\left(g^{\infty, o}\right)^{-1} K_{\mathbb{A}, I^{o}}^{\infty, o} g^{\infty, o}\right), h_{o}^{e t}\left(K_{o, m} \cap\left(g_{o}^{e t}\right)^{-1} K_{o, m} g_{o}^{e t}\right), d_{o}\left(1+\Pi_{o, h}^{s+1} \mathcal{D}_{o, h}\right)\right] \\
=\left[h^{\infty, o}\left(g^{\infty, o}\right)^{-1} K_{\mathbb{A}, I^{o}}^{\infty, o} h_{o}^{e t}\left(g_{o}^{e t}\right)^{-1} K_{o, m}, d_{o}\left(1+\Pi_{o, h}^{s+1} \mathcal{D}_{o, h}\right)\right]
\end{aligned}
$$

III.1.3.1. Remarque. - Dans cette description l'action de

$$
\left(g_{o}^{c}, \delta_{o}, \sigma_{o}\right) \in \mathcal{N}_{o} \subset G L_{h}\left(F_{o}\right) \times D_{o, h}^{\times} \times W_{F_{o}}
$$

$\operatorname{sur} M_{(\tilde{F}, \tilde{\Pi})}$ est donnée par la multiplication à gauche de $\delta_{o}$ sur la composante $D_{o, h}^{\times} /\left(1+\Pi_{o, h}^{s+1} \mathcal{D}_{o, h}\right)$.

III.1.3.2. Définition. - Pour $r>0, \bar{g}_{\infty} \in\left(\bar{D}_{\infty}^{\times} / \varpi_{\infty}^{\mathbb{Z}}\right)$ et $\delta_{o} \in \mathcal{D}_{o, h}^{\times}$, on note

$$
\operatorname{Fix}_{r}^{=h}\left(\bar{g}_{\infty}, \delta_{o}, g_{o}^{c}, g^{\infty, \tilde{o}}, s\right)
$$

l'ensemble $\left\{m \in M\left(g^{\infty, \tilde{o}}, s\right)\left(\overline{\mathbb{F}}_{q}\right) /\left(g_{o}^{c}, \Pi_{o, h}^{-\operatorname{val}\left(\operatorname{det}\left(g_{o}^{c}\right)\right)-r} \delta_{o}, \operatorname{Frob}_{o}^{r}\right)\left(c_{1}(m)\right) \cdot \bar{g}_{\infty} \bar{K}_{\infty}=c_{2}(m)\right\}$.

III.1.3.3. Lemme. - (cf. [19] lemme 11.1) Les ensembles $\operatorname{Fix}_{r}^{=h}\left(\delta_{o}, g_{o}^{c}, g^{\infty, \tilde{o}}, s\right)$ pour $r>0$ sont finis et chacun de ces points fixes est de multiplicité 1.

III.1.3.4 - On décompose ces ensembles, suivant leur $(D, \infty, o)$-type

$$
\operatorname{Fix}_{r}^{=h}\left(\bar{g}_{\infty}, \delta_{o}, g_{o}^{c}, g^{\infty, \tilde{o}}, s\right)=\coprod_{(\tilde{F}, \tilde{\Pi})} \operatorname{Fix}_{r}^{=h}\left(\bar{g}_{\infty}, \delta_{o}, g_{o}^{c}, g^{\infty, \tilde{o}}, s\right)_{(\tilde{F}, \tilde{\Pi})}
$$

où la réunion porte sur les $(D, \infty, o)$-type associés à la strate $h$.

Soit $(\tilde{F}, \tilde{\Pi})$ un $(D, \infty, o)$-type associé à la strate $h$ et soit $\Delta$ l'algèbre à division centrale sur $F$ correspondante. On note $\Delta_{\natural}^{\times}$un système de représentants des classes de conjugaisons dans $\Delta^{\times}$et pour $\delta \in \Delta^{\times}$, soit $\Delta_{\delta}^{\times}$le centralisateur de $\delta$ dans $\Delta^{\times}$.

III.1.3.5. Proposition. - L'ensemble $\operatorname{Fix}_{r}^{=h}\left(\bar{g}_{\infty}, \delta_{o}, g_{o}^{c}, g^{\infty, \tilde{o}}, s\right)_{(\tilde{F}, \tilde{\Pi})}$ est la réunion disjointe des doubles classes

$$
\coprod_{\delta \in \Delta_{\natural}^{\times}} \Delta_{\delta}^{\times}\left[\bar{h}_{\infty} \bar{K}_{\infty}, h^{\infty, o} K_{\mathbb{A}, I^{o}}^{\infty, o}, h_{o}^{e t} K_{o, m}, d_{o}\left(1+\Pi_{o, h}^{s+1} \mathcal{D}_{o, h}\right)\right]
$$

telles que

$$
\left\{\begin{array}{l}
\left(\bar{h}_{\infty}\right)^{-1} \delta \bar{h}_{\infty} \in \bar{g}_{\infty} \bar{K}_{\infty} \\
\left(h^{\infty, o}\right)^{-1} \delta h^{\infty, o} \in K_{\mathbb{A}, I^{o}}^{\infty, o} g^{\infty, o} K_{\mathbb{A}, I^{o}}^{\infty, o} \\
\left(h_{o}^{e t}\right)^{-1} \delta h_{o}^{e t} \in K_{o, m} g_{o}^{e t} K_{o, m} \\
\delta \in \Pi_{o, h}^{r+\operatorname{val}\left(\operatorname{det}\left(g_{o}^{c}\right)\right)} \delta_{o}^{-1}\left(1+\Pi_{o, h}^{s+1} \mathcal{D}_{o, h}\right)
\end{array}\right.
$$


Démonstration. - D'après la description des correspondances de Hecke donnée ci-dessus,

$$
\begin{aligned}
\Delta^{\times}\left[\bar{h}_{\infty} \bar{K}_{\infty}, h^{\infty, o}\left(K_{\mathbb{A}, I^{o}}^{\infty, o} \cap\left(g^{\infty, o}\right)^{-1} K_{\mathbb{A}, I^{o}}^{\infty, o} g^{\infty, o}\right),\right. & \\
& \left.h_{o}^{e t}\left(K_{o, m} \cap\left(g_{o}^{e t}\right)^{-1} K_{o, m} g_{o}^{e t}\right), d_{o}\left(1+\Pi_{o, h}^{s+1} \mathcal{D}_{o, h}\right)\right]
\end{aligned}
$$

appartient à $\operatorname{Fix}_{r}^{=h}\left(\delta_{o}, g_{o}^{c}, g^{\infty, \tilde{o}}, s\right)_{(\tilde{F}, \tilde{\Pi})}$ si et seulement si il existe $\delta \in \Delta^{\times}, \bar{k}_{\infty} \in \bar{K}_{\infty}, k^{\infty, o} \in K_{\mathbb{A}, I^{o}}^{\infty, o}$, $k_{o}^{e t} \in K_{o, m}$ et $k_{s} \in\left(1+\Pi_{o, h}^{s+1} \mathcal{D}_{o, h}\right)$ tels que

$$
\left\{\begin{array}{l}
\bar{h}_{\infty} \bar{g}_{\infty}=\delta \bar{h}_{\infty} \bar{k}_{\infty} \\
h^{\infty, o}=\delta h^{\infty, o}\left(g^{\infty, o}\right)^{-1} k^{\infty, o} \\
h_{o}^{e t}=\delta h_{o}^{e t}\left(g_{o}^{e t}\right)^{-1} k_{o}^{e t} \\
d_{o}=\delta \Pi_{o, h}^{-r-\operatorname{val}\left(\operatorname{det}\left(g_{o}^{c}\right)\right)} \delta_{o} d_{o} k_{s}
\end{array}\right.
$$

soit, si et seulement si, il existe $\delta \in \Delta^{\times}$tel que

$$
\left\{\begin{array}{l}
\left(\bar{h}_{\infty}\right)^{-1} \delta \bar{h}_{\infty} \in \bar{g}_{\infty} \bar{K}_{\infty} \\
\left(h^{\infty, o}\right)^{-1} \delta h^{\infty, o} \in K_{\mathbb{A}, I^{o}}^{\infty} g^{\infty, o} \\
\left(h_{o}^{e t}\right)^{-1} \delta h_{o}^{e t} \in K_{o, m} g_{o}^{e t} \\
\delta \in \Pi_{o, h}^{r+\operatorname{val}\left(\operatorname{det}\left(g_{o}^{c}\right)\right)} \delta_{o}^{-1}\left(1+\Pi_{o, h}^{s+1} \mathcal{D}_{o, h}\right)
\end{array}\right.
$$

en particulier, on a $o(\operatorname{rn}(\delta))=r+\operatorname{val}\left(\operatorname{det} g_{o}^{c}\right)$. L'application

$$
\begin{gathered}
h^{\infty, o}\left(K_{\mathbb{A}, I^{o}}^{\infty, o} \cap\left(g^{\infty, o}\right)^{-1} K_{\mathbb{A}, I^{o}}^{\infty} g^{\infty, o}\right) \\
\left(\text { resp. } h_{o}^{e t}\left(K_{o, m} \cap\left(g_{o}^{e t}\right)^{-1} K_{o, m} g_{o}^{e t}\right) \mapsto h_{o}^{e t} K_{\mathbb{A}, m}^{\infty, o}\right)
\end{gathered}
$$

de l'ensemble des classes satisfaisant

$$
\left(h^{\infty, o}\right)^{-1} \delta h^{\infty, o} \in K_{\mathbb{A}, I^{o}}^{\infty, o} g^{\infty, o}\left(\operatorname{resp} .\left(h_{o}^{e t}\right)^{-1} \delta h_{o}^{e t} \in K_{o, m} g_{o}^{e t}\right)
$$

dans l'ensemble des classes vérifiant

$$
\left(h^{\infty, o}\right)^{-1} \delta h^{\infty, o} \in K_{\mathbb{A}, I^{o}}^{\infty, o} g^{\infty, o} K_{\mathbb{A}, I^{o}}^{\infty, o}\left(\text { resp. }\left(h_{o}^{e t}\right)^{-1} \delta h_{o}^{e t} \in K_{o, m} g_{o}^{e t} K_{o, m}\right)
$$

est clairement bijective. Le résultat découle alors du lemme suivant.

III.1.3.6. Lemme. - Pour tout $h^{\infty, o} \in\left(D_{\mathbb{A}}^{\infty, o}\right)^{\times}, h_{o}^{e t} \in G L_{d-h}\left(F_{o}\right)$ et $d_{o} \in D_{o, h}^{\times}$, le seul élément $\delta \in \Delta^{\times}$tel que

est l'identité.

$$
\left\{\begin{array}{l}
\left(h^{\infty, o}\right)^{-1} \delta h^{\infty, o} \in K_{\mathbb{A}, I^{o}}^{\infty} \\
\left(h_{o}^{e t}\right)^{-1} \delta h_{o}^{e t} \in K_{o, m} \\
o(\operatorname{rn}(\delta))=0
\end{array}\right.
$$

Démonstration. - cf. [20] (3.2.6).

III.1.4. Intégrales orbitales. - Pour tout $\delta \in \Delta^{\times}$, soit

$$
\left(D_{\mathbb{A}}^{\infty, o}\right)_{\delta}^{\times} \quad\left(\text { resp. } G L_{d-h}\left(F_{o}\right)_{\delta}, \quad \operatorname{resp} .\left(D_{o, h}\right)_{\delta}^{\times}, \quad \operatorname{resp} .\left(\bar{D}_{\infty}^{\times}\right)_{\delta}\right)
$$

le centralisateur de $\delta$ dans $\left(D_{\mathbb{A}}^{\infty, o}\right)^{\times}$(resp. $G L_{d-h}\left(F_{o}\right)$, resp. $D_{o, h}^{\times}$, resp. $\left.\bar{D}_{\infty}^{\times}\right)$. On fixe une mesure de Haar,

$$
d h^{\infty, o} \quad\left(\text { resp. } d h_{o}^{e t}, \quad \text { resp. } d \bar{h}_{\infty}\right)
$$

$\operatorname{sur}\left(D_{\mathbb{A}}^{\infty, o}\right)^{\times}\left(\right.$resp. $G L_{d-h}\left(F_{o}\right)$, resp. $\left.\bar{D}_{\infty}^{\times} / \varpi_{\infty}^{\mathbb{Z}}\right)$ normalisée par

$$
\operatorname{vol}\left(K_{\mathbb{A}, I}^{\infty, o}, d h^{\infty, o}\right)=1 \quad\left(\operatorname{resp} \cdot \operatorname{vol}\left(K_{o, m}, d h_{o}^{e t}\right)=1 \operatorname{resp} . \operatorname{vol}\left(\bar{K}_{\infty}, d \bar{h}_{\infty}\right)=1\right) .
$$

Soit

$$
d h_{\delta}^{\infty, o} \quad\left(\text { resp. } d h_{o, \delta}^{e t}, \quad \text { resp. } d \bar{h}_{o, \delta}, \quad \text { resp. } d \bar{h}_{\infty, \delta}\right)
$$


une mesure de Haar sur $\left(D_{\mathbb{A}}^{\infty, o}\right)_{\delta}^{\times}$(resp. $G L_{d-h}\left(F_{o}\right)_{\delta}$, resp. $\left(D_{o, h}\right)_{\delta}^{\times}$, resp. $\left.\left(\bar{D}_{\infty}^{\times}\right)_{\delta} / \varpi_{\infty}^{\mathbb{Z}}\right)$ et soit $d \delta^{\prime}$ la mesure de comptage sur $\Delta_{\delta}^{\times}$. Soit alors

$$
f^{\infty, o} \quad\left(\text { resp. } f_{o}^{e t}, \quad \text { resp. } \bar{f}_{\infty}\right)
$$

la fonction caractéristique de

$$
K_{\mathbb{A}, I^{o}}^{\infty, o} g^{\infty, o} K_{\mathbb{A}, I^{o}}^{\infty, o} \quad\left(\text { resp. } K_{o, m} g_{o}^{e t} K_{o, m}, \quad \text { resp. } \bar{g}_{\infty} \bar{K}_{\infty}\right)
$$

dans $\left(D_{\AA}^{\infty, o}\right)^{\times}$(resp. $G L_{d-h}\left(F_{o}\right)$, resp. $\left.\bar{D}_{\infty}^{\times} / \varpi_{\infty}^{\mathbb{Z}}\right)$. On introduit alors les intégrales orbitales

$$
\begin{gathered}
O_{\delta}\left(\bar{f}_{\infty}, d \bar{h}_{\infty, \delta}\right)=\int_{\left(\bar{D}_{\infty}^{\times}\right)_{\delta} \backslash \bar{D}_{\infty}^{\times}} \bar{f}_{\infty}\left(\left(\bar{h}_{\infty}\right)^{-1} \delta \bar{h}_{\infty}\right) \frac{d \bar{h}_{\infty}}{d \bar{h}_{\infty, \delta}}, \\
O_{\delta}\left(f^{\infty, o}, d h_{\delta}^{\infty, o}\right)=\int_{\left(D_{\mathbb{A}}^{\infty, o}\right)_{\delta}^{\times} \backslash\left(D_{\mathbb{A}}^{\infty, o}\right)^{\times}} f^{\infty, o}\left(\left(h^{\infty, o}\right)^{-1} \delta h^{\infty, o}\right) \frac{d h^{\infty, o}}{d h_{\delta}^{\infty, o}}, \\
O_{\delta}\left(f_{o}^{e t}, d h_{o, \delta}^{e t}\right)=\int_{G L_{d-h}\left(F_{o}\right)_{\delta} \backslash G L_{d-h}\left(F_{o}\right)} f_{o}^{e t}\left(\left(h_{o}^{e t}\right)^{-1} \delta h_{o}^{e t}\right) \frac{d h_{o}^{e t}}{d h_{o, \delta}^{e t}},
\end{gathered}
$$

Ces intégrales sont absolument convergentes et on introduit le volume $V$ défini par

$$
\operatorname{vol}\left(\Delta_{\delta}^{\times} \backslash\left[\left(\left(\bar{D}_{\infty}^{\times}\right)_{\delta} / \varpi_{\infty}^{\mathbb{Z}}\right) \times\left(D_{\mathbb{A}}^{\infty, o}\right)_{\delta}^{\times} \times G L_{d-h}\left(F_{o}\right)_{\delta}\right], \frac{d h_{\delta}^{\infty, o} d h_{o, \delta}^{e t}}{d \delta^{\prime}}\right)
$$

Pour tout $\delta \in \Delta^{\times}$, l'inclusion d'algèbres $\Delta^{\infty, o} \hookrightarrow D_{\mathbb{A}}^{\infty, o}$ (resp. $\Delta_{o}^{e t} \hookrightarrow \mathbb{M}_{d-h}\left(F_{o}\right)$, resp. $\Delta_{o}^{c} \hookrightarrow$ $D_{o, h}$, resp. $\left.\Delta \hookrightarrow \bar{D}_{\infty}^{o p}\right)$, induit un isomorphisme de groupe $\left(\Delta^{\infty, o}\right)_{\delta}^{\times} \simeq\left(D_{\mathbb{A}}^{\infty, o}\right)_{\delta}^{\times}$(resp. $\left(\Delta_{o}^{e t}\right)_{\delta}^{\times} \simeq$ $G L_{d-h}\left(F_{o}\right)_{\delta}$, resp. $\left(\Delta_{o}^{c}\right)_{\delta}^{\times} \simeq\left(D_{o, h}\right)_{\delta}^{\times}$, resp. $\left.\left(\Delta_{\infty}^{\times}\right)_{\delta} \simeq\left(\bar{D}_{\infty}^{\times}\right)_{\delta}\right)$. En particulier la mesure de Haar $d \bar{h}_{\infty, \delta} \times d h_{\delta}^{\infty, o} \times d h_{o, \delta}^{e t}$ induit une mesure de Haar $d h_{\delta} \operatorname{sur}\left(\Delta_{\mathbb{A}}^{\times}\right)_{\delta} / \varpi_{\infty}^{\mathbb{Z}}$.

III.1.4.1. Lemme. - Le volume $V$ est égal au volume

$$
\operatorname{vol}\left(\Delta_{\delta}^{\times} \backslash\left(\Delta_{\mathbb{A}}^{\times}\right)_{\delta} / \varpi_{\infty}^{\mathbb{Z}}, \frac{d h_{\delta}}{d \delta^{\prime}}\right)
$$

Démonstration. - cf. [20] (3.3.4).

III.1.4.2 - Soit $\operatorname{Lef}_{r}^{=h}\left(\bar{g}_{\infty}, \delta_{o}, g_{o}^{c}, g^{\infty, \tilde{o}}, s\right)_{(\tilde{F}, \tilde{\Pi})}$ le cardinal de $\operatorname{Fix}_{r}^{=h}\left(\bar{g}_{\infty}, \delta_{o}, g_{o}^{c}, g^{\infty, \tilde{o}}, s\right)_{(\tilde{F}, \tilde{\Pi})}$. D'après ce qui précède $\operatorname{Lef}_{r}^{=h}\left(\bar{g}_{\infty}, \delta_{o}, g_{o}^{c}, g^{\infty, \tilde{o}}, s\right)_{(\tilde{F}, \tilde{\Pi})}$ est égal à

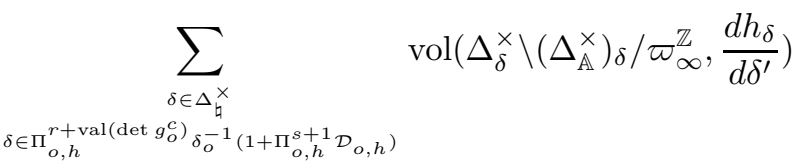

$$
\begin{aligned}
& O_{\delta}\left(\bar{f}_{\infty}, d \bar{h}_{\infty, \delta}\right) O_{\delta}\left(f^{\infty, o}, d h_{\delta}^{\infty, o}\right) O_{\delta}\left(f_{o}^{e t}, d h_{o, \delta}^{e t}\right)
\end{aligned}
$$

III.1.4.3. Lemme. - (cf. [20] (3.4)) La construction de la fin du paragraphe (III.1.1) définit une bijection naturelle

$$
\left\{\gamma \in D_{\natural}^{\times} / \gamma \text { est elliptique en } \infty \text { et de type } h \text { en o }\right\} \stackrel{\sim}{\longrightarrow} \coprod_{(\tilde{F}, \tilde{\Pi})}\left\{\delta \in \Delta_{\natural}^{\times}\right\}
$$

où $(\tilde{F}, \tilde{\Pi})$ décrit l'ensemble des $(D, \infty, o)$-type associé à la strate $h$.

III.1.4.4 - Soit alors $\gamma \in D_{\natural}^{\times}$elliptique en $\infty$ et de type $h$ en $o$ et soit $((\tilde{F}, \tilde{\Pi}), \delta)$ le triplet correspondant.

- On peut voir $\bar{D}_{\infty}^{\times}$comme une forme intérieure de $D_{\infty}^{\times}=G L_{d}\left(F_{\infty}\right)$ et si $\bar{\gamma} \in \bar{D}_{\infty}^{\times}$est le transfert de $\gamma \in D_{\infty}^{\times}$défini à conjugaison près, $\bar{\gamma}$ et l'image de $\delta \in \Delta^{\times}$dans $\bar{D}_{\infty}^{\times}$sont conjugués dans $\bar{D}_{\infty}^{\times}$; on peut alors identifier le centralisateur $\left(\bar{D}_{\infty}^{\times}\right)_{\bar{\gamma}}$ de $\bar{\gamma}$ dans $\bar{D}_{\infty}^{\times}$avec $\left(\Delta_{\infty}^{\times}\right)_{\delta}$.

- Comme $\Delta_{\mathbb{A}}^{\infty, o}$ est le centralisateur de $\tilde{F}$ dans $D_{\mathbb{A}}^{\infty, o}$ avec $\tilde{F} \subset F^{\prime}=F[\gamma]=\tilde{F}[\delta] \subset \Delta$, le centralisateur $\left(D_{\mathbb{A}}^{\infty, o}\right)_{\gamma}^{\times}$de $\gamma$ dans $\left(D_{\mathbb{A}}^{\infty, o}\right)^{\times}$coïncide avec $\left(\Delta_{\mathbb{A}}^{\infty, o}\right)_{\delta}^{\times}$. 
- De même comme $\Delta_{o}^{e t}\left(\right.$ resp. $\Delta_{o}^{c}$ ) est le centralisateur de $\tilde{F}_{o}^{\tilde{o}}$ (resp. $\left.\tilde{F}_{\tilde{o}}\right)$ dans $\mathbb{M}_{d-h}\left(F_{o}\right)$ (resp. $\left.D_{o, h}\right)$, le centralisateur $G L_{d-h}\left(F_{o}\right)_{\gamma_{o}^{e t}}\left(\operatorname{resp} .\left(D_{o, h}\right)_{\gamma_{o}^{c}}^{\times}\right)$de $\gamma_{o}^{\text {et }}$ (resp. $\left.\gamma_{o}^{c}\right)$ dans $G L_{d-h}\left(F_{o}\right)$ (resp. $\left.D_{o, h}^{\times}\right)$coïncide avec $\left(\Delta_{o}^{e t}\right)_{\delta}^{\times}\left(\operatorname{resp} .\left(\Delta_{o}^{c}\right)_{\delta}^{\times}\right)$.

III.1.4.5 - La mesure de Haar $d h_{\delta}=d \bar{h}_{\infty, \delta} d h_{\delta}^{\infty, o} \times d h_{o, \delta}^{e t} \times d \bar{h}_{o, \delta}$ sur $\left(\Delta^{\times}\right)_{\delta}$ définit alors une mesure de Haar $d h_{\gamma}^{o}$ (resp. $d h_{o, \gamma}^{e t}$, resp. $\left.d \bar{h}_{o, \gamma}\right) \operatorname{sur}\left(D_{\mathbb{A}}^{o}\right)_{\gamma}^{\times}\left(\operatorname{resp} . G L_{d-h}\left(F_{o}\right)_{\gamma}\right.$, resp. $\left.\left(D_{o, h}\right)_{\gamma}^{\times}\right)$. Soit comme précédemment $\gamma \in D_{\natural}^{\times}$elliptique en $\infty$ et de type $h$ en $o$ et soit $((\tilde{F}, \tilde{\Pi}), \delta)$ le triplet associé. Le centralisateur $D_{\gamma}^{\times}$de $\gamma$ dans $D^{\times}$est une forme intérieure du centralisateur $\Delta_{\delta}^{\times}$de $\delta$ dans $\Delta^{\times}$.

III.1.4.6. Lemme. - On a

$$
\operatorname{vol}\left(\Delta_{\delta}^{\times} \backslash\left(\Delta_{\mathbb{A}}^{\times}\right)_{\delta} / \varpi_{\infty}^{\mathbb{Z}}, \frac{d \delta_{\mathbb{A}, \delta}}{d \delta^{\prime}}\right)=\operatorname{vol}\left(D_{\gamma}^{\times} \backslash\left(D_{\mathbb{A}}^{\times}\right)_{\gamma} / \varpi_{\infty}^{\mathbb{Z}}, \frac{d h_{\mathbb{A}, \gamma}}{d h_{\gamma}}\right)
$$

Démonstration. - cf. [20] (3.5).

Soit $D_{\natural_{h}}^{\times}$un système de représentants des classes de conjugaisons de $D^{\times}$elliptiques en $\infty$ et de type $h$ en $o$.

Remarque : - Soit $\gamma \in D_{\sharp_{h}}^{\times}$et $\delta \in \Delta_{\natural}^{\times}$l'élément qui lui est associé d'après le lemme (III.1.4.3). L'image de $\delta$ par l'inclusion $\left(D^{\infty, o}\right)^{\times} \hookrightarrow\left(D_{\mathbb{A}}^{\infty, o}\right)^{\times}$est conjuguée à $\gamma \in D^{\times} \subset\left(D_{\mathbb{A}}^{\infty, o}\right)^{\times}$. De même en notant $\delta_{o}^{e t}\left(\operatorname{resp} . \bar{\delta}_{o}\right)$ l'image de $\delta$ dans $\Delta_{o}^{e t} \hookrightarrow \mathbb{M}_{d-h}\left(F_{o}\right)$ (resp. $\Delta_{o}^{c} \hookrightarrow D_{o, h}$ ), si $g_{o}^{c}$ est un élément semi-simple de $G L_{h}\left(F_{o}\right)$ de même polynôme caractéristique que $\delta_{o}^{c}$, alors $\left(g_{o}^{e t}, g_{o}^{c}\right) \in G L_{d}\left(F_{o}\right)$ est conjugué à $\gamma_{o} \in D_{o}^{\times}$.

- Soit $g_{o}^{e t} \in G L_{d-h}\left(F_{o}\right)$ (resp. $\bar{g}_{o} \in D_{o, h}^{\times}$) dans le support de $f_{o}^{e t}$ (resp. de $\bar{f}_{o}$ ). Si $g_{o}^{c}$ est un élément semi-simple de $G L_{h}\left(F_{o}\right)$ de même polynôme caractéristique que $\bar{g}_{o}$, le centralisateur de $\left(g_{o}^{e t}, g_{o}^{c}\right)$ dans $G L_{d}\left(F_{o}\right)$ est le même que dans $G L_{d-h}\left(F_{o}\right) \times G L_{h}\left(F_{o}\right)$; il suffit en effet de remarquer que l'on a pris $r>0$ pour la définition de $\bar{f}_{o}$. Ainsi étant donnée une mesure de Haar $d h_{\left(g_{o}^{e t}, g_{o}\right)}$ sur $G L_{d}\left(F_{o}\right)_{\left(g_{o}^{e t}, g_{o}^{c}\right)}$, on lui associe les mesures de Haar $d h_{g_{o}^{e t}}$ et $d h_{g_{o}^{c}}$. On obtient alors la proposition suivante.

III.1.4.7. Proposition. - On considère, comme précédemment, les mesures de Haar $d \bar{h}_{\infty}$, $d h^{\infty}$. Pour $\gamma \in D_{\bigsqcup_{h}}^{\times}$, on fixe une mesure de Haar $d h_{\mathbb{A}, \gamma}=d h_{\infty, \gamma} \times d h_{\gamma}^{\infty}$ sur le centralisateur $\left(D_{\mathbb{A}}^{\times}\right)_{\gamma} / \varpi_{\infty}^{\mathbb{Z}}$ dans $D_{\mathbb{A}}^{\times} / \varpi_{\infty}^{\mathbb{Z}}$ et soit dh $h_{\gamma}$ la mesure de comptage sur $D_{\gamma}^{\times}$. Soit $\bar{\gamma} \in \bar{D}_{\infty}^{\times}$, défini à conjugaison près, le transfert de $\gamma$; son centralisateur $\left(\bar{D}_{\infty}^{\times}\right)_{\bar{\gamma}}$ dans $\bar{D}_{\infty}^{\times}$est une forme intérieure de $\left(D_{\infty}^{\times}\right)_{\gamma}$; soit alors $d \bar{h}_{\infty, \bar{\gamma}}$ le transfert de $d h_{\infty, \gamma}$, la mesure de Haar sur $\left(\bar{D}_{\infty}^{\times}\right)_{\bar{\gamma}} / \varpi_{\infty}^{\mathbb{Z}}$. On note

$$
\epsilon_{\infty}(\gamma)=(-1) \frac{d}{\left[F_{\infty}[\gamma]: F_{\infty}\right]}-1
$$

le signe de Kottwitz à l'infini de $\gamma$.

En posant $f^{\infty}=f^{\infty, o} f_{o}^{\text {et }}$, le nombre de Lefschetz $\operatorname{Lef}_{r}^{=h}\left(\bar{g}_{\infty}, \delta_{o}, g_{o}^{c}, g^{\infty, \tilde{o}}, s\right)$ est alors donné par

$$
\begin{gathered}
\sum_{\gamma \in D_{\sharp_{h}}^{\times} \cap C_{h}\left(\Pi_{o, h}^{\mathrm{val}\left(\operatorname{det} g_{o}^{c}\right)+r} \delta_{o}^{-1}\right)} \epsilon_{\infty}(\gamma) \operatorname{vol}\left(D_{\gamma}^{\times} \backslash\left(D_{\mathbb{A}}\right)_{\gamma}^{\times} / \varpi_{\infty}^{\mathbb{Z}}, \frac{d h_{\mathbb{A}, \gamma}}{d h_{\gamma}}\right) \\
O_{\bar{\gamma}}\left(\bar{f}_{\infty}, d \bar{h}_{\infty, \bar{\gamma}}\right) O_{\gamma^{o}}\left(f^{o}, d h_{\gamma}^{o}\right) O_{g_{o}^{e t}}\left(f_{o}^{e t}, d h_{g_{o}^{e t}}\right)
\end{gathered}
$$

où : - $\left.C_{h}\left(\Pi_{o, h}^{\alpha} \delta_{o}^{-1}\right)\right)$ désigne l'ensemble des $\gamma$ conjugués à un élément de la forme $\left(g_{o}^{e t}, g_{o}^{d}\right)$ avec $g_{o}^{e t} \in G L_{d-h}\left(F_{o}\right)$ et $g_{o}^{d} \in G L_{h}\left(F_{o}\right)$ semi-simple de même polynôme caractéristique que $\Pi_{o, h}^{\alpha} \delta_{o}^{-1}$;

- dh $h_{g_{o}^{e t}}$ et $d h_{g_{o}^{c}}$ sont respectivement les mesures de Haar sur $G L_{d-h}\left(F_{o}\right)_{g_{o}^{e t}}$ et $G L_{h}\left(F_{o}\right)$ associées à la mesure de Haar $d h_{o, \gamma}$ par le procédé rappelé ci-dessus. 
III.1.5. Formule des traces de Lefschetz. — On fixe une représentation irréductible

$$
\rho_{\infty}: \bar{D}_{\infty}^{\times} / \varpi_{\infty}^{\mathbb{Z}} \longrightarrow G L(L)
$$

sur un $\overline{\mathbb{Q}}_{l}$-espace vectoriel de dimension finie $L$, qui est définie sur une extension finie $E_{\lambda}$ de $\mathbb{Q}_{l}$ dans $\overline{\mathbb{Q}}_{l}$ et qui est continue pour la topologie pro-finie sur $\bar{D}_{\infty}^{\times}$et la topologie $l$-adique sur $G L(L)$. Ainsi $\rho_{\infty}$ se factorise à travers un quotient fini $\left(\bar{D}_{\infty}^{\times} / \varpi_{\infty}^{\mathbb{Z}}\right) / \bar{K}_{\infty}$, pour $\bar{K}_{\infty}$ un sous-groupe ouvert normal. Le revêtement $\widetilde{\mathcal{J}_{I^{o}}=h}(s) \longrightarrow \mathcal{J}_{I^{o}, m}^{=h}(s)$ et $\rho_{\infty}$ définissent un $\overline{\mathbb{Q}}_{l}$-faisceau localement constant $\mathcal{L}_{\rho_{\infty}} \operatorname{sur} \mathcal{J}_{I^{o}, m}^{=h}(s)$.

III.1.5.1 - Soit de même $\tau_{o}$ une représentation irréductible admissible de $D_{o, h}^{\times}$et $\mathcal{F}_{\tau_{o}}$ le système local sur $\mathcal{I}_{I^{o}, m}^{=h}$ associé à la restriction de $\tau_{o}$ à $\mathcal{D}_{o, h}^{\times}$et au revêtement d'Igusa de seconde espèce $\mathcal{J}_{I^{o}, m}^{=h}(\infty) \longrightarrow \mathcal{I}_{I^{o}, m}^{=h}$. Soit $\mathcal{H}_{I^{o}, m}^{\infty, \tilde{o}}$ la $\mathbb{Q}$-algèbre des fonctions localement constantes à support compact

$$
f^{\infty}:\left(D_{\mathbb{A}}^{\infty, o}\right)^{\times} \times G L_{d-h}\left(F_{o}\right) \longrightarrow \mathbb{Q}
$$

qui sont $K_{I^{o}}^{\infty, o} \times K_{o, m}$-bi-invariantes par translations à droite et à gauche; le produit est donné par le produit de convolution avec la mesure de Haar $d h^{\infty, o} \times d h_{o}^{e t}$. Une base de $\mathcal{H}_{I^{o}, m}^{\infty, \tilde{o}}$ est donnée par les fonctions caractéristiques

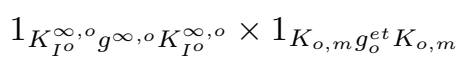

des doubles classes $K_{I^{o}}^{\infty, o} g^{\infty, o} K_{I^{o}}^{\infty, o} \subset\left(D_{\mathbb{A}}^{\infty, o}\right)^{\times}$(resp. $K_{o, m} g_{o}^{e t} K_{o, m} \subset G L_{d-h}\left(F_{o}\right)$ ), où $g^{\infty, o}$ (resp. $\left.g_{o}^{e t}\right)$ décrit un système de représentants de ces doubles classes. Pour tout $\left(g^{\infty, o}, g_{o}^{e t}\right) \in\left(D_{\mathbb{A}}^{\infty, o}\right)^{\times} \times$ $G L_{d-h}\left(F_{o}\right)$, on a une correspondance de Hecke

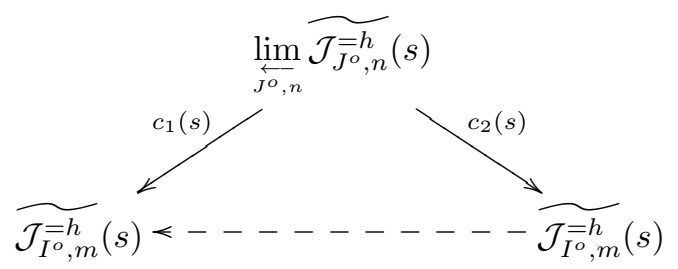

Cette correspondance agit sur chaque

$$
H_{h, I^{o}, m, \rho_{\infty}, \tau_{o}}^{i}:=H_{c}^{i}\left(\mathcal{I}_{I^{o}, m}^{=h} \otimes_{\kappa(o)} \bar{\kappa}(o), \mathcal{L}_{\rho_{\infty}} \otimes \mathcal{F}_{\tau_{o}}\right)
$$

et cette action dépend seulement des doubles classes

$$
K_{I^{o}}^{\infty, o} g^{\infty, o} K_{I^{o}}^{\infty, o} \times K_{o, m} g_{o}^{e t} K_{o, m}
$$

III.1.5.2 - On obtient ainsi une action de $\mathcal{H}_{I^{o}, m}^{\infty, \tilde{o}}$ sur $H_{h, I^{o}, m, \rho_{\infty}, \tau_{o}}^{i}$. D'après la remarque (III.1.3.1), $C_{c}\left(D_{o, h}^{\times} / \mathcal{D}_{o, h}^{\times}\right)$agit sur $H_{h, I^{o}, m, \rho_{\infty}, \tau_{o}}^{i}$ et son action commute à celle de $\mathcal{H}_{I^{o}, m}^{\infty, \tilde{o}}$. Dans le groupe de Grothendieck de $\mathcal{H}_{I^{o}, m}^{\infty, \tilde{o}} \times C_{c}\left(D_{o, h}^{\times} / \mathcal{D}_{o, h}^{\times}\right)$, on note

$$
\left[H_{h, I^{o}, m, \rho_{\infty}, \tau_{o}}^{*}\right]:=\sum_{i=0}^{2 d-2 h}(-1)^{d-h-i}\left[H_{h, I^{o}, m, \rho_{\infty}, \tau_{o}}^{i}\right]
$$

Avec $\bar{K}_{\infty}$ choisi comme ci-dessus, on note aussi

$$
\left[{\widetilde{H_{h, I^{o}, m, s}}}^{*}\right]:=\sum_{i=0}^{2 d-2 h}(-1)^{d-h-i}\left[H_{c}^{i}\left(\widetilde{\mathcal{J}_{I^{o}, m} h(s)} \otimes_{\kappa(o)} \bar{\kappa}(o), \overline{\mathbb{Q}}_{l}\right)\right]
$$

dans le groupe de Grothendieck de $\left(\bar{D}_{\infty}^{\times} / \varpi_{\infty}^{\mathbb{Z}}\right) \times \mathcal{H}_{I^{o}, m}^{\infty, \tilde{o}} \times \widetilde{\mathcal{N}}_{o}$. On notera bien que $\widetilde{\mathcal{N}}_{o} / \mathcal{D}_{o, h}^{\times}$est isomorphe à $G L_{h}\left(F_{o}\right) \times W_{F_{o}}$. Soit $W$ (resp. $V$ ) l'espace vectoriel sous-jacent à $\tau_{o}$ (resp. $\left.\rho_{\infty}\right)$ et soit $s \geqslant 0$ tel que $\tau_{o}$ est trivial sur $\left(1+\Pi_{o, h}^{s+1} \mathcal{D}_{o, h}\right)$, alors par définition des systèmes locaux,

$$
H_{c}^{i}\left(\mathcal{I}_{I^{o}, m}^{=h} \otimes_{\kappa(o)} \bar{\kappa}(o), \mathcal{L}_{\rho_{\infty}} \otimes \mathcal{F}_{\tau_{o}}\right)
$$


est isomorphe à

$$
\left.\left(H_{c}^{i} \widetilde{\left(\widetilde{\mathcal{J}_{I^{o}}}, m\right.}(s), \overline{\mathbb{Q}}_{l}\right) \otimes(V \otimes W)\right)^{\left(\left(\bar{D}_{\infty}^{\times} / \varpi_{\infty}^{\mathbb{Z}}\right) / \bar{K}_{\infty}\right) \times \mathcal{D}_{o, h}^{\times} /\left(1+\Pi_{o, h}^{s+1} \mathcal{D}_{o, h}\right)}
$$

où $\mathcal{D}_{o, h}^{\times} /\left(1+\Pi_{o, h}^{s+1} \mathcal{D}_{o, h}\right)\left(\right.$ resp. $\left.\left(\bar{D}_{\infty}^{\times} / \varpi_{\infty}^{\mathbb{Z}}\right) / \bar{K}_{\infty}\right)$ agit sur $W($ resp. $v)$ via $\tau_{o}$ (resp. $\left.\rho_{\infty}\right)$. En particulier, on a

$$
\begin{gathered}
\operatorname{Tr}\left(1_{n} \times 1_{K_{\mathbb{A}, I^{o}}^{\infty, o} g^{\infty, o} K_{\mathbb{A}, I^{o}}^{\infty} \times} \times 1_{\left.K_{o, m} g_{o}^{e t} K_{o, m},\left[H_{h, I^{o}, m, \rho_{\infty}, \tau_{o}}^{*}\right]\right)=}\right. \\
\frac{1}{\left|\mathcal{D}_{o, h}^{\times}:\left(1+\Pi_{o, h}^{s+1} \mathcal{D}_{o, h}\right)\right|\left|\left(\bar{D}_{o}^{\times} / \varpi_{\infty}^{\mathbb{Z}}\right) / \bar{K}_{\infty}\right|} \\
\sum_{\delta_{o} \in \mathcal{D}_{o, h}^{\times} /\left(1+\Pi_{o, h}^{s+1} \mathcal{D}_{o, h}\right) \bar{g}_{\infty} \bar{K}_{\infty} \in\left(\bar{D}_{\infty}^{\times} / \varpi_{\infty}^{\mathbb{Z}}\right) / \bar{K}_{\infty}} \operatorname{Tr}\left(\rho_{\infty}\left(\bar{g}_{\infty}\right)\right) \operatorname{Tr}\left(\tau_{o}\left(\Pi_{o, h}^{n} \delta_{o}\right)\right) \\
\operatorname{Tr}\left(\bar{g}_{\infty} \times\left(g_{o}^{c}, \Pi_{o, h}^{n} \delta_{o}, \operatorname{Frob}_{o}^{r}\right) \times 1_{\left.K_{\mathbb{A}, I^{o}}^{\infty} g^{\infty, o} K_{\mathbb{A}, I^{o}}^{\infty} \times 1_{K_{o, m} g_{o}^{e t} K_{o, m}},[\underbrace{}_{h, I^{o}, m, s}]\right)}^{*}\right)
\end{gathered}
$$

où $g_{o}^{c} \in G L_{h}\left(F_{o}\right)$ et $r \in \mathbb{Z}$ sont tels que $\left(g_{o}^{c}, \Pi_{o, h}^{n}, \operatorname{Frob}_{o}^{r}\right) \in \widetilde{\mathcal{N}}_{o}$. En appliquant la formule des traces de Lefschetz, on obtient la proposition suivante

III.1.5.3. Proposition. - Avec les notations précédentes,

$$
\operatorname{Tr}\left(1_{n} \times 1_{K_{\mathbb{A}, I^{o}}^{\infty, o} g^{\infty, o} K_{\mathbb{A}, I^{o}}^{\infty, o}}^{\infty} \times 1_{K_{o, m} g_{o}^{e t} K_{o, m}},\left[H_{h, I^{o}, m, \rho_{\infty}, \tau_{o}}^{*}\right]\right)
$$

est donné par la formule

$$
\begin{aligned}
& \frac{1}{\left|\mathcal{D}_{o, h}^{\times}:\left(1+\Pi_{o, h}^{s+1} \mathcal{D}_{o, h}\right)\right|\left|\left(\bar{D}_{\infty}^{\times} / \varpi_{\infty}^{\mathbb{Z}}\right) / \bar{K}_{\infty}\right|} \sum_{\delta_{o} \in \mathcal{D}_{o, h}^{\times} /\left(1+\Pi_{o, h}^{s+1} \mathcal{D}_{o, h}\right)} \sum_{\bar{g}_{\infty} \bar{K}_{\infty} \in\left(\bar{D}_{\infty}^{\times} / \varpi_{\infty}^{\mathbb{Z}}\right) / \bar{K}_{\infty}} \operatorname{Lef}_{r}^{=h}\left(\bar{g}_{\infty}, \delta_{o}, g_{o}^{c}, g^{\infty, \tilde{o}}, s\right) \operatorname{Tr}\left(\tau_{o}\left(\Pi_{o, h}^{n} \delta_{o}\right)\right) \operatorname{Tr}\left(\rho_{\infty}\left(\bar{g}_{\infty}\right)\right)
\end{aligned}
$$

soit en remplaçant $\operatorname{Lef}_{r}^{=h}\left(\bar{g}_{\infty}, \delta_{o}, g_{o}, g^{\infty, \tilde{o}}, s\right)$ par sa valeur :

$$
\begin{array}{r}
\sum_{\gamma \in D_{\natural_{h}}^{\times} \cap C_{h}\left(\Pi_{o, h}^{\operatorname{val}\left(\operatorname{det} g_{o}^{c}\right)+r} \delta_{o}^{-1}\right)} \epsilon_{\infty}(\gamma) \operatorname{vol}\left(D_{\gamma}^{\times} \backslash\left(D_{\mathbb{A}}\right)_{\gamma}^{\times} /\left(\varpi_{\infty}^{\mathbb{Z}}\right), \frac{d h_{\mathbb{A}, \gamma}}{d h_{\gamma}}\right) \\
O_{\bar{\gamma}}\left(\bar{f}_{\infty}, d \bar{h}_{\infty, \gamma}\right) O_{\gamma}\left(f^{\infty, o}, d h_{\gamma}^{\infty, o}\right) O_{g_{o}^{e t}}\left(f_{o}^{e t}, d h_{g_{o}^{e t}} \frac{\operatorname{Tr} \tau_{o}\left(\Pi_{o, h}^{-\alpha} \delta_{o}\right)}{\operatorname{vol}\left(\mathcal{D}_{o, h}^{\times}, d \bar{h}_{o}\right)}\right.
\end{array}
$$

ò̀ :

- les mesures de Haar $d h^{\infty}, d h_{\mathbb{A}, \gamma}, d h_{\gamma}^{\infty}, d h_{\gamma}$, et $d \bar{h}_{\infty, \gamma}$ sont choisis comme précédemment, - $\bar{h}_{\infty}$ est arbitraire avec

$$
\bar{f}_{\infty}=\frac{\xi_{\rho_{\infty}}}{\operatorname{vol}\left(\bar{D}_{\infty}^{\times} / \varpi_{\infty}^{\mathbb{Z}}, d \bar{h}_{\infty}\right)}
$$

où $\xi_{\rho_{\infty}}$ est le caractère de $\rho_{\infty}$.

Remarque : Le produit $\bar{f}_{\infty} d \bar{h}_{\infty}$ est indépendant du choix de $d \bar{h}_{\infty}$.

\section{III.1.6. Transfert des intégrales orbitales. -}

III.1.6.1. Définition. - Une représentation $\pi_{x}$ de $G L_{d}\left(F_{x}\right)$ est dite de carré intégrable (resp. essentiellement de carré intégrable) si $\pi_{x}$ (resp. s'il existe un caractère $\psi_{x}$ tel que $\psi_{x} \otimes \pi_{x}$ ) a un coefficient matriciel de carré intégrable sur $G L_{d}\left(F_{x}\right) / F_{x}^{\times}$. 
A la place $\infty$ : On suit de très près le paragraphe (13.8) de [19]. D'après [1] , il existe une unique représentation irréductible $\pi_{\infty}$ de $D_{\infty}^{\times} / \varpi_{\infty}^{\mathbb{Z}}$ essentiellement de carré intégrable qui est déterminée par la relation suivante sur la restriction des caractères aux éléments elliptiques réguliers; si $\gamma \in D_{\infty}^{\times} / \varpi_{\infty}^{\mathbb{Z}}$ est elliptique régulier ${ }^{(6)}$ correspondant à un élément $\bar{\gamma} \in \bar{D}_{\infty}^{\times} / \omega_{\infty}^{\mathbb{Z}}$ par transfert, alors

$$
\xi_{\pi_{\infty}}(\gamma)=(-1)^{d-1} \xi_{\rho_{\infty}}(\bar{\gamma})
$$

D'après [24], $\pi_{\infty}$ est de la forme $\operatorname{St}_{2 t+1}\left(\pi_{\infty}^{\prime}\right)$, où $\pi_{\infty}^{\prime}$ est une représentation cuspidale de $G L_{d^{\prime}}\left(F_{\infty}\right)$ avec $d=d^{\prime}(2 t+1)$ et $t \in \frac{1}{2} \mathbb{Z}$, où l'on rappelle que $\mathrm{St}_{2 t+1}\left(\pi_{\infty}^{\prime}\right)$ est l'unique sous-représentation irréductible de la représentation induite normalisée (cf. ci-après)

$$
\pi_{\infty}^{\prime}(t) \times \cdots \times \pi_{\infty}^{\prime}(-t)
$$

En outre cette dernière induite possède un unique quotient irréductible que l'on note $\operatorname{Speh}_{2 t+1}\left(\pi_{\infty}^{\prime}\right)$.

La proposition suivante est bien connue (cf. par exemple [14] lemme I.3.4 (3))

III.1.6.2. Proposition. - Il existe une fonction $f_{\pi_{\infty}}$ sur $D_{\infty}^{\times} / \varpi_{\infty}^{\mathbb{Z}}$, localement constante, à support compact, vérifiant les propriétés suivantes :

(i) Les intégrales orbitales non elliptiques de $f_{\pi_{\infty}}$ sont nulles; pour $\gamma \in D_{\infty}^{\times}$elliptique, on a

$$
O_{\gamma}\left(f_{\pi_{\infty}}, d h_{\infty, \gamma}\right)=\epsilon_{\infty}(\bar{\gamma}) O_{\bar{\gamma}}\left(\bar{f}_{\infty}, d \bar{h}_{\infty, \gamma}\right)
$$

où $\bar{f}_{\infty}$ et les mesures de Haar sont définies comme au paragraphe précédent.

(ii) Pour une représentation irréductible $\tilde{\pi}_{\infty}$ de $D_{\infty}^{\times} / \varpi_{\infty}^{\mathbb{Z}}$, on a

$$
\operatorname{Tr} \tilde{\pi}_{\infty}\left(f_{\pi_{\infty}}\right)=\left\{\begin{array}{l}
1 \text { si } \tilde{\pi}_{\infty} \simeq \pi_{\infty}=\operatorname{St}_{2 t+1}\left(\pi_{\infty}^{\prime}\right) \\
(-1)^{2 t} \text { si } \tilde{\pi}_{\infty} \simeq \operatorname{Speh}_{2 t+1}\left(\pi_{\infty}^{\prime}\right) \\
0 \text { sinon }
\end{array}\right.
$$

A la place o : On reprend textuellement les résultats de [14] VI.5 On rappelle que $P_{h, d} \subset$ $G L_{d}$ est le sous-groupe parabolique constitué des matrices triangulaires supérieures par blocs de Levi $G L_{h} \times G L_{d-h}$. Soit alors $N_{h, d}$ son radical unipotent. On note $N_{h, d}^{o p}$ le radical unipotent du parabolique opposé $P_{h, d}^{o p}$ de $P_{h, d}$. Pour $\pi_{o}$ une représentation irréductible admissible de $G L_{d}\left(F_{o}\right)$ et $P$ un parabolique de radical unipotent $N$, le module de Jacquet $J_{N}\left(\pi_{o}\right)$ est la représentation admissible $\pi_{o, N} \otimes \delta_{P}^{1 / 2}$ du groupe $(P / N)\left(F_{o}\right)$ dont l'espace sous-jacent est l'espace des $N\left(F_{o}\right)$ coinvariants de l'espace de $\pi_{o}$ et

$$
\delta_{P}(h)=\left|\operatorname{det}\left(\operatorname{ad}(h)_{\mid \operatorname{LieN}}\right)\right|_{F_{o}} .
$$

Si $\pi_{o}$ est une représentation admissible de $(P / N)\left(F_{o}\right)$, on note

$$
n-\operatorname{Ind}_{P\left(F_{o}\right)}^{G L_{d}\left(F_{o}\right)}\left(\pi_{o}\right):=\operatorname{Ind}_{P\left(F_{o}\right)}^{G L_{d}\left(F_{o}\right)}\left(\pi_{o} \otimes \delta_{P}^{1 / 2}\right) .
$$

Pour $\pi_{o}$ une représentation irréductible admissible essentiellement de carré intégrable de $G L_{d}\left(F_{o}\right)$, de caractère central $\psi_{\pi_{o}}$, Deligne, Kazhdan et Vigneras (cf. [1] ) montrent l'existence d'une fonction $\phi_{\pi_{o}} \in C_{c}^{\infty}\left(G L_{d}\left(F_{o}\right), \psi_{\pi_{o}}^{-1}\right)$, que l'on appelle un pseudo-coefficient de $\pi_{o}$, vérifiant les propriétés suivantes :

$-\operatorname{Tr} \pi_{o}\left(\phi_{\pi_{o}}\right)=\operatorname{vol}\left(D_{o, d}^{\times} / F_{o}^{\times}\right){ }^{(7)} ;$

- si $P$ est un sous-groupe parabolique de $G L_{d}$ de Levi $G L_{d_{1}} \times \cdots \times G L_{d_{s}}, s>1$. Supposons donnés des représentations irréductibles admissibles essentiellement de carré intégrable $\pi_{o, i}$ de $G L_{d_{i}}\left(F_{o}\right)$ tels que $\psi_{\pi_{o, 1}} \cdots \psi_{\pi_{o, s}}=\psi_{\pi_{o}}$, alors

$$
\operatorname{Tr}\left(n-\operatorname{Ind}_{P\left(F_{o}\right)}^{G L_{d}\left(F_{o}\right)}\left(\pi_{o, 1} \times \pi_{o, s}\right)\right)\left(\phi_{\pi_{o}}\right)=0 ;
$$

\footnotetext{
${ }^{(6)}$ i.e. $F_{\infty}[\gamma]$ est une extension séparable de degré $d$ de $F_{\infty}$

(7) où l'on considère sur $D_{o, d}^{\times}$le transfert de la mesure de Haar sur $G L_{d}\left(F_{o}\right)$
} 
- si $\gamma \in G L_{d}\left(F_{o}\right)$ est un élément semi-simple non elliptique alors

$$
O_{\gamma}^{G L_{d}\left(F_{o}\right)}\left(\phi_{\pi_{o}}\right)=0
$$

(cf. [14] $\S \mathrm{I} .3$ )

- si $\gamma \in G L_{d}\left(F_{o}\right)$ est elliptique semi-simple et si $\delta \in D_{o, d}^{\times}$a le même polynôme caractéristique que $\gamma$ alors (cf. [14] lemme I.3.1)

$$
O_{\gamma}^{G L_{d}\left(F_{o}\right)}\left(\phi_{\pi_{o}}\right)=(-1)^{d\left(1-\left[F_{o}(\gamma): F_{o}\right]^{-1}\right)} \operatorname{vol}\left(D_{o, d}^{\times} / Z_{D_{o, d}^{\times}}(\delta)\right) \operatorname{Tr} \mathrm{JL}^{-1}\left(\pi_{o}^{\vee}\right)(\delta)
$$

Soient $d h_{o}^{e t}$ et $d h_{o}$ des mesures de Haar sur respectivement $G L_{d-h}\left(F_{o}\right)$ et $G L_{d}\left(F_{o}\right)$.

III.1.6.3. Proposition. - (cf. [14] lemmes VI.5.1 et VI.5.2) Soient $\tau_{o}$ une représentation irréductible de $D_{o, h}^{\times}$et $\phi_{o}^{\text {et }} \in C_{c}^{\infty}\left(G L_{d-h}\left(F_{o}\right)\right)$; il existe alors une fonction

$$
I P C_{\tau_{o}}\left(\phi_{o}^{e t} ; d h_{o}^{e t}\right) \in C_{c}^{\infty}\left(G L_{d}\left(F_{o}\right)\right)
$$

telle que

(1) $O_{\gamma}\left(I P C_{\tau_{o}}\left(\phi_{o}^{e t} ; d h_{o}^{e t}\right)\right)$ est nulle si $\gamma$ n'appartient pas à $C_{h}\left(\Pi_{o, h}^{-\alpha} \delta_{o}\right)$ et sinon est égal à

$$
(-1)^{h-1} O_{g_{o}^{e t}}^{G L_{d-h}\left(F_{o}\right)}\left(\phi_{o}^{e t}, d h_{o, g_{o}^{e t}}^{e t}\right) \frac{\operatorname{Tr} \tau_{o}\left(\Pi_{o, h}^{-\alpha} \delta_{o}\right)}{\operatorname{vol}\left(\mathcal{D}_{o, h}^{\times}, d \bar{h}_{o}\right)}
$$

(2) En outre si $\pi_{o}$ est une représentation irréductible admissible de $G L_{d}\left(F_{o}\right)$ et si

$$
\left[J_{N_{h, d}}\left(\pi_{o}\right) \otimes \delta_{P_{h, d}}^{1 / 2}\right]=\sum_{\alpha, \beta} m_{\alpha, \beta}[\alpha \otimes \beta]
$$

où $\alpha$ (resp. $\beta)$ décrit l'ensemble des représentations irréductibles admissibles de $G L_{h}\left(F_{o}\right)$ (resp. $\left.G L_{d-h}\left(F_{o}\right)\right)$, alors

$$
\operatorname{Tr} \pi_{o}\left(I P C_{\tau_{o}}\left(\phi_{o}^{e t}, d h_{o}^{e t}\right)=\right.
$$

$$
\sum_{\alpha, \beta, \psi} \operatorname{Tr} \psi\left(\bar{\phi}_{o}\right) \frac{m_{\alpha, \beta}}{\operatorname{vol}\left(D_{o, h}^{\times} / F_{o}^{\times}, d \bar{h}_{o}\right)^{-1}} \operatorname{Tr} \alpha\left(\phi_{\mathrm{JL}\left(\tau_{o} \otimes \psi^{-1}\right)}\right) \operatorname{Tr} \beta\left(\phi_{o}^{e t}\right)
$$

où $\alpha$ (resp. $\beta$, resp. $\psi$ ) décrit les représentations irréductibles admissibles de $G L_{h}\left(F_{o}\right)$ (resp. $G L_{d-h}\left(F_{o}\right)$, resp. $\left.D_{o, h}^{\times} / \mathcal{D}_{o, h}^{\times}\right)$; la somme sur $\psi$ porte sur les $\psi$ tels que $\alpha$ et $\tau_{o} \otimes \psi^{-1}$ ont le même caractère central.

Démonstration. - Celle-ci est strictement similaire à celle de loc. cit. avec les modifications suivantes. En premier lieu on note que $\gamma$ est associé à $\Pi_{o, h}^{\alpha} \delta_{o}^{-1}$ alors que l'on considère $\operatorname{Tr} \tau_{o}\left(\Pi_{o, h}^{-\alpha} \delta_{o}\right)$; en outre $g_{o}^{c}$ induit la multiplication par $-\operatorname{val}\left(\operatorname{det} g_{o}^{c}\right)$ sur $\mathbb{Z}$, à comparer avec val( $\left.\operatorname{det} g_{o}^{c}\right)$ dans loc. cit.. On se retrouve alors avec $\mathrm{JL}\left(\tau_{o} \otimes \psi^{-1}\right)$ au lieu de $\mathrm{JL}\left(\tau_{o}^{\vee} \otimes \psi\right)$.

III.1.6.4 — On introduit alors

$$
\operatorname{Red}_{\tau_{o}}^{h}: \operatorname{Groth}\left(G L_{d}\left(F_{o}\right)\right) \longrightarrow \operatorname{Groth}\left(D_{o, h}^{\times} / \mathcal{D}_{o, h}^{\times} \times G L_{d-h}\left(F_{o}\right)\right)
$$

défini comme la composition des deux homomorphismes suivant.

- En premier lieu, on a un homomorphisme

$$
\begin{aligned}
& \operatorname{Groth}\left(G L_{d}\left(F_{o}\right)\right) \longrightarrow \operatorname{Groth}\left(G L_{h}\left(F_{o}\right) \times G L_{d-h}\left(F_{o}\right)\right) \\
& {\left[\pi_{o}\right] \mapsto\left[J_{N_{h, d}}\left(\pi_{o}\right) \otimes \delta_{P_{h, d}}^{1 / 2}\right]}
\end{aligned}
$$

- Ensuite on a un homomorphisme

$$
\begin{aligned}
& \operatorname{Groth}\left(G L_{h}\left(F_{o}\right) \times G L_{d-h}\left(F_{o}\right)\right) \longrightarrow \operatorname{Groth}\left(D_{o, h}^{\times} / \mathcal{D}_{o, h}^{\times} \times G L_{d-h}\left(F_{o}\right)\right) \\
& {[\alpha \otimes \beta] \mapsto \sum_{\psi} \operatorname{vol}\left(D_{o, h}^{\times} / F_{o}^{\times}, d \bar{h}_{o}\right)^{-1} \operatorname{Tr} \alpha\left(\phi_{\mathrm{JL}\left(\tau_{o} \otimes \psi^{-1}\right)}\right)[\psi \otimes \beta],}
\end{aligned}
$$

où $\psi$ décrit les caractères de $D_{o, h}^{\times} / \mathcal{D}_{o, h}^{\times}$tels que $\alpha$ et $\tau_{o} \otimes \psi^{-1}$ ont le même caractère central et où l'on considère des mesures de Haar associées sur $G L_{h}\left(F_{o}\right)$ et $D_{o, h}^{\times}$. 
III.1.6.5. Corollaire. — Pour $\pi_{o}$ une représentation irréductible admissible de $G L_{d}\left(F_{o}\right)$, on a

$$
\operatorname{Tr} \pi_{o}\left(I P C_{\tau_{o}}\left(\phi_{o}^{e t}, d h_{o}^{e t}\right)\right)=\operatorname{Tr} \operatorname{Red}_{\tau_{o}}^{h}\left(\pi_{o}\right)\left(\phi_{o}^{e t}\right)
$$

où $\phi_{o}^{e t} \in C_{c}^{\infty}\left(G L_{d-h}\left(F_{o}\right)\right)$.

D'après la proposition (III.1.5.3) et en remarquant que $h\left[F_{\infty}^{\prime}: F_{\infty}\right]=d\left[F_{o}^{\prime}: F_{o}\right]$ avec les notations précédentes, on obtient le résultat suivant.

III.1.6.6. Théorème. — Avec les notations précédentes, on pose

$$
f=f_{\pi_{\infty}} f^{\infty, o} I P C_{\tau_{o}}\left(f_{o}^{e t}\right) .
$$

On a alors

$$
\begin{aligned}
& \operatorname{Tr}\left(1_{n} \times 1_{K_{\mathbb{A}, I}^{\infty, o} g \infty, o}^{\infty} K_{\mathbb{A}, I}^{\infty, o} \times 1_{K_{o, m} g_{o}^{e t} K_{o, m}}, H_{h, I^{o}, m, \rho_{\infty}, \tau_{o}}^{*}\right)= \\
& \sum_{\gamma \in D_{\natural}^{\times}} \operatorname{vol}\left(D_{\gamma}^{\times} \backslash\left(D_{\mathbb{A}}\right)_{\gamma}^{\times} /\left(\varpi_{\infty}^{\mathbb{Z}}\right), \frac{d h_{\mathbb{A}, \gamma}}{d h_{\gamma}}\right) O_{\gamma}\left(f, d h_{\mathbb{A}, \gamma}\right)
\end{aligned}
$$

III.1.7. Formule des traces de Selberg. — Soit $\mathcal{A}\left(D^{\times} \backslash D_{\mathbb{A}}^{\times} /\left(\varpi_{\infty}^{\mathbb{Z}}\right)\right)$ l'espace des fonctions localement constantes muni de la représentation régulière à droite de $D_{\mathbb{A}}^{\times} /\left(\varpi_{\infty}^{\mathbb{Z}}\right)$. Comme $D$ est une algèbre à division, $D^{\times} \backslash D_{\mathbb{A}}^{\times} /\left(\varpi_{\infty}^{\mathbb{Z}}\right)$ est compact, de sorte que

$$
\mathcal{A}\left(D^{\times} \backslash D_{\mathbb{A}}^{\times} /\left(\varpi_{\infty}^{\mathbb{Z}}\right)\right)=\bigoplus_{\Pi} m(\Pi) \Pi
$$

avec $m(\Pi)$ fini et où $\Pi$ décrit l'ensemble des représentations irréductibles admissibles de $D_{\mathbb{A}}^{\times} /\left(\varpi_{\infty}^{\mathbb{Z}}\right)$. Si la multiplicité $m(\Pi)$ n'est pas nulle, la représentation $\Pi$ est dite automorphe. L'opérateur induit par une fonction localement constante à support compact sur $D_{\mathbb{A}}^{\times} /\left(\varpi_{\infty}^{\mathbb{Z}}\right)$, a une trace :

$$
\begin{aligned}
\operatorname{Tr}\left(f ; \mathcal{A}\left(D^{\times} \backslash D_{\mathbb{A}}^{\times} /\left(\varpi_{\infty}^{\mathbb{Z}}\right)\right)\right) & =\sum_{\Pi} m(\Pi) \operatorname{Tr} \Pi(f) \\
& =\sum_{\gamma \in D_{\natural}^{\times}} \operatorname{vol}\left(D_{\gamma}^{\times} \backslash\left(D_{\mathbb{A}}\right)_{\gamma}^{\times} /\left(\varpi_{\infty}^{\mathbb{Z}}\right), \frac{d h_{\mathbb{A}, \gamma}}{d h_{\gamma}}\right) O_{\gamma}\left(f, d h_{\mathbb{A}, \gamma}\right)
\end{aligned}
$$

III.1.7.1. Proposition. — Pour tout $r>0$, on a l'égalité

$$
\operatorname{Tr}\left(1_{n} \times 1_{K_{\mathbb{A}, I}^{\infty, o} g^{\infty, o} K_{\mathbb{A}, I}^{\infty, o}} \times 1_{K_{o, m} g_{o}^{e t} K_{o, m}}, H_{h, I^{o}, m, \rho_{\infty}, \tau_{o}}^{*}\right)=\sum_{\Pi} m(\Pi) \operatorname{Tr} \Pi(f)
$$

III.1.7.2 - On note $\left[H_{h, \rho_{\infty}, \tau_{o}}^{i}\right]$ l'élément du groupe de Grothendieck

$$
\operatorname{Groth}\left(\left(D_{\mathbb{A}}^{\infty, o}\right)^{\times} G L_{d-h}\left(F_{o}\right) \times D_{o, h}^{\times} / \mathcal{D}_{o, h}^{\times}\right)
$$

défini par la limite inductive sur $I^{o}$ et $m$ des $\left[H_{h, I^{o}, m, \rho_{\infty}, \tau_{o}}^{i}\right]$. En vertu de la proposition précédente, on a le corollaire suivant.

III.1.7.3. Corollaire. — Soit $\Pi$ une représentation automorphe de $D_{\mathbb{A}}^{\times}$, alors dans le groupe de Grothendieck $\operatorname{Groth}\left(G L_{d-h}\left(F_{o}\right) \times\left(D_{o, h}^{\times} / \mathcal{D}_{o, h}^{\times}\right)\right)$, on a

$$
\left[H_{h, \rho_{\infty}, \tau_{o}}^{*}\left(\Pi^{\infty, o}\right)\right]= \begin{cases}m(\Pi) \operatorname{Red}_{\tau_{o}}^{h}\left(\Pi_{o}\right) & \text { si } \Pi_{\infty} \simeq \operatorname{St}_{2 t+1}\left(\pi_{\infty}^{\prime}\right) \text { ou } \operatorname{Speh}_{2 t+1}\left(\pi_{\infty}^{\prime}\right) \\ 0 & \text { sinon }\end{cases}
$$




\section{III.2. Application à la cohomologie du modèle local}

III.2.1. Calcul de $\sum_{i}(-1)^{i} \widetilde{\Psi_{F_{o}}^{d, i}}\left(\mathrm{JL}^{-1}\left(\mathrm{St}_{s}\left(\pi_{o}\right)\right)\right)$. - Dans [5], pour une représentation cuspidale $\pi_{o}$ de $G L_{d}\left(F_{o}\right)$, on montre que

$$
\widetilde{\mathcal{U}_{F_{o}}^{d, i}}\left(\pi_{o}\right)=\left\{\begin{array}{l}
\mathrm{JL}^{-1}\left(\pi_{o}\right) \otimes L_{d}\left(\pi_{o}\right)\left(\frac{1-d}{2}\right) \text { pour } i=d-1 \\
0 \text { si } i \neq d-1
\end{array}\right.
$$

où $L_{d}$ (resp. JL) est la bijection de Langlands (resp. de Jacquet-Langlands) de l'ensemble des classes d'équivalences des représentations irréductibles essentiellement de carré intégrables de $G L_{d}\left(F_{o}\right)$ dans l'ensemble des classes d'équivalences des représentations de dimension $d$ de $W_{F_{o}}$ (resp. des représentations irréductibles admissibles de $D_{o, d}^{\times}$). Le but de ce paragraphe est d'obtenir le pendant du théorème VII.1.5 de [14], à savoir.

III.2.1. Théorème. - (cf. [14] théorème VII.1.5) Soit $d=s g$ avec $s$ et $g$ des entiers positifs, et soit $\pi_{o}$ une représentation irréductible cuspidale de $G L_{g}\left(F_{o}\right)$, alors

$$
\left.\widetilde{\left[\mathcal{U}_{F_{o}}^{d, *}\right.}\left(\mathrm{JL}^{-1}\left(\mathrm{St}_{s}\left(\pi_{o}\right)\right)\right)\right]=\sum_{j=1}^{s}(-1)^{s-j}[\overleftarrow{j-1}, \overrightarrow{s-j}]_{\pi_{o}} \otimes L_{g}\left(\pi_{o}\right)\left(-\frac{d-s+2(j-1)}{2}\right)
$$

Démonstration. - Dans le groupe de Grothendieck de $\left(D_{\mathbb{A}}^{\infty}\right)^{\times} \times W_{F_{o}}$, on note

$$
\begin{gathered}
H_{\eta_{o}, \rho_{\infty}}^{i}=\sum_{\Pi_{\infty}} \Pi^{\infty} \otimes W_{\rho_{\infty}, i}\left(\Pi^{\infty}\right) \\
{\left[H_{\eta_{o}, \rho_{\infty}}^{*}\right]:=\sum_{i=0}^{2 d-2}(-1)^{d-1-i}\left[\varliminf_{I}^{\lim } H^{i}\left(M_{I, \eta_{o}}, \mathcal{L}_{\rho_{\infty}}\right)\right]=\sum_{\Pi^{\infty}}\left[\Pi^{\infty}\right]\left[W_{\rho_{\infty}}^{*}\left(\Pi^{\infty}\right)\right]}
\end{gathered}
$$

où la somme porte sur les représentations irréductibles automorphes de $\left(D_{\mathbb{A}}^{\infty}\right)^{\times}$. On rappelle alors l'un des résultats principaux de [19].

III.2.2. Théorème. - Si $\Pi$ est une représentation automorphe de $D_{\mathbb{A}}^{\times}$telle que $\Pi_{\infty} \simeq \mathrm{St}_{\infty}$ et telle qu'il existe deux places $x_{1}, x_{2}$ de $X^{\prime}$ distinctes de $\infty$ et o avec $\Pi_{x_{i}}$ cuspidales pour $i=1,2$, alors

$$
\left[W_{1_{\infty}}^{*}\left(\Pi^{\infty}\right)_{\mid W_{o}}\right] \simeq\left[L_{d}\left(\Pi_{o}\right)\right]\left(\frac{1-d}{2}\right)
$$

où $L_{d}$ désigne la correspondance de Langlands. ${ }^{(8)}$

III.2.1.1 - La suite spectrale des cycles évanescents donne alors l'égalité

$$
\left[H_{\eta_{o}, \rho_{\infty}}^{*}\right]=\sum_{0 \leqslant j \leqslant d-1}(-1)^{j}\left[\varliminf_{I}^{\lim } H^{*}\left(M_{I, \bar{s}_{o}}, R^{j} \Psi_{\eta_{o}}\left(\mathcal{L}_{\rho_{\infty}}\right)\right)\right]
$$

Cette égalité combinée à la suite spectrale associée à la stratification donne l'égalité suivante

$$
\left[H_{\eta_{o}, \rho_{\infty}}^{*}\right]=\sum_{\substack{0 \leqslant j \leqslant-1 \\ 1 \leqslant h \leqslant d}}(-1)^{j}\left[{\underset{I}{\leftarrow}}_{I} H_{c}^{*}\left(M_{I, s_{o}}^{=h}, R^{j} \Psi_{\eta_{o}}\left(\mathcal{L}_{\rho_{\infty}}\right)\right)\right]
$$

D'après [5], pour $\mathcal{F}$ un faisceau sur $M_{\bar{I}, s_{o}}^{=h}$, on a

$$
\lim _{I} H_{c}^{i}\left(M_{I, \bar{s}_{o}}^{=h}, \mathcal{F}\right)=\operatorname{Ind}_{P_{h, d}^{o p}\left(F_{o}\right)}^{G L_{d}\left(F_{o}\right)} \underset{I}{\lim _{I}} H_{c}^{i}\left(M_{I, \bar{s}_{o}, 1}^{=h}, \mathcal{F}\right)
$$

On obtient ainsi l'égalité

$$
\left[H_{\eta_{o}, \rho_{\infty}}^{*}\right]=\sum_{\substack{0 \leqslant j \leqslant d-1 \\ 1 \leqslant h \leqslant d}}(-1)^{j}\left[\operatorname{Ind}_{P_{h, d}^{o p}\left(F_{o}\right)}^{G L_{d}\left(F_{o}\right)} \lim _{I} H_{c}^{*}\left(M_{I, s_{o}, 1}^{=h}, R^{j} \Psi_{\eta_{o}}\left(\mathcal{L}_{\rho_{\infty}}\right)\right)\right]
$$

${ }^{(8)}$ On a même la nullité des $W_{1_{\infty}, i}\left(\Pi^{\infty}\right)$ pour $i \neq d-1$ et alors l'égalité en tant que représentations et pas seulement des semi-simplifiées. 
D'après le corollaire (I.5.3.8) et le lemme (I.5.2.1) du chapitre 3, on a

$$
h\left[H_{c}^{i}\left(M_{I, \bar{s}_{o}, 1}^{=h}, R^{j} \Psi_{\eta_{o}}\left(\mathcal{L}_{\rho_{\infty}}\right)\right)\right]=\bigoplus_{\tau_{o} \in \mathfrak{C}_{h}} \frac{h}{e_{\tau_{o}}}\left[H_{c}^{i}\left(M_{I, \bar{s}_{o}, 1}^{=h}, \mathcal{L}_{\rho_{\infty}} \otimes \mathcal{F}_{\tau_{o}}\right)\right] *_{d_{h}}\left[\widetilde{\mathcal{U}_{F_{o}, n}^{h, j}}\left(\tau_{o}\right)\right]
$$

où $n$ est la multiplicité de $o$ dans $I$, où

$$
d_{h}:\left\{\begin{array}{l}
G L_{h}\left(F_{o}\right) \times W_{F_{o}} \longrightarrow\left(D_{\mathbb{A}}^{\infty, o}\right)^{\times} \times G L_{d-h}\left(F_{o}\right) \times D_{o, h}^{\times} / \mathcal{D}_{o, h}^{\times} \\
\left(g_{o}^{c}, \sigma_{o}\right) \longmapsto\left(1,1, \delta_{o}\right)
\end{array}\right.
$$

où $\delta_{o}$ est tel que $\operatorname{val}\left(\operatorname{rn}\left(\delta_{o}\right)\right)=-\operatorname{val}\left(\operatorname{det}\left(g_{o}^{c}\right)\right)-\operatorname{deg}\left(\sigma_{o}\right)$ et où pour $\pi_{i}$ une représentation de $G_{i}$ pour $i=1,2$ et $d: G_{2} \longrightarrow Z\left(G_{1}\right)$, on note $\pi_{1} \otimes_{d} \pi_{2}$ la représentation de $G_{1} \times G_{2}$ définie par

$$
\left(\pi_{1} \otimes_{d} \pi_{2}\right)\left(g_{1}, g_{2}\right)=\pi_{1}\left(g_{1} d\left(g_{2}\right)\right) \otimes \pi_{2}\left(g_{2}\right)
$$

dans le groupe de Grothendieck de $G_{1} \times G_{2}$, on note $\left[\pi_{1}\right] *_{d}\left[\pi_{2}\right]$ l'image de $\left[\pi_{1} \otimes_{d} \pi_{2}\right]$. Cette égalité est vue dans

$$
\operatorname{Groth}\left(\left(D_{\mathbb{A}}^{\infty, o}\right)^{\times} \times G L_{d-h}\left(F_{o}\right) \times G L_{h}\left(F_{o}\right) \times W_{F_{o}}\right)
$$

via l'application évidente

$$
\operatorname{Groth}\left(\left(D_{\mathbb{A}}^{\infty, o}\right)^{\times} \times G L_{d-h}\left(F_{o}\right) \times D_{o, h}^{\times} / \mathcal{D}_{o, h}^{\times}\right) \longrightarrow \operatorname{Groth}\left(\left(D_{\mathbb{A}}^{\infty, o}\right)^{\times} \times G L_{d-h}\left(F_{o}\right)\right)
$$

Soient alors $g$ un diviseur de $d=s g$ et $\pi_{o}$ une représentation irréductible cuspidale de $G L_{g}\left(F_{o}\right)$. On considère $\Pi$ une représentation irréductible de $D_{\mathbb{A}}^{\times}$vérifiant les conditions du théorème (III.2.2) et telle que $\Pi_{o} \simeq \operatorname{St}_{s}\left(\pi_{o}\right)^{(9)}$.

III.2.1.2. Lemme. - On a

$$
\operatorname{Red}_{\mathrm{JL}^{-1}\left(\mathrm{St}_{s}\left(\pi_{o}\right)\right)}\left([\overleftarrow{s-1}]_{\pi_{o}}\right)=e_{\mathrm{JL}^{-1}\left(\mathrm{St}_{s}\left(\pi_{o}\right)\right)}[\overleftarrow{s-l-1}]_{\pi_{o}\left(\frac{l(g-1)}{2}\right)} \otimes \Xi^{\frac{(s-l)(g-1)}{2}}
$$

où $\Xi$ est le caractère multiplicatif de $\mathbb{Z}$ tel que $\Xi(1)=\frac{1}{p}$.

Démonstration. — Le résultat découle directement de [24] 2.2. En effet on a

$$
J_{N_{l g, d}}\left([\overleftarrow{s-1}]_{\pi_{o}}=[\overleftarrow{l-1}]_{\pi_{o}\left(\frac{(s-l)}{2}\right)} \otimes[\overleftarrow{s-l-1}]_{\pi_{o}\left(\frac{-l}{2}\right)}\right.
$$

de sorte qu'après multiplication par $\delta_{P_{l g, d}}^{1 / 2}$ on obtient

$$
[\overleftarrow{l-1}]_{\pi_{o}\left(-\frac{(s-l)(g-1)}{2}\right)} \otimes[\overleftarrow{s-l-1}]_{\pi_{o}\left(\frac{l(g-1)}{2}\right)}
$$

d'où le résultat.

Ainsi en combinant (III.2.1.4), (III.2.1.5) avec les lemmes précédents, on obtient

$$
\begin{aligned}
{[\overleftarrow{s-1}]_{\pi_{o}} \otimes\left[L_{g}\left(\pi_{o}\right) \otimes \mathrm{Sp}_{s}\right] } & = \\
& \left.\sum_{l=1}^{s} \widetilde{\mathcal{U}_{F_{o}}^{l g, *}}\left(\mathrm{JL}^{-1}\left(\operatorname{St}_{l}\left(\pi_{o}\right)\right)\right)\right]\left(-\frac{(s-l)(g-1)}{2}\right) \overrightarrow{\times}[\overleftarrow{s-l-1}]_{\pi_{o}}
\end{aligned}
$$

où $\left(-\frac{(s-l)(g-1)}{2}\right)$ est la torsion sur la partie galoisienne ${ }^{(10)}$ et $\left[\mathrm{Sp}_{s}\right]=1\left(\frac{1-s}{2}\right)+\cdots+1\left(\frac{s-1}{2}\right)$. On suppose alors, par récurrence sur $s$, que pour tout $1 \leqslant l<s$, on ait

$$
\left.\widetilde{\mathcal{U}_{F_{o}}^{l g, *}}\left(\mathrm{JL}^{-1}\left(\mathrm{St}_{l}\left(\pi_{o}\right)\right)\right)\right]=\sum_{r=0}^{l-1}(-1)^{r}[\overleftarrow{l-1-r}, \vec{r}]_{\pi_{o}} \otimes L_{g}\left(\pi_{o}\right)\left(-\frac{l(g-1)+2(l-r-1)}{2}\right)
$$

${ }^{(9)}$ pour l'existence d'une telle globalisation cf. [15]

${ }^{(10)}$ Celle sur $G L_{l g}\left(F_{o}\right)$ est contenu dans le symbole $\vec{x}$. 
On réinjecte ces égalités dans (III.2.1.6) ce qui donne en rassemblant selon les poids :

$$
\begin{aligned}
& \left.\widetilde{\mathcal{U}_{F_{o}}^{d, *}}\left(\mathrm{JL}^{-1}\left(\mathrm{St}_{s}\left(\pi_{o}\right)\right)\right)\right]=\sum_{l=0}^{s-1} L_{g}\left(\pi_{o}\right)\left(-\frac{s(g-1)+2 l}{2}\right) \\
& {[\overleftarrow{s-1}]_{\pi_{o}}-\sum_{k=0}^{s-l-1}(-1)^{k}[\overleftarrow{l-1}, \vec{k}]_{\pi_{o}} \overrightarrow{\times}[\overleftarrow{s-l-k-1}]_{\pi_{o}}}
\end{aligned}
$$

d'où le résultat en utilisant le lemme suivant.

III.2.1.3. Lemme. - Pour tout $0<l<s$, on a l'égalité suivante dans le groupe de Grothendieck des représentations admissibles de $G L_{d}\left(F_{o}\right)$ :

$$
\sum_{r=0}^{s-l-1}(-1)^{r}[\overleftarrow{l-1}, \vec{r}]_{\pi_{o}} \overrightarrow{\times}[\overleftarrow{s-l-r-1}]_{\pi_{o}}=[\overleftarrow{s-1}]_{\pi_{o}}+(-1)^{s-l-1}[\overleftarrow{l-1}, \overrightarrow{s-l}]_{\pi_{o}}
$$

Démonstration. - Soit $a_{k}=\sum_{r=0}^{k}(-1)^{r}[\overleftarrow{l-1}, \vec{r}]_{\pi_{o}} \overrightarrow{\times}[\overleftarrow{s-l-r-1}]_{\pi_{o}}$ et montrons par récurrence sur $k$ de 0 à $s-l-1$ que $a_{k}=[\overleftarrow{s-1}]_{\pi_{o}}+(-1)^{k}[\overleftarrow{l-1}, \overrightarrow{k+1}, \overleftarrow{s-l-k-1}]_{\pi_{o}}$. Le résultat est clairement vérifié pour $k=0$ supposons le vrai au rang $k-1$ et traitons le cas de $k$ soit

$$
\begin{aligned}
a_{k}= & a_{k-1}+(-1)^{k}[\overleftarrow{l-1}, \vec{k}]_{\pi_{o}} \overrightarrow{\times}[\overleftarrow{s-l-k-1}]_{\pi_{o}} \\
= & {[\overleftarrow{s-1}]_{\pi_{o}}+(-1)^{k-1}\left([\overleftarrow{l}-1, \overleftrightarrow{k}, \overleftarrow{s-l-k}]_{\pi_{o}}-\right.} \\
& {[\overleftarrow{l-1}, \overleftrightarrow{k}, \overleftarrow{s-l-k}]_{\pi_{o}}-\left[\overleftarrow{l-1}, \overleftrightarrow{\left.k+1, \overleftrightarrow{s-l-k-1}]_{\pi_{o}}\right)}\right.} \\
= & {[\overleftarrow{s-1}]_{\pi_{o}}+(-1)^{k}[\overleftarrow{l-1}, \overrightarrow{k+1}, \overleftarrow{s-l-k-1}]_{\pi_{o}} }
\end{aligned}
$$

III.2.2. Retour sur le cas Iwahori. - D'après le corollaire (II.2.7), pour tout $0 \leqslant i<d$ et toute représentation irréductible admissible $\tau_{o}$ de $D_{o, d}^{\times},\left(\Psi_{F_{o}}^{d, i}\right)^{\mathrm{Iw}}$ est pur de poids $2 i$. Ainsi d'après (III.2.1), il est égal à $[\overleftarrow{i}, \overrightarrow{d-i-1}]_{1} \otimes|c \mathrm{cl}|^{-i}$, ce qui redonne bien le théorème (II.2.5). 


\section{CHAPITRE IV}

\section{FILTRATION DE MONODROMIE DES CYCLES ÉVANESCENTS}

\section{Introduction}

0.1. - Soit $K$ un corps local complet d'égale caractéristique $p$, d'anneau des entiers $\mathcal{O}_{K}$. Pour un entier $d$ strictement positif fixé, on introduit le groupe $D_{K, d}^{\times}$(resp. $\left.W_{K}\right)$ des éléments inversibles de "l"'algèbre à division centrale sur $K$ d'invariant $1 / d$ (resp. le groupe de Weil de $K$ ). Pour un nombre premier $l \neq p$, Langlands (resp. Jacquet-Langlands) a (resp. ont) conjecturé l'existence d'une bijection $L_{d}$ (resp. d'une injection JL) entre les $\overline{\mathbb{Q}}_{l}$-représentations irréductibles admissibles de $G L_{d}(K)$ et les représentations $l$-adiques indécomposables de $W_{K}$ (resp. entre les représentations admissibles irréductibles de $D_{K, d}^{\times}$et les représentations essentiellement de carré intégrable de $\left.G L_{d}(K)\right)$ qui sont compatibles à la formation des fonctions $L$ de paires ${ }^{(1)}$.

A l'aide de la cohomologie étale Deligne a construit une série de représentations $\mathcal{U}_{K}^{d, i}$ du produit de ces trois groupes. Pour $d=2$ et $\rho$ une représentation irréductible admissible de $D_{K, d}^{\times}$telle que $\pi:=\mathrm{JL}(\rho)$ est une représentation cuspidale de $G L_{d}(K)$, Carayol, dans [8], montre que la composante $\rho$-isotypique $\mathcal{U}_{K}^{2,1}(\rho)$ de $\mathcal{U}_{K}^{2,1}$ réalise les correspondances de Langlands et de JacquetLanglands, i.e.

$$
\mathcal{U}_{K}^{2,1}\left(\mathrm{JL}^{-1}(\pi)^{\vee}\right) \simeq \pi \otimes L_{d}(\pi)^{\vee}\left(-\frac{d-1}{2}\right)
$$

Dans [5], en égale caractéristique $p$, je traite le cas $d$ quelconque.

En outre pour $d=2$, Carayol décrit également les $\mathcal{U}_{K}^{2,1}(\rho)$ pour $\rho$ quelconque. Le but premier de ce travail est de faire de même pour $d$ quelconque, i.e. calculer les $\mathcal{U}_{K}^{d, i}(\rho)$ pour $\rho$ une représentation irréductible admissible de $D_{K, d}^{\times}$. Dans le cas où $\rho$ est la représentation triviale, rappelons que d'après le théorème (II.2.5), on a

Théorème 1 Pour $0 \leqslant i \leqslant d-1$, on a

$$
\mathcal{U}_{K}^{d, i}(1)=\pi_{i} \otimes 1(-i)
$$

où $\pi_{i}$ est l'unique quotient irréductible de l'induite parabolique

$$
\operatorname{Ind}_{P_{d-i, d}(K)}^{G L_{d}(K)} 1 \otimes \mathrm{St}_{i}
$$

où $P_{d-i, d}$ est le parabolique standard associée aux $d-i$ premiers vecteurs et $\mathrm{St}_{i}$ est la représentation de Steinberg de $G L_{i}(K)$.

L'énoncé du cas général, théorème (IV.3.1.1), s'énonce de manière similaire en faisant intervenir les correspondances de Langlands et Jacquet-Langlands. Une autre formulation du résultat revient à dire qu'il n'y a pas d'annulation dans l'expression, cf. le théorème (III.2.1), de la représentation virtuelle $\sum_{i=0}^{d-1}(-1)^{i}\left[\mathcal{U}_{K}^{d, i}(\rho)\right]$ où $\mathcal{U}_{K}^{d, d-i}(\rho)$, pour $1 \leqslant i \leqslant d$, y sera alors donné par le $i$-ème terme de plus haut poids.

(1) Pour un énoncé précis, cf. [16] 
0.2. - La preuve du théorème 1 dans le cas général procède par globalisation via l'étude des

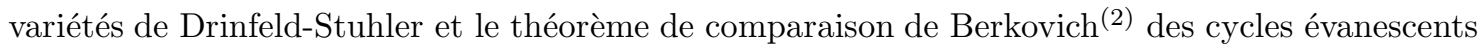
locaux et globaux.

Soit donc $X$ une courbe projective lisse, irréductible et géométriquement connexe définie sur le corps fini à $q=p^{r}$ éléments $\mathbb{F}_{q}$, et soit $F$ son corps des fonctions. On fixe deux places distinctes $\infty$ et $o$ de $X$ que l'on peut supposer par simplification, rationnelles sur $\mathbb{F}_{q}$, de sorte que le complété $F_{o}$ du localisé en $o$ de $F$ est isomorphe au corps local précédemment noté $K$. On note $A$ l'anneau des fonctions sur $X$, régulières en dehors de $\infty$. Étant donné un entier $d \geqslant 1$, on fixe une algèbre à division centrale $D$ sur $F$ de dimension $d^{2}$, non ramifiée en $\infty$ et $o$, ainsi qu'un ordre maximal $\mathcal{D}$. Dans [19], les auteurs construisent pour un idéal non trivial $I$ de $A$, un schéma $M_{I}$ défini sur $F$, classifiant les $\mathcal{D}$-faisceaux elliptiques sur $X$, munis d'une structure de niveau $I$. Pour $o \notin V(I)$, $M_{I}$ a un modèle entier $M_{I, o}$ lisse sur le complété $\mathcal{O}_{o}$ de $A$ en la place $o$. Un tel modèle non lisse dans le cas où $o \in V(I)$ est construit dans [5]. Les schémas $M_{I, o}$ sont naturellement munis d'une action, par correspondances, de $\left(D_{\mathbb{A}}^{\infty}\right)^{\times}$.

0.3. - On s'intéresse alors à la fibre spéciale $M_{I, s_{o}}$ de $M_{I, o}$. Dans [5], je stratifie $M_{I, s_{o}}$ par des sous-schémas localement fermés $M_{I, s_{o}}^{=h}$ pour $1 \leqslant h \leqslant d$, de pure dimension $d-h$ tels que l'on ait un équivalent du théorème de Serre-Tate pour les $\mathcal{D}$-faisceaux elliptiques à savoir : le complété de l'hensélisé strict de l'anneau local de $M_{I, o}$ en un point géométrique de $M_{I, s_{o}}^{=h}$ est isomorphe à $\operatorname{Def}_{n}^{h}\left[\left[x_{1}, \cdots, x_{d-h}\right]\right]$ où $n$ est la multiplicité de $o$ dans $I$ et $\operatorname{Def}_{n}^{h}$ représente le foncteur des déformations de niveau $n$ d'un $\mathcal{O}_{o}$-module formel de hauteur $h$ sur $\overline{\mathbb{F}}_{p}$. Par ailleurs pour $1 \leqslant h<d$, il existe un sous-schéma fermé $M_{I, s_{o}, 1}^{=h}$ de $M_{I, s_{o}}^{=h}$ stable sous les correspondances associées aux éléments du sous-groupe parabolique $P_{h, d}^{o p}\left(F_{o}\right)$ de $G L_{d}\left(F_{o}\right)$ (cf. la définition (II.1.1.1)) et tel que

$$
M_{I, s_{o}}^{=h}=M_{I, s_{o}, 1}^{=h} \times_{P_{h, d}^{o p}\left(\mathcal{O}_{o} / \mathcal{M}_{o}^{n}\right)} G L_{d}\left(\mathcal{O}_{o} / \mathcal{M}_{o}^{n}\right)
$$

où $n$ est la multiplicité de $o$ dans $I$ : on dit que les strates non supersingulières sont géométriquement induites.

0.4. - Suivant [14], on introduit sur chacune des strates $M_{I, s_{o}, 1}^{=h}$, des systèmes locaux $\mathcal{F}_{\tau_{o}}$ associés aux représentations $\tau_{o}$ du groupe des inversibles $D_{o, h}^{\times}$de l'algèbre à division centrale sur $F_{o}$ d'invariant $1 / h$. On décrit alors la restriction des cycles évanescents $R^{i} \Psi_{\eta_{o}}\left(\overline{\mathbb{Q}}_{l}\right)$ à la strate $M_{I, s_{o}}^{=h}$ en fonction des $\mathcal{F}_{\tau_{o}}$ et des cycles évanescents locaux $\Psi_{F_{o}, n}^{h, i}$, cf. (IV.2.2.12). D'après le théorème de comparaison de Berkovich, le théorème local se déduit de la connaissance de la fibre en un point supersingulier des $R^{i} \Psi_{\eta_{o}}\left(\overline{\mathbb{Q}}_{l}\right)$.

0.5. - Le complexe $R \Psi_{\eta_{o}}\left(\overline{\mathbb{Q}}_{l}\right)[d-1]$ est vu comme un faisceau pervers muni d'une filtration de monodromie dont on notera $g r_{k}$ les gradués. Le deuxième résultat concerne la description des gradués $g r_{k}$ dans la catégorie des faisceaux pervers sur $M_{I, s_{o}}$ munis d'une action compatible de $\left(D_{\mathbb{A}}^{\infty}\right)^{\times} \times W_{o}$. Pour $1 \leqslant l g \leqslant d$, et pour $\pi_{o}\left(\right.$ resp. $\left.\Pi_{l}\right)$ une représentation irréductible cuspidale de $G L_{g}\left(F_{o}\right)$ (resp. quelconque de $G L_{l g}\left(F_{o}\right)$ ), on introduit le faisceau $H T\left(g, l, \pi_{o}, \Pi_{l}\right)$ sur la strate $M_{I, s_{o}}^{=l g}$, "induit" à partir du système local $\mathcal{F}_{\mathrm{JL}^{-1}\left(\mathrm{St}_{l}\left(\pi_{o}\right)\right)} \otimes \Pi_{l}$ sur la composante $M_{I, s_{o}, 1}^{=l g}$.

Les composantes $\pi_{o}$-isotypiques $g r_{k, \pi_{o}}$ des $g r_{k}$, cf. la proposition (IV.4.1.3), se décrivent alors au moyen des faisceaux pervers $j_{!_{*}}^{\geqslant l g} H T\left(g, l, \pi_{o}, \mathrm{St}_{l}\left(\pi_{o}\right)\right)[d-l g]$, cf. le théorème (IV.4.4.4), où $j \geqslant l g$ désigne l'injection de la strate $M_{I, s_{o}}^{=l g}$; en ce qui concerne la partie associée à $\pi_{o}$ triviale l'énoncé est le suivant :

Théorème 2 Les faisceaux pervers $g r_{k, 1_{o}}$ sont nuls pour $k \geqslant d$ et sinon on a

$$
g r_{k, 1_{o}},=\bigoplus_{\substack{|k|<l \leqslant d \\ l \equiv k-1 \bmod 2}} j_{! *}^{\geqslant l} H T\left(1, l, 1_{o}, \mathrm{St}_{l}\right)\left(-\frac{l g+k-1}{2}\right)[d-l]
$$

${ }^{(2)}$ en fait sur une version raffinée fournie par Fargues, cf. le théorème de l'appendice de [6] 
L'énoncé pour $\pi_{o}$ quelconque est similaire et fait intervenir les correspondances de Langlands et Jacquet-Langlands.

0.6. - En utilisant le théorème de monodromie-poids, la preuve du théorème 2 découle de la connaissance du théorème 1 pour toutes les hauteurs $h<d$ ainsi que de la description des restrictions aux strates des faisceaux des cycles évanescents en fonction des systèmes locaux $\mathcal{F}_{\tau_{o}}$ comme rappelé en (0.4.). Par ailleurs le théorème 1 en hauteur $d$, découle d'après le théorème de comparaison de Berkovich-Fargues, du calcul des germes aux points supersinguliers des faisceaux de cohomologie des $g r_{k}$.

On raisonne alors par récurrence en supposant connus ${ }^{(3)}$ les $\mathcal{U}_{F_{o}}^{h, i}$ du modèle de Deligne-Carayol de hauteur $h$ pour tout $1 \leqslant h<d$. On en déduit alors le théorème 2 . Par ailleurs on sait déterminer tous les faisceaux de cohomologie $h^{i} g r_{k}$ des $g r_{k}$ en dehors des points supersinguliers. La technique repose sur l'étude de la suite spectrale associée à la filtration de monodromie :

$$
E_{1}^{i, j}=h^{i+j} g r_{-i} \Rightarrow R^{i+j+d-1} \Psi_{\eta_{o}}\left(\overline{\mathbb{Q}_{l}}\right)
$$

en essayant d'en deviner les termes initiaux, l'aboutissement étant connu. En outre en utilisant la perversité des $g r_{k}$ ainsi que la compatibilité de $R \Psi_{\eta_{o}}$ à la dualité de Verdier, on obtient un contrôle sur les germes aux points supersinguliers des $h^{i} g r_{k}$. On étudie ensuite la suite spectrale des cycles évanescents dont à nouveau on essaie de deviner les termes initiaux alors que l'aboutissement est connu, en utilisant en particulier le théorème de Lefschetz difficile. Le contrôle obtenu précédemment nous permet alors de prouver le théorème 1 et on explicite la suite spectrale de monodromie (IV.0.2.7).

0.7. - En ce qui concerne les résultats globaux que l'on obtient, citons

- la description "explicite" des gradués pour la filtration de monodromie du faisceau pervers des cycles évanescents ainsi que de la suite spectrale associée;

- la détermination des extensions intermédiaires des systèmes locaux d'Harris-Taylor;

- le calcul de tous les groupes de cohomologie des différents faisceaux ou complexes de faisceaux qui sont introduits.

0.8. - Les résultats obtenus dans le cadre des variétés de Drinfeld s'adaptent aussi en caractéristique mixte dans le cadre des variétés de Shimura de type PEL étudiées dans [14]; c'est ce travail qui est effectué dans [6]. Le fait est que tous les arguments reposent de façon formelle sur les propriétés géométriques du paragraphe (IV.1.2) et les propriétés cohomologiques du paragraphe (IV.2.2) ; nous avons ainsi donné dans l'appendice A, le dictionnaire entre nos notations et celles de [14] en caractéristique mixte et rappelé où se trouvaient dans loc. cit. les propriétés cohomologiques que l'on utilise dans le paragraphe (IV.2.2). La différence essentielle réside dans le fait qu'en caractéristique mixte, nous ne disposons pas à priori de monodromie-poids. Ainsi dans le texte nous avons, chaque fois que nécessaire, indiqué des preuves qui n'utilisent pas cette propriété. Au final dans la situation de [14], on obtient alors une preuve de la conjecture de monodromie-poids versions faisceautique et cohomologique.

0.9. - Comme conséquence directe de ces calculs on obtient une correspondance de JacquetLanglands globale entre les algèbres à divisions $D$ et $\bar{D}$, résultat qui d'après I. Badulescu, peut s'obtenir aisément à partir de la formule des traces simples. En outre on montre que les composantes locales des représentations automorphes de $D_{\mathbb{A}}^{\times}$sont celles prévues par l'existence, en général encore conjecturale, d'une correspondance de Jacquet-Langlands globale avec $G L_{d}(\mathbb{A})$ à partir des résultats de Moeglin-Waldspurger sur les représentations de carré intégrable modulo le centre de $G L_{d}(\mathbb{A})$ et la conjecture de Ramanujan-Peterson prouvée par Lafforgue. Ainsi dans les cas

(3) En fait on suppose plutôt connu les gradués locaux $g r_{h, k, l o c}$ de la filtration de monodromie-locale du complexe des cycles évanescents $\Psi_{F_{O}}^{h, \bullet}$. 
défavorables où il n'existe pas encore une telle correspondance de Jacquet-Langlands, on obtient la description des composantes locales des représentations automorphes de $D_{\mathbb{A}}^{\times}$vérifiant $\operatorname{Hyp}(\infty)$ et donc en particulier la conjecture de Ramanujan-Peterson.

0.10. - Décrivons succinctement le contenu des divers paragraphes. On commence par des rappels sur les données géométriques locales et globales tirées des chapitres précédents dans le cas d'égale caractéristique, et de [14] dans celui de la caractéristique mixte, cf. l'appendice A.

En ce qui concerne les données globales, outre le fait que les strates non supersingulières soient géométriquement induites, la donnée fondamentale est celle des systèmes locaux d'Harris-Taylor $\mathcal{F}\left(g, l, \pi_{o}\right)$ sur la strate $l g$, attachés aux représentations $\mathrm{JL}^{-1}\left(\operatorname{St}_{l}\left(\pi_{o}\right)\right)$ des inversibles $D_{o, l g}^{\times}$de l'algèbre à division centrale sur $F_{o}$ d'invariant $1 / \mathrm{lg}$. La propriété essentielle que l'on utilisera sur ces systèmes locaux est l'isomorphisme (IV.2.2.12).

On redonne en outre, d'après [5], la description de l'ensemble des points supersinguliers et on définit pour tout diviseur $g$ de $d=s g$, le faisceau $\mathcal{F}\left(g, s, \pi_{o}\right)$ à support sur les points supersinguliers. On notera par ailleurs que les $\mathcal{F}\left(g, l, \pi_{o}\right)$ ne sont pas à priori irréductibles car ils proviennent de la restriction à $\mathcal{D}_{o, l g}^{\times}$de $\mathrm{JL}^{-1}\left(\mathrm{St}_{l}\left(\pi_{o}\right)\right)$.

0.11. - Au deuxième paragraphe, on explicite le lien entre $\Psi_{F_{o}}^{h, i}$ et $\mathcal{U}_{F_{o}}^{h, i}$ et on introduit l'entier $e_{\pi_{o}}$ qui est le cardinal de la classe d'équivalence inertielle, définition (IV.2.1), de $\pi_{o}$. En particulier $e_{\pi_{o}}$ est égal au nombre de sous-représentations irréductibles de la restriction à $\mathcal{D}_{o, l g}^{\times}$de $\mathrm{JL}^{-1}\left(\operatorname{St}_{l}\left(\pi_{o}\right)\right)$.

On rappelle alors le théorème (3.2.4) de [5] qui décrit les parties cuspidales des groupes de cohomologie des modèles locaux de Deligne-Carayol. A ce propos, pour avoir un énoncé exact dans loc. cit., il faut considérer l'action naturelle de $G L_{d}\left(F_{o}\right)$ sur $\mathcal{U}_{F_{o}}^{d, i}$, tordue par $g_{o} \mapsto{ }^{t} g_{o}^{-1}$; on introduira un tilde pour marquer cette modification. On rappelle alors le théorème (VII.1.5) de [14] qui donne le calcul de la somme alternée, dans le groupe de Grothendieck des $G L_{d}\left(F_{o}\right) \times W_{o^{-}}$ modules, $\sum_{i}(-1)^{i} \widetilde{\mathcal{U}_{F_{o}}^{d, i}}\left(\mathrm{JL}^{-1}\left(\mathrm{St}_{s}\left(\pi_{o}\right)\right)\right)$.

En global l'isomorphisme (IV.2.2.12) se traduit cohomologiquement par la proposition (IV.2.2.1). On introduit selon [19], pour toute représentation $\rho_{\infty}$ du groupe des inversibles $\bar{D}_{\infty}^{\times}$de "l"'algèbre à division centrale sur $F_{\infty}$ d'invariant $-1 / d$, le système local $\mathcal{L}_{\rho_{\infty}}$ sur les variétés $M_{I, o}$. On considère alors les groupes de cohomologie du produit tensoriel de ce dernier avec un système local d'Harris-Taylor. Le résultat fondamental qui résulte des arguments de comptage de points est la proposition (IV.2.2.4) qui calcule la somme alternée des groupes de cohomologie à support compact des systèmes locaux d'Harris-Taylor, dans le groupe de Grothendieck des $\left(D_{\mathbb{A}}^{\infty}\right)^{\times} \times \mathbb{Z}$-modules, où pour tout $h, \mathbb{Z}$ est identifié au quotient $D_{o, h}^{\times} / \mathcal{D}_{o, h}^{\times}$via la valuation de la norme réduite. On introduit pour cela le caractère $\Xi$ de $\mathbb{Z} \longrightarrow \overline{\mathbb{Q}}_{l}^{\times}$défini par $\Xi(1)=\frac{1}{p}$.

Ainsi les représentations automorphes $\Pi$ qui interviennent dans cette description ainsi que dans celle de la cohomologie de la fibre générique à valeurs dans $\mathcal{L}_{\rho_{\infty}}$, vérifient des conditions $\operatorname{Hyp}(\infty)$ à la place $\infty$ que l'on donne à la définition (IV.2.2.2) : en résumé pour $\rho_{\infty}=\mathrm{JL}^{-1}\left(\operatorname{St}_{s}\left(\pi_{\infty}\right)\right)$, il faut que $\Pi_{\infty}$ soit isomorphe à $\operatorname{St}_{s}\left(\pi_{\infty}\right)$ ou à $\operatorname{Speh}_{s}\left(\pi_{\infty}\right)$.

0.12. - Au troisième paragraphe le théorème (IV.3.1.1) est la motivation initiale de ce travail, i.e. décrire chacun des $\widetilde{\mathcal{U}_{F_{o}}^{d, i}}\left(\mathrm{JL}^{-1}\left(\mathrm{St}_{s}\left(\pi_{o}\right)\right)\right)$. Finalement on obtient un résultat plus précis, théorème (IV.3.2.3), qui est la description des gradués de la filtration de monodromie-locale définie par Fargues dans l'appendice de [6] et de la suite spectrale correspondante. Le fait primordial démontré dans par Laurent Fargues est le théorème de comparaison à la Berkovich qui implique qu'en tout point géométrique de $M_{I, s_{o}}^{=h}$, la filtration et la suite spectrale correspondante, induite par la monodromie globale sur le germe en ce point du complexe des cycles évanescents, donne la suite spectrale de filtration de monodromie-locale du complexe des cycles évanescents du modèle de 
Deligne-Carayol pour la hauteur $h$. On référera à ce résultat comme le théorème de comparaison de Berkovich-Fargues.

0.13. - Le quatrième paragraphe est consacré aux énoncés globaux. On commence, après avoir fait quelques rappels sur les faisceaux pervers $§$ IV.4.1, par découper, proposition (IV.4.1.3), nos faisceaux pervers selon leur composantes isotypiques pour le sous-groupe d'inertie $I_{o}$ et on note ainsi $g r_{k, \pi_{o}}$ (resp. $g r_{k, \rho_{\infty}, \pi_{o}}$ ) la composante $L_{g}\left(\pi_{o}\right)_{\mid I_{o}}$-isotypiques du gradué $g r_{k}$ (resp. $g r_{k, \rho_{\infty}}$ ) pour la filtration de monodromie du faisceau pervers $R \Psi_{\eta_{o}}\left(\overline{\mathbb{Q}}_{l}\right)[d-1]\left(\operatorname{resp} . R \Psi_{\eta_{o}}\left(\mathcal{L}_{\rho_{\infty}}\right)[d-1]\right.$ pour $\rho_{\infty}$ une représentation irréductible de $\left.\bar{D}_{\infty}^{\times}\right)$, où $\pi_{o}$ est une représentation irréductible cuspidale de $G L_{g}\left(F_{o}\right)$ avec $1 \leqslant g \leqslant d$. La représentation $L_{g}\left(\pi_{o}\right)_{\mid I_{o}}$ n'étant pas irréductible, $V_{\pi_{o}}$ est le facteur direct de $V$ sur lequel l'action de $I_{o}$ se décompose en une somme de sous-représentations irréductibles qui sont aussi des sous-représentations de $L_{g}\left(\pi_{o}\right)_{\mid I_{o}}$.

On introduit ensuite $\S \mathrm{IV} .4 .2$, certaines catégories de faisceaux pervers de Hecke qui fourniront le cadre catégoriel des différents complexe de faisceaux que nous considérerons dans la suite et on donne, définition (IV.4.3.1), un certain nombre de notations attachées aux systèmes locaux d'Harris-Taylor $\mathcal{F}\left(g, l, \pi_{o}\right)_{1}$ et aux faisceaux induits $H T\left(g, l, \pi_{o}, \Pi_{l}\right)$ qui leurs sont associés sur la strate $M_{I, s_{o}}^{=l g}$ où $\Pi_{l}$ est une représentation de $G L_{l g}\left(F_{o}\right)$ qui sera le plus souvent elliptique de type $\pi_{o}$. On introduit alors le groupe de Grothendieck $\mathfrak{G}$ des faisceaux pervers de Hecke sur la tour des $M_{I, s_{o}}$ munis d'une action compatible de $\left(D_{\mathbb{A}}^{\infty}\right)^{\times} \times W_{o}$.

On énonce alors les théorèmes globaux qui précisent, théorème (IV.4.4.1) (resp. (IV.4.4.4)), les faisceaux pervers simples de $R \Psi_{\eta_{o}}\left(\mathcal{L}_{\rho_{\infty}}\right)[d-1]$ (resp. des $g r_{k, \rho_{\infty}, \pi_{o}}$ ) en termes des faisceaux pervers $j_{!_{*}}^{\geqslant l g} H T\left(g, l, \pi_{o}, \mathrm{St}_{l}\left(\pi_{o}\right)\right)[d-l g] \otimes L_{g}\left(\pi_{o}\right)\left(-\frac{l g-1+k}{2}\right)$ avec $1 \leqslant l \leqslant d / g$ (resp. $|k|<l \leqslant s_{g}$ et $l \equiv k-1 \bmod 2)$.

D'après le théorème de comparaison de Berkovich-Fargues, le théorème local (IV.3.1.1) se déduit alors du calcul, théorème (IV.4.4.7), des faisceaux de cohomologie des $j_{! *}^{\geqslant l g} \mathcal{F}\left(g, l, \pi_{o}\right)$ et de la détermination, théorème (IV.4.4.10), de la suite spectrale de monodromie associée.

On donne ensuite le schéma de la preuve qui procède par récurrence en supposant connu (IV.3.2.3) pour tout $d^{\prime}<d$. On renvoie le lecteur à §IV.4.5 pour un apercu des implications logiques entre les divers énoncés locaux et globaux.

0.14. - Dans le cinquième paragraphe, on démontre le théorème (IV.4.4.1), i.e. on donne l'image de $R \Psi_{\eta_{o}}\left(\overline{\mathbb{Q}}_{l}\right)[d-1]$ dans le groupe de Grothendieck $\mathfrak{G}$ des faisceaux pervers de Hecke sur la tour des $M_{I, s_{o}}$. La preuve procède en plusieurs étapes. Tout d'abord de la description (IV.2.2.12) des restrictions aux strates $M_{I, s_{o}}^{=h}$ des $R^{i} \Psi_{\eta_{o}}\left(\overline{\mathbb{Q}}_{l}\right)$ et du calcul (IV.2.2.13) de $\sum_{i}(-1)^{i}\left[\mathcal{U}_{F_{o}}^{d, i}\right]$, on en déduit, proposition (IV.5.2.2), l'égalité suivante où on a posé $s_{g}=\left\lfloor\frac{d}{g}\right\rfloor$, la partie entière de $d / g$ :

$$
\begin{aligned}
{\left[R \Psi_{\eta_{o}}\left(\overline{\mathbb{Q}}_{l}\right)[d-1]\right] } & =\sum_{g=1}^{d} \sum_{\pi_{o} \in \operatorname{Cusp}_{o}(g)} \frac{1}{e_{\pi_{o}}} \sum_{i=1}^{s_{g}} \sum_{l=i}^{s_{g}}(-1)^{l-i} \\
& {\left[j_{!}^{\geqslant l g} H T\left(g, l, \pi_{o},[\overleftarrow{l-1}, \overrightarrow{l-i}]_{\pi_{o}}\right)[d-l g] \otimes L_{g}\left(\pi_{o}\right)\left(-\frac{l g-2+2 i-l}{2}\right)\right] }
\end{aligned}
$$

L'étape suivante consiste alors à exprimer l'image des faisceaux pervers qui interviennent dans le membre de droite de l'égalité ci-dessus soit :

$$
\begin{aligned}
{\left[j_{!}^{\geqslant l g} H T\left(g, l, \pi_{o},[\overleftarrow{l-1}]_{\pi_{o}}\right)[d-l g]\right] } & = \\
& \sum_{i=0}^{s_{g}-l} j_{! *}^{\geqslant l^{\prime} g} H T\left(g, l+i, \pi_{o},[\overleftarrow{l-1}]_{\pi_{o}} \overrightarrow{\times}[\overleftarrow{l-1}]_{\pi_{o}}\right)[d-(l+i) g] \otimes \Xi^{i(g-1) / 2}
\end{aligned}
$$

Pour prouver cette dernière égalité, on raisonne par récurrence descendante sur la dimension des supports des faisceaux pervers simples dans $j_{!}^{\geqslant l g} H T\left(g, l, \pi_{o},[\overleftarrow{l-1}]_{\pi_{o}}\right)[d-l g]$ en réinjectant 
(IV.0.2.9) pour les différents $l$, avec $g$ et $\pi_{o}$ fixés, dans (IV.0.2.8). On argumente tout d'abord sur le fait que le membre de droite de (IV.0.2.8) doit s'écrire comme une somme à coefficients positifs de faisceaux pervers simples, autoduale pour la dualité de Verdier.

On invoque ensuite le théorème de comparaison de Berkovich-Fargues afin d'utiliser l'hypothèse de récurrence sur les modèles locaux en hauteur $h<d$, afin d'obtenir des renseignements sur les germes aux points non supersinguliers de ces faisceaux pervers simples. On démontre alors, proposition (IV.5.3.3), le résultat hors des points supersinguliers au sens où les égalités du théorème précédent et de (IV.0.2.9) sont vraies si on rajoute une somme alternée de faisceaux pervers à support sur les points supersinguliers. Le fait est qu'on utilise vraiment (IV.2.2.12) et pas seulement un calcul de somme alternée des restrictions des faisceaux des cycles évanescents, ce qui explique l'indétermination au niveau des points supersinguliers.

Le paragraphe (IV.5.4) est consacré à la détermination de ces faisceaux pervers ponctuels qui nous manquent. Une idée naive est que pour connaître un faisceau ponctuel, on peut commencer par calculer son groupe de cohomologie $H^{0}$. Étant donné un tel faisceau pervers ponctuel $\mathcal{P}$ à déterminer, nous verrons en fait que la connaissance de son $H^{0}$ suffit à le déterminer complètement : en effet grâce à (IV.5.2.18), nous montrerons que $\mathcal{P}$ contient $\mathcal{F}\left(g, s, \pi_{o}\right) \otimes\left(\Pi_{l} \overrightarrow{\times}[\overleftarrow{s-l-1}]_{\pi_{o}}\right) \otimes$ $\Xi^{(s-l)(g-1) / 2}$ avec

$$
H^{0}\left(\mathcal{F}\left(g, s, \pi_{o}\right) \otimes \Pi_{l} \overrightarrow{\times}[\overleftarrow{s-l-1}]_{\pi_{o}}\right) \otimes \Xi^{(s-l)(g-1) / 2}=H^{0}(\mathcal{P})
$$

de sorte que $\mathcal{P}=\mathcal{F}\left(g, s, \pi_{o}\right) \otimes \Pi_{l} \overrightarrow{\times}[\overleftarrow{s-l-1}]_{\pi_{o}} \otimes \Xi^{(s-l)(g-1) / 2}$.

On commence alors par calculer les groupes de cohomologie des faisceaux pervers d'HarrisTaylor, ou tout du moins leur $\Pi^{\infty, o}$-partie, pour $\Pi$ automorphe vérifiant $\operatorname{Hyp}(\infty)$ avec $\Pi_{o}=\operatorname{St}_{s}\left(\pi_{o}\right)$ de sorte que, remarque (IV.5.4.4), la condition $\operatorname{Hyp}(\infty)$ implique que $\Pi_{\infty}$ est isomorphe à $\operatorname{St}_{s^{\prime}}\left(\pi_{\infty}\right)$ pour un diviseur $s^{\prime}$ de $d=s^{\prime} g^{\prime}$ et $\pi_{\infty}$ une représentation irréductible cuspidale de $G L_{g^{\prime}}\left(F_{\infty}\right)$. On montre à la proposition (IV.5.4.1) que ceux-ci sont alors tous nuls de sorte que, d'après la proposition (IV.5.3.3), l'égalité (IV.2.2.13) fournit, corollaire (IV.5.4.6), le $H^{0}$ des faisceaux ponctuels manquant ainsi que leur détermination. Le théorème (IV.4.4.1) découle alors directement de ces résultats, cf. le corollaire (IV.5.4.8).

0.15. - Le sixième paragraphe est consacré, proposition (IV.2.2.1), au calcul des faisceaux de cohomologies des faisceaux pervers $j_{! *}^{\geqslant l g} H T\left(g, l, \pi_{o}, \Pi_{l}\right)[d-l g]$ en fonction des extensions par zéro $j_{!}^{\geqslant l^{\prime} g} H T\left(g, l^{\prime}, \pi_{o}, \Pi_{l} \overrightarrow{\times}\left[\overrightarrow{l^{\prime}-l-1}\right]_{\pi_{o}}\right) \otimes \Xi^{\left(l^{\prime}-l\right)(g-1) / 2}$. D'après la proposition (IV.5.3.3), on peut procéder par récurrence en utilisant la suite spectrale (IV.4.1.17) dont l'aboutissement est connu sauf au niveau des points supersinguliers.

Ainsi ces faisceaux de cohomologie ne sont pas complètement déterminés aux points supersinguliers mais il ne reste qu'un nombre réduit de possibilités, cf. le lemme (IV.6.2.5), que l'on peut obtenir, d'après (IV.5.3.3), par récurrence en utilisant par exemple la suite spectrale (IV.6.2.20).

On donne ensuite, proposition (IV.7.3.1), une preuve de monodromie-poids. En égale caractéristique ce résultat est connu et nous l'utilisons à maintes reprises, cependant, nous en démontrons un cas particulier qui dans la situation de caractéristique mixte fournira une preuve complète du théorème de monodromie-poids pour les variétés de Shimura de [14]. On remarque tout d'abord que pour $s>2$, le raisonnement par récurrence implique le résultat de sorte qu'il reste à initialiser la récurrence et donc à prouver que pour $s=2$, la monodromie n'est pas triviale, en particulier sur la cohomologie. En égale caractéristique le résultat est connu d'après [19] tandis qu'en caractéristique il est connu pour $s=2$ et $g=1$ d'après [8]. Le principe, qui nous a été suggéré par M. Harris, est de s'y ramener via un changement de base adéquat, cf. appendice A. (4)

${ }^{(4)}$ Notons par ailleurs que le cas général, i.e. $g=1$ et $s$ quelconque a récemment été prouvé directement par Yoshida et Taylor. 
0.16. - Au septième paragraphe, on prouve les théorèmes globaux sous la proposition (IV.7.1.1). D'après le théorème de comparaison de Berkovich-Fargues la connaissance du théorème local (IV.3.2.3) revient à celle des germes aux points supersinguliers des faisceaux de cohomologie des faisceaux pervers d'Harris-Taylor ainsi que les flèches correspondantes dans la suite spectrale (IV.4.1.17). Si nous disposions du théorème (IV.3.2.3) pour $d$, le raisonnement de la preuve de la proposition (IV.6.2.3) nous permettrait de déterminer complètement les faisceaux de cohomologie des $g r_{k}$. Par souci d'efficacité nous montrons, proposition (IV.7.1.2), qu'il suffit, en utilisant l'opérateur de monodromie $N$, en fait de connaitre les parties de poids $s(g-1)$ de l'aboutissement de la suite spectrale de monodromie-locale de (IV.3.2.3). On est alors ramené à prouver la proposition (IV.7.1.1), i.e. à déterminer les parties de poids $s(g-1) \operatorname{des} \widetilde{\mathcal{U}_{F_{o}}^{d, i}}$.

0.17. - Le huitième paragraphe est consacré à la preuve de la proposition (IV.7.1.1). Celle-ci repose sur l'étude de la suite spectrale des cycles évanescents. Pour $\Pi$ une représentation irréductible automorphe de $D_{\mathbb{A}}^{\times}$telle que $\Pi_{o} \simeq \operatorname{St}_{s}\left(\pi_{o}\right)$ avec $\pi_{o}$ cuspidale, la $\Pi^{\infty, o}$-partie de son aboutissement est connue d'après [19], ou peut-être rapidement recalculée à partir de la proposition (IV.5.4.1). La détermination de cet aboutissement nous restreint, corollaire (IV.8.1.3), alors le nombre de possibilités pour les $\widetilde{\mathcal{U}_{F_{o}}^{d, i}}\left(\mathrm{JL}^{-1}\left(\mathrm{St}_{s}\left(\pi_{o}\right)\right)\right)$.

En utilisant une propriété, théorème (IV.8.2.1), d'invariance des $\mathcal{U}_{F_{o}}^{d, \bullet}$ sous l'involution de Zelevinski, il est alors possible de prouver la proposition (IV.7.1.1). Cependant la preuve de celle-ci repose sur tout ou partie du théorème de comparaison de Faltings à partir d'un énoncé similaire du coté espace de Drinfeld, ce qui dépasse le cadre de ce texte. Par ailleurs la preuve, dans le cas de la caractéristique mixte, de la conjecture de monodromie-poids version cohomologique demande une étude des $\Pi^{\infty}$-parties des divers groupes de cohomologie, pour $\Pi$ automorphe vérifiant $\operatorname{Hyp}(\infty)$ et telle que $\Pi_{o} \simeq \operatorname{Speh}_{s}\left(\pi_{o}\right)$. La $\Pi^{\infty, o}$-partie de la cohomologie de la fibre générique n'est pas à priori connue. On se propose dans un premier temps de la calculer, ou tout du moins les bouts de poids $s(g-1)$, le cas général étant traité, de manière indépendante, à la proposition (V.1.1).

On calcule tout d'abord, proposition (IV.8.3.4), les $\Pi^{\infty, o}$-parties des groupes de cohomologie des faisceaux pervers d'Harris-Taylor. On en déduit alors, corollaire (IV.8.3.5), la connaissance des $\Pi^{\infty, o}$-parties de poids $s(g-1)$ des groupes de cohomologie de la fibre générique. Pour ce faire on utilise le théorème de Lefschetz difficile. On étudie alors, proposition (IV.8.3.8), les $\Pi^{\infty, o}$-parties des termes $E_{2}^{p, q}$ de la suite spectrale des cycles évanescents.

On montre, qu'à travers les suites spectrales associées à la stratification, qu'en ce qui concerne les bouts de poids $s(g-1)$, seule la strate supersingulière contribue de sorte que l'on se retrouve dans une situation similaire à celle de [5] dans le cas cuspidal, où les bouts de poids $s(g-1)$ des $E_{2}^{p, q}\left[\Pi^{\infty, o}\right]$ sont nuls pour $p \neq 0$. Cette constatation découle du contrôle, lemme (IV.8.3.10), des contributions des strates non supersingulières. La proposition (IV.7.1.1), découle alors directement de la connaissance des $\Pi^{\infty, o}$-parties de l'aboutissement de la suite spectrale des cycles évanescents et du fait qu'en ce qui concerne celles de poids $s(g-1)$, celles-ci ne proviennent que des points supersinguliers.

Note : pour les lecteurs qui n'ont pas lu les chapitres précédents, on leur conseille de lire toutefois le $\S I I .1$ qui rappelle la combinatoire sur les représentations elliptiques de $G L_{d}\left(F_{o}\right)$ due à Zelevinski (cf. $[\mathbf{2 4}])$.

\section{IV.1. Rappels des données géométriques}

IV.1.1. Définitions. - - Soit cl : $W_{o} \longrightarrow F_{o}^{\times}$le morphisme de la théorie du corps de classe qui envoie les frobenius géométriques sur les uniformisantes, i.e. $\operatorname{val}\left(\mathrm{cl}\left(\operatorname{Fr}_{o}\right)\right)=-1$.

- Pour $c_{o} \in W_{o}$, on notera $\operatorname{deg}\left(c_{o}\right):=\operatorname{val}\left(\operatorname{cl}\left(c_{o}\right)\right)$. 
- Étant donnés une représentation complexe $\sigma_{o}$ (resp. $\left.\pi_{o}\right)$ de $W_{o}$ (resp. de $G L_{d}\left(F_{o}\right)$ ) et un entier $r$, on notera $\sigma_{o}(r)\left(\right.$ resp. $\left.\pi_{o}(r)\right)$ la représentation $\sigma_{o} \otimes|\mathrm{cl}|^{r}\left(\right.$ resp. $\left.\pi_{o} \otimes|\operatorname{det}|^{r}\right)$.

IV.1.1. Le modèle local de Deligne-Carayol. - Pour tout $n \geqslant 0$, Def ${ }_{n}^{d}$ représente ${ }^{(5)}$ le foncteur des déformations de niveau $n$, par isogénies, du $\mathcal{O}_{o}$-module formel de hauteur $d$ sur $\overline{\mathbb{F}}_{q}$. $I V .1 .1 .1$ - Soit alors $\Psi_{F_{o}, n}^{d, i}$ le $\overline{\mathbb{Q}}_{l}$-espace vectoriel de dimension finie obtenu via la théorie de Berkovich comme le $i$-ème foncteur des cycles évanescents associé au morphisme structural

$$
\operatorname{Spf} \operatorname{Def}_{n}^{d} \longrightarrow \operatorname{Spf} \hat{\mathcal{O}}_{o}^{n r} .
$$

Cet espace vectoriel est muni entre autre d'une action de $G L_{d}\left(\mathcal{O}_{o}\right)$ qui se factorise par le morphisme surjectif naturel $G L_{d}\left(\mathcal{O}_{o}\right) \longrightarrow G L_{d}\left(\mathcal{O}_{o} / \mathcal{M}_{o}^{n}\right)$ et on pose

$$
\Psi_{F_{o}}^{d, i}=\underset{n}{\lim } \Psi_{F_{o}, n}^{d, i}
$$

de sorte que pour $K_{o, n}:=\operatorname{Ker}\left(\mathcal{O}_{o}^{\times} \longrightarrow\left(\mathcal{O}_{o} / \mathcal{M}_{o}^{n}\right)^{\times}\right), \Psi_{F_{o}, n}^{d, i}=\left(\Psi_{F_{o}}^{d, i}\right)^{K_{o, n}}$. On introduit le groupe $\mathcal{N}_{o}$ (resp. $\left.\mathcal{N}_{o}^{\prime}\right)$ défini comme le noyau de

$$
\left(g_{o}, \delta_{o}, c_{o}\right) \in G L_{d}\left(F_{o}\right) \times D_{o, d}^{\times} \times W_{o} \mapsto \operatorname{val}\left(\operatorname{det}\left(g_{o}^{-1}\right) \operatorname{rn}\left(\delta_{o}\right) \operatorname{cl}\left(c_{o}\right)\right) \in \mathbb{Z}
$$

(resp. composé avec la projection canonique $\mathbb{Z} \longrightarrow \mathbb{Z} / d \mathbb{Z}$ ).

Remarque : Parfois on précisera la dimension en notant $\mathcal{N}_{o}(d)$.

$I V$.1.1.2 - Pour $\xi_{o}$ un caractère d'ordre fini de $F_{o}^{\times}$, on note $\Psi_{F_{o}, \xi_{o}}^{d, i}$ la $\xi_{o}^{\prime}$-composante isotypique où $\xi_{o}^{\prime}$ est la restriction de $\xi_{o}$ à $\mathcal{O}_{o}^{\times}$. Ainsi $\Psi_{F_{o}, \xi_{o}}^{d, i}\left(\right.$ resp. $\left.\Psi_{F_{o}}^{d, i}\right)$ est muni d'une action de $\mathcal{N}_{o}^{\prime}$ (resp. $\left.\operatorname{de} \mathcal{N}_{o}\right)$.

IV.1.1.3 - Dans la définition de $\operatorname{Def}_{n}^{d}$, il est agréable de considérer plutôt les déformations

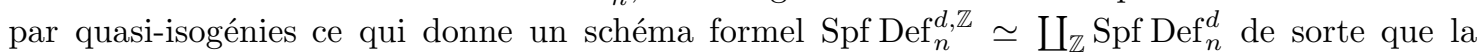
construction précédente fourni des $\overline{\mathbb{Q}}_{l}$-espaces vectoriels $\mathcal{U}_{F_{o}, n}^{d, i} \simeq\left(\mathcal{U}_{F_{o}}^{d, i}\right)^{K_{o, n}}$ où

$$
\mathcal{U}_{F_{o}, \xi_{o}}^{d, i}:=\operatorname{Ind}_{\mathcal{N}_{o}}^{G L_{d}\left(F_{o}\right) \times D_{o, d}^{\times} \times W_{F_{o}}} \Psi_{F_{o}, \xi_{o}}^{d, i}
$$

est une représentation de $G L_{d}\left(F_{o}\right) \times D_{o, d}^{\times} \times W_{o}$.

IV.1.1.4 - Pour toute représentation admissible irréductible $\tau_{o}$ de $D_{o, d}^{\times}$de caractère central $\xi_{o}$, la réciprocité de Frobenius donne que la composante isotypique $\mathcal{U}_{F_{o}, \xi_{o}}^{d, i}\left(\tau_{o}\right)$ est isomorphe à $\operatorname{Hom}_{\mathcal{D}_{o, d}^{\times}}\left(\operatorname{res}_{\mathcal{D}_{o, d}^{\times}}^{D_{o, d}^{\times}} \tau_{o}, \Psi_{F_{o}, \xi_{o}^{-1}}^{d, i}\right)$ où l'action de $\left(g_{o}^{c}, \sigma_{o}\right)$ est donnée par celle de $\left(g_{o}, \delta_{o}, \sigma_{o}\right) \in \mathcal{N}_{o}^{\prime}$ pour $\delta_{o} \in D_{o, d}^{\times}$quelconque.

IV.1.2. Les variétés globales et les systèmes locaux d'Harris-Taylor. - Par souci de simplicité, les notations et le contexte sont ceux de [19] et [5], i.e. le cas d'égale caractéristique. Les résultats que nous rappelons sont une traduction de ceux de [14] et peuvent être trouvés dans le chapitre I. Il se trouve que les preuves qui suivent reposent formellement sur les propriétés cohomologiques ci-après de sorte que les résultats que nous obtenons sont également valides en caractéristique mixte dans le cadre de $[\mathbf{1 4}]$; nous donnerons dans l'appendice A le dictionnaire entre nos notations et celle de [14] et nous préciserons où trouver les propriétés cohomologiques que nous utilisons; pour de plus amples détails on renvoie à [6]. Les données sont alors :

(1) une tour de schémas $\left(M_{I, o}\right)_{I}$ définis sur le trait $\operatorname{Spec} \mathcal{O}_{o}$ et indexés par les idéaux $I$ de $A$; cette tour est munie d'une action, par correspondances, du groupe $\left(D_{\AA}^{\infty}\right)^{\times}$;

(2) pour $\rho_{\infty}$ une représentation irréductible du groupe des inversibles $\bar{D}_{\infty}^{\times}$de l'algèbre à division centrale sur $F_{\infty}$ d'invariant $-1 / d$, un système local $\mathcal{L}_{\rho_{\infty}}$ sur les $M_{I, o}$;

(5) cf. [9] ou [5] 
(3) une stratification de la fibre spéciale $M_{I, s_{o}}$, par des sous-schémas fermés $M_{I, s_{o}}^{\geqslant h}$, pour $1 \leqslant$ $h \leqslant d$, de pure dimension $d-h$, telle que ${ }^{(6)}$ le complété de l'anneau local de $M_{I, o}$ en tout point géométrique de $M_{I, s_{o}}^{=h}$ est isomorphe à $\operatorname{Def}_{n}^{h}\left[\left[x_{1}^{n}, \cdots, x_{d-h}^{n}\right]\right]$. Par ailleurs les inclusions $j \geqslant h: M_{I, s_{o}}^{=h} \hookrightarrow M_{I, s_{o}}^{\geqslant h}$ sont affines;

(4) les strates non supersingulières, i.e. $h \neq d$, sont induites, au sens où il existe un sous-schéma fermé $M_{I, s_{o}, 1}^{=h}$ de $M_{I, s_{o}}^{=h}$ muni d'une action par correspondances de $P_{h, d}\left(F_{o}\right)$ telle que

$$
M_{I^{o} \mathcal{M}_{o}^{n}, s_{o}}^{=h}=M_{I^{o} \mathcal{M}_{o}^{n}, s_{o}, 1}^{=h} \times_{P_{h, d}^{o p}\left(\mathcal{O}_{o} / \mathcal{M}_{o}^{n}\right)} G L_{d}\left(\mathcal{O}_{o} / \mathcal{M}_{o}^{n}\right),
$$

où l'action de $\left(\begin{array}{cc}g_{o}^{c} & 0 \\ * & g_{o}^{e t}\end{array}\right)$ se factorise par $\left(-\operatorname{val}\left(\operatorname{det}\left(g_{o}^{c}\right)\right), g_{o}^{e t}\right) \in \mathbb{Z} \times G L_{d-h}\left(F_{o}\right)$ et où Frob agit via $\left(-1, I_{d-h}\right)$. Pour tout $g \in G L_{d}\left(F_{o}\right)$ d'image $a \in G L_{d}\left(F_{o}\right) / P_{h, d}\left(F_{o}\right)$, on notera $M_{I, s_{o}, a}^{=h}$ l'image par $g$ de $M_{I, s_{o}, 1}^{=h}$ et $P_{h, d, a}$ le parabolique correspondant;

(5) pour $\tau_{o}$ une représentation irréductible de $\bar{D}_{o, h}^{\times}$, un système local $\mathcal{F}_{\tau_{o}, I, a}$ sur $M_{\bar{I}, s_{o}, a}^{=h}$; on note $\mathcal{F}_{\tau_{o}, I}:=\mathcal{F}_{\tau_{o}, I, a} \times_{P_{h, d, a}\left(\mathcal{O}_{o} / \mathcal{M}_{o}^{n}\right)} G L_{d}\left(\mathcal{O}_{o} / \mathcal{M}_{o}^{n}\right)$ où $n=\operatorname{mult}_{o}(I)$;

(6) Soit $\bar{D}$ l'algèbre à division centrale sur $F$ telle que $\bar{D}_{\mathbb{A}}^{\infty, o} \simeq D_{\mathbb{A}}^{\infty, o}$ et $\bar{D}_{o}$ (resp. $\bar{D}_{\infty}$ ) est l'algèbre à division centrale sur $F_{o}\left(\right.$ resp. sur $\left.F_{\infty}\right)$ d'invariant $1 / d($ resp. $-1 / d)$. Pour tout idéal $I$ de $A$, l'ensemble des points supersinguliers $M_{I, s_{o}}^{d}\left(\overline{\mathbb{F}}_{q}\right)$ est en bijection avec le $\left(D_{\mathbb{A}}^{\infty}\right)^{\times} \times W_{o^{-}}$ ensemble

$$
\bar{D}^{\times} \backslash\left[\left(\bar{D}_{\mathbb{A}}^{\infty, o}\right)^{\times} / K_{\mathbb{A}, I}^{\infty, o} \times \mathbb{Z}\right]
$$

où l'action de $c_{o} \in W_{o}$ est donnée par la translation de $-\operatorname{deg}\left(c_{o}\right)$ sur la composante $\mathbb{Z}$ et où l'action de $g^{\infty} \in\left(D_{\mathbb{A}}^{\infty}\right)^{\times}$est donnée par la correspondance

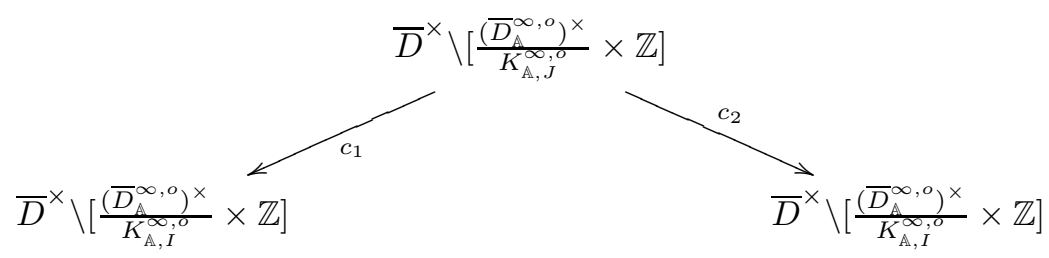

où $J$ est un idéal de $A$ tel que $K_{\mathbb{A}, J}^{\infty, o} \subset K_{\mathbb{A}, I}^{\infty, o} \cap\left(g^{\infty, o}\right)^{-1} K_{\mathbb{A}, I}^{\infty, o} g^{\infty, o}$, avec $c_{1}$ (resp. $\left.c_{2}\right)$ induit par l'inclusion $K_{\mathbb{A}, J}^{\infty, o} \subset K_{\mathbb{A}, I}^{\infty, o}$ (resp. par la multiplication à droite de $\left(g^{\infty, o}\right)^{-1} \operatorname{sur}\left(D_{\mathbb{A}}^{\infty, o}\right)^{\times}$) et la translation de $-\operatorname{val}\left(\operatorname{det}\left(g_{o}\right)\right)$ sur la composante $\mathbb{Z}$.

IV.1.2.1. Définition. - Pour tout diviseur $g$ de $d=s g$ et toute représentation irréductible cuspidale $\pi_{o}$ de $G L_{g}\left(F_{o}\right)$, on notera $\mathcal{F}\left(g, s, \pi_{o}, I\right)$ le faisceau concentré aux points supersinguliers

$$
\begin{gathered}
\bar{D}^{\times} \backslash\left[\left(\bar{D}_{\mathbb{A}}^{\infty, o}\right)^{\times} / K_{\mathbb{A}, I}^{\infty, o} \times\left(\mathbb{Z} \times\left(\mathrm{JL}^{-1}\left([\overleftarrow{s-1}]_{\pi_{o}}\right)\right)^{K_{o}^{n}}\right)\right] \\
\downarrow \\
M_{I, s_{o}}^{d}\left(\overline{\mathbb{F}}_{q}\right)=\bar{D}^{\times} \backslash\left[\left(\bar{D}_{\mathbb{A}}^{\infty, o}\right)^{\times} / K_{\mathbb{A}, I}^{\infty, o} \times \mathbb{Z}\right]
\end{gathered}
$$

avec $n=\operatorname{mult}_{o}(I)$ et où l'action diagonale de $\bar{D}^{\times}$est donnée par translation à droite $\operatorname{sur}\left(\bar{D}_{\mathbb{A}}^{\infty, o}\right)^{\times}$, par translation de valeur - val $\operatorname{rn}($.$) sur \mathbb{Z}$ et par l'action naturelle sur $\mathrm{JL}^{-1}\left([\stackrel{\leftrightarrow}{s-1}]_{\pi_{o}}\right)$.

${ }^{(6)}$ C'est l'équivalent du théorème de Serre-Tate. 


\section{IV.2. Rappels des propriétés cohomologiques}

IV.2.1. Définition. - Soit $\tau_{o}$ une représentation irréductible de $D_{o, h}^{\times}$, sa restriction à $\mathcal{D}_{o, h}^{\times}$est une somme de représentations irréductibles

$$
\rho_{o, 1} \oplus \cdots \oplus \rho_{o, e_{\tau_{o}}}
$$

et on notera $e_{\tau_{o}}$ le nombre de celles ci. Étant donnée une représentation irréductible $\rho_{o}$ de $\mathcal{D}_{o, h}^{\times}$, soient alors $\tau_{o}$ et $\tau_{o}^{\prime}$ des sous-représentations irréductibles de l'induite de $\mathcal{D}_{o, h}^{\times}$à $D_{o, h}^{\times}$de $\rho_{o}$ : d'après la réciprocité de Frobenius, ce sont exactement celles telles que leur restriction à $\mathcal{D}_{o, h}^{\times}$ contienne $\rho_{o}$. On en déduit alors que $\tau_{o}$ et $\tau_{o}^{\prime}$ sont inertiellement équivalentes, i.e. $\tau_{o}^{\prime} \simeq \tau_{o} \otimes \xi_{o}$ avec $\xi_{o}: \delta \mapsto x^{v(\operatorname{det} \delta)}$ pour $x \in \overline{\mathbb{Q}}_{l}^{\times}$. On note $\mathfrak{C}_{h}$ l'ensemble des classes d'équivalences inertielles des représentations admissibles et irréductibles du groupe $D_{o, h}^{\times}$. De la même façon, deux représentations $\pi_{o}$ et $\pi_{o}^{\prime}$ de $G L_{g}\left(F_{o}\right)$ seront dites inertiellement équivalentes, s'il existe un caractère $\xi: \mathbb{Z} \longrightarrow \mathbb{Q}_{l}^{\times}$tel que $\pi_{o} \simeq \pi_{o}^{\prime} \circ \xi \circ$ valo det et on notera Cusp $_{g}$ l'ensemble des classes d'équivalence inertielle des représentations cuspidales de $G L_{g}\left(F_{o}\right)$ ainsi que $e_{\pi_{o}}$ le cardinal de la classe d'équivalence inertielle de $\pi_{o}$.

IV.2.2. Définition. - Soit $\sigma_{o}$ une représentation irréductible de $W_{o}$. Une représentation de $I_{o}$ sera dite $\sigma_{o}$-isotypique, si ses sous-représentations irréductibles sont aussi des sous-représentations irréductibles de la restriction à $I_{o}$ de $\sigma_{o}$.

Remarque : Si une représentation de $I_{o}$ est $\sigma_{o}$-isotypique et $\sigma_{o}^{\prime}$-isotypique alors $\sigma_{o}$ et $\sigma_{o}^{\prime}$ sont inertiellement équivalentes.

IV.2.3. Lemme. - Pour tout entier $g$ et toute représentation irréductible cuspidale $\pi_{o}$ de $G L_{g}\left(F_{o}\right), e_{\mathrm{JL}^{-1}\left([\overleftarrow{s-1}]_{\pi_{o}}\right)}=e_{\pi_{o}}$

Démonstration. - On rappelle que l'entier en question correspond au nombre de caractères $\xi_{o}$ de $\mathbb{Z}$ tels que $\tau_{o} \simeq \tau_{o} \otimes \xi_{o}$ o valo det en notant $\tau_{o}=\mathrm{JL}^{-1}\left([\overleftarrow{s-1}]_{\pi_{o}}\right)$; par Jacquet-Langlands c'est aussi le nombre de caractères $\xi_{o}$ de $\mathbb{Z}$ tels que

$$
[\overleftarrow{s-1}]_{\pi_{o}} \simeq[\overleftarrow{s-1}]_{\pi_{o}} \otimes\left(\xi_{o} \circ \text { val } \circ \text { det }\right) \simeq[\overleftarrow{s-1}]_{\pi_{o} \otimes\left(\xi_{o} \circ \text { val } \circ \text { det }\right)}
$$

et donc au nombre de caractères $\xi_{o}$ tels que $\pi_{o} \simeq \pi_{o} \otimes \xi_{o} \circ$ val $\circ$ det, d'où le résultat.

IV.2.1. Sur le modèle local. - Soit $\tau_{o}$ une représentation admissible irréductible de $D_{o, h}^{\times}$. L'espace que l'on souhaite étudier est le $\left(G L_{h}\left(F_{o}\right) \times W_{F_{o}}\right)$-module

$$
\operatorname{Hom}_{D_{o, h}^{\times}}\left(\tau_{o}, \mathcal{U}_{F_{o}}^{h, i}\right)=\mathcal{U}_{F_{o}}^{h, i}\left(\tau_{o}\right) \simeq \Psi_{F_{o}}^{h, i}\left(\tau_{o}\right):=\operatorname{Hom}_{\mathcal{D}_{o, h}^{\times}}\left(\tau_{o}, \Psi_{F_{o}}^{h, i}\right)
$$

IV.2.1.1 - Pour tout $\tau_{o}$, on a un morphisme naturel de $\mathcal{N}_{o}$-modules :

$$
\mathcal{U}_{F_{o}}^{h, i}\left(\tau_{o}\right) \otimes \tau_{o} \longrightarrow \Psi_{F_{o}}^{h, i}
$$

qui envoie $f \otimes v$ sur $f(v)$. On note $\Psi_{F_{o}}^{h, i}\left[\tau_{o}\right]$ l'image de ce morphisme et soit $\Psi_{F_{o}, m}^{h, i}\left[\tau_{o}\right]$ la préimage de $\Psi_{F_{o}}^{h, i}\left[\tau_{o}\right]$ dans $\Psi_{F_{o}, m}^{h, i}$. Le sous-module $\Psi_{F_{o}}^{h, i}\left[\tau_{o}\right]$ ne dépend que de la classe d'équivalence inertielle de $\tau_{o}$. Le groupe $\mathcal{D}_{o, h}^{\times}$étant compact, on a

$$
\Psi_{F_{o}}^{h, i}=\bigoplus_{\tau_{o} \in \mathfrak{C}_{h}} \Psi_{F_{o}}^{h, i}\left[\tau_{o}\right]
$$

Soit $\Delta_{\tau_{o}}$ un ensemble d'éléments de $D_{o, h}^{\times}$tel que les congruences des val(det $\delta$ ) pour $\delta \in \Delta_{\tau_{o}}$ forment un système de représentants de $\mathbb{Z} / e_{\tau_{o}} \mathbb{Z}$. L'application

$$
\begin{aligned}
\mathcal{U}_{F_{o}}^{h, i}\left(\tau_{o}\right) \otimes \tau_{o} & \longrightarrow \bigoplus_{\delta \in \Delta_{\tau_{o}}} \Psi_{F_{o}}^{h, i}\left[\tau_{o}\right]^{\delta} \\
f \otimes v & \mapsto\left(f\left(\delta^{-1} v\right)\right)_{\delta}
\end{aligned}
$$


où $\Psi_{F_{o}}^{h, i}\left[\tau_{o}\right]^{\delta}$ est l'espace $\Psi_{F_{o}}^{h, i}\left[\tau_{o}\right]$ muni de la structure de $\mathcal{N}_{o}$-module où $\left(g_{o}, \delta_{o}, c_{o}\right)$ agit via $\left(g_{o}, \delta^{-1} \delta_{o} \delta, c_{o}\right)$, est un isomorphisme de $\mathcal{N}_{o}$-modules.

IV.2.1.2. Définition. - On notera $\widetilde{\mathcal{U}_{F_{o}}^{h, i}\left(\tau_{o}\right)}$ l'espace $\mathcal{U}_{F_{o}}^{h, i}\left(\tau_{o}\right)$ où l'action de $G L_{h}\left(F_{o}\right)$ est tordue par l'application $g_{o} \mapsto{ }^{t} g_{o}^{-1}$.

IV.2.1.3. Théorème. - (cf. [5] théorème (3.2.4)) Pour toute représentation irréductible cuspidale $\pi_{o}$ de $G L_{d}\left(F_{o}\right), \widetilde{\mathcal{U}_{F_{o}}^{d, i}}\left(\mathrm{JL}^{-1}\left(\pi_{o}\right)\right)$ est nul pour $i \neq d-1$ et

$$
\widetilde{\mathcal{U}_{F_{o}}^{d, d-1}}\left(\mathrm{JL}^{-1}\left(\pi_{o}\right)\right) \simeq \pi_{o} \otimes L_{g}\left(\pi_{o}\right)\left(-\frac{d-1}{2}\right)
$$

IV.2.1.4. Théorème. - (cf. [14] théorème VII.1.5, ou le théorème (III.2.1)) Pour tout diviseur $g$ de $d=s g$ et toute représentation irréductible cuspidale $\pi_{o}$ de $G L_{g}\left(F_{o}\right)$, on a

$$
\begin{aligned}
& \sum_{i=0}^{d-1}(-1)^{i}\left[\widetilde{\mathcal{U}_{F_{o}}^{d, d-1}-i}\left(\mathrm{JL}^{-1}\left([\overleftarrow{s-1}]_{\pi_{o}}\right)\right)\right]= \\
& \sum_{i=1}^{s}(-1)^{i}[\overleftarrow{s-1-i}, \vec{i}]_{\pi_{o}} \otimes L_{g}\left(\pi_{o}\right)\left(-\frac{d+s-2-2 i}{2}\right)
\end{aligned}
$$

ou de manière équivalente

$$
\begin{aligned}
& \sum_{i=0}^{d-1}(-1)^{i}\left[\mathcal{U}_{F_{o}}^{d, d-1-i}\left(\mathrm{JL}^{-1}\left([\overleftarrow{s-1}]_{\pi_{o}}\right)\right)\right]= \\
& \sum_{i=1}^{s}(-1)^{i}[\vec{i}, \overleftarrow{s-1-i}]_{\pi_{o}} \otimes L_{g}\left(\pi_{o}\right)\left(-\frac{d+s-2-2 i}{2}\right)
\end{aligned}
$$

IV.2.2. Sur les systèmes locaux d'Harris-Taylor. — Dans la suite nous ne considérerons plus les schémas sur $\mathbb{F}_{p}, M_{I, s_{o}}, M_{I, s_{o}}^{=h} \ldots$ mais plutôt les $\overline{\mathbb{F}}_{p}$-schémas $M_{I, s_{o}} \times_{\mathbb{F}_{p}} \overline{\mathbb{F}}_{p}, M_{I, s_{o}}^{=h} \times_{\mathbb{F}_{p}} \overline{\mathbb{F}}_{p} \ldots$ Afin de ne pas alourdir encore plus les énoncés, nous garderons les mêmes notations, par exemple $M_{I, s_{o}}$ désignera ce que l'on devrait noter $M_{I, s_{o}} \times_{\mathbb{F}_{p}} \overline{\mathbb{F}}_{p}$.

Les systèmes locaux $\mathcal{F}_{\tau_{o}}$ d'Harris-Taylor sont tels que la restriction à $M_{I, s_{o}, 1}^{=h}$ du $i$-ème faisceau des cycles évanescents $R^{i} \Psi_{\eta_{o}, I}\left(\overline{\mathbb{Q}}_{l}\right)$ vérifie

$$
\left(R^{i} \Psi_{\eta_{o}, I}\left(\overline{\mathbb{Q}}_{l}\right)\right)_{M_{I, s_{o}, 1}^{h}}^{h} \simeq \bigoplus_{\tau_{o} \in \mathfrak{C}_{h}}\left(\mathcal{F}_{\tau_{o}, I} \otimes \widetilde{\mathcal{U}_{F_{o}, n}^{h, i}\left(\tau_{o}\right)}\right)^{h / e_{\tau_{o}}}
$$

où $n=\operatorname{mult}_{o}(I)$ et où l'action l'action d'un élément $\left(g^{\infty, o}, g_{o}^{e t}, g_{o}^{c}, r, c_{o}\right) \in\left(D_{\mathbb{A}}^{\infty, o}\right)^{\times} \times G L_{d-l g}\left(F_{o}\right) \times$ $G L_{l g}\left(F_{o}\right) \times \mathbb{Z} \times W_{o}$ est donnée par l'action naturelle de

$$
\left.\left(g^{\infty, o}, g_{o}^{e t}, r-\operatorname{val}\left(\operatorname{det} g_{o}^{c}\right)-\operatorname{deg}\left(c_{o}\right)\right)\right) \in\left(D_{\mathbb{A}}^{\infty, o}\right)^{\times} \times G L_{d-l g}\left(F_{o}\right) \times \mathbb{Z}
$$

sur la tour des $\left(\mathcal{F}_{\tau_{o}, I}\right)_{I}$ au dessus de $M_{I, s_{o}, 1}^{=l g}$, et par celle de $\left(g_{o}^{c}, c_{o}\right)$ sur la tour des $\left.\left(\widetilde{\mathcal{U}_{F_{o}, n}^{h, i}\left(\tau_{o}\right.}\right)\right)_{n}$. L'isomorphisme (IV.2.2.12) implique alors la proposition suivante.

IV.2.2.1. Proposition. - Pour tout $i, j$, on a un isomorphisme canonique

$$
H_{c}^{j}\left(M_{I, s_{o}, 1}^{=h}, R^{i} \Psi_{\eta_{o}, I}\left(\mathcal{L}_{\rho_{\infty}}\right)\right)^{h} \simeq \bigoplus_{\tau_{o} \in \mathfrak{C}_{h}}\left(H_{c}^{j}\left(M_{I, s_{o}, 1}^{=h}, \mathcal{F}_{\tau_{o}, I} \otimes \mathcal{L}_{\rho_{\infty}}\right) \otimes \mathcal{U}_{F_{o}, m}^{h, i}\left(\tau_{o}\right)\right)^{h / e_{\tau_{o}}}
$$

avec $m=$ mult $_{o}(I)$ et tel que l'action de $\left(g^{\infty, o}, g_{o}^{c}, g_{o}^{e t}, \sigma_{o}\right) \in\left(D_{\mathbb{A}}^{\infty, o}\right)^{\times} \times G L_{h}\left(F_{o}\right) \times G L_{d-h}\left(F_{o}\right) \times W_{F_{o}}$ sur la limite inductive ${ }^{(7)}$ du membre de gauche, induit l'action de

$$
\left(g^{\infty, o}, g_{o}^{e t},-\operatorname{val}\left(\operatorname{det} g_{o}^{c}\right)-\operatorname{deg}\left(\sigma_{o}\right)\right) \otimes\left(g_{o}^{c}, \sigma_{o}\right)
$$

(7) indexée par les idéaux $I=I^{o} \mathcal{M}_{o}^{m}$ 
sur la limite inductive du membre de droite.

Remarque : Les systèmes locaux $\mathcal{F}_{\tau_{o}, I}$ ne sont pas irréductibles mais plutôt une somme directe de $e_{\tau_{o}}$ systèmes locaux irréductibles. La complexité de l'écriture de (IV.2.2.12), est la contre-partie de la simplicité de la description de l'action de $G L_{d}\left(F_{o}\right) \times W_{o}$ qui tient au fait que l'on a fait apparaître $\widetilde{\mathcal{U}_{F_{o}}^{h, i}}\left(\tau_{o}\right)$ plutôt que $\operatorname{Hom}_{\mathcal{D}_{o, h}^{\times}}\left(\rho_{o}, \widetilde{\Psi_{F_{o}}^{h, i}}\right)$ avec $\rho_{o}$ une représentation irréductible de $I_{o}$ de sorte que ce dernier est seulement muni d'une action de $\widetilde{\mathcal{N}}_{o} \cap\left(G L_{h}\left(F_{o}\right) \times D_{o, h}^{\times}\right)$.

IV.2.2.2. Définition. - Dans la suite, pour $\rho_{\infty}$ une représentation irréductible de $\bar{D}_{\infty}^{\times}$, qui est "l" 'algèbre à division centrale sur $F_{\infty}$ d'invariant $-1 / d$, on considère pour une représentation automorphe $\Pi$ de $D_{\mathbb{A}}^{\times}$, l'hypothèse $\operatorname{Hyp}\left(\rho_{\infty}\right)$ suivante : si $\rho_{\infty}=J L^{-1}\left(\operatorname{St}_{s}\left(\pi_{\infty}\right)\right.$ pour $\pi_{\infty}$ une représentation irréductible cuspidale de $G L_{g}\left(F_{\infty}\right)$ pour $d=s g$, alors $\Pi_{\infty}$ est soit isomorphe à $\overleftarrow{(s)}_{\pi_{\infty}}$ soit à $\overrightarrow{(s}_{\pi_{\infty}}$. Plus généralement on notera $\operatorname{Hyp}(\infty)$ l'hypothèse sur $\Pi$ qu'il existe $\rho_{\infty}$ tel que $\Pi$ vérifie $\operatorname{Hyp}\left(\rho_{\infty}\right)$.

Remarque : Dans le cas de caractéristique mixte la condition $\mathrm{Hyp}(\infty)$ est rappelée dans l'appendice A.

On note $\left[H_{h, \rho_{\infty}, \tau_{o}}^{*}\right]:=\sum_{i}(-1)^{i} \underset{I}{\underset{I}{\lim }}\left[H_{c}^{i}\left(M_{I, s_{o}, 1}^{=h}, \mathcal{F}_{\tau_{o}, I} \otimes \mathcal{L}_{\rho_{\infty}}\right)\right]$ dans le groupe de Grothendieck des représentations admissibles de $\left(D_{\mathbb{A}}^{\infty, o}\right)^{\times} \times G L_{d-h}\left(F_{o}\right) \times \mathbb{Z}$.

IV.2.2.3. Définition. — Pour tout entier $h$, on considère l'identification canonique $D_{o, h}^{\times} / \mathcal{D}_{o, h}^{\times} \longrightarrow$ $\mathbb{Z}$ défini par la valuation de la norme réduite. On notera $\Xi: \mathbb{Z} \longrightarrow \overline{\mathbb{Q}}_{l}^{\times}$le caractère défini par $\Xi(1)=\frac{1}{p}$

IV.2.2.4. Proposition. — Pour $\Pi$ une représentation de $D_{\mathbb{A}}^{\times}$, on a alors

$$
\left[H_{h, \rho_{\infty}, \tau_{o}}^{*}\left(\Pi^{\infty, o}\right)\right]= \begin{cases}\epsilon(\Pi) m(\Pi) \operatorname{Red}_{\tau_{o}}^{h}\left(\Pi_{o}\right) & \text { si } \Pi_{\infty} \text { vérifie } \operatorname{Hyp}(\infty) \\ 0 & \text { sinon }\end{cases}
$$

où $\epsilon(\Pi)$ est un signe qui dépend de $\Pi, m(\Pi)$ est la multiplicité de $\Pi$ dans l'espace des formes automorphes et $\operatorname{Red}_{\tau_{o}}^{h}: \operatorname{Groth}\left(G L_{d}\left(F_{o}\right)\right) \longrightarrow \operatorname{Groth}\left(D_{o, h}^{\times} / \mathcal{D}_{o, h}^{\times} \times G L_{d-h}\left(F_{o}\right)\right)$ est défini comme la composition des deux homomorphismes suivant :

- en premier lieu, on a un homomorphisme

$$
\begin{aligned}
& \operatorname{Groth}\left(G L_{d}\left(F_{o}\right)\right) \longrightarrow \operatorname{Groth}\left(G L_{h}\left(F_{o}\right) \times G L_{d-h}\left(F_{o}\right)\right) \\
& {\left[\pi_{o}\right] \mapsto\left[J_{P_{h, d}}\left(\pi_{o}\right) \otimes \delta_{P_{h, d}}^{1 / 2}\right]}
\end{aligned}
$$

- ensuite on a un homomorphisme

$$
\begin{aligned}
& \operatorname{Groth}\left(G L_{h}\left(F_{o}\right) \times G L_{d-h}\left(F_{o}\right)\right) \longrightarrow \operatorname{Groth}\left(D_{o, h}^{\times} / \mathcal{D}_{o, h}^{\times} \times G L_{d-h}\left(F_{o}\right)\right) \\
& {[\alpha \otimes \beta] \mapsto \sum_{\psi} \operatorname{vol}\left(D_{o, h}^{\times} / F_{o}^{\times}, d \bar{h}_{o}\right)^{-1} \operatorname{Tr} \alpha\left(\phi_{\mathrm{JL}\left(\tau_{o} \otimes \psi^{-1}\right)}\right)[\psi \otimes \beta],}
\end{aligned}
$$

où $\psi$ décrit les caractères de $\mathbb{Z} \simeq D_{o, h}^{\times} / \mathcal{D}_{o, h}^{\times}$tels que $\alpha$ et $\tau_{o} \otimes \psi^{-1}$ ont le même caractère central et où l'on considère des mesures de Haar associées sur $G L_{h}\left(F_{o}\right)$ et $D_{o, h}^{\times}$.

Remarque : En égale caractéristique on a $\epsilon(\Pi)=1\left(\operatorname{resp} . \epsilon(\Pi)=(-1)^{s-1}\right)$ si $\Pi \simeq \operatorname{St}_{s}\left(\pi_{\infty}\right)($ resp. $\left.\Pi_{\infty} \simeq \operatorname{Speh}_{s}\left(\pi_{\infty}\right)\right)$ pour $\pi_{\infty}$ une représentation irréductible cuspidale de $G L_{g}\left(F_{\infty}\right)$ avec $d=s g$. En caractéristique mixte, $\epsilon(\Pi)=1$ (resp. $\epsilon(\Pi)=(-1)^{s-1}$ ) s'il existe une place $x$ tel que $\Pi_{x}$ est de carré intégrable (resp. $\Pi_{x} \simeq \operatorname{Speh}_{s}\left(\pi_{x}\right)$ pour $\pi_{x}$ irréductible cuspidale de $G L_{g}\left(F_{x}\right)$ avec $d=s g$ ). (8)

${ }^{(8)}$ L'auteur n'est pas certain qu'en caractéristique mixte, le cas $\Pi_{x} \simeq \operatorname{Speh}_{s}\left(\pi_{x}\right)$ soit connu, de sorte qu'on en donnera une preuve. 
Pour toute représentation irréductible $\rho_{\infty}$ de $\bar{D}_{\infty}^{\times}$, on note $\mathcal{C}_{\bar{D}, \rho_{\infty}}^{\infty}$ la composante $\rho_{\infty}$-isotypique de $C^{\infty}\left(\bar{D}^{\times} \backslash\left(\bar{D}_{\mathbb{A}}\right)^{\times}\right)$.

IV.2.2.5. Proposition. - Pour tout $i$, on a un isomorphisme $\left(D_{\mathbb{A}}^{\infty}\right)^{\times} \times W_{\text {o-équivariant }}$

$$
\lim _{\vec{I}} H^{0}\left(M_{I, s_{o}}^{d}, R^{i} \Psi_{\eta_{o}, I}\left(\mathcal{L}_{\rho_{\infty}}\right)\right) \simeq \operatorname{Hom}_{\bar{D}_{o}^{\times}}\left(\left(\mathcal{C}_{\bar{D}, \rho_{\infty}}^{\infty}\right)^{\vee}, \widetilde{\mathcal{U}_{F_{o}}^{d, i}}\right)
$$

\section{IV.3. Énonces des théorèmes locaux}

IV.3.1. Groupes de cohomologie des modèles de Deligne-Carayol. - Considérons le complexe à flèches nulles

$$
\begin{array}{r}
M L^{\bullet}(s):=\left([\overrightarrow{s-1}]_{1_{o}} \otimes|\mathrm{cl}|^{(s-1) / 2},[\overleftarrow{1}, \overrightarrow{s-2}]_{1_{o}} \otimes|\mathrm{cl}|^{(s-3) / 2}, \cdots,[\overleftarrow{s-1}]_{1_{o}} \otimes|\mathrm{cl}|^{(1-s) / 2}\right) \\
\otimes \mathrm{JL}^{-1}\left(\mathrm{St}_{s}\left(1_{o}\right)\right) \otimes L_{g}\left(1_{o}\right)((1-s) / 2)
\end{array}
$$

où $[\overleftarrow{s-1}]_{1}$ est placé en degré 0 . Par définition, pour $\pi_{o}$ une représentation cuspidale de $G L_{g}\left(F_{o}\right)$, on pose

$$
\begin{array}{r}
\pi_{o} \diamond M L \cdot(s):=\left([\overrightarrow{s-1}]_{\pi_{o}} \otimes|\mathrm{cl}|^{(s-1) / 2},[\overleftarrow{1}, \overrightarrow{s-2}]_{\pi_{o}} \otimes|\mathrm{cl}|^{(s-3) / 2}, \cdots,[\overleftarrow{s-1}]_{\pi_{o}} \otimes|\mathrm{cl}|^{(1-s) / 2}\right) \\
\otimes \mathrm{JL}^{-1}\left(\mathrm{St}_{s}\left(\pi_{o}\right)\right) \otimes L_{g}\left(\pi_{o}\right)((1-s g) / 2)
\end{array}
$$

IV.3.1.1. Théorème. - Pour tout d, on a

$$
\widetilde{\mathcal{U}_{F_{o}}^{d, d-1+}}=\bigoplus_{\substack{g \mid d \\ d=s g}} \bigoplus_{\pi_{o} \in \mathrm{Cusp}_{g}} \pi_{o} \diamond M L^{\bullet}(s)
$$

où $\operatorname{Cusp}_{g}$ désigne l'ensemble des classes d'équivalence des représentations irréductibles cuspidales de $G L_{g}\left(F_{o}\right)$.

$I V .3 .1 .2$ - Autrement dit, pour $\pi_{o}$ une représentation irréductible cuspidale de $G L_{g}\left(F_{o}\right)$ on a :

$$
\widetilde{\mathcal{U}_{F_{o}}^{d, d-s+i}}\left(\mathrm{JL}^{-1}\left(\mathrm{St}_{s}\left(\pi_{o}\right)\right)\right) \simeq\left\{\begin{array}{cl}
L_{g}\left(\pi_{o}\right)\left(-\frac{d-s+2 i}{2}\right) \otimes[\overleftarrow{i}, \overrightarrow{s-i-1}]_{\pi_{o}} & 0 \leqslant i<s \\
0 & i<0
\end{array}\right.
$$

où $[\overleftarrow{i}, \overrightarrow{s-i-1}]_{\pi_{o}}$ est l'unique quotient irréductible de l'induite

Remarque : De manière équivalente on a

$$
\operatorname{Ind}_{P_{i g, s g}\left(F_{o}\right)^{o p}}^{G L_{s g}\left(F_{o}\right)} \operatorname{St}_{i}\left(\pi_{o}\left(-\frac{(s-i)(g-1)}{2}\right)\right) \otimes \operatorname{Speh}_{s-i}\left(\pi_{o}\left(\frac{i(g-1)}{2}\right)\right) .
$$

$$
\mathcal{U}_{F_{o}}^{d, d-s+i}\left(\mathrm{JL}^{-1}\left(\mathrm{St}_{s-1}\left(\pi_{o}\right)\right)\right) \simeq L_{g}\left(\pi_{o}\right)\left(-\frac{d-s+2 i}{2}\right) \otimes[\overrightarrow{s-1-i}, \overleftarrow{i}]_{\pi_{o}^{\vee}}
$$

IV.3.2. Filtration de monodromie-locale. - On rappelle le théorème principal de l'appendice de $[6]$, fournie par Laurent Fargues.

IV.3.2.1. Théorème-définition. - (cf. le théorème principal de l'appendice de [6]) Soit $X \rightarrow$ Spec $\mathcal{O}_{o}$ un morphisme propre d'un schéma $X$ de type fini de dimension d sur le trait $\operatorname{Spec} \mathcal{O}_{o}$. Le complexe des cycles évanescents $R \Psi_{\eta_{o}}\left(\mathbb{Q}_{l}\right)[d-1]$ est un faisceau pervers muni d'une action de $W_{o}$ qui fournit une filtration de monodromie dont on notera $\mathrm{gr}_{k}$ les gradués et on considère la suite spectrale $E_{1}^{i, j}=h^{i+j} g_{-i} \Rightarrow R^{i+j+d-1} \Psi_{\eta_{o}}\left(\mathbb{Q}_{l}\right)$. Pour tout point géométrique $x$ de la fibre spéciale $X_{s_{o}}$, en considérant les germes en $x$ des $h^{i} g_{k}$, on obtient une suite spectrale

$$
E_{1, x}^{i, j}=\left(h^{i+j} g r_{-i}\right)_{x} \Rightarrow R^{i+j+d-1} \Psi_{\eta_{o}}\left(\mathbb{Q}_{l}\right)_{x}
$$

dont la nature est purement locale de sorte que si $Y \rightarrow \operatorname{Spec} \mathcal{O}_{o}$ est un autre schéma avec un point y tel que le complété formel de l'anneau local de $Y$ en y est isomorphe, en tant que $\mathcal{O}_{o}$-schéma 
formel, au complété formel de l'anneau local de $X$ en $x$, alors pour tout $r \geqslant 1$, on a $E_{r, x}^{i, j}=E_{r, y}^{i, j}$. La filtration ainsi obtenue sera dite de monodromie-locale.

$I V$.3.2.2 - Pour tout $s \geqslant 1$, on introduit une suite de bicomplexe $M L E_{r}^{i, j}(s)$ définit comme suit : - pour $r=1, M L E_{1}^{i, j}(s)$ est nul pour $|j| \geqslant s$ ou $j \equiv s \bmod 2$ ou $i+j>0$ ou $i<(1-s-j) / 2$. Sinon pour $j=1-s+2 r$ avec $0 \leqslant r<s$ et $i=s-1-2 r-k$ avec $0 \leqslant k \leqslant(s-1-j) / 2$, on a

$$
M L E_{1}^{s-1-2 r-k, 1-s+2 r}(s)=\left.[\overleftarrow{s-1-k}]_{1_{o}} \overrightarrow{\times}[\overrightarrow{k-1}]_{1_{o}} \otimes|c|\right|^{(s-1-2 r) / 2}
$$

- les flèches $d_{1}^{i, j}$ se déduisent des suites exactes courtes (IV.3.2.15);

- pour tout $r \geqslant 2, M L E_{r}^{i, j}(s)=M L E_{2}^{i, j}(s)$ est nul pour $|j| \geqslant s$ ou $j \equiv s \bmod 2$ ou $2 i \neq 1-s-j$, et

$$
M L E_{2}^{1-s+r, 1-s+2 r}=[\overleftarrow{s-1-r}, \vec{r}]_{1_{o}} \otimes|\mathrm{cl}|^{-(s-1-2 r) / 2}
$$

Par exemple pour $s=4$ on a représenté à la figure (1) les $M L E_{1}^{i, j}(4)$ et $M L E_{\infty}^{i, j}(4)$.

Nous allons en fait prouver l'énoncé suivant dont découle directement le théorème (IV.3.1.1).

IV.3.2.3. Théorème. - La filtration de monodromie-locale du complexe $\widetilde{\mathcal{U}_{F_{o}, n}^{d, \bullet}}$ est équivariante pour l'action de l'algèbre de Hecke $\mathcal{H}_{n}=\mathcal{C}^{\infty}\left(K_{o, n} \backslash G L_{d}\left(F_{o}\right) / K_{o, n}\right)$ et celle de $I_{o}$; en outre pour $n$ variant, les filtrations de monodromie locale forment des systèmes inductifs compatibles à l'action des correspondances de Hecke. Les termes $E_{r, g r, l o c}^{i, j}$ de la suite spectrale associée

$$
E_{1, g r, l o c}^{i, j}=h^{i+j} g r_{d, n, l o c,-i} \Rightarrow \widehat{\mathcal{U}_{F_{o}, n}^{d, d-1+i+j}}
$$

vérifient

$$
E_{\bullet, g r, l o c}^{\bullet, d-1+\bullet}\left(\frac{d-1}{2}\right)=\bigoplus_{g \mid d=s g} \bigoplus_{\pi_{o} \in \operatorname{Cusp}_{g}} J L^{-1}\left(\operatorname{St}_{s}\left(\pi_{o}\right)\right) \otimes\left(\pi_{o} \diamond M L E_{\bullet}^{\bullet, \bullet}(s)\right)
$$

$I V .3 .2 .4$ - Autrement dit, les gradués $g r_{d, n, l o c, k}$ vérifient les propriétés suivantes :

(1) pour tout $k, g r_{d, n, l o c, k}$ est une somme directe

$$
\bigoplus_{\substack{g, d=s g \\ \pi_{o} \in \operatorname{Cusp}}} g r_{d, n, l o c, k, \pi_{o}}
$$

où en tant que $I_{o}$-module, $g r_{d, n, l o c, k, \pi_{o}}$ est $L_{g}\left(\pi_{o}\right)$-isotypique au sens de la définition (IV.2.2) (9)

(2) les $g r_{d, n, l o c, k, \pi_{o}}$ sont nuls pour $|k| \geqslant s$ et pour $|k|<s$, ses groupes de cohomologie $h^{i} g r_{d, n, l o c, k, \pi_{o}}$ sont nuls pour $i<d-s+|k|$ et pour $i \not \equiv d-s+k \bmod 2$; pour $d-s+|k| \leqslant$ $i=d-s+|k|+2 r \leqslant d-1, h^{d-s+|k|+2 r} g r_{d, l o c, k, \pi_{o}}$ est l'espace des vecteurs invariants sous $K_{o, n} \subset G L_{d}\left(F_{o}\right)$ de l'espace suivant :

$$
\mathrm{JL}^{-1}\left([\overleftarrow{s-1}]_{\pi_{o}}\right) \otimes[\overleftarrow{|k|+2 r}]_{\pi_{o}} \overrightarrow{\times}[\overrightarrow{s-2-2 r-|k|}]_{\pi_{o}} \otimes L_{g}\left(\pi_{o}\right)\left(-\frac{i+k}{2}\right)
$$

(3) la suite spectrale $E_{1, g r, l o c, \pi_{o}}^{i, j}=h^{i+j} g r_{d, n, l o c,-i, \pi_{o}} \Rightarrow \widetilde{\mathcal{U}_{F_{o}, n}^{d, i+j}}$ dégénère en $E_{2}$ et ses $d_{1}^{i, j}$ se déduisent des suites exactes courtes

$$
0 \rightarrow[\overleftarrow{l-1}, \overrightarrow{s-l}]_{\pi_{o}} \rightarrow[\overleftarrow{l-1}]_{\pi_{o}} \overrightarrow{\times}[\overrightarrow{s-l-1}]_{\pi_{o}} \rightarrow[\overleftarrow{l}, \overrightarrow{s-l-1}]_{\pi_{o}} \rightarrow 0
$$

\footnotetext{
${ }^{(9)}$ Sans utiliser le fait que les $L_{g}\left(\pi_{o}\right)$ décrivent l'ensemble des représentations irréductibles de $W_{o}$, l'énoncé dit que pour $\sigma_{o}$ qui ne serait pas de la forme $L_{g}\left(\pi_{o}\right), g r_{d, l o c, k, \sigma_{o}}$ est nul.
} 


\section{IV.4. Énonces des théorèmes globaux}

IV.4.1. Rappels sur les faisceaux pervers. - Pour $X$ un schéma séparé de type fini sur un corps, on considère la $t$-structure perverse autoduale sur $D_{c}^{b}\left(X, \overline{\mathbb{Q}}_{l}\right)$. On reprend les notations et les résultats de $[\mathbf{2}]$ : en particulier quand il n'y a pas de confusion relativement à $X$, on note pour $a \in \mathbb{Z},{ }^{p} \tau^{\leqslant a},{ }^{p} \tau^{\geqslant a}$ les foncteurs de troncations de $D_{c}^{b}:=D_{c}^{b}\left(X, \overline{\mathbb{Q}}_{l}\right)$ dans $D^{\leqslant a}:=D_{c}^{b}\left(X, \overline{\mathbb{Q}}_{l}\right)^{\leqslant a}$ et $D^{\geqslant a}:=D_{c}^{b}\left(X, \overline{\mathbb{Q}}_{l}\right) \geqslant a$, Perv $:=D^{\geqslant 0} \cap D^{\leqslant 0},{ }^{p} H^{0}:={ }^{p} \tau^{\leqslant 0} p_{\tau} \geqslant 0: D_{c}^{b} \longrightarrow$ Perv.

IV.4.1.1. Définition. - Soit $\sigma_{o}$ une représentation irréductible de $W_{o}$. Un faisceau pervers $P$ irréductible, de la forme $j_{\text {!* }} \mathcal{L}$ pour $j: U \hookrightarrow X$ localement fermé et $\mathcal{L}$ un système local sur $U$, muni d'une action de $I_{o}$, sera dit $\sigma_{o}$-isotypique si $\mathcal{L}$ l'est au sens de la définition (IV.2.2).

IV.4.1.2. Lemme. - Soient $\sigma_{o, 1}$ et $\sigma_{o, 2}$ des représentations irréductibles non inertiellement équivalentes de $W_{o}$ et soient $P_{1}, P_{2}$ des faisceaux pervers muni d'une action de $I_{o}$, respectivement $\sigma_{o, 1}$ et $\sigma_{o, 2}$ isotypique. Si $\varphi: P_{1} \longrightarrow P_{2}$ est un morphisme de faisceau pervers $I_{o}$-équivariant, pour tout $i$, on $a h^{i} \varphi=0$.

Démonstration. - C'est immédiat.

Le lemme (5.3.6) de [2] donne alors la décomposition en somme directe suivante.

$I V$.4.1.3. Proposition. - Tout faisceau pervers $P$ sur $X$ muni d'une action de $I_{o}$ s'écrit comme une somme directe

$$
\bigoplus_{\sigma_{o}} P_{\sigma_{o}}
$$

où la somme porte sur les classes d'équivalence inertielle des représentations irréductibles de $W_{o}$ et où $P_{\sigma_{o}}$ est $\sigma_{o}$-isotypique au sens de la définition (IV.2.2). En particulier pour tout point géométrique $x: \operatorname{Spec} K \longrightarrow X,\left(P_{\sigma_{o}}\right)_{x}$ est une représentation $\sigma_{o}$-isotypique.

IV.4.1.4. Corollaire. - Soit $P$ un faisceau pervers sur $X$ muni d'une action compatible de $W_{o}$. On considère sa filtration de monodromie et on note $\operatorname{gr}_{k} P$ son gradué de poids $k$. La suite spectrale

$$
E_{1, g r, g l o b}^{i, j}=h^{i+j} g r_{-i} P \Longrightarrow h^{i+j} P
$$

est la somme directe sur les classes d'équivalence inertielle des représentations irréductibles $\sigma_{o}$ de $W_{o}$ des suites spectrales

$$
E_{1, g r, g l o b, \sigma_{o}}^{i, j}=h^{i+j} g_{-i} P_{\sigma_{o}} \Longrightarrow h^{i+j} P_{\sigma_{o}}
$$

$I V .4 .1 .5$ - Le complexe des cycles évanescents $R \Psi_{\eta_{o}, I}\left(\overline{\mathbb{Q}}_{l}\right)[d-1]$ sur $M_{I, s_{o}}$ est un faisceau pervers muni d'une action de $W_{F_{o}}$; on considère alors sa filtration de monodromie et on note $g r_{k, I}$ son

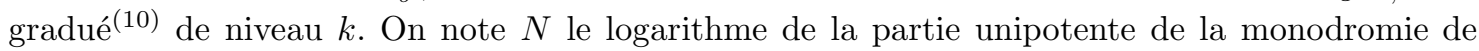
sorte qu'en particulier on a

$$
N^{k}: g r_{k, I} \simeq g r_{-k, I} .
$$

On pose $g r_{k}=\left(g r_{k, I}\right)_{I}$, et on dispose alors de la suite spectrale

$$
E_{1, I, g r, g l o b}^{i, j}=h^{i+j}\left(g r_{-i, I}\right) \Rightarrow h^{i+j}\left(R \Psi_{\eta_{o}, I}\left(\overline{\mathbb{Q}}_{l}\right)\right)[d-1]=R^{i+j+d-1} \Psi_{\eta_{o}, I}\left(\overline{\mathbb{Q}}_{l}\right)
$$

équivariante sous l'action de $\left(D_{\mathbb{A}}^{\infty}\right)^{\times} \times W_{o}$, qui, d'après le corollaire précédent, se scinde en une somme directe portant sur les classes d'équivalence inertielle des représentations irréductibles $\sigma_{o}$ de $W_{o}$ :

$$
E_{1, I, g r, g l o b, \sigma_{o}}^{i, j}=h^{i+j} g r_{-i, I, \sigma_{o}} \Rightarrow h^{i+j}\left(R \Psi_{\eta_{o}, I, \sigma_{o}}\left(\overline{\mathbb{Q}}_{l}\right)\right)[d-1]=R^{i+j+d-1} \Psi_{\eta_{o}, I, \sigma_{o}}\left(\overline{\mathbb{Q}}_{l}\right)
$$

${ }^{(10)}$ en égale caractéristique, les gradués $g r_{k, I}$ de la filtration de monodromie sont purs de poids $k+d-1$, i.e. les gradués de la filtration de monodromie sont purs. 
IV.4.1.6. Définition. - Pour $\rho_{\infty}$ une représentation irréductible de $\bar{D}_{\infty}^{\times}$, on notera $g r_{k, I, \rho_{\infty}}$ le gradué de $R \Psi_{\eta_{o}, I}\left(\mathcal{L}_{\rho_{\infty}}\right)$ soit $g r_{k, I, \rho_{\infty}} \simeq g r_{k, I} \otimes \mathcal{L}_{\rho_{\infty}}$. Pour $\sigma_{o}$ une représentation irréductible de $W_{o}$, on notera $g r_{k, I, \rho_{\infty}, \sigma_{o}}:=g r_{k, I, \sigma_{o}} \otimes \mathcal{L}_{\rho_{\infty}}$. Pour $\pi_{o}$ une représentation irréductible cuspidale de $G L_{g}\left(F_{o}\right)$, on notera $g r_{k, I, \pi_{o}}$ (resp. $g r_{k, I, \rho_{\infty}, \pi_{o}}$ ) pour $g r_{k, I, L_{g}\left(\pi_{o}\right)}$ (resp. $g r_{k, I, \rho_{\infty}, L_{g}\left(\pi_{o}\right)}$ ) et de manière générale on mettra un indice $\pi_{o}$ en lieu et place de $L_{g}\left(\pi_{o}\right)$.

IV.4.2. Définition de la catégorie des faisceaux pervers de Hecke sur $X_{\mathcal{I}}$. - Il s'agit ici de donner le cadre catégoriel pour les énoncés et les preuves des résultats des paragraphes suivants; ce qui suit m’a été suggéré par Jean-François Dat.

$I \boldsymbol{V}$.4.2.1. Définition. - On considère un groupe $G$ et on considère un ensemble $\mathcal{I}$ tel qu'à tout $I \in \mathcal{I}$ soit associé un sous-groupe compact $\mathcal{K}_{I}$ de $\tilde{G}$. On met sur $\mathcal{I}$ une relation d'ordre partiel $J \subset I$ si et seulement si $\mathcal{K}_{J}$ est un sous-groupe distingué de $\mathcal{K}_{I}$. Un schéma de Hecke pour $(G, \mathcal{I})$, est un système projectif de schémas

$$
X_{\mathcal{I}}=\left(X_{I}\right)_{I \in \mathcal{I}}
$$

relativement à des morphismes finis $r_{J, I}: X_{J} \longrightarrow X_{I}$ de restriction du niveau, tel que pour tout $g \in G$ et tout $J \subset I$ tels que $g^{-1} \mathcal{K}_{J} g \subset \mathcal{K}_{I}$, on dispose d'un morphisme fini de schémas

$$
[g]_{J, I}: X_{J} \longrightarrow X_{I}
$$

vérifiant les propriétés suivantes :

- pour tout $g \in \mathcal{K}_{I}$ et tout $J \subset I,[g]_{J, I}: X_{J} \longrightarrow X_{I}$ est égal au morphisme de restriction du niveau $r_{J, I}$

- pour tout $g, g^{\prime} \in G$, et tout $K \subset J \subset I$ tels que $g^{-1} \mathcal{K}_{J} g \subset \mathcal{K}_{I}$ et $\left(g^{\prime}\right)^{-1} \mathcal{K}_{K} g^{\prime} \subset \mathcal{K}_{J}$, on a

$$
[g]_{J, I} \circ\left[g^{\prime}\right]_{K, J}: X_{K} \longrightarrow X_{J} \longrightarrow X_{I}
$$

est égal à $\left[g g^{\prime}\right]_{K, I}$.

- pour tout $J \subset I, X_{J} / \mathcal{K}_{I} \simeq X_{I}$, où $g \in \mathcal{K}_{I}$ agit sur $X_{J}$ via $[g]_{J, J} ;$ autrement dit $r_{J, I}: X_{J} \longrightarrow$ $X_{I}$ est un bon quotient de $X_{J}$ par $\mathcal{K}_{I} / \mathcal{K}_{J}$.

Exemples : $\mathcal{I}$ est l'ensemble des idéaux $I$ de $A$, avec $X_{I}=M_{I, o}, M_{I, s_{o}}$ avec $G=\left(D_{\mathbb{A}}^{\infty}\right)^{\times}$ou $X_{I}=M_{I, s_{o}, 1}^{=h}, M_{I, s_{o}, 1}^{\geqslant h}$ avec $G=\left(D_{\mathbb{A}}^{\infty, o}\right)^{\times} \times P_{h, d}\left(F_{o}\right)$.

IV.4.2.2. Définition. - Soit $X_{\mathcal{I}}=\left(X_{I}\right)_{I}$ un schéma de Hecke pour $(G, \mathcal{I})$, on définit alors la catégorie $\mathrm{FPH}_{G}\left(X_{\mathcal{I}}\right)$ (resp. $\mathrm{FH}_{G}\left(X_{\mathcal{I}}\right)$ ) des faisceaux pervers (resp. des faisceaux) de Hecke sur $X_{\mathcal{I}}$ comme la catégorie dont les objets sont les systèmes $\left(\mathcal{F}_{I}\right)_{I \in \mathcal{I}}$ où $\mathcal{F}_{I}$ est un faisceau pervers (resp. faisceau) sur $X_{I}$ tels que

- pour tout $g \in G$ et $J \subset I$ tel que $g^{-1} \mathcal{K}_{J} g \subset \mathcal{K}_{I}$, on dispose d'un morphisme de faisceau sur $X_{I}$ :

$$
u_{J, I}(g): \mathcal{F}_{I} \longrightarrow[g]_{J, I, *} \mathcal{F}_{J}
$$

soumise à la condition de cocycle habituelle

$$
u_{K, I}\left(g^{\prime} g\right)=[g]_{J, I, *}\left(u_{K, J}\left(g^{\prime}\right)\right) \circ u_{J, I}(g)
$$

- pour tout $g \in \mathcal{K}_{I}, u_{J, I}(g)$ se factorise par les $\mathcal{K}_{I}$ invariants, i.e. induit une flèche $\mathcal{F}_{I} \longrightarrow$ $r_{J, I, *} \mathcal{F}_{J}^{\mathcal{K}_{I}}$ où $\mathcal{F}_{J}^{\mathcal{K}_{I}}$ désigne le sous-faisceau de $\mathcal{F}_{J}$ invariant par tous les $u_{J, J}(g)$ où $g$ décrit $\mathcal{K}_{I}$.

Les flèches sont alors les systèmes $\left(f_{I}\right)_{I \in \mathcal{I}}$ avec $f_{I}: \mathcal{F}_{I} \longrightarrow \mathcal{F}_{I}^{\prime}$ tel que le diagramme suivant soit commutatif :

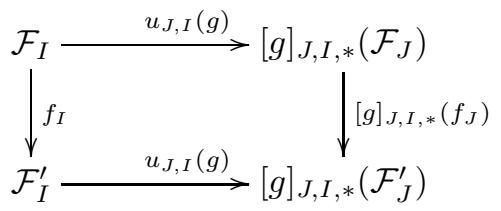


IV.4.2.3. Proposition. - Pour tout schéma de Hecke $X_{\mathcal{I}}$, la catégorie $\mathrm{FH}_{G}\left(X_{\mathcal{I}}\right)$ (resp. $\mathrm{FPH}_{G}\left(X_{\mathcal{I}}\right)$ ) est abélienne (resp. abélienne et artinienne).

Démonstration. - Elle ne présente aucune difficulté. Montrons par exemple l'existence d'un noyau pour $f=\left(f_{I}: \mathcal{F}_{I} \rightarrow \mathcal{F}_{I}^{\prime}\right)_{I}$. Les catégories à niveau fini étant abélienne, soit pour tout $I, \mathcal{G}_{I}$ un noyau de $f_{I}$. On a alors le diagramme commutatif suivant :

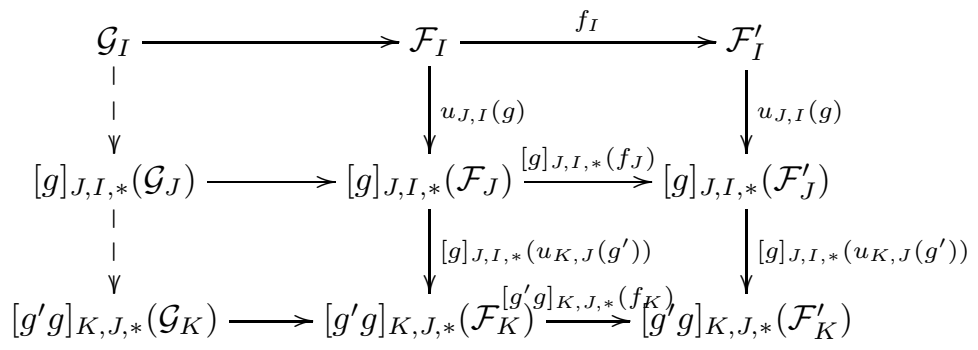

De la propriété universelle du noyau $\mathcal{G}_{J}\left(\right.$ resp. $\left.\mathcal{G}_{K}\right)$, on en déduit une flèche

$$
u_{J, I}(g): \mathcal{G}_{I} \longrightarrow[g]_{J, I, *}\left(\mathcal{G}_{J}\right)
$$

$$
\left(\text { resp. }[g]_{K, J, *}\left(u_{K, J}\left(g^{\prime}\right)\right):[g]_{J, I, *}\left(\mathcal{G}_{J}\right) \longrightarrow\left[g^{\prime} g\right]_{K, J, *}\left(\mathcal{G}_{K}\right)\right)
$$

la propriété de cocycle pour $\mathcal{G}$ découle alors de celles pour $\mathcal{F}$ et $\mathcal{F}^{\prime}$ via la commutativité du diagramme précédent. De la même façon, en utilisant la propriété universelle du noyau et la commutativité du diagramme suivant

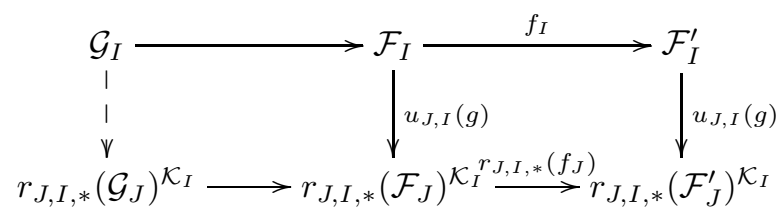

on obtient que pour tout $g \in \mathcal{K}_{I}, u_{J, I}(g)$ induit une flèche $\mathcal{G}_{I} \longrightarrow r_{J, I, *} \mathcal{G}_{J}^{\mathcal{K}_{I}}$.

Remarque: Dans la suite du texte, les faisceaux de Hecke que l'on considérera seront tels que pour $g \in \mathcal{K}_{I}, u_{J, I}(g)$ induit un isomorphisme $\mathcal{F}_{I} \longrightarrow r_{J, I, *} \mathcal{F}_{J}^{\mathcal{K}_{I}}$, de sorte que par exemple, les invariants sous $\mathcal{K}_{I}$ de la cohomologie en niveau $J$, est isomorphe à la cohomologie en niveau $I$. Cette propriété est clairement conservée par passage au noyau mais ne l'est pas pour les conoyaux. Cela étant, on remarquera que les constructions des paragraphes suivants se font toujours par des noyaux à partir d'une flèche entre deux faisceaux vérifiant cette propriété additionnelle de sorte que tous les faisceaux que l'on construit la vérifient aussi.

IV.4.2.4. Notation. - Pour $\mathcal{F}=\left(\mathcal{F}_{I}\right)_{I \in \mathcal{I}}$ un objet de $\operatorname{FPH}_{G}\left(X_{\mathcal{I}}\right)$ ou de $\mathrm{FH}_{G}\left(X_{\mathcal{I}}\right)$, on notera $H^{i}(\mathcal{F})$ pour la limite inductive $\underset{\vec{I}}{\lim _{\vec{I}}} H^{i}\left(X_{I}, \mathcal{F}_{I}\right)$.

IV.4.2.5. Proposition. - Soient $\bar{X}_{\mathcal{I}}, X_{\mathcal{I}}$ et $Y_{\mathcal{I}}$ des schémas de Hecke pour $(G, \mathcal{I})$ tels que $j_{\mathcal{I}}: X_{\mathcal{I}} \hookrightarrow \bar{X}_{\mathcal{I}}$ soit un système projectif d'inclusions affines $G$-équivariantes et $Y_{\mathcal{I}}=\bar{X}_{\mathcal{I}} \backslash X_{\mathcal{I}}$. On dispose alors des foncteurs

$$
j_{!}, R j_{*}, j_{! *}: \mathrm{FPH}_{G}\left(X_{\mathcal{I}}\right) \longrightarrow \mathrm{FPH}_{G}\left(\bar{X}_{\mathcal{I}}\right) \quad{ }^{p} j^{*}={ }^{p} j^{!}: \mathrm{FPH}_{G}\left(\bar{X}_{\mathcal{I}}\right) \longrightarrow \mathrm{FPH}_{G}\left(X_{\mathcal{I}}\right)
$$

et pour $i: Y_{\mathcal{I}}:=\bar{X}_{\mathcal{I}}-X_{\mathcal{I}} \hookrightarrow \bar{X}_{\mathcal{I}}$ des foncteurs

$$
i_{*}=i_{!}: \mathrm{FPH}_{G}\left(Y_{\mathcal{I}}\right) \longrightarrow \mathrm{FPH}_{G}\left(\bar{X}_{\mathcal{I}}\right) \quad{ }^{p} i^{*},{ }^{p} R i^{!}: \mathrm{FPH}_{G}\left(\bar{X}_{\mathcal{I}}\right) \longrightarrow \mathrm{FPH}_{G}\left(Y_{\mathcal{I}}\right)
$$

Démonstration. - On commence par rappeler la proposition suivante tirée de [2]. 
IV.4.2.6. Proposition. - (cf. proposition 1.3.17 de [2]) Pour tout foncteur exact $f$, on note ${ }^{p} f$ pour ${ }^{p} H^{0} f$ comme foncteur sur la catégorie des faisceaux pervers.

(i) Si $f$ est t-exact à gauche (resp. à droite), ${ }^{p} f$ est alors exact à gauche (resp. à droite). Par ailleurs pour $\mathcal{F}$ dans $D^{\geqslant 0}$ (resp. $D^{\leqslant 0}$ ), on a

$$
{ }^{p} f\left({ }^{p} H^{0} \mathcal{F}\right) \stackrel{\sim}{\longrightarrow}{ }^{p} H^{0} f(\mathcal{F}) \quad\left(\text { resp. }{ }^{p} H^{0} f(\mathcal{F}) \stackrel{\sim}{\longrightarrow}{ }^{p} f\left({ }^{p} H^{0} \mathcal{F}\right)\right) .
$$

(ii) Soit $\left(f^{+}, f_{+}\right)$une paire de foncteurs exacts adjoints; pour que $f^{+}$soit $t$-exact à droite, il faut et il suffit que $f_{+}$soit $t$-exact à gauche et dans ce cas $\left({ }^{p} f^{+},{ }^{p} f_{+}\right)$forment une paire de foncteurs adjoints.

(iii) Si $f_{1}: D_{c, 1}^{b} \rightarrow D_{c, 2}^{b}$ et $f_{2}: D_{c, 2}^{b} \rightarrow D_{c, 3}^{b}$ sont t-exacts à gauche (resp. à droite), alors $f_{2} \circ f_{1}$ l'est aussi et ${ }^{p}\left(f_{2} \circ f_{1}\right)={ }^{p} f_{2} \circ f_{1}$.

On rappelle que $j_{!}, R j_{*}, j^{*}, i_{*}$ sont $t$-exacts et donc égaux à leur version perverse alors que $i^{*}$ est $t$-exact à droite. De même les morphismes $r_{J, I}$ et $[g]_{J, I}$ étant finis, $r_{J, I}^{*}$ (resp. $[g]_{J, I, *}$ ) est $t$-exact à droite (resp. $t$-exact) de sorte que $\left({ }^{p} r_{J, I}^{*},{ }^{p} r_{J, I, *}\right)$ et $\left({ }^{p}[g]_{J, I, *}=[g]_{J, I, *},{ }^{p}[g]_{J, I}^{!}\right)$forment des paires de foncteurs adjoints avec ${ }^{p} r_{J, I}^{*}$ et ${ }^{p}[g]_{J, I}^{!}$(resp. ${ }^{p} r_{J, I, *}$, resp. ${ }^{p}[g]_{J, I, *}$ ) exacts à droite (resp. à gauche, resp. exact). Pour $J \subset I$, considérons le diagramme suivant :

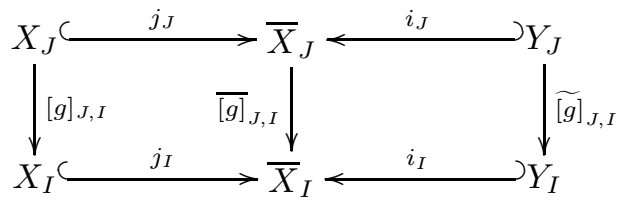

Soient alors $\left(\mathcal{F}_{I}\right)_{I \in \mathcal{I}}$ un objet de $\operatorname{FPH}\left(X_{\mathcal{I}}\right)$ et $g, J, I$ avec $u_{J, I}(g): \mathcal{F}_{I} \longrightarrow[g]_{J, I, *}\left(\mathcal{F}_{I}\right)$. Par application de $R j_{\mathcal{I}, *}\left(\right.$ resp. $\left.j_{\mathcal{I}, !}\right)$, on obtient

$$
\begin{gathered}
R j_{I, *} \mathcal{F}_{I} \stackrel{R j_{I, *}\left(u_{J_{J} I}(g)\right)}{\longrightarrow} R j_{I, *}[g]_{J, I, *}\left(\mathcal{F}_{J}\right)=\overline{[g}_{J, I, *}\left(R j_{J, *} \mathcal{F}_{J}\right) \\
\text { (resp. } \left.j_{I, !} \mathcal{F}_{I}^{j_{I, !}\left(u_{J, I}(g)\right)} j_{I, !}[g]_{J, I, !}\left(\mathcal{F}_{J}\right)=\overline{[g}_{J, I, *}\left(j_{J, !} \mathcal{F}_{J}\right)\right)
\end{gathered}
$$

En ce qui concerne $j_{\mathcal{I}, ! *}$, on considère le diagramme commutatif suivant :

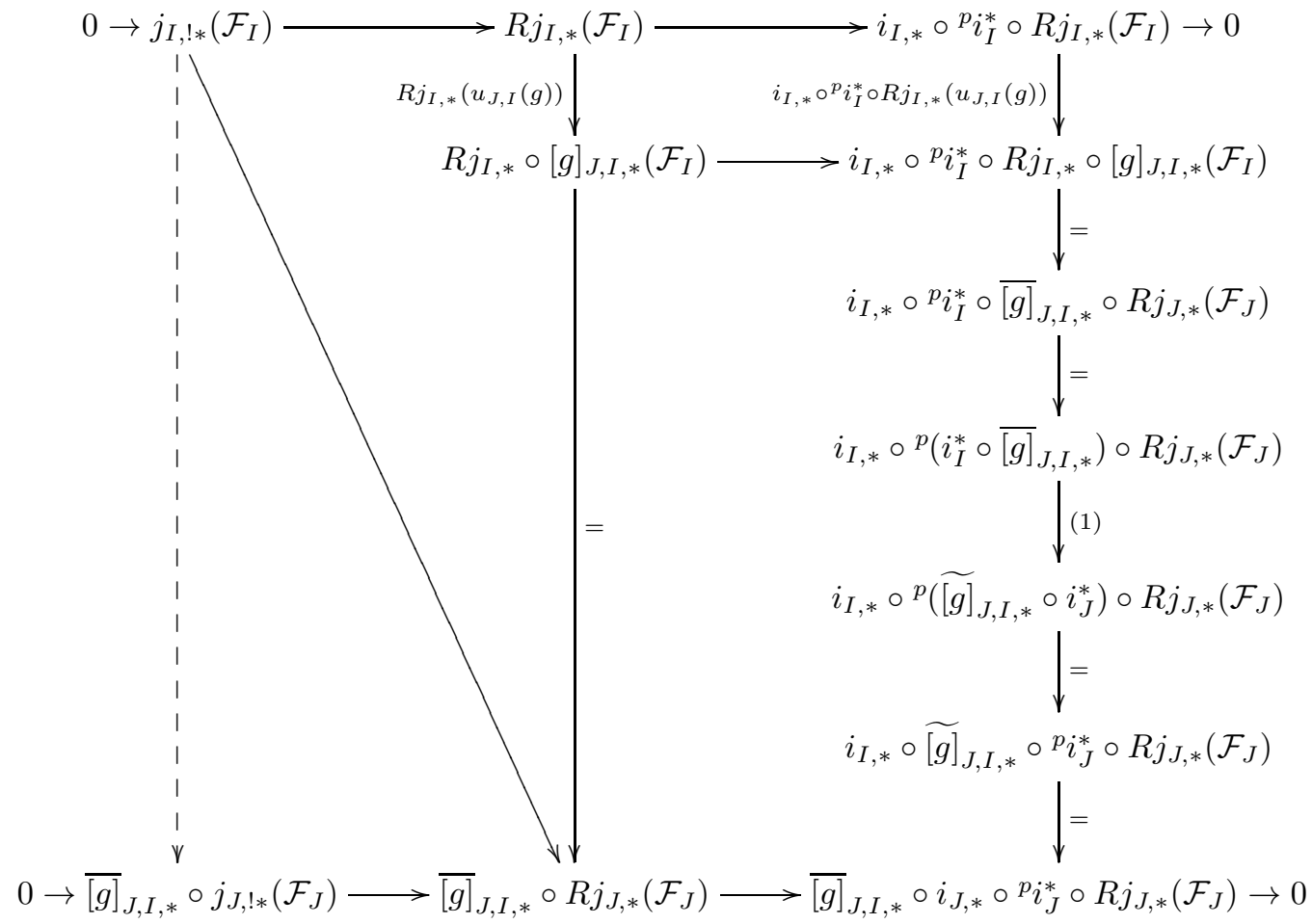


où la flèche notée (1) s'obtient à partir du diagramme commutatif suivant

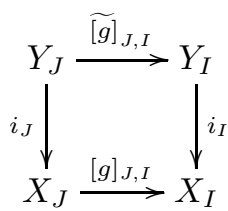

comme le morphisme de changement de base $\bar{i}_{I}^{*} \circ[g]_{J, I, *} \longrightarrow[g]_{J, I, *} \circ i_{J}^{*}$.

Les cas de $i_{*},{ }^{p} i^{*}$ sont traités ci dessus et ceux de ${ }^{p} j^{*}$ et ${ }^{p} R i^{!}$se traitent de manière strictement similaire.

Exemples : étant donné un système local $\mathcal{L}_{\emptyset}$ sur $X_{\emptyset}$, soit pour $I \in \mathcal{I}, \mathcal{L}_{I}=r_{I, \emptyset}^{*} \mathcal{L}_{\emptyset} ;\left(\mathcal{L}_{I}[d]\right)_{I \in \mathcal{I}}$ est alors un objet de $\mathrm{FPH}_{G}\left(X_{\mathcal{I}}\right)$, où $d$ est la dimension de $X_{\mathcal{I}}$. Si en outre les $X_{I}$ sont la fibre générique d'un $S$-schéma $\mathcal{X}_{I}$ de dimension relative $d-1$, pour $S$ un trait, alors $\left(R \Psi_{\eta}\left(\mathcal{L}_{I}\right)[d-1]\right)_{I \in \mathcal{I}}$ est un objet de $\mathrm{FPH}_{G}\left(\bar{X}_{\mathcal{I}}\right)$ où $\bar{X}_{I}$ désigne la fibre spéciale de $X_{I}$.

\section{IV.4.3. Notations. -}

IV.4.3.1. Définitions. - - Pour $1 \leqslant g \leqslant d$, on notera dans la suite $s_{g}:=\left\lfloor\frac{d}{g}\right\rfloor$, la partie entière de $d / g$.

- On introduit les injections

$$
i^{h}: M_{I, s_{o}}^{\geqslant h} \hookrightarrow M_{I, s_{o}}, \quad j^{\geqslant h}: M_{I, s_{o}}^{=h} \hookrightarrow M_{I, s_{o}}^{\geqslant h} \quad k_{1}^{=h}: M_{I, s_{o}, 1}^{=h} \hookrightarrow M_{I, s_{o}}^{=h}
$$

On omet volontairement l'indice I dans les notations afin de ne pas encore alourdir le texte surtout qu'en général le contexte est clair.

- On notera $\operatorname{FPH}\left(M_{s_{o}}\right)$ (resp. $\operatorname{FPH}\left(M_{o}\right)$, resp. $\operatorname{FPH}\left(M_{s_{o}}^{=h}\right)$, resp. $\operatorname{FPH}\left(M_{s_{o}}^{\geqslant h}\right)$ ) la catégorie des faisceaux pervers de Hecke sur la tour des $M_{I, s_{o}}$ (resp. $M_{I, o}$, resp. $M_{I, s_{o}}^{=h}$, resp. $M_{I, s_{o}}^{\geqslant h}$ ) avec $G=\left(D_{\mathbb{A}}^{\infty}\right)^{\times}$; on notera aussi $\operatorname{FPH}\left(M_{s_{o}, 1}^{=h}\left(\right.\right.$ resp. $\left.\operatorname{FPH}\left(M_{s_{o}, 1}^{\geqslant h}\right)\right)$ la catégorie des faisceaux pervers de Hecke sur la tour des $M_{I, s_{o}, 1}^{=h}\left(\right.$ resp. $\left.M_{I, s_{o}, 1}^{\geqslant h}\right)$ avec $G=\left(D_{\mathbb{A}}^{\infty, o}\right)^{\times} \times P_{h, d}\left(F_{o}\right)$. On utilisera des notations similaires pour les faisceaux de Hecke.

- Un système projectif de faisceaux pervers ou pas, non nul $\mathcal{F}=\left(\mathcal{F}_{I}\right)_{I} \in \operatorname{FPH}\left(M_{s_{o}}^{=h}\right)$ (ou dans $\left.\mathrm{FH}\left(M_{s_{o}}\right)\right)$ sera dit induit s'il est de la forme

$$
\left(k_{1}^{=h, *} \mathcal{F}_{I}\right)_{I \in \mathcal{I}} \times_{P_{h, d}^{o p}\left(F_{o}\right)} G L_{d}\left(F_{o}\right)
$$

où $\times_{P_{h, d}^{o p}\left(F_{o}\right)} G L_{d}\left(F_{o}\right): \operatorname{FPH}\left(M_{s_{o}, 1}^{=h}\right) \longrightarrow \operatorname{FPH}\left(M_{s_{o}}^{=h}\right)$ est le foncteur qui à un faisceau $\left(\mathcal{F}_{0, I}\right)_{I}$ associe $\left(\mathcal{F}_{0, I} \times_{P_{h, d}^{o p}\left(\mathcal{O}_{o}\right)} G L_{d}\left(\mathcal{O}_{o}\right)\right)_{I}$ de sorte que pour tout $g \in G L_{d}\left(F_{o}\right)$, la correspondance associée induit un isomorphisme de $g^{*} \mathcal{F}_{\bar{g}} \sim \mathcal{F}_{0}$ où $\mathcal{F}_{\bar{g}}$ est la restriction de $\mathcal{F}$ à la composante $\left(M_{I, s_{o}, \bar{g}}^{=h}\right)_{I}$ où $\bar{g}$ est l'image de $g$ dans le quotient $G L_{d}\left(F_{o}\right) / P_{h, d}\left(F_{o}\right)$.

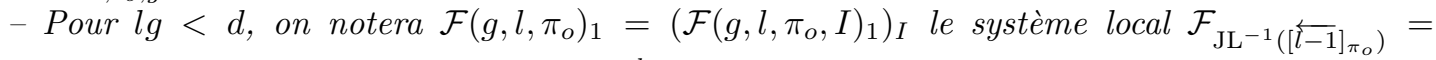
$\left(\mathcal{F}_{\mathrm{JL}^{-1}\left([\overleftarrow{l-1}]_{\pi_{o}}\right), I}\right)_{I}$ sur la tour des $\left(M_{I, s_{o}, 1}^{=l g}\right)_{I}$ et soit $\mathcal{F}\left(g, l, \pi_{o}\right)=\left(\mathcal{F}\left(g, l, \pi_{o}, I\right)\right)_{I}$ le faisceau induit associé, défini donc sur la tour des $\left(M_{I, s_{o}}^{=l g}\right)_{I}$ :

$$
\mathcal{F}\left(g, l, \pi_{o}\right)[d-l g] \in \operatorname{FPH}\left(M_{s_{o}}^{=l g}\right)
$$

- Pour $1 \leqslant g \leqslant d$, un $G L_{d}\left(F_{o}\right) \times W_{o}$-faisceau sur la tour $\left(M_{I, s_{o}}^{=l g}\right)_{I}$ sera dit de type $H T(g, l)$ s'il est induit, et si sa restriction $\grave{a}\left(M_{I, s_{o}, 1}^{=l g}\right)_{I}$ est de la forme

$$
\left(\mathcal{F}\left(g, l, \pi_{o}, I\right)_{1} \otimes \Pi_{o}^{K_{o, n}} \otimes \xi\right)_{I}
$$

pour un certain triplet $\left(\Pi_{o}, \pi_{o}, \xi\right)$ où :

- $n=\operatorname{mult}_{o}(I)$

- $\pi_{o}$ est une représentation irréductible cuspidale de $G L_{g}\left(F_{o}\right)$, 
- $\Pi_{o}$ est une représentation de $G L_{l g}\left(F_{o}\right)$

- et $\xi$ un caractère de $\mathbb{Z}$,

telle que l'action d'un élément $\left(g^{\infty, o}, g_{o}^{e t}, g_{o}^{c}, c_{o}\right) \in\left(D_{\mathbb{A}}^{\infty, o}\right)^{\times} \times G L_{d-l g}\left(F_{o}\right) \times G L_{l g}\left(F_{o}\right) \times W_{o}$ soit donnée par l'action naturelle de

$$
\left(g^{\infty, o}, g_{o}^{e t},-\operatorname{val}\left(\operatorname{det} g_{o}^{c}\right)-\operatorname{deg}\left(c_{o}\right)\right) \in\left(D_{\mathbb{A}}^{\infty, o}\right)^{\times} \times G L_{d-l g}\left(F_{o}\right) \times \mathbb{Z}
$$

sur $\mathcal{F}_{\mathrm{JL}^{-1}\left([\overleftarrow{l-1}]_{\pi_{o}}\right)}$ au dessus de $\left(M_{I, s_{o}, 1}^{=l g}\right)_{I}$, et par celle de $\left(g_{o}^{c}, c_{o}\right)$ sur $\Pi_{o} \otimes \xi$. On le notera alors

$H T\left(g, l, \pi_{o}, \Pi_{o}, \xi\right)=\left(H T\left(g, l, \pi_{o}, \Pi_{o}, \xi, I\right)\right)_{I}$,

$$
H T\left(g, l, \pi_{o}, \Pi_{o}, \xi\right)[d-l g] \in \operatorname{FPH}\left(M_{s_{o}}^{=l g}\right)
$$

et on omettra $\xi$ si celui-ci est trivial. De même on notera

$H T_{\rho_{\infty}}\left(g, l, \pi_{o}, \Pi_{o}\right)=\left(H T_{\rho_{\infty}}\left(g, l, \pi_{o}, \Pi_{o}, I\right)\right)_{I}$,

$$
H T_{\rho_{\infty}}\left(g, l, \pi_{o}, \Pi_{o}\right)[d-l g] \in \operatorname{FPH}\left(M_{s_{o}}^{=l g}\right)
$$

le faisceau $H T\left(g, l, \pi_{o}, \Pi_{o}\right) \otimes \mathcal{L}_{\rho_{\infty}}$.

- Pour $\pi_{o}$ une représentation irréductible cuspidale de $G L_{g}\left(F_{o}\right)$ et $1 \leqslant l \leqslant s_{g}$, on note

$$
\mathcal{P}\left(g, l, \pi_{o}\right)=\left(\mathcal{P}\left(g, l, \pi_{o}, I\right)\right)_{I} \in \mathrm{FPH}\left(M_{s_{o}}\right)
$$

le faisceau pervers défini comme l'extension intermédiaire $j_{!_{*}}^{\geqslant l g}$ du faisceau pervers sur $M_{I, s_{o}}^{=l g}$, $H T\left(g, l, \pi_{o},[\overleftarrow{l-1}]_{\pi_{o}}\right)[d-l g] \otimes L_{g}\left(\pi_{o}\right)$, qui est donc pur de poids $d-l g$ pour $\pi_{o}$ unitaire ${ }^{(11)}$ et qui ne dépend que de la classe d'équivalence inertielle de $\pi_{o}$.

IV.4.3.2. Remarque. - $\mathcal{F}\left(g, l, \pi_{o}\right)$ et $H T\left(g, l, \pi_{o}, \Pi_{o}\right)$ (resp. $\left.\mathcal{P}\left(g, l, \pi_{o}\right)\right)$ se présentent sous la forme d'une somme directe de $e_{\pi_{o}}$ systèmes locaux (resp. faisceaux pervers) irréductibles :

$$
\mathcal{F}\left(g, l, \pi_{o}\right)=\bigoplus_{i=1}^{e_{\pi_{o}}} \mathcal{F}\left(g, l, \rho_{o, i}\right) \quad\left(\text { resp. } \mathcal{P}\left(g, l, \pi_{o}\right)=\bigoplus_{i=1}^{e_{\pi_{o}}} \mathcal{P}\left(g, l, \rho_{o, i}\right)\right)
$$

donnés par la restriction de $\mathrm{JL}^{-1}\left([\overleftarrow{l-1}]_{\pi_{o}}\right)$ à $\mathcal{D}_{o, l g}^{\times}$qui s'écrit comme une somme directe $\bigoplus_{i=1}^{e_{\pi_{o}}} \rho_{o, i}$ de représentations irréductibles, de sorte que la différence entre les faisceaux $\mathcal{F}\left(g, l, \rho_{o, i}\right)($ resp. $\left.\mathcal{P}\left(g, l, \rho_{o, i}\right)\right)$ provient de l'action de $\mathcal{D}_{o, l g}^{\times} \subset \mathcal{N}_{o}$ donnée sur chacun comme dans la formule (IV.2.1).

IV.4.3.3. Définition. — Soit $\mathfrak{G}=\left(\mathfrak{G}_{I}\right)_{I}$ le système projectif de groupes de Grothendieck associé à $\operatorname{FPH}\left(M_{I, s_{o}}\right)$.

\section{IV.4.4. Filtration de monodromie. -}

IV.4.4.1. Théorème. - Soit $1 \leqslant g \leqslant d$ et $s_{g}$ la partie entière de $d / g$. Pour $\pi_{o}$ une représentation irréductible cuspidale de $G L_{g}\left(F_{o}\right)$, on a l'égalité suivante dans $\mathfrak{G}$ :

$$
e_{\pi_{o}}\left[R \Psi_{\eta_{o}, \pi_{o}}\left(\overline{\mathbb{Q}}_{l}[d-1]\right)\right]=\sum_{k=1-s_{g}}^{s_{g}-1} \sum_{\substack{|k|<l \leqslant s_{g} \\ l \equiv k-1 \bmod 2}} \mathcal{P}\left(g, l, \pi_{o}\right)\left(-\frac{l g+k-1}{2}\right),
$$

où la torsion concerne l'action de $W_{o}$.

$I V .4 .4 .2$ - Pour tout $s$, soit $M G r_{k}(s)$ le faisceau pervers nul pour $|k| \geqslant s$ et égal à

$$
\bigoplus_{\substack{|k|<l \leqslant s \\ l \equiv k-1 \bmod 2}} j_{!_{*}}^{\geqslant l} \mathcal{F}\left(1, l, 1_{o}\right)[d-l] \otimes[\overleftarrow{l-1}]_{1_{o}} \otimes L_{g}\left(1_{o}\right)(-(l-1+k) / 2)
$$

${ }^{(11)}$ sinon on rajoute le poids de $L_{g}\left(\pi_{o}\right)$ 
Concrètement on peut lire la définition des $M G r_{\bullet}(s)$ dans un repère $(l, k)$ en marquant sur la ligne $k=k_{0}$ les $l$ tels que $j_{! *}^{\geqslant l} \mathcal{F}\left(1, l, 1_{o}\right)[d-l] \otimes[\overleftarrow{l-1}]_{1_{o}} \otimes L_{g}\left(1_{o}\right)(-(l-1+k) / 2)$ soit un constituant de $M G r_{k_{0}}(s)$; par exemple pour $s=4$ on obtient la figure (1).

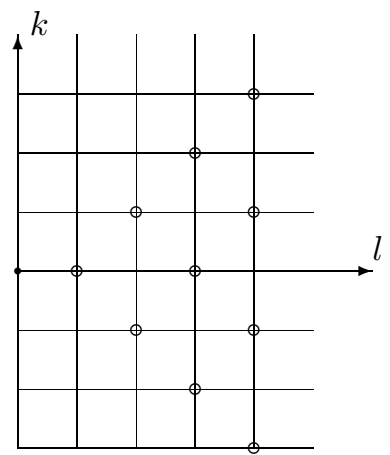

FigURE $1 . \quad M G r_{\bullet}(4)$

$I V .4 .4 .3$ - Pour tout représentation cuspidale $\pi_{o}$ de $G L_{g}\left(F_{o}\right)$, on pose

$$
\begin{aligned}
\pi_{o} \diamond\left(j_{! *}^{\geqslant l} \mathcal{F}\left(1, l, 1_{o}\right)[d-l] \otimes[\overleftarrow{l-1}]_{1_{o}} \otimes L_{g}\left(1_{o}\right)(-(l-1+k) / 2)\right):= \\
\quad j_{! *}^{\geqslant l g} \mathcal{F}\left(g, l, \pi_{o}\right)[d-l g] \otimes[\overleftarrow{l-1}]_{\pi_{o}} \otimes L_{g}\left(\pi_{o}\right)(-(l g-1+k) / 2)
\end{aligned}
$$

Le théorème (IV.4.4.4) se reformule alors sous la forme

IV.4.4.4. Théorème. - Soit $1 \leqslant g \leqslant d$ et $s_{g}$ la partie entière de $d / g$. Pour $\pi_{o}$ une représentation irréductible cuspidale de $G L_{g}\left(F_{o}\right)$, on a

$$
e_{\pi_{o}} g r_{\bullet}, \pi_{o}=\pi_{o} \diamond M G r_{\bullet}\left(s_{g}\right)
$$

$I V .4 .4 .5$ - Autrement dit, pour $1 \leqslant g \leqslant d$ et $\pi_{o}$ une représentation irréductible cuspidale de $G L_{g}\left(F_{o}\right)$, pour tout $|k|<s_{g}, e_{\pi_{o}} g r_{k, \pi_{o}}$ est égale à

$$
\bigoplus_{\substack{|k|<l \leqslant s g \\ l \equiv k-1 \bmod 2}} \mathcal{P}\left(g, l, \pi_{o}\right)\left(-\frac{l g+k-1}{2}\right),
$$

dans $\operatorname{FPH}\left(M_{s_{o}}\right)$, où la torsion concerne l'action de $W_{o}$.

IV.4.4.6. Définition. - Pour $1 \leqslant g \leqslant d$, et on fixe une représentation $\pi_{o}$ irréductible cuspidale de $G L_{g}\left(F_{o}\right)$, que l'on notera $\xi_{o}$ pour $g=1$. Pour tout $1 \leqslant l \leqslant s_{g}, \Pi_{l}$ désigne une représentation quelconque de $G L_{l g}\left(F_{o}\right)$, qui dans les applications sera elliptique de type $\pi_{o}$.

$I V \cdot 4 \cdot 4.7$. Théorème. - Pour $g, \pi_{o}$ et $\Pi_{l}$ comme ci-dessus, on considère la restriction à la tour $\operatorname{des}\left(M_{I, s_{o}}^{=h}\right)_{I}$ de $h^{i} j_{l_{*}}^{\geqslant l g} H T\left(g, l, \pi_{o}, \Pi_{l}\right)[d-l g]$;

- elle est nulle pour $h$ ne s'écrivant pas sous la forme $(l+a) g$ avec $0 \leqslant a \leqslant s_{g}-l$;

- pour $h=(l+a) g$ avec $0 \leqslant a \leqslant s_{g}-l$, elle est nulle pour $i \neq l g-d+a(g-1)$ et sinon elle est isomorphe dans $\mathrm{FH}\left(M_{s_{o}}\right) \grave{a}{ }^{(12)}$

$$
H T\left(g, l+a, \pi_{o}, \Pi_{l} \overrightarrow{\times}[\overrightarrow{a-1}]_{\pi_{o}}\right) \otimes \Xi^{\frac{a(g-1)}{2}}
$$

${ }^{(12)}$ cf. la définition (IV.2.2.3) 
On en déduit alors les corollaires suivant.

IV.4.4.8. Corollaire. - Pour $g>1, h^{i} j_{! *}^{\geqslant l g} H T\left(g, l, \pi_{o}, \Pi_{l}\right)[d-l g]$ est nul pour $i$ ne s'écrivant pas sous la forme $l g-d+a(g-1)$ avec $0 \leqslant a \leqslant s_{g}-l$ et pour un tel $i=l g-d+a(g-1)$ il est isomorphe dans $\mathrm{FH}\left(M_{s_{o}}\right) \grave{a}$

$$
j_{!}^{\geqslant(l+a) g} H T\left(g, l+a, \pi_{o}, \Pi_{l} \overrightarrow{\times}[\overrightarrow{a-1}]_{\pi_{o}}\right) \otimes \Xi^{\frac{a(g-1)}{2}} .
$$

IV.4.4.9. Corollaire. - Pour $\pi_{o}$ une représentation irréductible cuspidale de $G L_{g}\left(F_{o}\right)$ pour $1 \leqslant g \leqslant d$, on $a$

(i) pour $g>1,\left(h^{i} g r_{k, \pi_{o}}\right)^{e_{\pi_{o}}}$ est une somme directe sur tous les couples $(l, a)$ tels que $-d+l g+$ $a(g-1)=i$ et $l \equiv k-1 \bmod 2$ des faisceaux induits dans $\mathrm{FH}\left(M_{s_{o}}\right)$ :

$$
j_{!}^{\geqslant(l+a) g} H T\left(g, l+a, \pi_{o},[\overleftarrow{l-1}]_{\pi_{o}} \overrightarrow{\times}[\overrightarrow{a-1}]_{\pi_{o}}\right) \otimes L_{g}\left(\pi_{o}\right)\left(-\frac{l g-1+k+a(g-1)}{2}\right)
$$

(ii) pour $g=1$, la restriction de $h^{i} g r_{k, \xi_{o}}$ pour $i \equiv k-1 \bmod 2$, à la tour des $M_{I, s_{o}}^{=l}$ pour $l \geqslant d+i$ est égale au faisceau induit dans $\mathrm{FH}\left(M_{s_{o}}\right)$

$$
H T\left(1, l, \xi_{o},[\overleftarrow{i-1}]_{\xi_{o}} \overrightarrow{\times}[\overrightarrow{l-i-1}]_{\xi_{o}}\right) \otimes \xi_{o}\left(-\frac{d+i-1+k}{2}\right)
$$

et nulle dans tous les autres cas.

Le point (2) du théorème (IV.3.2.3) et donc l'énoncé de (IV.3.1.1), découle alors du résultat suivant. On introduit les bicomplexes

$$
M G E_{\bullet}^{\bullet}, \bullet(s)=\bigoplus_{l=1}^{s} j_{!}^{\geqslant l} \mathcal{F}\left(1, l, 1_{o}\right)[d-l] \otimes M L E_{\bullet}^{\bullet, \bullet}(l)
$$

IV.4.4.10. Théorème. — Soit $1 \leqslant g \leqslant d$ et $s_{g}$ la partie entière de $d / g$. Pour $\pi_{o}$ une représentation irréductible cuspidale de $G L_{g}\left(F_{o}\right)$, on a

$$
e_{\pi_{o}} E_{\bullet, g r, g l o b, \pi_{o}}^{\bullet, \bullet}\left(\frac{d-1}{2}\right)=\pi_{o} \diamond M G E_{\bullet}^{\bullet, \bullet}\left(s_{g}\right)
$$

$I V .4 \cdot 4 \cdot 11$ - Autrement dit la suite spectrale (IV.4.1.17) dégénère en $E_{2}$ et les applications $d_{1}^{i, j}$ sont induites par les suites exactes (IV.3.2.15).

IV.4.5. Schéma de la preuve : par récurrence. — Remarquons tout d'abord que le théorème (IV.4.4.4) découle directement de (IV.4.4.1) en utilisant monodromie-poids (MP), i.e. la pureté des gradués de la filtration de monodromie. D'après le théorème de comparaison de Berkovich-Fargues $(\mathrm{BF})$, le théorème (IV.3.2.3) découle directement de la détermination des germes en un point supersingulier des gradués de la filtration de monodromie du complexe des cycles évanescents de

\begin{tabular}{|c|c|}
\hline $\begin{array}{l}\text { (IV.4.4.1) } \\
\Downarrow \mathrm{MP} \\
(\mathrm{IV} .4 .4 .4)+\end{array}$ & $(\mathrm{IV} .4 .4 .7)+(\mathrm{IV} .4 .4 .10)$ \\
\hline (IV.3.2.3) & $\begin{array}{l}\Downarrow \mathrm{BF} \\
\text { en hauteur } \leqslant d\end{array}$ \\
\hline (IV.3.1.1) & $\begin{array}{l}\Downarrow \\
\text { en hauteur } \leqslant d\end{array}$ \\
\hline
\end{tabular}
la variété de Drinfeld-Stuhler et de la suite spectrale associée, soit donc des théorèmes (IV.4.4.4), (IV.4.4.7) et (IV.4.4.10). Par ailleurs, comme on l'a déjà noté, le théorème (IV.3.1.1) découle directement de (IV.3.2.3) puisqu'il s'agit de l'aboutissement de la suite spectrale associée à la filtration de monodromie-locale. On résume ces implications dans le tableau suivant.

Le principe est alors de raisonner par récurrence en supposant connu (IV.3.2.3) pour tout $d^{\prime}<d$. 
$I V$.4.5.1. Remarque. - A priori, il nous faut travailler à niveau fini, pour tous les idéaux $I$ de $A$ et montrer que les résultats obtenus sont compatibles aux correspondances de Hecke; cependant devant l'immédiateté de ces vérifications et afin de ne pas alourdir encore les preuves, on raisonne souvent sur le système inductif total.

$I V$ 4.5.2 - Dans un premier temps le théorème de comparaison de Berkovich-Fargues (BF) et la description (HT) des faisceaux des cycles évanescents en fonction des systèmes locaux d'HarrisTaylor (cf. (IV.2.2.12)), nous permet, §IV.5, de montrer le théorème (IV.4.4.1).

- On montre ensuite, proposition (IV.6.2.1), le théorème (IV.4.4.7) hors des points supersinguliers et on obtient un contrôle $(\mathrm{C})$ sur ce qui peut se passer au niveau de ces derniers.

— Selon le même principe, si on disposait de (IV.3.2.3) pour la hauteur $d$, on en déduirait (IV.4.4.7) et (IV.4.4.10). On démontre, $\S I V .7$, comment la connaissance des parties de poids minimal de (IV.3.1.1) permet d'obtenir (IV.4.4.7), proposition (IV.7.1.2) ${ }^{(13)}$, (IV.4.4.10), §IV.7.2 et même (IV.4.4.4) dans le cas Iwahori sans utiliser monodromie-poids, §IV.7.3 ce qui, en caractéristique mixte, nous permettra de prouver monodromie-poids en toute généralité en se ramenant, par un changement de base automorphe adéquat, au cas Iwahori.

- Reste alors, §IV.8.1.24, à calculer les parties de poids minimal de (IV.3.1.1) ce qui se fait via l'étude de la suite spectrale des cycles évanescents (SSCE) à travers les suites spectrales associées à la stratification (SSS) en utilisant le contrôle (C) sur ce qui peut se passer au niveau des points supersinguliers. Evidemment tout ceci passe par le calcul, ou au moins du contrôle, des groupes de cohomologie $(\mathrm{CHT})$ des systèmes locaux d'Harris-Taylor, qui sont distillés au fur et à mesure et dont nous détaillons l'enchainement logique après le tableau récapitulatif suivant.

\begin{tabular}{|c|c|c|}
\hline $\begin{array}{l}\S I V .5:(\text { IV.3.2.3) } \\
\text { en hauteur } d^{\prime}<d\end{array}$ & $\begin{array}{c}\Longrightarrow \\
\mathrm{BF}+\mathrm{HT}\end{array}$ & (IV.4.4.1) \\
\hline $\begin{array}{l}\S I V .6:(\text { IV.3.2.3) } \\
\text { en hauteur } d^{\prime}<d\end{array}$ & $\begin{array}{l}\mathrm{BF}+\mathrm{HT} \\
\Longrightarrow \\
(\mathrm{IV} .6 .2 .1)\end{array}$ & $\begin{array}{l}\text { (IV.4.4.7) hors des points supersinguliers } \\
+\mathrm{C}=\text { contrôle aux points supersinguliers }\end{array}$ \\
\hline $\begin{array}{l}\text { (IV.3.2.3) } \\
\text { en hauteur } d^{\prime} \leqslant d\end{array}$ & $\begin{array}{c}\Longrightarrow \\
(\mathrm{IV} .7 .1 .2)\end{array}$ & $(\mathrm{IV} .4 .4 .7)+(\mathrm{IV} .4 .4 .10)$ \\
\hline $\begin{array}{l}\oint \text { IV.7 : }(\text { IV.3.1.1) } \\
\text { en hauteur } d^{\prime}<d \\
\text { et en poids minimal } \\
\text { pour la hauteur } d\end{array}$ & $\Longrightarrow$ & $\left\{\begin{array}{l}(\text { IV.4.4.7) :£IV.7.1 proposition (IV.7.1.2) } \\
\text { (IV.4.4.10) : } \text { IV.7.2 } \\
\text { (IV.4.4.4): } \S I V .7 .3 \text { sans MP }\end{array}\right.$ \\
\hline$\S I V .8$ & $\begin{array}{l}\Longrightarrow \\
\mathrm{SSCE}+\mathrm{SSS} \\
+\mathrm{C}+\mathrm{CHT}\end{array}$ & $\begin{array}{l}\text { poids minimal de (IV.3.1.1) } \\
\text { en hauteur } d\end{array}$ \\
\hline
\end{tabular}

$I V .4 .5 .3$ - En ce qui concerne le rôle des divers calculs de groupes de cohomologie, voici leur enchaînement logique. Pour commencer on calcule exclusivement des composantes $\Pi^{\infty, o}$-isotypiques pour $\Pi$ une représentation irréductible automorphe de $D_{\mathbb{A}}^{\times}$vérifiant $\operatorname{Hyp}(\infty)$, car dans tous les autres cas tous les calculs donnent des résultats nuls. On considère plus particulièrement deux situations selon que $\Pi_{o}$ est de la forme $[\overleftarrow{s-1}]_{\pi_{o}}$ ou $[\overrightarrow{s-1}]_{\pi_{o}}$ pour $\pi_{o}$ une représentation cuspidale de $G L_{g}\left(F_{o}\right)$ avec $d=s g$. Pour alléger les notations, on notera $H^{i}\left(j_{!}^{\geqslant l g}\right)$ pour la composante $\Pi^{\infty, o}$ isotypique du $i$-ème groupe de cohomologie du complexe $j_{!}^{\geqslant l g} \mathcal{F}\left(g, l, \pi_{o}\right)[d-l g]$ et on utilisera une notation similaire pour $H^{i}\left(j_{! *}^{\geqslant l g}\right)$.

$\overline{(13) \text { de sorte que }}($ IV .4 .4 .7$)=($ IV .6 .2 .1$)+($ IV .7 .1 .2$)$ 
$I V .4 .5 .4$ - La situation est largement plus simple dans le cas où $\Pi_{o}=[\overleftarrow{s-1}]_{\pi_{o}}$. On commence, proposition (IV.5.4.1), par calculer les $H^{i}\left(j_{!_{*}}^{\geqslant l g}\right)$ et on trouve que ceux-ci sont tous nuls si $l g \neq d$. En utilisant (IV.2.2.13), on en déduit alors, corollaire (IV.5.4.3), le calcul des $H^{i}\left(j_{!}^{\geqslant l g}\right)$ qui sont nuls pour $i \neq 0$. Ces calculs permettent de compléter, corollaire (IV.5.4.6), aux points supersinguliers, la détermination des constituants simples du faisceau pervers $j_{l}^{\geqslant l g} \mathcal{F}\left(g, l, \pi_{o}\right)[d-l g]$ obtenu, corollaire (IV.5.3.12), sur les autres strates grâce à la description des faisceaux des cycles évanescents en fonction des systèmes locaux d'Harris-Taylor ainsi qu'au théorème de comparaison de BerkovichFargues. On en déduit alors, corollaire (IV.5.4.9), le calcul des $\Pi^{\infty, o}$-parties des groupes de cohomologie $H_{c}^{j}\left(M_{I, s_{o}}^{=h}, R^{i} \Psi_{\eta_{o}, I}\left(\mathcal{L}_{\rho_{\infty}}\right)\right)$. A ce moment ci, on dispose du théorème (IV.4.4.4) de sorte que l'on peut calculer via la suite spectrale des poids SSP, corollaire (IV.8.1.1), les $\Pi^{\infty, o}$-parties des groupes de cohomologie $H_{\eta_{o}, \rho_{\infty}}^{i}$ de la fibre générique. On en déduit alors, proposition (IV.8.1.2), les $\Pi^{\infty, o}$-parties des termes $E_{2}^{p, q}$ de la suite spectrale des cycles évanescents qui nous permet d'obtenir, corollaire (IV.8.1.3), une première liste de possibilités pour les $\widetilde{\mathcal{U}_{F_{o}}^{d, i}}\left(\mathrm{JL}^{-1}\left(\operatorname{St}_{s}\left(\pi_{o}\right)\right)\right.$ ).

$I V .4 .5 .5$ - On considère alors $\Pi_{o}=[\overrightarrow{s-1}]_{\pi_{o}}$ et on calcule, proposition (IV.8.3.4), les $H^{i}\left(j_{! *}^{\geqslant l g}\right)$ qui sont non nuls pour $i \equiv s-l \bmod 2$ et $|i| \leqslant s-l$. De ce calcul et de la connaissance des constituants simples des $j_{!}^{\geqslant l g} \mathcal{F}\left(g, l, \pi_{o}\right)[d-l g]$, on en déduit, lemme (IV.8.3.10), un contrôle sur les $H^{i}\left(j_{!}^{\geqslant l g}\right)$. On étudie ensuite la suite spectrale des cycles évanescents. On commence par en déterminer l'aboutissement, corollaire (IV.8.3.5) et proposition (V.1.1), en utilisant le calcul des $H^{i}\left(j_{! *}^{\geqslant l g}\right)$ ainsi que le théorème (IV.4.4.4). En utilisant le théorème de Lefschetz difficile ainsi que le corollaire (IV.8.1.3) qui contrôle ce qui se passe au niveau des points supersinguliers, on en déduit, proposition (IV.8.3.9) et corollaire (V.1.2), le calcul des $H^{i}\left(j_{!}^{\geqslant l g}\right)$. Le résultat est qu'en ce qui concerne les parties de poids minimal, $s(g-1)$, tout ce passe au niveau des points supersinguliers. L'étude de la suite spectrale des cycles évanescents fournit alors la partie de poids $s(g-1)$ du théorème (IV.3.1.1) qui d'après ce que l'on a vu, implique les théorèmes globaux (IV.4.4.7) et (IV.4.4.10) qui d'après le théorème de comparaison de Berkovich-Fargues, impliquent le théorème local (IV.3.2.3), ce qui complète la démonstration. On résume la discussion précédente dans le tableau suivant.

IV.4.5.6. Remarque. - Afin de ne pas multiplier les situations, nous n'avons considéré dans nos énoncés que les faisceaux induits notés $H T\left(g, l, \pi_{o}, \Pi_{l}\right)$ à partir des systèmes locaux $\mathcal{F}\left(g, l, \pi_{o}\right)_{1} \otimes \Pi_{l}$. Ceux-ci sont munis d'une action par correspondances de $\left(D_{\mathbb{A}}^{\infty}\right)^{\times} \times \mathbb{Z} ;$ cependant quand on les considère comme des constituants de $R \Psi_{\eta_{o}}\left(\overline{\mathbb{Q}}_{l}\right)$, on les voit comme munis d'une action par correspondances de $\left(D_{\mathbb{A}}^{\infty}\right)^{\times} \times W_{o}$. Le lien entre les deux situations est donnée par $c_{o} \in W_{o} \mapsto-\operatorname{deg}\left(c_{o}\right) \in \mathbb{Z}$. Afin de distinguer les deux situations, l'action de $W_{o}$ sera toujours accompagnée d'un $L_{g}\left(\pi_{o}\right)$ tandis que pour $\mathbb{Z}$ il sera toujours question du caractère $\Xi$ (cf. plus loin).

- Par ailleurs nous ne considérons pas par la suite l'action totale de $W_{o}$ mais plutôt ses frobenius semi-simplifiés.

Avant de rentrer dans la preuve proprement dite, on rappelle la proposition (I.5.3.10) du chapitre I.

IV.4.5.7. Proposition. - Soit $1 \leqslant l g<d$ et soit $\pi_{o}$ une représentation irréductible cuspidale de $G L_{g}\left(F_{o}\right)$. Pour $1 \leqslant i \leqslant l$ et pour tout $j$,

$$
\lim _{\vec{I}} H_{c}^{j}\left(M_{I, s_{o}}^{=l g}, H T_{\rho_{\infty}}\left(g, l, \pi_{o}, \Pi_{l}, I\right) \otimes L_{g}\left(\pi_{o}\right)\right)
$$

en tant que représentation de $G L_{d}\left(F_{o}\right) \times W_{o}$ est de la forme

$$
\bigoplus_{\xi}\left(\operatorname{Ind}_{P_{l g, d}^{\mathrm{oo}}\left(F_{o}\right)}^{G F_{o}\left(F_{o}\right)} \Pi_{l} \circ \xi(\operatorname{val}(\text { det })) \otimes \pi_{\xi}\right) \otimes L_{g}\left(\pi_{o} \circ \xi(\operatorname{val}(\operatorname{det}))\right)
$$




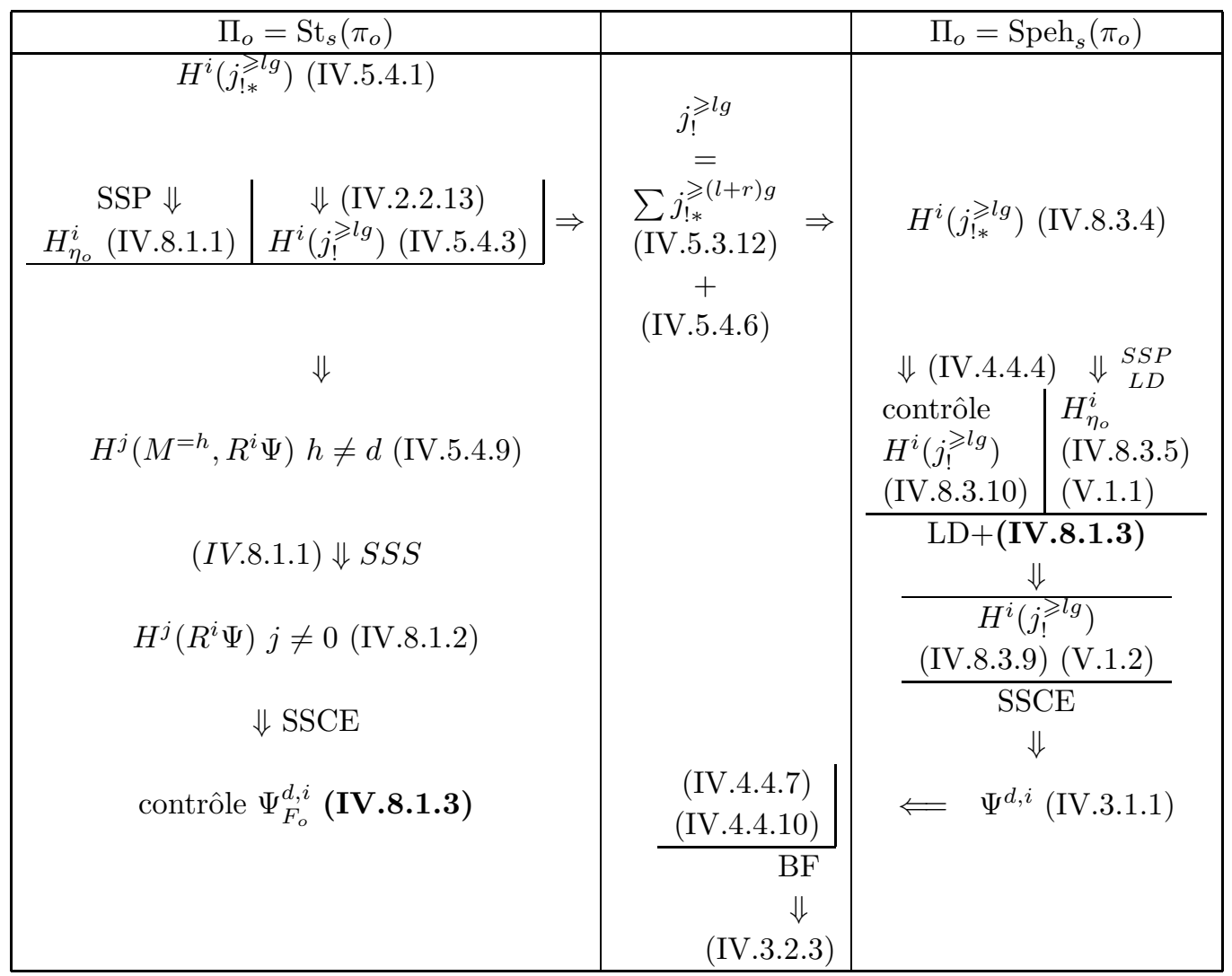

où $\xi$ décrit les caractères $\mathbb{Z} \longrightarrow \overline{\mathbb{Q}}_{l}^{\times}$et $\pi_{\xi}$ est une représentation de $G L_{d-l g}\left(F_{o}\right)$.

\section{IV.5. Étude dans le groupe de Grothendieck $\mathfrak{G}$}

IV.5.1. Groupe de Grothendieck des faisceaux pervers : généralités. - Soit $X$ un $\mathbb{F}_{q^{-}}$ schéma $X$. On rappelle que la catégorie $\operatorname{Perv}(X)$ des faisceaux pervers sur $X$ est noethérienne et artinienne, ses objets simples étant de la forme $j_{!_{*}} \mathcal{L}$ où $\mathcal{L}$ est un système local irréductible sur $j: U \hookrightarrow X$, où $U$ est un ouvert d'un fermé de $X$.

Pour $P$ un objet de $\operatorname{Perv}(X)$, sa dimension $n$ est par définition la plus grande dimension des supports $U$ des faisceaux pervers simples constituant $P$ de sorte que $-n=\min \left\{i / h^{i} P \neq 0\right\}$ et $h^{-n} P$ a un support de dimension $n$.

IV.5.1.1. Définition. - Étant donnée une catégorie triangulée $A$ localement petite, on considère son groupe de Grothendieck $K(A)$ défini comme le groupe libre engendré par les classes d'isomorphismes d'objets de $A$ quotienté par les relations :

- $A[1]=-A$

- $A=B+C$ pour tout triangle distingué $B \longrightarrow A \longrightarrow C \stackrel{+1}{\longrightarrow}$.

IV.5.1.2. Proposition. - Soit $\left(\mathcal{D}^{\leqslant 0}, \mathcal{D}^{\geqslant 0}\right)$ une catégorie dérivée d'une catégorie abélienne $\mathcal{A}$, munie d'une t-structure non dégénérée : on note $\mathcal{C}$ son coeur qui est alors une catégorie abélienne de groupe de Grothendieck $\operatorname{Groth}(\mathcal{C})$. L'application qui à un objet $\mathcal{F}$ de $\mathcal{D}$ associe

$$
\sum_{i}(-1)^{i}\left[{ }^{p} h^{i} \mathcal{F}\right] \in \operatorname{Groth}(\mathcal{C})
$$

induit un isomorphisme du groupe de Grothendieck $K(\mathcal{D})$ de la catégorie triangulée $\mathcal{D}$, sur $\operatorname{Groth}(\mathcal{C})$. 
Démonstration. - Pour tout objet $\mathcal{G}$ de $\mathcal{D}$ et pour tout $n \in \mathbb{Z}$, on a un triangle distingué

$$
\tau_{\leqslant n} \mathcal{G} \longrightarrow \mathcal{G} \longrightarrow \tau_{\geqslant n+1} \mathcal{G} \stackrel{+1}{\longrightarrow}
$$

de sorte que si $\mathcal{G}$ est un objet de $\mathcal{D} \geqslant n$, on a $[\mathcal{G}]=(-1)^{n}\left[{ }^{p} h^{n} \mathcal{G}\right]+\left[\tau_{n+1} \mathcal{G}\right]$ car $\tau_{\leqslant n} \mathcal{G}=\tau_{\leqslant n} \tau_{\geqslant n} \mathcal{G}=$ $\left({ }^{p} h^{n} \mathcal{G}\right)[n]$. Soit alors $a$ et $b$ tel que $\mathcal{F}$ soit un objet de $\mathcal{D}^{[a, b]}$. En appliquant ce qui précède à $\tau_{\geqslant n} \mathcal{F}$ pour $n$ variant de $a$ à $b$, on obtient l'égalité

$$
[\mathcal{F}]=\sum_{i}(-1)^{i}\left[{ }^{p} h^{i} \mathcal{F}\right]
$$

de sorte que l'application $\sum_{i} \alpha_{i}\left[P_{i}\right] \in \operatorname{Groth}(\mathcal{C}) \mapsto \sum_{i} \alpha_{i}\left[P_{i}\right] \in K(\mathcal{D})$ est inverse de celle de l'énoncé.

IV.5.1.3. Corollaire. - Soit $D_{b}^{c}\left(X, \overline{\mathbb{Q}}_{l}\right)$ la catégorie dérivée des $\overline{\mathbb{Q}}_{l}$-complexes constructibles sur un $\mathbb{F}_{q}$-schéma $X$ que l'on muni de la t-structure de perversité autoduale (resp. de la t-structure triviale) de coeur la catégorie $\operatorname{Perv}(X)$ (resp. Const $(X)$ ) des faisceaux pervers (resp. des faisceaux constructibles) sur $X$. Pour tout objet $\mathcal{F}$ de $D_{b}^{c}\left(X, \overline{\mathbb{Q}}_{l}\right)$, son image dans $\operatorname{Groth}(\operatorname{Perv}(X))$ est déterminée par son image dans $\operatorname{Groth}(\operatorname{Const}(X))$.

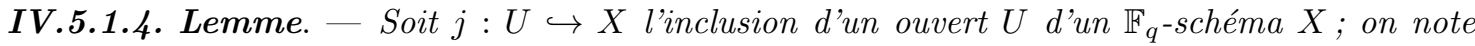
$i: Z \hookrightarrow X$ le fermé complémentaire. L'image d'un objet $\mathcal{F}$ de $D_{b}^{c}\left(X, \overline{\mathbb{Q}}_{l}\right)$ dans $\operatorname{Groth}(\operatorname{Const}(X))$ est déterminée par l'image de $j^{*} \mathcal{F}$ dans $\operatorname{Groth}(\operatorname{Const}(U))$ et celle de $i^{*} \mathcal{F}$ dans $\operatorname{Groth}(\operatorname{Const}(Z))$.

Démonstration. — Le résultat découle directement de l'existence du triangle distingué $j ! j^{*} \mathcal{F} \longrightarrow$ $\mathcal{F} \longrightarrow i_{*} i^{*} \mathcal{F} \stackrel{+1}{\longrightarrow}$.

On en déduit alors la proposition suivante.

IV.5.1.5. Proposition. — Soit X un schéma muni d'une stratification

$$
X^{\geqslant d} \subset X^{\geqslant d-1} \subset \cdots \subset X^{\geqslant 1}=X
$$

et soit $\mathcal{P}$ un faisceau pervers sur $X$. Alors l'image de $\mathcal{P}$ dans le groupe de Grothendieck des faisceaux pervers sur $X$ est déterminée par l'image de $\sum_{i}(-1)^{i}\left[h^{i}(\mathcal{P})_{\mid X=h}\right.$ dans le groupe de Grothendieck des faisceaux localement constant sur $X^{=h}:=X^{\geqslant h}-X^{\geqslant h-1}$ pour tout $1 \leqslant h \leqslant d$.

IV.5.2. Image dans $\mathfrak{G}$ de $R \Psi_{\eta_{o}}\left(\overline{\mathbb{Q}}_{l}\right)[d-1]$. -

IV.5.2.1. Proposition. - Les complexes $R j_{*}^{\geqslant l g} \mathcal{F}\left(g, l, \pi_{o}\right)_{0}[d-l g]$ et $j_{!}^{\geqslant l g} \mathcal{F}\left(g, l, \pi_{o}\right)_{0}[d-l g]$ sont des faisceaux pervers.

Démonstration. - Le résultat découle du fait que $j^{\geqslant l g}$ est une inclusion affine.

IV.5.2.2. Proposition. — Pour $\pi_{o}$ une représentation irréductible cuspidale de $G L_{g}\left(F_{o}\right)$, on a l'égalité suivante dans $\mathfrak{G}$ :

$$
\begin{aligned}
& e_{\pi_{o}}\left[R \Psi_{\eta_{o}, \pi_{o}}\left(\overline{\mathbb{Q}}_{l}\right)[d-1]\right]=\sum_{i=1}^{s_{g}} \sum_{l=i}^{s_{g}}(-1)^{l-i} \\
& {\left[j_{!}^{\geqslant l g} H T\left(g, l, \pi_{o},[\overleftarrow{i-1}, \overrightarrow{l-i}]_{\pi_{o}}\right) \otimes L_{g}\left(\pi_{o}\right)\left(-\frac{l g-2+2 i-l}{2}\right)\right] }
\end{aligned}
$$

Démonstration. — L'isomorphisme (IV.2.2.12) et l'égalité (IV.2.1.10) déterminent pour tout $0 \leqslant$ $h<d$, la somme suivante

$$
\sum_{i}(-1)^{i}\left[R^{i} \Psi_{v, \mid M_{\bar{I}, s_{O}}^{=h}}\right]
$$


dans le groupe de Grothendieck de la catégorie abélienne des faisceaux constructibles sur $M_{I, s_{o}}^{=h}$. Le résultat découle alors de la proposition (IV.5.1.5).

IV.5.3. Décomposition dans $\mathfrak{G}$ des $j_{!}^{\geqslant l g} \mathcal{F}\left(g, l, \pi_{o}\right)[d-l g]$. — Rappelons le théorème de comparaison de Berkovich-Fargues

IV.5.3.1. Théorème. - Soit z un point géométrique de $M_{I, s_{o}}^{=h}$. Le germe en z de $R \Psi_{\eta_{o}, I}\left(\overline{\mathbb{Q}}_{l}\right)$ est égal à $\widetilde{\Psi_{F_{o}, n}^{h}}$ en tant qu'objet de la catégorie dérivée filtrée $\mathbb{D}^{b} F\left(\overline{\mathbb{Q}}_{l}\right)$, avec $n=\operatorname{mult}_{o}(I)$.

Ainsi l'hypothèse de récurrence sur la filtration de monodromie-locale des modèles de DeligneCarayol de hauteur strictement inférieure à $d$, donne le corollaire suivant.

IV.5.3.2. Corollaire. - Pour tout point géométrique $z_{I}$ de $M_{I, s_{o}}^{=h}$, le germe en $z_{I}$ de $h^{i} g r_{k, \pi_{o}, I}$ vérifie les propriétés suivantes:

(1) il est nul si h n'est pas divisible par $g$;

(2) pour $h=l g$, ils sont nuls pour $|k| \geqslant l$ quelque soit $i$;

(3) pour $h=l g$ et $|k|<l$ :

(i) ils sont nuls pour $i<l g-d-l+1+|k|$ ou pour $i \not \equiv l g-d-l+1+k \bmod 2$ ou pour $i>l g-d$;

(ii) pour $l g-d-l+1+|k| \leqslant i=l g-d-l+1+|k|+2 r \leqslant l g-d$, la fibre en $z_{I}$ de $h^{i} g r_{k, \pi_{o}, I}$ est naturellement munie d'une action de $\mathcal{N}_{o}(h) \cap \mathcal{H}_{o, n}(h)$ où $\mathcal{N}_{o}(h) \subset G L_{h}\left(F_{o}\right) \times D_{o, h}^{\times} \times W_{o}$ est défini comme au $\S I V .1 .1$, où $G L_{h}\left(F_{o}\right)$ est vu naturellement comme un sous-groupe du Levi $G L_{h}\left(F_{o}\right) \times G L_{d-h}\left(F_{o}\right)$ de $G L_{d}\left(F_{o}\right)$ et où $\mathcal{H}_{o, n}(h)$ est l'algèbre de Hecke associée à $K_{o, n}(h) \subset G L_{h}\left(\mathcal{O}_{o}\right)$ avec $n=\operatorname{mult}_{o}(I)$. On obtient alors que la fibre en $z_{I}$ de $\left(h^{i} g r_{k, \pi_{o}, I}\right)^{e_{\pi_{o}}}$ est isomorphe aux invariants sous $K_{o, n}$ de

$$
\mathrm{JL}^{-1}\left([\overleftarrow{l-1}]_{\pi_{o}}\right) \otimes[\overleftarrow{|k|+2 r}]_{\pi_{o}} \overrightarrow{\times}[\overrightarrow{l-2 r-|k|-2}]_{\pi_{o}} \otimes L_{g}\left(\pi_{o}\right)\left(-\frac{l(g-1)+|k|+k+2 r}{2}\right)
$$

en tant que $\mathcal{N}_{o}(h) \cap \mathcal{H}_{o, n}(h)$ module.

Remarque : En ce qui concerne le dernier point du corollaire précédent, et donc le fait que la filtration locale soit équivariante, on peut la déduire du théorème de comparaison de BerkovichFargues avec les strates des variétés de Drinfeld-Stuhler en rang $d^{\prime}<d$ en supposant connus, par récurrence, les théorèmes globaux pour celles-ci.

IV.5.3.3. Proposition. - Pour tout $1 \leqslant l \leqslant s_{g}$, on a l'égalité dans $\mathfrak{G}$

$$
j_{!}^{\geqslant l g} H T\left(g, l, \pi_{o}, \Pi_{l}\right)[d-l g]=\sum_{i=l}^{s_{g}} j_{! *}^{\geqslant i g} H T\left(g, i, \pi_{o}, \Pi_{l} \overrightarrow{\times}[\overleftarrow{i-l-1}]_{\pi_{o}}\right)[d-i g] \otimes \Xi^{\frac{(l-i)(g-1)}{2}} \oplus P_{!, l}
$$

où $P_{!, l}$ est une somme de faisceaux pervers concentrés aux points supersinguliers.

Démonstration. - Dans $\mathfrak{G}$, on écrit $j_{!}^{\geqslant l g} H T\left(g, l, \pi_{o}, \Pi_{l}\right)[d-l g]$ sous la forme $\sum_{i} A_{\pi_{o}, l, i}\left(\Pi_{l}\right)$ où les $A_{\pi_{o}, l, i}\left(\Pi_{l}\right)$ sont une somme à coefficients positifs de faisceaux pervers simples de poids $d-l g-i$, que l'on appellera les constituants de $j_{!}^{\geqslant l g} H T\left(g, l, \pi_{o}, \Pi_{l}\right)[d-l g]$. On raisonne par récurrence descendante sur la dimension des faisceaux pervers qui interviennent, en traitant tous les $l$ par récurrence de $s_{g}$ à 1 et en travaillant sur les systèmes inductifs comme indiqué dans la remarque (IV.4.5.1).

IV.5.3.4. Lemme. - Pour tout $1 \leqslant l \leqslant s_{g}, j_{! *}^{\geqslant l g} H T\left(g, l, \pi_{o}, \Pi_{l}\right)[d-l g]$ est le seul constituant de dimension $d-l g$ de $j_{!}^{\geqslant l g} H T\left(g, l, \pi_{o}, \Pi_{l}\right)[d-l g]$. Par ailleurs tous les autres constituants de $j_{!}^{\geqslant l g} H T\left(g, l, \pi_{o}, \Pi_{l}\right)[d-l g]$ sont de poids strictement inférieur à $d-l g$. 
Démonstration. — On a la suite exacte courte de faisceaux pervers

$$
0 \rightarrow P_{\pi_{o}, l, 0}\left(\Pi_{l}\right) \longrightarrow j_{!}^{\geqslant l g} H T\left(g, l, \pi_{o}, \Pi_{l}\right)[d-l g] \longrightarrow j_{!_{*}}^{\geqslant l g} H T\left(g, l, \pi_{o}, \Pi_{l}\right)[d-l g] \rightarrow 0
$$

où $P_{\pi_{o}, l, 0}$ est égale à $i \geqslant l g, * j_{!_{*}}^{\geqslant l g} H T\left(g, l, \pi_{o}, \Pi_{l}\right)[d-l g-1]$ qui est donc de poids inférieur ou égal à $d-l g-1$ et de dimension strictement inférieure à $d-l g$, d'où le résultat.

D'après le lemme précédent, tous les faisceaux pervers qui interviennent dans l'écriture de $j_{!}^{\geqslant l g} H T\left(g, l, \pi_{o}, \Pi_{l}\right)[d-l g]$ sont de dimension inférieure ou égale à $d-l g$. Ainsi pour $h>d-g$, il n'y a aucun constituant de dimension $h$ dans les $j_{!}^{\geqslant l g} H T\left(g, l, \pi_{o}, \Pi_{l}\right)[d-l g]$ pour $1 \leqslant l \leqslant s_{g}$ et pour $h=d-g, j_{!_{*}}^{\geqslant g} H T\left(g, 1, \pi_{o}, \Pi_{1}\right)[d-g]$ est le seul constituant de dimension $d-g$ pour $l=1$. Supposons donc que pour tout $h>h_{0}$, les constituants des $j_{!}^{\geqslant l g} H T\left(g, l, \pi_{o}, \Pi_{l}\right)[d-l g]$ pour $1 \leqslant l \leqslant s_{g}$, sont ceux prédits par l'énoncé et traitons la dimension $h_{0}$.

IV.5.3.5. Lemme. — Supposons la proposition (IV.5.3.3) vérifiée pour les faisceaux pervers de dimension strictement supérieure à $0<h_{0}<d-g$, i.e. pour tout $1 \leqslant l \leqslant s_{g}$, les constituants de dimension strictement plus grande que $h_{0}$ des $j_{!}^{\geqslant l g} H T\left(g, l, \pi_{o}, \Pi_{l}\right)[d-l g]$ sont les

$$
j_{! *}^{\geqslant(l+r) g} H T\left(g, l+r, \pi_{o}, \Pi_{l} \overrightarrow{\times}[\overleftarrow{r-1}]_{\pi_{o}}\right)[d-l g] \otimes \Xi^{\frac{r(g-1)}{2}}
$$

avec $d-(l+r) g>h_{0}$. Pour tout $k$, l'image dans $\mathfrak{G}$ de $e_{\pi_{o}} g r_{k, \pi_{o}}$ est alors égale à

$$
\sum_{\substack{|k|<l<\left(d-h_{0}\right) / g \\ l \equiv k-1 \bmod 2}} \mathcal{P}\left(g, l, \pi_{o}\right)\left(-\frac{l g+k-1}{2}\right)+P_{k, h_{0}}
$$

où $P_{k, h_{0}}=\left(P_{k, h_{0}, I}\right)_{I}$ est une somme de faisceaux pervers simples de dimension inférieure ou égale $\grave{a} h_{0}$ à support dans la tour des $\left(M_{I, s_{o}}^{\geqslant d-h_{0}}\right)_{I}$, i.e. la fibre de $h^{i} P_{k, h_{0}, I}$ en tout point géométrique de $M_{I, s_{o}}^{=h}$ est nulle pour $h<d-h_{0}$ et pour tout $i$.

Démonstration. — D'après (IV.5.2.2), chacun $^{(14)}$ des $\mathcal{P}\left(g, l, \pi_{o}\right)\left(-\frac{l g+k-1}{2}\right)$, pour $d-l g>h_{0}$ est un constituant d'un $e_{\pi_{o}} g r_{r, \pi_{o}}$; il s'agit alors de montrer que $r=k$. Le résultat découle directement via le théorème de comparaison de Berkovich-Fargues, de l'hypothèse de récurrence sur le théorème local (IV.3.2.3). En effet, pour $l g<d$ les $\pi_{o}$-parties des $h^{0} g r_{l g, l o c, k}$ sont pures de poids $l g-1+k$. L'équivalent du théorème de Serre-Tate et le théorème de Berkovich-Fargues impliquent alors que la fibre en tout point géométrique $z$ de la strate $l g$ de $h^{l g-d} g r_{k, \pi_{o}}$ est pure de poids $l g-1+k$. Or la fibre en un point géométrique de la strate $l g$ de $h^{l g-d} \mathcal{P}\left(g, l, \pi_{o}\right)\left(-\frac{l g+k-1}{2}\right)$ est de poids $l g-1+k$. Ainsi pour $l=1, \mathcal{P}\left(g, 1, \pi_{o}\right)((1-g) / 2)$ est un constituant de $g r_{0, \pi_{o}}$ car tous les autres faisceaux pervers qui interviennent sont de dimension strictement plus petite.

On raisonne alors par récurrence sur $l$; on suppose que pour tout $l<l_{0}<\left(d-h_{0}\right) / g$, et tout $|k|<l-1, \mathcal{P}\left(g, l, \pi_{o}\right)\left(-\frac{l g+k-1}{2}\right)$ est un constituant de $g r_{k, \pi_{o}}$ et traitons le cas de $l_{0}$. D'après ce qui précède on en déduit que pour tout $|k|<l_{0}, \mathcal{P}\left(g, l_{0}, \pi_{o}\right)\left(-\frac{l_{0} g-1+k}{2}\right)$ est un constituant de $g r_{r, \pi_{o}}$ avec $r \geqslant k$; en effet dans le cas contraire la filtration par le poids de $g r_{r}$ donnerait une suite exacte courte

$$
0 \rightarrow P \longrightarrow g r_{r, \pi_{o}} \longrightarrow \mathcal{P}\left(g, l_{0}, \pi_{o}\right)\left(-\frac{l_{0} g-1+k}{2}\right) \rightarrow 0
$$

modulo des faisceaux pervers de dimension strictement plus petite, ce qui donnerait une suite exacte longue

$$
\cdots h^{l_{0} g-d} g r_{r, \pi_{o}} \longrightarrow h^{l_{0} g-d} \mathcal{P}\left(g, l_{0}, \pi_{o}\right)\left(-\frac{l_{0} g-1+k}{2}\right) \longrightarrow h^{l_{0} g-d+1} P \ldots
$$

$\overline{{ }^{(14)} \text { On rappelle, }}$ cf. la remarque (IV.4.3.2), que les $\mathcal{P}\left(g, l, \pi_{o}\right)$ ne sont pas irréductibles; cependant les arguments fonctionnent de manière strictement identique pour tous ses constituants simples car la seule différence entre ceux-ci provient de l'action de $\mathcal{D}_{o, l g}^{\times}$. 
or $h^{l g-d+1} P$ a un support de dimension strictement plus petite que $d-l_{0} g$ de sorte qu'il existerait un point géométrique de $M_{I, s_{o}}^{=l_{0} g}$ tel que la fibre de $h^{l_{0} g-d} g r_{r, \pi_{o}}$ serait de poids $l_{0} g-1+k$ ce qui n'est pas. Ainsi en particulier pour $k=l_{0}-1$, il existe $r \geqslant l_{0}-1$ tel que $g r_{r, \pi_{o}}$ soit de dimension $d-l_{0} g$. Pour la même raison que ci-dessus, on en déduit qu'il s'agit de $r=l_{0}-1$. On utilise alors l'opérateur $N$ qui finit de placer les $\mathcal{P}\left(g, l_{0}, \pi_{o}\right)\left(-\frac{l_{0} g+k-1}{2}\right)$ sur les $g r_{k, \pi_{o}}$, d'où la récurrence.

Par ailleurs on remarque qu'en tout point géométrique $z$ de $M_{I, s_{o}}^{\leqslant d-h_{0}}$, la fibre en $z$ des faisceaux de cohomologie de $g r_{k, \pi_{o}, I}-P_{k, h_{0}, I}$ est égale à celle du modèle local i.e., d'après le théorème de comparaison de Berkovich-Fargues à celle de $g r_{k, \pi_{o}, I}$ de sorte que celle de $P_{k, h_{0}, I}$ est nulle.

Retour à la preuve de la proposition (IV.5.3.3) :

le principe est d'étudier l'égalité (IV.5.2.18) en utilisant le lemme précédent ainsi que (IV.5.3.4) pour $j_{!}^{\geqslant}{ }^{l_{0} g} H T\left(g, l_{0}, \pi_{o}, \Pi_{l_{0}}\right)\left[d-l_{0} g\right]$ dont le seul constituant de dimension supérieur ou égal à $d-l_{0} g$ est $j_{! *}^{\geqslant} l_{0} g T\left(g, l_{0}, \pi_{o}, \Pi_{l_{0}}\right)\left[d-l_{0} g\right]$.

Note : on suppose, afin de simplifier la rédaction, que $\Pi_{l}$ est elliptique de type $\pi_{o}$.

IV.5.3.6. Lemme. - Pour tout $1 \leqslant l \leqslant s_{g}$, soit $A_{\pi_{o}, l, r}\left(\Pi_{l}\right)$ un constituant de

$$
j_{!}^{\geqslant l g} H T\left(g, l, \pi_{o}, \Pi_{l}\right)[d-l g]
$$

de poids $d-l g-r$ et de dimension $h_{0}$. Il existe alors des entiers $k$ et $t \geqslant 0$ ainsi que $\Pi_{l}^{\prime}$ une représentation elliptique de type $\pi_{o}$, et donc de même support cuspidal que $\Pi_{l}$, telle que $A_{\pi_{o}, l, r}\left(\Pi_{l}^{\prime}\right) \otimes L_{g}\left(\pi_{o}\right)\left(-\frac{l(g+1)-2(t+1)}{2}\right)$ soit un constituant de $e_{\pi_{o}} g r_{k, \pi_{o}}{ }^{(15)}$.

Démonstration. - Soit $A_{\pi_{o}, l, r}\left(\Pi_{l}\right)$ un constituant de $j_{!}^{\geqslant l g} H T\left(g, l, \pi_{o}, \Pi_{l}\right)[d-l g]$ de sorte que $A_{\pi_{o}, l, r}\left([\overleftarrow{l-1}]_{\pi_{o}}\right) \otimes L_{g}\left(\pi_{o}\right)\left(-\frac{l(g+1)-2}{2}\right)$ apparaît dans le membre de droite de l'égalité de la proposition (IV.5.2.2). On considère alors $\delta$ maximal tel qu'il existe une représentation elliptique $\Pi_{l}^{\prime}$ de type $\pi_{o}$ de $G L_{l g}\left(F_{o}\right)$ tel que $A_{\pi_{o}, l, r}\left(\Pi_{l}^{\prime}\right) \otimes L_{g}\left(\pi_{o}\right)\left(-\frac{\delta}{2}\right)$ apparaisse dans le membre de droite de l'égalité de la proposition (IV.5.2.2). On remarque alors qu'il y apparaît avec un coefficient positif et aucun négatif de sorte qu'il reste dans la somme ce qui implique le résultat. En effet s'il apparaissait avec un coefficient négatif, ce serait comme constituant d'un $j_{!}^{\geqslant l^{\prime} g} H T\left(g, l^{\prime}, \pi_{o},\left[\overleftarrow{l^{\prime}-r-1}, \vec{r}\right]_{\pi_{o}}\right)[d-$ $\left.l^{\prime} g\right] \otimes L_{g}\left(\pi_{o}\right)\left(-\frac{l^{\prime}(g+1)-2(r+1)}{2}\right)$ pour $r$ impair positif et on note que $j_{!}^{\geqslant l^{\prime} g} H T\left(g, l^{\prime}, \pi_{o},\left[\overleftarrow{l^{\prime}-1}\right]_{\pi_{o}}\right)[d-$ $\left.l^{\prime} g\right] \otimes L_{g}\left(\pi_{o}\right)\left(-\frac{l^{\prime}(g+1)-2}{2}\right)$ contiendrait un $A_{\pi_{o}, l, r}\left(\Pi_{l}^{\prime \prime}\right) \otimes L_{g}\left(\pi_{o}\right)\left(-\frac{\delta+2 r}{2}\right)$ pour $\Pi_{l}^{\prime \prime}$ une représentation de même support cuspidal que $\Pi_{l}^{\prime}$, contredisant la maximalité de $\delta$.

- Supposons dans un premier temps que $h_{0}$ n'est pas de la forme $d-l g$ et qu'il existe $l, r$ tel que $A_{\pi_{o}, l, r}\left(\Pi_{l}\right)$ contienne un faisceau pervers simple de dimension $h_{0}$. Le lemme précédent implique alors qu'il existe $k \geqslant 0$ tel que, avec les notations du lemme (IV.5.3.5), $P_{k, h_{0}}$ ait un constituant simple, disons $B_{\pi_{o}, k}$, de dimension $h_{0}$ et de poids $d-1+t$ pour un certain entier $t$. On raisonne alors comme dans la preuve du lemme (IV.5.3.5). Étant de dimension inférieure ou égale à $h_{0}$, $P_{k, h_{0}}$ est alors de dimension $h_{0}$ de sorte que $h^{-h_{0}} P_{k, h_{0}}$ est de dimension $h_{0}$. En outre comme $h^{-h_{0}} P_{k, h_{0}, I}$, d'après le lemme (IV.5.3.5), est à support dans $M_{I, s_{o}}^{\geqslant d-h_{0}}$, on en déduit que pour tout point générique de $M_{I, s_{o}}^{=d-h_{0}}$, la fibre en ce point de $h^{-h_{0}} P_{k, h_{0}, I}$ est non nulle de sorte qu'il existe un point géométrique $z_{I}$ de $M_{I, s_{o}}^{=d-h_{0}}$ tel que la fibre en $z_{I}$ de $h^{-h_{0}} P_{k, h_{0}, I}$ soit non nulle. Par ailleurs, les $\pi_{o}$-parties des $h^{-h_{0}} \mathrm{gr}_{k, l o c}$ du modèle local de hauteur $d-h_{0}$ sont toutes nulles, on en déduit, d'après le théorème de comparaison de Berkovich-Fargues, que la fibre en $z$ de $h^{-h_{0}} g r_{k, \pi_{o}}$ est nulle

${ }^{(15)}$ En utilisant monodromie-poids, on obtient $k=l-1-2 t$. 
ce qui implique que $t \neq k$ : en effet sinon $e_{\pi_{o}} g r_{k, \pi_{o}}$ est une extension de

$$
\left(\bigoplus_{\substack{|k|<l<\left(d-h_{0}\right) / g \\ l \equiv k-1 \bmod 2}} \mathcal{P}\left(g, l, \pi_{o}\right)\left(-\frac{l g+k-1}{2}\right)\right) \oplus B_{k, \pi_{o}}
$$

par des faisceaux pervers de dimension inférieure ou égale à $h_{0}$ ce qui implique que $\left(h^{-h_{0}} g r_{k, \pi_{o}}\right)^{e_{\pi_{o}}}$ admettrait $h^{-h_{0}} B_{k, h_{0}}$ comme facteur direct. Selon le même principe, on doit même avoir $t<k$. Or d'après la pureté des $g r_{k}$, on doit avoir $t=k$ d'où le résultat. Sans utiliser monodromie-poids, on raisonne alors comme suit.

IV.5.3.7. Lemme. - Soit $\mathcal{P}$ un constituant de $R \Psi_{\eta_{o}, \pi_{o}}\left(\overline{\mathbb{Q}}_{l}[d-1]\right)$. On en déduit alors que $D \mathcal{P}(1-d)$ est un constituant de $R \Psi_{\eta_{o}, \pi_{o}^{\vee}}\left(\overline{\mathbb{Q}}_{l}[d-1]\right)$.

Démonstration. - Le résultat découle simplement de la compatibilité de $R \Psi_{\eta_{o}}$ avec la dualité de Verdier, soit

$$
D R \Psi_{\eta_{o}}\left(\overline{\mathbb{Q}}_{l}[d-1]\right) \simeq R \Psi_{\eta_{o}}\left(D \overline{\mathbb{Q}}_{l}[d-1]\right)
$$

et du fait que la fibre générique de $M_{I, o}$ est lisse de sorte que $D \overline{\mathbb{Q}}_{l}[d-1] \simeq \overline{\mathbb{Q}}_{l}[d-1](1-d)$.

On considère alors un constituant simple de dimension $h_{0}$ de poids maximal, disons $d-1+r$ de $R \Psi_{\eta_{o}, \pi_{o}}\left(\overline{\mathbb{Q}}_{l}\right)[d-1]$. C'est alors un constituant de $g r_{k, \pi_{o}}$ pour $k>r$ d'après ce qui précède. Par ailleurs en utilisant le lemme (IV.5.3.7), on obtient un constituant simple de dimension $h_{0}$ de $g r_{-k, \pi_{o}}$ et de poids $d-1-r$, de sorte que l'opérateur de monodromie $N^{k}$ fournit un constituant simple de dimension $h_{0}$ de $g r_{k, \pi_{o}}$ et de poids $d-1-r+2 k>d-1+r$, d'où la contradiction par maximalité de $r$.

- Supposons désormais que $h_{0}=d-l_{0} g$ et supposons avoir montré par récurrence que pour tout $l_{1} \leqslant l \leqslant s_{g}$, le seul constituant de dimension $d-l_{0} g$ de $j_{!}^{\geqslant l g} H T\left(g, l, \pi_{o}, \Pi_{l}\right)[d-l g]$, est $j_{!_{*}}^{\geqslant l_{0} g} H T\left(g, l_{0} g, \pi_{o}, \Pi_{l} \overrightarrow{\times}\left[\overleftarrow{l_{0}-l-1}\right]_{\pi_{o}}\right)\left[d-l_{0} g\right] \otimes \Xi^{\frac{\left(l_{0}-l\right)(g-1)}{2}}$. Le résultat est vérifié pour $l_{1}=l_{0}$ supposons le donc vérifié jusqu'au rang $l_{1}$ et traitons le cas de $l_{1}-1$. On étudie alors les faisceaux pervers de dimension $d-l_{0} g$ dans le membre de droite de (IV.5.2.18), en particulier ceux de poids $d-l_{0}$, ce qui donne $j_{!_{*}}^{\geqslant l_{0} g} H T\left(g, l_{0}, \pi_{o}, \Pi\right)\left[d-l_{0} g\right] \otimes L_{g}\left(\pi_{o}\right)\left(-\frac{l_{0}(g-1)}{2}\right)$ avec

$$
(-1)^{l_{0}-1} \Pi=\left[\overrightarrow{l_{0}-1}\right]_{\pi_{o}}-\sum_{i=1}^{l_{0}-l_{1}}(-1)^{i}\left[\overrightarrow{l_{0}-i-1}\right]_{\pi_{o}} \overrightarrow{\times}[\overleftarrow{i-1}]_{\pi_{o}}
$$

soit $\Pi=(-1)^{l_{1}-1}\left[\overrightarrow{l_{1}-1}, \overleftarrow{l_{0}-l_{1}}\right]_{\pi_{o}}$. Or ce dernier ne peut pas apparaître dans le membre de gauche de (IV.5.2.18). En effet si $l_{1}$ est pair c'est évident car à gauche il ne peut y avoir que des coefficients positifs. Sinon de manière générale, on raisonne comme suit. Le lemme (IV.5.3.7) donnerait que $j_{!_{*}}^{\geqslant l_{0} g} H T\left(g, l_{0}, \pi_{o}^{\vee},\left[\overleftarrow{l_{0}-l_{1}}, \overleftrightarrow{l_{1}-1}\right]_{\pi_{o}^{\vee}}\right)\left[d-l_{0} g\right] \otimes L_{g}\left(\pi_{o}^{\vee}\right)\left(-\frac{l_{0}(g+1)-2}{2}\right)$ serait un constituant de $e_{\pi_{o}}\left[R \Psi_{\eta_{o}, \pi_{o}}\left(\overline{\mathbb{Q}}_{l}\right)[d-1]\right]$ qui, vu le poids, ne pourrait provenir que de

$$
j_{!}^{\geqslant l_{0} g} H T\left(g, l_{0}, \pi_{o}^{\vee},\left[\overleftarrow{l_{0}-1}\right]_{\pi_{o}^{\vee}}\right)\left[d-l_{0} g\right] \otimes L_{g}\left(\pi_{o}^{\vee}\right)\left(-\frac{l_{0}(g+1)-2}{2}\right),
$$

ce qui n'est pas d'après le lemme (IV.5.3.4). ${ }^{(16)}$.

Ainsi $j_{!_{*}}^{\geqslant l_{0} g} H T\left(g, l_{0}, \pi_{o},\left[\overrightarrow{l_{1}-1}, \overleftarrow{l_{0}-l_{1}}\right]_{\pi_{o}}\right)\left[d-l_{0} g\right] \otimes L_{g}\left(\pi_{o}\right)\left(-\frac{l_{0}(g-1)}{2}\right)$ doit être un constituant d'un $j_{!}^{\geqslant l g} H T\left(g, l, \pi_{o},[\overleftarrow{i}, \overrightarrow{l-i-1}]_{\pi_{o}}\right)[d-l g] \otimes L_{g}\left(\pi_{o}\right)\left(-\frac{l(g-1)+2 i}{2}\right)$ pour $0 \leqslant i<l$ et $l>l_{0}$ et $l \equiv l_{1}-1 \bmod 2$. Le résultat, i.e. $l=l_{1}-1$ et $r=0$, découle alors des trois lemmes suivants.

${ }^{(16)}$ En utilisant la pureté des gradués de la filtration de monodromie, on peut argumenter comme suit : le faisceau pervers en question serait alors un constituant de $e_{\pi_{o}} g r_{1-l_{0}, \pi_{o}}$ et donc $j_{! *}^{\geqslant} l_{0} g H T\left(g, l_{0}, \pi_{o},\left[\overrightarrow{l_{1}-1}, \overleftarrow{l_{0}-l_{1}}\right]_{\pi_{o}}\right) \otimes$ $L_{g}\left(\pi_{o}\right)\left(-\frac{l_{0}(g+1)-2}{2}\right)$ serait un constituant de $e_{\pi_{o}}\left[g r_{l_{0}-1, \pi_{o}}\right]$ qui ne pourrait être obtenu que via $j_{!}^{\geqslant} l_{0} g$ HT $\left(g, l, \pi_{o},\left[\overleftarrow{l_{0}-1}\right]_{\pi_{o}}\right)\left[d-l_{0} g\right] \otimes L_{g}\left(\pi_{o}\right)\left(-\frac{l_{0}(g+1)-2}{2}\right)$ ce qui ne se peut pas d'après le lemme (IV.5.3.4) 
IV.5.3.8. Lemme. - Pour un point géométrique $z$ de $M_{I, s_{o}, 1}^{=l_{0},}$ les fibres en $z$ des faisceaux de cohomologies des $j_{!_{*}}^{\geqslant l g} H T\left(g, l, \pi_{o}, \Pi_{l}\right)[d-l g] \otimes L_{g}\left(\pi_{o}\right)$, pour $l \geqslant l_{0}$, sont, en tant que représentation de $G L_{l_{0} g}\left(F_{o}\right) \times G L_{d-l_{0} g}\left(F_{o}\right) \times W_{o}$ de la forme

$$
\bigoplus_{\xi}\left(\Pi_{l}(\xi) \times \pi_{\xi}\right) \otimes \pi_{\xi}^{\prime} \otimes L_{g}\left(\pi_{o}\right)(\xi)
$$

où $\xi$ décrit les caractères de $\mathbb{Z}$ et $\pi_{\xi}$ (resp. $\pi_{\xi}^{\prime}$ ) est une représentation de $G L_{\left(l_{0}-l\right) g}\left(F_{o}\right)$ (resp. $\left.G L_{d-l_{0} g}\left(F_{o}\right)\right)$.

Démonstration. - C'est évident en utilisant que les strates sont induites, i.e.

$$
j_{!_{*}}^{\geqslant l g} H T\left(g, l, \pi_{o}, \Pi_{l}\right)=j_{1, ! *}^{\geqslant l g} \mathcal{F}\left(g, l, \pi_{o}\right)_{1} \otimes \Pi_{l} \times_{P_{l g, l_{0} g, d}\left(F_{o}\right)} P_{l_{0} g, d}\left(F_{o}\right)
$$

en tant que $P_{l_{0} g, d}\left(F_{o}\right) \times \mathbb{Z}$-module. Les torsions découlent alors de l'action telle qu'elle est décrite au $§ I V .2 .2$.

IV.5.3.9. Lemme. - Supposons que pour tout $1 \leqslant l \leqslant l_{0}$, les constituants simples de dimension strictement supérieure à $d-l_{0} g$ des $j_{! *}^{\geqslant l g} H T\left(g, l, \pi_{o}, \Pi_{l}\right)[d-l g]$ sont ceux prévus par la proposition (IV.5.3.3) et supposons qu'il existe $l<l_{0}$ tel que

$$
j_{!}^{\geqslant l g} H T\left(g, l, \pi_{o},[\overleftarrow{r}, \overrightarrow{l-r-1}]_{\pi_{o}}\right)[d-l g] \otimes L_{g}\left(\pi_{o}\right)\left(-\frac{l(g-1)+2 r}{2}\right)
$$

admette $j_{!_{*}}^{\geqslant l_{0} g} H T\left(g, l_{0}, \pi_{o},\left[\overrightarrow{l_{1}-1}, \overleftarrow{1}, \overleftarrow{l_{0}-l_{1}-1}\right]_{\pi_{o}}\right)\left[d-l_{0} g\right] \otimes L_{g}\left(\pi_{o}\right)\left(-\frac{l_{0}(g-1)}{2}\right)$ comme constituant On a alors $l \leqslant l_{1}$ et $r=0$.

Démonstration. - On considère la filtration par le poids de

$$
j_{!}^{\geqslant l g} H T\left(g, l, \pi_{o},[\overleftarrow{r}, \overrightarrow{l-r-1}]_{\pi_{o}}\right)[d-l g] \otimes L_{g}\left(\pi_{o}\right)\left(-\frac{l(g-1)+2 r}{2}\right)
$$

et la suite spectrale qui s'en déduit

$$
E_{1}^{i, j}=h^{i+j} g r_{-i}(l, r) \Rightarrow h^{i+j} j_{!}^{\geqslant l g} H T\left(g, l, \pi_{o},[\overleftarrow{r}, \overrightarrow{l-r-1}]_{\pi_{o}}\right)[d-l g] \otimes L_{g}\left(\pi_{o}\right)\left(-\frac{l(g-1)+2 r}{2}\right)
$$

où $g r_{k}(l, r)$ est le gradué de poids $k$ de $j_{!}^{\geqslant l g} H T\left(g, l, \pi_{o},[\overleftarrow{r}, \overrightarrow{l-r-1}]_{\pi_{o}}\right)[d-l g] \otimes L_{g}\left(\pi_{o}\right)\left(-\frac{l(g-1)+2 r}{2}\right)$. D'après le lemme précédent toutes les fibres aux points géométriques de $M_{I, s_{o}, 1}^{=l_{0} g}$ des $E_{1}^{i, j}$ avec $i+j<l_{0} g-d$ sont, en tant que $G L_{l_{0} g}\left(F_{o}\right) \times W_{o}$-module, de la forme

$$
\bigoplus_{\xi}[\overleftarrow{r}, \overrightarrow{l-r-1}]_{\pi_{o}(\xi)} \times \pi_{\xi} \otimes L_{g}\left(\pi_{o}(\xi)\right)\left(-\frac{l(g-1)+2 r}{2}\right)
$$

alors que celles de $j_{! *}^{\geqslant l_{0} g} H T\left(g, l_{0}, \pi_{o},\left[\overrightarrow{l_{1}-1}, \overleftarrow{1}, \overleftarrow{l_{0}-l_{1}-1}\right]_{\pi_{o}}\right)\left[d-l_{0} g\right] \otimes L_{g}\left(\pi_{o}\right)\left(-\frac{l_{0}(g-1)}{2}\right)$ sont de la forme

$$
\bigoplus_{\xi}\left[\overrightarrow{l_{1}-1}, \overleftarrow{1}, \overleftarrow{l_{0}-l_{1}-1}\right]_{\pi_{o}(\xi)} \times \pi_{\xi}^{\prime} \otimes L_{g}\left(\pi_{o}(\xi)\right)\left(-\frac{l(g-1)}{2}\right)
$$

En remarquant que les $E_{1}^{i, l_{0} g-d+1-i}$ ont un support de dimension strictement inférieur à $d-l_{0} g$ et que les $E_{\infty}^{n}$ sont nuls pour $n \neq l g-d$, on en déduit alors que $r=0$ et $l \leqslant l_{1}$.

IV.5.3.10. Lemme. - Pour tout $1 \leqslant l<l_{0}$ et toute représentation $\Pi$ elliptique de type $\pi_{o}$ de $G L_{\left(l_{0}-l\right) g}\left(F_{o}\right)$ distincte de $\left[\overleftarrow{l_{0}-l-1}\right]_{\pi_{o}}, j_{!}^{\geqslant l g} H T\left(g, l, \pi_{o}, \Pi_{l}\right)[d-l g]$ ne contient pas

$$
j_{! *}^{\geqslant l_{0} g} H T\left(g, l_{0}, \pi_{o}, \Pi_{l} \overrightarrow{\times} \Pi\right)[d-l g] \otimes \Xi^{\left(l_{0}-l\right)(g-1) / 2} .
$$


Démonstration. — Dans le cas contraire considérons $l$ minimal pour cette propriété. En l'appliquant à $\Pi_{l}=[\overrightarrow{l-1}]_{\pi_{o}}$, on en déduit que

$$
j_{!_{*}}^{\geqslant l_{0} g} H T\left(g, l_{0}, \pi_{o},\left[\overrightarrow{l-1}, \overleftarrow{1}, \overleftarrow{l_{0}-l-1}\right)[d-l g] \otimes L_{g}\left(\pi_{o}\right)\left(-\frac{l_{0}(g-1)}{2}\right)\right.
$$

reste dans le membre de droite de (IV.5.2.18), où $\left[\overleftrightarrow{l_{0}-l-1}\right]_{\pi_{o}}$ désigne $\Pi$. En effet il y apparaît via $j_{!_{*}}^{\geqslant l g} H T\left(g, l, \pi_{o},[\overrightarrow{l-1}]_{\pi_{o}}\right)[d-l g] \otimes L_{g}\left(\pi_{o}\right)\left(-\frac{l(g-1)}{2}\right)$ et n'est pas compensé car d'après le lemme précédent ce ne pourrait qu'être pour un $l^{\prime}<l$ ce qui contredirait la minimalité de $l$. Si le signe est négatif on obtient directement la contradiction, sinon on argument comme suit : en utilisant la pureté (resp. sans utiliser la pureté), par application de $N^{l_{0}-1}$ (resp. de la dualité de Verdier), on en déduit que $e_{\pi_{o}}\left[g r_{l_{0}-1, \pi_{o}}\right]$ (resp. $e_{\pi_{o}}\left[g r_{k, \pi_{o}}\right]$ pour un certain $k$ ) devrait contenir

$$
j_{! *}^{\geqslant l_{0} g} H T\left(g, l_{0}, \pi_{o},\left\lceil[\overrightarrow{l-1}]_{\pi_{o}} \overrightarrow{\times} \Pi_{l}\right\rceil\right)\left[d-l_{0} g\right] \otimes L_{g}\left(\pi_{o}\right)\left(-\frac{l_{0}(g+1)-2}{2}\right)
$$

(resp. $\left.j_{!_{*}}^{\geqslant l_{0} g} H T\left(g, l_{0}, \pi_{o}^{\vee},\left\lceil[\overrightarrow{l-1}]_{\pi_{o}} \overrightarrow{\times} \Pi_{l}\right\rceil^{\vee}\right)\left[d-l_{0} g\right] \otimes L_{g}\left(\pi_{o}^{\vee}\right)\left(-\frac{l_{0}(g+1)-2}{2}\right)\right)$ qui est de poids $d+l_{0}-2$. Or tous les constituants de dimension strictement supérieur à $d-l_{0} g$ de $R \Psi_{\eta_{o}, \pi_{o}}\left(\overline{\mathbb{Q}}_{l}\right)[d-1]$ (resp. de $\left.R \Psi_{\eta_{o}, \pi_{o}^{\vee}}\left(\overline{\mathbb{Q}}_{l}\right)[d-1]\right)$ sont de poids strictement inférieur à $d+l_{0}-2$ de sorte qu'il existerait un point géométrique de $M_{I, s_{o}}^{=l_{0} g}$ tel que la fibre de $h^{l_{0} g-d} g r_{l_{0}-1, \pi_{o}}\left(\right.$ resp. $\left.h^{l_{0} g-d} g r_{k, \pi_{o}}\right)$ en ce point admettrait un facteur direct de poids $d+l_{0}-2$ de la forme $\left\lceil[\overrightarrow{l-1}]_{\pi_{o}} \overrightarrow{\times} \Pi_{l}\right\rceil\left(\right.$ resp. $\left.\left\lceil[\overrightarrow{l-1}]_{\pi_{o}} \overrightarrow{\times} \Pi_{l}\right\rceil^{\vee}\right)$ ce qui n'est pas d'après le corollaire (IV.5.3.2).

Fin de la preuve de la proposition (IV.5.3.3) : pour tout $k$, on pose dans $\mathfrak{G}$ :

$$
Q_{k, \pi_{o}, l_{0}}:=e_{\pi_{o}} g r_{k, \pi_{o}}-\sum_{\substack{|k|<l \leqslant l_{0} \\ l \equiv k-1 \bmod 2}} \mathcal{P}\left(g, l, \pi_{o}\right)\left(-\frac{l g+k-1}{2}\right)
$$

Le corollaire (IV.5.3.2) et ce qui précède, prouvent alors que pour tout point générique $z$ de dimension supérieure ou égale à $d-l_{0} g, \sum_{i}(-1)^{i}\left(h^{i} Q_{k, \pi_{o}, l_{0}}\right)_{z}$ est nulle. On en déduit donc d'après la proposition (IV.5.1.5), que $Q_{k, \pi_{o}, l_{0}}$ est de dimension strictement inférieure à $d-l_{0} g$, ce qui conclut la récurrence.

IV.5.3.11. Définition. - Pour $l \leqslant l^{\prime}$ et $\Pi_{l}$ une représentation de $G L_{l g}\left(F_{o}\right)$, on introduit les faisceaux pervers purs de $\operatorname{FPH}\left(M_{s_{o}}\right){ }^{(17)}$

$$
\mathcal{P}_{-}\left(g, l^{\prime}, \pi_{o}, l, \Pi_{l}\right):=j_{! *}^{\geqslant l^{\prime} g} H T\left(g, l^{\prime}, \pi_{o}, \Pi_{l} \overrightarrow{\times}\left[\overleftarrow{l^{\prime}-l-1}\right]_{\pi_{o}}\right)\left[d-l^{\prime} g\right] \otimes \Xi^{\frac{\left(l^{\prime}-l\right)(g-1)}{2}}
$$

purs de poids $d-l^{\prime} g-2\left(l^{\prime}-l\right)$ et

$$
\mathcal{P}_{+}\left(g, l^{\prime}, \pi_{o}, l, \Pi_{l}\right):=j_{! *}^{\geqslant l^{\prime} g} H T\left(g, l^{\prime}, \pi_{o}, \Pi_{l} \overleftarrow{\times}\left[\overleftarrow{l^{\prime}-l-1}\right]_{\pi_{o}}\right)\left[d-l^{\prime} g\right] \otimes \Xi^{\frac{\left(l^{\prime}-l\right)(g+1)}{2}}
$$

purs de poids $d-l^{\prime} g+2\left(l^{\prime}-l\right)$.

La filtration par le poids donne alors le corollaire suivant.

IV.5.3.12. Corollaire. — (i) Il existe, pour $0 \leqslant i \leqslant s_{g}-l$, des faisceaux pervers $P_{\pi_{o}, l, i}\left(\Pi_{l}\right) \in$ $\mathrm{FPH}\left(M_{s_{o}}\right)$ tels que l'on ait, dans $\mathrm{FPH}\left(M_{s_{o}}\right)$, les suites exactes suivantes :

$$
\begin{gathered}
0 \rightarrow P_{\pi_{o}, l, 0}\left(\Pi_{l}\right) \longrightarrow j_{!}^{\geqslant l g} H T\left(g, l, \pi_{o}, \Pi_{l}\right)[d-l g] \longrightarrow j_{!_{*}}^{\geqslant l g} H T\left(g, l, \pi_{o}, \Pi_{l}\right)[d-l g] \rightarrow 0 \\
0 \rightarrow P_{\pi_{o}, l, 1}\left(\Pi_{l}\right) \longrightarrow P_{\pi_{o}, l, 0}\left(\Pi_{l}\right) \longrightarrow \mathcal{P}_{-}\left(g, l+1, \pi_{o}, l, \Pi_{l}\right) \oplus A_{\pi_{o}, l, 0}\left(\Pi_{l}\right) \rightarrow 0
\end{gathered}
$$

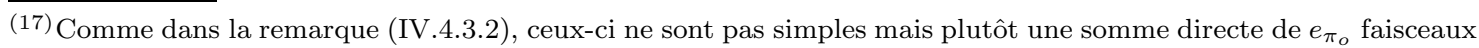
pervers simples où la différence entre eux provient de l'action de $\mathcal{D}_{o, l g}^{\times}$. Par ailleurs pour ce qui est des poids cf. la remarque (IV.4.5.6). 


$$
\begin{aligned}
0 \rightarrow P_{\pi_{o}, l, r}\left(\Pi_{l}\right) \longrightarrow P_{\pi_{o}, l, r-1}\left(\Pi_{l}\right) \longrightarrow & \mathcal{P}_{-}\left(g, l+r, \pi_{o}, l, \Pi_{l}\right) \oplus A_{\pi_{o}, l, r-1}\left(\Pi_{l}\right) \rightarrow 0 \\
& \cdots \\
0 \rightarrow P_{\pi_{o}, l, s_{g}-l}\left(\Pi_{l}\right) \longrightarrow P_{\pi_{o}, l, s_{g}-l-1}\left(\Pi_{l}\right) \longrightarrow & \mathcal{P}_{-}\left(g, s_{g}, \pi_{o}, l, \Pi_{l}\right) \oplus A_{\pi_{o}, l, s_{g}-l-1}\left(\Pi_{l}\right) \longrightarrow 0
\end{aligned}
$$

avec $A_{\pi_{o}, l, i}\left(\Pi_{l}\right)$, pour $1 \leqslant i \leqslant s_{g}-l$, des faisceaux pervers concentrés aux points supersinguliers purs de poids $d-l g-i-1$ et $P_{\pi_{o}, l, s_{g}-l}\left(\Pi_{l}\right)$ un faisceau pervers concentré aux points supersinguliers de poids inférieur ou égal à $d-l g-s_{g}+l$.

(ii) Dualement pour la dualité de Verdier, le complexe $\mathrm{Rj}_{*}^{\geqslant l g} H T\left(g, l, \pi_{o}, \Pi_{l}\right)[d-l g]$ est un objet de $\operatorname{FPH}\left(M_{s_{o}}\right)$ qui s'insère dans les suites exactes courtes

$$
\begin{gathered}
0 \rightarrow j_{!_{*}}^{\geqslant l g} H T\left(g, l, \pi_{o}, \Pi_{l}\right)[d-l g] \longrightarrow R j_{*}^{\geqslant l g} H T\left(g, l, \pi_{o}, \Pi_{l}\right)[d-l g] \\
0 \rightarrow D A_{\pi_{o}, l, 0}\left(\Pi_{l}\right)(l g-d) \oplus \mathcal{P}_{+}\left(g, l+1, \pi_{o}, l, \Pi_{l}\right) \longrightarrow \\
D P_{\pi_{o}, l, 0}\left(\Pi_{l}\right)(l g-d) \\
\cdots \\
0 D P_{\pi_{o}, l, 0}\left(\Pi_{l}\right)(l g-d) \rightarrow 0 \\
0 P_{\pi_{o}, l, 1}\left(\Pi_{l}\right)(l g-d) \rightarrow 0 \\
0 A_{\pi_{o}, l, r-1}\left(\Pi_{l}\right)(l g-d) \oplus \mathcal{P}_{+}\left(g, l+r, \pi_{o}, l, \Pi_{l}\right) \longrightarrow \\
D P_{\pi_{o}, l, r-1}\left(\Pi_{l}\right)(l g-d) \longrightarrow D P_{\pi_{o}, l, r}\left(\Pi_{l}\right)(l g-d) \rightarrow 0 \\
\cdots \\
0 \rightarrow D A_{\pi_{o}, l, s_{g}-l-1}\left(\Pi_{l}\right)(l g-d) \oplus \mathcal{P}_{+}\left(g, s_{g}, \pi_{o}, l, \Pi_{l}\right) \longrightarrow \\
D P_{\pi_{o}, l, s_{g}-l-1}\left(\Pi_{l}\right)(l g-d) \longrightarrow D P_{\pi_{o}, l, s_{g}-l}\left(\Pi_{l}\right)(l g-d) \rightarrow 0
\end{gathered}
$$

Remarque : Hors des points supersinguliers, nous avons montré que les $g r_{k}$ étaient purs de poids $d-1+k$, ce qui n'a rien d'impressionnant puisque finalement on l'a déduite de la pureté locale qui nous est donnée d'après l'hypothèse de récurrence. Nous verrons à la proposition (IV.7.3.2) comment la démontrer pour les faisceaux pervers supportés par les points supersinguliers.

IV.5.3.13. Corollaire. - Pour tout $|k|<s_{g}$, l'image de $e_{\pi_{o}} g r_{k, \pi_{o}}$ dans $\mathfrak{G}$ est égale à

$$
\left(\sum_{\substack{|k|<l \leqslant s g \\ l \equiv k-1 \bmod 2}} \mathcal{P}\left(g, l, \pi_{o}\right)\left(-\frac{l g+k-1}{2}\right)\right)+P_{k}
$$

où comme ci-dessus, $P_{k}$ est une somme de faisceaux pervers concentrés aux points supersinguliers. Dans tous les autres cas $\mathrm{gr}_{k, \pi_{o}}$ est de dimension nulle, concentré aux points supersinguliers.

IV.5.4. Étude aux points supersinguliers des $j_{!}^{\geqslant l g} \mathcal{F}\left(g, l, \pi_{o}\right)[d-l g]$. — Le but de ce paragraphe est de déterminer les faisceaux pervers ponctuels non précisés dans la proposition (IV.5.3.3). On rappelle que le raisonnement du paragraphe précédent ne s'appliquait pas au niveau des points supersinguliers car nous n'y connaissons pas l'aboutissement de (IV.4.1.17). Une idée naive est que pour connaître un faisceau ponctuel, on peut commencer par calculer son groupe de cohomologie $H^{0}$.

IV.5.4.1. Proposition. - Soit $1 \leqslant g<d$ ne divisant pas $d$ (resp. $g$ divisant $d=s g$ ), et soit $\Pi$ une représentation globale de $D_{\mathbb{A}}^{\times}$telle que $\Pi$ vérifie $\operatorname{Hyp}\left(\rho_{\infty}\right)$ avec $\Pi_{o} \simeq \operatorname{St}_{s}\left(\pi_{o}\right)$ pour $\pi_{o}$ une représentation irréductible cuspidale de $G L_{g}\left(F_{o}\right)$. Pour tout $i$ et $1 \leqslant l \leqslant s_{g}$ (resp. $1 \leqslant l<s$ ), la composante $\Pi^{\infty, o}$-isotypique des groupes de cohomologie des faisceaux pervers $j_{! *}^{\geqslant l g} H T_{\rho_{\infty}}\left(g, l, \pi_{o}, \Pi_{l}\right)[d-l g]$ est nulle, soit avec les notations de (IV.4.2.4) :

$$
H^{i}\left(j_{! *}^{\geqslant l g} H T_{\rho_{\infty}}\left(g, l, \pi_{o}, \Pi_{l}\right)[d-l g]\right)\left[\Pi^{\infty, o}\right]=0 .
$$


Démonstration. - On raisonne par récurrence pour $l$ variant de $s-1$ à 1 ; l'initialisation de la récurrence se fait d'elle même dans la preuve de l'induction qui suit. On reprend les suites exactes courtes de faisceaux pervers du corollaire (IV.5.3.12). On montre tout d'abord, par récurrence sur $i$ de $s-l-1$ à 0 , que pour tout $j \neq 0$, les groupes de cohomologie $H^{j}\left(P_{\pi_{o}, l, i}\left(\Pi_{l}\right) \otimes \mathcal{L}_{\rho_{\infty}}\right)\left[\Pi^{\infty, o}\right]$ sont nuls et que

$$
\begin{aligned}
H^{0}\left(P_{\pi_{o}, l, i}\left(\Pi_{l}\right) \otimes \mathcal{L}_{\rho_{\infty}}\right)\left[\Pi^{\infty, o}\right] & = \\
& \sum_{k=i}^{s_{g}-l-1} H^{0}\left(A_{\pi_{o}, l, k}\left(\Pi_{l}\right) \otimes \mathcal{L}_{\rho_{\infty}}\right)\left[\Pi^{\infty, o}\right]+H^{0}\left(P_{\pi_{o}, l, s_{g}-l}\left(\Pi_{l}\right) \otimes \mathcal{L}_{\rho_{\infty}}\right)\left[\Pi^{\infty, o}\right]
\end{aligned}
$$

Le résultat est clairement vrai pour $i=s-l-1$ car $P_{\pi_{o}, l, s-l-1}\left(\Pi_{l}\right)$ est un faisceau pervers ponctuel. Supposons donc le résultat acquis jusqu'au rang $i+1$ et montrons le au rang $i$. La suite exacte longue de cohomologie associée à

$$
0 \rightarrow P_{\pi_{o}, l, i}\left(\Pi_{l}\right) \longrightarrow P_{\pi_{o}, l, i-1}\left(\Pi_{l}\right) \longrightarrow \mathcal{P}_{-}\left(g, l+i, \pi_{o}, l, \Pi_{l}\right) \oplus A_{\pi_{o}, l, i-1}\left(\Pi_{l}\right) \rightarrow 0
$$

fournit, pour $j \neq 0$, les isomorphismes

$$
H^{j}\left(P_{\pi_{o}, l, i}\left(\Pi_{l}\right) \otimes \mathcal{L}_{\rho_{\infty}}\right)\left[\Pi^{\infty, o}\right] \simeq H^{j}\left(P_{\pi_{o}, l, i-1}\left(\Pi_{l}\right) \otimes \mathcal{L}_{\rho_{\infty}}\right)\left[\Pi^{\infty, o}\right]
$$

car d'après l'hypothèse de récurrence portant sur les $l$,

$$
H^{j}\left(\mathcal{P}\left(g, l+i, \pi_{o}, l, \Pi_{l}\right) \otimes \mathcal{L}_{\rho_{\infty}}\right)\left[\Pi^{\infty, o}\right]
$$

est nul ce qui implique la nullité de

$$
H^{j}\left(\mathcal{P}_{-}\left(g, l+i, \pi_{o}, l, \Pi_{l}\right) \otimes \mathcal{L}_{\rho_{\infty}}\right)\left[\Pi^{\infty, o}\right]
$$

Par ailleurs pour $j=0$, on a la suite exacte courte

$$
\begin{aligned}
& 0 \rightarrow H^{0}\left(P_{\pi_{o}, l, i}\left(\Pi_{l}\right) \otimes \mathcal{L}_{\rho_{\infty}}\right)\left[\Pi^{\infty, o}\right] \longrightarrow H^{0}\left(P_{\pi_{o}, l, i-1}\left(\Pi_{l}\right) \otimes \mathcal{L}_{\rho_{\infty}}\right) \\
& \longrightarrow H^{0}\left(A_{\pi_{o}, l, i-1}\left(\Pi_{l}\right) \otimes \mathcal{L}_{\rho_{\infty}}\right) \rightarrow 0
\end{aligned}
$$

d'où le résultat.

On considère alors la suite exacte longue de cohomologie associée à

$$
0 \rightarrow P_{\pi_{o}, l, 0}\left(\Pi_{l}\right) \longrightarrow j_{!}^{\geqslant l g} H T\left(g, l, \pi_{o}, \Pi_{l}\right)[d-l g] \longrightarrow j_{!_{*}}^{\geqslant l g} H T\left(g, l, \pi_{o}, \Pi_{l}\right)[d-l g] \rightarrow 0
$$

qui s'écrit

$$
0 \rightarrow H^{-1}\left(j_{!}^{\geqslant l g}\right) \longrightarrow H^{-1}\left(j_{! *}^{\geqslant l g}\right) \longrightarrow H^{0}\left(P_{\pi_{o}, l, s-l-1}\right) \longrightarrow H^{0}\left(j_{!}^{\geqslant l g}\right) \longrightarrow H^{0}\left(j_{! *}^{\geqslant l g}\right) \rightarrow 0
$$

et pour tout $i \neq-1,0, H^{i}\left(j_{!}^{\geqslant l g}\right) \simeq H^{i}\left(j_{!_{*}}^{\geqslant l g}\right)$ où pour alléger les notations, on a omis d'écrire $H T_{\rho_{\infty}}\left(g, l, \pi_{o}, \Pi_{l}\right)[d-l g]$ ainsi que $\left[\Pi^{\infty, o}\right]$. On en déduit alors que pour $i \neq 0, H^{i}\left(j_{\text {! }}^{\geqslant l g}\right)$ est pur de poids $d-1+i$ alors que $H^{0}\left(j_{!}^{\geqslant l g}\right)$ est mixte de poids inférieur ou égal à $d-1$. Le calcul de la somme alternée $\sum_{i}(-1)^{i} H^{i}\left(j_{!}^{\geqslant l g}\right)$, laquelle est nulle pour $g \nmid d$ et pour $g \mid d$ est constituée d'un seul terme de poids $d-1-(s-l)$, implique alors la nullité des $H^{i}\left(j_{!}^{\geqslant l g}\right)$ pour $i>0$ et celle des $H^{i}\left(j_{!_{*}}^{\geqslant l g} H T_{\rho_{\infty}}\left(g, l, \pi_{o}, \Pi_{l}\right)[d-l g]\right)\left[\Pi^{\infty, o}\right]$ pour $i \geqslant 0$ et pour tout $\pi_{o}$. La dualité de Poincaré donne alors la nullité des $H^{i}\left(j_{!_{*}}^{\geqslant l g} H T_{\rho_{\infty}^{\vee}}\left(g, l, \pi_{o}^{\vee}, \Pi_{l}^{\vee}\right)[d-l g]\right)\left[\Pi^{\infty, o}\right]$ pour tout $\pi_{o}$ et tout $i \leqslant 0$ et donc finalement la nullité des $H^{i}\left(j_{!_{*}}^{\geqslant l g} H T_{\rho_{\infty}}\left(g, l, \pi_{o}, \Pi_{l}\right)[d-l g]\right)\left[\Pi^{\infty, o}\right]$ pour tout $i$ et tout $\pi_{o}$.

Remarque : Il est possible de faire des calculs strictement similaires pour $\Pi_{o}$ quelconque. Par exemple, au lemme (IV.7.3.2) on traite le cas de $\Pi_{o} \simeq \operatorname{St}_{n_{1}}\left(\xi_{1}\right) \boxplus \cdots \boxplus \operatorname{St}_{n_{r}}\left(\xi_{r}\right)$, le cas général étant traité dans la preuve du théorème (V.4.1).

$I V .5 .4 .2$ - D'après le corollaire (IV.5.3.12) et en remarquant qu'un faisceau pervers ponctuel n'a de la cohomologie qu'en degré zéro, les corollaires suivants découlent directement de (IV.2.2.13). 
IV.5.4.3. Corollaire. - Pour g divisant $d=s g$ (resp. $1 \leqslant g<d$ ne divisant pas $d$ ), soit $\Pi$ une représentation irréductible de $D_{\mathbb{A}}^{\times}$vérifiant $\operatorname{Hyp}\left(\rho_{\infty}\right)$ telle que $\Pi_{o} \simeq \operatorname{St}_{s}\left(\pi_{o}\right)$. On a alors :

(i) pour tout $i \neq d-l g$ (resp. pour tout $i$ ), $H_{c}^{i}\left(M_{I, s_{o}, 1}^{=l g}, \mathcal{F}\left(g, l, \pi_{o}\right)_{1} \otimes \mathcal{L}_{\rho_{\infty}}\right)\left[\Pi^{\infty, o}\right]$ et donc $H_{c}^{i}\left(M_{I, s_{o}}^{=l g}, \mathcal{F}\left(g, l, \pi_{o}, I\right) \otimes \mathcal{L}_{\rho_{\infty}}\right)\left[\Pi^{\infty, o}\right]$ sont nuls et pour $i=d-l g$

$$
\begin{gathered}
\lim _{\vec{I}} H_{c}^{d-l g}\left(M_{I, s_{o}, 1}^{=l g}, \mathcal{F}\left(g, l, \pi_{o}, I\right)_{1} \otimes \mathcal{L}_{\rho_{\infty}}\right)\left[\Pi^{\infty, o}\right]= \\
m(\Pi) \overleftarrow{s-l-1}]_{\pi_{o}(l(g-1) / 2)} \otimes\left(\Xi^{\frac{(s-l)(g-1)}{2}} \bigoplus_{\xi \in \mathfrak{A}\left(\pi_{o}\right)} \xi^{-1}\right) \\
\underset{\lim _{I}}{\rightarrow} H_{c}^{d-l g}\left(M_{I, \bar{s}_{o}}^{=l g}, H T_{\rho_{\infty}}\left(g, l, \pi_{o}, \Pi_{l}, I\right)\right)\left[\Pi^{\infty, o}\right] \\
=m(\Pi)\left(\Pi_{l} \overrightarrow{\times}[\overleftarrow{s-l-1}]_{\pi_{o}}\right) \otimes\left(\Xi^{(s-l)(g-1) / 2} \bigoplus_{\xi \in \mathfrak{A}\left(\pi_{o}\right)} \xi^{-1}\right)
\end{gathered}
$$

en tant que représentation de $G L_{(s-l) g}\left(F_{o}\right) \times \mathbb{Z}$ et de $G L_{d}\left(F_{o}\right) \times \mathbb{Z}$, où $\mathfrak{A}\left(\pi_{o}\right)$ est l'ensemble des caractères $\xi: \mathbb{Z} \longrightarrow \mathbb{Q}_{l}^{\times}$, tels que $\pi_{o} \otimes \xi^{-1} \circ \operatorname{val}($ det $) \simeq \pi_{o}$ et $m(\Pi)$ est la multiplicité de $\Pi$ dans l'espace des formes automorphes.

(ii) pour tout $1 \leqslant l \leqslant s$, on a

$$
\begin{aligned}
H^{0}\left(j_{!}^{\geqslant l g} H T\left(g, l, \pi_{o}, \Pi_{l}\right)[d-l g] \otimes L_{g}\left(\pi_{o}\right)\right) & \\
& =m(\Pi) e_{\pi_{o}}\left(\Pi_{l} \overrightarrow{\times}[\overleftarrow{s-l-1}]_{\pi_{o}}\right) \otimes L_{g}\left(\pi_{o}\right)|-|^{-(s-l)(g-1) / 2}
\end{aligned}
$$

IV.5.4.4. Remarque. - Si $\Pi$ vérifie $\operatorname{Hyp}(\infty)$, la condition $\Pi_{o} \simeq[\overleftarrow{s-1}]_{\pi_{o}}$ pour $\pi_{o}$ une représentation irréductible cuspidale de $G L_{g}\left(F_{\infty}\right)$, implique qu'il existe une représentation irréductible cuspidale $\pi_{\infty}$ de $G L_{g^{\prime}}\left(F_{\infty}\right)$ avec $d=s^{\prime} g^{\prime}$ telle que $\Pi_{\infty} \simeq\left[\overleftarrow{s^{\prime}-1}\right]_{\pi_{\infty}}$ et donc $\Pi$ vérifie $\operatorname{Hyp}\left(\rho_{\infty}\right)$ avec $\rho_{\infty}=\mathrm{JL}^{-1}\left(\left[\overleftarrow{s^{\prime}-1}\right]_{\pi_{\infty}}\right)$

$I V .5 .4 .5$ - On rappelle que $\bar{D}$ est une algèbre à division centrale sur $F$ dont les invariants locaux en les places $x$ de $F$ distinctes de $\infty$ et $o$ sont égaux à ceux de $D$ et $\bar{D}_{o} \simeq D_{o, d}$ (resp. $\bar{D}_{\infty}$ ) est l'algèbre à division centrale sur $F_{o}\left(\right.$ resp. $\left.F_{\infty}\right)$ d'invariant $1 / d($ resp. $-1 / d)$. Pour la preuve de l'énoncé suivant on considère une représentation automorphe irréductible $\Pi$ de $D_{\mathbb{A}}^{\times}$telle que :

$-\Pi_{\infty} \simeq[\overleftarrow{s-1}]_{\pi_{\infty}}$ pour $\pi_{\infty}$ une représentation cuspidale de $G L_{d}\left(F_{\infty}\right)$ et $\Pi_{o} \simeq[\overleftarrow{s-1}]_{\pi_{o}} ;$

$-m(\Pi)=1$;

- l'ensemble $\mathfrak{A}_{\bar{D}^{\times}}(\Pi)$ des représentations irréductibles automorphes $\bar{\Pi}$ de $\bar{D}_{\mathbb{A}}^{\times}$telle que $\bar{\Pi}^{\infty, o} \simeq$ $\Pi^{\infty, o}$ est réduit à un élément avec $m(\bar{\Pi})=1, \bar{\Pi}_{o} \simeq \mathrm{JL}^{-1}\left(\Pi_{o}\right)$ et $\bar{\Pi}_{\infty} \simeq \mathrm{JL}^{-1}\left([\overleftarrow{s-1}]_{\pi_{\infty}}\right)$. L'existence d'une telle représentation $\Pi$ est assurée par Henniart (cf. [15] annexe A-4).

IV.5.4.6. Corollaire. - Pour tout $1 \leqslant l \leqslant s_{g}$, les faisceaux pervers $A_{\pi_{o}, l, i}\left(\Pi_{l}\right) d u$ corollaire (IV.5.3.12) sont tous nuls et pour $g$ ne divisant pas $d$ (resp. $g \mid d=s g) P_{\pi_{o}, l, s_{g}-l}\left(\Pi_{l}\right)$ est nul (resp. $P_{\pi_{o}, l, s-l-1}\left(\Pi_{l}\right)$ est le faisceau pervers ponctuel de support l'ensemble des points supersinguliers tel que $P_{\pi_{o}, l, s-l-1}\left(\Pi_{l}\right)$ est isomorphe à $\left.\mathcal{F}\left(g, s, \pi_{o}\right)\left(-\frac{(s-l)(g-1)}{2}\right) \otimes\left(\Pi_{l} \overrightarrow{\times}[\overleftarrow{s-l-1}]_{\pi_{o}}\right)\right)$.

Démonstration. - D'après la proposition précédente on a

$$
\begin{gathered}
\sum_{i=0}^{s_{g}-l-1} \lim _{\vec{I}} H^{0}\left(M_{I, s_{o}}, A_{\pi_{o}, l, i}\left(\Pi_{l}\right) \otimes \mathcal{L}_{\rho_{\infty}}\right)\left[\Pi^{\infty, o}\right]+ \\
\lim _{\vec{I}} H^{0}\left(M_{I, s_{o}}, P_{\pi_{o}, l, s_{g}-l}\left(\Pi_{l}\right) \otimes \mathcal{L}_{\rho_{\infty}}\right)\left[\Pi^{\infty, o}\right]= \\
\underset{\underset{I}{\lim }}{\lim ^{d-l g}\left(M_{I, s_{o}}, j_{!}^{\geqslant l g} H T_{\rho_{\infty}}\left(g, l, \pi_{o}, \Pi_{l}, I\right)\right)\left[\Pi^{\infty, o}\right] .}
\end{gathered}
$$


Le membre de droite est d'après (IV.2.2.13) soit nul, pour $g$ ne divisant pas $d$, soit de poids $d-l g-s_{g}+l$ pour $g$ divisant $d$.

- Les faisceaux pervers $A_{\pi_{o}, l, i}\left(\Pi_{l}\right)$, pour $0 \leqslant i<s_{g}-l$ étant de dimension zéro et purs de poids $d-l g-i$, la nullité de $H^{0}\left(M_{I, s_{o}, 1}^{\geqslant l g}, A_{\pi_{o}, l, i}\left(\Pi_{l}\right) \otimes \mathcal{L}_{\rho_{\infty}}\right)\left[\Pi^{\infty, o}\right]$ implique celle des $A_{\pi_{o}, l, i}\left(\Pi_{l}\right)$.

- De même pour $g$ ne divisant pas $d, P_{\pi_{o}, l, s-l}$, faisceau pervers concentré aux points supersinguliers, est nul car son groupe de cohomologie en degré 0 l'est.

- Pour $g$ divisant $d$, on rappelle, cf. le corollaire (IV.5.4.3), que

$$
H_{c}^{i}\left(M_{I, s_{o}, 1}^{=l g}, \mathcal{F}\left(g, l, \pi_{o}, \Pi_{l}, I\right)_{1} \otimes \mathcal{L}_{\rho_{\infty}}\right)\left[\Pi^{\infty, o}\right]
$$

est nul pour $i \neq d-l g$, et

$$
\begin{aligned}
\left.\lim _{\vec{I}} H_{c}^{d-l g}\left(M_{I, s_{o}, 1}^{=l g}, \mathcal{F}\left(g, l, \pi_{o}\right)_{1} \otimes \mathcal{L}_{\rho_{\infty}}\right)\right)\left[\Pi^{\infty, o}\right] & = \\
& m(\Pi) \bigoplus_{\xi \in \mathfrak{A}\left(\pi_{o}\right)}[\overleftarrow{s-l-1}]_{\pi_{o}(l(g-1) / 2)} \otimes \Xi^{\frac{(s-l)(g-1)}{2}} \xi
\end{aligned}
$$

où $\mathfrak{A}\left(\pi_{o}\right)$ est l'ensemble des caractères $\xi: \mathbb{Z} \longrightarrow \mathbb{Q}_{l}^{\times}$, tels que $\pi_{o} \otimes \xi^{-1} \circ \operatorname{val}($ det $) \simeq \pi_{o}$. Ainsi pour toute représentation elliptique $\Pi_{l}$ de type $\pi_{o}$ de $G L_{l g}\left(F_{o}\right)$,

$$
\begin{aligned}
\lim _{\vec{I}} H^{0}\left(M_{I, s_{o}}^{\geqslant l g}, j_{!}^{\geqslant l g} H T_{\rho_{\infty}}\left(g, l, \pi_{o}, \Pi_{l}, I\right)[d-l g]\right)\left[\Pi^{\infty, o}\right] & = \\
& \left.m(\Pi) e_{\pi_{o}} \Pi_{l} \overrightarrow{\times} \overleftarrow{s-l-1}\right]_{\pi_{o}} \otimes \Xi^{\frac{(s-l)(g-1)}{2}}
\end{aligned}
$$

en tant que représentation de $G L_{d}\left(F_{o}\right) \times \mathbb{Z}$. On étudie alors comme précédemment l'égalité (IV.5.2.18) et tout particulièrement les faisceaux pervers de dimension nulle. En ce qui concerne les faisceaux pervers simples de poids $s(g+1)-4$, le membre de droite de (IV.5.2.18) est égal à

$$
\begin{aligned}
\left(P_{\pi_{o}, s-1,1}\left([\overleftarrow{s-2}]_{\pi_{o}}\right)\left(-\frac{(s-1)(g+1)-2}{2}\right)-\right. & \\
& \left.\mathcal{F}\left(g, s, \pi_{o}\right) \otimes[\overleftarrow{s-2}, \overrightarrow{1}]_{\pi_{o}}\right) \otimes L_{g}\left(\pi_{o}\right)\left(-\frac{s(g+1)-4}{2}\right)
\end{aligned}
$$

de sorte que $P_{\pi_{o}, s-1,1}\left([\overleftarrow{s-2}]_{\pi_{o}}\right)$ contient $\mathcal{F}\left(g, s, \pi_{o}\right)\left(-\frac{g-1}{2}\right) \otimes[\overleftarrow{s-2}, \overrightarrow{1}]_{\pi_{o}}$ et donc, vu que les strates sont induites, $\mathcal{F}\left(g, s, \pi_{o}\right)\left(-\frac{g-1}{2}\right) \otimes[\overleftarrow{s-2}]_{\pi_{o}} \overrightarrow{\times}[\overleftarrow{0}]_{\pi_{o}}$. Par ailleurs d'après (IV.2.2.14), on a le lemme suivant

IV.5.4.7. Lemme. - Pour toute représentation $\Pi_{s}$ de $G L_{d}\left(F_{o}\right)$, on a

$$
\underset{\vec{I}}{\lim _{\vec{I}}} H^{0}\left(M_{I, s_{o}}^{d}, \mathcal{F}\left(g, s, \pi_{o}, I\right) \otimes \mathcal{L}_{\rho_{\infty}} \otimes \Pi_{s}\right)=e_{\pi_{o}} \mathcal{C} \bar{D}_{, \rho_{\infty}}\left[\mathrm{JL}^{-1}\left(\overleftarrow{(s)}_{\pi_{o}}\right)\right] \otimes \Pi_{s}
$$

de sorte que sa partie $\bar{\Pi}^{\infty, o}$-isotypique est $e_{\pi_{o}} m(\bar{\Pi}) \Pi_{s}$.

En appliquant ce lemme à $\Pi_{s}=\Pi_{s-1} \overrightarrow{\times}[\overleftarrow{0}]_{\pi_{o}}$, et en utilisant les propriétés imposées à $\Pi$, on obtient que la partie $\bar{\Pi}^{\infty, o}$-isotypique de $\lim _{\rightarrow} H^{0}\left(M_{I, s_{o}}^{d}, \mathcal{F}\left(g, s, \pi_{o}\right) \otimes \Pi_{s-1} \overrightarrow{\times}[\overleftarrow{0}]_{\pi_{o}}\right)$ est égale à la partie $\Pi^{\infty, o}$-isotypique de $\lim _{\vec{I}} H_{c}^{0}\left(M_{I, s_{o}}^{=(s-1) g}, H T\left(g, s-1, \pi_{o}, \Pi_{s-1}\right)[g]\right.$ qui d'après ce qui précède est égale à celle de $\lim _{\rightarrow} H^{0}\left(M_{I, s_{o}}, P_{\pi_{o}, s-1,1}\left(\Pi_{s-1}\right)\right)$. On en déduit donc que $P_{\pi_{o}, s-1,1}\left(\Pi_{s-1}\right)$ est égal à $\mathcal{F}\left(g, s, \pi_{o}\right)\left(-\frac{g-1}{2}\right) \otimes \Pi_{s-1} \overrightarrow{\times}[\overleftarrow{0}]_{\pi_{o}}$

On raisonne alors par récurrence sur $l$ de $s-1$ à 1 , en supposant que pour tout $l>l_{0}$, $P_{\pi_{o}, l, s-l-1}\left(\Pi_{l}\right)$ est isomorphe à $\mathcal{F}\left(g, s, \pi_{o}\right)\left(-\frac{(s-l)(g-1)}{2}\right) \otimes \Pi_{l} \overrightarrow{\times}[\overleftarrow{s-l-1}]_{\pi_{o}}$. On regarde alors les 
faisceaux pervers de dimension nulle et de poids $s(g+1)-2\left(s-l_{0}+1\right)$ dans l'égalité (IV.5.2.18), soit

$$
\begin{aligned}
P_{\pi_{o}, l_{0}, s-l_{0}-1}\left(\left[\overleftarrow{l_{0}-1}\right]_{\pi_{o}}\right) \otimes\left(-\frac{l_{0}(g+1)-2}{2}\right)+\mathcal{F}\left(g, s, \pi_{o}\right) \otimes & \\
& \left(\sum_{i=1}^{s-l_{0}}(-1)^{i}\left[\overleftarrow{l_{0}-1}, \vec{i}\right]_{\pi_{o}} \overrightarrow{\times}\left[\overleftarrow{s-l_{0}-i-2}\right]_{\pi_{o}}\right) \otimes L_{g}\left(\pi_{o}\right)\left(-\frac{s(g+1)-2\left(s-l_{0}+1\right)}{2}\right)
\end{aligned}
$$

ce qui donne

$$
P_{\pi_{o}, l_{0}, s-l_{0}-1}\left(\left[\overleftarrow{l_{0}-1}\right]_{\pi_{o}}\right)-\mathcal{F}\left(g, s, \pi_{o}\right) \otimes\left[\overleftarrow{l_{0}-1}, \overrightarrow{1}, \overleftarrow{s-l_{0}-1}\right]_{\pi_{o}} \otimes L_{g}\left(\pi_{o}\right)\left(-\frac{\left(s-l_{0}\right)(g-1)}{2}\right)
$$

positif. On en déduit alors que $P_{\pi_{o}, l_{0}, s-l_{0}-1}\left(\left[\overleftarrow{l_{0}-1}\right]_{\pi_{o}}\right)$ contient

$$
\mathcal{F}\left(g, s, \pi_{o}\right)\left(-\frac{\left(s-l_{0}\right)(g-1)}{2}\right) \otimes\left[\overleftarrow{l_{0}-1}, \overrightarrow{1}, \overleftarrow{s-l_{0}-1}\right]_{\pi_{o}}
$$

et donc vu le caractère induit des strates, $P_{\pi_{o}, l_{0}, s-l_{0}-1}\left(\Pi_{l_{0}}\right)$ contient

$$
\mathcal{F}\left(g, s, \pi_{o}\right)\left(-\frac{\left(s-l_{0}\right)(g-1)}{2}\right) \otimes \Pi_{l_{0}} \overrightarrow{\times}\left[\overleftarrow{s-l_{0}-1}\right]_{\pi_{o}} .
$$

L'égalité provient alors de l'égalité des $H^{0}$ comme dans le cas $l_{0}=s-1$, traité ci-avant, d'où le résultat.

IV.5.4.8. Corollaire. - Le théorème (IV.4.4.1) est vrai.

Démonstration. - En effet le résultat découle directement de la proposition (IV.5.2.2) en réinjectant les égalités de la proposition (IV.5.3.3) où les $P_{!, l}$ sont nuls d'après le corollaire (IV.5.4.6). On obtient ainsi pour tout $1 \leqslant i \leqslant s_{g}$ :

$$
\begin{gathered}
\sum_{l=i}^{s_{g}}(-1)^{l-i} j_{!}^{\geqslant l g} H T\left(g, l, \pi_{o},[\overleftarrow{i-1}, \overrightarrow{l-i}]_{\pi_{o}}\right)[d-l g] \otimes L_{g}\left(\pi_{o}\right)\left(-\frac{l g-2+2 i-l}{2}\right)= \\
\sum_{l=i}^{s_{g}}(-1)^{l-i} \sum_{r=0}^{s_{g}-l} j_{! *}^{\geqslant(l+r) g} H T\left(g, l+r, \pi_{o},[\overleftarrow{i-1}, \overleftrightarrow{l-i}]_{\pi_{o}} \overrightarrow{\times}[\overleftarrow{r-1}]_{\pi_{o}}\right)[d-(l+r) g] \\
\otimes L_{g}\left(\pi_{o}\right)\left(-\frac{(l+r)(g-1)+2(i-1)}{2}\right)
\end{gathered}
$$

laquelle somme est alors égale à

$$
\sum_{l=i}^{s_{g}} j_{! *}^{\geqslant l g} H T\left(g, l, \pi_{o}, \Pi_{l, i}\right)[d-l g] \otimes L_{g}\left(\pi_{o}\right)\left(-\frac{l(g-1)+2(i-1)}{2}\right)
$$

où

avec

$$
\Pi_{l, i}:=\sum_{r=i}^{l}(-1)^{r-i}[\overleftarrow{\imath-1}, \overrightarrow{r-i}]_{\pi_{o}} \vec{x}[\overleftarrow{l-r-1}]_{\pi_{o}}=[\overleftarrow{l-1}]_{\pi_{o}}
$$

$$
\begin{aligned}
& e_{\pi_{o}}\left[R \Psi_{\eta_{o}, \pi_{o}}\left(\overline{\mathbb{Q}}_{l}\right)[d-1]\right]=\sum_{i=1}^{s_{g}} \sum_{l=i}^{s_{g}} j_{! *}^{\geqslant l g} H T\left(g, l, \pi_{o}, \Pi_{l, i}\right)[d-l g] \otimes \\
& L_{g}\left(\pi_{o}\right)\left(-\frac{l(g-1)+2(i-1)}{2}\right) \\
& =\sum_{l=1}^{s_{g}} j_{! *}^{\geqslant l g} H T\left(g, l, \pi_{o},[\overleftarrow{l-1}]_{\pi_{o}}\right) \otimes \\
& L_{g}\left(\pi_{o}\right)\left(-\frac{l g-1}{2}\right)\left(\sum_{\substack{k \equiv l-1 \mid \bmod 2 \\
k=l}}\left(-\frac{k}{2}\right)\right.
\end{aligned}
$$

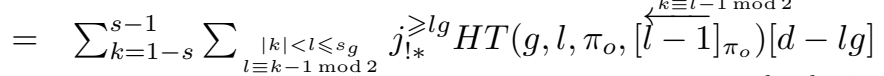

$$
\begin{aligned}
& \otimes L_{g}\left(\pi_{o}\right)\left(-\frac{l g+k-1}{2}\right)
\end{aligned}
$$

d'où le résultat. 
IV.5.4.9. Corollaire. - Pour tout $g \mid d=s g$, et $\pi_{o}$ une représentation cuspidale de $G L_{g}\left(F_{o}\right)$, on considère les groupes de cohomologie $H_{c}^{j}\left(M_{\bar{I}, \bar{s}_{o}}^{=h}, R^{i} \Psi_{\eta_{o}, I, \pi_{o}}\left(\mathcal{L}_{\rho_{\infty}}\right)\right)$ ainsi que leur limite inductive sur tous les idéaux $I$ de $A$, limite que l'on notera $H_{=h, i, \pi_{o}, \rho_{\infty}}^{j}$. Pour $\Pi$ une représentation automorphe de $D_{\mathbb{A}}^{\times}$vérifiant $\operatorname{Hyp}\left(\rho_{\infty}\right)$, on a les résultats suivant:

(1) pour $g$ ne divisant pas $d$, les $H_{=h, i, \pi_{o}, \rho_{\infty}}^{j}\left[\Pi^{\infty, o}\right]$ sont nuls pour tous $j, h, i$;

(2) pour $g$ divisant $d=s g$ et $\Pi_{o} \simeq[\stackrel{\leftrightarrow}{s-1}]_{\pi_{o}}$, les $H_{=h, i, \pi_{o}, \rho_{\infty}}\left[\Pi^{\infty, o}\right]$ sont nuls pour $h$ qui n'est pas de la forme $l g$ avec $1 \leqslant l \leqslant s$;

(3) pour $g$ divisant $d=s g$ et $\Pi_{o} \simeq[\overleftarrow{s-1}]_{\pi_{o}}$, les $H_{=l g, i, \pi_{o}, \rho_{\infty}}^{j}\left[\Pi^{\infty, o}\right]$ sont nuls pour $j \neq d-l g$;

(4) pour $g$ divisant $d=s g$ et $\Pi_{o} \simeq[\overleftarrow{s-1}]_{\pi_{o}}$, les $H_{=l g, i, \pi_{o}, \rho_{\infty}}^{d-l g}\left[\Pi^{\infty, o}\right]$ sont nuls pour $i$ ne vérifiant pas $l(g-1) \leqslant i \leqslant l g-1$. Si $1 \leqslant l<s$ et $i=l g-r$ avec $1 \leqslant r \leqslant l, H_{=l g, l g-r, \pi_{o}, \rho_{\infty}}^{d-l g}\left[\Pi^{\infty, o}\right]$ est isomorphe $\grave{a}$

$$
m(\Pi)\left([\overleftarrow{l-r}, \overrightarrow{r-1}]_{\pi_{o}} \overrightarrow{\times}[\overleftarrow{s-l-1}]_{\pi_{o}}\right) \otimes L_{g}\left(\pi_{o}\right)\left(-\frac{s(g-1)-2(r-l)}{2}\right)
$$

en tant que représentation de $G L_{d}\left(F_{o}\right) \times W_{o}$, où $m(\Pi)$ est la multiplicité de $\Pi$ dans l'espace des formes automorphes.

Démonstration. - On rappelle (cf. la proposition (IV.2.2.1)) que pour tout $i, j$, on a un isomorphisme canonique

$$
H_{c}^{j}\left(M_{\bar{I}, s_{o}, 1}^{=h}, R^{i} \Psi_{\eta_{o}}\left(\mathcal{L}_{\rho_{\infty}}\right)\right)^{h} \simeq \bigoplus_{\tau_{o} \in \mathfrak{C}_{h}}\left(H_{c}^{j}\left(M_{\bar{I}, s_{o}, 1}^{=h}, \mathcal{F}_{\tau_{o}} \otimes \mathcal{L}_{\rho_{\infty}}\right) \otimes \widetilde{\mathcal{U}_{F_{o}, m}^{h, i}\left(\tau_{o}\right)}\right)^{h / e_{\tau_{o}}}
$$

de sorte que les résultats découle directement du corollaire (IV.5.4.3).

\section{IV.6. Faisceaux de cohomologie des $j_{!_{*}}^{\geqslant l g} \mathcal{F}\left(g, l, \pi_{o}\right)$}

Rappelons, cf §IV.4.5, que d'après le théorème de comparaison de Berkovich-Fargues, la filtration de monodromie-locale du complexe $\Psi_{F_{o}}^{d}$ sera donnée par les germes en un point supersinguliers des faisceaux de cohomologie des $g r_{k}$. On propose alors de calculer tous les faisceaux de cohomologie des $g r_{k}$. D'après la proposition (IV.5.3.3) précisée par le corollaire (IV.5.4.6), il nous suffit de déterminer les faisceaux de cohomologie des $j_{!_{*}}^{>l g} \mathcal{F}\left(g, l, \pi_{o}\right)[d-l g]$. Nous verrons que cela ne pose aucun problème en dehors des points supersinguliers car on peut utiliser l'hypothèse de récurrence dans le théorème (IV.3.2.3). Au niveau des points supersinguliers on ne dispose d'aucun renseignement local, cependant la proposition (IV.5.3.3) nous permet de restreindre efficacement les possibilités pour les germes en un point supersingulier de ces faisceaux de cohomologie.

IV.6.1. Une écriture dans $\mathfrak{G}$ de $j_{! *}^{\geqslant l g} \mathcal{F}\left(g, l, \pi_{o}\right)[d-l g]$. -

IV.6.1.1. Lemme. - Pour tout $1 \leqslant l \leqslant s_{g}$, on a l'égalité dans $\mathfrak{G}$ :

$$
\begin{aligned}
\mathcal{P}\left(g, l, \pi_{o}\right)=\sum_{r=0}^{s_{g}-l}(-1)^{r} j_{!}^{\geqslant(l+r) g} H T\left(g, l+r, \pi_{o},[\overleftarrow{l-1}]_{\pi_{o}} \overrightarrow{\times}[\overrightarrow{r-1}]_{\pi_{o}}\right)[d-(l+r) g] & \\
& \otimes L_{g}\left(\pi_{o}\right)\left(-\frac{r(g-1)}{2}\right)
\end{aligned}
$$

Démonstration. - On démontre le résultat par récurrence sur $l$ de $s_{g}$ à 1 , en utilisant les corollaires (IV.5.3.12) et (IV.5.4.6). Le cas $l=s_{g}$ est directement donné par loc. cit. Supposons le résultat 
acquis jusqu'au rang $l+1$ et traitons le cas de $l$. D'après loc. cit. on a

$$
\begin{aligned}
& j_{!}^{\geqslant l g} H T\left(g, l, \pi_{o},[\overleftarrow{l-1}]_{\pi_{o}}\right)[d-l g] \otimes L_{g}\left(\pi_{o}\right)=\mathcal{P}\left(g, l, \pi_{o}\right) \\
& \quad+\sum_{i=1}^{s-l} j_{! *}^{\geqslant(l+i) g} H T\left(g, l+i, \pi_{o},[\overleftarrow{l-1}]_{\pi_{o}} \overrightarrow{\times}[\overleftarrow{i-1}]_{\pi_{o}}\right)[d-(l+i) g] \otimes L_{g}\left(\pi_{o}\right)\left(-\frac{i g-i}{2}\right)
\end{aligned}
$$

D'après l'hypothèse de récurrence on a

$$
\begin{array}{r}
j_{! *}^{\geqslant(l+i) g} H T\left(g, l+i, \pi_{o},[\overleftarrow{l-1}]_{\pi_{o}} \overrightarrow{\times}[\overleftarrow{i-1}]_{\pi_{o}}\right)[d-(l+i) g] \otimes L_{g}\left(\pi_{o}\right)\left(-\frac{i g-i}{2}\right)= \\
\sum_{r=0}^{s-l-i}(-1)^{r} j_{!}^{\geqslant(l+i+r) g} H T\left(g, l+i+r, \pi_{o},[\overleftarrow{l-1}]_{\pi_{o}} \overrightarrow{\times}[\overleftarrow{i-1}]_{\pi_{o}} \overrightarrow{\times}[\overrightarrow{r-1}]_{\pi_{o}}\right)[d-(l+i+r) g] \\
\otimes L_{g}\left(\pi_{o}\right)\left(-\frac{(i+r) g-i-r}{2}\right)
\end{array}
$$

de sorte que (IV.6.1.19) s'écrit

$$
\begin{aligned}
\mathcal{P}\left(g, l, \pi_{o}\right)- & j_{!}^{\geqslant l g} H T\left(g, l, \pi_{o},[\overleftarrow{l-1}]_{\pi_{o}}\right)[d-l g] \otimes L_{g}\left(\pi_{o}\right)= \\
& \sum_{r=1}^{s-l}(-1)^{r} j_{!}^{\geqslant(l+r) g} H T\left(g, l+r, \pi_{o},[\overleftarrow{l-1}]_{\pi_{o}} \overrightarrow{\times} \Pi_{r}\right)[d-(l+r) g] \otimes L_{g}\left(\pi_{o}\right)\left(-\frac{(r g-r}{2}\right)
\end{aligned}
$$

où $\Pi_{r}=(-1)^{r-1}[\overleftarrow{r-1}]_{\pi_{o}}+\sum_{i=1}^{r-1}(-1)^{i-1}[\overleftarrow{i-1}]_{\pi_{o}} \overrightarrow{\times}[\overrightarrow{r-i-1}]_{\pi_{o}}=[\overrightarrow{r-1}]_{\pi_{o}}$ d'où le résultat.

IV.6.2. Filtration de monodromie-locale en hauteur non maximale. - On rappelle que d'après l'hypothèse de récurrence, le théorème (IV.3.2.3) est connu pour les modèles locaux de Deligne-Carayol de hauteur strictement inférieure à $d$. Ainsi l'aboutissement de la suite spectrale (IV.4.1.17) est connue en dehors des points supersinguliers tandis qu'on ne connaît que les germes en des points non supersinguliers des termes $E_{1}$ (cf. le théorème-définition (IV.3.2.1)).

IV.6.2.1. Proposition. - Pour $g \neq 1$ et $1 \leqslant l \leqslant s_{g}$, les faisceaux de cohomologie $h^{i} \mathcal{P}\left(g, l, \pi_{o}\right)$ sont nuls pour tout $i<l-s_{g}$ et $i$ qui n'est pas de la forme $l g-d+a(g-1)$. Pour $i=l g-d+a(g-1)$ avec $0 \leqslant a<s_{g}-l$, ils sont égaux dans $\mathrm{FH}\left(M_{s_{o}}\right) \grave{a}$

$$
j_{!}^{\geqslant(l+a) g} H T\left(g, l+a, \pi_{o},[\overleftarrow{l-1}]_{\pi_{o}} \overrightarrow{\times}[\overrightarrow{a-1}]_{\pi_{o}}\right) \otimes L_{g}\left(\pi_{o}\right)\left(-\frac{a(g-1)}{2}\right) .
$$

Démonstration. - Pour $i<l g-d$, les $h^{i} \mathcal{P}\left(g, l, \pi_{o}\right)$ sont tous nuls car $\mathcal{P}\left(g, l, \pi_{o}\right)$ est de dimension $d-l g$. On a la suite exacte courte de faisceaux pervers

$$
0 \rightarrow P_{\pi_{o}, l, 0}\left(\pi_{o}\right) \otimes L_{g}\left(\pi_{o}\right) \longrightarrow j_{!}^{\geqslant g} \mathcal{F}\left(g, l, \pi_{o}, \pi_{o}\right)[d-l g] \otimes L_{g}\left(\pi_{o}\right) \longrightarrow \mathcal{P}\left(g, l, \pi_{o}\right) \rightarrow 0
$$

où $P_{\pi_{o}, 1,0}\left(\pi_{o}\right)$ est un faisceau pervers de dimension $d-(l+1) g$ de sorte que

$$
h^{l g-d} j_{! *}^{\geqslant g} \mathcal{F}\left(g, 1, \pi_{o}\right)[d-g]=j_{!}^{\geqslant g} \mathcal{F}\left(g, 1, \pi_{o}\right) .
$$

Le principe est alors d'utiliser le théorème de comparaison de Berkovich-Fargues couplé avec le lemme (IV.6.1.1).

Remarquons tout d'abord que d'après le corollaire (IV.5.3.13), pour tout $k, g r_{k, \pi_{o}}$ est pur hors des points supersinguliers, de sorte qu'en ce qui concerne les strates non supersingulières, on a

$$
e_{\pi_{o}} h^{i} g r_{k, \pi_{o}}=\bigoplus_{\substack{|k|<l \leqslant s g \\ l \equiv k-1 \bmod 2}} h^{i} \mathcal{P}\left(g, l, \pi_{o}\right)\left(-\frac{l g-1+k}{2}\right)
$$

Ainsi d'après le corollaire (IV.5.3.2), on en déduit le lemme suivant.

IV.6.2.2. Lemme. — Les $h^{i} \mathcal{P}\left(g, l, \pi_{o}\right)$ vérifient les propriétés suivantes : 
- hormis les points supersinguliers, ils sont à support dans les strates $M_{I, s_{o}}^{=l^{\prime} g}$ pour $l \leqslant l^{\prime} \leqslant s_{g}$ avec $l^{\prime} g-d-l^{\prime}+l-1^{\prime} \leqslant i \leqslant l^{\prime} g-d$ et $i \equiv l^{\prime} g-d-l^{\prime}+l-1 \bmod 2$;

- pour $i=l^{\prime} g-d-l^{\prime}+l-1+2 r$, la fibre en un point géométrique de $M_{I, s_{o}}^{=l^{\prime} g}$ de $h^{i} \mathcal{P}\left(g, l, \pi_{o}\right)$ est un facteur direct de

$$
[\overleftarrow{l+2 r-1}]_{\pi_{o}} \overrightarrow{\times}\left[\overline{l^{\prime}-l-2 r-1}\right]_{\pi_{o}} \otimes L_{g}\left(\pi_{o}\right)\left(-\frac{l^{\prime}(g-1)+2(l-1)+2 r}{2}\right)
$$

Soit $z$ un point géométrique de $M_{I, s_{o}}^{=l^{\prime} g}$. Les strates étant induites, on en déduit que la fibre en $z$ de $h^{i} \mathcal{P}\left(g, l, \pi_{o}\right)\left(-\frac{l(g-1)}{2}\right)$ est de la forme :

$$
\bigoplus_{\xi}\left([\overleftarrow{l-1}]_{\pi_{o} \circ \xi\left(-\left(l^{\prime}-l\right)(g-1) / 2\right)} \overrightarrow{\times} \pi_{\xi}\right) \otimes L_{g}\left(\pi_{o} \circ \xi\right)\left(-\frac{l(g-1)}{2}\right)
$$

où $\pi_{\xi}$ est une représentation de $G L_{\left(l^{\prime}-l\right) g}\left(F_{o}\right)$. Pour $\xi=\Xi^{\left(\left(l^{\prime}-l\right)(g-1)+2 r\right) / 2}$ avec $r>0$, on remarque que si $[\overleftarrow{l-1}]_{\pi_{o}(r)} \overrightarrow{\times} \pi_{\xi}$ contient un des deux constituants de

$$
[\overleftarrow{l+2 r-1}]_{\pi_{o}} \overrightarrow{\times}\left[\overrightarrow{l^{\prime}-l-2 r-1}\right]_{\pi_{o}}
$$

alors il contient aussi tous les constituants de

$$
\left([\overleftarrow{l-1}]_{\pi_{o}} \overrightarrow{\times}\left[\overline{l^{\prime}-l-2 r-1}\right]_{\pi_{o}}\right) \overleftarrow{\times}[\overleftarrow{2 r-1}]_{\pi_{o}}
$$

alors que, par exemple $\left[\overleftarrow{2 r-1}, \overrightarrow{1}, \overleftarrow{l-1}, \overrightarrow{l^{\prime}-l-2 r}\right]_{\pi_{o}} \otimes L_{g}\left(\pi_{o}\right)\left(-\frac{l^{\prime}(g-1)+2 r}{2}\right)$ n'est pas un constituant de $e_{\pi_{o}} h^{l^{\prime} g-d-l^{\prime}+l-1+2 r} g r_{1-l, \pi_{o}}$ d'où la contradiction.

On en déduit ainsi que $h^{l^{\prime} g-d-l^{\prime}+l-1} \mathcal{P}\left(g, l, \pi_{o}\right)$ est le seul faisceau de cohomologie ayant un support d'intersection non vide avec la strate $l^{\prime} g$. Le raisonnement étant valide pour tout $l^{\prime}$, on en déduit aussi que le support de $h^{l^{\prime} g-d-l^{\prime}+l-1} \mathcal{P}\left(g, l, \pi_{o}\right)$ est contenu dans la strate $l^{\prime} g$. Le lemme (IV.6.1.1) donne alors que la restriction à la strate $l^{\prime} g$ de $h^{l^{\prime} g-d-l^{\prime}+l-1} \mathcal{P}\left(g, l, \pi_{o}\right)$ est isomorphe à $H T\left(g, l^{\prime}, \pi_{o},[\overleftarrow{l-1}]_{\pi_{o}} \overrightarrow{\times}\left[\overrightarrow{l^{\prime}-l-1}\right]_{\pi_{o}}\right) \otimes L_{g}\left(\pi_{o}\right)\left(-\frac{\left(l^{\prime}-l\right)(g-1)}{2}\right)$, de sorte que

$$
\begin{aligned}
h^{l^{\prime} g-d-l^{\prime}+l-1} \mathcal{P}\left(g, l, \pi_{o}\right) \simeq j_{!}^{\geqslant l^{\prime} g} H T\left(g, l^{\prime}, \pi_{o},[\overleftarrow{l-1}]_{\pi_{o}} \overrightarrow{\times}\left[\overrightarrow{l^{\prime}-l-1}\right]_{\pi_{o}}\right) & \\
& \otimes L_{g}\left(\pi_{o}\right)\left(-\frac{\left(l^{\prime}-l\right)(g-1)}{2}\right) .
\end{aligned}
$$

IV.6.2.3. Proposition. - Pour $g=1$, les $h^{i} \mathcal{P}\left(1, l, \xi_{o}\right)$, sont nuls pour $i \neq l-d$ et pour $i=$ $l-d$ leur restriction à $M_{I, s_{o}}^{=l+r}$, pour $0 \leqslant r<d-l$ est égale dans $\mathrm{FH}\left(M_{s_{o}}^{=l+r}\right) \dot{a} H T(1, l+$ $\left.r, \xi_{o},[\overleftarrow{l-1}]_{\xi_{o}} \overrightarrow{\times}[\overrightarrow{r-1}]_{\xi_{o}}\right)$.

Démonstration. — Elle est strictement identique à celle pour $g \neq 1$, en considérant à chaque étape les restrictions aux strates $M_{I, s_{o}}^{=l+r}$.

IV.6.2.4. Proposition. - Pourg ne divisant pas $d, h^{s_{g} g-d-\left(s_{g}-l\right)} \mathcal{P}\left(g, l, \pi_{o}\right)$ est, dans $\mathrm{FH}\left(M_{s_{o}}\right)$, isomorphe ̀̀

$$
j_{!}^{\geqslant s_{g} g} H T\left(g, s_{g}, \pi_{o},\left([\overleftarrow{l-1}]_{\pi_{o}} \overrightarrow{\times}\left[\overrightarrow{s_{g}-l-1}\right]_{\pi_{o}}\right)\right) \otimes L_{g}\left(\pi_{o}\right)\left(-\frac{\left(s_{g}-l\right)(g-1)}{2}\right) .
$$

Par ailleurs les $h^{i} \mathcal{P}\left(g, l, \pi_{o}\right)$ sont nuls pour $s_{g} g-d-\left(s_{g}-l\right)<i \leqslant 0$.

Démonstration. - Tant qu'on est en dehors des points supersinguliers, les arguments précédents s'adaptent, en utilisant le théorème de comparaison de Berkovich-Fargues avec la connaissance de la filtration de monodromie-locale des modèles de Deligne-Carayol en hauteur strictement inférieure 
à $d$. En ce qui concerne les points supersinguliers, on raisonne par récurrence sur $l$ de $s_{g}$ à 1 . Pour $l=s_{g}$, le corollaire (IV.5.3.12), joint au corollaire (IV.5.4.6), donne

$$
\mathcal{P}\left(g, s_{g}, \pi_{o}\right)=j_{!}^{\geqslant s_{g} g} H T\left(g, s_{g}, \pi_{o},\left[\overleftarrow{s_{g}-1}\right]_{\pi_{o}}\right)\left[d-s_{g} g\right] \otimes L_{g}\left(\pi_{o}\right)
$$

d'où le résultat. Supposons donc le résultat acquis jusqu'au rang $l+1$ et traitons le cas de $l$. On considère les suites exactes courtes du corollaire (IV.5.3.12), où l'on rappelle que d'après (IV.5.4.6), les $A_{\pi_{o}, l, g}$ sont nuls :

$$
\begin{aligned}
& 0 \rightarrow P_{\pi_{o}, l, 0}\left([\overleftarrow{l-1}]_{\pi_{o}}\right) \longrightarrow j_{!}^{\geqslant l g} H T\left(g, l, \pi_{o},[\overleftarrow{l-1}]_{\pi_{o}}\right)[d-l g] \longrightarrow \\
& j_{! *}^{\geqslant l g} H T\left(g, l, \pi_{o},[\overleftarrow{l-1}]_{\pi_{o}}\right)[d-l g] \rightarrow 0 \\
& 0 \rightarrow P_{\pi_{o}, l, s_{g}-l}\left([\overleftarrow{l-1}]_{\pi_{o}}\right) \longrightarrow P_{\pi_{o}, l, s_{g}-l-1}\left([\overleftarrow{l-1}]_{\pi_{o}}\right) \longrightarrow \mathcal{P}_{-}\left(g, s_{g}, \pi_{o}, l,[\overleftarrow{l-1}]_{\pi_{o}}\right) \rightarrow 0
\end{aligned}
$$

où $P_{\pi_{o}, l, s_{g}-l}\left([\overleftarrow{l-1}]_{\pi_{o}}\right)$ est le faisceau pervers nul. On démontre alors par récurrence sur $r$, de $s_{g}-l$ à 0 , que le germe en un point supersingulier de $h^{i} P_{\pi_{o}, l, r}\left([\overleftarrow{l-1}]_{\pi_{o}}\right)$ est nul pour tout $i$, d'où le résultat.

En direction du théorème (IV.4.4.7), on a le résultat suivant.

IV.6.2.5. Lemme. - Pour $g \neq 1$ divisant $d=s g$ (resp. $g=1$ ), la fibre en un point supersingulier de $h^{i} \mathcal{P}\left(g, l, \pi_{o}\right)$ (resp. de $h^{i} \mathcal{P}\left(1, l, \xi_{o}\right)$ ) est nulle pour $i<l-s$. Par ailleurs les $h^{l-s+i} \mathcal{P}\left(g, l, \pi_{o}\right)$ sont concentrés aux points supersinguliers pour $0 \leqslant i<s-l$, de fibre (resp. la fibre en un point supersingulier de $h^{l-s+i} \mathcal{P}\left(1, l, \xi_{o}\right)$ est), en tant que $G L_{d}\left(F_{o}\right) \times W_{o}$-module, un sous-espace, éventuellement nul, de

$$
[\overleftarrow{l-1}]_{\pi_{o}} \overrightarrow{\times}[\overrightarrow{s-l-2-i}]_{\pi_{o}} \overrightarrow{\times}[\overleftarrow{i}]_{\pi_{o}} \otimes L_{g}\left(\pi_{o}\right)\left(-\frac{(s-l)(g-1)}{2}\right) .
$$

Démonstration. - Nous ne traitons que le cas $g \neq 1$, le raisonnement pour $g=1$ étant strictement identique. On raisonne par récurrence sur $l$ de $s$ à 1 , le cas $l=s$ étant trivial. Supposons donc le résultat acquis jusqu'au rang $l+1$ et traitons le cas de $l$. On considère alors les suites exactes courtes de faisceaux pervers de (IV.5.3.12). On remarque tout d'abord que toutes les fibres en un point supersingulier des faisceaux de cohomologie des $\mathcal{P}_{-}\left(g, l+r, \pi_{o}, l, \Pi_{l}\right)$ sont de poids $(s-l)(g-1)$ de sorte qu'il en est de même pour les $P_{\pi_{o}, l, r}\left(\Pi_{l}\right)$ et donc pour $\mathcal{P}\left(g, l, \pi_{o}\right)$. Le résultat découle alors de l'étude des suites exactes longues associées. Une façon plus visuelle et plus directe d'obtenir le résultat est de considérer l'isomorphisme $D j_{! *}^{\geqslant l g} \mathcal{F}\left(g, l, \pi_{o}\right)[d-l g] \simeq j_{!_{*}}^{\geqslant l g} \mathcal{F}\left(g, l, \pi_{o}^{\vee}\right)[d-l g](d-l g)$ et de regarder la suite spectrale

$$
E_{2}^{p, q}=R^{p} \operatorname{Hom}\left(h^{-q} \mathcal{P}\left(g, l, \pi_{o}\right), K_{s_{o}}\right) \Rightarrow h^{p+q} \mathcal{P}\left(g, l, \pi_{o}^{\vee}\right)(d-1)
$$

où $K_{s_{o}}$ désigne le complexe dualisant sur $M_{I, s_{o}}{ }^{(18)}$

On rappelle que pour un faisceau $\mathcal{L}$ sur $M_{I, s_{o}}^{=(l+r) g}$, par adjonction on a

$$
R H \operatorname{Hom}\left(i_{*}^{(l+r) g} j_{!}^{\geqslant(l+r) g} \mathcal{L}, f^{!} \overline{\mathbb{Q}}_{l}\right) \simeq i_{*}^{(l+r) g} R j_{*}^{\geqslant(l+r) g} R H \operatorname{Hom}\left(\mathcal{L}, j^{\geqslant(l+r) g, !} i^{(l+r) g, !} f^{!} \overline{\mathbb{Q}}_{l}\right)
$$

et comme $M_{I, s_{o}}^{=(l+r) g}$ est lisse, on a

$$
j \geqslant(l+r) g, ! i^{(l+r) g, !} f^{!} \overline{\mathbb{Q}}_{l} \simeq \overline{\mathbb{Q}}_{l}[2(d-(l+r) g)](d-(l+r) g)
$$

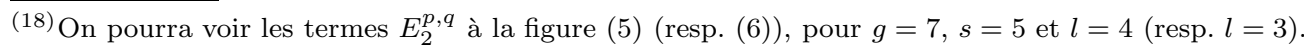


soit pour $p \geqslant-2(d-(l+r) g)$,

$$
\begin{aligned}
& R^{p} \operatorname{Hom}\left(j _ { ! } ^ { \geqslant ( l + r ) g } H T \left(g, l+a, \pi_{o},\right.\right. \\
& {\left.\left.[\overleftarrow{l-1}]_{\pi_{o}} \overrightarrow{\times}[\overleftarrow{a-1}]_{\pi_{o}}\right) \otimes|\mathrm{cl}|^{-a(g-1) / 2}, K_{s_{o}}\right) } \\
& \simeq\left(i _ { * } ^ { ( l + a ) g } R ^ { p + 2 ( d - ( l + a ) g ) } j _ { * } ^ { \geqslant ( l + a ) g } H T \left(g, l+a, \pi_{o}^{\vee},\right.\right. \\
& {\left.\left.[\overleftarrow{l-1}]_{\pi_{o}^{\vee}} \overleftarrow{\times}[\overleftarrow{a-1}]_{\pi_{o}^{\vee}}\right) \otimes|\mathrm{cl}|^{-a(g+1) / 2}\right)(l g-d) }
\end{aligned}
$$

Ainsi pour $z$ un point supersingulier, $\left(E_{2}^{p, q}\right)_{z}$, pour $q$ de la forme $d-l g-a(g-1)($ resp. $q=a+1)$ avec $0 \leqslant a<s-l$, et $p=-(s-l-a) g-i$ avec $0 \leqslant i \leqslant s-l-a$ (resp. $p=0)$, s'il est non nul, est mixte de poids $(s-l-a-r)(g-1) / 2+(a+r)(g+1) / 2$ avec $0 \leqslant r<s-l-a$ si $p<-(s-l-a) g$ et pur de poids $(s-l)(g+1) / 2$ si $p=(s-l-a) g$. Dans ce dernier cas on obtient alors

$$
[\overleftarrow{l-1}]_{\pi_{o}} \overrightarrow{\times}[\overrightarrow{s-l-a-1}]_{\pi_{o}} \overleftarrow{\times}[\overleftarrow{a-1}]_{\pi_{o}} \otimes|\mathrm{cl}|^{-(s-l)(g+1) / 2}
$$

(resp. $\left(h^{-q} j_{!_{*}}^{\geqslant l g} \mathcal{F}\left(g, l, \pi_{o}\right)_{0}\right)_{z}^{\vee}(-l g) \otimes|\mathrm{cl}|^{-(s-l)(g+1) / 2}$ où le dual est pris en tant que représentation de $\left.G L_{(s-l) g}\left(F_{o}\right)\right)$. Dans la figure $(2)$ on représente ces $\left(E_{2}^{p, q}\right)_{z}$ de poids $(s-l)(g+1)$. Le résultat découle alors du fait que les $E_{\infty}^{i}$ sont tous nuls pour $i \geqslant 0$.

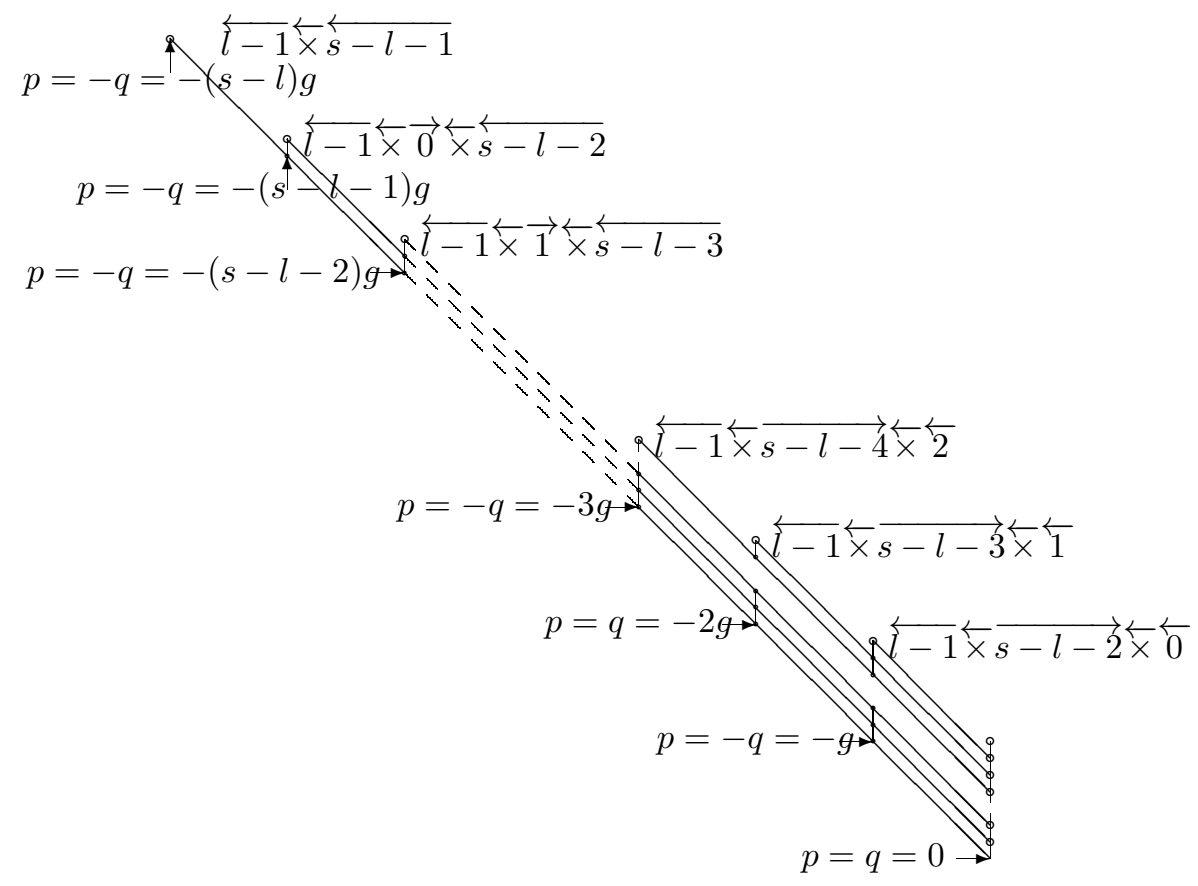

Figure 2. parties de poids $(s-l)(g+1)$ de la fibre aux points supersinguliers des $E_{2}^{p, q}$ de la suite spectrale (IV.6.2.20)

\section{IV.7. Preuve des théorèmes globaux sous IV.7.1.1}

On rappelle, cf. $\S$ IV.4.5, que le théorème (IV.3.2.3) découle, d'après le théorème de comparaison de Berkovich-Fargues, des théorèmes globaux (IV.4.4.4), (IV.4.4.7) et (IV.4.4.10). En outre le théorème (IV.3.2.3) implique, grâce à (IV.4.4.10) via Berkovich-Fargues, le théorème (IV.3.1.1). Par ailleurs le principe de la preuve de la proposition (IV.6.2.1) montre que (IV.3.2.3) implique les théorèmes globaux. Le but de ce paragraphe est de montrer que le théorème (IV.3.1.1) implique 
les théorèmes globaux (IV.4.4.4), (IV.4.4.7) et (IV.4.4.10) et donc le théorème local (IV.3.2.3). Par souci d'efficacité, on montrera, en utilisant l'opérateur $N$, qu'il nous suffit en fait de connaître les parties de poids $s(g-1)$ de (IV.3.1.1).

\section{IV.7.1. Preuve de (IV.4.4.7) sous (IV.7.1.1). -}

IV.7.1.1. Proposition. - Pour tout diviseur $g$ de $d=s g$ et $\pi_{o}$ une représentation irréductible cuspidale de $G L_{g}\left(F_{o}\right)$, la partie de poids $d-s$ de $\mathcal{U}_{F_{o}, \xi_{o}}^{d, d-s+i}\left(\mathrm{JL}^{-1}\left([\overleftarrow{s-1}]_{\pi_{o}}\right)\right)$ est nulle pour $0<i<s$ et égale $\grave{a}[\overrightarrow{s-1}]_{\pi_{o}} \otimes L_{g}\left(\pi_{o}\right)\left(-\frac{d-s}{2}\right)$ pour $i=0$.

La preuve de cette proposition sera donnée au paragraphe suivant; montrons comment le théorème (IV.3.2.3) en découle. On commence par prouver le théorème (IV.4.4.7) qui d'après la proposition (IV.6.2.1) découle alors de la proposition suivante.

IV.7.1.2. Proposition. - Sous le résultat de la proposition (IV.7.1.1), pour $g \neq 1$ divisant $d, h^{l-s+i} \mathcal{P}\left(g, l, \pi_{o}\right)$ est nul pour $i \neq 0$ et sinon est concentré aux points supersinguliers de fibre isomorphe $\grave{a}[\overleftarrow{l-1}]_{\pi_{o}} \overrightarrow{\times}[\overrightarrow{s-l-1}]_{\pi_{o}} \otimes L_{g}\left(\pi_{o}\right)\left(-\frac{(s-l)(g-1)}{2}\right)$ pour $i=0$.

Démonstration. - D'après le lemme (IV.6.2.5) tous les germes en un point supersingulier des $\mathcal{P}\left(g, l, \pi_{o}\right)$, sont de poids $(s-l)(g-1)$. Le raisonnement de la preuve de la proposition (IV.6.2.1) s'applique alors tel quel en étudiant les $\mathcal{P}\left(g, l, \pi_{o}\right)\left(-\frac{l(g-1)}{2}\right)$ pour $1 \leqslant l \leqslant s$ et en utilisant la connaissance des parties de poids $s(g-1)$ de (IV.3.2.3).

IV.7.2. Preuve de (IV.4.4.10) sous (IV.7.1.1). — On considère la suite spectrale (IV.4.1.17) associée à la filtration de monodromie. D'après la proposition précédente et le point (ii) de la proposition (IV.5.3.3), le germe en un point supersingulier $z$ de $E_{1}^{i, j}$ vérifie les propriétés suivantes (cf. la figure (1) dans le cas $s=4$ et $g=3$ ) :

- il est nul pour $i, j$ ne vérifiant pas la condition suivante : il existe $0 \leqslant k \leqslant s-1$ tel que $j=1-s+2 k$ et $-k \leqslant i \leqslant s-1-2 k$;

- pour $j=1-s+2 k$ avec $0 \leqslant k \leqslant s-1$, le germe en $z$ de $E_{1}^{s-1-2 k-r, 1-s+2 k}$ pour $0 \leqslant r \leqslant s-1-k$ est isomorphe à

$$
[\overleftarrow{s-r-1}]_{\pi_{o}} \overrightarrow{\times}[\overrightarrow{r-1}]_{\pi_{o}} \otimes L_{g}\left(\pi_{o}\right)\left(-\frac{s(g-1)+2 k}{2}\right) .
$$

En outre d'après (IV.7.1.1), pour $j=1-s$ et pour tout $0 \leqslant i<s-1$, l'application $d_{1}^{i, 1-s}$ : $E_{1}^{i, 1-s} \longrightarrow E_{1}^{i+1,1-s}$ induit en $z$ la flèche non triviale

$$
[\overleftarrow{i}]_{\pi_{o}} \overrightarrow{\times}[\overrightarrow{s-i-2}]_{\pi_{o}} \longrightarrow[\overleftarrow{i+1}]_{\pi_{o}} \overrightarrow{\times}[\overrightarrow{s-i-3}]_{\pi_{o}}
$$

dont le noyau est $[\overleftarrow{i}, \overrightarrow{s-i-1}]_{\pi_{o}}$ et le conoyau est $[\overleftarrow{i+2}, \overrightarrow{s-i-3}]_{\pi_{o}}$. Le logarithme $N^{k}$ de la partie unipotente de la monodromie induit un diagramme commutatif

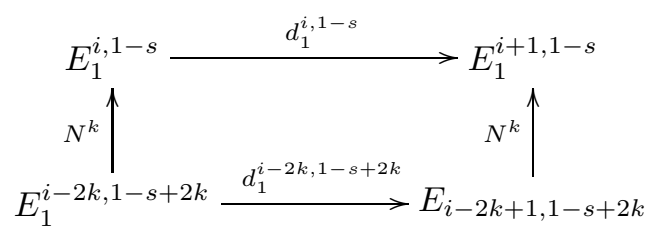

En remarquant que pour $0 \leqslant k \leqslant-i, N^{k}$ induit des isomorphismes $E_{1}^{i-2 k, 1-s+2 k} \simeq E_{1}^{i, 1-s}$ et $E_{1}^{i+1-2 k, 1-s+2 k} \simeq E_{1}^{i+1,16 s}$, on en déduit que la flèche $d_{1}^{i-2 k, 1-s+2 k}$ est aussi induite par la flèche non triviale

$$
[\overleftarrow{i}]_{\pi_{o}} \overrightarrow{\times}[\overrightarrow{s-i-2}]_{\pi_{o}} \longrightarrow[\overleftarrow{i+1}]_{\pi_{o}} \overrightarrow{\times}[\overrightarrow{s-i-1}]_{\pi_{o}}
$$


Finalement pour tout $1 \leqslant l^{\prime}<l \leqslant s$ et tout $k \equiv l^{\prime}-1 \bmod 2$ avec $|k| \leqslant l^{\prime}-1$, les flèches

$$
h^{-d+l g-\left(l-l^{\prime}\right)} g r_{k, \pi_{o}} \longrightarrow h^{-d+l g-\left(l-l^{\prime}-1\right)} g r_{k-1, \pi_{o}}
$$

de la suite spectrale (IV.4.1.17), sont non nulles, et se déduisent des suites exactes suivantes

$$
\begin{aligned}
0 \rightarrow\left[\overleftarrow{l^{\prime}-1}, \overrightarrow{l-l^{\prime}}\right]_{\pi_{o}} \rightarrow\left[\overleftarrow{l^{\prime}-1}\right]_{\pi_{o}} \overrightarrow{\times}\left[\overrightarrow{l-l^{\prime}-1}\right]_{\pi_{o}} \rightarrow & \\
& \rightarrow\left[\overleftarrow{l^{\prime}}\right]_{\pi_{o}} \overrightarrow{\times}\left[\overleftrightarrow{l-l^{\prime}-2}\right]_{\pi_{o}} \rightarrow\left[\overleftarrow{l^{\prime}+1}, \overrightarrow{l-l^{\prime}-2}\right]_{\pi_{o}} \rightarrow 0
\end{aligned}
$$

pour $l^{\prime}<l-1$ et pour $l^{\prime}=l-1$ de la suite exacte courte :

$$
0 \rightarrow[\overleftarrow{l-2}, \overrightarrow{1}]_{\pi_{o}} \rightarrow[\overleftarrow{l-2}]_{\pi_{o}} \overrightarrow{\times}[\overrightarrow{0}]_{\pi_{o}} \rightarrow[\overleftarrow{l-1}]_{\pi_{o}} \rightarrow 0
$$

d'où le résultat.

Remarque : On verra plus loin (cf. les remarques (IV.8.3.6) et (IV.8.4.1)) qu'on pourrait en fait montrer (IV.3.2.3) directement, sans utiliser l'opérateur de monodromie $N$.

IV.7.2.1. Corollaire. - Pour $0 \leqslant i \leqslant d-l g$ et $g>1, R^{i} j_{*}^{\geqslant l g} H T\left(g, l, \pi_{o}, \Pi_{l}\right)[d-l g]$ est, dans $\mathrm{FH}\left(M_{s_{o}}\right)$, une somme directe sur tous les couples $(n, r)$ tels que $n g+r(g-1)=i$ des faisceaux de type $H T(g, l+n+r)$

$$
j_{!}^{\geqslant(l+n+r) g} H T\left(g, l+n+r, \pi_{o},\left(\Pi_{l} \overrightarrow{\times}[\overleftarrow{n-1}]_{\pi_{o}}\right) \overleftarrow{\times}[\overrightarrow{r-1}]_{\pi_{o}}\right) \otimes \Xi^{\frac{n(g+1)+r(g-1)}{2}}
$$

Pour $g=1, R^{i} j_{*}^{\geqslant h} H T\left(1, h, \xi_{o}, \Pi_{h}\right)[d-h]$ est à support dans la tour des $M_{I, s_{o}}^{\geqslant h+i}$ et sa restriction $\grave{a} M_{I, s_{o}}^{=h+i+r}$ est le faisceau de type $H T(1, h+i+r)$ :

$$
H T\left(1, h+i+r, \xi_{o},\left(\Pi_{h} \overrightarrow{\times}[\overleftarrow{i-1}]_{\xi_{o}}\right) \overleftarrow{\times}[\overleftarrow{r-1}]_{\xi_{o}}\right) \otimes \Xi^{i}
$$

IV.7.3. Pureté de la filtration de monodromie de $R \Psi_{\eta_{o}}\left(\overline{\mathbb{Q}}_{l}\right)$. — La proposition suivante correspond au théorème (IV.4.4.4) qui rappelons le découle, en utilisant la pureté de la filtration de monodromie, du théorème (IV.4.4.1) qui est prouvé au corollaire (IV.5.4.8). Ce paragraphe n'a d'intérêt que par son application au cas de la caractéristique mixte.

IV.7.3.1. Proposition. - Pour tout $|k|<s_{g}, e_{\pi_{o}} g r_{k, \pi_{o}}$ est, dans $\operatorname{FPH}\left(M_{s_{o}}\right)$, égal à

$$
\sum_{\substack{|k|<l \leqslant s_{g} \\ l \equiv k-1 \bmod 2}} \mathcal{P}\left(g, l, \pi_{o}\right)\left(-\frac{l g+k-1}{2}\right)
$$

Dans tous les autres cas $\mathrm{gr}_{k, \pi_{o}}$ est nul.

Démonstration. - Par rapport au corollaire (IV.5.3.13), il s'agit de traiter les points supersinguliers et donc, d'après le corollaire (IV.5.4.6) de montrer que pour tout $r \equiv s-1 \bmod 2$ et $|r| \leqslant s-1, \mathcal{P}\left(g, s, \pi_{o}\right)\left(-\frac{s g+r-1}{2}\right)$ est un constituant de $e_{\pi_{o}} g r_{r, \pi_{o}}{ }^{(19)}$

On commence par remarquer, en utilisant la partie unipotente $N$ de la monodromie, qu'il suffit en fait de montrer que $\mathcal{P}\left(g, s, \pi_{o}\right)\left(-\frac{s(g-1)}{2}\right)$ est un constituant de $e_{\pi_{o}} g r_{1-s, \pi_{o}}$. Considérons alors $\mathcal{P}\left(g, s, \pi_{o}\right)\left(-\frac{s(g-1)}{2}\right)$ et soit $r$ tel qu'il soit un constituant de $e_{\pi_{o}} g r_{r, \pi_{o}}$. Soit $z_{I}$ un point géométrique de $M_{I, s_{o}}^{=d}$. D'après la proposition (IV.7.1.2), les germes en $z_{I}$ des $h^{-1} g r_{k, I, \pi_{o}}$ sont tous de poids strictement plus grand que $s(g-1)$ sauf pour $k=2-s$. Par ailleurs le germe en $z_{I}$ de $R^{d-1} \Psi_{\eta_{o}, I, \pi_{o}}\left(\overline{\mathbb{Q}_{l}}\right)$ n'a aucun constituant de poids $s(g-1)$ de sorte que $r \leqslant 2-s$.

${ }^{(19)}$ On remarquera que le raisonnement suivant est valable pour toutes les strates de sorte qu'en raisonnant par récurrence l'argument du corollaire (IV.5.3.13) en utilisant le théorème de comparaison de Berkovich-Fargues n'était pas strictement nécessaire. 
- Si on avait $r \leqslant-s$, la partie unipotente $N$ de la monodromie donnerait que

$$
\mathcal{P}\left(g, s, \pi_{o}\right)\left(-\frac{s(g-1)-2 r}{2}\right)
$$

serait un constituant de $e_{\pi_{o}} R \Psi_{\eta_{o}, \pi_{o}}\left(\overline{\mathbb{Q}_{l}}\right)[d-1]$ ce qui n'est pas d'après (IV.5.2.2) (IV.5.3.3) et (IV.5.4.6), car $1-s-2 r>s-1$.

- Supposons $r=2-s$ : pour $s>2$, par application de la dualité de Verdier et de l'opérateur de monodromie $N$, on en déduit que $\mathcal{P}\left(g, s, \pi_{o}\right) \otimes L_{g}\left(\pi_{o}\right)\left(-\frac{s(g+1)-2}{2}\right)$ et $\mathcal{P}\left(g, s, \pi_{o}\right)\left(-\frac{s(g+1)-4}{2}\right)$ sont des constituants de $e_{\pi_{o}} g r_{s-2, \pi_{o}}$. Ainsi par une nouvelle application de $N, \mathcal{P}\left(g, s, \pi_{o}\right)\left(-\frac{s(g+1)-4}{2}\right)$ est aussi un constituant de $e_{\pi_{o}} g r_{s-5, \pi_{o}}$ et donc devrait apparaître deux fois ce qui n'est pas. Dans le cas $s=2$, la situation défavorable correspondrait à $\mathcal{P}\left(g, 2, \pi_{o}\right)(-g)$ et $\mathcal{P}\left(g, 2, \pi_{o}\right)(1-g)$ constituants de $e_{\pi_{o}} g r_{0, \pi_{o}}$ de sorte que dans la cohomologie globale, la monodromie serait nulle ce qui est en contradiction avec la proposition suivante pour $s=2$.

IV.7.3.2. Proposition. - Pour tout diviseur $g$ de $d=s g$ et toute représentation irréductible cuspidale de $G L_{g}\left(F_{o}\right)$, il existe une représentation irréductible automorphe $\Pi$ de $D_{\mathbb{A}}^{\times}$vérifiant $\operatorname{Hyp}(\infty)$ telle que $\Pi_{o} \simeq[\overleftarrow{s-1}]_{\pi_{o}}$ telle que l'opérateur de monodromie $N$ sur $H_{\eta_{o}}^{d-1}\left[\Pi^{\infty}\right]$ soit non nul.

Démonstration. - En égale caractéristique, le résultat est connu d'après [19]. En caractéristique mixte, le principe, qui nous a été suggéré par M. Harris, est de se ramener au cas Iwahori par changement de base résoluble. Ledit changement de base est expliqué dans l'appendice A, traitons alors le cas Iwahori. Notons que récemment Yoshida et Taylor, cf. [23], ont rédigé ce résultat qui par ailleurs m'avait été expliqué par A. Genestier. Cependant dans notre cas, vu que l'on ne s'intéresse qu'au cas $s=2$ et donc $g=1$, le résultat a déjà été prouvé par Carayol, cf. [8].

En particulier on obtient la proposition suivante.

IV.7.3.3. Proposition. - Soit $\Pi$ une représentation irréductible automorphe de $D_{\mathbb{A}}^{\times}$telle que $\Pi_{o}$ est l'induite irréductible $\left[\overleftarrow{s_{1}-1}\right]_{\xi_{1}} \boxplus \cdots \boxplus\left[\overleftarrow{s_{r}-1}\right]_{\xi_{r}}$ où les $\xi_{i}$ sont des caractères de $F_{o}^{\times}$. En tant que représentation de $W_{o}, H_{\eta_{o}}^{d-1}\left[\Pi^{\infty}\right]$ est la somme directe des

$$
\bigoplus_{i=1}^{r} \operatorname{Sp}_{s_{i}} \otimes \xi_{i}
$$

où $\mathrm{Sp}_{s}$ est la représentation de dimension $s,|-|^{(1-s) / 2} \oplus \cdots \oplus|-|^{(s-1) / 2}$ où l'indice de nilpotence de l'opérateur de monodromie $N$ est égal à s.

Démonstration. - Le cas $s=2$ et $g=1$ de la proposition (IV.7.3.2) est prouvé par Carayol dans [8] de sorte que la proposition précédente est vraie pour $g=1$ et $s$ quelconque. On étudie ensuite la suite spectrale

$$
E_{1}^{i, j}\left[\Pi^{\infty}\right]=H^{i+j}\left(g r_{-i}\right)\left[\Pi^{\infty}\right] \Rightarrow H_{\eta_{o}}^{i+j}\left[\Pi^{\infty}\right]
$$

Cette étude est faite dans le cas général au théorème (V.4.1) ; le point est qu'en ce qui concerne les $\Pi^{\infty}$-parties, nous ne devons considérer que les représentations $\pi_{o}$ de $G L_{1}\left(F_{o}\right)$, i.e. le cas $g=1$, qui rappelons le est connu grâce à Carayol, d'où le résultat.

Remarque : Pour des résultats généraux sur les composantes locales $\Pi_{o}$ ainsi que sur la partie $\Pi^{\infty}$-isotypique des groupes de cohomologie de la fibre générique, on renvoie respectivement aux théorèmes (V.3.1) et (V.4.1). 


\section{IV.8. Étude de la suite spectrale des cycles évanescents}

Le but de ce paragraphe est de prouver la proposition (IV.7.1.1). Le principe de la preuve est d'étudier la suite spectrale des cycles évanescents en y intégrant, via les suites spectrales associées à la stratification, la connaissance des $H_{c}^{i}\left(M_{I, s_{o}}^{=l g}, \mathcal{F}\left(g, l, \pi_{o}\right) \otimes \mathcal{L}_{\rho_{\infty}}\right)$.

IV.8.1. Cas où $\Pi_{o} \simeq \operatorname{St}_{s}\left(\pi_{o}\right)$. - Soit $1 \leqslant g \leqslant d$ et $\pi_{o}$ une représentation irréductible cuspidale de $G L_{g}\left(F_{o}\right)$. On fixe dans la suite une représentation automorphe irréductible $\Pi$ de $D_{\mathbb{A}}^{\times}$vérifiant $\operatorname{Hyp}\left(\rho_{\infty}\right)$ et telle que $\Pi_{o} \simeq[\overleftarrow{s-1}]_{\pi_{o}}$ pour $\pi_{o}$ une représentation irréductible cuspidale de $G L_{g}\left(F_{o}\right)$ et on note $m(\Pi)$ la multiplicité de $\Pi$ dans l'espace des formes automorphes. On commence par un résultat déjà présent dans [19].

IV.8.1.1. Corollaire. - Les groupes de cohomologie de la fibre générique $H_{\eta_{o}, \rho_{\infty}}^{i}\left[\Pi^{\infty, o}\right]$ sont nuls pour $i \neq d-1$ et

$$
H_{\eta_{o}, \rho_{\infty}}^{d-1}\left[\Pi^{\infty, o}\right]=[\overleftarrow{s-1}]_{\pi_{o}} \otimes L_{d}\left([\overleftarrow{s-1}]_{\pi_{o}}\right)\left(-\frac{s g-1}{2}\right)
$$

Démonstration. - On considère la suite spectrale

$$
E_{1, \rho_{\infty}}^{i, j}\left[\Pi^{\infty, o}\right]:=H^{i+j}\left(g r_{-i, \rho_{\infty}}\right)\left[\Pi^{\infty, o}\right] \Rightarrow H_{\eta_{o}, \rho_{\infty}}^{i+j+d-1}\left[\Pi^{\infty, o}\right]
$$

On rappelle qu'en utilisant la pureté des $g r_{k}$, le corollaire (IV.5.4.8) implique le théorème (IV.4.4.4). Sans utiliser monodromie-poids, en caractéristique mixte, le théorème (IV.4.4.4) découle de la proposition (IV.7.3.2). La proposition (IV.5.4.1) donne alors la nullité de $E_{1, \rho_{\infty}}^{i, j}\left[\Pi^{\infty, o}\right]$ pour : $i+j \neq 0$, ou $|i| \geqslant s$ ou $i \equiv s \bmod 2$. Pour $|i|<s$ et $i=s-1-2 r$, on a

$$
E_{\infty, \rho_{\infty}}^{s-1-2 r, 2 r+1-s}\left[\Pi^{\infty, o}\right]=E_{1, \rho_{\infty}}^{s-1-2 r, 2 r+1-s}\left[\Pi^{\infty, o}\right]=[\overleftarrow{s-1}]_{\pi_{o}} \otimes L_{g}\left(\pi_{o}\right)\left(-\frac{s(g-1)+2 r}{2}\right)
$$

d'où le résultat.

IV.8.1.2. Proposition. - Pour tout $0 \leqslant i \leqslant d-1$, les $G L_{d}\left(F_{o}\right) \otimes W_{o}$-modules

$$
H^{j}\left(R^{i} \Psi_{\eta_{o}, \pi_{o}, \rho_{\infty}}\left(\overline{\mathbb{Q}}_{l}\right)\right)\left[\Pi^{\infty, o}\right]
$$

vérifient les propriétés suivantes :

(1) ils sont nuls si g n'est pas un diviseur de $d$;

(2) pour $g$ un diviseur de $d=s g$, ils sont nuls si $j$ n'est pas de la forme $d-l g$ pour $1 \leqslant l \leqslant s$;

(3) pour $g$ un diviseur de $d=s g$ et $j=d-l g$ avec $1 \leqslant l \leqslant s$, ils sont nuls si $i$ n'est pas de la forme $l g-r$ avec $1 \leqslant r \leqslant l$;

(4) pourg un diviseur de $d=$ sg et $1 \leqslant l<s, H^{d-l g}\left(R^{l g-r} \Psi_{\eta_{o}, I, \pi_{o}, \rho_{\infty}}\left(\overline{\mathbb{Q}}_{l}\right)\right)\left[\Pi^{\infty, o}\right]$ est isomorphe $\grave{a}$

$$
m(\Pi)[\overleftarrow{l-r}, \overrightarrow{r-1}]_{\pi_{o}} \overrightarrow{\times}[\overleftarrow{s-l-1}]_{\pi_{o}} \otimes L_{g}\left(\pi_{o}\right)\left(-\frac{s(g-1)+2(l-r)}{2}\right)
$$

Démonstration. - On utilise la suite spectrale associée à la stratification

$$
\begin{aligned}
E_{1, I, \pi_{o}, \rho_{\infty}}^{p, q ; i}=H_{c}^{p+q}\left(M_{I, \bar{s}_{o}}^{=p-1}, R^{i} \Psi_{\eta_{o}, I, \pi_{o}, \rho_{\infty}}\left(\overline{\mathbb{Q}}_{l}\right)\right) & \\
& \Rightarrow H_{c}^{p+q}\left(M_{I, \bar{s}_{o}}, R^{i} \Psi_{\eta_{o}, I, \pi_{o}, \rho_{\infty}}\left(\overline{\mathbb{Q}}_{l}\right)\right)
\end{aligned}
$$

On rappelle que d'après le corollaire (IV.5.4.9), $E_{1, I, \pi_{o}, \rho_{\infty}}^{p, q i}\left[\Pi^{\infty, o}\right]$ est non nul si et seulement si :

- $g$ est un diviseur de $d=s g$,

$-p-1=\lg$ pour $1 \leqslant l \leqslant s$,

$-p+q=d-l g$,

$-i=l g-r$ avec $1 \leqslant r \leqslant l$. 
Les points (1), (2) et (3) en découlent alors directement. Par ailleurs on a

$$
\begin{aligned}
\underset{I}{\lim } E_{1, I, \pi_{o}, \rho_{\infty}}^{l g+1, d-2 l g-1 ; l g-r}\left[\Pi^{\infty, o}\right] \simeq m(\Pi)[\overleftarrow{l-r}, \overrightarrow{r-1}]_{\pi_{o}} \overrightarrow{\times}[\overleftarrow{s-l-1}]_{\pi_{o}} & \\
& \otimes L_{g}\left(\pi_{o}\right)\left(-\frac{s(g-1)-2(r-l)}{2}\right)
\end{aligned}
$$

de sorte que pour tout $k \geqslant 1$, les flèches $d_{k}^{p, q ; i}: E_{k, \pi_{o}, \rho_{\infty}}^{p, q ; i}\left[\Pi^{\infty, o}\right] \longrightarrow E_{k, \pi_{o}, \rho_{\infty}}^{p+k, p_{i}}\left[\Pi^{\infty, o}\right]$ de (IV.8.1.23) sont toutes nulles. En effet pour que $E_{k, \pi_{o}, \rho_{\infty}}^{p, q i}\left[\Pi^{\infty, o}\right]$ (resp. $\left.E_{k, \pi_{o}, \rho_{\infty}}^{p+k, q+k-1 ; i}\left[\Pi^{\infty, o}\right]\right)$ soit non nul, il faut qu'il existe $1 \leqslant l_{1} \leqslant s$ et $1 \leqslant r_{1} \leqslant l_{1}$ (resp. $1 \leqslant l_{2} \leqslant s$ et $1 \leqslant r_{2} \leqslant l_{2}$ ) avec

$$
\begin{gathered}
(p, q, i)=\left(l_{1} g+1, d-2 l_{1} g-1, l_{1} g-r_{1}\right) \\
\left(\text { resp. }(p+k, q+k-1, i)=\left(l_{2} g+1, d-2 l_{2} g-1, l_{2} g-r_{2}\right)\right) .
\end{gathered}
$$

Ce qui donne $1=3 g\left(l_{2}-l_{1}\right)$; on voit alors que pour tout $k \geqslant 1, E_{k, \pi_{o}, \rho_{\infty}}^{p, q i}\left[\Pi^{\infty, o}\right]$ et $E_{k, \pi_{o}, \rho_{\infty}}^{p+k, q+k-1}\left[\Pi^{\infty, o}\right]$ ne peuvent pas être tous deux non nuls de sorte que $E_{\infty, I, \pi_{o}, \rho_{\infty}}^{p, q ; i}\left[\Pi^{\infty, o}\right]=E_{1, I, \pi_{o}, \rho_{\infty}}^{p, q ; i}\left[\Pi^{\infty, o}\right]$ d'où le résultat d'après le corollaire (IV.5.4.9).

IV.8.1.3. Corollaire. - La partie de poids $d-s$ de $\widetilde{\mathcal{U}_{F_{o}}^{d, d-i}}\left(\mathrm{JL}^{-1}\left([\overleftarrow{s-1}]_{\pi_{o}}\right)\right)$ est un constituant de $\Pi_{i} \otimes L_{g}\left(\pi_{o}\right)\left(-\frac{d-s}{2}\right)$ où

$$
\Pi_{i}=\left\{\begin{array}{cl}
{[\overleftrightarrow{s-1}]_{\pi_{o}}} & \text { pour } i=1 \\
{[\overrightarrow{0}]_{\pi_{o}} \overrightarrow{\times}[\overleftrightarrow{s-2}]_{\pi_{o}}} & \text { pour } i=2 \\
\cdots & \\
{[\overrightarrow{i-2}]_{\pi_{o}} \overrightarrow{\times}[\overleftarrow{s-i}]_{\pi_{o}}} & \text { pour } i \\
\cdots & \\
{[\overrightarrow{s-2}]_{\pi_{o}} \overrightarrow{\times}[\overleftarrow{0}]_{\pi_{o}}} & \text { pour } i=s
\end{array}\right.
$$

En outre $\widetilde{\mathcal{U}_{F_{o}}^{d, d-s}}\left(\mathrm{JL}^{-1}\left([\overleftarrow{s-1}]_{\pi_{o}}\right)\right)$ contient $[\overrightarrow{s-1}]_{\pi_{o}} \otimes L_{g}\left(\pi_{o}\right)\left(-\frac{s(g-1)}{2}\right)$ et la partie de poids $s(g-1)$ de $\sum_{i=1}^{s}(-1)^{i} \widetilde{\mathcal{U}_{F_{o}}^{d, d-i}}\left(\mathrm{JL}^{-1}\left([\overleftarrow{s-1}]_{\pi_{o}}\right)\right)$ est égale à $(-1)^{s}[\overrightarrow{s-1}]_{\pi_{o}} \otimes L_{g}\left(\pi_{o}\right)\left(-\frac{s(g-1)}{2}\right)$.

Démonstration. - On étudie la $\Pi^{\infty, o}$-partie de la suite spectrale des cycles évanescents pour $\Pi$ vérifiant les propriétés du début de ce paragraphe:

$$
E_{2, \pi_{o}, \rho_{\infty}}^{p, q}\left[\Pi^{\infty, o}\right]=\underset{I}{\lim _{I}} H^{p}\left(M_{I, \bar{s}_{o}}, R^{q} \Psi_{\eta_{o}, I, \pi_{o}, \rho_{\infty}}\left(\overline{\mathbb{Q}}_{l}\right)\right)\left[\Pi^{\infty, o}\right]
$$

$$
\Rightarrow H_{\eta_{o}, \pi_{o}, \rho_{\infty}}^{p+q}\left[\Pi^{\infty, o}\right]
$$

IV.8.1.4. Lemme. - Pour tout $1<r<s$, la partie de poids $s(g-1)$ de $E_{2, \pi_{o}, \rho_{\infty}}^{0, d-r}\left[\Pi^{\infty, o}\right]$ est un constituant de $E_{2, \pi_{o}, \rho_{\infty}}^{d-(r-1) g,(r-1)(g-1)}\left[\Pi^{\infty, o}\right]$, i.e. de

$$
[\overrightarrow{r-2}]_{\pi_{o}} \overrightarrow{\times}[\overleftrightarrow{s-r}]_{\pi_{o}} \otimes L_{g}\left(\pi_{o}\right)\left(-\frac{s(g-1)}{2}\right)
$$

celle de $E_{2, \pi_{o}}^{0, d-1}\left[\Pi^{\infty, o}\right]$ est nulle.

Démonstration. - D'après la proposition précédente, les $E_{2, \pi_{o}, \rho_{\infty}}^{p, q}\left[\Pi^{\infty, o}\right]$ de poids $s(g-1)$ pour $p \neq 0$, non nuls, sont

$$
E_{2, \pi_{o}, \rho_{\infty}}^{d-r g, r(g-1)}\left[\Pi^{\infty, o}\right] \simeq[\overrightarrow{r-1}]_{\pi_{o}} \overrightarrow{\times}[\overleftrightarrow{s-r-1}]_{\pi_{o}} \otimes L_{g}\left(\pi_{o}\right)\left(-\frac{s(g-1)}{2}\right)
$$

Or, d'après le corollaire (IV.8.1.1), pour $1 \leqslant r<s$, la partie de poids $s(g-1)$ de $E_{\infty, \pi_{o}, \rho_{\infty}}^{d-r}\left[\Pi^{\infty, o}\right]$ est nulle d'où le résultat. 
IV.8.1.5. Lemme. - Pour $g>1$, le terme $E_{2, \pi_{o}}^{0, d-r}\left[\Pi^{\infty, o}\right]$ de la suite spectrale des cycles évanescents est égal à $\operatorname{Hom}_{\bar{D}_{o}}\left(\left(\mathcal{C} \frac{\infty}{D}\right)^{\vee}, \widetilde{\mathcal{U}_{F_{o}}^{d, d-r}}\right)\left[\Pi^{\infty, o}\right]$.

Démonstration. — On étudie la suite spectrale associée à la stratification pour $R^{d-r} \Psi_{\eta_{o}, \pi_{o}, \rho_{\infty}}\left(\overline{\mathbb{Q}}_{l}\right)$. D'après la proposition précédente, pour tout $1 \leqslant l<s, H_{c}^{0}\left(M_{I, s_{o}}^{=l g}, R^{d-r} \Psi_{\eta_{o}, I, \pi_{o}, \rho_{\infty}}\left(\overline{\mathbb{Q}}_{l}\right)\right)\left[\Pi^{\infty, o}\right]$ est nul; pour $g \neq 1$, il en est de même de $H_{c}^{1}\left(M_{I, s_{o}}^{=l g}, R^{d-r} \Psi_{\eta_{o}, I, \pi_{o}, \rho_{\infty}}\left(\overline{\mathbb{Q}}_{l}\right)\right)\left[\Pi^{\infty, o}\right]$, d'où le résultat.

IV.8.1.6. Lemme. - Pour $\left.g=1, \operatorname{Hom}_{\bar{D}_{o}^{\times}}\left(\left(\mathcal{C}_{\bar{D}}^{\infty}\right)^{\vee}, \widetilde{\mathcal{U}_{F_{o}}^{d, d-r}}\right)\left[\xi_{o}\right)\right]$ est un constituant de

$$
[\overrightarrow{r-2}]_{\xi_{o}} \overrightarrow{\times}[\overleftarrow{d-r}]_{\xi_{o}} \otimes \xi_{o}
$$

Démonstration. - Pour $g=1$, on remarque que le seul $H_{c}^{1}\left(M_{I, s_{o}}^{=h}, R^{d-r} \Psi_{\eta_{o}, I, \xi_{o}, \rho_{\infty}}\left(\overline{\mathbb{Q}}_{l}\right)\right)$ ayant une partie de poids 0 non nulle, est pour $h=d-1$ et $r=d$, de $\Pi^{\infty, o}$-composante isotypique égale à $[\overrightarrow{d-2}]_{\xi_{o}} \overrightarrow{\times}[\overleftarrow{0}]_{\xi_{o}} \otimes \xi_{o}$ ce qui correspond à la contribution de $E_{2, \xi_{o}, \rho_{\infty}}^{1,0}\left[\Pi^{\infty, o}\right]$ dans le lemme (IV.8.1.4), d'où le résultat.

Ainsi pour tout $1 \leqslant i \leqslant s$, la partie de poids $d-s$ de $\widetilde{\mathcal{U}_{F_{o}}^{d, d-i}}\left(\mathrm{JL}^{-1}\left([\overleftarrow{s-1}]_{\pi_{o}}\right)\right)$ est un constituant de $\Pi_{i} \otimes L_{g}\left(\pi_{o}\right)\left(-\frac{d-s}{2}\right)$ où $\Pi_{i}$ est comme dans l'énoncé. Par ailleurs on remarque que $E_{2, \pi_{o}, \rho_{\infty}}^{d-s+1,(s-1)(g-1)}\left[\Pi^{\infty, o}\right]$ contient $[\overrightarrow{s-1}]_{\pi_{o}} \otimes L_{g}\left(\pi_{o}\right)\left(-\frac{s(g-1)}{2}\right)$ alors que $E_{2, \pi_{o}}^{d-s+2,(s-2)(g-1)}\left[\Pi^{\infty, o}\right]$ non; on en déduit alors que $\widetilde{\mathcal{U}_{F_{o}}^{d, d-i}}\left(\mathrm{JL}^{-1}\left(\pi_{o}\right)\right)$ contient $[\overrightarrow{s-1}]_{\pi_{o}} \otimes L_{g}\left(\pi_{o}\right)\left(-\frac{s(g-1)}{2}\right)$. Le calcul sur la somme alternée correspond à (IV.2.1.10).

IV.8.2. Involution de Zelevinski et première preuve de (IV.7.1.1). — Il est possible de prouver la proposition (IV.7.1.1) sans plus d'étude cohomologique en utilisant le résultat suivant.

IV.8.2.1. Théorème. — Pour toute représentation irréductible cuspidale $\pi_{o}$ de $G L_{g}\left(F_{o}\right)$, on a, pour tout $s \geqslant 1$ et pour tout $i$, un isomorphisme canonique

$$
\left(\mathcal{U}_{F_{o}, l, s g}^{s g-1+\bullet}\left(\mathrm{JL}^{-1}\left(\operatorname{St}_{s}\left(\pi_{o}\right)\right)\right)\right)^{\vee, \iota}(d-1) \simeq \mathcal{U} F_{o}, l, s g^{s g+s-2-2 \bullet}\left(\mathrm{JL}^{-1}\left(\operatorname{St}_{s}\left(\pi_{o}^{\vee}\right)\right)\right)
$$

où dans le membre de droite l'exposant $\vee, \iota$ désigne le dual composé avec l'involution de Zelevinski, $\iota$, sur $G L_{s g}\left(F_{o}\right)$.

Remarque : Laurent Fargues a une preuve de ce résultat qui utilise tout ou partie l'isomorphisme de Faltings, à partir d'un résultat similaire du coté de l'espace de Drinfeld.

Ainsi avec les notations du corollaire (IV.8.1.3), s'il existait $1 \leqslant i \leqslant s$ tel que $\Pi_{i}$ admette un constituant autre que $[\overrightarrow{s-1}]_{\pi_{o}}$, on en déduirait que $\mathcal{U}_{F_{o}, l, s g}^{s g-s-1+i}\left(\mathrm{JL}^{-1}\left([\overleftarrow{s-1}]_{\pi_{o}}\right)\right)$ aurait un constituant de poids $s g+s-2$ autre que $[\overleftarrow{s-1}]_{\pi_{o}^{\vee}}$. Or, d'après les corollaires (IV.5.3.12) et (IV.5.4.6), pour tout $k$, les germes aux points supersinguliers des $h^{i} g r_{k, \pi_{o}^{\vee}}$ sont tous de poids strictement plus petit que $s g+s-2$ ou alors égales à $[\overleftarrow{s-1}]_{\pi_{o}^{\vee}} \otimes \operatorname{rec}_{F_{o}}\left(\pi_{o}\right)\left(-\frac{s g+s-2}{2}\right)$, d'où le résultat.

IV 8.3. Cas $\Pi_{o} \simeq \operatorname{Speh}_{s}\left(\pi_{o}\right)$. — Nous allons prouver la proposition (IV.7.1.1) sans utiliser le théorème (IV.8.2.1). Pour cela nous revenons à l'étude de la suite spectrale des cycles évanescents. On peut remarquer que la connaissance des $E_{2, \pi_{o}, \rho_{\infty}}^{p, q}\left[\Pi^{\infty, o}\right]$ de la suite spectrale (IV.8.1.24) ne nous fournit pas le théorème local. La figure (3) illustre ce fait dans le cas $s=2$, où pour $\pi_{o}$ une représentation irréductible cuspidale de $G L_{d / 2}\left(F_{o}\right)$, l'on n'arrive pas à exclure le cas :

$$
-\widetilde{\mathcal{U}_{F_{o}}^{d, d-1}}\left(\mathrm{JL}^{-1}\left([\overleftarrow{1}]_{\pi_{o}}\right)\right)=[\overleftarrow{1}]_{\pi_{o}} \otimes\left(L_{g}\left(\pi_{o}\right)\left(-\frac{d-1}{2}\right) \otimes \mathrm{Sp}_{2}\right)
$$




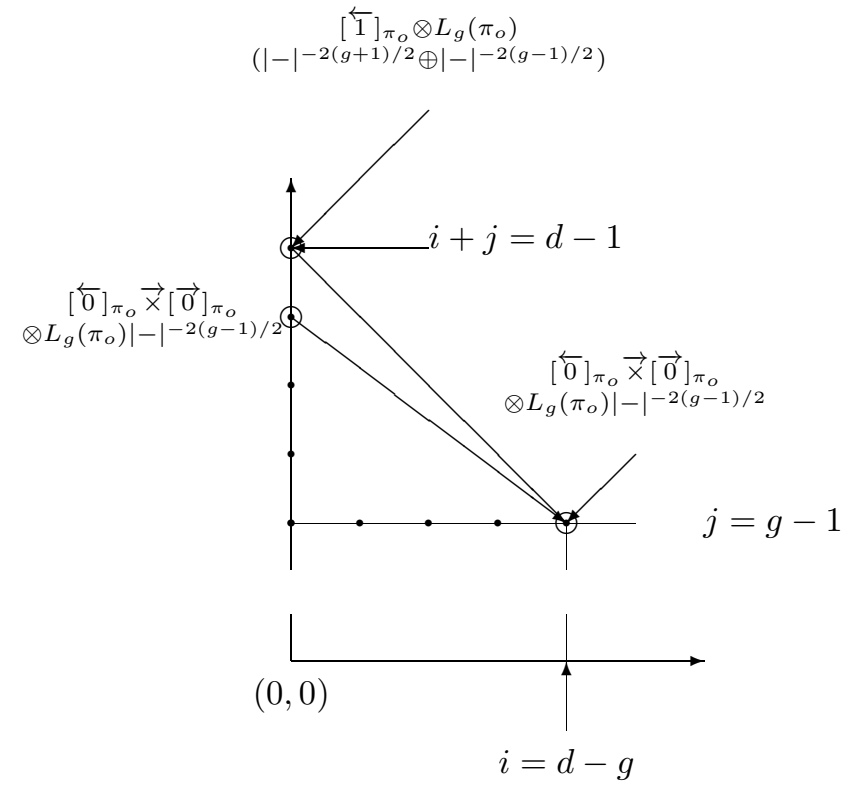

Figure 3. $\quad E_{2}^{p, q}\left[\Pi^{\infty, o}\right]$ de (IV.8.1.24) pour $\Pi_{o} \simeq \mathrm{St}_{s}\left(\pi_{o}\right)$ compatible avec l'aboutissement

$$
-\widetilde{\mathcal{U}_{F_{o}}^{d, d-2}}\left(\mathrm{JL}^{-1}\left([\overleftarrow{1}]_{\pi_{o}}\right)\right)=[\overleftarrow{0}]_{\pi_{o}} \overrightarrow{\times}[\overleftarrow{0}]_{\pi_{o}} \otimes L_{g}\left(\pi_{o}\right)\left(-\frac{d-2}{2}\right)
$$

Si la flèche indiquée est un isomorphisme, on obtient bien le bon aboutissement.

$I V .8 .3 .1$ - Dans le cas où l'on considère une représentation automorphe $\Pi$ de $D_{\mathbb{A}}^{\times}$vérifiant $\operatorname{Hyp}\left(\rho_{\infty}\right)$ et telle que $\Pi_{o} \simeq[\overrightarrow{s-1}]_{\pi_{o}}$, on calculera les termes $E_{2, \rho_{\infty}}^{p, q}\left[\Pi^{\infty, o}\right]$ de la suite spectrale des cycles évanescents et on montrera alors que le théorème local en découle. Dans la figure (4), on illustre comment on exclut le cas défavorable ci-avant, en remarquant que $[\overleftarrow{0}]_{\pi_{o}} \overrightarrow{\times}[\overleftarrow{0}]_{\pi_{o}}$ n'est pas isomorphe à $[\overleftarrow{0}]_{\pi_{o}} \overleftarrow{\times}[\overleftarrow{0}]_{\pi_{o}}$, contredisant le théorème de Lefschetz difficile. On pourra aussi se référer aux figures (3) et (4) qui détaillent pour $s=4$ et $g=2$, les $E_{2, \pi_{o}}^{p, q}\left[\Pi^{\infty, o}\right]$ respectivement dans les cas $\Pi_{\infty}=[\overleftarrow{s-1}]_{\pi_{\infty}}$ et $\Pi_{\infty}=[\overrightarrow{s-1}]_{\pi_{\infty}}$.

IV.8.3.2. Remarque. - Si $\Pi$ vérifie $\operatorname{Hyp}\left(\rho_{\infty}\right)$, la condition $\Pi_{o} \simeq[\overrightarrow{s-1}]_{\pi_{o}}$ pour $\pi_{o}$ une représentation irréductible cuspidale de $G L_{g}\left(F_{o}\right)$, implique qu'il existe une représentation irréductible cuspidale $\pi_{\infty}$ de $G L_{g^{\prime}}\left(F_{\infty}\right)$ avec $d=s^{\prime} g^{\prime}$ telle que $\Pi_{\infty} \simeq\left[\overleftarrow{s^{\prime}-1}\right]_{\pi_{\infty}}$ et donc $\Pi$ vérifie $\operatorname{Hyp}\left(\rho_{\infty}\right)$ avec $\rho_{\infty}=\mathrm{JL}^{-1}\left(\left[\overleftarrow{s^{\prime}-1}\right]_{\pi_{\infty}}\right)$ et $s=s^{\prime}$

$I V .8 .3 .3$ - On considère ${ }^{(20)}$ dans la suite $\Pi$ une représentation irréductible automorphe de $D_{\mathbb{A}}^{\times}$ vérifiant $\operatorname{Hyp}\left(\rho_{\infty}\right)$ et telle que $\Pi_{o} \simeq[\overrightarrow{s-1}]_{\pi_{o}}$, pour $\pi_{o}$ une représentation irréductible cuspidale de $G L_{g}\left(F_{o}\right)$. On suppose par ailleurs que l'ensemble des représentations irréductibles automorphes $\bar{\Pi}$ de $\bar{D}_{\mathbb{A}}^{\times}$telles que $\bar{\Pi}^{\infty, o} \simeq \Pi^{\infty, o}$ est réduite à une unique représentation, en particulier $m(\Pi)=$ $m(\bar{\Pi})=1$, et $\bar{\Pi}_{o} \simeq \mathrm{JL}^{-1}\left([\overleftarrow{s-1}]_{\pi_{o}}\right)$ et $\bar{\Pi}_{\infty} \simeq \mathrm{JL}^{-1}\left([\overleftarrow{s-1}]_{\pi_{\infty}}\right)$. L'objectif est de calculer les termes $E_{2, \rho_{\infty}}^{p, q}\left[\Pi^{\infty, o}\right]$ de la suite spectrale des cycles évanescents.

IV.8.3.4. Proposition. - Pour tout $1 \leqslant l \leqslant s$,

$$
H^{i}\left(j_{!_{*}}^{\geqslant l g} H T_{\rho_{\infty}}\left(g, l, \pi_{o},[\overleftarrow{l-1}]_{\pi_{o}}\right)[d-l g]\right)\left[\Pi^{\infty, o}\right]
$$

${ }^{(20)}$ cf. aussi les conditions qui précèdent le corollaire (IV.5.4.6) 


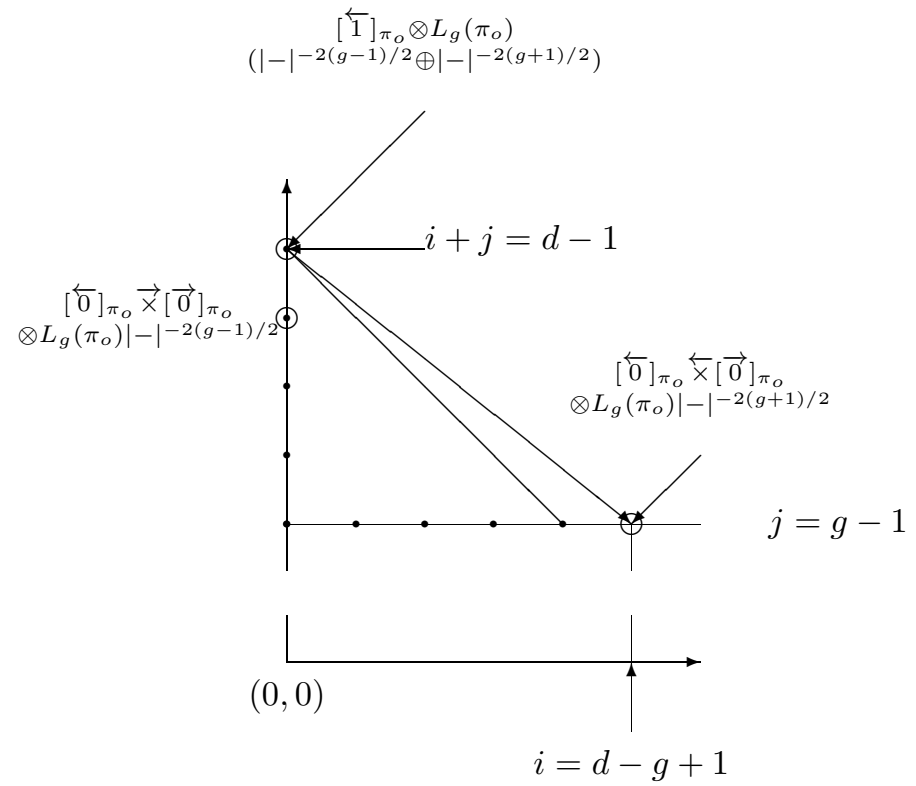

Figure 4. $E_{2, \pi_{o}}^{p, q}\left[\Pi^{\infty, o}\right]$ de (IV.8.1.24) pour $\Pi_{\infty} \simeq \operatorname{Speh}_{s}\left(\pi_{\infty}\right)$ non compatible avec l'aboutissement

est nul pour $|i|>s-l$ ou $i \not \equiv s-l \bmod 2$ et sinon, il est isomorphe $\grave{a}$

$$
\left([\overleftarrow{l-1}]_{\pi_{o}} \overrightarrow{\times}\left[\frac{\overrightarrow{s-l-i}}{2}-1\right]_{\pi_{o}}\right) \overleftarrow{\times}\left[\frac{\overrightarrow{s-l+i}}{2}-1\right]_{\pi_{o}} \otimes\left(\Xi^{\frac{(s-l) g+i}{2}} \otimes \bigoplus_{\xi \in \mathfrak{A}\left(\pi_{o}\right)} \xi^{-1}\right)
$$

en tant que représentation de $G L_{d}\left(F_{o}\right) \times \mathbb{Z}$, où $\mathfrak{A}\left(\pi_{o}\right)$ est l'ensemble des caractères $\xi: \mathbb{Z} \longrightarrow \mathbb{Q}_{l}^{\times}$, tels que $\pi_{o} \otimes \xi^{-1} \circ \operatorname{val}(\operatorname{det}) \simeq \pi_{o}$.

Démonstration. - On raisonne par récurrence pour $l$ variant de $s$ à 1 , le cas $l=s$ est donné par le lemme (IV.5.4.7) et les conditions imposées ci-dessus à $\Pi$. Les suites exactes courtes

$$
\begin{aligned}
0 \rightarrow P_{\pi_{o}, l, 0}\left([\overleftarrow{l-1}]_{\pi_{o}}\right) \longrightarrow j_{!}^{\geqslant l g} \operatorname{HT}\left(g, l, \pi_{o},[\overleftarrow{l-1}]_{\pi_{o}}\right)[d-l g] & \\
& \longrightarrow j_{! *}^{\geqslant l g} H T\left(g, l, \pi_{o},[\overleftarrow{l-1}]_{\pi_{o}}\right)[d-l g] \rightarrow 0 \\
0 \rightarrow P_{\pi_{o}, l, i}\left([\overleftarrow{l-1}]_{\pi_{o}}\right) \longrightarrow P_{\pi_{o}, l, i-1}\left([\overleftarrow{l-1}]_{\pi_{o}}\right) & \longrightarrow \mathcal{P}_{-}\left(g, l+i, \pi_{o}, l,[\overleftarrow{l-1}]_{\pi_{o}}\right) \rightarrow 0
\end{aligned}
$$

pour $1 \leqslant i \leqslant s-l-1$, fournissent

$$
\begin{aligned}
\sum_{i}(-1)^{i} H^{i}\left(j_{!_{*}}^{\geqslant l g}\right. & \left.H T_{\rho_{\infty}}\left(g, l, \pi_{o},[\overleftarrow{l-1}]_{\pi_{o}}\right)[d-l g]\right)\left[\Pi^{\infty, o}\right]= \\
& \sum_{r=1}^{s-l}(-1)^{r} \sum_{i}(-1)^{i} H^{i}\left([\overleftarrow{l-1}]_{\pi_{o}} \overrightarrow{\times}[\overleftarrow{r-1}]_{\pi_{o}}\right)\left[\Pi^{\infty, o}\right] \otimes \Xi^{r / 2}+ \\
& \sum_{i}(-1)^{i} H^{i}\left(j_{!}^{\geqslant l g} H T_{\rho_{\infty}}\left(g, l, \pi_{o},[\overleftarrow{l-1}]_{\pi_{o}}\right)[d-l g]\right)\left[\Pi^{\infty, o}\right]
\end{aligned}
$$

où par simplification, on écrit $H^{i}\left([\overleftarrow{l-1}]_{\pi_{o}} \overrightarrow{\times}[\overleftarrow{r-1}]_{\pi_{o}}\right)$ pour

$$
\lim _{\underset{I}{I}} H^{i}\left(M_{I, s_{o}}^{\geqslant(l+r) g}, j_{! *}^{\geqslant(l+r) g} H T_{\rho_{\infty}}\left(g, l+r, \pi_{o},[\overleftarrow{l-1}]_{\pi_{o}} \overrightarrow{\times}[\overleftarrow{r-1}]_{\pi_{o}}\right)[d-(l+r) g]\right)
$$


D'après l'hypothèse de récurrence et (IV.2.2.13), pour tout $i \equiv 1 \bmod 2$, la partie de poids $(s-l)(g-1)+i$ du membre de droite de (IV.8.3.25) est nulle; il en est de même pour $i>2(s-l)$ ou $i<0$, tandis que pour $0 \leqslant i=2 k<2(s-l)$ (resp. $i=2(s-l))$ celle-ci est égale à

$$
\sum_{r=1}^{s-l-k} H^{l-s+2 k+r}\left([\overleftarrow{l-1}]_{\pi_{o}} \overrightarrow{\times}[\overleftarrow{r-1}]_{\pi_{o}}\right)\left[\Pi^{\infty, o}\right] \otimes \Xi^{r / 2}
$$

(resp. à la partie de poids $(s-l)(g+1)$ de (IV.2.2.13)), ce qui donne $\pi \otimes\left(\Xi^{\frac{s(g-1)+2 k}{2}} \otimes \bigoplus_{\xi \in \mathfrak{A}\left(\pi_{o}\right)} \xi^{-1}\right)$ où

$$
\begin{aligned}
\pi= & \left([ \overleftarrow { l - 1 } ] _ { \pi _ { o } } \vec { \times } \left((-1)^{s-l-k}[\overleftrightarrow{s-l-k-1}]_{\pi_{o}}\right.\right. \\
& \left.\left.+\sum_{r=1}^{s-l-k-1}(-1)^{r-1}[\overleftrightarrow{r-1}]_{\pi_{o}} \overrightarrow{\times}[\overrightarrow{s-l-r-k-1}]_{\pi_{o}}\right)\right) \overleftarrow{\times}[\overrightarrow{k-1}]_{\pi_{o}} \\
= & \left([\overleftrightarrow{l l-1}]_{\pi_{o}} \overrightarrow{\times}[\overleftrightarrow{s-l-k-1}]_{\pi_{o}}\right) \overleftarrow{\times}[\overrightarrow{k-1}]_{\pi_{o}} \\
& \left(\text { resp. }[\overleftrightarrow{l-1}]_{\pi_{o}} \overleftarrow{\times}[\overrightarrow{s-l-1}]_{\pi_{o}}\right)
\end{aligned}
$$

Or $H^{i}\left(j_{! *}^{\geqslant l g} H T_{\rho_{\infty}}\left(g, l, \pi_{o},[\overleftarrow{l-1}]_{\pi_{o}}\right)[d-l g]\right)$ est pur de poids $(s-l) g+i$ de sorte que son semi-simplifié est égal à celui de l'énoncé. On conclut alors à l'égalité des représentations, et pas seulement de leur semi-simplifiée, en remarquant que les strates étant induites, l'espace précédent, en tant que représentation de $G L_{d}\left(F_{o}\right)$ est de la forme

$$
\operatorname{Ind}_{P_{l g, d}^{o p}\left(F_{o}\right)}^{G L_{d}\left(F_{o}\right)}[\overleftarrow{l-1}]_{\pi_{o}((s-l) g+i) / 2} \otimes \pi^{\prime}
$$

pour une certaine représentation $\pi^{\prime}$ de $G L_{d-l g}\left(F_{o}\right)$.

IV.8.3.5. Corollaire. - Pour tout $i \neq d-s$, la partie de poids $s(g-1)$ de $H_{\eta_{o}, \rho_{\infty}}^{i}\left[\Pi^{\infty, o}\right]$ est nulle alors que pour $i=d-s$ elle est égale $\grave{a}[\overrightarrow{s-1}]_{\pi_{o}} \otimes L_{g}\left(\pi_{o}\right)\left(-\frac{s(g-1)}{2}\right)$.

IV.8.3.6. Remarque. - En fait on peut à ce stade déterminer complètement les $H_{\eta_{o}, \rho_{\infty}}^{i}$, cependant comme on l'a déjà remarqué seule la connaissance des parties de poids $s(g-1)$ nous est nécessaire. Pour un énoncé complet, on pourra voir la proposition (V.1.1).

Démonstration. - On écrit $i$ sous la forme $d-1-\delta$ et on étudie la suite spectrale

$$
E_{1, \rho_{\infty}}^{i, j}:=H^{i+j}\left(g r_{-i, \rho_{\infty}}\right) \Rightarrow H_{\eta_{o}, \rho_{\infty}}^{d-1+i+j}
$$

qui, d'après la pureté, dégénère en $E_{2}$. On pourra se référer à la figure (2) où l'on a représenté les $H^{i}\left(g r_{k, \rho_{\infty}}\right)\left[\Pi^{\infty, o}\right]$ pour $s=4$. D'après les propositions (IV.5.3.3) (ii) et (IV.7.3.1), on a

$$
e_{\pi_{o}} H^{i}\left(g r_{k, \rho_{\infty}}\right)\left[\Pi^{\infty, o}\right]=\bigoplus_{\substack{\mid k<l \leqslant s \\ l \equiv k+1 \bmod 2}} H^{i}\left(\mathcal{P}\left(g, l, \pi_{o}\right)\left(-\frac{l g-1+k}{2}\right)\right)\left[\Pi^{\infty, o}\right]
$$

Ainsi d'après la proposition (IV.8.3.4), pour $\delta>0$, la partie de poids $s(g-1)$ de $H_{\eta_{o}, \rho_{\infty}}^{d-1+\delta}\left[\Pi^{\infty, o}\right]$ est nulle; précisément pour $\delta>0$, les poids de $H_{\eta_{o}, \rho_{\infty}}^{d+1+\delta}\left[\Pi^{\infty, o}\right]$ sont parmi les $k=s(g-1)+2 \delta+2 r$ avec $0 \leqslant r<s-\delta$ et sa partie de poids $s(g-1)+2 \delta$, que l'on notera avec un indice, est un quotient de

$$
\begin{aligned}
& E_{1, \rho_{\infty}}^{1-s+\delta, s-1}\left[\Pi^{\infty, o}\right]_{s(g-1)+2 \delta}=\frac{1}{e_{\pi_{o}}} H^{\delta}\left(\mathcal{P}\left(g, s-\delta, \pi_{o}\right)\left(-\frac{(s-\delta)(g-1)}{2}\right)\right) \\
&=[\overleftarrow{s-\delta-1}]_{\pi_{o}} \overleftarrow{\times}[\overrightarrow{\delta-1}]_{\pi_{o}} \otimes L_{g}\left(\pi_{o}\right)\left(-\frac{s(g-1)+2 \delta}{2}\right)
\end{aligned}
$$


Par ailleurs, pour $\delta>0$, d'après (IV.8.3.4), la partie de poids $s(g-1)$ de $H_{\eta_{o}, \rho_{\infty}}^{d-s-\delta}\left[\Pi^{\infty, o}\right]$ est un sous-quotient de

$$
\begin{aligned}
E_{1, \rho_{\infty}}^{1-s+\delta, s-1-2 \delta}\left[\Pi^{\infty, o}\right]_{s(g-1)}=\frac{1}{e_{\pi_{o}}} H^{-\delta}(\mathcal{P}(g, s & \left.\left.-\delta, \pi_{o}\right)\left(-\frac{(s-\delta)(g-1)}{2}\right)\right) \\
& =[\overleftarrow{s-\delta-1}]_{\pi_{o}} \overrightarrow{\times}[\overrightarrow{\delta-1}]_{\pi_{o}} \otimes L_{g}\left(\pi_{o}\right)\left(-\frac{s(g-1)}{2}\right)
\end{aligned}
$$

On utilise alors le théorème de Lefschetz difficile dont on rappelle l'énoncé ci-après.

IV.8.3.7. Théorème. - (cf. [19] 14.19) Il existe une classe $h \in H_{\eta_{o}, \rho_{\infty}}^{2}(1)$, invariante sous les actions de $\left(D_{\mathbb{A}}^{\infty}\right)^{\times}$et $W_{o}$, telle que les applications itérées du cup produit

$$
h^{i}: H_{\eta_{o}, \rho_{\infty}}^{d-1-i} \longrightarrow H_{\eta_{o}, \rho_{\infty}}^{d-1+i}(i)
$$

sont des isomorphismes.

- Ainsi pour $s>2$, on observe que si la partie de poids $s(g-1)$ de $H_{\eta_{o}}^{d-1-\delta}\left[\Pi^{\infty, o}\right]$, pour $1<\delta<s-1$, est non nulle, ses constituants sont de la forme $[\overleftrightarrow{s-2}, \overrightarrow{1}]_{\pi_{o}} \otimes L_{g}\left(\pi_{o}\right)\left(-\frac{s(g-1)}{2}\right)$ alors qu'un constituant non nul de poids $s(g-1)+2 \delta$ de $H_{\eta_{o}, \rho_{\infty}}^{d-1+2 \delta}\left[\Pi^{\infty, o}\right]$, s'il existe, est de la forme $[\overleftrightarrow{s-2}, \overleftarrow{1}]_{\pi_{o}} \otimes L_{g}\left(\pi_{o}\right)\left(-\frac{s(g-1)+2 \delta}{2}\right)$. La contradiction découle alors du théorème de Lefschetz difficile et de l'observation de l'orientation de la dernière flèche.

- Pour $\delta=1$ et $s>2$, la partie de poids $s(g-1)+2$ de $H_{\eta_{o}, \rho_{\infty}}^{d}\left[\Pi^{\infty, o}\right]$ est un quotient de $[\overleftarrow{s-2}]_{\pi_{o}} \overleftarrow{\times}[\overrightarrow{0}]_{\pi_{o}} \otimes L_{g}\left(\pi_{o}\right)\left(-\frac{s(g-1)+2}{2}\right)$ alors que la partie de poids $s(g-1)$ de $H_{\eta_{o}, \rho_{\infty}}^{d-2}\left[\Pi^{\infty, o}\right]$ est un constituant de $[\overleftarrow{s-2}]_{\pi_{o}} \overrightarrow{\times}[\overrightarrow{0}]_{\pi_{o}} \otimes L_{g}\left(\pi_{o}\right)\left(-\frac{s(g-1)}{2}\right)$. La contradiction découle alors du théorème de Lefschetz difficile et du fait que $[\overleftarrow{s-1}]_{\pi_{o}}$ n'est pas un quotient de $[\overleftarrow{s-2}]_{\pi_{o}} \overleftarrow{\times}[\overrightarrow{0}]_{\pi_{o}}$

- Pour $\delta=s-1$ et $s \geqslant 2$, on observe que $E_{2, \rho_{\infty}}^{d-s}\left[\Pi^{\infty, o}\right]=H_{\eta_{o}, \rho_{\infty}}^{d-s}\left[\Pi^{\infty, o}\right]$ est un sous-espace de

$$
E_{1, \rho_{\infty}}^{0,1-s}\left[\Pi^{\infty, o}\right]=\frac{1}{e_{\pi_{o}}} H^{1-s}\left(\mathcal{P}\left(g, 1, \pi_{o}\right)\right)=[\overleftarrow{0}]_{\pi_{o}} \overrightarrow{\times}[\overrightarrow{s-2}]_{\pi_{o}} \otimes L_{g}\left(\pi_{o}\right)\left(-\frac{s(g-1)}{2}\right)
$$

alors que $E_{2, \rho_{\infty}}^{d-s}\left[\Pi^{\infty, o}\right]=H_{\eta_{o}, \rho_{\infty}}^{d+s-2}\left[\Pi^{\infty, o}\right]$ est un quotient de

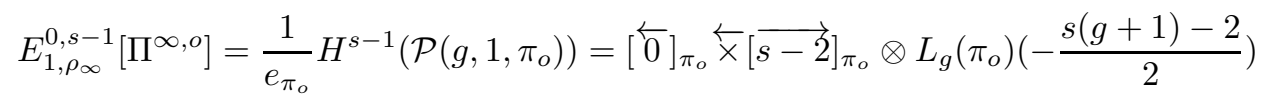

Ainsi d'après Lefschetz difficile, s'ils sont non nuls, ils doivent être égaux à $[\overrightarrow{s-1}]_{\pi_{o}}$. Par ailleurs on remarque que ce dernier n'est pas un constituant de $H_{\eta_{o}, \rho_{\infty}}^{d-s+1}\left[\Pi^{\infty, o}\right]$, ni de $H_{\eta_{o}, \rho_{\infty}}^{d+s-3}\left[\Pi^{\infty, o}\right]$, de sorte que $[\overrightarrow{s-1}]_{\pi_{o}} \otimes L_{g}\left(\pi_{o}\right)\left(-\frac{s(g-1)}{2}\right)$ est effectivement un constituant de $H_{\eta_{o}, \rho_{\infty}}^{d-s}$, d'où le résultat.

IV.8.3.8. Proposition. - Pour tout $p \neq 0$ et tout $q$, les parties de poids $s(g-1)$ des $E_{2, \rho_{\infty}}^{p, q}\left[\Pi^{\infty, o}\right]$ de la suite spectrale des cycles évanescents

$$
E_{2, \rho_{\infty}}^{p, q}=\underset{I}{\lim _{\longrightarrow}} H^{p}\left(M_{I, \bar{s}_{o}}, R^{q} \Psi_{\eta_{o}, I}\left(\mathcal{L}_{\rho_{\infty}}\right)\right) \Rightarrow H_{\eta_{o}, \rho_{\infty}}^{p+q}
$$

sont nulles.

Démonstration. - Le principe est d'étudier les $E_{2, \rho_{\infty}}^{p, q}\left[\Pi^{\infty, o}\right]$ via les suites spectrales associées à la stratification

$$
E_{1, I, \rho_{\infty}}^{p, q ; i}=H_{c}^{p+q}\left(M_{I, \bar{s}_{o}}^{=p-1}, R^{i} \Psi_{\eta_{o}, I}\left(\mathcal{L}_{\rho_{\infty}}\right)\right) \Rightarrow H_{c}^{p+q}\left(M_{I, \bar{s}_{o}}, R^{i} \Psi_{\eta_{o}, I}\left(\mathcal{L}_{\rho_{\infty}}\right)\right)
$$

Ainsi le résultat découle simplement de la proposition suivante.

IV.8.3.9. Proposition. - Pour tout $1 \leqslant l<s$ et pour tout $i$, la partie de poids $(s-l)(g-1)$ de $H_{c}^{i}\left(M_{I, s_{o}}^{=l g}, \mathcal{F}\left(g, l, \pi_{o}, I\right) \otimes \mathcal{L}_{\rho_{\infty}}\right)\left[\Pi^{\infty, o}\right]$ est nulle. 
Démonstration. - Il s'agit dans un premier temps d'étudier les parties de poids $(s-l)(g-1)$ des $H_{c}^{i}\left(M_{I, s_{o}}^{=l g}, H T_{\rho_{\infty}}\left(g, l, \pi_{o}, \Pi_{l}, I\right)\right)\left[\Pi^{\infty, o}\right]$ pour une représentation $\Pi_{l}$ quelconque de $G L_{l g}\left(F_{o}\right)$.

IV.8.3.10. Lemme. - Pour tout $1 \leqslant l<s$, les

$$
\lim _{\vec{I}} H^{i}\left(M_{I, s_{o}}^{\geqslant l g},\left(j_{!}^{\geqslant l g} H T_{\rho_{\infty}}\left(g, l, \pi_{o}, \Pi_{l}, I\right)[(s-l) g]\right)\right)\left[\Pi^{\infty, o}\right]
$$

vérifient les propriétés suivantes :

(i) ils sont de la forme $\bigoplus_{\xi}\left(\operatorname{Ind}_{P_{l g, d}^{\mathrm{o}}\left(F_{o}\right)}^{G L_{d}\left(F_{o}\right)}\left(\Pi_{l} \otimes \xi\right) \otimes \pi_{\xi}\right) \otimes \xi^{-1}$, en tant que représentation de $G L_{d}\left(F_{o}\right) \times \mathbb{Z}$, où $\xi$ décrit les caractères $\mathbb{Z} \longrightarrow \overline{\mathbb{Q}}_{l}^{\times}$et $\pi_{\xi}$ est une représentation de $G L_{(s-l) g}\left(F_{o}\right)$;

(ii) ils sont nuls pour $|i| \geqslant s-l+1$;

(iii) ils sont en général mixtes de poids $(s-l)(g+1)-2(k-1)$ pour $1 \leqslant k \leqslant s-l+1$ vérifiant $s-l-2(k-1) \leqslant i \leqslant s-l-(k-1)$;

(iv) soit $l-s \leqslant i_{0}<0$, le plus petit indice $i$ tel que la partie de poids $(s-l)(g-1)$ de

$$
\left.\lim _{\vec{I}} H^{i}\left(M_{I, s_{o}}^{\geqslant l g}, j_{!}^{\geqslant l g} H T_{\rho_{\infty}}\left(g, l, \pi_{o}, \Pi_{l}, I\right)[(s-l) g]\right)[(s-l) g]\right)\left[\Pi^{\infty, o}\right]
$$

soit non nulle ${ }^{(21)}$. Cette dernière est alors égale à

$$
\Pi_{l} \overrightarrow{\times}\left[\overleftrightarrow{i_{0}-l+s-1}, \overrightarrow{-i_{0}}\right]_{\pi_{o}} \otimes\left(\Xi^{\frac{(s-l)(g-1)}{2}} \otimes \bigoplus_{\xi \in \mathfrak{A}\left(\pi_{o}\right)} \xi^{-1}\right) .
$$

Démonstration. - Le point (i) correspond à la proposition (I.5.3.10) qui découle directement de l'action de $G L_{l g}\left(F_{o}\right)$ sur la strate via val(det) et du fait que les strates non supersingulières sont induites.

Pour les points (ii)-(iii), on considère les suites exactes

$$
0 \rightarrow P_{\pi_{o}, l, i+1}\left(\Pi_{l}\right) \longrightarrow P_{\pi_{o}, l, i}\left(\Pi_{l}\right) \longrightarrow \mathcal{P}_{-}\left(g, l+i+1, \pi_{o}, l, \Pi_{l}\right) \rightarrow 0
$$

et on reprend les notations simplifiées de la preuve de la proposition (IV.8.3.4) en notant $P_{\pi_{o}, l,-1, \rho_{\infty}}\left(\Pi_{l}\right):=j_{!}^{\geqslant l g} H T_{\rho_{\infty}}\left(g, l, \pi_{o}, \Pi_{l}\right)[d-l g]$. Par facilité on notera $P_{\pi_{o}, l, i}\left(\Pi_{l}\right)$ pour $P_{\pi_{o}, l, i}\left(\Pi_{l}\right) \otimes \mathcal{L}_{\rho_{\infty}}$. Le résultat découle alors directement du cas $i=-1$ dans le lemme suivant.

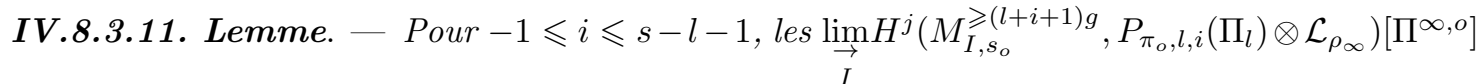
vérifient les points suivants :

(1) ils sont nuls pour $|j| \geqslant s-l-i$;

(2) pour $|j|<s-l-i$, ils sont mixtes de poids $(s-l)(g+1)-2(k+i)$ pour $1 \leqslant k \leqslant s-l-i$ vérifiant $s-l-i-1-2(k-1) \leqslant j \leqslant s-l-i-1-(k-1)$;

Démonstration. - (1)-(2) On raisonne par récurrence descendante, le cas $i=s-l-1$ étant évident car $P_{\pi_{o}, l, s-l-1}\left(\Pi_{l}\right)$ est le faisceau concentré aux points supersinguliers

$$
\mathcal{F}\left(g, s, \pi_{o}\right) \otimes \Pi_{l}(-(s-l) / 2) \times[\overleftarrow{s-l-1}]_{\pi_{o}(l / 2)} \otimes|c 1|^{-\frac{(s-l)(g-1)}{2}}
$$

Supposons donc le résultat acquis jusqu'au rang $i+1$ et traitons le cas de $P_{\pi_{o}, l, i}\left(\Pi_{l}\right)$. On considère la suite exacte longue de cohomologie associée à la suite exacte courte (IV.8.3.29). D'après l'hypothèse de récurrence les $H^{j}\left(P_{\pi_{o}, l, i+1}\left(\Pi_{l}\right)\right)$ sont nuls pour $|j| \geqslant s-l-i-1$ et d'après la proposition

${ }^{(21)}$ Si un tel $i_{0}$, n'existe pas l'énoncé est vide. 
(IV.8.3.4), les $H^{j}\left(\mathcal{P}_{-}\left(g, l+i+1, \pi_{o}, l, \Pi_{l}\right)\right)$ sont nuls pour $|j| \geqslant s-l-i$, d'où le point (1). En ce qui concerne le point (2), la suite exacte longue en question s'écrit alors

$$
\begin{aligned}
& 0 \rightarrow H^{-(s-l-i-1)}\left(P_{\pi_{o}, l, i}\left(\Pi_{l}\right)\right) \longrightarrow H^{-(s-l-i-1)}\left(j_{! *}^{\geqslant(l+i+1) g}\right) \longrightarrow \\
& H^{-(s-l-i-2)}\left(P_{\pi_{o}, l, i+1}\left(\Pi_{l}\right)\right) \longrightarrow H^{-(s-l-i-2)} P_{\pi_{o}, l, i}\left(\Pi_{l}\right) \longrightarrow 0 \cdots \\
& \cdots 0 \rightarrow H^{(s-l-i-1)-2 r}\left(P_{\pi_{o}, l, i+1}\left(\Pi_{l}\right)\right) \longrightarrow H^{(s-l-i-1)-2 r}\left(P_{\pi_{o}, l, i}\left(\Pi_{l}\right)\right) \longrightarrow \\
& H^{(s-l-i-1)-2 r}\left(j_{! *}^{\geqslant(l+i+1) g}\right) \longrightarrow H^{(s-l-i-1)-2 r+1}\left(P_{\pi_{o}, l, i+1}\left(\Pi_{l}\right)\right) \\
& \longrightarrow H^{(s-l-i-1)-2 r+1}\left(P_{\pi_{o}, l, i}\left(\Pi_{l}\right)\right) \rightarrow 0 \\
& \cdots \\
& 0 \rightarrow H^{(s-l-i-1)-2}\left(P_{\pi_{o}, l, i+1}\left(\Pi_{l}\right)\right) \longrightarrow H^{(s-l-i-1)-2}\left(P_{\pi_{o}, l, i}\left(\Pi_{l}\right)\right) \longrightarrow \\
& H^{(s-l-i-1)-2}\left(j_{! *}^{\geqslant(l+i+1) g}\right) \longrightarrow H^{s-l-i-2}\left(P_{\pi_{o}, l, i+1}\left(\Pi_{l}\right)\right) \longrightarrow H^{s-l-i-2}\left(P_{\pi_{o}, l, i}\left(\Pi_{l}\right)\right) \rightarrow 0 \\
& 0 \rightarrow H^{s-l-i-1}\left(P_{\pi_{o}, l, i}\left(\Pi_{l}\right)\right) \longrightarrow H^{s-l-i-1}\left(j_{! *}^{\geqslant(l+i+1) g}\right) \rightarrow 0
\end{aligned}
$$

On rappelle que $H^{j}\left(j_{! *}^{\geqslant(l+i+1) g}\right)$ est pur de poids $(s-l) g-(i+1)+j$ de sorte qu'en utilisant l'hypothèse de récurrence, les $H^{j}\left(P_{\pi_{o}, l, i}\left(\Pi_{l}\right)\right)$ sont de poids $(s-l)(g+1)-2(i+1+k-1)$ avec $1 \leqslant k \leqslant s-l-i$.

Soit alors $j$ de la forme $s-l-1-i-(2 r+1)$ avec $0 \leqslant 2 r+1 \leqslant 2(s-l-1-i)$; la suite exacte longue ci-dessus montre alors que les poids de $H^{j}\left(P_{\pi_{o}, l, i}\left(\Pi_{l}\right)\right)$ sont ceux de $H^{j}\left(P_{\pi_{o}, l, i+1}\left(\Pi_{l}\right)\right)$, i.e. $(s-l)(g+1)-2(i+1+k)$ pour $1 \leqslant k \leqslant s-l-i-1$ vérifiant $-2(k-1) \leqslant j-(s-l-2-i) \leqslant-(k-1)$. Le changement de variable $k^{\prime}=k+1$ donne alors le résultat, i.e. $H^{j}\left(P_{\pi_{o}, l, i}\left(\Pi_{l}\right)\right)$ est de poids $(s-l)(g+1)-2\left(i+k^{\prime}\right)$ avec $1 \leqslant k^{\prime} \leqslant s-l-i$ vérifiant $-2\left(k^{\prime}-1\right)+1 \leqslant j-(s-l-1-i) \leqslant-\left(k^{\prime}-1\right)$ soit ce qui est prévu car $j-(s-l-1-i)$ est impair ${ }^{(22)}$.

Pour $j$ de la forme $s-l-1-i-2 r$ avec $0 \leqslant 2 r \leqslant 2(s-l-1-i)$, la suite exacte longue précédente montre que les poids de $H^{j}\left(P_{\pi_{o}, l, i}\left(\Pi_{l}\right)\right)$ sont, à priori, ceux de $H^{j}\left(P_{\pi_{o}, l, i+1}\left(\Pi_{l}\right)\right)$ ainsi que celui de $H^{j}\left(j_{! *}^{\geqslant(l+i+1) g}\right)$ soit $(s-l)(g+1)-2(i+1+r)$. D'après l'hypothèse de récurrence, $H^{j}\left(P_{\pi_{o}, l, i}\left(\Pi_{l}\right)\right)$ est de poids $(s-l)(g+1)-2(i+1+k)$ avec $1 \leqslant k \leqslant s-l-i-1$ vérifiant $-2(k-1) \leqslant$ $j-(s-l-2-i) \leqslant-(k-1)$, de sorte que $H^{j}\left(P_{\pi_{o}, l, i}\left(\Pi_{l}\right)\right)$ est de poids $(s-l)(g+1)-2\left(i+k^{\prime}\right)$ avec $1 \leqslant k^{\prime} \leqslant s-l-i$ vérifiant $-2\left(k^{\prime}-1\right)+1 \leqslant j-(s-l-1-i) \leqslant-\left(k^{\prime}-1\right)$ soit ce qui est prévu car le cas $j-(s-l-1-i)=-2\left(k^{\prime}-1\right)$ est justement donné par le poids de $H^{j}\left(j_{! *}^{\geqslant(l+i+1) g}\right)$ soit $(s-l)(g+1)-2(i+1+r)$.

Suite de la preuve du lemme (IV.8.3.10) : (iv) Dans la suite on ne considère que les parties de poids $(s-l)(g-1)$. D'après le lemme (IV.8.3.11), $H^{l-s}\left(P_{\pi_{o}, l, 0}\left(\Pi_{l}\right)\right)$ est nul de sorte que la suite exacte longue (IV.8.3.30) s'écrit

$$
\begin{aligned}
0 \rightarrow H^{l-s}\left(j_{!}^{\geqslant l g}\right) \longrightarrow \Pi_{l} \overrightarrow{\times}[\overrightarrow{s-l-1}]_{\pi_{o}} \otimes & \left(\Xi^{(s-l)(g-1) / 2} \otimes \bigoplus_{\xi \in \mathfrak{A}\left(\pi_{o}\right)} \xi^{-1}\right) \\
& \longrightarrow H^{1+l-s}\left(P_{\pi_{o}, l, 0}\left(\Pi_{l}\right)\right) \longrightarrow H^{1+l-s}\left(j_{!}^{\geqslant l g}\right) \rightarrow 0 \rightarrow \cdots
\end{aligned}
$$

Le cas $i_{0}=l-s$ découle alors du fait que les strates sont induites, i.e. si $H^{l-s}\left(j_{!}^{\geqslant l g}\right)$ est un sous-espace de $\Pi_{l} \overrightarrow{\times}[\overrightarrow{s-l-1}]_{\pi_{o}} \otimes\left(\Xi^{\frac{(s-l)(g-1)}{2}} \otimes \bigoplus_{\xi \in \mathfrak{A}\left(\pi_{o}\right)} \xi^{-1}\right)$ alors il est égal à tout l'espace.

$\left.\overline{{ }^{(22)} \text { Le cas } j-(s}-l-1-i\right)=-2\left(k^{\prime}-1\right)$ n'est pas à considérer. 
Pour $i_{0}=1+l-s,($ IV.8.3.30) s'écrit

$$
\begin{aligned}
0 \rightarrow \Pi_{l} \overrightarrow{\times}[\overrightarrow{s-l-1}]_{\pi_{o}} \otimes\left(\Xi^{\frac{(s-l)(g-1)}{2}} \otimes \bigoplus_{\xi \in \mathfrak{A}\left(\pi_{o}\right)} \xi^{-1}\right) \longrightarrow \\
H^{1+l-s}\left(P_{\pi_{o}, l, 0}\left(\Pi_{l}\right)\right) \longrightarrow H^{1+l-s}\left(j_{!}^{\geqslant l g}\right) \rightarrow 0
\end{aligned}
$$

tandis que celle associée à la suite exacte courte

$$
0 \rightarrow P_{\pi_{o}, l, 1}\left(\Pi_{l}\right) \longrightarrow P_{\pi_{o}, l, 0}\left(\Pi_{l}\right) \longrightarrow \mathcal{P}_{-}\left(g, l+1, \pi_{o}, l, \Pi_{l}\right) \rightarrow 0
$$

s'écrit

$$
0 \rightarrow H^{1+l-s}\left(P_{\pi_{o}, l, 0}\left(\Pi_{l}\right)\right) \longrightarrow \Pi_{l} \overrightarrow{\times}[\overrightarrow{0}]_{\pi_{o}} \overrightarrow{\times}[\overrightarrow{s-l-2}]_{\pi_{o}} \otimes\left(\Xi^{\frac{(s-l)(g-1)}{2}} \otimes \bigoplus_{\xi \in \mathfrak{A}\left(\pi_{o}\right)} \xi^{-1}\right) \rightarrow \cdots
$$

Ainsi si $H^{1+l-s}\left(j_{!}^{\geqslant l g}\right)$ est non nul, alors $H^{1+l-s}\left(P_{\pi_{o}, l, 0}\left(\Pi_{l}\right)\right)$ contient strictement

$$
\left.\Pi_{l} \overrightarrow{\times} \overrightarrow{(s-l)}\right)_{\pi_{o}} \otimes\left(\Xi^{\frac{(s-l)(g-1)}{2}} \otimes \bigoplus_{\xi \in \mathfrak{A}\left(\pi_{o}\right)} \xi^{-1}\right)
$$

et étant de la forme $\Pi_{l} \overrightarrow{\times} \pi \otimes\left(\Xi \frac{(s-l)(g-1)}{2} \otimes \bigoplus_{\xi \in \mathfrak{A}\left(\pi_{o}\right)} \xi^{-1}\right)$ ainsi qu'un sous-espace de

$$
\Pi_{l} \overrightarrow{\times}[\overrightarrow{0}]_{\pi_{o}} \overrightarrow{\times}[\overrightarrow{s-l-2}]_{\pi_{o}} \otimes\left(\Xi^{\frac{(s-l)(g-1)}{2}} \otimes \bigoplus_{\xi \in \mathfrak{A}\left(\pi_{o}\right)} \xi^{-1}\right)
$$

on en déduit qu'il est égal à ce dernier de sorte que $H^{1+l-s}\left(j_{!}^{\geqslant l g}\right)$ est isomorphe à

$$
\Pi_{l} \overrightarrow{\times}[\overleftarrow{1}, \overrightarrow{s-l-1}]_{\pi_{o}} \otimes\left(\Xi^{\frac{(s-l)(g-1)}{2}} \otimes \bigoplus_{\xi \in \mathfrak{A}\left(\pi_{o}\right)} \xi^{-1}\right)
$$

d'où le résultat.

Par ailleurs on remarque de la même façon que si $i_{0}>1+l-s$, la partie de poids $(s-l)(g-1)$ de $H^{1+l-s}\left(P_{\pi_{o}, l, 0}\left(\Pi_{l}\right)\right)$ est alors égale à

$$
\Pi_{l} \overrightarrow{\times}[\overrightarrow{s-l-1}]_{\pi_{o}} \otimes\left(\Xi^{\frac{(s-l)(g-1)}{2}} \otimes \bigoplus_{\xi \in \mathfrak{A}\left(\pi_{o}\right)} \xi^{-1}\right) .
$$

Supposons alors $i_{0} \geqslant 2+l-s$. La suite exacte longue (IV.8.3.30) donne l'égalité pour tout $i \geqslant 2$, des parties de poids $(s-l)(g-1)$ de $H^{l-s+i}\left(P_{\pi_{o}, l, 0}\left(\Pi_{l}\right)\right)$ et de $H^{l-s+i}\left(j_{!}^{\geqslant l g}\right)$. On va montrer que, pour tout $2 \leqslant i \leqslant i_{0}-(l-s)$, la partie de poids $(s-l)(g-1)$ de $H^{l-s+r}\left(P_{\pi_{o}, l, i-2}\left(\Pi_{l}\right)\right)$ est nulle pour $i \leqslant r<i_{0}-l+s$ et que celle de $H^{i+l-s-1}\left(P_{\pi_{o}, l, i-2}\left(\Pi_{l}\right)\right)$ est égale à $\Pi_{l} \overrightarrow{\times}[\overleftrightarrow{i-1}, \overrightarrow{s-l-i}]_{\pi_{o}} \otimes$ $\left(\Xi^{\frac{(s-l)(g-1)}{2}} \otimes \bigoplus_{\xi \in \mathfrak{A}\left(\pi_{o}\right)} \xi^{-1}\right)$. D'après ce que l'on vient de voir, c'est vrai pour $i=2$. Supposons donc le résultat acquis jusqu'au rang $i$ et traitons le cas de $i+1$. La suite exacte longue de cohomologie associée à la suite exacte courte de faisceaux pervers

$$
0 \rightarrow P_{\pi_{o}, l, i-1}\left(\Pi_{l}\right) \longrightarrow P_{\pi_{o}, l, i-2}\left(\Pi_{l}\right) \longrightarrow \mathcal{P}_{-}\left(g, l+i-1, \pi_{o}, l, \Pi_{l}\right) \rightarrow 0
$$

s'écrit

$$
\begin{array}{r}
0 \rightarrow H^{i-1+l-s}\left(P_{\pi_{o}, l, i-2}\left(\Pi_{l}\right)\right) \longrightarrow \Pi_{l} \overrightarrow{\times}[\overleftarrow{i-2}]_{\pi_{o}} \overrightarrow{\times}[\overrightarrow{s-l-i}]_{\pi_{o}} \otimes\left(\Xi^{\frac{(s-l)(g-1)}{2}} \otimes \bigoplus_{\xi \in \mathfrak{A}\left(\pi_{o}\right)} \xi^{-1}\right) \\
\longrightarrow H^{i+l-s}\left(P_{\pi_{o}, l, i-1}\left(\Pi_{l}\right)\right) \longrightarrow H^{i+l-s}\left(P_{\pi_{o}, l, i-2}\left(\Pi_{l}\right)\right) \rightarrow 0 \cdots \quad \text { (IV.8. }
\end{array}
$$

ainsi que l'égalité des parties de poids $(s-l)(g-1)$ des espaces $H^{i+l-s+r}\left(P_{\pi_{o}, l, i-1}\left(\Pi_{l}\right)\right)$ et $H^{i+l-s+r}\left(P_{\pi_{o}, l, i-2}\left(\Pi_{l}\right)\right)$ pour tout $r>0$. La nullité de la partie de poids $(s-l)(g-1)$ de $H^{i+l-s}\left(P_{\pi_{o}, l, i-2}\left(\Pi_{l}\right)\right)$ et l'isomorphisme

$$
H^{i+l-s-1}\left(P_{\pi_{o}, l, i-2}\left(\Pi_{l}\right)\right) \simeq \Pi_{l} \overrightarrow{\times}[\overleftarrow{i-2}, \overrightarrow{s-l-i+1}]_{\pi_{o}} \otimes\left(\Xi^{\frac{(s-l)(g-1)}{2}} \otimes \bigoplus_{\xi \in \mathfrak{A}\left(\pi_{o}\right)} \xi^{-1}\right)
$$

donne le cas $i+1$. 
Traitons désormais le cas $i=i_{0}-l+s$. On pose $i_{1}=i_{0}-l+s-2$ et considérons alors la suite exacte longue de cohomologie associée à

$$
0 \rightarrow P_{\pi_{o}, l, i_{1}+1}\left(\Pi_{l}\right) \longrightarrow P_{\pi_{o}, l, i_{1}}\left(\Pi_{l}\right) \longrightarrow \mathcal{P}_{-}\left(g, l+i_{1}+1, \pi_{o}, l, \Pi_{l}\right) \rightarrow 0
$$

qui s'écrit

$$
\begin{aligned}
0 \rightarrow H^{i_{0}-1}\left(P_{\pi_{o}, l, i_{1}}\left(\Pi_{l}\right)\right) \longrightarrow & \Pi_{l} \overrightarrow{\times}\left[\overleftarrow{i_{1}}\right]_{\pi_{o}} \overrightarrow{\times}\left[\overrightarrow{s-l-i_{1}-2}\right]_{\pi_{o}} \otimes\left(\Xi^{\frac{(s-l)(g-1)}{2}} \otimes \bigoplus_{\xi \in \mathfrak{A}\left(\pi_{o}\right)} \xi^{-1}\right) \\
& \longrightarrow H^{i_{0}}\left(P_{\pi_{o}, l, i_{1}+1}\left(\Pi_{l}\right)\right) \longrightarrow H^{i_{0}}\left(P_{\pi_{o}, l, i_{1}}\left(\Pi_{l}\right)\right) \rightarrow 0 \cdots \quad \text { IV } 8
\end{aligned}
$$

avec $H^{i_{0}}\left(P_{\pi_{o}, l, i_{1}}\left(\Pi_{l}\right)\right)=H^{i_{0}}\left(j_{!}^{\geqslant l g}\right)$ non nul par hypothèse. La suite exacte longue associée à

$$
0 \rightarrow P_{\pi_{o}, l, i_{1}+2}\left(\Pi_{l}\right) \longrightarrow P_{\pi_{o}, l, i_{1}+1}\left(\Pi_{l}\right) \longrightarrow \mathcal{P}_{-}\left(g, i_{1}+2, \pi_{o}, l, \Pi_{l}\right) \rightarrow 0
$$

s'écrit

$$
\begin{aligned}
0 \rightarrow H^{i_{0}}\left(P_{\pi_{o}, l, i_{1}+1}\left(\Pi_{l}\right)\right) \longrightarrow \Pi_{l} \overrightarrow{\times}\left[\overleftarrow{i_{1}+1}\right]_{\pi_{o}} \overrightarrow{\times}\left[\overrightarrow{s-l-i_{1}-3}\right]_{\pi_{o}} \\
\otimes\left(\Xi^{\frac{(s-l)(g-1)}{2}} \otimes \bigoplus_{\xi \in \mathfrak{A}\left(\pi_{o}\right)} \xi^{-1}\right) \longrightarrow \cdots
\end{aligned}
$$

Ainsi si on veut que $H^{i_{0}}\left(P_{\pi_{o}, l, i_{1}}\left(\Pi_{l}\right)\right)$ soit non nul, il faut que $H^{i_{0}}\left(P_{\pi_{o}, l, i_{1}+1}\left(\Pi_{l}\right)\right)$ soit égal à $\Pi_{l} \overrightarrow{\times}\left[\overleftarrow{i_{1}+1}\right]_{\pi_{o}} \overrightarrow{\times}\left[\overrightarrow{s-l-i_{1}-3}\right]_{\pi_{o}} \otimes\left(\Xi^{\frac{(s-l)(g-1)}{2}} \otimes \bigoplus_{\xi \in \mathfrak{A}\left(\pi_{o}\right)} \xi^{-1}\right)$ et donc

$$
H^{i_{0}}\left(P_{\pi_{o}, l, i_{1}+1}\left(\Pi_{l}\right)\right) \simeq \Pi_{l} \overrightarrow{\times}\left[\overleftarrow{i_{1}+2}, \overrightarrow{s-l-i_{1}-3}\right]_{\pi_{o}} \otimes\left(\Xi^{\frac{(s-l)(g-1)}{2}} \otimes \bigoplus_{\xi \in \mathfrak{A}\left(\pi_{o}\right)} \xi^{-1}\right)
$$

d'où le résultat.

Retour sur la preuve de la proposition (IV.8.3.9) :

Remarque : Si on savait que les strates $M_{I, s_{o}}^{=h}$ étaient affines, la proposition (IV.8.3.9) découlerait directement du point (iii) du lemme (IV.8.3.10) pour $k=s-l+1$ car seul $H_{c}^{d-l g}\left(M_{I, s_{o}}^{=h}, \mathcal{F}\left(g, l, \pi_{o}, I\right) \otimes\right.$ $\left.\mathcal{L}_{\rho_{\infty}}\right)\left[\Pi^{\infty, o}\right]$ pourrait être de poids $l-s$ ce qui serait contradictoire avec (IV.2.2.13). Ne disposant pas de l'affinité des strates $M_{I, s_{o}}^{=h}$, on entre plus précisément dans la combinatoire.

On raisonne par récurrence sur $l$ de 1 à $s_{g}$ puis pour $l$ fixé, par récurrence sur $i$ de $l-s$ à 0 . L'initialisation de la récurrence pour $l=1$ se traite comme le passage de $l$ à $l+1$; on suppose alors le résultat acquis pour tout $1 \leqslant l^{\prime}<l^{(23)}$.

- Pour $i=l-s$, si l'espace en question était non nul, on aurait d'après le point (iv) du lemme (IV.8.3.10),

$$
\begin{aligned}
& \lim _{\vec{I}} H_{c}^{(s-l)(g-1)}\left(M_{I, s_{o}}, H T_{\rho_{\infty}}\left(g, l, \pi_{o}, \Pi_{l}\right)\right)\left[\Pi^{\infty, o}\right]= \\
& \Pi_{l} \overrightarrow{\times}[\overrightarrow{s-l-1}]_{\pi_{o}} \otimes\left(\Xi^{\frac{(s-l)(g-1)}{2}} \otimes \bigoplus_{\xi \in \mathfrak{A}\left(\pi_{o}\right)} \xi^{-1}\right) .
\end{aligned}
$$

On considère alors la suite spectrale (IV.8.1.23) pour $i=l(g-1)$. On remarque que toutes les parties de poids $s(g-1)$ de $E_{1}^{p, q ; l(g-1)}\left[\Pi^{\infty, o}\right]$ sont nulles pour $p-1 \neq l g$; en effet pour $p-1<l g$ cela découle de l'hypothèse de récurrence et pour $p-1>l g$ du fait que $l^{\prime}(g-1)>l(g-1)$ pour $p-1=l^{\prime} g$. On obtient alors

$$
\begin{aligned}
\underset{I}{\lim } H^{(s-l)(g-1)}\left(M_{I, \bar{s}_{o}}, R^{l(g-1)} \Psi_{\eta_{o}, I, \pi_{o}}\left(\mathcal{L}_{\rho_{\infty}}\right)\right)\left[\Pi^{\infty, o}\right] & \\
& {[\overleftarrow{l-1}]_{\pi_{o}} \vec{x}[\overrightarrow{s-l-1}]_{\pi_{o}} \otimes L_{g}\left(\pi_{o}\right)\left(-\frac{s(g-1)}{2}\right) }
\end{aligned}
$$

${ }^{(23)}$ Pour $l=1$, l'hypothèse de récurrence est vide. 
On considère alors la suite spectrale (IV.8.1.24), de sorte que $E_{2, \rho_{\infty}}^{(s-l)(g-1), l(g-1)}\left[\Pi^{\infty, o}\right]$ est isomorphe à l'espace ci-dessus. Or comme d'après l'hypothèse de récurrence toutes les parties de poids $s(g-1)$ $\operatorname{des} E_{2, \rho_{\infty}}^{p, q}\left[\Pi^{\infty, o}\right]$ pour $q<l(g-1)$ sont nulles et que d'après le lemme (IV.8.3.10) il en est de même pour $p+q<d-s$, on en déduit que $E_{\infty, \rho_{\infty}}^{d-s}\left[\Pi^{\infty, o}\right]$ admettrait $[\overleftarrow{l-1}]_{\pi_{o}} \overrightarrow{\times}[\overrightarrow{s-l-1}]_{\pi_{o}} \otimes$ $L_{g}\left(\pi_{o}\right)\left(-\frac{s(g-1)}{2}\right)$ comme sous-espace ce qui n'est pas d'après la proposition (IV.8.3.8).

- Supposons donc le résultat vérifié pour tout $l-s \leqslant i=l-s+\delta \leqslant i_{0}<-1$ et traitons le cas de $i_{0}$ D'après le point (iv) du lemme (IV.8.3.10), on obtiendrait

$$
[\overleftarrow{l-1}]_{\pi_{o}} \overrightarrow{\times}[\overleftarrow{\delta}, \overrightarrow{s-l-1-\delta}]_{\pi_{o}} \otimes\left(\Xi^{\frac{(s-l)(g-1)}{2}} \otimes \bigoplus_{\xi \in \mathfrak{A}\left(\pi_{o}\right)} \xi^{-1}\right)
$$

Comme précédemment, la suite spectrale (IV.8.1.23) pour $i=l(g-1)$, donne que la partie de poids $s(g-1)$ de $E_{2, \rho_{\infty}}^{(s-l)(g-1)+\delta, l(g-1)}\left[\Pi^{\infty, o}\right]$ dans la suite spectrale (IV.8.1.24) est isomorphe à

$$
[\overleftarrow{l-1}]_{\pi_{o}} \overrightarrow{\times}[\overleftarrow{\delta}, \overrightarrow{s-l-1-\delta}]_{\pi_{o}} \otimes L_{g}\left(\pi_{o}\right)\left(-\frac{s(g-1)}{2}\right)
$$

On remarque alors que $E_{\infty, \rho_{\infty}}^{d-s+\delta}\left[\Pi^{\infty, o}\right]$ admettrait $[\overleftarrow{l+\delta}, \overrightarrow{s-l-1-\delta}]_{\pi_{o}} \otimes L_{g}\left(\pi_{o}\right)\left(-\frac{s(g-1)}{2}\right)$ comme sous-espace car ce dernier n'apparaît pas dans les $E_{2, \rho_{\infty}}^{p, q}\left[\Pi^{\infty, o}\right]$ pour $q<l(g-1)$ d'après l'hypothèse de récurrence, ni pour $q=l^{\prime}(g-1)>l(g-1)$ d'après le lemme (IV.8.3.10) (i) ainsi que le corollaire (IV.8.1.3).

IV.8.4. Preuve de IV.7.1.1. - Il s'agit donc de prouver la proposition (IV.7.1.1). En étudiant les parties de poids $s(g-1)$ des composantes $\Pi^{\infty, o}$ isotypiques pour $\Pi$ irréductible automorphe tel que $\Pi_{o} \simeq \operatorname{Speh}_{s}\left(\pi_{o}\right)$, on s'est ramené d'après le paragraphe précédent, à une situation similaire à celle de [5], i.e. dans la suite spectrale des cycles évanescents, seuls les points supersinguliers contribuent. La fin de la preuve procède alors exactement comme dans loc. cit.

Précisément, la partie de poids $s(g-1)$ de $\widetilde{\mathcal{U}_{F_{o}}^{d, d-s}}\left(\mathrm{JL}^{-1}\left([\overleftarrow{s-1}]_{\pi_{o}}\right)\right)$, d'après le corollaire (IV.8.1.3), est un constituant de $[\overrightarrow{s-2}]_{\pi_{o}} \overrightarrow{\times}[\overrightarrow{0}]_{\pi_{o}} \otimes L_{g}\left(\pi_{o}\right)\left(-\frac{s(g-1)}{2}\right)$. On considère alors la suite spectrale (IV.8.3.28) pour $i=d-s$, où, d'après le corollaire (IV.5.4.9), tous les $E_{1, \rho_{\infty}}^{p, q ; d-s}$ sont nuls pour $p+q \neq 0$ ou $p-1 \neq d$ de sorte que le terme $E_{2, \rho_{\infty}}^{0, d-s}\left[\Pi^{\infty, o}\right]$ de (IV.8.3.27), et donc $E_{\infty, \rho_{\infty}}^{0, d-s}\left[\Pi^{\infty, o}\right]$ d'après (IV.8.3.9), est égal à cet espace qui est donc, d'après (IV.8.3.8), égal à $[\overrightarrow{s-1}]_{\pi_{o}} \otimes L_{g}\left(\pi_{o}\right)\left(-\frac{s(g-1)}{2}\right)$.

On suppose avoir montré par récurrence que pour tout $0 \leqslant r<r_{0}<s-1$, les parties de poids $s(g-1)$ de $\mathcal{U}_{F_{o}} \widetilde{d, d-s+r}\left(\mathrm{JL}^{-1}\left([\overleftarrow{s-1}]_{\pi_{o}}\right)\right)$ sont comme prévues, i.e. nulles pour $r \neq 0$, et égales à $[\overrightarrow{s-1}]_{\pi_{o}} \otimes L_{g}\left(\pi_{o}\right)\left(-\frac{s(g-1)}{2}\right)$ pour $r=0$. D'après le corollaire (IV.8.1.3), la partie de poids $s(g-1)$ de $\mathcal{U}_{F_{o}}^{\widehat{d, d-s+r_{0}}}\left(\mathrm{JL}^{-1}\left([\overleftarrow{s-1}]_{\pi_{o}}\right)\right)$ est un constituant de $\left[\overrightarrow{s-2-r_{0}}\right]_{\pi_{o}} \overrightarrow{\times}\left[\overleftarrow{r_{0}}\right]_{\pi_{o}} \otimes L_{g}\left(\pi_{o}\right)\left(-\frac{s(g-1)}{2}\right)$. Or comme la partie de poids $s(g-1)$ de $\sum_{i=0}^{s-1}(-1) i \widetilde{\mathcal{U}_{F_{o}}^{d, d-s+i}}\left(\mathrm{JL}^{-1}\left([\overleftarrow{s-1}]_{\pi_{o}}\right)\right)$ est égale à $[\overrightarrow{s-1}]_{\pi_{o}} \otimes$ $L_{g}\left(\pi_{o}\right)\left(-\frac{s(g-1)}{2}\right)$, en remarquant, d'après le corollaire (IV.8.1.3), que

$$
\left[\overrightarrow{s-1-r_{0}}, \overleftarrow{r_{0}+1}\right]_{\pi_{o}} \otimes L_{g}\left(\pi_{o}\right)\left(-\frac{s(g-1)}{2}\right)
$$

ne peut pas être un constituant de $\widetilde{\mathcal{U}_{F_{o}}^{d, d-s+r}}\left(\mathrm{JL}^{-1}\left([\overleftarrow{s-1}]_{\pi_{o}}\right)\right)$ pour $r>r_{0}$, on en déduit que si la partie de poids $s(g-1)$ de $\mathcal{U}_{F_{o}}^{\widehat{d, d-s+r_{0}}}\left(\mathrm{JL}^{-1}\left([\overleftarrow{s-1}]_{\pi_{o}}\right)\right)$ est non nulle, elle est alors égale à $\left[\overrightarrow{s-2-r_{0}}, \overleftarrow{r_{0}+2}\right]_{\pi_{o}} \otimes L_{g}\left(\pi_{o}\right)\left(-\frac{s(g-1)}{2}\right)$. Comme précédemment ce dernier espace est aussi égal à la partie de poids $s(g-1)$ de $E_{\infty, \rho_{\infty}}^{d-s+r_{0}}\left[L_{g}\left(\pi_{o}\right)\right]\left[\Pi^{\infty, o}\right]$ ce qui n'est pas d'après (IV.8.3.8).

Finalement le cas $r_{0}=s-1$ est donné en utilisant que la partie de poids $s(g-1)$ de $\sum_{i=0}^{s-1}(-1)^{i} \mathcal{U}_{F_{o}}^{d, d-s+i}\left(\mathrm{JL}^{-1}\left([\overleftarrow{s-1}]_{\pi_{o}}\right)\right)$ est égale à $[\overrightarrow{s-1}]_{\pi_{o}} \otimes L_{g}\left(\pi_{o}\right)\left(-\frac{s(g-1)}{2}\right)$. 
IV.8.4.1. Remarque. - Comme dans la remarque (IV.8.3.6), on aurait pu traiter tous les poids $\operatorname{des} \widetilde{\mathcal{U}_{F_{o}}^{d, i}}$. Il suffit pour cela de montrer un analogue du point (iv) de la proposition (IV.8.3.9) pour tous les poids, ce qui ne pose aucune difficulté sauf qu'on ne se retrouve pas dans une situation aussi favorable où seuls les points supersinguliers contribuent. 


\section{CHAPITRE V}

\section{COMPLÉMENTS SUR LA COHOMOLOGIE GLOBALE ET APPLICATIONS}

\section{Introduction}

On donne quelques applications des calculs du chapitre précédent.

0.1. - On commence, proposition (V.1.1), par calculer les $\Pi^{\infty, o}$-parties des groupes de cohomologie de la fibre générique pour $\Pi$ automorphe tel que $\Pi_{o} \simeq \operatorname{Speh}_{s}\left(\pi_{o}\right)$, alors que nous n'avions traité, corollaire (IV.8.3.5), que celles de poids $s(g-1){ }^{(1)}$

0.2. - On en déduit ensuite, proposition (V.2.1), une correspondance de Jacquet-Langlands entre les représentations automorphes de $D_{\mathbb{A}}^{\times}$et celles de $\bar{D}_{\mathbb{A}}^{\times}$: d'après les experts ce résultat s'obtient aisément à partir de la formule des traces simples.

0.3. - On décrit, proposition (V.3.1), les composantes locales des représentations automorphes de $D_{\mathbb{A}}^{\times}$: au final en égale caractéristique, corollaire (V.3.4), on obtient ce que l'on déduirait des résultats de Moeglin-Waldspurger sur les représentations de carré intégrable de $G L_{d}(\mathbb{A})$ et de la conjecture de Ramanujan-Peterson, prouvée par Lafforgue dans ce cadre, si on disposait d'une correspondance de Jacquet-Langlands globale entre $G L_{d}(\mathbb{A})$ et $D_{\mathbb{A}}^{\times}$. (2)

0.4. - On conclut enfin, théorème (V.4.1), par la preuve, qui n'a d'intérêt qu'en caractéristique mixte, de la conjecture de monodromie-poids globale, i.e. les gradués de la filtration de monodromie des groupes de cohomologie de la fibre générique sont purs.

\section{V.1. Preuve de la conjecture (14.21) de [LRS]}

V.1.1. Proposition. - (cf. [19] (14.21)) Soient g un diviseur de $d=$ sg et $\pi_{o}$ (resp. $\left.\pi_{\infty}\right)$ une représentation irréductible cuspidale unitaire de $G L_{g}\left(F_{o}\right)$ (resp. de $G L_{g}\left(F_{\infty}\right)$ ). On considère une représentation automorphe $\Pi$ de $D_{\mathbb{A}}^{\times}$telle que $\Pi_{o} \simeq[\overrightarrow{s-1}]_{\pi_{o}}\left(\right.$ resp. $\left.\Pi_{\infty} \simeq[\overrightarrow{s-1}]_{\pi_{\infty}}\right)$. Le $G L_{d}\left(F_{o}\right) \times W_{o}$-module $H_{\eta_{o}, \rho_{\infty}}^{d+s-2-2 i}\left[\Pi^{\infty, o}\right]$ est alors isomorphe $\grave{a}$

$$
[\overrightarrow{s-1}]_{\pi_{o}} \otimes L_{g}\left(\pi_{o}\right)\left(-\frac{d+s-2-2 i}{2}\right)
$$

pour $0 \leqslant i<s$ et $\rho_{\infty}:=\mathrm{JL}^{-1}\left([\overleftarrow{s-1}]_{\pi_{\infty}}\right)$.

Démonstration. - La proposition (IV.8.3.8) joint au théorème de Lefschetz difficile implique que pour tout $0 \leqslant r \leqslant s-1, H_{\eta_{o}, \rho_{\infty}}^{d-s+2 r}\left[\Pi^{\infty, o}\right]$ admet $[\overrightarrow{s-1}]_{\pi_{o}} \otimes L_{g}\left(\pi_{o}\right)\left(-\frac{s(g-1)+2 r}{2}\right)$ comme facteur direct. On reprend la suite spectrale (IV.8.3.26) en utilisant la proposition (IV.8.3.4) ainsi que

(1) Ce résultat correspond à la conjecture 14.21 de [19].

(2) En particulier, on en déduit que l'hypothèse 14.23 de [19] est vérifiée. 
le théorème de Lefschetz difficile. On pourra se reporter à la figure (2) où l'on a représenté les $H^{i}\left(\operatorname{gr}_{k, \rho_{\infty}}\right)\left[\Pi^{\infty, o}\right]$ dans le cas $s=4$. On rappelle tout d'abord que pour $1<\delta<s$, on a

$$
\begin{aligned}
& H^{-\delta}\left(g r_{k, \rho_{\infty}}\right)\left[\Pi^{\infty, o}\right]=\bigoplus_{\substack{|k|<l \leqslant s \\
l=k+1 \bmod 2 \\
l \equiv s+\delta \bmod 2}}\left([\overleftarrow{l-1}]_{\pi_{o}} \overrightarrow{\times}\left[\frac{\overrightarrow{s-l+\delta-2}}{2}\right]_{\pi_{o}}\right) \overleftarrow{\times}\left[\frac{\overrightarrow{s-l-\delta-2}}{2}\right]_{\pi_{o}} \\
& \otimes L_{g}\left(\pi_{o}\right)\left(-\frac{d-1-\delta+k}{2}\right) \\
& H^{\delta}\left(g r_{k, \rho_{\infty}}\right)\left[\Pi^{\infty, o}\right]=\bigoplus_{\substack{\mid k<l \leq s \\
l \equiv k+1 \text { mod } 2 \\
l \equiv s+\delta \text { mod } 2}}\left([\overleftarrow{l-1}]_{\pi_{o}} \overrightarrow{\times}\left[\frac{s-l-\delta-2}{2}\right]_{\pi_{o}}\right) \overleftarrow{\times}\left[\frac{s-l+\delta-2}{2}\right]_{\pi_{o}} \\
& \otimes L_{g}\left(\pi_{o}\right)\left(-\frac{d-1+\delta+k}{2}\right)
\end{aligned}
$$

On remarque ainsi que tous les $[\overrightarrow{s-1}]_{\pi_{o}}$ des $E_{1, \rho_{\infty}}^{p, q}\left[\Pi^{\infty, o}\right]$ de (IV.8.3.26) restent dans l'aboutissement; il nous faut alors montrer que tous les autres disparaissent. Dans la suite on ne considérera plus les $[\overrightarrow{s-1}]_{\pi_{o}}$.

Prenons dans un premier temps $\delta>1$, de sorte qu'outre les $[\overrightarrow{s-1}]_{\pi_{o}}$, les éventuels constituants non nuls de $E_{\infty, \rho_{\infty}}^{d-s-\delta}\left[\Pi^{\infty, o}\right]$ (resp. de $E_{\infty, \rho_{\infty}}^{d-s+\delta}\left[\Pi^{\infty, o}\right]$ ) sont de la forme

$$
[\vec{r}, \overleftarrow{l-1}, \overrightarrow{s-l-r}]_{\pi_{o}} \otimes L_{g}\left(\pi_{o}\right)\left(-\frac{d-1-\delta-k}{2}\right)
$$

avec $s-l-r=r+\delta, r+\delta+1, r+\delta-1$ (resp. $s-l-r=r-\delta, r-\delta-1, r-\delta+1$ ) et certains entiers $k$ qu'il n'est pas nécessaire de préciser. Pour $\delta \geqslant 2$, on remarque que les ensembles $\{\delta, \delta+1, \delta-1\}$ et $\{-\delta,-\delta+1,-\delta-1\}$ sont disjoints de sorte que d'après le théorème de Lefschetz difficile, l'éventuel constituant est forcément nul. ${ }^{(3)}$

Pour $\delta=1$, le raisonnement est plus fin et demande de distinguer les sous-espaces des quotients dans nos induites. Si on reprend le raisonnement précédent dans le cas $\delta=1$, le théorème de Lefschetz difficile impose que les éventuels constituants de $H_{\eta_{o}, \rho_{\infty}}^{d-1 \pm 1}\left[\Pi^{\infty, o}\right]$ sont de la forme

$$
\left[\frac{\overrightarrow{s-l}}{2}, \overleftarrow{l-1}, \frac{\overrightarrow{s-l}}{2}\right]_{\pi_{o}} \otimes L_{g}\left(\pi_{o}\right)\left(-\frac{d-1-k}{2}\right)
$$

pour $1 \leqslant l \leqslant s, 0 \leqslant r \leqslant(s-l) / 2$ et certains entiers $k$ qu'il n'est pas nécessaire de préciser. On revient sur (V.1.0.33) et sur la suite spectrale (IV.8.3.26). Ainsi on a

$$
\begin{aligned}
E_{1, \rho_{\infty}}^{-k,-1+k}\left[\Pi^{\infty, o}\right]= & \bigoplus_{\substack{|k|<l \leq s \\
l=k+1 \bmod 2 \\
l \equiv s-1 \bmod 2}}\left([\overleftarrow{l-1}]_{\pi_{o}} \overrightarrow{\times}\left[\frac{s-l-1}{2}\right]_{\pi_{o}}\right) \overleftarrow{\times}\left[\frac{\overrightarrow{s-l-3}}{2}\right]_{\pi_{o}} \\
E_{1, \rho_{\infty}}^{-k-1,-1+k}\left[\Pi^{\infty, o}\right]= & \otimes L_{g}\left(\pi_{o}\right)\left(-\frac{d-2+k}{2}\right) \\
\bigoplus_{\substack{\mid l k<l \leq s \\
l \equiv k+1 \bmod 2 \\
l \equiv s-2 \bmod 2}}\left([\overleftarrow{l}]_{\pi_{o}} \overrightarrow{\times}\left[\frac{\overrightarrow{s-l}}{2}\right]_{\pi_{o}}\right) \overleftarrow{\times}\left[\frac{\overrightarrow{s-l-4}}{2}\right]_{\pi_{o}} & \otimes L_{g}\left(\pi_{o}\right)\left(-\frac{d-2+k}{2}\right)
\end{aligned}
$$

\footnotetext{
(3) On peut aussi argumenter en remarquant que ce ne sont pas des constituants locaux licites d'une représentation automorphe alors que dans l'aboutissement seules celles ci doivent apparaître.
} 


$$
\begin{aligned}
E_{1, \rho_{\infty}}^{-k+1,-1+k}\left[\Pi^{\infty, o}\right]= & \bigoplus_{\substack{l k \mid<l \leqslant s \\
l=k+1 \text { mod } 2 \\
l \equiv s \bmod 2}}\left([\overleftarrow{l-1}]_{\pi_{o}} \overrightarrow{\times}\left[\frac{\overrightarrow{s-l-2}}{2}\right]_{\pi_{o}}\right) \overleftarrow{\times}\left[\frac{\overrightarrow{s-l-2}}{2}\right]_{\pi_{o}} \\
& \otimes L_{g}\left(\pi_{o}\right)\left(-\frac{d-2+k}{2}\right)
\end{aligned}
$$

Pour $k=s-1$, on remarque que la partie de poids $s(g+1)-2$ de $H_{\eta_{o}, \rho_{\infty}}^{d-2}\left[\Pi^{\infty, o}\right]$ est un sous-espace de $[\overleftarrow{s-2}]_{\pi_{o}} \overrightarrow{\times}[\overrightarrow{0}]_{\pi_{o}} \otimes L_{g}\left(\pi_{o}\right)\left(-\frac{s(g+1)-2}{2}\right)$. Or $[\overleftarrow{s-1}]_{\pi_{o}}$ n'étant pas un sous espace de $[\overleftarrow{s-2}]_{\pi_{o}} \overrightarrow{\times}[\overrightarrow{0}]_{\pi_{o}}$, on en déduit, en utilisant (V.1.0.35), que la partie de poids $s(g+1)-2$ de $H_{\eta_{o}, \rho_{\infty}}^{d-2}\left[\Pi^{\infty, o}\right]$ est nulle.

Pour $0 \leqslant k \leqslant s-3$, on a :

- $E_{1, \rho_{\infty}}^{-k,-1+k}\left[\Pi^{\infty, o}\right]=E_{1, \rho_{\infty}}^{-k-2, k+1}\left[\Pi^{\infty, o}\right] \otimes|-|^{2} \oplus V_{k,-1} \otimes L_{g}\left(\pi_{o}\right)\left(-\frac{d-2+k}{2}\right)$, avec

$$
\begin{gathered}
V_{k,-1}=\left([\overleftarrow{k}]_{\pi_{o}} \overrightarrow{\times}\left[\frac{\overrightarrow{s-k-2}}{2}\right]_{\pi_{o}}\right) \overleftarrow{\times}\left[\frac{\overrightarrow{s-k-4}}{2}\right]_{\pi_{o}} ; \\
-E_{1, \rho_{\infty}}^{-k-1,-1+k}\left[\Pi^{\infty, o}\right]=E_{1, \rho_{\infty}}^{-k-3, k+1}\left[\Pi^{\infty, o}\right] \otimes|-|^{2} \oplus V_{k,-2} \otimes L_{g}\left(\pi_{o}\right)\left(-\frac{d-2+k}{2}\right), \text { avec } \\
V_{k,-2}=\left([\overleftarrow{k+1}]_{\pi_{o}} \overrightarrow{\times}\left[\frac{\overrightarrow{s-k-2}}{2}\right]_{\pi_{o}}\right) \overleftarrow{\times}\left[\frac{\overrightarrow{s-k-6}}{2}\right]_{\pi_{o}} ; \\
-E_{1, \rho_{\infty}}^{-k+1,-1+k}\left[\Pi^{\infty, o}\right]=E_{1, \rho_{\infty}}^{-k-1, k+1}\left[\Pi^{\infty, o}\right] \otimes|-|^{2} \oplus V_{k, 0} \otimes L_{g}\left(\pi_{o}\right)\left(-\frac{d-2+k}{2}\right) \text { avec } V_{0,0}=0 \text { et } \\
V_{k, 0}=\left([\overleftarrow{k-1}]_{\pi_{o}} \overrightarrow{\times}\left[\frac{\overrightarrow{s-k-4}}{2}\right]_{\pi_{o}}\right) \overleftarrow{\times}\left[\frac{\overrightarrow{s-k-4}}{2}\right]_{\pi_{o}} \text { pour } 0<k \leqslant s-3 .
\end{gathered}
$$

On suppose alors par récurrence que

$$
E_{\infty}^{-k-2, k+1}\left[\Pi^{\infty, o}\right]=\left(\operatorname{Ker} d_{1}^{-k-2, k+1} / \operatorname{Im} d_{1}^{-k-3, k+1}\right)\left[\Pi^{\infty, o}\right]
$$

est nulle; l'opérateur de monodromie $N$ implique alors que $E_{\infty}^{-k,-1+k}\left[\Pi^{\infty, o}\right]$ est égal à Ker $d_{+} / \operatorname{Im} d_{-} \otimes L_{g}\left(\pi_{o}\right)\left(-\frac{d-2+k}{2}\right)$ avec

$$
V_{k,-2} \stackrel{d_{-}}{\longrightarrow} V_{k,-1} \stackrel{d_{+}}{\longrightarrow} V
$$

Ainsi d'après (V.1.0.35), si $E_{\infty}^{-k,-1+k}\left[\Pi^{\infty, o}\right]$ était non nul, il serait égal à

$$
\left[\frac{\overrightarrow{s-k}}{2}, \overleftarrow{k-1}, \frac{\overrightarrow{s-k}}{2}\right]_{\pi_{o}} \otimes L_{g}\left(\pi_{o}\right)\left(-\frac{d-2+k}{2}\right) .
$$

En remarquant qu'aucun des constituants de $\left[\frac{\overrightarrow{s-k}}{2}, \overleftarrow{k-1}\right]_{\pi_{o}} \overrightarrow{\times}\left[\frac{\overrightarrow{s-k-2}}{2}\right]_{\pi_{o}}$ n'est un constituant de $V_{k,-2}$, on en déduit que quelque soit $d_{-}$, on a une surjection

$$
V_{k,-1} / \operatorname{Im} d_{-} \rightarrow\left[\frac{\overrightarrow{s-k}}{2}, \overleftarrow{k-1}\right]_{\pi_{o}} \overrightarrow{\times}\left[\frac{\overrightarrow{s-k-2}}{2}\right]_{\pi_{o}} \otimes L_{g}\left(\pi_{o}\right)\left(-\frac{d-2+k}{2}\right)
$$

alors que $\left[\frac{\overrightarrow{s-k}}{2}, \overleftarrow{k-1}, \frac{\overrightarrow{s-k}}{2}\right]_{\pi_{o}}$ n'est pas un sous-espace de $\left[\frac{\overrightarrow{s-k}}{2}, \overleftarrow{k-1}\right]_{\pi_{o}} \overrightarrow{\times}\left[\frac{\overrightarrow{s-k-2}}{2}\right]_{\pi_{o}}$ de sorte que $E_{\infty}^{-k,-1+k}\left[\Pi^{\infty, o}\right]$ est nul.

Pour $1-s \leqslant k \leqslant 0$, on peut, dans le cas d'égale caractéristique, conclure en utilisant les cas $0 \leqslant k \leqslant s-1$ et la pureté des $H_{\eta_{o}, \rho_{\infty}}^{i}$, dont les gradués pour la filtration de monodromie sont purs. Sinon on raisonne de manière strictement identique en étudiant les suites exactes

$$
E_{1, \rho_{\infty}}^{-k-1, k+1} \stackrel{d_{1}^{-k-1, k+1}}{\longrightarrow} E_{1, \rho_{\infty}}^{-k, k+1} \stackrel{d_{1}^{-k, k+1}}{\longrightarrow} E_{1, \rho_{\infty}}^{-k+1, k+1}
$$

et en remplaçant l'étude des sous-espaces par celle des quotients.

On donne alors l'amélioration suivante de (IV.2.2.13) qui constitue le pendant du corollaire (IV.5.4.3) dans le cas $\Pi_{\infty}=[\overrightarrow{s-1}]_{\pi_{\infty}}$. 
V.1.2. Corollaire. - Soit $\Pi$ une représentation irréductible automorphe de $D_{\mathbb{A}}^{\times}$telle que $\Pi_{\infty} \simeq$ $[\overrightarrow{s-1}]_{\pi_{\infty}}$ où $\pi_{\infty}$ est une représentation cuspidale de $\left.G L_{g}\left(F_{\infty}\right)\right)$ avec $d=s g$. On suppose que $\Pi_{o} \simeq[\overrightarrow{s-1}]_{\pi_{o}}$ où $\pi_{o}$ est une représentation irréductible cuspidale unitaire de $G L_{g}\left(F_{o}\right)$. Si

$$
\left[H_{h, \mathrm{JL}-1}^{i}\left([\overleftarrow{s-1}]_{\pi_{\infty}}\right), \tau_{o}\left(\Pi^{\infty, o}\right)\right]
$$

muni de son action naturelle de $G L_{d}\left(F_{o}\right)$ comme dans [19], est non nul dans le groupe de Grothendieck $\operatorname{Groth}\left(G L_{d-h}\left(F_{o}\right) \times\left(D_{o, h}^{\times} / \mathcal{D}_{o, h}^{\times}\right)\right)$, on a alors

- $\tau_{o}=\mathrm{JL}^{-1}\left([\overleftarrow{s-1}]_{\pi_{o}}\right)$;

$-h=l g$ avec $1 \leqslant l \leqslant s$ et $i=(s-l)(g+1)$.

Dans ce cas, ils sont donnés par

$$
\begin{aligned}
\lim _{\vec{I}} H_{c}^{(s-l)(g+1)}\left(M_{I, s_{o}, 1}^{=l g}, \mathcal{F}_{\tau_{o}} \otimes\right. & \left.\mathcal{L}_{\rho_{\infty}}\right)\left[\Pi^{\infty, o}\right] \simeq \\
& m(\Pi)[\overrightarrow{s-l-1}]_{\pi_{o}(l(g+1) / 2)} \otimes\left(\Xi^{\frac{(s-l)(g+1)}{2}} \bigoplus_{\xi \in \mathfrak{D}\left(\pi_{o}\right)} \xi^{-1}\right)
\end{aligned}
$$

en tant que représentation de $G L_{(s-l) g}\left(F_{o}\right) \times\left(D_{o, h}^{\times} / \mathcal{D}_{o, h}^{\times}\right)$, où $\mathfrak{D}\left(\pi_{o}\right)$ est l'ensemble des caractères $\xi$ de $\mathbb{Z} \simeq D_{o, h}^{\times} / \mathcal{D}_{o, h}^{\times}$tels que

$$
\mathrm{JL}^{-1}\left([\overleftarrow{s-1}]_{\pi_{o}}\right) \otimes \xi^{-1} \simeq \mathrm{JL}^{-1}\left([\overleftarrow{s-1}]_{\pi_{o}}\right)
$$

Démonstration. - On rappelle que d'après le lemme (IV.8.3.10), les composantes $\Pi^{\infty, o_{-}}$ isotypiques $\operatorname{des} H_{c}^{i}\left(M_{I, s_{o}}^{=l g}, \mathcal{F}\left(g, l, \pi_{o}\right) \otimes \mathcal{L}_{\rho_{\infty}}\right) \otimes \Pi_{l}$ sont mixtes de poids $(s-l)(g-1)+2 k$ avec $0 \leqslant k<s-l$ et de la forme

$$
\left(\Pi_{l} \overrightarrow{\times} \pi_{+}\right) \overleftarrow{\times} \pi_{-} \otimes L_{g}\left(\pi_{o}\right)\left(-\frac{(s-l)(g-1)+2 k}{2}\right)
$$

où $\pi_{+}\left(\right.$resp. $\left.\pi_{-}\right)$est une représentation elliptique de $G L_{(s-l-k) g}\left(F_{o}\right)$ (resp. de $G L_{k g}\left(F_{o}\right)$ ). On raisonne alors par récurrence sur $k$, de 0 à $s-l-2$, afin de montrer que pour tout $i$, les parties de poids $(s-l)(g-1)+2 k$ des $H_{c}^{i}\left(M_{I, s_{o}}^{=l g}, \mathcal{F}\left(g, l, \pi_{o}, I\right) \otimes \mathcal{L}_{\rho_{\infty}}\right)\left[\Pi^{\infty, o}\right]$ sont nulles. Le cas $k=0$ a été traité dans la proposition (IV.8.3.9). Supposons le résultat acquis jusqu'au rang $k<s-l-2$ et montrons le au rang $k+1$. On raisonne alors par récurrence sur $l$ de 1 à $s-1$. L'initialisation de la récurrence se prouve comme le cas général; on suppose donc le résultat acquis jusqu'au rang $l<s-2$ et prouvons le au rang $l+1$.

Supposons qu'il existe $j$ tel que la partie de poids $(s-l)(g-1)+2(k+1)$ de $H_{c}^{j}\left(M_{I, s_{o}}^{=l g}, \mathcal{F}\left(g, l, \pi_{o}, I\right) \otimes\right.$ $\left.\mathcal{L}_{\rho_{\infty}}\right)\left[\Pi^{\infty, o}\right]$ soit non nulle. On étudie ensuite la suite spectrale (IV.8.3.28) associée à la stratification pour $i=l(g-1)$. Ainsi la partie de poids $m_{k}:=s(g-1)+2(k+1)$ de $E_{1, \rho_{\infty}}^{l g+1, j-l g-1 ; l(g-1)}\left[\Pi^{\infty, o}\right]$ est non nulle et de la forme $\left([\overrightarrow{l-1}]_{\pi_{o}} \overrightarrow{\times} \pi_{+}\right) \overleftarrow{\times} \pi_{-} \otimes L_{g}\left(\pi_{o}\right)\left(-\frac{m_{k}}{2}\right)$, où $\pi_{+}$(resp. $\pi_{-}$) est une représentation elliptique de $G L_{(s-l-k) g}\left(F_{o}\right)$ (resp. de $G L_{k g}\left(F_{o}\right)$ ). Or d'après l'hypothèse de récurrence pour tout $1 \leqslant l^{\prime}<l$, les parties de poids $m_{k}=\left(s-l^{\prime}\right)(g-1)+l^{\prime}(g-1)+2(k+1)$ des $E_{1, \rho_{\infty}}^{l^{\prime} g+1, j^{\prime}-l^{\prime} g-1 ; l(g-1)}\left[\Pi^{\infty, o}\right]$ sont nulles; en effet celles-ci sont données par les parties de poids $\left(s-l^{\prime}\right)(g-1)+2 k^{\prime}$ avec $s(g-1)+2(k+1)=\left(s-l^{\prime}\right)(g-1)+l^{\prime}(g-1)+2 \delta+2 k^{\prime}$ avec $l(g-1)=l^{\prime}(g-1)+\delta, 0<\delta<l^{\prime}<l$, soit $k^{\prime}=k+1-\delta$. Pour $l^{\prime}>l$, les parties de poids $m_{k}$ des $E_{1, \rho_{\infty}}^{l^{\prime} g+1, j^{\prime}-l^{\prime} g-1 ; l(g-1)}\left[\Pi^{\infty, o}\right]$ sont nulles car $l(g-1)$ ne s'écrit pas sous la forme $l^{\prime}(g-1)+\delta$ avec $0 \leqslant \delta<l^{\prime}$.

On étudie alors la suite spectrale (IV.8.3.27) des cycles évanescents. D'après ce qui précède, la partie de poids $m_{k}$ de $E_{2, \rho_{\infty}}^{j, l(g-1)}\left[\Pi^{\infty, o}\right]$ est de la forme $\left([\overrightarrow{l-1}]_{\pi_{o}} \overrightarrow{\times} \pi_{+}\right) \overleftarrow{\times} \pi_{-} \otimes L_{g}\left(\pi_{o}\right)\left(-\frac{m_{k}}{2}\right)$. Par ailleurs les parties de poids $m_{k}$ des $E_{2, \rho_{\infty}}^{j+r+1, l(g-1)-r}\left[\Pi^{\infty, o}\right]$, pour $r \geqslant 1$ sont nulles; en effet celles-ci proviendraient à travers la suite spectrale (IV.8.3.28), des parties de poids $\left(s-l^{\prime}\right)(g-1)+2 k^{\prime}$ de 


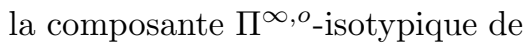

$$
H_{c}^{j+r-1}\left(M_{I, s_{o}}^{=l^{\prime} g}, \mathcal{F}\left(g, l^{\prime}, \pi_{o}\right) \otimes \mathcal{L}_{\rho_{\infty}}\right) \otimes\left[\overleftarrow{k^{\prime}}, \overrightarrow{l-1-k^{\prime}}\right]_{\pi_{o}}
$$

pour $1 \leqslant l^{\prime}<l$ avec $s(g-1)+2(k+1)=\left(s-l^{\prime}\right)(g-1)+l^{\prime}(g-1)+2 \delta+2 k^{\prime}$, soit $k^{\prime}=k+1-\delta$ avec $0<\delta<l^{\prime}$ qui sont nulles d'après l'hypothèse de récurrence. En ce qui concerne les parties de poids $m_{k}$ des $E_{2, \rho_{\infty}}^{j-r-1, l(g-1)+r}\left[\Pi^{\infty, o}\right]$ pour $r>0$, elles proviennent à nouveau des parties de poids $\left(s-l^{\prime}\right)(g-1)+2 k^{\prime}$ des composantes $\Pi^{\infty, o}$-isotypiques des $H_{c}^{j-r-1}\left(M_{I, s_{o}}^{=l^{\prime} g}, \mathcal{F}\left(g, l^{\prime}, \pi_{o}\right) \otimes \mathcal{L}_{\rho_{\infty}}\right) \otimes$ $\left[\overleftarrow{k^{\prime}}, \overrightarrow{l-1-k^{\prime}}\right]_{\pi_{o}}$ pour $1 \leqslant l^{\prime} \leqslant s$ avec $k^{\prime}=k+1-\delta$ et $0 \leqslant \delta=\left(l-l^{\prime}\right)(g-1)+r<l^{\prime}$, ce qui impose d'après l'hypothèse de récurrence $l^{\prime}>l$, avec $\delta=0$ et $k^{\prime}=k+1$. On remarque alors que

$$
[\overleftrightarrow{k}, \overrightarrow{l-1}, \overleftarrow{1}, \overleftrightarrow{s-l-k-1}]_{\pi_{o}} \otimes L_{g}\left(\pi_{o}\right)\left(-\frac{s(g-1)+2(k+1)}{2}\right)
$$

serait un constituant de $E_{\infty, \rho_{\infty}}^{j}\left[\Pi^{\infty, o}\right]$, ce qui n'est pas d'après la proposition (V.1.1).

Remarque : On pourra voir la figure (4), où l'on a représenté pour $s=4$ et $g=2$, les $E_{2}^{p, q}\left[\Pi^{\infty, o}\right]$ de la suite spectrale des cycles évanescents.

\section{V.2. Correspondances de Jacquet-Langlands globales}

Badulescu m'a expliqué que les résultats de ce paragraphe peuvent s'obtenir aisément via la formule des traces simples. Nous avons tout de même souhaité montrer comment ils découlaient de nos calculs.

On rappelle que $D$ et $\bar{D}$ sont des algèbres à division sur un corps global $F$ d'égale caractéristique $p$, telles que :

- pour tout place $x \neq \infty, o, D_{x} \simeq \bar{D}_{x}$;

$-D_{\infty}^{\times} \simeq G L_{d}\left(F_{\infty}\right)$ et $\operatorname{inv}_{\infty} \bar{D}^{\times}=-1 / d$;

- $D_{o}^{\times} \simeq G L_{d}\left(F_{o}\right)$ et $\operatorname{inv}_{o} \bar{D}^{\times}=1 / d$.

V.2.1. Proposition. - On fixe un diviseur $g$ de $d=s g$ ainsi qu'une représentation irréductible cuspidale unitaire $\pi_{\infty}$ de $G L_{g}\left(F_{\infty}\right)$. Il existe alors une bijection dite de Jacquet-Langlands entre : - les représentations irréductibles automorphes $\bar{\Pi}$ de $\bar{D}_{\mathbb{A}}^{\times}$telles que $\bar{\Pi}_{\infty} \simeq \mathrm{JL}^{-1}\left([\overleftarrow{s-1}]_{\pi_{\infty}}\right)$, - les représentations irréductibles automorphes $\Pi$ de $D_{\mathbb{A}}^{\times}$vérifiant l'une des deux conditions suivantes :

(a) $\Pi_{\infty}$ est isomorphe $\grave{a}[\overleftarrow{s-1}]_{\pi_{\infty}}$ et $\Pi_{o}$ est une représentation de carré intégrable;

(b) $\Pi_{\infty} \simeq[\overrightarrow{s-1}]_{\pi_{\infty}}$ et $\Pi_{o} \simeq[\overrightarrow{s-1}]_{\pi_{o}}$ pour $\pi_{o}$ une représentation irréductible cuspidale de $G L_{g}\left(F_{o}\right)$.

compatible aux correspondances de Jacquet-Langlands locales, soit $\bar{\Pi}^{\infty, o} \simeq \Pi^{\infty, o}$ et $\Pi_{o} \simeq \mathrm{JL}\left(\bar{\Pi}_{o}\right)$ dans le cas (a) et $\Pi_{o} \simeq{ }^{t} \mathrm{JL}\left(\bar{\Pi}_{o}\right)$ dans le cas (b) où ${ }^{t} \pi$ désigne la représentation duale associée $\grave{a}$ $\pi$ pour la dualité de Zelevinski. En outre on a $m(\Pi)=m(\bar{\Pi})$.

Par ailleurs soit $\Pi^{o}$ une représentation de $\left(D_{\AA}^{o}\right)^{\times}$telle que pour toute représentation $\Pi_{o}$ de $G L_{d}\left(F_{o}\right)$ avec $\Pi:=\Pi^{o} \Pi_{o}$ vérifiant $\operatorname{Hyp}(\infty), \Pi_{o}$ n'est pas de la forme $[\overleftarrow{s-1}]_{\pi_{o}}$ ou $[\overrightarrow{s-1}]_{\pi_{o}}$ pour $\pi_{o}$ une représentation irréductible cuspidale de $G L_{g}\left(F_{o}\right)$ avec $d=s g$. Alors il n'existe pas de représentation irréductible automorphe $\bar{\Pi}$ de $\bar{D}_{\mathbb{A}}^{\times}$telle que $\bar{\Pi}^{\infty, o} \simeq \Pi^{\infty, o}$.

Démonstration. - Posons $\rho_{\infty}=\mathrm{JL}^{-1}\left([\overleftarrow{s-1}]_{\pi_{\infty}}\right)$.

- Soit $\Pi$ une représentation automorphe de $D_{\mathbb{A}}^{\times}$telle que $\Pi_{\infty} \simeq[\overleftarrow{s-1}]_{\pi_{\infty}}$ et $\Pi_{o} \simeq\left[\overleftarrow{s^{\prime}-1}\right]_{\pi_{o}}$ pour une certaine représentation cuspidale unitaire $\pi_{o}$ de $G L_{g^{\prime}}\left(F_{o}\right)$. D'après la proposition (IV.5.4.1), 
pour tout $1 \leqslant l<s^{\prime}$, on a

$$
\begin{aligned}
& \lim _{\vec{I}} H^{0}\left(M_{I, s_{o}}^{\geqslant l g^{\prime}}, j_{!}^{\geqslant l g^{\prime}} H T_{\rho_{\infty}}\left(g^{\prime}, l, \pi_{o}, \Pi_{l}, I\right)\left[d-l g^{\prime}\right]\right)\left[\Pi^{\infty, o}\right]= \\
& \quad m(\Pi) \Pi_{l} \overrightarrow{\times}\left[\overleftarrow{s^{\prime}-l-1}\right]_{\pi_{o}} \otimes\left(\Xi^{\frac{\left(s^{\prime}-l\right)\left(g^{\prime}-1\right)}{2}} \otimes \bigoplus_{\xi \in \mathfrak{A}\left(\pi_{o}\right)} \xi^{-1}\right)
\end{aligned}
$$

Par ailleurs ce dernier est aussi égal à

$$
\lim _{\vec{I}} H^{0}\left(M_{I, s_{o}}^{d}, \mathcal{F}\left(g^{\prime}, s^{\prime}, \pi_{o}, I\right) \otimes \mathcal{L}_{\rho_{\infty}} \otimes \Pi_{l} \overrightarrow{\times}\left[\overleftarrow{s^{\prime}-l-1}\right]_{\pi_{o}}\right)\left[\Pi^{\infty, o}\right]
$$

$$
\otimes\left(\Xi^{\frac{\left(s^{\prime}-l\right)\left(g^{\prime}-1\right)}{2}} \otimes \bigoplus_{\xi \in \mathfrak{A}\left(\pi_{o}\right)} \xi^{-1}\right)
$$

qui d'après le lemme (IV.5.4.7) est égal à

$$
\sum_{\bar{\Pi}} \Pi_{l} \overrightarrow{\times}\left[\overleftarrow{s^{\prime}-l-1}\right]_{\pi_{o}} \otimes\left(\Xi^{\frac{\left(s^{\prime}-l\right)\left(g^{\prime}-1\right)}{2}} \otimes \bigoplus_{\xi \in \mathfrak{A}\left(\pi_{o}\right)} \xi^{-1}\right)
$$

où $\bar{\Pi}$ décrit les représentations irréductibles automorphes de $\bar{D}_{\mathbb{A}}^{\times}$telles que $\bar{\Pi}^{\infty, o} \simeq \Pi^{\infty, o}, \bar{\Pi}_{\infty} \simeq$ $\mathrm{JL}^{-1}\left([\overleftarrow{s-1}]_{\pi_{\infty}}\right)$ et $\bar{\Pi}_{o} \simeq \mathrm{JL}^{-1}\left(\left[\overleftarrow{s^{\prime}-1}\right]_{\pi_{o}}\right)$

- De la même façon, soit $\Pi$ telle que $\Pi_{\infty} \simeq[\overrightarrow{s-1}]_{\pi_{\infty}}$ et $\Pi_{o} \simeq\left[\overrightarrow{s^{\prime}-1}\right]_{\pi_{o}}$. On reprend la preuve de la proposition (IV.8.3.4). Pour $l=s^{\prime}$, le lemme (IV.5.4.7) donne

$$
\lim _{\vec{I}} H^{0}\left(M_{I, s_{o}}^{s^{\prime} g^{\prime}}, \mathcal{F}\left(g^{\prime}, s^{\prime}, \pi_{o}, I\right) \otimes\left[\overleftarrow{s^{\prime}-1}\right]_{\pi_{o}}\right)\left[\Pi^{\infty, o}\right]=
$$

$$
\left(\sum_{\bar{\Pi} \in \mathfrak{U}_{\bar{D}}\left(\Pi^{\infty}, o\right)} m(\bar{\Pi})\right)\left[\overleftarrow{s^{\prime}-1}\right]_{\pi_{o}} \otimes \bigoplus_{\xi \in \mathfrak{A}\left(\pi_{o}\right)} \xi^{-1}
$$

où $\mathfrak{U}_{\bar{D}}\left(\Pi^{\infty, o}\right)$ désigne l'ensemble des $\bar{\Pi}$ automorphes telles que $\bar{\Pi}^{\infty, o} \simeq \Pi^{\infty, o}, \bar{\Pi}_{\infty} \simeq \mathrm{JL}^{-1}\left([\overleftarrow{s-1}]_{\pi_{\infty}}\right)$ et $\bar{\Pi}_{o} \simeq\left[\overleftarrow{s^{\prime}-1}\right]_{\pi_{o}}$. Par ailleurs pour $l=s^{\prime}-1$, on trouve que

$$
\begin{aligned}
\sum_{i}(-1)^{i} H^{i}\left(j_{! *}^{\geqslant\left(s^{\prime}-1\right) g^{\prime}}\right)= & \left(-\left(\sum_{\bar{\Pi} \in \mathfrak{U} \bar{D}_{(\Pi \infty, o)}} m(\bar{\Pi})\right)\left[\overleftarrow{s^{\prime}-2}\right]_{\pi_{o}} \overrightarrow{\times}[\overleftarrow{0}]_{\pi_{o}} \otimes \Xi^{-1 / 2}\right. \\
& \left.-\left(\sum_{\Pi \in \mathfrak{U}_{D}(\Pi \infty, o)} m(\Pi)\right)\left[\overleftarrow{s^{\prime}-2}\right]_{\pi_{o}} \overleftarrow{\times}[\overleftarrow{0}]_{\pi_{o}} \otimes \Xi^{1 / 2}\right)\left(\Xi^{g^{\prime} / 2} \otimes \underset{\xi \in \mathfrak{A}\left(\pi_{o}\right)}{\bigoplus} \xi^{-1}\right)
\end{aligned}
$$

D’après la pureté, le terme de gauche de l'égalité ci-dessus doit être égal à

$$
-H^{-1}\left(j_{!_{*}}^{\geqslant\left(s^{\prime}-1\right) g^{\prime}}\right)-H^{1}\left(j_{!_{*}}^{\geqslant\left(s^{\prime}-1\right) g^{\prime}}\right)
$$

de sorte que la dualité de Verdier donne le résultat.

- Supposons que $\Pi_{o}$ ne soit ni de la forme $\left[\overleftarrow{s^{\prime}-1}\right]_{\pi_{o}}$ ni $\left[\overrightarrow{s^{\prime}-1}\right]_{\pi_{o}}$ pour tout diviseur $s^{\prime}$ de $d=s^{\prime} g^{\prime}$ et toute représentation cuspidale $\pi_{o}$ de $G L_{g^{\prime}}\left(F_{o}\right)$. Supposons qu'il existe une représentation $\bar{\Pi}$ telle que $\bar{\Pi}^{\infty, o} \simeq \Pi^{\infty, o}$ et soit $s^{\prime}, \pi_{o}$ tel que $\bar{\Pi}_{o} \simeq \mathrm{JL}^{-1}\left(\left[\overleftarrow{s^{\prime}-1}\right]_{\pi_{o}}\right)$. Le lemme (IV.5.4.7) donne comme précédemment

$$
H^{0}\left(\mathcal{F}\left(g, s^{\prime}, \pi_{o}\right) \otimes\left[\overleftarrow{s^{\prime}-1}\right]_{\pi_{o}}\right) \simeq\left(\sum_{\left.\bar{\Pi} \in \mathfrak{U} \bar{D}^{\left(\Pi^{\infty}, o\right.}\right)} m(\bar{\Pi})\right)\left[\overleftarrow{s^{\prime}-1}\right]_{\pi_{o}} \otimes \bigoplus_{\xi \in \mathfrak{A}\left(\pi_{o}\right)} \xi^{-1}
$$


de sorte que

$$
\begin{aligned}
& \sum_{i}(-1)^{i} H^{i}\left(j_{! *}^{\geqslant\left(s^{\prime}-1\right) g^{\prime}}\right)\left[\Pi^{\infty, o}\right]=\left(-\left(\sum_{\Pi \in \mathfrak{U}_{D}\left(\Pi^{\infty}, o\right.} m(\Pi)\right)\left(\operatorname{Ind}_{P_{d-g^{\prime}, d}\left(F_{o}\right)}^{G L_{d}\left(F_{o}\right)}\left[\overleftarrow{s^{\prime}-2}\right]_{\pi_{o}} \otimes\right.\right. \\
&\left.\left.\left.\quad \operatorname{red}_{\mathrm{JL}^{-1}\left[\overleftarrow{s^{\prime}-2}\right]_{\pi_{o}}}^{d-g^{\prime}}\left(\Pi_{o}\right)\right)+\left(\sum_{\bar{\Pi} \in \mathfrak{U}_{\bar{D}}\left(\Pi^{\infty}, o\right.} m(\bar{\Pi})\right)\left[\overleftarrow{s^{\prime}-2}\right]_{\pi_{o}} \overrightarrow{\times}[\overleftarrow{0}]_{\pi_{o}} \otimes \Xi^{\left(g^{\prime}-1\right) / 2}\right) \otimes \bigoplus_{\xi \in \mathfrak{A}\left(\pi_{o}\right)} \xi^{-1}\right)
\end{aligned}
$$

La condition de pureté et la compatibilité à la dualité de Verdier, impose alors que $\Pi_{o}$ est soit isomorphe à $\left[\overleftarrow{s^{\prime}-1}\right]_{\pi_{o}}$ soit à $\left[\overrightarrow{s^{\prime}-1}\right]_{\pi_{o}}$ d'où la contradiction.

Ce dernier raisonnement montre en outre qu'étant donné une représentation $\bar{\Pi}$ comme dans l'énoncé, il lui correspond une représentation $\Pi$ vérifiant les conditions de l'énoncé. Dans le cas où $\Pi_{o} \simeq\left[\overrightarrow{s^{\prime}-1}\right]_{\pi_{o}}$, l'égalité $s=s^{\prime}$ découle du corollaire (V.3.3).

\section{V.3. Composantes locales des représentations automorphes vérifiant $\operatorname{Hyp}(\infty)$}

Les résultats ci-dessous sont bien connus dans le cas où il existe une correspondance de JacquetLanglands vers $G L_{d}(F)$, à partir des travaux de Moeglin-Waldspurger. C'est le cas par exemple s'il existe une place $x$ telle que $D_{x}$ est une algèbre à division sur $F_{x}$.

V.3.1. Proposition. - Soit $\Pi$ une représentation irréductible automorphe de $D_{\mathbb{A}}^{\times}$vérifiant $\operatorname{Hyp}\left(\rho_{\infty}\right)$. Pour tout $0 \leqslant i<d$, il existe alors un réel $\delta$ ainsi que des entiers $n_{i}{ }^{(4)}$ tels que :

$-\sum_{i=0}^{d-1} n_{i}(d-i)=d$,

- pour $i$ tel que $n_{i} \neq 0, n_{j}=0$ pour $j \equiv i+1 \bmod 2$, tels que pour toute place o telle que $D_{o}^{\times} \simeq G L_{d}\left(F_{o}\right)$, il existe :

- des entiers $t_{j}>1, g_{j}, 1 \leqslant j \leqslant u$, et $t_{k}^{\prime}, g_{k}^{\prime}, 1 \leqslant k \leqslant u^{\prime}$, avec $\sum_{j=1}^{u} t_{j} g_{j}+\sum_{k=1}^{u^{\prime}} t_{k}^{\prime} g_{k}^{\prime}=d$,

- des représentations irréductibles cuspidales $\pi_{o, j}$ et $\pi_{o, k}^{\prime}$ de respectivement $G L_{g_{j}}\left(F_{o}\right)$ et $G L_{g_{k}^{\prime}}\left(F_{o}\right)$ telles que $\pi_{o, j}(\delta)$ et $\pi_{o, k}^{\prime}(\delta)$ soient unitaires

vérifiant les conditions suivantes:

- pour tout $0 \leqslant i<d-1, \sum_{k} / t_{k}^{\prime}=d-1-i=g_{k}^{\prime}=n_{i} ;$

- $\Pi_{o}$ est l'induite irréductible $\left[\overleftarrow{t_{1}-1}\right]_{\pi_{o, 1}} \times \cdots \times\left[\overleftarrow{t_{u}-1}\right]_{\pi_{o, u}} \times\left[\overrightarrow{t_{1}^{\prime}-1}\right]_{\pi_{o, 1}^{\prime}} \times \cdots \times\left[\overrightarrow{t_{u^{\prime}}^{\prime}-1}\right]_{\pi_{o, u^{\prime}}^{\prime}}$, avec :

- si $u>0$ alors pour tout $j, s_{j} \equiv 1 \bmod 2$;

- si $u=0$ alors tous les $s_{i}$ ont la même parité donnée par $(-1)^{s_{i}-1}=\epsilon(\Pi)$.

On en déduit en particulier les corollaires suivant.

V.3.2. Corollaire. - Pour toute représentation automorphe de $D_{\mathbb{A}}^{\times}$vérifiant $\operatorname{Hyp}\left(\rho_{\infty}\right)$, la conjecture de Ramanujan-Peterson est vérifiée.

V.3.3. Corollaire. - Soit $\Pi$ une représentation irréductible automorphe de $D_{\mathbb{A}}^{\times}$vérifiant $\operatorname{Hyp}\left(\rho_{\infty}\right)$ et supposons qu'il existe une place $o_{0}$ telle que $D_{o_{0}}^{\times} \simeq G L_{d}\left(F_{o_{0}}\right)$ et $\Pi_{o_{0}}$ tempérée, i.e. avec les notations du théorème précédent $t_{i}^{\prime}=1$ pour tout $1 \leqslant i \leqslant u^{\prime}$ (resp. $\Pi_{o_{0}} \simeq[\overrightarrow{s-1}]_{\pi_{o_{0}}}$ pour $\pi_{o_{0}}$ une représentation cuspidale de $G L_{g}\left(F_{o_{0}}\right)$ avec $\left.d=s g\right)$. On en déduit alors, en utilisant les notations du théorème précédent, que pour toute place o non ramifiée, pour tout $1 \leqslant k \leqslant u^{\prime}$ (resp. pour tout $1 \leqslant j \leqslant u) t_{k}^{\prime}=0$ (resp. $t_{j}=0$ ), i.e. $\Pi_{o}$ est tempérée et $n_{i}=0$ pour tout $0 \leqslant i<d-1$ (resp. $n_{i}=0$ pour $i \neq s$ et $\Pi_{o} \simeq[\overrightarrow{s-1}]_{\pi_{o, 1}^{\prime} \times \cdots \times \pi_{o, u^{\prime}}^{\prime}}$ ). Dans le cas d'égale caractéristique, on en

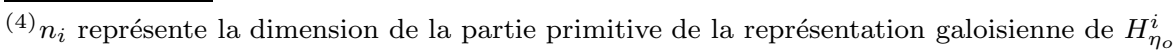


déduit en outre que $\Pi_{\infty} \simeq\left[\overleftarrow{s^{\prime}-1}\right]_{\pi_{\infty}}$ (resp. $\left.\Pi_{\infty} \simeq[\overrightarrow{s-1}]_{\pi_{\infty}}\right)$ avec $\rho_{\infty}$ cuspidale de $G L_{g^{\prime}}\left(F_{\infty}\right)$ (resp. de $G L_{g}\left(F_{\infty}\right)$ i.e. le même s et $g$ que celui en la place $\left.o_{0}\right)$ avec $d=s^{\prime} g^{\prime}$ (resp. $d=s g$ ).

V.3.4. Corollaire. - En égale caractéristique, soit $\Pi$ une représentation irréductible automorphe de $D_{\mathbb{A}}^{\times}$telle que $\Pi_{\infty}$ est de la forme $[\overleftarrow{s-1}]_{\pi_{\infty}}$ (resp. $[\overrightarrow{s-1}]_{\pi_{\infty}}$ ) pour $\pi_{\infty}$ une représentation irréductible cuspidale de $G L_{g}\left(F_{\infty}\right)$ avec $d=s g$. Pour toute place o où $D_{o}^{\times} \simeq G L_{d}\left(F_{o}\right)$, la composante locale $\Pi_{o}$ est tempérée, i.e. de la forme $\left[\overleftarrow{t_{1}-1}\right]_{\pi_{o, 1}} \times \cdots \times\left[\overleftarrow{t_{r}-1}\right]_{\pi_{o, u}}$ (resp. de la forme $\left.[\overrightarrow{s-1}]_{\pi_{o, 1}} \times \cdots \times[\overrightarrow{s-1}]_{\pi_{o, u^{\prime}}}\right)$.

Remarque : On en déduit en particulier que l'hypothèse 14.23 de [19] est vérifiée.

Démonstration. - On reprend les notations de la proposition (V.3.1). Dans le cas $\Pi_{\infty} \simeq$ $[\overleftarrow{s-1}]_{\pi_{\infty}}$, si $u>0$ (resp. $u=0$ ) la représentation galoisienne

$$
\begin{gathered}
\sigma(\Pi):=|\mathrm{cl}|^{(d-1) / 2} \bigoplus_{i \equiv 1 \bmod 2} H_{\eta_{o}, \rho_{\infty}}^{d-1+i}\left[\Pi^{\infty}\right] \\
\text { (resp. } \left.\sigma(\Pi):=|\mathrm{cl}|^{(d-1) / 2} \bigoplus_{i \equiv \epsilon(\Pi)+1 \bmod 2} H_{\eta_{o}, \rho_{\infty}}^{d-1+i}\left[\Pi^{\infty}\right]\right)
\end{gathered}
$$

est telle qu'en toute place $x \neq \infty$ telle que $D_{x}^{\times} \simeq G L_{d}\left(F_{x}\right), \sigma(\Pi)_{x}$ est la représentation de Langlands $L_{d}\left(\Pi_{x}\right)$ associée à la composante locale $\Pi_{x}$ de $\Pi$. Il est alors connu qu'il en est de même à la place $\infty$ de sorte que $\sigma(\Pi)$ est irréductible ce qui implique qu'il existe un unique $i$ tel que $H_{\eta_{o}, \rho_{\infty}}^{d-1+i}\left[\Pi^{\infty}\right]$ soit non nul : la dualité implique qu'il s'agit de $i=0$, d'où le résultat.

Dans le cas où $\Pi_{\infty} \simeq[\overrightarrow{s-1}]_{\pi_{\infty}}$, on considère $\sigma(\Pi):=\bigoplus_{i \equiv s-1 \bmod 2} H_{\eta_{o}, \rho_{\infty}}^{d+i}\left[\Pi^{\infty}\right]$. Le même raisonnement que précédemment s'applique où cette fois $\sigma(\Pi)_{\infty}$ est la somme directe de $s$ représentation irréductible de dimension $g$. Or d'après le théorème de Cebotarev, $\sigma(\Pi)=\bigoplus_{i \equiv s-1 \bmod 2} V_{d-1+i}$ où $V_{d-1+i}=\bigoplus_{\substack{0 \leqslant r \leqslant d-1 \\ r \equiv s-1 \bmod 2}} W_{r}$ où $W_{r}$ est de dimension $n_{r}$. D'après la pureté on en déduit alors que chacun des $V_{d-1+i}$ pour $i \equiv s-1 \bmod 2$ et $|i|<s$ est de dimension $g$ de sorte que tous les $n_{j}$ sont nuls sauf $n_{d-s}=g$, d'où le résultat.

Remarque : Dans le cas où l'on dispose de la correspondance de Jacquet-Langlands globale entre $D_{\mathbb{A}}^{\times}$et $G L_{d}(\mathbb{A})$, ces résultats sont compatibles avec ceux de Moeglin-Waldspurger. En effet soit JL(П) la représentation de $G L_{d}$ correspondante qui est donc de carré intégrable modulo le centre. Si JL $(\Pi)_{\infty} \simeq[\overleftarrow{s-1}]_{\pi_{\infty}}$ pour $\pi_{\infty}$ irréductible cuspidale, alors JL(ח) est cuspidale de sorte que ses composantes locale $\mathrm{JL}(\Pi)_{o}$ sont génériques et même tempérée d'après la conjecture de RamanujanPeterson prouvée dans ce cadre par Lafforgue. Si JL $(\Pi)_{\infty} \simeq[\overrightarrow{s-1}]_{\pi_{\infty}}$ alors $\mathrm{JL}(\Pi)$ ne peut être cuspidale car $[\overrightarrow{s-1}]_{\infty}$ n'est pas générique de sorte que d'après Moeglin-Waldspurger, JL(П) est de la forme $[\overrightarrow{s-1}]_{\tilde{\Pi}}$ pour $\tilde{\Pi}$ une représentation cuspidale de $G L_{g}(\mathbb{A})$.

Démonstration. - de la proposition (V.3.1) : A torsion par un caractère près, $\Pi_{o}$ est unitaire et donc d'après la classification de Tadic de la forme

$$
\left[\overrightarrow{s_{1}-1}\right]_{\left[\overleftarrow{t_{1}-1}\right]_{\pi_{o, 1}\left(\lambda_{1}\right)}} \times \cdots \times\left[\overrightarrow{s_{u}-1}\right]_{\left[\overleftarrow{t_{u}-1}\right]_{\pi_{o, u}(\lambda u)}}
$$

où $\pi_{o, i}$ sont des représentations irréductibles cuspidales unitaires de $G L_{g_{i}}\left(F_{o}\right)$ avec $\left.\lambda_{i} \in\right] \frac{-1}{2}, \frac{1}{2}[$ et $\sum_{i=1}^{u} g_{i} s_{i} t_{i}=d$ et où on induit par rapport aux paraboliques opposés aux paraboliques standards. L'induite étant irréductible, l'ordre des facteurs n'importe pas. Soit alors $r \geqslant 1$ tel que $\pi_{o}:=\pi_{o, 1}\left(\lambda_{1}\right) \simeq \cdots \simeq \pi_{o, r}\left(\lambda_{r}\right)$ et $\pi_{o, i}\left(\lambda_{i}\right)$ n'est pas isomorphe à $\pi_{o}$ pour $i>r$. On note $l_{0}:=\max _{1 \leqslant i \leqslant r}\left\{s_{i}, t_{i}\right\}$. On note $g$ l'entier tel que $\pi_{o}$ est une représentation cuspidale de $G L_{g}\left(F_{o}\right)$. Par ailleurs soit $0 \leqslant k_{1}$ (resp. $k_{1} \leqslant k_{2}$, resp. $k_{2} \leqslant k_{3} \leqslant r$ ) tel que pour tout $1 \leqslant i \leqslant k_{1}$ (resp. $k_{1}<i \leqslant k_{2}$, resp. $k_{2}<i \leqslant k_{3}$ ) on ait $s_{i}=t_{i}=l_{0}$ (resp. $t_{i}<s_{i}=l_{0}$, resp. $s_{i}<t_{i}=l_{0}$ ) et $\max _{k_{3}<i \leqslant r}\left\{t_{i}, s_{i}\right\}<l_{0}$. 
V.3.5. Lemme. - Pour tout $\max \{s, t\}<l \leqslant s t, \operatorname{red}_{\mathrm{JL}^{-1}\left([\overleftarrow{l-1}]_{\pi_{o}}\right)}\left([\overrightarrow{s-1}]_{[\overleftarrow{t-1}]_{\pi_{o}}}\right)=(0)$.

Démonstration. - Le résultat découle essentiellement de $[\mathbf{2 4}]$ et en particulier du lemme suivant.

V.3.6. Lemme. - Pour tout $s, t$, les constituants de $J_{N_{g, 2 g}, \ldots, s t g}\left([\overrightarrow{s-1}]_{[\overleftarrow{t-1}]_{\pi_{o}}}\right)$ sont de la forme $\pi_{o}\left(\frac{t+s-2}{2}-\sigma(1)\right) \otimes \cdots \otimes \pi_{o}\left(\frac{t+s-2}{2}-\sigma(s t)\right)$ où le multi-ensemble $R:=\{\sigma(1), \cdots, \sigma($ st $)\}$ est tel que $\left\{\pi_{o}\left(\frac{t+s-2}{2}-r\right) / r \in R\right\}$ soit égal au support cuspidal de $[\overrightarrow{s-1}]_{[\overleftarrow{t-1}]_{\pi_{o}}}$. Pour tout $0 \leqslant i \leqslant s t-1$, on pose $\sigma^{-1}(i)=\left\{n_{i}(1)<n_{i}(2)<\cdots\right\}$. On a alors les conditions suivantes :

(i) pour tout $0 \leqslant k<s-1$ et $0 \leqslant i<t$, on a $n_{t-1+k-i}(\min (k+1, i+1))>n_{t+k-i}(\min (k+$ $2, i+1))$;

(ii) pour tout $0 \leqslant k<s$ et tout $0 \leqslant i<j<t$, on a $n_{t-1+k-i}(\min (k+1, i+1))>$ $n_{t-1+k-j}(\min (k+1, j+1))$.

Remarque : Quand on regarde le support cuspidal de

$$
\overbrace{[\overleftarrow{t-1}]_{\pi_{o}\left(\frac{s-1}{2}\right)} \boxplus \cdots[\overleftarrow{t-1}]_{\pi_{o}\left(\frac{1-s}{2}\right)}}^{s}
$$

dans l'ordre de gauche à droite, pour tout $0 \leqslant k<s-1$ et $0 \leqslant i<t$, le $\pi_{o}\left(\frac{t+s-2}{2}-(t-1+k-i)\right)$ du $k$-ème facteur est le $\min (k+1, i+1)$-ème. La condition (ii) du lemme affirme que dans un même facteur, on doit prendre les $\pi_{o}(i)$ de gauche à droite, tandis que la condition (i) précise que le $\pi_{o}(i)$ du $k$-ème facteur arrive après le $\pi_{o}(i+1)$ du $k+1$-ème facteur.

Démonstration. - En remarquant que $\left([\overrightarrow{s-1}]_{[\overleftarrow{t-1}]_{\pi_{o}}}\right)$ s'injecte dans les induites

$$
\left.[\overleftarrow{t-1}]_{\pi_{o}\left(\frac{s-1}{2}\right)} \times[\overrightarrow{s-2}]_{[\overleftarrow{t-1}]_{\pi_{o}(-1 / 2)}} \quad[\overleftarrow{t-1}]_{\pi_{o}\left(\frac{s-1}{2}\right)} \boxplus[\overleftarrow{t-1}]_{\pi_{o}\left(\frac{s-3}{2}\right)} \times \overrightarrow{(s-2)}\right)_{[\overleftarrow{t-1}]_{\pi_{o}(-1)}}
$$

on se ramène aisément, par récurrence sur $s$, au cas $s=2$ soit à étudier $[\overleftarrow{t-1}]_{\pi_{o}(1 / 2)} \boxplus$ $[\overleftarrow{t-1}]_{\pi_{o}(-1 / 2)}$. Or dans le groupe de Grothendieck, cette dernière est égale à

$$
[\overleftarrow{t-1}]_{\pi_{o}(1 / 2)} \times[\overleftarrow{t-1}]_{\pi_{o}(-1 / 2)}-[\overleftarrow{t}]_{\pi_{o}} \times[\overleftarrow{t-2}]_{\pi_{o}}
$$

La condition (ii) constitue l'unique restriction sur les $\sigma$ pour l'induite totale $[\overleftarrow{t-1}]_{\pi_{o}(1 / 2)} \times$ $[\overleftarrow{t-1}]_{\pi_{o}(-1 / 2)}$. Il suffit alors de remarquer que les $\sigma$ vérifiant (i) et pas (ii) sont obtenues à partir de $[\overleftarrow{t}]_{\pi_{o}} \times[\overleftarrow{t-2}]_{\pi_{o}}$, d'où le résultat.

Ainsi si on obtenait un constituant de la forme $[\overleftarrow{l-1}]_{\pi_{o}} \otimes \pi$, on en déduirait, par transitivité du foncteur de Jacquet, avec les notations du lemme précédent, que la bijection $\sigma_{0}$ telle que $\sigma_{0}(i)=\sigma(1)-i+1$ pour $1 \leqslant i \leqslant l$ vérifierait les conditions (i) et (ii) ce qui impose $l \leqslant t$.

De même si on obtenait $[\overrightarrow{l-1}]_{\pi_{o}} \otimes \pi$, la bijection $\sigma_{0}$ telle que $\sigma_{0}(i)=\sigma_{0}(1)+i-1$ pour $1 \leqslant i \leqslant l$ vérifierait les conditions (i) et (ii) ce qui impose $l \leqslant s$, d'où le résultat.

On en déduit alors que pour tout $l>l_{0}, \operatorname{red}_{\mathrm{JL}^{-1}\left(\left[\overleftarrow{l-1}_{\pi_{o}}\right)\right.}\left(\Pi_{o}\right)$ est nul de sorte que d'après (IV.2.2.13), que pour tout $l_{0}<l \leqslant s, \sum_{i}(-1)^{i} H^{i}\left(j_{!}^{\geqslant l g} H T_{\rho_{\infty}}\left(g, l, \pi_{o}, \Pi_{l}\right)\right)\left[\Pi^{\infty}\right]=0$. D'après le lemme (IV.6.1.1), on en déduit alors que $H^{i}\left(j_{!_{*}}^{\geqslant l g} H T_{\rho_{\infty}}\left(g, l, \pi_{o}, \Pi_{l}\right)\right)\left[\Pi^{\infty}\right]$ est nul, pour tout $i$.

V.3.7. Lemme. - Soit $l:=\max \{s, t\}$. Si $l>t$ (resp. $l>s)$, alors

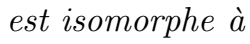

$$
\operatorname{red}_{\mathrm{JL}^{-1}\left([\overleftarrow{l-1}]_{\pi_{o}}\right)}\left([\overrightarrow{s-1}]_{[\overleftarrow{t-1}]_{\pi_{o}}}\right)
$$

$$
\begin{aligned}
& \left.(-1)^{l-1} \Xi^{\frac{(t-1)(s g-1)}{2}} \otimes[\overrightarrow{s-1}]_{[\overleftarrow{t-2}]_{\pi_{o}\left(\frac{s g-1}{2}\right)}}\right) \\
& \left(\text { resp. } \Xi^{\frac{(s-1)(t g+1)}{2}} \otimes[\overrightarrow{s-2}]_{\left.[\overleftarrow{t-1}]_{\pi_{o}\left(\frac{t g+1}{2}\right)}\right)}\right.
\end{aligned}
$$


Dans le cas $l=s=t$, on obtient la somme des deux termes ci-dessus.

Démonstration. - L'argument, d'après le lemme (V.3.6), est le même que celui de la preuve du lemme (V.3.5).

Ainsi d'après (IV.2.2.13), on en déduit que

$$
\epsilon(\Pi) \sum_{i}(-1)^{i} H^{i}\left(j_{!}^{\geqslant l_{0} g} H T_{\rho_{\infty}}\left(g, l_{0}, \pi_{o}, \Pi_{l}\right)\left[d-l_{0} g\right]\right)\left[\Pi^{\infty}\right]
$$

qui d'après ce qui précède est égal à $\epsilon(\Pi) \sum_{i}(-1)^{i} H^{i}\left(j_{!_{*}}^{\geqslant l_{0} g} H T_{\rho_{\infty}}\left(g, l, \pi_{o}, \Pi_{l}\right)\left[d-l_{0} g\right]\right)$, est donné par

$$
\begin{aligned}
& \Xi^{\frac{d-l_{0} g}{2}}\left(\left(\Xi^{\frac{l_{0}-1}{2}}(-1)^{l_{0}-1} \Pi_{l}\left(\frac{1-l_{0}}{2}\right) \times\left(\left[\overrightarrow{l_{0}-2}\right]_{\left[\overleftarrow{l_{0}-1}\right]_{\pi_{o}(1 / 2)}}\right)^{k_{1}}+\right.\right. \\
& \left.\Xi^{-\frac{l_{0}-1}{2}}\left(\Pi_{l}\left(\frac{l_{0}-1}{2}\right) \times\left[\overrightarrow{l_{0}-1}\right]_{\left[\overleftarrow{l_{0}-2}\right]_{\pi_{o}(-1 / 2)}}\right)^{k_{1}}\right) \times\left[\overrightarrow{s_{2}-1}\right]_{\left[\overleftarrow{t_{2}-1}\right]_{\pi_{o, 2}\left(\lambda_{2}\right)}} \times \cdots \times\left[\overrightarrow{s_{u}-1}\right]_{\left[\overleftarrow{t_{u}-1}\right]_{\pi_{o}, u}\left(\lambda_{u}\right)}+ \\
& \sum_{i=k_{1}+1}^{k_{2}} \Xi^{-\frac{t_{i}-1}{2}}(-1)^{l_{0}-1}\left(\left[\overrightarrow{s_{1}-1}\right]_{\left[\overleftarrow{t_{1}-1}\right]_{\pi_{o, 1}\left(\lambda_{1}\right)}} \times \cdots \times\left[\overrightarrow{s_{i-1}-1}\right]_{\left[\overleftarrow{t_{i-1}-1}\right]_{\pi_{o, i-1}\left(\lambda_{i-1}\right)}} \times\right. \\
& \Pi_{l}\left(\frac{t_{i}-1}{2}\right) \times\left[\overrightarrow{l_{0}-1}\right]_{\left[\overleftarrow{t_{i}-2}\right]_{\pi_{o}(1 / 2)}} \times \\
& \left.\left[\overrightarrow{s_{i+1}-1}\right]_{\left[\overleftarrow{t_{i+1}-1}\right]_{\pi_{o, i+1}\left(\lambda_{i+1}\right)}} \times \cdots \times\left[\overrightarrow{s_{u}-1}\right]_{\left[\overleftarrow{t_{u}-1}\right]_{\pi_{o, u}(\lambda u)}}\right)+ \\
& \sum_{i=k_{2}+1}^{k_{3}} \Xi^{\frac{s_{i}-1}{2}}\left(\left(\left[\overrightarrow{s_{1}-1}\right]_{\left[\overleftarrow{t_{1}-1}\right]_{\pi_{o, 1}\left(\lambda_{1}\right)}} \times \cdots \times\left[\overrightarrow{s_{i-1}-1}\right]_{\left[\overleftarrow{t_{i-1}-1}\right]_{\pi_{o, i-1}\left(\lambda_{i-1}\right)}} \times\right.\right. \\
& \left.\Pi_{l}\left(\frac{1-s_{i}}{2}\right) \times\left[\overrightarrow{s_{i}-2}\right]_{\left[\overleftarrow{l_{0}-1}\right]_{\pi_{o}(-1 / 2)}}\right) \times \\
& \left.\left[\overrightarrow{s_{i+1}-1}\right]_{\left[\overleftarrow{t_{i+1}-1}\right]_{\pi_{o, i+1}\left(\lambda_{i+1}\right)}} \times \cdots \times\left[\overrightarrow{s_{u}-1}\right]_{\left[\overleftarrow{t_{u}-1}\right]_{\pi_{o, u}\left(\lambda_{u}\right)}}\right)
\end{aligned}
$$

En utilisant que pour tout $i, H^{i}\left(j_{!_{*}}^{\geqslant l_{0} g} H T_{\rho_{\infty}}\left(g, l_{0}, \pi_{o}, \Pi_{l}\right)\left[d-l_{0} g\right]\right)$ est pur de poids $d-l_{0} g+i$, on en déduit alors que ceux-ci sont alors isomorphes à

$$
\begin{aligned}
& \Pi_{l}\left(\frac{1-l_{0}}{2}\right) \times\left(\left[\overrightarrow{l_{0}-1}\right]_{\left[\overleftarrow{l_{0}-2}\right]_{\pi_{o}(1 / 2)}}\right)^{k_{1}} \times\left[\overrightarrow{s_{2}-1}\right]_{\left[\overleftarrow{t_{2}-1}\right]_{\pi_{o, 2}\left(\lambda_{2}\right)}} \times \cdots \times\left[\overrightarrow{s_{u}-1}\right]_{\left[\overleftarrow{t_{u}-1}\right]_{\pi_{o, u}\left(\lambda_{u}\right)}} \\
& \text { pour } i=1-l_{0} \\
& \Pi_{l}\left(\frac{l_{0}-1}{2}\right) \times\left(\left[\overrightarrow{l_{0}-2}\right]_{\left[\overleftarrow{l_{0}-1}\right]_{\pi_{o}(-1 / 2)}}\right)^{k_{1}} \times\left[\overrightarrow{s_{2}-1}\right]_{\left[\overleftarrow{t_{2}-1}\right]_{\pi_{o, 2}\left(\lambda_{2}\right)}} \times \cdots \times\left[\overrightarrow{s_{u}-1}\right]_{\left[\overleftarrow{t_{u}-1}\right]_{\pi_{o, u}\left(\lambda_{u}\right)}} \\
& \text { pour } i=l_{0}-1 \\
& \bigoplus_{k_{1}<k \leqslant k_{2} / 1-t_{k}=i}(-1)^{l_{0}-1}\left(\left[\overrightarrow{s_{1}-1}\right]_{\left[\overleftarrow{t_{1}-1}\right.}\right]_{\pi_{o, 1}\left(\lambda_{1}\right)} \times \cdots \times\left[\overrightarrow{s_{i-1}-1}\right]_{\left[\overleftarrow{t_{i-1}-1}\right]_{\pi_{o, i-1}\left(\lambda_{i-1}\right)}} \times \\
& \left.\Pi_{l}\left(\frac{t_{i}-1}{2}\right) \times\left[\overrightarrow{l_{0}-1}\right]_{\left[\overleftarrow{t_{i}-2}\right]_{\pi_{o}(1 / 2)}} \times\left[\overrightarrow{s_{i+1}-1}\right]_{\left[\overleftarrow{t_{i+1}-1}\right]_{\pi_{o, i+1}\left(\lambda_{i+1}\right)}} \times \cdots \times\left[\overrightarrow{s_{u}-1}\right]_{\left[\overleftarrow{t_{u}-1}\right]_{\pi_{o, u}\left(\lambda_{u}\right)}}\right) \\
& \text { pour } 0 \leqslant-i<l_{0}-1 \\
& \bigoplus_{k_{2}<k \leqslant k_{3}}\left(\left(\left[\overrightarrow{s_{1}-1}\right]_{\left[\overleftarrow{t_{1}-1}\right]_{\pi_{o, 1}\left(\lambda_{1}\right)}} \times \cdots \times\left[\overrightarrow{s_{i-1}-1}\right]_{\left[\overleftarrow{t_{i-1}-1}\right]_{\pi_{o, i-1}\left(\lambda_{i-1}\right)}} \times\right.\right.
\end{aligned}
$$

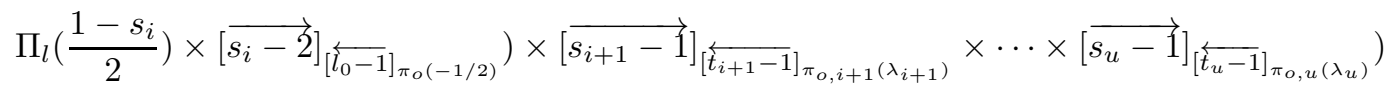

$$
\begin{aligned}
& \text { pour } 0 \leqslant i<l_{0}-1
\end{aligned}
$$


Si on veut que ces résultats soient compatibles à la dualité de Verdier, il faut alors que pour tout $i, \min \left\{s_{i}, t_{i}\right\}=1$. En outre s'il existe $i$ tel que $t_{i}>1$ alors $\epsilon(\Pi)=1$ et pour tout $j, s_{j} \equiv 1 \bmod 2$. Si pour tout $i, t_{i}=1$ alors tous les $s_{i}$ ont la même parité donnée par $(-1)^{s_{i}-1}=\epsilon(\Pi)$.

Par ailleurs les $H^{i}\left(j_{! *}^{\geqslant l g} H T_{\rho_{\infty}}\left(g, l, \pi_{o},[\overleftarrow{l-1}]_{\pi_{o}}\right)[d-l g]\right) \otimes L_{g}\left(\pi_{o}\right)(1-l g)$ servent à calculer la cohomologie de la fibre générique via la suite spectrale des poids. On en déduit alors que tous les poids $L_{g}\left(\pi_{o, i}\left(\lambda_{i}\right)\right)$ s'obtiennent à partir de ceux obtenus par la cohomologie de la fibre générique par une translation entière, d'où le résultat.

\section{V.4. Pureté de la filtration de monodromie des $H_{\eta_{o}}^{i}$}

V.4.1. Théorème. - Pour toute représentation irréductible $\Pi$ et pour tout $i$, les gradués, $g r_{k, \rho_{\infty}}\left[\Pi^{\infty}\right]$ de la filtration de monodromie de $H_{\eta_{o}, \rho_{\infty}}\left[\Pi^{\infty}\right]$ sont purs de poids $d-1+k^{(5)}$. En fait pour $i \neq d-1$, l'opérateur de monodromie $N$ agit trivialement sur les $H_{\eta_{o}, \rho_{\infty}}^{i}$.

Remarque : Il s'agit donc de prouver la conjecture de monodromie-poids, dite globale, résultat déjà connu en égale caractéristique d'après Deligne. On ne cherche pas ici à prouver ce résultat dans cette situation, puisqu'à de nombreuses reprises nous avons déjà utilisé la pureté des gradués de la filtration de monodromie. La preuve qui suit n'a donc de sens qu'en caractéristique mixte.

Démonstration. - Soit $o$ une place non ramifiée. Remarquons tout d'abord que s'il n'existe pas de $\Pi_{o}^{\prime}{ }^{(6)}$ tel que $\Pi^{o} \Pi_{o}^{\prime}$ ne vérifie pas $\operatorname{Hyp}(\infty)$, alors tous les $H_{\eta_{o}}^{i}\left[\Pi^{\infty}\right]$ sont nuls. Considérons alors $\Pi$ irréductible vérifiant $\operatorname{Hyp}(\infty)$ de sorte que, d'après ce qui précède, $\Pi_{o}$ est de la forme

$$
\left[\overrightarrow{s_{1}-1}\right]_{\pi_{o, 1}} \times \cdots \times\left[\overrightarrow{s_{u}-1}\right]_{\pi_{o, u}} \times\left[\overleftarrow{t_{1}-1}\right]_{\pi_{o, 1}^{\prime}} \times \cdots \times\left[\overleftarrow{t_{v}-1}\right]_{\pi_{o, v}^{\prime}}
$$

avec $\pi_{o, i}$ et $\pi_{o, j}^{\prime}$ irréductibles cuspidales unitaires de respectivement $G L_{g_{i}}\left(F_{o}\right)$ et $G L g_{j}^{\prime}\left(F_{o}\right)$. ${ }^{(7)}$ On étudie alors la suite spectrale

$$
E_{1}^{i, j}=H^{i+j}\left(g r_{-i, \rho_{\infty}}\right) \Rightarrow H_{\eta_{o}, \rho_{\infty}}^{i+j+d-1}
$$

D'après le théorème (IV.4.4.4), les $H^{r}\left(g r_{k, \rho_{\infty}}\right)\left[\Pi^{\infty, o}\right]$ se calculent à partir des $H^{r}\left(\mathcal{P}\left(g_{i}, l, \pi_{o, i}\right)\right)\left[\Pi^{\infty, o}\right]$ pour $1 \leqslant i \leqslant u$ et $1 \leqslant l g_{i} \leqslant d$ ainsi que des $H^{r}\left(\mathcal{P}\left(g_{i}^{\prime}, l, \pi_{o, i}^{\prime}\right)\right)\left[\Pi^{\infty, o}\right]$ pour $1 \leqslant i \leqslant u^{\prime}$ et $1 \leqslant l g_{i}^{\prime} \leqslant d$. Le calcul de ces derniers s'obtient par récurrence descendante sur $l$ via le calcul des

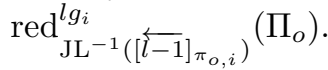

A priori, pour une preuve rigoureuse on devrait introduire des notations comme dans la preuve de la proposition (V.3.1) en regroupant les $\pi_{o, i}$ qui sont isomorphes, puis en les triant selon leur $s_{i}$. Cependant comme le foncteur de Jacquet est additif sur les induites et vu que les $H^{i}\left(j_{!_{*}}^{\geqslant l g} H T\left(g, l, \pi_{o}, \Pi_{l}\right)\right)\left[\Pi^{\infty, o}\right]$ se calculent à partir de ceux-ci, par récurrence, on remarque alors que les divers $\pi_{o, i}$ n'interagissent pas entre eux de sorte que chacun des $H^{i}\left(j_{! *}^{\geqslant l g} H T\left(g, l, \pi_{o}, \Pi_{l}\right)[d-\right.$ $l g])\left[\Pi^{\infty, o}\right]$ sera de la forme

$$
\bigoplus_{\substack{k / \pi_{o, k} \simeq \pi_{o} \\ s_{k} \geqslant l}} H\left(i, \pi_{o}, l, s_{k},-\right) \oplus \bigoplus_{\substack{k / \pi_{o, k}^{\prime} \simeq \pi_{o} \\ t_{k} \geqslant l}} H\left(i, \pi_{o}, l, t_{k},+\right)
$$

où $H\left(i, \pi_{o}, l, s_{k},-\right)$ (resp. $\left.H\left(i, \pi_{o}, l, t_{k},+\right)\right)$ correspond au calcul de

$$
H^{i}\left(j_{!_{*}}^{\geqslant l g} H T\left(g, l, \pi_{o}, \Pi_{l}\right)[d-l g]\right)\left[\Pi^{\infty, o}\right]
$$

effectué comme si $k$ est tel que pour tout $1 \leqslant i \neq k \leqslant u$ et pour tout $1 \leqslant j \leqslant u^{\prime}$ (resp. pour tout $1 \leqslant i \leqslant u$ et $\left.1 \leqslant j \neq k \leqslant u^{\prime}\right), \pi_{o, i}$ et $\pi_{o, j}^{\prime}$ ne sont pas isomorphes à $\pi_{o}$. Plaçons nous alors dans une

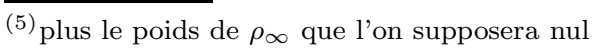

${ }^{(6)}$ On peut en outre supposer que ce dernier soit de même support cuspidal que $\Pi_{O}$

(7) En outre tous les $s_{i}$ ont la même congruence modulo 2. 
telle situation, i.e. $\Pi_{o} \simeq[\overleftarrow{s-1}]_{\pi_{o}} \times \pi_{o, 1}$ (resp. $\left.\Pi_{o} \simeq[\overrightarrow{s-1}]_{\pi_{o}} \times \pi_{o, 1}\right)$ où $\pi_{o, 1}$ est une représentation irréductible de $G L_{d-s g}\left(F_{o}\right)$ de support cuspidal disjoint de la droite associée à $\pi_{o}$ irréductible cuspidale de $G L_{g}\left(F_{o}\right)$. Les calculs sont alors exactement similaires à ceux déjà effectués, rappelons rapidement comment cela s'articule.

- pour $l>s$, les $H^{i}\left(j_{!_{*}}^{\geqslant l g} H T\left(g, l, \pi_{o}, \Pi_{l}\right)\right)\left[\Pi^{\infty, o}\right]$ sont nuls : on procède par récurrence descendante sur $l$ de $s_{g}$ à $s+1$. Le cas $l=s_{g}$ quand $s_{g} g=d$ découle du lemme (IV.5.4.7) en utilisant le théorème (V.2.1). Dans les autres situations le résultat découle du fait que $\operatorname{red}_{\mathrm{JL}^{-1}\left(\left[\overleftarrow{l l-1}_{\pi_{o}}\right)\right.}^{l g}\left(\Pi_{o}\right)$ est nul;

- pour $l=s$, on a $H^{0}\left(j_{! *}^{\geqslant s^{\prime} g} H T\left(g, s^{\prime}, \pi_{o},[\overleftarrow{s-1}]_{\pi_{o}}\right)[d-s g]\right)\left[\Pi^{\infty, o}\right] \simeq \Pi_{o}{ }^{(8)}$ : le résultat découle directement du point précédent et du fait que $\operatorname{red}_{\mathrm{JL}^{-1}\left([\overleftarrow{s-1}]_{\pi_{o}}\right)}\left(\Pi_{o}\right)=\pi_{o, 1}(s g / 2) \otimes \Xi^{\frac{d-s g}{2}}$;

- pour $1 \leqslant l<s$ et $\Pi_{o} \simeq[\overleftarrow{s-1}]_{\pi_{o}} \times \pi_{o, 1}$, tous les $H^{i}\left(j_{! *}^{\geqslant l g} H T\left(g, l, \pi_{o},[\overleftarrow{l-1}]_{\pi_{o}}\right)\right)\left[\Pi^{\infty, o}\right]$ sont nuls : la preuve est strictement identique à celle de la proposition (IV.5.4.1);

- pour $1 \leqslant l<s$ et $\Pi_{o} \simeq[\overrightarrow{s-1}]_{\pi_{o}} \times \pi_{o, 1}$, les $H^{i}\left(j_{!_{*}}^{\geqslant l g} H T\left(g, l, \pi_{o},[\overleftarrow{l-1}]_{\pi_{o}}\right)[d-l g]\right)\left[\Pi^{\infty, o}\right]$ sont nuls pour $|i|>s-l$ ou $i \equiv s-l-1 \bmod 2$. Dans les autres cas, il est isomorphe à

$$
\left([\overleftarrow{l-1}]_{\pi_{o}} \overrightarrow{\times}\left[\frac{\overrightarrow{s-l+i-4}}{2}\right]_{\pi_{o}}\right) \overleftarrow{\times}\left[\frac{\overrightarrow{s-l-i-4}}{2}\right]_{\pi_{o}} \otimes\left(\Xi^{\frac{(s-l) g+i}{2}} \otimes \bigoplus_{\xi \in \mathfrak{A}\left(\pi_{o}\right)} \xi^{-1}\right)
$$

La preuve est identique à celle de la proposition (IV.8.3.4).

On revient alors à l'étude de la suite spectrale (V.4.0.40) dont on vient de déterminer les termes $E_{1}^{i, j}\left[\Pi^{\infty, o}\right]$. La détermination des flèches $d_{1}^{i, j}$ se fait alors comme dans la preuve de la proposition (V.1.1) car à nouveau les divers $\pi_{o, i}$ n'interagissent pas entre eux dans les arguments de dualité. Considérons en effet le cas de deux contributions : si celles-ci correspondent à des Steinberg généralisées alors tout est concentré sur les $H^{0}$ de sorte que toutes les flèches sont nulles. Supposons donc avoir à traiter le cas de deux Speh ${ }^{(9)}$. Si elles sont de même longueur, on se retrouve alors dans la situation de la preuve de (V.1.1) mais avec des multiplicité 2, ce qui ne change en rien les arguments. Supposons les alors de longueur distinctes, $l^{\prime}<l$ et reprenons les arguments de la preuve de (V.1.1). Tant qu'on étudie des $H^{i}\left(g r_{k}\right)$ avec $\min \{|i|,|k|\}>l$, il n'y a rien à rajouter car n'intervient que la composante de plus grande longueur. Dans les autres cas, l'argument consiste à comparer par dualité les termes $H^{i}$ et $H^{-i}$. Pour $i>0, H^{i}$ (resp. $H^{-i}$ ) est une somme directe constituée de représentations de la forme

$$
[\overrightarrow{l-1}]_{\pi_{o}} \times\left(\left[\overrightarrow{r_{1}-1}\right]_{\pi_{o}} \overrightarrow{\times}\left[\overleftarrow{l^{\prime}-r_{1}-r_{2}-1}\right]_{\pi_{o}} \vec{\otimes}\left[\overrightarrow{r_{2}-1}\right]_{\pi_{o}}\right)
$$

avec $r_{2} \leqslant l^{\prime} / 2$ (resp. $\left.r_{1} \leqslant l^{\prime} / 2\right)(10)$

$$
\left(\left[\overrightarrow{r_{1}-1}\right]_{\pi_{o}} \overrightarrow{\times}\left[\overleftrightarrow{l-r_{1}-r_{2}-1}\right]_{\pi_{o}} \overrightarrow{\times}\left[\overrightarrow{r_{2}-1}\right]_{\pi_{o}}\right) \times\left[\overrightarrow{l^{\prime}-1}\right]_{\pi_{o}}
$$

avec $r_{2} \leqslant l / 2$ (resp. $r_{1} \leqslant l / 2$ ). On remarque alors que les composants de (V.4.0.41) sont disjoints de ceux de (V.4.0.42), sauf éventuellement $[\overrightarrow{l-1}]_{\pi_{o}} \times\left[\overrightarrow{l^{\prime}-1}\right]_{\pi_{o}}$. Ce sont donc les seuls qui peuvent rester dans l'aboutissement ${ }^{(11)}$. Pour voir que ces derniers restent effectivement dans l'aboutissement il suffit de remarquer qu'ils n'apparaissent que dans les termes $H^{i}\left(g r_{0}\right)\left[\Pi^{\infty, o}\right]$ de sorte qu'il ne peut y avoir de simplifications, d'où le résultat.

${ }^{(8)}$ Dans le cas respé on utilise que $(-1)^{s-1}=\epsilon(\Pi)$.

(9) Le cas d'une Speh avec une St se traite de la même façon.

(10) On peut être plus précis, cf. la proposition (IV.8.3.4).

(11) On le savait déjà car seules les représentations automorphes subsistent dans l'aboutissement. 


\section{APPENDICE A}

\section{CAS DE LA CARACTÉRISTIQUE MIXTE}

Il s'agit dans un premier temps de donner la correspondance entre nos notations et celles de $[14]$.

\begin{tabular}{|c|c|}
\hline égale caractéristique : & caractéristique mixte : [14] \\
\hline$F$ & $F$ \\
\hline$o$ & $\omega$ \\
\hline$\Psi_{F_{o}, m}^{d, i}$ & $\Psi_{F_{\omega}, l, d, m}^{i}$ \\
\hline$L_{g}$ & $\operatorname{rec}_{F_{\omega}}^{v}$ \\
\hline $\mathrm{cl}$ & $\operatorname{Art}_{F_{\omega}}^{-1}$ \\
\hline$D_{\mathbb{A}}^{\times}$ & $G_{\tau}(\mathbb{A})$ \\
\hline$\left(D_{\mathbb{A}}^{\infty}\right)^{\times}$ & $G\left(\mathbb{A}^{\infty}\right)=G_{\tau}\left(\mathbb{A}^{\infty}\right)$ \\
\hline$M_{I, o}$ & $X_{U}$ \\
\hline$M_{I, s_{o}}$ & $\bar{X}_{U}$ \\
\hline$M_{\bar{I}, s_{o}}^{=h}$ & $\bar{X}_{U}^{(d-h)}$ \\
\hline$P_{h, d}$ & $P_{d-h}$ \\
\hline$M_{I^{o} \mathcal{M}^{n}, s_{o}, 1}^{=h}$ & $\bar{X}_{U^{p}, m, M}^{(d-h)}$ \\
\hline $\begin{array}{c}\mathcal{F}_{\tau_{o}} \\
\left(D^{\infty}, 0\right) \times\end{array}$ & $\mathcal{F}_{\tau_{o}^{\vee}}$ \\
\hline$\left(D_{\mathbb{A}}^{\infty, o}\right)^{\times}$ & $G\left(\mathbb{A}^{\infty, p}\right) \times \mathbb{Q}_{p}^{\times} \times \prod_{i=2}^{r}\left(B_{\omega_{i}}^{o p}\right)^{\times}$ \\
\hline$\rho_{\infty}$ & $\xi$ \\
\hline$P_{h, d}$ & $P_{h}^{\text {op }}$ \\
\hline
\end{tabular}

où on $\mathrm{a}$

$$
\begin{gathered}
G\left(\mathbb{Q}_{p}\right) \simeq \mathbb{Q}_{p}^{\times} \times G L_{d}\left(F_{\omega}\right) \times \prod_{i=2}^{r}\left(B_{\omega_{i}}^{o p}\right)^{\times} \\
G\left(\mathbb{A}^{\infty}\right)=G\left(\mathbb{A}^{\infty, p}\right) \times \mathbb{Q}_{p}^{\times} \times G L_{d}\left(F_{\omega}\right) \times \prod_{i=2}^{r}\left(B_{\omega_{i}}^{o p}\right)^{\times}
\end{gathered}
$$

L'algèbre $\bar{D}$ associée aux points supersinguliers est $H_{z}$ (cf. [14] p.153, qui dans [13] p.33 et p.62 est noté $I_{x}$ ) où $z$ est un point de la strate basique (groupe des quasi-isogénies du triplet $(A, \lambda, i)$ associé à $z$, cf. loc. cit.).

A.0.0.1 - Notes sur les induites: dans le but de garder la même combinatoire avec les mêmes notations, il convient de faire les modifications suivantes aux définitions du §II.1.1.

- comme précisé plus haut, on considère les paraboliques standards (en caractéristique mixte) en lieu et place des paraboliques opposés (en égale caractéristique) en particulier dans la définition de $\operatorname{red}_{\tau_{o}}^{h}$; 
- pour $\pi_{1}$ (resp. $\pi_{2}$ ) une représentation de $G L_{n_{1}}\left(F_{o}\right)$ (resp. $\pi_{1} \times \pi_{2}$ désigne l'induite

$$
\operatorname{Ind}_{P_{n_{1}, n_{1}+n_{2}}\left(F_{o}\right)}^{G L_{n_{1}+n_{2}}\left(F_{o}\right)} \pi_{1}\left(n_{2}\right) \otimes \pi_{2}\left(-n_{1}\right) .
$$

Dans le cas où $n_{1}=l_{1} g$ et $n_{2}=l_{2} g, \pi_{1} \overrightarrow{\times} \pi_{2}$ (resp. $\pi_{1} \overleftarrow{\times} \pi_{2}$ ) désigne l'induite normalisée $\pi_{1}\left(-l_{2}\right) \times \pi_{2}\left(l_{1}\right)\left(\right.$ resp. $\left.\pi_{1}\left(l_{2}\right) \times \pi_{2}\left(-l_{1}\right)\right)$;

- soit $g$ un diviseur de $d=s g$ et $\pi_{o}$ une représentation irréductible cuspidale de $G L_{g}\left(F_{o}\right)$. Pour $1 \leqslant h \leqslant d$, le foncteur de Jacquet vérifie les propriétés suivantes :

- si $g$ ne divise pas $h$, alors

$$
J_{N_{h, d}^{\mathrm{op}}}\left([\overleftarrow{s-1}]_{\pi_{o}}\right)=J_{N_{h, d}^{\mathrm{op}}}\left([\overrightarrow{s-1}]_{\pi_{o}}\right)=(0)
$$

- si $h=l g$ alors

$$
\begin{aligned}
& J_{N_{l g, s g}^{\mathrm{op}}}\left([\overleftarrow{s-1}]_{\pi_{o}}\right)=[\overleftarrow{l-1}]_{\pi_{o}((l-s) / 2)} \otimes[\overleftarrow{s-l-1}]_{\pi_{o}(l / 2)} \\
& J_{N_{l g, s g}^{\mathrm{op}}}\left([\overrightarrow{s-1}]_{\pi_{o}}\right)=[\overrightarrow{l-1}]_{\pi_{o}((s-l) / 2)} \otimes[\overrightarrow{s-l-1}]_{\pi_{o}(-l / 2)}
\end{aligned}
$$

- $V\left(\pi_{o}, s\right)$ est ici l'induite $\pi_{o}\left(\frac{1-s}{2}\right) \times \cdots \times \pi_{o}\left(\frac{s-1}{2}\right)$. La représentation de Steinberg généralisée $\operatorname{St}_{s}\left(\pi_{o}\right)\left(\operatorname{resp} . \operatorname{Speh}_{s}\left(\pi_{o}\right)\right)$ est encore notée $[\overleftarrow{s-1}]_{\pi_{o}}\left(\right.$ resp. $\left.[\overrightarrow{s-1}]_{\pi_{o}}\right)$ et de manière générale on reprend les notations de $\S$ II.1.1 de sorte que par rapport à la remarque (II.1.1.6), les flèches correspondent aux orientations du graphe

$$
\circ^{\frac{1-s}{2}} \rightarrow \circ \cdots \rightarrow \circ^{\frac{s-1}{2}}
$$

numéroté donc dans l'ordre inverse par rapport à loc. cit. de sorte que les orientations sont cette fois-ci compatibles aux foncteurs de Jacquet pour les paraboliques standards. On notera par ailleurs que ce l'on note, par exemple, $[\overleftarrow{l-1}, \overrightarrow{s-l}]_{\pi_{o}}$ dans ce paragraphe, est noté $[\overrightarrow{s-l}, \overleftarrow{l-1}]_{\pi_{o}}$ au $\S I I .1 .1$ de sorte que, par exemple, les énoncés locaux en égale caractéristique et en caractéristique mixte, bien que noté de la manière similaire avec nos notations, sont en fait duaux.

A.0.0.2 - On a ainsi : $\bar{X}_{U^{p}, m}^{(d-h)}=\bar{X}_{U^{p}, m, M}^{(d-h)} \times_{P_{h, d}\left(\mathcal{O}_{o} / \mathcal{M}_{o}^{n}\right)} G L_{d}\left(\mathcal{O}_{o} / \mathcal{M}_{o}^{n}\right)$ et donc

$$
\lim _{\vec{U}} H_{c}^{i}\left(\bar{X}_{U^{p}, m}^{(d-h)}, \mathcal{L}_{\xi}\right)=\operatorname{Ind}_{P_{h, d}\left(F_{o}\right)}^{G L_{d}\left(F_{o}\right)} \lim _{\vec{U}} H_{c}^{i}\left(\bar{X}_{U^{p}, m, M}^{(d-h)}, \mathcal{L}_{\xi}\right)
$$

où les $\bar{X}_{U^{p}, m, M}^{(d-h)}{ }^{(1)}$ sont munis par correspondances d'une action de $\mathbb{Z} \times G L_{d-h}\left(F_{o}\right)$ de sorte que l'action de $\left(\left(\begin{array}{cc}g_{o}^{c} & * \\ 0 & g_{o}^{e t}\end{array}\right), c_{o}\right) \in P_{h, d}\left(F_{o}\right) \times W_{o}$ est donnée par l'action de $\left(\operatorname{val}\left(\operatorname{det} g_{o}^{c}\right)-\operatorname{deg} c_{o}, g_{o}^{e t}\right) \in$ $\mathbb{Z} \times G L_{d-h}\left(F_{o}\right)$.

A.0.0.3 - En ce qui concerne les propriétés cohomologiques associées aux systèmes locaux d'Harris-Taylor du paragraphe (IV.2.2), elles sont présentes dans [14], soit :

(a) l'isomorphisme (IV.2.2.12) avec l'action de $G\left(\mathbb{A}^{\infty}\right)$, est donné au corollaire IV.2.3 et IV.2.4 en utilisant la formule du bas de la page 138. L'action de $\mathbb{Z}$ dans l'énoncé de (IV.2.2.12) découle de l'action naturelle de $\mathbb{Z}$ sur les variétés d'Igusa de première espèce, cf. loc. cit. p.122;

(b) la proposition (IV.2.2.1) correspond au corollaire IV.2.8 de [14], où donc l'action d'un élément

$$
\left(g^{p}, g_{p, 0}, g_{\omega}^{0}, g_{\omega}^{e t}, g_{\omega_{i}}, \sigma\right) \in
$$

$$
G\left(\mathbb{A}^{\infty, p}\right) \times \mathbb{Q}_{p}^{\times} \times G L_{n-h}\left(F_{\omega}\right) \times G L_{h}\left(F_{\omega}\right) \times \prod_{i=2}^{r}\left(B_{\omega_{i}}^{o p}\right)^{\times} \times W_{F_{\omega}}
$$

\footnotetext{
${ }^{(1)}$ On rappelle que pour tout $U$, il y a un morphisme radiciel $I_{U^{p}, m}^{(h)} \longrightarrow \bar{X}_{U^{p}, m, M}^{(d-h)}$.
} 
est donnée par l'action de

$$
\left(g^{p}, g_{p, 0} p^{f_{1}\left(w\left(w(\sigma)-\operatorname{det} g_{\omega}^{0}\right)\right)}, \delta, g_{\omega}^{e t}, g_{\omega_{i}}\right) \times\left(g_{\omega}^{0}, \sigma\right) \in G^{(h)}\left(\mathbb{A}^{\infty}\right) / \mathcal{O}_{D_{F_{\omega}, n-h}}^{\times} \times G L_{d-h}\left(F_{\omega}\right) \times W_{F_{\omega}}
$$

avec

$$
G^{(h)}\left(\mathbb{A}^{\infty}\right)=G\left(\mathbb{A}^{\infty, o}\right) \times \mathbb{Q}_{p}^{\times} \times D_{F_{\omega}, n-h}^{\times} \times G L_{h}\left(F_{\omega}\right) \times \prod_{i=2}^{r}\left(B_{\omega_{i}}^{o p}\right)^{\times}
$$

où $\delta \in D_{F_{\omega}, n-h}^{\times}$est tel que $w(\operatorname{det} \delta)=w(\sigma)-w\left(\operatorname{det} g_{\omega}^{0}\right)$ et $\mathcal{O}_{D_{F_{\omega}, n-h}}$ est l'ordre maximal de $D_{F_{\omega}, n-h}$;

Remarque : On notera que les signes opposés dans $w(\sigma)-w\left(\operatorname{det} g_{\omega}^{0}\right)$ sont compatibles à l'utilisation des paraboliques standards $P_{h, d}$ dans les formules d'induction tout au long de la preuve, cf. par exemple la proposition (I.5.3.10). Les preuves sont par ailleurs strictement similaires en modifiant les calculs de $\operatorname{red}_{\tau_{o}}^{h}$. Dans certaines formules où n'apparaît pas les notations $\overleftarrow{x}$ ou $\overrightarrow{\times}$ qui intègrent les modifications à effectuer entre égale et inégale caractéristique, il faut rajouter un signe devant la torsion pour l'action du groupe linéaire; en particulier la formule (V.1.0.39) doit se modifier comme suit :

$$
\begin{aligned}
\lim _{\vec{I}} H_{c}^{d-l g}\left(M_{I, s_{o}, 1}^{=l g}, \mathcal{F}\left(g, l, \pi_{o}\right)_{1} \otimes \mathcal{L}_{\rho_{\infty}}\right)\left[\Pi^{\infty, o}\right]= & \\
& m(\Pi) \bigoplus_{\xi \in \mathfrak{A}\left(\pi_{o}\right)}[\overleftarrow{s-l-1}]_{\pi_{o}(-l(g-1) / 2)} \otimes \Xi^{(s-l)(g-1) / 2}
\end{aligned}
$$

de sorte qu'en tant que $G L_{d}\left(F_{o}\right) \times W_{o}$-module, on a

$$
\begin{aligned}
& \lim _{\vec{I}} H_{c}^{d-l g}\left(M_{I, s_{o}}^{=l g}, H T_{\rho_{\infty}}\left(g, l, \pi_{o},[\overleftarrow{l-1}]_{\pi_{o}}\right)\right)\left[\Pi^{\infty, o}\right] \otimes \Xi^{\frac{l g-1}{2}}= \\
& m(\Pi) e_{\pi_{o}} \operatorname{Ind}_{P_{l g, d}\left(F_{o}\right)}^{G L_{d}\left(F_{o}\right)}[\overleftarrow{l-1}]_{\pi_{o}((s-l)(g-1) / 2)} \otimes[\overleftarrow{s-l-1}]_{\pi_{o}(-l(g-1) / 2)} \otimes \Xi^{\frac{d-s+l-1}{2}} \\
& \simeq m(\Pi) e_{\pi_{o}}[\overleftarrow{l-1}]_{\pi_{o}} \overrightarrow{\times}[\overleftarrow{s-l-1}]_{\pi_{o}} \otimes \Xi^{\frac{d-s+l-1}{2}}
\end{aligned}
$$

où l'on notera bien que $\mathcal{F}\left(g, l, \pi_{o}\right)$ est le faisceau induit à partir du système local d'HarrisTaylor, $\mathcal{F}_{\mathrm{JL}^{-1}\left(\mathrm{St}_{s}\left(\pi_{o}\right)^{\vee}\right)}$, de même que $\mathcal{P}\left(g, l, \pi_{o}\right)$ est l'extension intermédiaire de $\mathcal{F}\left(g, l, \pi_{o}\right)[d-$ $l g] \otimes[\overleftarrow{l-1}]_{\pi_{o}} \otimes L_{g}\left(\pi_{o}\right)^{\vee}$. De manière générale il faut aussi remplacer $\mathrm{JL}^{-1}(\bullet)$ par $\mathrm{JL}^{-1}(\bullet)^{\vee}$.

(c) la condition $\operatorname{Hyp}(\infty)$ est la suivante $: \Pi_{\infty}$ est cohomologique pour une certaine représentation algébrique $\xi$ sur $\mathbb{C}$ de la restriction des scalaires de $F$ à $\mathbb{Q}$ de $G L_{g}$, i.e. il existe $i$ tel que

$$
H^{i}\left(\left(\operatorname{Lie} G_{\tau}(\mathbb{R})\right) \otimes_{\mathbb{R}} \mathbb{C}, U_{\tau}, \Pi_{\infty} \otimes \xi^{\prime}\right) \neq(0)
$$

où $U_{\tau}$ est un sous-groupe compact modulo le centre de $G_{\tau}(\mathbb{R})$, maximal, cf. [14] p.92, et où $\xi^{\prime}$ est le caractère sur $\mathbb{C}$ associé à $\xi$ via un isomorphisme $\overline{\mathbb{Q}}_{l} \simeq \mathbb{C}$ fixé. On dira que $\Pi$ vérifie $\operatorname{Hyp}(\xi)$ si $\Pi$ vérifie $\operatorname{Hyp}(\infty)$ pour $\xi$.

(d) l'équivalent de la proposition (IV.2.2.4) correspond à la deuxième identité fondamentale, théorème V.5.4, où Red est définie p.182.

Retour sur la preuve de la proposition (IV.7.3.2). — : (d'après une idée de M. Harris) on rappelle que dans [14], la somme alternée de la cohomologie de la variété globale à valeur dans $\mathcal{L}_{\xi}$, dans le groupe de Grothendieck correspondant, est écrite sous la forme

$$
\sum_{\pi^{\infty}} \pi^{\infty} \otimes\left[R_{\xi}\left(\pi^{\infty}\right)\right] \quad\left[R_{\xi}\left(\pi^{\infty}\right)\right]=\sum_{i}(-1)^{i} R_{\xi}^{i}\left(\pi^{\infty}\right)
$$

où $\pi^{\infty}$ décrit l'ensemble des représentations irréductibles de $G\left(\mathbb{A}^{\infty}\right)$. Par ailleurs, corollaire VI.2.7 de loc. cit., s'il existe une représentation irréductible $\pi_{\infty}$ de $G_{\tau}(\mathbb{R})$ telle que $\pi:=\pi^{\infty} \otimes \pi_{\infty}$ vérifie 
$\operatorname{Hyp}(\infty)$ avec $B C(\pi)=(\psi, \Pi)$ où $\mathrm{JL}(\Pi)$ est cuspidale, avec les notations de loc. cit., alors $R_{\xi}^{i}\left(\pi^{\infty}\right)$ est nul pour $i \neq d-1$.

Soit alors $\sigma_{\omega}=L_{g}\left(\pi_{\omega}\right)$ et soit $F_{\omega}^{\prime}$ une extension de $F_{\omega}$ telle que la restriction de $\sigma_{\omega}$ à $F_{\omega}^{\prime}$ soit non ramifiée. On rappelle que l'extension $F_{\omega}^{\prime} / F_{\omega}$ est résoluble; cf.[22] IV-2. On globalise alors la situation comme dans [14] : soit $F=F^{+} M$ où $M$ est un corps quadratique imaginaire dans lequel $p$ se décompose et soit $\left(F^{\prime}\right)^{+} / F^{+}$une extension résoluble de corps totalement réels telle que :

-la place $\omega$ de $F^{+}$soit inerte dans $\left(F^{\prime}\right)^{+}$;

- l'extension $\left(F^{\prime}\right)_{\omega}^{+} / F_{\omega}^{+}$est isomorphe à l'extension $F_{\omega}^{\prime} / F_{\omega}$.

On pose alors $F^{\prime}=M\left(F^{\prime}\right)^{+}$. D'après le corollaire VI.2.6 de loc. cit., on peut choisir une représentation automorphe cuspidale $\Pi$ de $G L_{d}\left(\mathbb{A}_{F}\right)$ telle que $\Pi$ vérifie :

$-\Pi^{c} \simeq \Pi^{\vee}$;

- $\Pi_{\infty}$ a le même caractère central qu'une représentation algébrique de $\operatorname{Res}_{\mathbb{Q}}^{G}\left(G L_{d}\right)$;

- $\Pi_{\omega} \simeq \operatorname{St}_{s}\left(\pi_{\omega} \otimes \psi\right)$ pour un certain caractère $\psi$ de $F_{\omega}^{\prime}$.

Soit alors le changement de base $\Pi^{\prime}:=B C_{F^{\prime} / F}(\Pi)$; d'après loc. cit. (théorèmes VI.1.1 et VI.2.9) on associe à $\Pi$ et $\Pi^{\prime}$ des représentations $\pi$ et $\pi^{\prime}$ de respectivement $G_{\tau}\left(\mathbb{A}_{F}\right)$ et $G_{\tau}\left(\mathbb{A}_{F^{\prime}}\right)$ qui vérifient $\operatorname{Hyp}(\infty)$ avec $\pi_{\omega}^{\prime} \simeq[\overleftarrow{s-1}]_{\zeta} \boxplus \cdots \boxplus[\overleftarrow{s-1}]_{\zeta}$, pour $\zeta$ un caractère de $F_{o}^{\prime}$. Par ailleurs d'après le théorème VII.1.9 de loc. cit. appliqué aux bonnes places de $F$, et en utilisant le théorème de densité de Cebotarev, on en déduit que la représentation galoisienne $R_{\xi^{\prime}}\left(\pi^{\prime}, \infty\right)$ est isomorphe à $R_{\xi}\left(\pi^{\infty}\right)_{\mid \operatorname{Gal}\left(\bar{F}^{\prime} / F^{\prime}\right)}$ avec

$$
R_{\xi}\left(\pi^{\prime}, \infty\right)_{\omega} \simeq L_{d}\left(\pi_{\omega}^{\prime}\right)^{\vee}=\left(\operatorname{Sp}_{s} \otimes \zeta^{-1}\right)^{g}
$$

de sorte que $R_{\xi}\left(\pi^{\infty}\right) \simeq \mathrm{Sp}_{s} \otimes L_{g}\left(\pi_{o}\right)^{\vee}$, d'où le résultat. 


\section{APPENDICE B}

\section{RÉCAPITULATIF DES CALCULS}

\section{B.1. sur les faisceaux}

(1) $-R \Psi_{\eta_{o}}\left(\mathcal{L}_{\rho_{\infty}}\right) \simeq R \Psi_{\eta_{o}}\left(\overline{\mathbb{Q}}_{l}\right) \otimes \mathcal{L}_{\rho_{\infty}} ;$

- $R \Psi_{\eta_{o}}\left(\overline{\mathbb{Q}}_{l}\right)[d-1]$ est un objet de $\operatorname{FPH}\left(M_{s_{o}}\right)$;

- $R \Psi_{\eta_{o}}\left(\overline{\mathbb{Q}}_{l}\right)[d-1]=\bigoplus_{\pi_{o} \in \operatorname{Cusp}(d)} R \Psi_{\eta_{o}, \pi_{o}}\left(\overline{\mathbb{Q}}_{l}\right)[d-1]$ avec les notations de la proposition (IV.4.1.3), où $\operatorname{Cusp}(d)$ désigne l'ensemble des classes d'équivalence inertielle des représentations irréductibles cuspidales de $G L_{g}\left(F_{o}\right)$ pour tout $1 \leqslant g \leqslant d$;

- $R \Psi_{\eta_{o}, \pi_{o}}\left(\overline{\mathbb{Q}}_{l}\right)[d-1]$ est mixte de poids $d-1$; sa filtration de monodromie est égale à celle par le poids au décalage de $d-1$ près, on note $g r_{k, \pi_{o}}$ le gradué de poids $k$ de sa filtration de monodromie de sorte qu'en particulier $N^{k}: g r_{k, \pi_{o}} \stackrel{\sim}{\longrightarrow} g r_{-k, \pi_{o}}$;

- pour $1 \leqslant g \leqslant d$ et $\pi_{o}$ une représentation irréductible cuspidale de $G L_{g}\left(F_{o}\right)$; pour tout $|k|<s_{g}:=\lfloor d / g\rfloor$, dans $\operatorname{FPH}\left(M_{s_{o}}\right)$ on a (cf. IV.4.4.4) :

$$
e_{\pi_{o}} g r_{k, \pi_{o}}=\bigoplus_{\substack{|k|<l \leqslant s_{g} \\ l \equiv k-1 \bmod 2}} \mathcal{P}\left(g, l, \pi_{o}\right)\left(-\frac{l g+k-1}{2}\right)
$$

où $\mathcal{P}\left(g, l, \pi_{o}\right):=j_{! *}^{\geqslant l g} H T\left(g, l, \pi_{o},[\overleftarrow{l-1}]_{\pi_{o}}\right)[d-l g] \otimes L_{g}\left(\pi_{o}\right)$;

- la suite spectrale des poids (IV.4.1.17) dégénère en $E_{2}$ et les applications $d_{1}^{i, j}$ sont induites par les suites exactes (IV.3.2.15).

(2) $j_{!_{*}}^{\geqslant l g} H T\left(g, l, \pi_{o}, \Pi_{l}\right)[d-l g] \in \operatorname{FPH}\left(M_{s_{o}}\right)$ est pur de poids $d-l g$ pour $\pi_{o}$ unitaire; ses faisceaux de cohomologies $h^{i} j_{!_{*}}^{\geqslant l g} H T\left(g, l, \pi_{o}, \Pi_{l}\right)[d-l g]$ sont, pour $g>1^{(1)}$, nuls pour $i$ ne s'écrivant pas sous la forme $l g-d+a(g-1)$ avec $0 \leqslant a \leqslant s_{g}-l$ et pour un tel $i=l g-d+a(g-1)$, isomorphe dans $\mathrm{FH}\left(M_{s_{o}}\right)$ à

$$
j_{!}^{\geqslant(l+a) g} H T\left(g, l+a, \pi_{o}, \Pi_{l} \overrightarrow{\times}[\overrightarrow{a-1}]_{\pi_{o}}\right) \otimes \Xi^{\frac{a(g-1)}{2}}
$$

(3) Pour tout $1 \leqslant l \leqslant s_{g}$, on a, cf. (IV.5.3.3), l'égalité dans $\mathfrak{G}$

$$
j_{!}^{\geqslant l g} H T\left(g, l, \pi_{o}, \Pi_{l}\right)[d-l g]=\sum_{i=l}^{s_{g}} j_{! *}^{\geqslant i g} H T\left(g, i, \pi_{o}, \Pi_{l} \overrightarrow{\times}[\overleftarrow{i-l-1}]_{\pi_{o}}\right)[d-i g] \otimes \Xi^{\frac{(l-i)(g-1)}{2}}
$$

Dualement on a

$R j_{*}^{\geqslant l g} H T\left(g, l, \pi_{o}, \Pi_{l}\right)[d-l g]=\sum_{i=l}^{s_{g}} j_{! *}^{\geqslant i g} H T\left(g, i, \pi_{o}, \Pi_{l} \overleftarrow{\times}[\overleftarrow{i-l-1}]_{\pi_{o}}\right)[d-i g] \otimes \Xi^{\frac{(l-i)(g+1)}{2}}$

On en déduit alors que, au moins pour $g>1$,

$\overline{(1) \text { pour } g=1 \mathrm{cf} .}$ (IV.4.4.7) 


$$
\begin{aligned}
R^{i} j_{*}^{\geqslant l g} H T\left(g, l, \pi_{o}, \Pi_{l}\right)[d-l g]= & \bigoplus_{(r, a) / i=(l+r+a) g-d-a} \\
& j_{!}^{\geqslant(l+r+a) g} H T\left(g, l+r+a, \pi_{o},\left(\Pi_{l} \overleftarrow{\times}[\overleftarrow{r-1}]_{\pi_{o}}\right) \overrightarrow{\times}[\overrightarrow{a-1}]_{\pi_{o}}\right) \otimes \Xi^{((r+a)(g-1)+2 r) / 2}
\end{aligned}
$$

\section{B.2. de groupe de cohomologie}

- Cas où $\Pi_{o}$ est de la forme $\operatorname{St}_{s}\left(\pi_{o}\right)$ :

(1) d'après la proposition (IV.5.4.1), $H^{i}\left(j_{! *}^{\geqslant l g} H T_{\rho_{\infty}}\left(g, l, \pi_{o}, \Pi_{l}\right)\right)\left[\Pi^{\infty}\right]$ est nul pour tout $i$.

(2) d'après le corollaire (IV.5.4.3), $H^{i}\left(j_{!}^{\geqslant l g} H T_{\rho_{\infty}}\left(g, l, \pi_{o}, \Pi_{l}\right)\right)\left[\Pi^{\infty}\right]$ est donné par

$$
\left\{\begin{array}{cl}
0 & \text { si } i \neq 0 \\
m(\Pi) \Pi_{l} \overrightarrow{\times}[\overleftarrow{s-l-1}]_{\pi_{o}} \otimes\left(\Xi^{\frac{(s-l)(g-1)}{2}} \otimes \bigoplus_{\xi \in \mathfrak{A}\left(\pi_{o}\right)} \xi^{-1}\right) & i=0
\end{array}\right.
$$

où $\mathfrak{A}\left(\pi_{o}\right)$ est l'ensemble des caractères $\xi: \mathbb{Z} \longrightarrow \mathbb{Q}_{l}^{\times}$, tels que $\pi_{o} \otimes \xi^{-1} \circ \operatorname{val}($ det $) \simeq \pi_{o}$.

(3) par application de la dualité de Verdier, $H^{i}\left(R j_{*}^{\geqslant l g} H T_{\rho_{\infty}}\left(g, l, \pi_{o}, \Pi_{l}\right)\right)\left[\Pi^{\infty}\right]$ est donné par

$$
\left\{\begin{array}{cl}
m(\Pi) \Pi_{l} \overleftarrow{\times}[\overleftarrow{s-l-1}]_{\pi_{o}} \otimes\left(\Xi^{(s-l)(g+1) / 2} \otimes \bigoplus_{\xi \in \mathfrak{A}\left(\pi_{o}\right)} \xi^{-1}\right) & \text { si } i \neq 0
\end{array}\right.
$$

- Cas où $\Pi_{o}$ est de la forme $\operatorname{Speh}_{s}\left(\pi_{o}\right)$ :

(1) d'après la proposition (IV.8.3.4), $H^{i}\left(j_{!_{*}}^{\geqslant l g} H T_{\rho_{\infty}}\left(g, l, \pi_{o}, \Pi_{l}\right)\right)\left[\Pi^{\infty}\right]$ est donné par

$$
\left\{\begin{array}{lr}
m(\Pi) \Pi_{l} \overrightarrow{\times}[\overrightarrow{s-l-1}]_{\pi_{o}} \otimes\left(\Xi^{\frac{(s-l)(g-1)}{2}} \otimes \bigoplus_{\xi \in \mathfrak{A}\left(\pi_{o}\right)} \xi^{-1}\right) & i=l-s \\
m(\Pi)\left(\Pi_{l} \overrightarrow{\times}\left[\frac{\overrightarrow{s-l-i}}{2}-1\right]_{\pi_{o}}\right) \overleftarrow{\times}\left[\frac{\overrightarrow{s-l+i}}{2}-1\right]_{\pi_{o}} \otimes\left(\Xi^{\frac{(s-l) g+i}{2}} \otimes \bigoplus_{\xi \in \mathfrak{A}\left(\pi_{o}\right)} \xi^{-1}\right) & l-s<i<s-l \\
m(\Pi) \Pi_{l} \overleftarrow{\times}[\overrightarrow{s-l-1}]_{\pi_{o}} \otimes\left(\Xi^{\frac{(s-l)(g+1)}{2}} \otimes \bigoplus_{\xi \in \mathfrak{A}\left(\pi_{o}\right)} \xi^{-1}\right) & i \equiv l-s \bmod 2 \\
& i=s-l
\end{array}\right.
$$

(2) d'après le corollaire (V.1.2), $H^{i}\left(j_{!}^{\geqslant l g} H T_{\rho_{\infty}}\left(g, l, \pi_{o}, \Pi_{l}\right)\right)\left[\Pi^{\infty}\right]$ est donné par

$$
\left\{\begin{array}{cl}
m(\Pi) \Pi_{l} \overleftarrow{\times}[\overrightarrow{s-l-1}]_{\pi_{o}} \otimes\left(\Xi \frac{(s-l)(g+1)}{2} \bigoplus_{\xi \in \mathfrak{A}\left(\pi_{o}\right)} \xi^{-1}\right) & i=s-l
\end{array}\right.
$$

(3) par application de la dualité de Verdier, $H^{i}\left(R j_{*}^{\geqslant l g} H T_{\rho_{\infty}}\left(g, l, \pi_{o}, \Pi_{l}\right)\right)\left[\Pi^{\infty}\right]$ est donné par

$$
\left\{\begin{array}{cl} 
& \text { si } i \neq 0 \\
m(\Pi) \Pi_{l} \overleftarrow{\times}[\overleftarrow{s-l-1}]_{\pi_{o}} \otimes\left(\Xi^{(s-l)(g+1) / 2} \otimes \bigoplus_{\xi \in \mathfrak{A}\left(\pi_{o}\right)} \xi^{-1}\right) & i=0
\end{array}\right.
$$

\section{B.3. sur la suite spectrale des cycles évanescents}

A partir du calcul des groupes de cohomologie à support compact des $H T\left(g, l, \pi_{o}, \Pi_{l}\right)$, l'isomorphisme (cf. la proposition (IV.2.2.1))

$$
H_{c}^{j}\left(M_{I, s_{o}, 1}^{=h}, R^{i} \Psi_{\eta_{o}}\left(\mathcal{L}_{\rho_{\infty}}\right)\right)^{h} \simeq \bigoplus_{\tau_{o} \in \mathfrak{C}_{h}}\left(H_{c}^{j}\left(M_{I, s_{o}, 1}^{=h}, \mathcal{F}_{\tau_{o}} \otimes \mathcal{L}_{\rho_{\infty}}\right) \otimes \widetilde{\left.\mathcal{U}_{F_{o}, m}^{h, i}\left(\tau_{o}\right)\right)^{h / e_{\tau_{o}}}}\right.
$$

permet de calculer les

$$
H_{=h, i, \pi_{o}, \rho_{\infty}}^{j}\left[\Pi^{\infty, o}\right]:=\underset{I}{\lim _{\longrightarrow}} H_{c}^{j}\left(M_{I, \bar{s}_{o}}^{=h}, R^{i} \Psi_{\eta_{o}, I, \pi_{o}}\left(\mathcal{L}_{\rho_{\infty}}\right)\right)
$$

Les suites spectrales associées à la stratification

$$
E_{1, I, \pi_{o}}^{p, q ; i}=H_{c}^{p+q}\left(M_{I, \bar{s}_{o}}^{=p-1}, R^{i} \Psi_{\eta_{o}, I, \pi_{o}}\left(\overline{\mathbb{Q}}_{l}\right)\right) \Rightarrow H_{c}^{p+q}\left(M_{I, \bar{s}_{o}}, R^{i} \Psi_{\eta_{o}, I, \pi_{o}}\left(\overline{\mathbb{Q}}_{l}\right)\right)
$$


permettent alors de calculer les termes $E_{2}^{i, j}\left[\Pi^{\infty, o}\right]$ de la suite spectrale des cycles évanescents. On résume les résultats obtenus dans

- le cas où $\Pi_{o} \simeq \operatorname{St}_{s}\left(\pi_{o}\right)$ : le corollaire (IV.5.4.9) donne tout d'abord :

B.3.0.4. Corollaire. - Les $H_{=h, i, \pi_{o}, \rho_{\infty}}\left[\Pi^{\infty, o}\right]$ vérifient les propriétés suivantes :

(1) pour $g$ divisant $d=s g$ et $\Pi_{o} \simeq[\overleftarrow{s-1}]_{\pi_{o}}$, les $H_{=h, i, \pi_{o}, \rho_{\infty}}\left[\Pi^{\infty, o}\right]$ sont nuls pour $h$ qui n'est pas de la forme $\lg$ avec $1 \leqslant l \leqslant s$;

(2) pour $g$ divisant $d=s g$ et $\Pi_{o} \simeq[\overleftarrow{s-1}]_{\pi_{o}}$, les $H_{=l g, i, \pi_{o}, \rho_{\infty}}^{j}\left[\Pi^{\infty, o}\right]$ sont nuls pour $j \neq d-l g$;

(3) pour $g$ divisant $d=$ sg et $\Pi_{o} \simeq[\overleftarrow{s-1}]_{\pi_{o}}$, les $H_{=l g, i, \pi_{o}, \rho_{\infty}}^{d-l g}\left[\Pi^{\infty, o}\right]$ sont nuls pour $i$ ne vérifiant pas $l(g-1) \leqslant i \leqslant l g-1$. Si $1 \leqslant l<s$ et $i=l g-r$ avec $1 \leqslant r \leqslant l$, on a

$$
H_{=l g, l g-r, \pi_{o}, \rho_{\infty}}^{d-l g}\left[\Pi^{\infty, o}\right] \simeq
$$

en tant que représentation de $G L_{d}\left(F_{o}\right) \times W_{o}$, où $m(\Pi)$ est la multiplicité de $\Pi$ dans l'espace des formes automorphes.

Ainsi, cf. la preuve de la proposition (IV.8.1.2), les suites spectrales associées à la stratification sont triviales, i.e. dégénèrent en $E_{1}$ de sorte que les aboutissements sont donnés par la proposition (IV.8.1.2) dont on rappelle l'énoncé ci-dessous :

B.3.0.5. Proposition. - Pour tout $0 \leqslant i \leqslant d-1$, les $H^{j}\left(R^{i} \Psi_{\eta_{o}, \pi_{o}}\left(\overline{\mathbb{Q}}_{l}\right)\right)\left[\Pi^{\infty, o}\right]$, en tant que $G L_{d}\left(F_{o}\right) \otimes W_{o}$-modules, vérifient les propriétés suivantes :

(1) ils sont nuls si $g$ n'est pas un diviseur de $d$;

(2) pour $g$ un diviseur de $d=s g$, ils sont nuls si $j$ n'est pas de la forme $d-l g$ pour $1 \leqslant l \leqslant s$;

(3) pour $g$ un diviseur de $d=s g$ et $j=d-l g$ avec $1 \leqslant l \leqslant s$, ils sont nuls si $i$ n'est pas de la forme $l g-r$ avec $1 \leqslant r \leqslant l$;

(4) pour $g$ un diviseur de $d=s g$ et $1 \leqslant l<s, H^{d-l g}\left(R^{l g-r} \Psi_{\eta_{o}, I, \pi_{o}}\left(\overline{\mathbb{Q}}_{l}\right)\right)\left[\Pi^{\infty, o}\right]$ est isomorphe $\grave{a}$

$$
m(\Pi)[\overleftarrow{l-r}, \overrightarrow{r-1}]_{\pi_{o}} \overrightarrow{\times}[\overleftarrow{s-l-1}]_{\pi_{o}} \otimes L_{g}\left(\pi_{o}\right)\left(-\frac{s(g-1)-2(r-l)}{2}\right)
$$

Enfin la suite spectrale des cycles évanescents dégénère en $E_{3}$ et l'aboutissement $H_{\eta_{o}}^{i}\left[\Pi^{\infty, o}\right]$ est donné par le corollaire (IV.8.1.1) :

B.3.0.6. Corollaire. - Les groupes de cohomologie de la fibre générique $H_{\eta_{o}}^{i}\left[\Pi^{\infty, o}\right]$ sont nuls pour $i \neq d-1$ et

$$
H_{\eta_{o}}^{d-1}\left[\Pi^{\infty, o}\right]=[\overleftarrow{s-1}]_{\pi_{o}} \otimes L_{g}\left([\overleftarrow{s-1}]_{\pi_{o}}\right)\left(-\frac{s g-1}{2}\right)
$$

- Cas où $\Pi_{o} \simeq \operatorname{Speh}_{s}\left(\pi_{o}\right)$ :

B.3.0.7. Corollaire. - Les $H_{=h, i, \pi_{o}, \rho_{\infty}}\left[\Pi^{\infty, o}\right]$ vérifient les propriétés suivantes :

(1) pour $g$ divisant $d=s g$ et $\Pi_{o} \simeq[\overrightarrow{s-1}]_{\pi_{o}}$, les $H_{=h, i, \pi_{o}, \rho_{\infty}}\left[\Pi^{\infty, o}\right]$ sont nuls pour $h$ qui n'est pas de la forme lg avec $1 \leqslant l \leqslant s$;

(2) pour $g$ divisant $d=$ sg et $\Pi_{o} \simeq[\overrightarrow{s-1}]_{\pi_{o}}$, les $H_{=l g, i, \pi_{o}, \rho_{\infty}}\left[\Pi^{\infty, o}\right]$ sont nuls pour $j \neq(s-$ l) $(g+1)$; 
(3) pour $g$ divisant $d=$ sg et $\Pi_{o} \simeq[\overrightarrow{s-1}]_{\pi_{o}}$, les $H_{=l g, i, \pi_{o}, \rho_{\infty}}^{(s-l)(g+1)}\left[\Pi^{\infty, o}\right]$ sont nuls pour $i$ ne vérifiant pas $l(g-1) \leqslant i \leqslant l g-1$. Si $1 \leqslant l<s$ et $i=l g-r$ avec $1 \leqslant r \leqslant l$, on a

$H_{=l g, l g-r, \pi_{o}, \rho_{\infty}}^{d-l g}\left[\Pi^{\infty, o}\right] \simeq$

$$
\left.m(\Pi)(\overleftarrow{[l-r}, \overrightarrow{r-1}]_{\pi_{o}} \overleftarrow{\times}[\overrightarrow{s-l-1}]_{\pi_{o}}\right) \otimes L_{g}\left(\pi_{o}\right)\left(-\frac{s(g+1)-2 r}{2}\right)
$$

en tant que représentation de $G L_{d}\left(F_{o}\right) \times W_{o}$, où $m(\Pi)$ est la multiplicité de $\Pi$ dans l'espace des formes automorphes.

Démonstration. - Le raisonnement est identique à celui de la preuve de la proposition (IV.8.1.2). On remarque alors que pour tout $k \geqslant 1$, les flèches

$$
d_{k}^{p, q ; i}: E_{k, \pi_{o}}^{p, q ; i}\left[\Pi^{\infty, o}\right] \longrightarrow E_{k, \pi_{o}}^{p+k, q+k-1}\left[\Pi^{\infty, o}\right]
$$

des suites spectrales associées à la stratification sont toutes nulles. En effet, d'après le corollaire (V.1.2), pour que $E_{k, \pi_{o}}^{p, q i}\left[\Pi^{\infty, o}\right]$ (resp. $E_{k, \pi_{o}}^{p+k, q+k-1 ; i}\left[\Pi^{\infty, o}\right]$ ) soit non nul, il faut qu'il existe $1 \leqslant l_{1} \leqslant s$ et $1 \leqslant r_{1} \leqslant l_{1}$ (resp. $1 \leqslant l_{2} \leqslant s$ et $\left.1 \leqslant r_{2} \leqslant l_{2}\right)$ avec

$$
\begin{gathered}
(p, q, i)=\left(l_{1} g+1, d-2 l_{1} g-1+s-l_{1}, l_{1} g-r_{1}\right) \\
\left(\text { resp. }(p+k, q+k-1, i)=\left(l_{2} g+1, d-2 l_{2} g-1+s-l_{2}, l_{2} g-r_{2}\right)\right) .
\end{gathered}
$$

Ce qui donne $1=(3 g+1)\left(l_{2}-l_{1}\right)$; on voit alors que pour tout $k \geqslant 1, E_{k, \pi_{o}}^{p, q ; i}\left[\Pi^{\infty, o}\right]$ et $E_{k, \pi_{o}}^{p+k, q+k-1}\left[\Pi^{\infty, o}\right]$ ne peuvent pas être tous deux non nuls de sorte que $E_{\infty, I, \pi_{o}}^{p, q ; i}\left[\Pi^{\infty, o}\right]=$ $E_{1, I, \pi_{o}}^{p, q ; i}\left[\Pi^{\infty, o}\right]$ d'où le résultat d'après le corollaire précédent.

Enfin la suite spectrale des cycles évanescents dégénère en $E_{3}$ et l'aboutissement $H_{\eta_{o}}^{i}\left[\Pi^{\infty, o}\right]$ est donnée par la proposition (V.1.1) dont on rappelle l'énoncé.

B.3.0.8. Proposition. - Les groupes de cohomologie de la fibre générique $H_{\eta_{o}, \rho_{\infty}}\left[\Pi^{\infty, o}\right]$ sont nuls pour $|d-1-i| \geqslant s$ ou pour $i \not \equiv d-s \bmod 2$ et sinon

$$
H_{\eta_{o}, \rho_{\infty}}^{d+s-2-2 i}\left[\Pi^{\infty, o}\right] \simeq[\overrightarrow{s-1}]_{\pi_{o}} \otimes L_{g}\left(\pi_{o}\right)\left(-\frac{d+s-2-2 i}{2}\right)
$$


APPENDICE C

FIGURES 

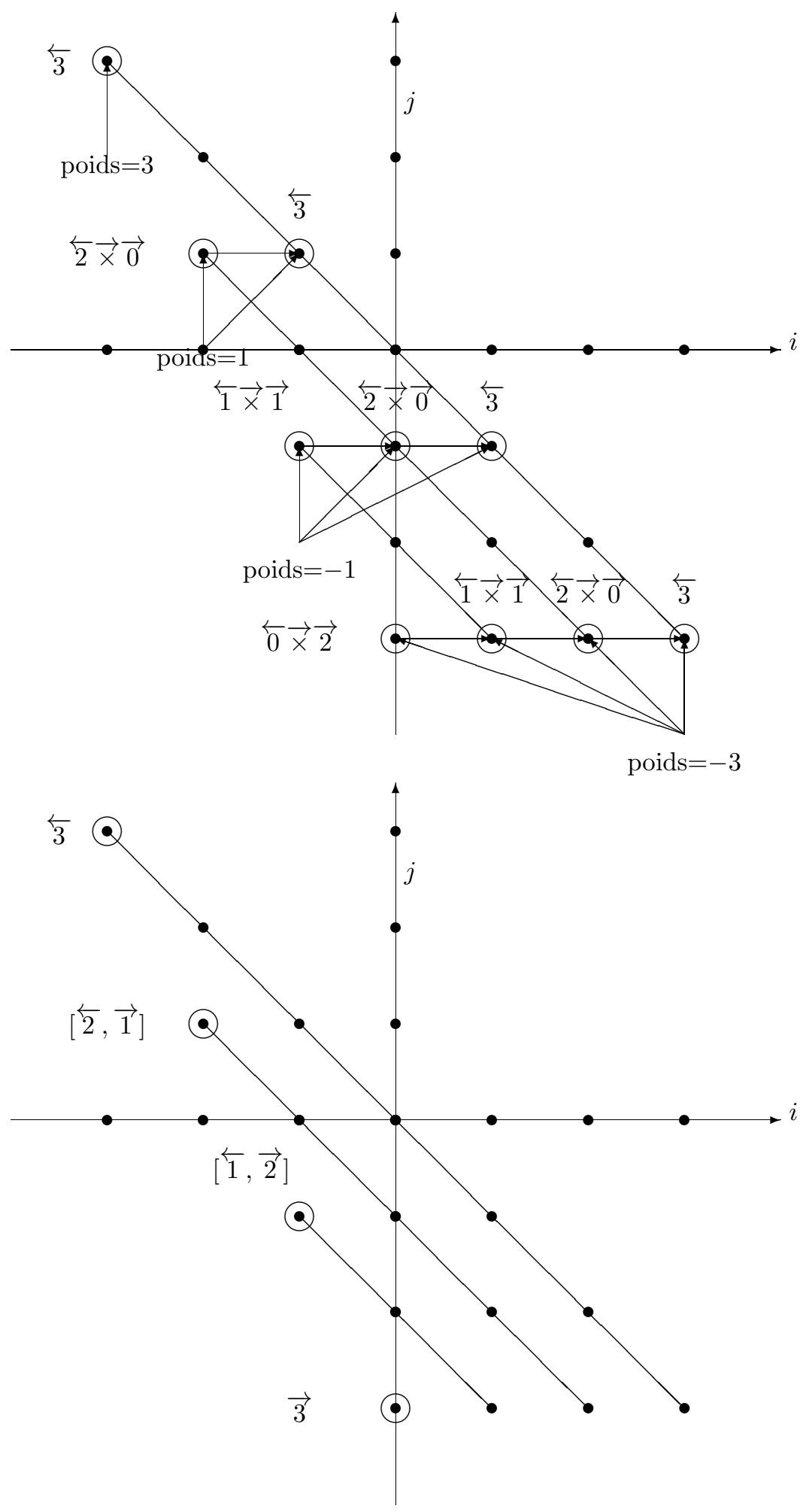

Figure 1. Termes $M L E_{\bullet}^{i, j}$ pour $s=4$ 

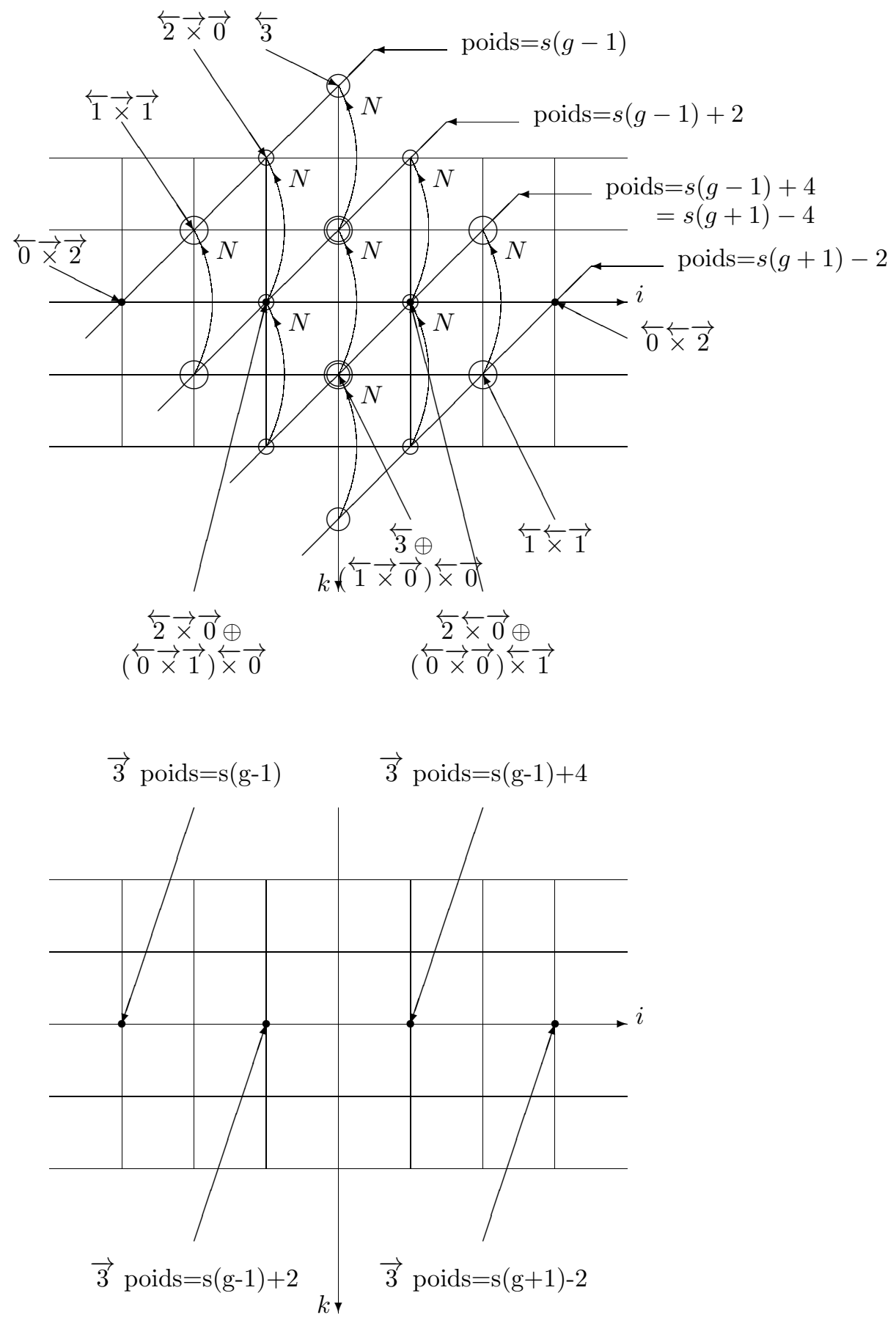

Figure 2. $H^{i}\left(g r_{k, \rho_{\infty}}\right)\left[\Pi^{\infty, o}\right]$ pour $\Pi_{\infty} \simeq \operatorname{Speh}_{4}\left(\pi_{\infty}\right)$ et $\Pi_{o} \simeq \operatorname{Speh}_{4}\left(\pi_{o}\right)$ avec $\pi_{\infty}$ (resp. $\pi_{o}$ ) une représentation cuspidale de $G L_{g}\left(F_{\infty}\right)$ (resp. $G L_{g}\left(F_{o}\right)$ ). Le dessin du bas représente le terme $E_{2}$ et donc l'aboutissement de la suite spectrale. 
Légende pour les figures (3) et (4):

- on indique les coordonnées $(p, q)$ telles que $E_{2}^{p, q}\left[\Pi^{\infty, o}\right]$ est non nulle avec $\Pi$ une représentation irréductible automorphe de $D_{\mathbb{A}}^{\times}$telle que $\Pi_{\infty} \simeq \operatorname{St}_{4}\left(\pi_{\infty}\right)\left(\operatorname{resp} . \operatorname{Speh}_{4}\left(\pi_{\infty}\right)\right)$ dans la figure 5 (resp. figure 6) pour $\pi_{\infty}$ une représentation cuspidale de $G L_{2}\left(F_{\infty}\right)$, et $\Pi_{o} \simeq \operatorname{St}_{4}\left(\pi_{o}\right)$ (resp. $\operatorname{Speh}_{4}\left(\pi_{o}\right)$ ) avec $\pi_{o}$ cuspidale de $G L_{2}\left(F_{o}\right)$. Les représentations obtenues sont des représentations elliptiques de type $\pi_{o}$. Pour chacune de ces coordonnées, on indique qu'elle est la strate qui la donne dans la suite spectrale de stratification correspondante;

- le nombre en dessous des $\overleftrightarrow{3}$ indique le poids;

- toutes les flèches indiquées sont les seules non triviales et découlent des suites exactes (IV.3.2.15);

- on note avec un cercle plus grand les coordonnées qui contribuent à l'aboutissement de la suite spectrale. 

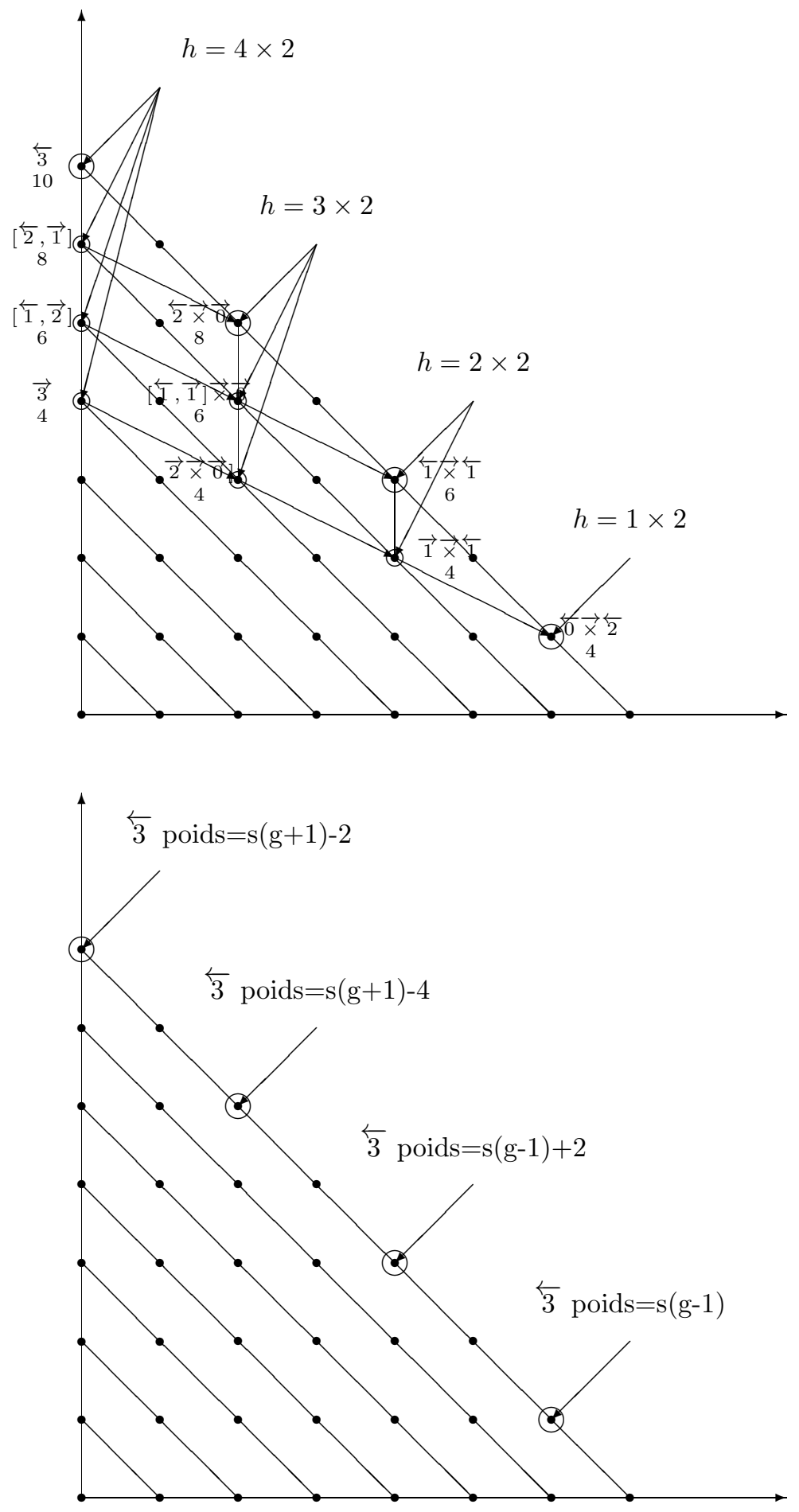

Figure 3. $s=4, g=2 ; E_{2}^{p, q}\left[\Pi^{\infty, o}\right]$ de la suite spectrale des cycles évanescents : cas $\Pi_{o}=\operatorname{St}_{4}\left(\pi_{o}\right)$. Le dessin du bas représente le terme $E_{3}$ et donc l'aboutissement de la suite spectrale. 

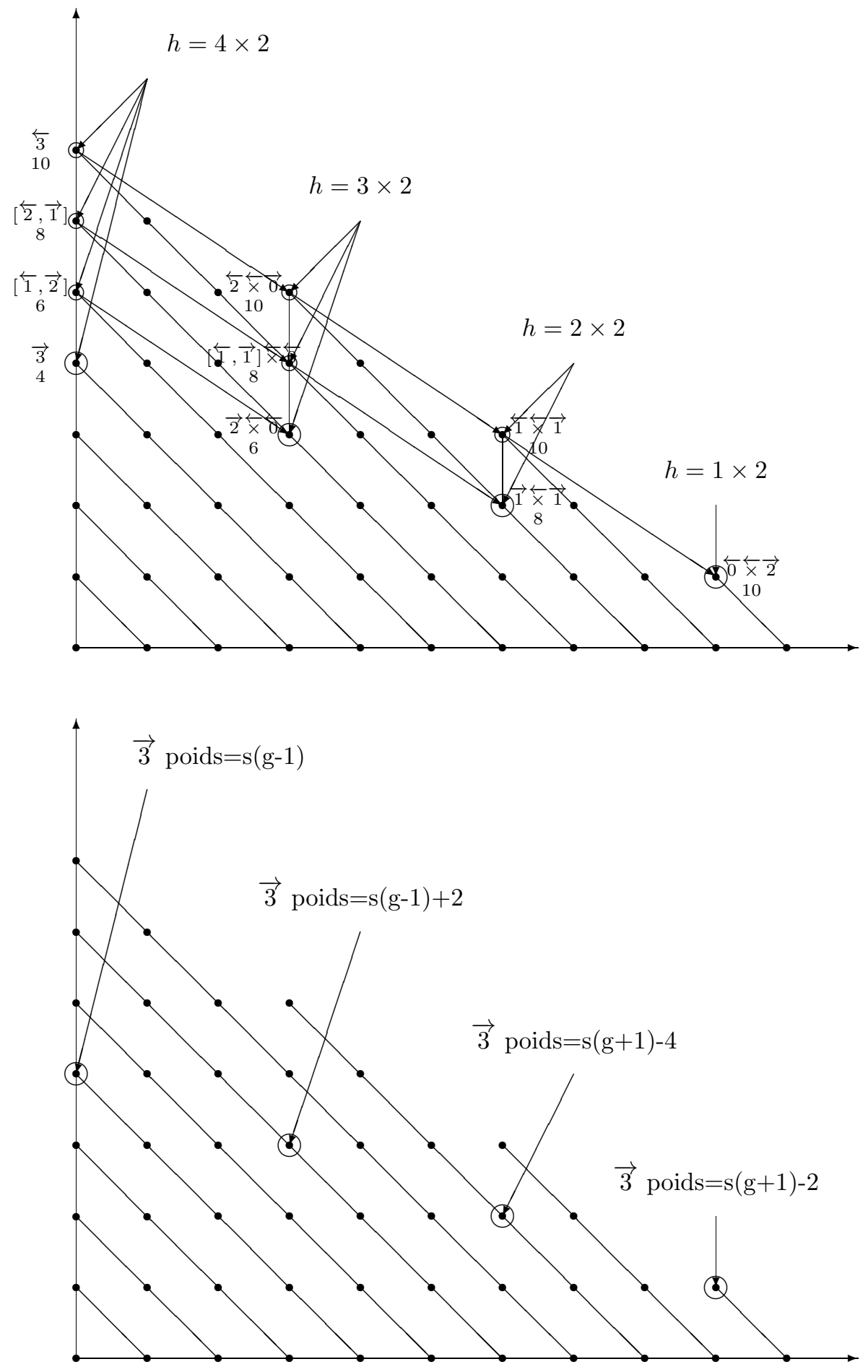

Figure 4. $s=4, g=2: E_{2}^{p, q}\left[\Pi^{\infty, o}\right]$ de la suite spectrale des cycles évanescents : cas $\Pi_{o}=\operatorname{Speh}_{s}\left(\pi_{o}\right)$. Le dessin du bas représente le terme $E_{3}$ et donc l'aboutissement de la suite spectrale. 
Légende pour les figures (5), (6) : le nombre qui accompagne les représentations indique le décalage à donner à la représentation de Galois associée; ainsi quand, dans les $E_{2}^{p, q}$ des suites

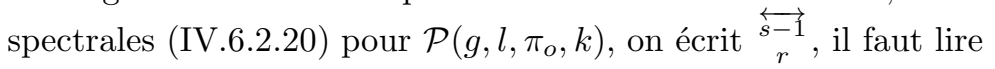

$$
j_{!}^{\geqslant l g} H T\left(g, l, \pi_{o},[\overleftrightarrow{s-1}]_{\pi_{o}}\right) \otimes L_{g}\left(\pi_{o}\right)\left(-\frac{i(g-1)+l-1-k+r}{2}\right) .
$$

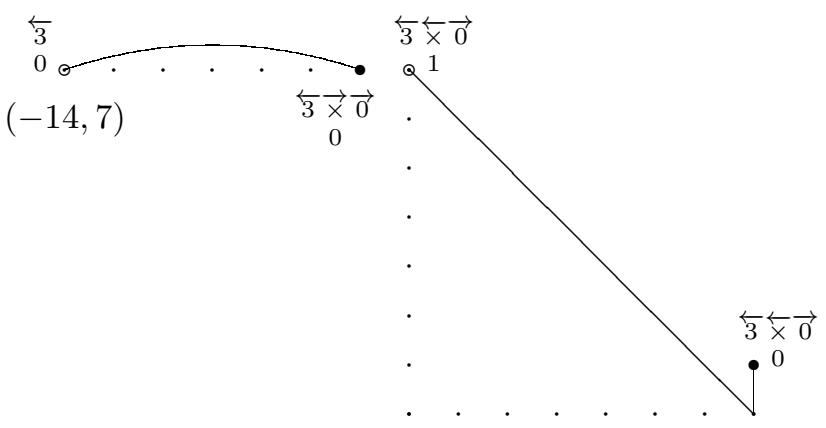

$(0,0)$

FIGURE 5. $g=7, s=5 ; E_{2}^{p, q}$ de la suite spectrale (IV.6.2.20) pour $l=4$

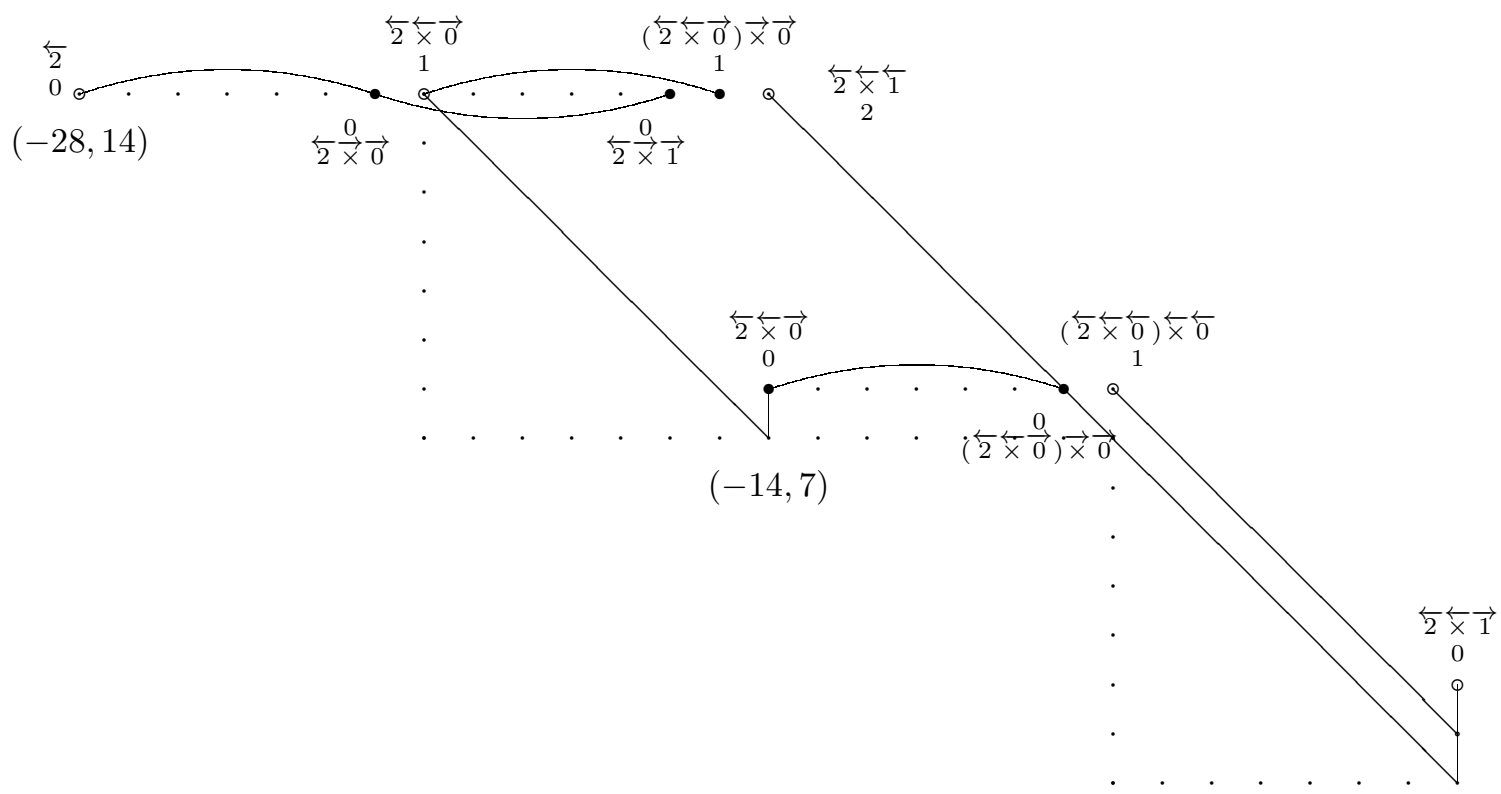

FiguRe 6. $g=7, s=5 ; E_{2}^{p, q}$ de la suite spectrale (IV.6.2.20) pour $l=3$ 



\section{BIBLIOGRAPHIE}

[1] Alexandru Ioan Badulescu. Correspondance de Jacquet-Langlands pour les corps locaux de caractéristique non nulle. Ann. Sci. École Norm. Sup. (4), 35(5) :695-747, 2002.

[2] A. A. Beilinson, J. Bernstein, and P. Deligne. Faisceaux pervers. In Analysis and topology on singular spaces, I (Luminy, 1981), volume 100 of Astérisque, pages 5-171. Soc. Math. France, Paris, 1982.

[3] V.G. Berkovich. Vanishing cycles for formal schemes. Invent. Math., 115(3) :539-571, 1994.

[4] V.G. Berkovich. Vanishing cycles for formal schemes. II. Invent. Math., 125(2) :367-390., 1996.

[5] P Boyer. Mauvaise réduction des variétés de Drinfeld et correspondance de Langlands locale. Invent. Math., 138(3):573-629, 1999.

[6] Pascal Boyer. Monodromie du faisceau pervers des cycles évanescents de quelques variétés de shimura simples et applications. Preprint, 2005.

[7] Glen E. Bredon. Sheaf theory. McGraw-Hill Book Co., New York, 1967.

[8] H. Carayol. Nonabelian Lubin-Tate theory. In Automorphic forms, Shimura varieties, and L-functions, volume 11 of Perspect. Math., pages 15-39. Academic Press, Boston, MA, 1990.

[9] V. G. Drinfel'd. Elliptic modules. Mat. Sb. (N.S.), 136(94) :594-627, 1974.

[10] Gerd Faltings. A relation between two moduli spaces studied by V. G. Drinfeld. In Algebraic number theory and algebraic geometry, volume 300 of Contemp. Math., pages 115-129. Amer. Math. Soc., Providence, RI, 2002.

[11] Laurent Fargues. Filtration de monodromie et cycles évanescents formels arxiv : math.AG/0511448

[12] Alain Genestier. Espaces symétriques de Drinfeld. Astérisque, (234) :ii+124, 1996.

[13] M. Harris. The local Langlands correspondence : Notes of (half) a course at the IHP, Spring 2000. A paraître.

[14] M. Harris, R. Taylor. The geometry and cohomology of some simple Shimura varieties, volume 151 of Annals of Mathematics Studies. Princeton University Press, Princeton, NJ, 2001.

[15] Guy Henniart. On the local Langlands conjecture for GL $(n)$ : the cyclic case. Ann. of Math. (2), 123(1):145-203, 1986.

[16] Guy Henniart. Sur la conjecture de Langlands locale pour $\mathrm{GL}_{n}$. J. Théor. Nombres Bordeaux, 13(1) :167-187, 2001. 21st Journées Arithmétiques (Rome, 2001).

[17] Luc Illusie. Autour du théorème de monodromie locale. In Périodes p-adiques, number 223 in Astérisque, 1994.

[18] N. Katz. Serre-Tate local moduli. In Algebraic surfaces (Orsay, 1976-78), volume 868 of Lecture Notes in Math., pages 138-202. Springer, Berlin, 1981. 
BIBLIOGRAPHIE

[19] G. Laumon, M. Rapoport, and U. Stuhler. D-elliptic sheaves and the Langlands correspondence. Invent. Math., 113(2) :217-338, 1993.

[20] Gérard Laumon. Cohomology of Drinfeld modular varieties. Part I, volume 41 of Cambridge Studies in Advanced Mathematics. Cambridge University Press, Cambridge, 1996. Geometry, counting of points and local harmonic analysis.

[21] P. Schneider and U. Stuhler. The cohomology of p-adic symmetric spaces. Invent. Math., 105(1) :47-122, 1991.

[22] Jean-Pierre Serre. Corps locaux. Hermann, Paris, 1968. Deuxième édition, Publications de l'Université de Nancago, No. VIII.

[23] Yoshida T. Taylor R. Compatibility of local and global langlands correspondences. Preprint, 2005.

[24] A. V. Zelevinsky. Induced representations of reductive $p$-adic groups. II. On irreducible representations of GL(n). Ann. Sci. École Norm. Sup. (4), 13(2) :165-210, 1980. 